

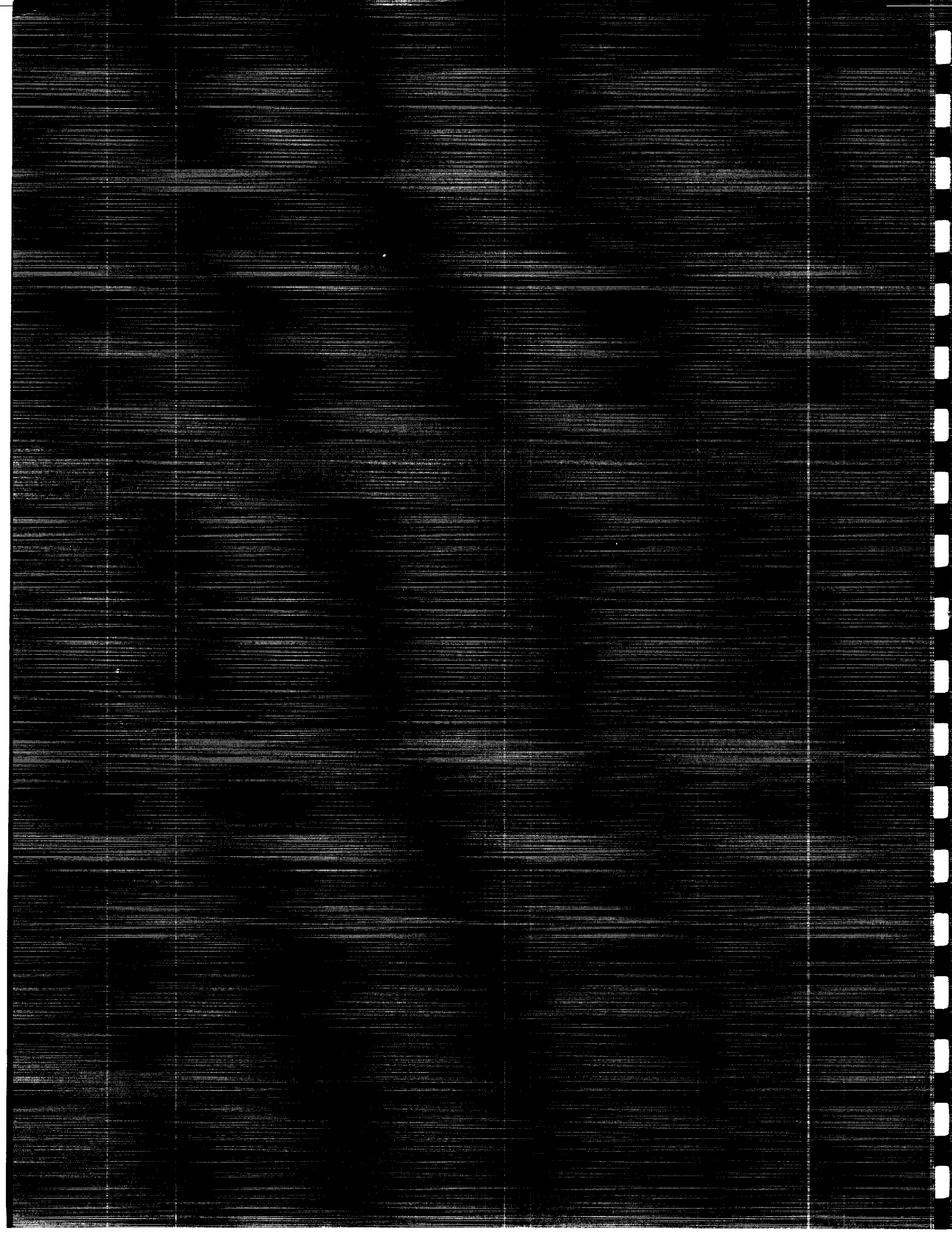




\title{
THE CHARGING OF COMPOSITES IN THE SPACE ENVIRONMENT
}

\author{
by \\ Steven A. Czepiela \\ Submitted to the Department of Aeronautics and Astronautics on August 22, 1997 in partial \\ fulfillment of the requirements for the Degree of Master of Science in Aeronautics and \\ Astronautics
}

\begin{abstract}
Deep dielectric charging and subsequent electrostatic discharge in composite materials used on spacecraft have become greater concerns since composite materials are being used more extensively as main structural components. Deep dielectric charging occurs when high energy particles penetrate and deposit themselves in the insulating material of spacecraft components. These deposited particles induce an electric field in the material, which causes the particles to move and thus changes the electric field. The electric field continues to change until a steady state is reached between the incoming particles from the space environment and the particles moving away due to the electric field. An electrostatic discharge occurs when the electric field is greater than the dielectric strength of the composite material. The goal of the current investigation is to investigate deep dielectric charging in composite materials and ascertain what modifications have to be made to the composite properties to alleviate any breakdown issues. A 1-D model was created. The model is given the space environment calculated using the Environmental Workbench software, the composite material properties, and the electric field and voltage boundary conditions. The output from the model is the charge density, electric field, and voltage distributions as functions of the depth into the material and time. Analysis using the model show that there should be no deep dielectric charging problem with conductive composites such as carbon fiber/ epoxy. With insulating materials such as glass fiber/ epoxy, Kevlar, and polymers, there is also no concern of deep dielectric charging problems with average day-to-day particle fluxes. However, problems can arise during geomagnetic substorms and solar particle events where particle flux levels increase by several orders of magnitude, and thus increase the electric field in the material by several orders of magnitude. Therefore, the second part of this investigation was an experimental attempt to measure the continuum electrical properties of a carbon fiber / epoxy composite, and to create a composite with tailorable conductivity without affecting its mechanical properties. The measurement of the conductivity and dielectric strength of carbon fiber / epoxy composites showed that these properties are surface layer dominated and difficult to measure. In the second experimental task, the conductivity of a glass fiber / epoxy composite was increased by 3 orders of magnitude, dielectric constant was increased approximately by a factor of 16 , with minimal change to the mechanical properties, by adding conductive carbon black to the epoxy.
\end{abstract}

Thesis Supervisor:

Title:
Hugh L. McManus

Associate Professor
Daniel E. Hastings

Professor 


\section{ACKNOWLEDGMENTS}

This thesis represents the culmination of the last two years of my life. However, without the help of many other people it would not have been possible. First, I would like to thank my advisors Prof. Hugh McManus and Prof. Daniel Hastings, without whom I would never have been able to complete this research. Their insight and knowledge was a great asset in helping me understand and learn about composite, the space environment, and their interaction.

I would also like to thank Paul, Mark, Carlos, and Prof. Dugundji for all their input during my TELAC presentations and literature reviews and for making sure that my work made sense and was up to par. Special thanks to Paul for instilling the fear in me which drove me to be able to give presentations effectively. Thanks to Ping for dealing with all my funding changes and calculations, of which there were several, and for taking care of all my requisitions I needed to order the materials required for my research. A special thanks to Deb, who was always there to set up meeting times with Hugh and to talk to while I spent days waiting on the 'Blue Chair'.

Two people who deserve a lot of thanks is Al Supple and John Kane, without whom no experimental research could be accomplished in TELAC. They were always more than willing to help explain how the lab equipment works and what the exact experimental procedures that need to be followed are. I would also like to thank Lenny

Rigione for letting me use and teaching me how to use a variety of material science 
equipment I used during my research. The same goes for Aaron Bent, who helped me with the experimental electrical work of my research and showed me how to use the lab equipment in AMSL. Thanks to my two UROPers Thad and Barbara, who never complained about any of the menial work I made them do, I hope you also learned a thing or two along the way.

As with any where you go, what defines the experience are the friends you make. Huge thanks go to guys I hung out with, whether it was just going out to eat, drinking, watching movies, or playing video games, Chris and the other guy (who wishes to remain anonymous) were always there having fun. Special thanks to Sharath, who was always around to show you how to get the most out of a night drinking; and to Bari, who taught me that a great way to end a day is with a good drink and a fine cigar. I would also like to thank Lauren for being around to talk to; Hari who learned on the way to VPI that to get any where you have to drive with the accelerator to the floor and change lanes in a blink of an eye, it sure was one hell of a drive. Thanks also to Tom, Dennis, and Kaustubh who tried unsuccessfully to master the art of managing the TELAC soda; to the SSL softball team, 'Go Chico's !'; to the MIT hockey team, the locker room talk was always refreshing and a great change of pace, maybe next year you'll win the championship; and to Coach Bill, who was always encouraging you to do your best, no matter what it was. I would also like to thank the rest of the grad students in TELAC, SSL, and AMSL for a great time. 
Finally I want to thank Kelly for always being available to talk to, and my Parents who have always been supportive of what I'm doing, thanks for giving me the support and encouragement to succeed. I couldn't have done it without you. 


\section{FOREWORD}

This work was conducted in the Technology Laboratory for Advanced Composites (TELAC) and the Space Systems Laboratory (SSL) in the Department of Aeronautics and Astronautics at the Massachusetts Institute of Technology. This work was sponsored by the NASA Lewis Research Center under NASA Contract NAS327550. 


\section{TABLE OF CONTENTS}

ABSTRACT

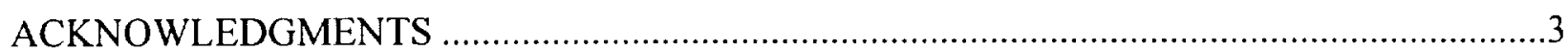

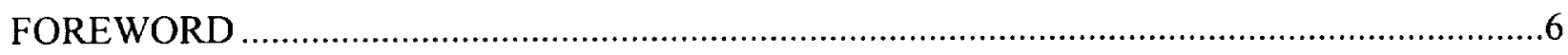

TABLE OF CONTENTS

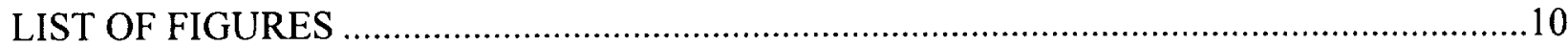

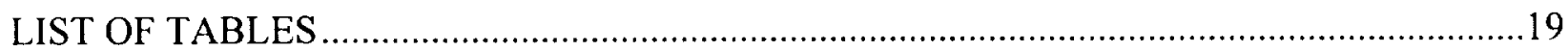

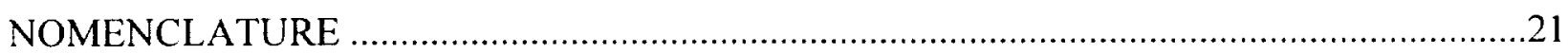

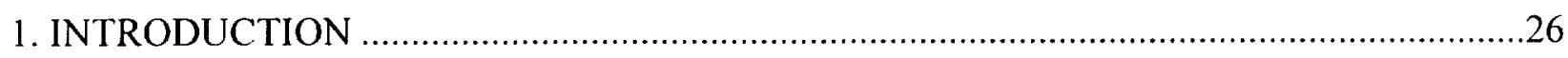

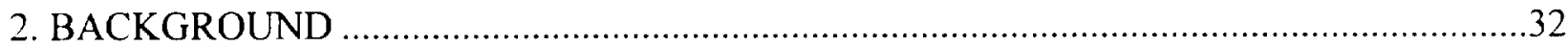

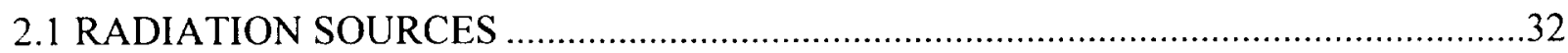

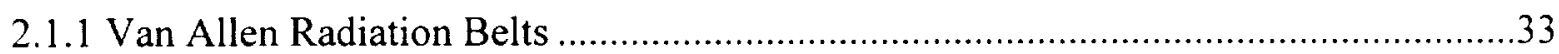

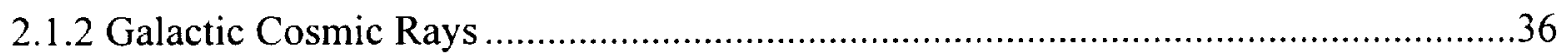

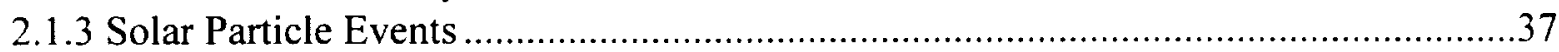

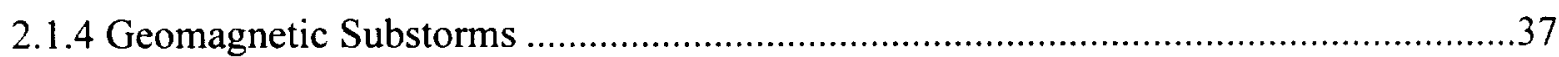

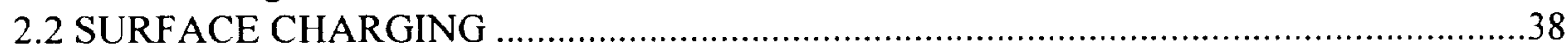

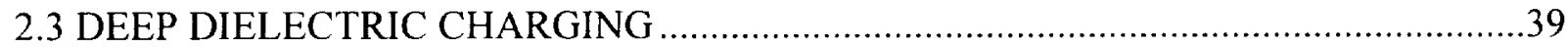

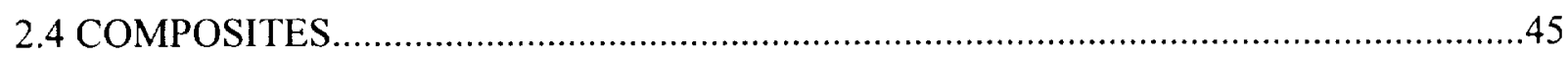

3. PROBLEM STATEMENT AND APPROACH

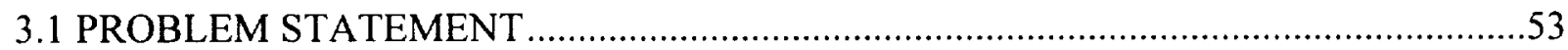

3.2 APPROACH

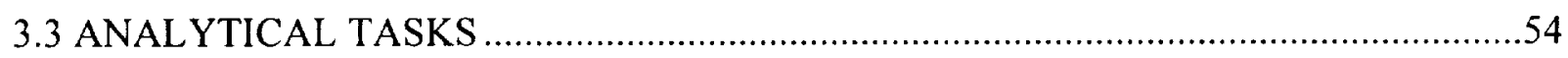

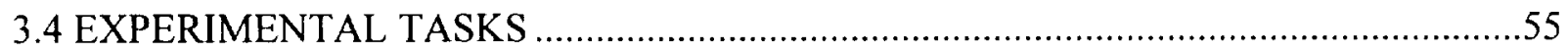

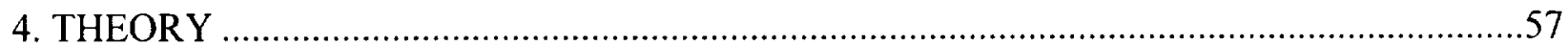

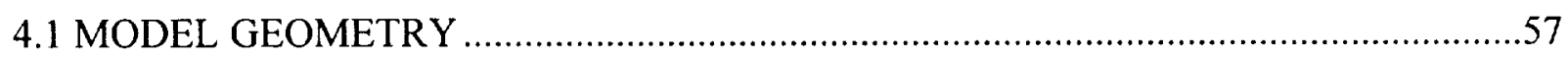

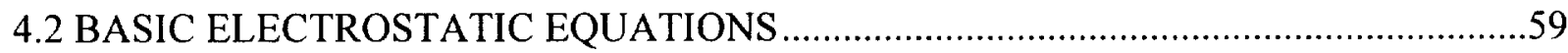

4.2.1 Conductivity In High Electric Fields ....................................................................61

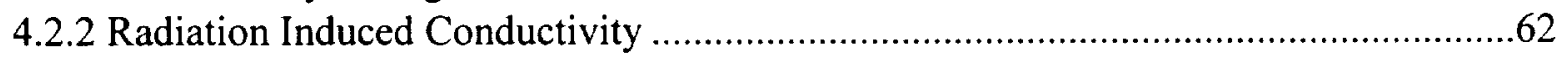

4.2.3 Delayed Conductivity ..........................................................................................63

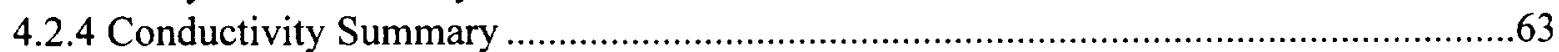

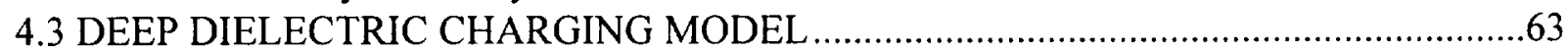

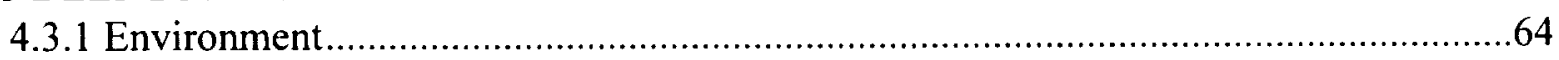

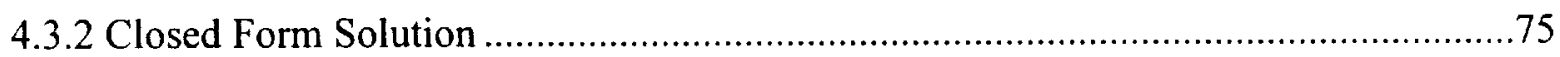

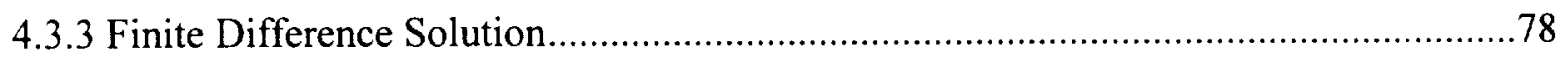

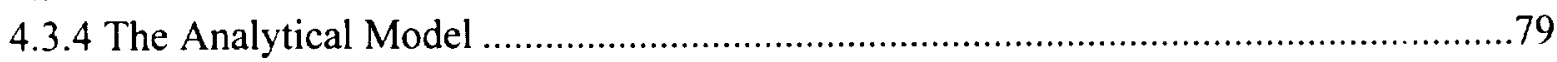




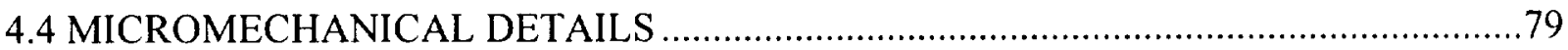

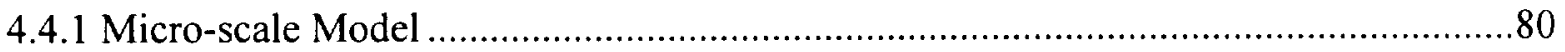

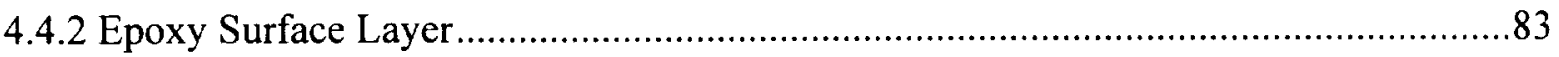

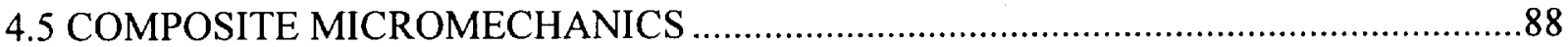

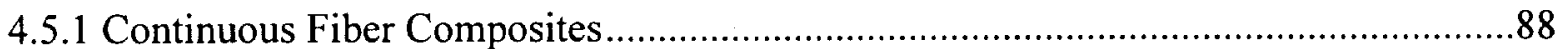

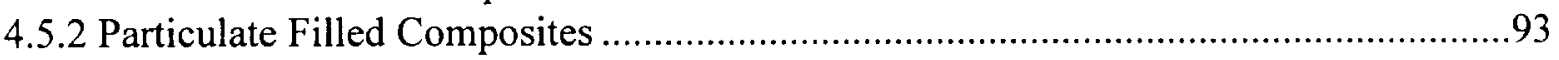

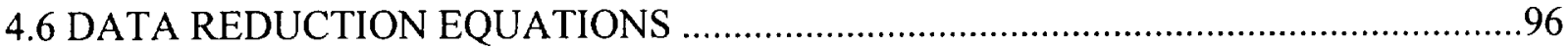

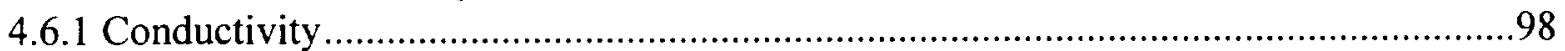

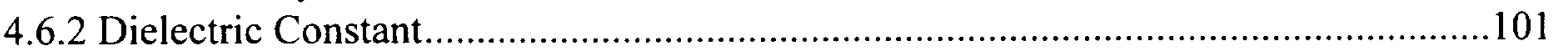

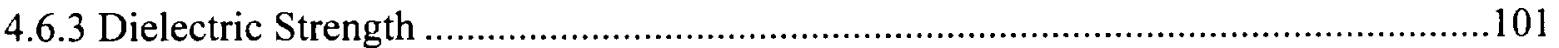

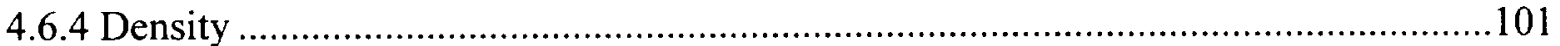

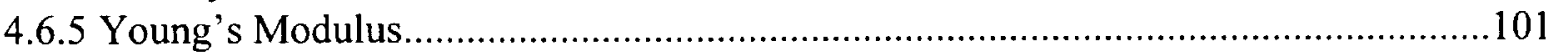

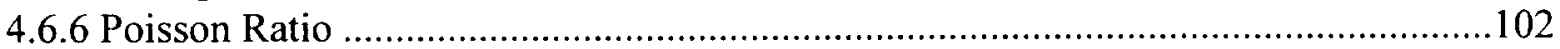

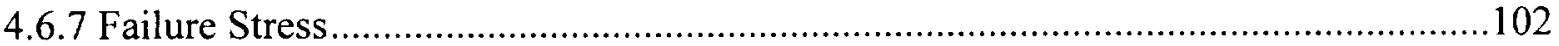

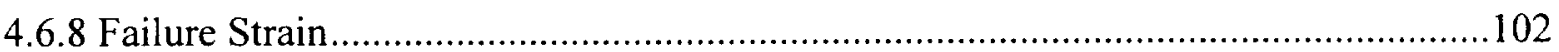

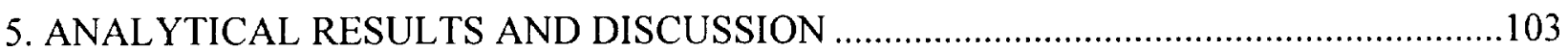

5.1 DEEP DIELECTRIC CHARGING MODEL ………...................................................103

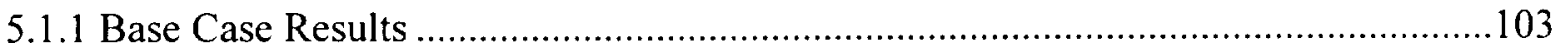

5.1.2 Base Case With High Field Conductivity ..................................................................112

5.1.3 Base Case with Radiation Induced Conductivity ……...............................................117

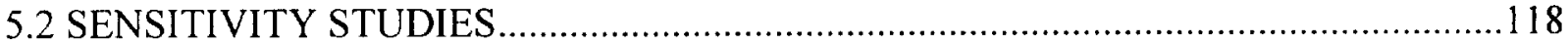

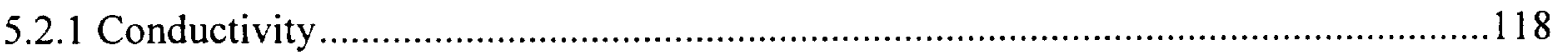

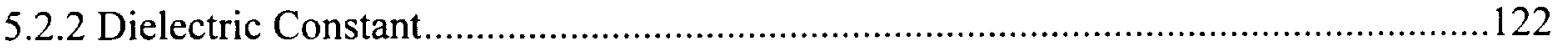

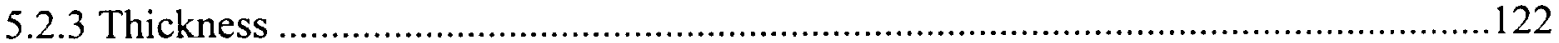

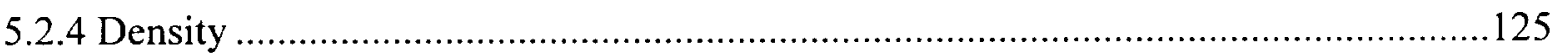

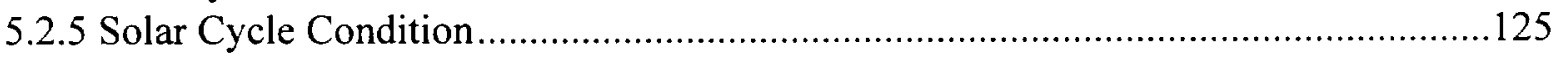

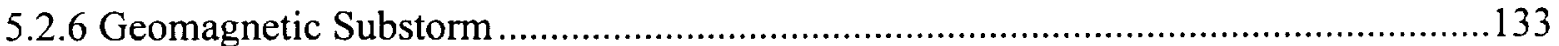

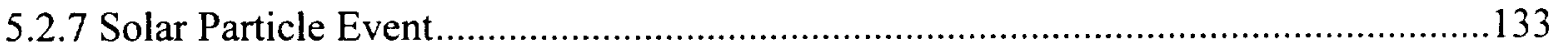

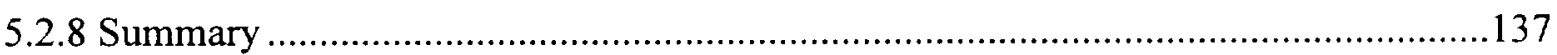

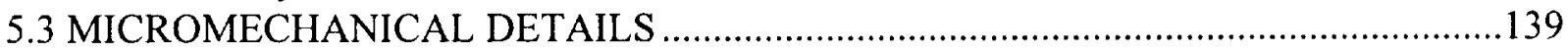

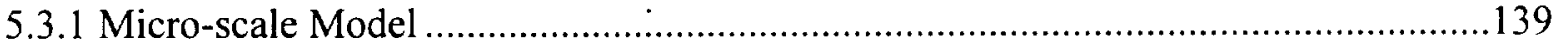

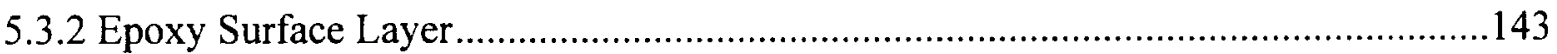

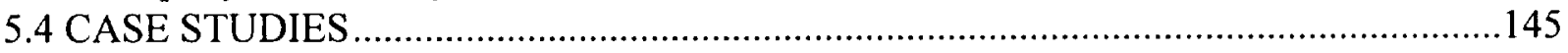

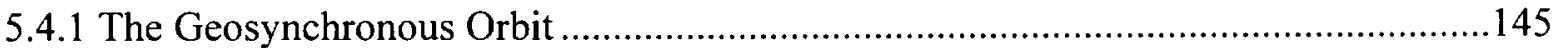

5.4.2 Global Positioning System Orbit …………….....................................................145

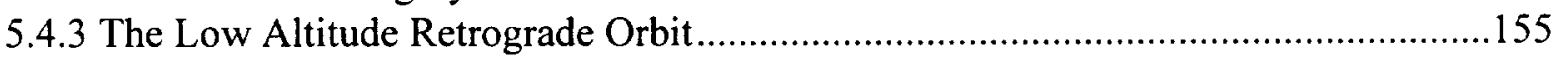

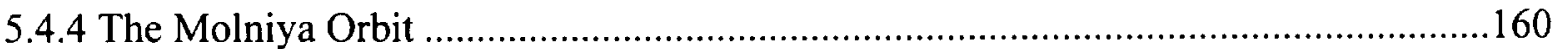

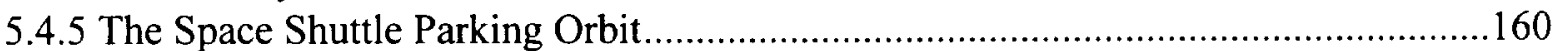

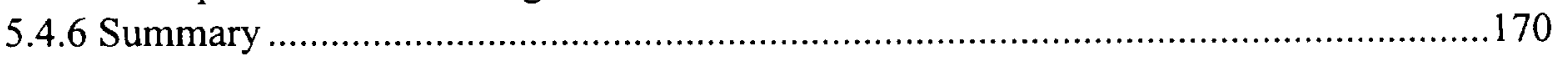

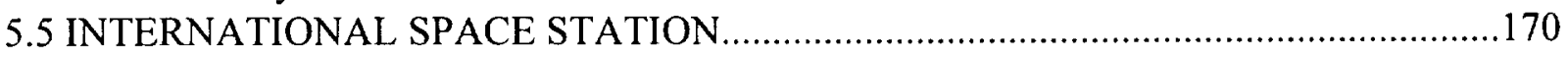

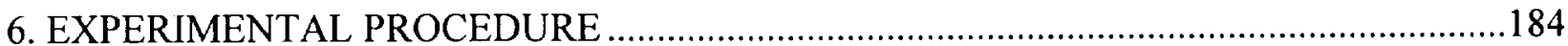

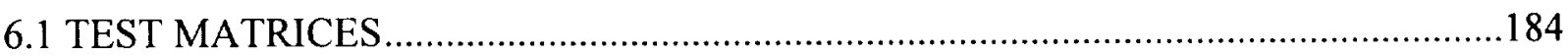

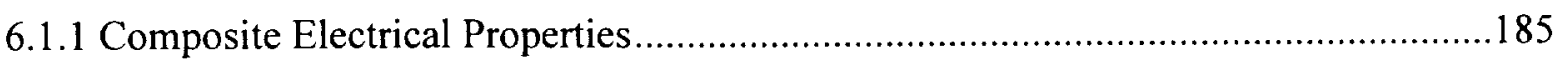




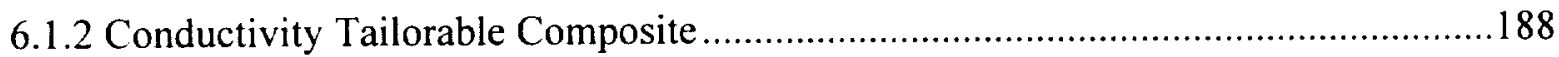

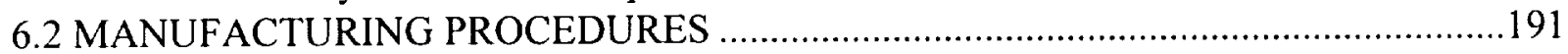

6.2.1 Carbon Fiber / Epoxy Prepreg Layup ................................................................191

6.2.2 Glass Fiber / Epoxy Wet Layup .............................................................................193

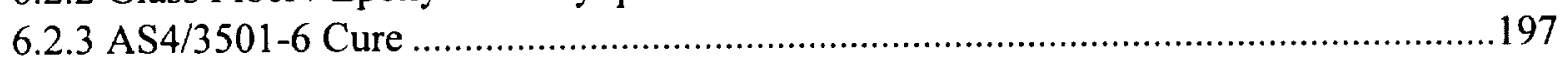

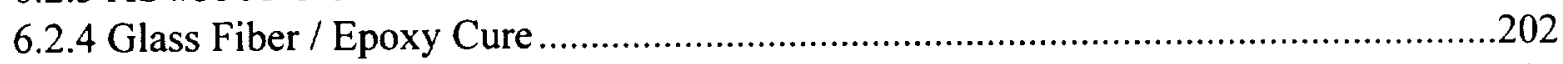

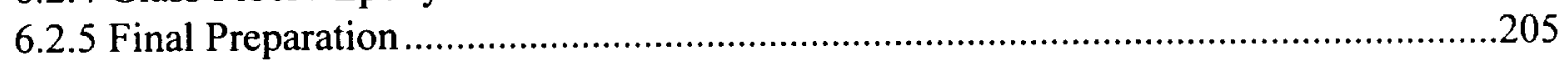

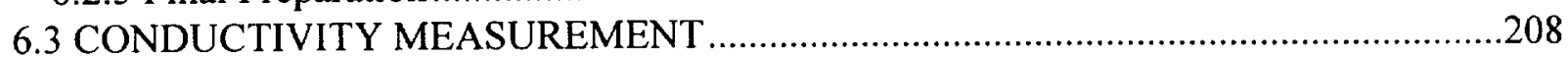

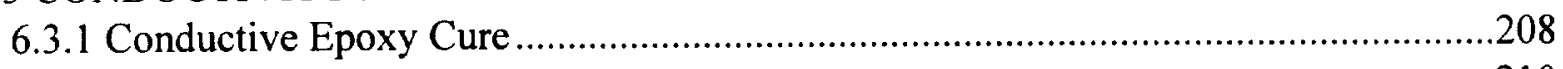

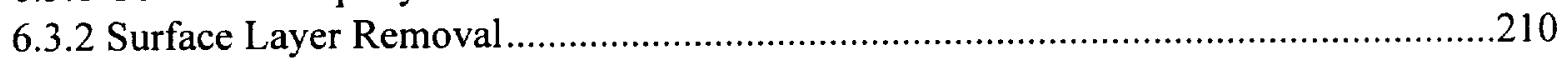

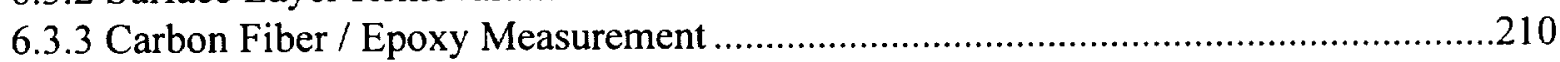

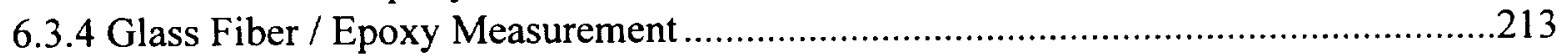

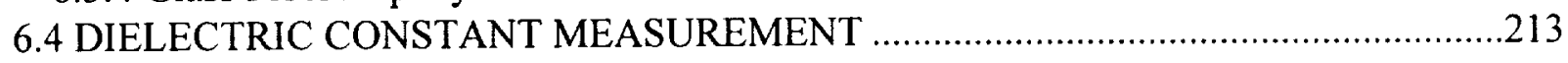

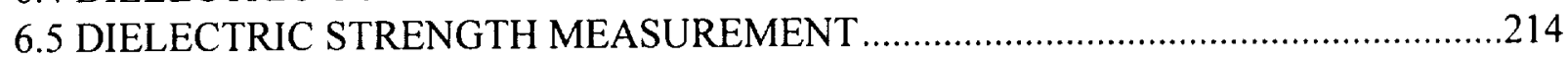

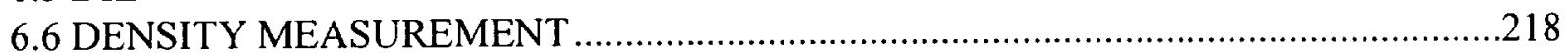

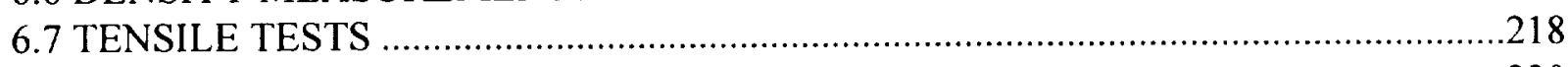

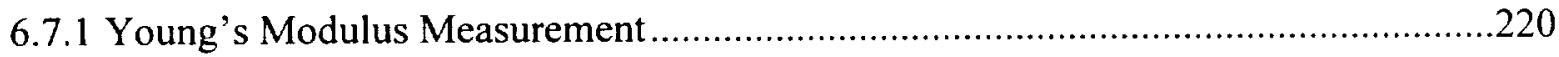

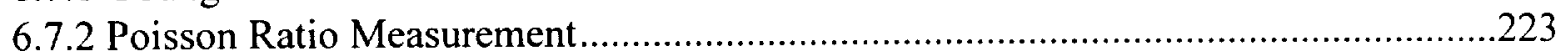

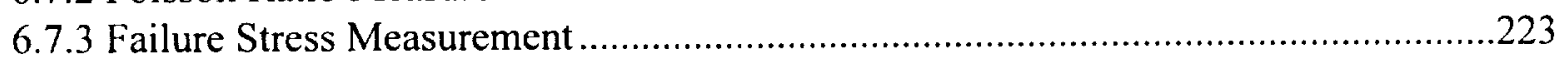

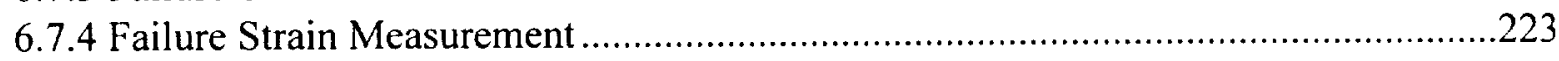

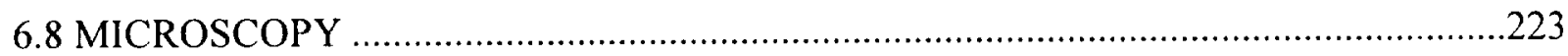

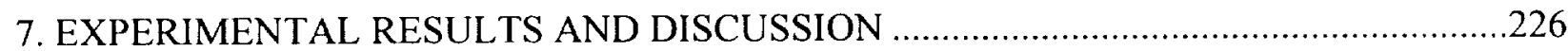

7.1 CARBON FIBER / EPOXY ELECTRICAL PROPERTIES ……..................................226

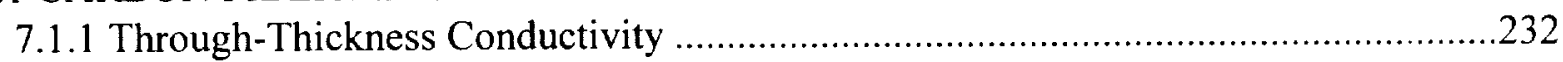

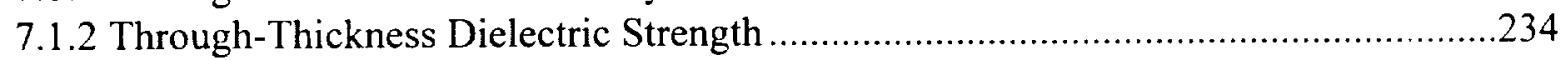

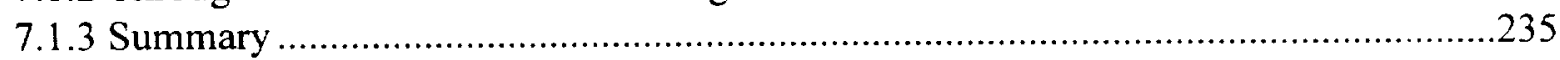

7.2 CONDUCTIVITY TAILORABLE GLASS FIBER / EPOXY ……................................236

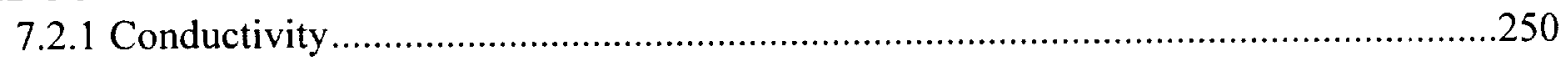

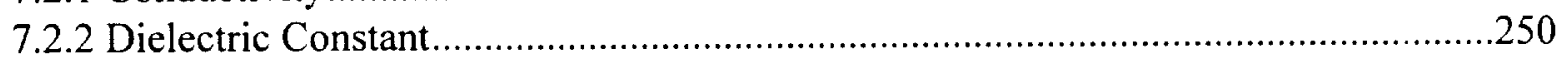

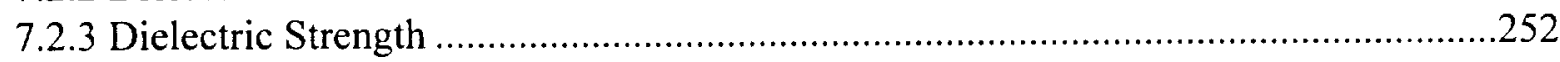

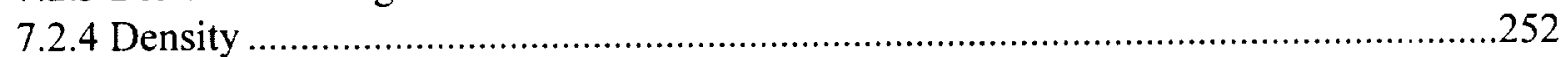

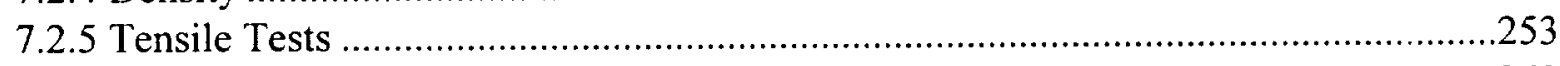

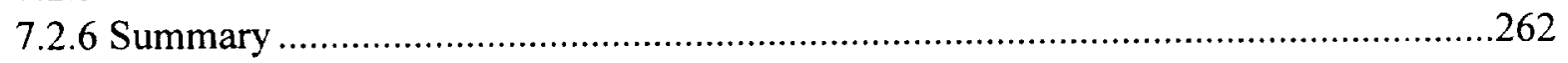

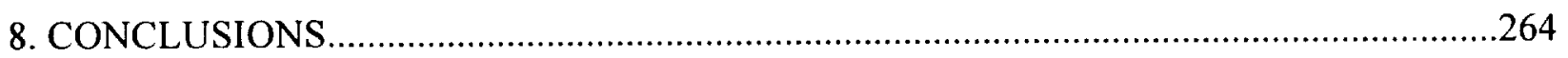

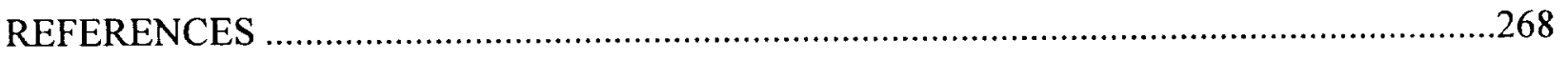

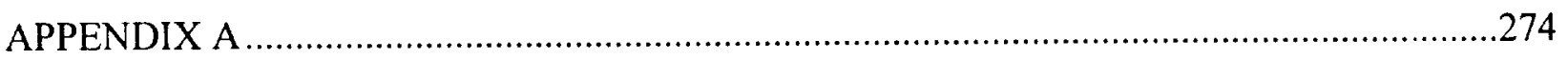

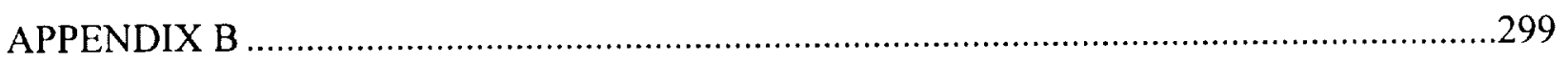

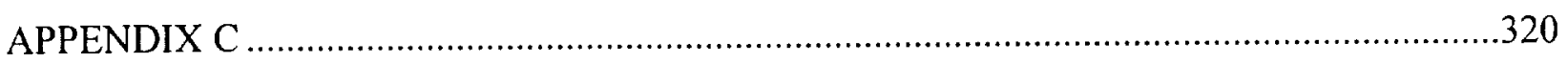




\section{LIST OF FIGURES}

Figure 1.1: Schematic of the deep dielectric charging model ................................28

Figure 2.1: $\quad$ Observed and one-year-ahead predicted sunspot numbers ......................34

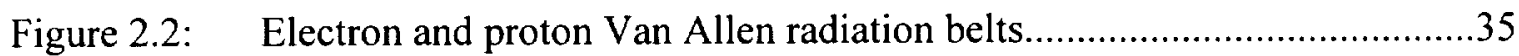

Figure 2.3: A local-time plot of the occurrence of anomalies on various spacecraft ..............................................................................4 40

Figure 2.4: Typical composite in the geometric coordinate system ........................47

Figure 2.5: Typical ply in the ply coordinate system ......................................48

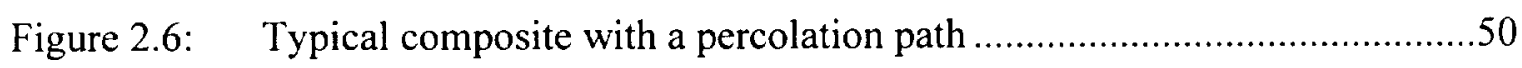

Figure 2.7: Typical composite with matrix rich surface layer...............................51

Figure 4.1: Geometry used in the CoDDCA model .........................................58

Figure 4.2: Stopping powers in silicon for various particles................................66

Figure 4.3: $\quad$ Electron and proton fluxes as a function of energy .............................67

Figure 4.4: Electron and proton fluxes as a function of depth ..............................68

Figure 4.5: Electron and proton LET as a function of energy ..............................69

Figure 4.6: Electron and proton penetration depth as a function of energy ................70

Figure 4.7: Electron and proton charge density rates as a function of depth..............71

Figure 4.8: Representation of how the particle per area data is converted into particle per volume data ............................................................... 73

Figure 4.9: Incoming charge density rate as a function of depth ............................74

Figure 4.10: Schematic of the fiber-matrix micro-scale model ...............................81

Figure 4.11: Schematic of the pure epoxy surface layer ......................................84

Figure 4.12: Schematic of the epoxy surface layer model .....................................86 
Figure 4.13: Various conductivity micromechanical models .................................94

Figure 4.14: Critical fiber volume fraction in conductive composites .......................95

Figure 4.15: Geometry of the electrical test sample ........................................97

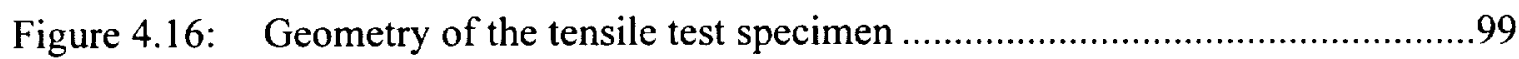

Figure 4.17: RC circuit model of high resistance conductivity samples ....................100

Figure 5.1: Closed form base case charge density..............................................106

Figure 5.2: Closed form solution base case electric field...................................107

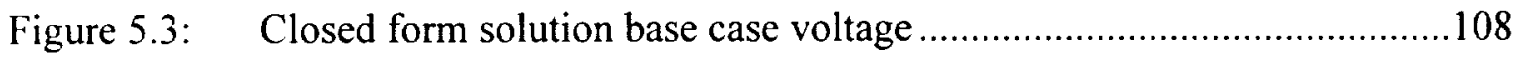

Figure 5.4: Finite difference solution base case charge density ...........................109

Figure 5.5: $\quad$ Finite difference solution base case electric field ............................110

Figure 5.6: Finite difference solution base case voltage .................................111

Figure 5.7: High field conductivity scaling factor.......................................113

Figure 5.8: High field conductivity scaling factor dielectric strength sensitivity ......115

Figure 5.9: High field conductivity scaling factor temperature sensitivity ...............116

Figure 5.10: Radiation induced conductivity exponent sensitivity ...........................119

Figure 5.11: Coefficient of radiation induced conductivity sensitivity .....................120

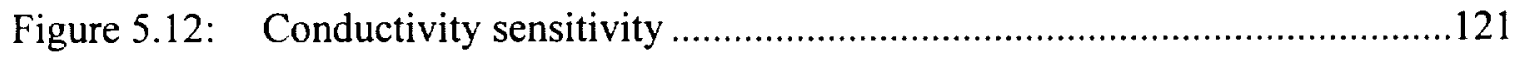

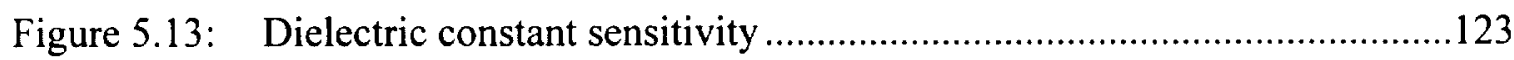

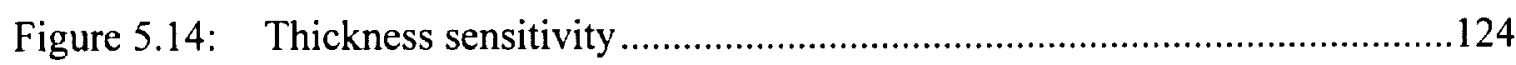

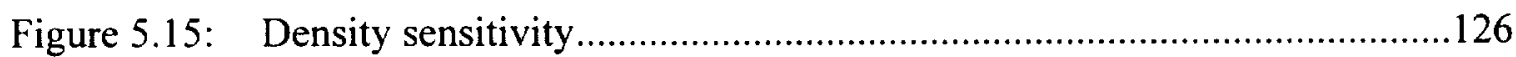

Figure 5.16: Electron and proton penetration depth density sensitivity ....................127

Figure 5.17: Steady-state electric field for various densities................................128 


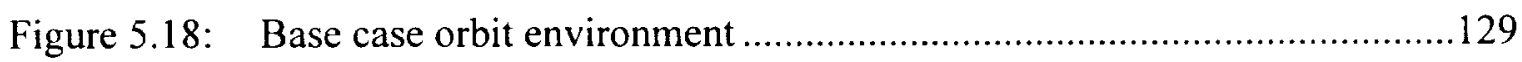

Figure 5.19: Solar condition charge density sensitivity ............................................130

Figure 5.20: Solar condition electric field sensitivity .............................................131

Figure 5.21: Solar condition voltage sensitivity .....................................................132

Figure 5.22: Geomagnetic substorm sensitivity ....................................................134

Figure 5.23: Electric field of base case with a substorm intensity of $1000 \ldots \ldots \ldots \ldots \ldots \ldots . .135$

Figure 5.24: Solar particle event sensitivity .........................................................136

Figure 5.25: Electric field of base case with a solar particle event intensity of

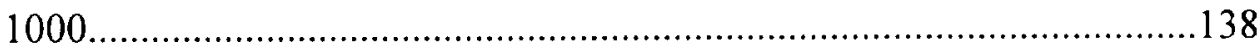

Figure 5.26: Electric field magnification around a fiber ............................................140

Figure 5.27: Schematic of an unlikely fiber arrangement which can lead to high field magnification ............................................................................. 142

Figure 5.28: Geosynchronous orbit environment ..................................................147

Figure 5.29: Geosynchronous orbit charge density distribution .................................148

Figure 5.30: Geosynchronous orbit electric field distribution....................................149

Figure 5.31: Geosynchronous orbit voltage distribution ..........................................150

Figure 5.32: Global Positioning System orbit environment ......................................151

Figure 5.33: Global Positioning System orbit charge density distribution ..................152

Figure 5.34: Global Positioning System orbit electric field distribution .....................153

Figure 5.35: Global Positioning System orbit voltage distribution...............................154

Figure 5.36: Low altitude retrograde orbit environment ..........................................156

Figure 5.37: Low altitude retrograde orbit charge density distribution........................157

Figure 5.38: Low altitude retrograde orbit electric field distribution..........................158 
Figure 5.39: Low altitude retrograde orbit voltage distribution

Figure 5.40: Molniya orbit environment .161

Figure 5.41: Molniya orbit charge density distribution. .162

Figure 5.42: Molniya orbit electric field distribution 163

Figure 5.43: Molniya orbit voltage distribution .164

Figure 5.44: Space shuttle parking orbit environment .165

Figure 5.45: Space shuttle parking orbit charge density distribution.

Figure 5.46: Space shuttle parking orbit electric field distribution .168

Figure 5.47: Space shuttle parking orbit voltage distribution .169

Figure 5.48: Space station orbit environment. .174

Figure 5.49: Space station Test Case No. 1 charge density distribution .175

Figure 5.50: $\quad$ Space station Test Case No. 1 electric field distribution 176

Figure 5.51: Space station Test Case No. 1 voltage distribution. .177

Figure 5.52: Space station charge density distribution. .179

Figure 5.53: Space station electric field distribution .180

Figure 5.54: Space station power off electric field distribution..... 182

Figure 5.55: Space station power off voltage distribution .183

Figure 6.1: Measurement locations for electrical property samples. 187

Figure 6.2: Measurement locations for tensile test specimen. 190

Figure 6.3: Schematic of AS4/3501-6 cure layup .194

Figure 6.4: Schematic of glass fiber / epoxy cure layup 198

Figure 6.5: Typical cure plate with bleeder paper rolls around laminate vessels.......199 
Figure 6.6: TELAC's Baron Blackshee autoclave .200

Figure 6.7: Illustration of the cure plate used for the AS4/3501-6 cure (top view)

Figure 6.8: AS4/3501-6 cure cycle .203

Figure 6.9: Illustration of the cure plate used for the Glass Fiber / Epoxy cure (top view) .204

Figure 6.10: Glass fiber / epoxy cure cycle .206

Figure 6.11: Schematic of the cutting patterns for the AS4/3501-6 laminates. 207

Figure 6.12: Schematic of the cutting patterns for the glass fiber / epoxy laminates... .209

Figure 6.13: Schematic of the conductivity sample holder 211

Figure 6.14: Experimental setup used for the conductivity measurements 212

Figure 6.15: Experimental setup used to measure the dielectric constant 215

Figure 6.16: Experimental setup used to measure the dielectric strength 216

Figure 6.17: Schematic of the dielectric strength sample holder

Figure 6.18: Experimental setup used to perform tensile tests .219

Figure 6.19: Illustration of the location of the strain gages on the tensile test sample .221

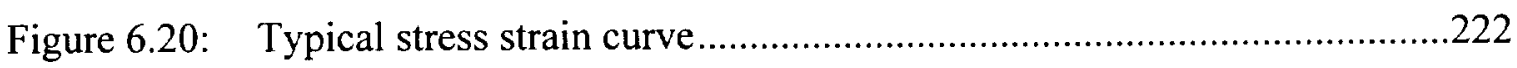

Figure 6.21: Typical transverse versus longitudinal strain curve .............................224

Figure 7.1: Unsanded carbon fiber / epoxy resistance data ................................227

Figure 7.2: Sanded carbon fiber / epoxy resistance data .....................................228

Figure 7.3: Unsanded carbon fiber / epoxy breakdown voltage data ......................229

Figure 7.4: Glass fiber/ epoxy conductivity .237 
Figure 7.5: Glass fiber / epoxy dielectric constant …….......................................238

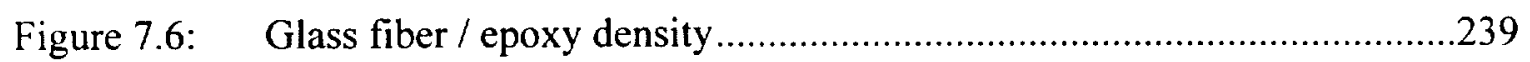

Figure 7.7: Glass fiber / epoxy Young's modulus....................................................240

Figure 7.8: Glass fiber / epoxy Poisson ratio .........................................................241

Figure 7.9: Glass fiber / epoxy failure stress ......................................................242

Figure 7.10: Glass fiber / epoxy failure strain .........................................................243

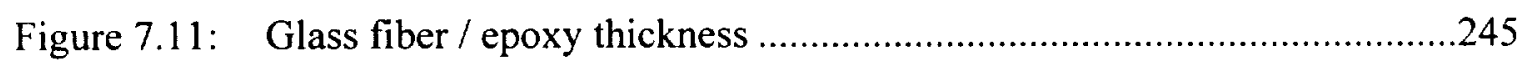

Figure 7.12: Glass fiber / epoxy surface layer thickness..........................................246

Figure 7.13: Micro-graphs of the surface layer of the conductivity-tailorable glass fiber / epoxy composite.................................................................248

Figure 7.14: Glass fiber / epoxy laminates ............................................................249

Figure 7.15: Schematic of unusual percolation paths which can lead to high

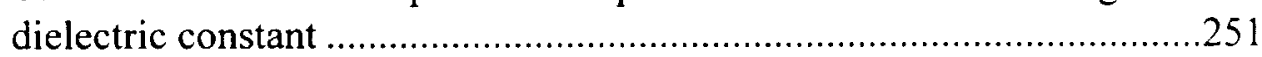

Figure 7.16: Broken $[0]_{4}$ glass fiber / epoxy tensile specimens …...........................255

Figure 7.17: Broken $0 \%$ carbon black tensile specimens ........................................256

Figure 7.18: Broken 5\% carbon black tensile specimens ........................................257

Figure 7.19: Broken $10 \%$ carbon black tensile specimens .......................................258

Figure 7.20: Broken 15\% carbon black tensile specimens .......................................259

Figure 7.21: Broken 20\% carbon black tensile specimens.......................................260

Figure B.1: High field conductivity charge density comparison with base case .........300

Figure B.2: High field conductivity electric field comparison with base case ...........301

Figure B.3: High field conductivity voltage comparison with base case....................302

Figure B.4: Radiation induced conductivity charge density comparison with

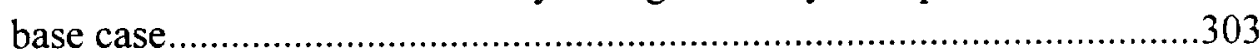


Figure B.5: Radiation induced conductivity electric field comparison with base case

Figure B.6: Radiation induced conductivity voltage comparison with base case ........305

Figure B.7: Electric field of base case with thickness of $0.01 \mathrm{~mm} \ldots \ldots \ldots \ldots \ldots \ldots \ldots \ldots \ldots \ldots . . .306$

Figure B.8: Electric field of base case with thickness of $0.1 \mathrm{~mm}$................................307

Figure B.9: Electric field of base case with thickness of $1 \mathrm{~mm}$.................................308

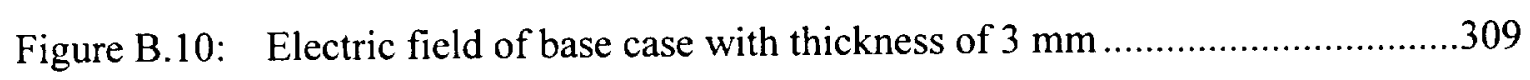

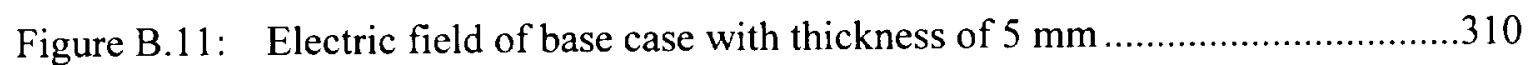

Figure B.12: Electric field of base case with thickness of $10 \mathrm{~mm} \ldots \ldots \ldots \ldots \ldots \ldots \ldots \ldots \ldots \ldots . . .311$

Figure B.13: Electric field of base case with a substorm intensity of $5 \ldots \ldots \ldots \ldots \ldots \ldots \ldots \ldots . . .312$

Figure B.14: Electric field of base case with a substorm intensity of $10 \ldots \ldots \ldots \ldots \ldots \ldots \ldots . . . .313$

Figure B.15: Electric field of base case with a substorm intensity of $100 \ldots \ldots \ldots \ldots \ldots \ldots . . .314$

Figure B.16: Electric field of base case with a substorm intensity of $1000 \ldots \ldots \ldots \ldots \ldots \ldots . . .315$

Figure B.17: Electric field of base case with a solar particle event intensity of 5 ........316

Figure B.18: Electric field of base case with a solar particle event intensity of $10 \ldots . . .317$

Figure B.19: Electric field of base case with a solar particle intensity of $100 \ldots \ldots \ldots \ldots . . .318$

Figure B.20: Electric field of base case with a solar particle event intensity of 1000 .

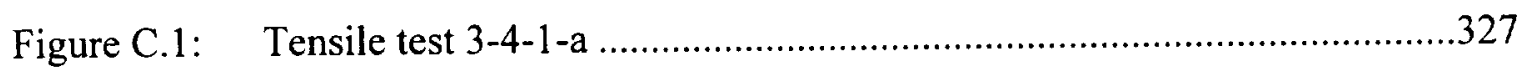

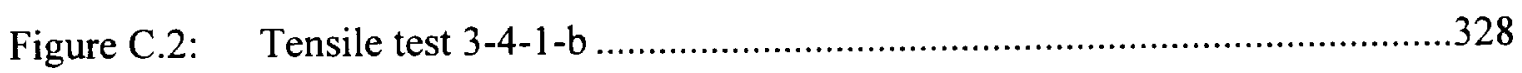

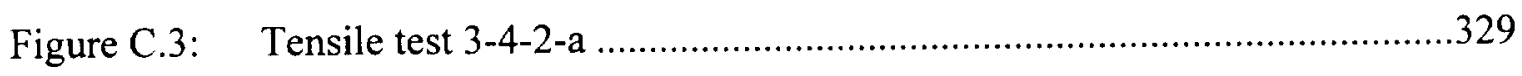

Figure C.4: Tensile test 3-4-2-b .........................................................................330

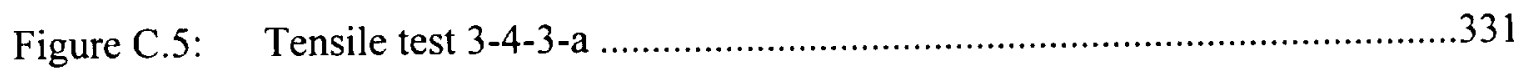




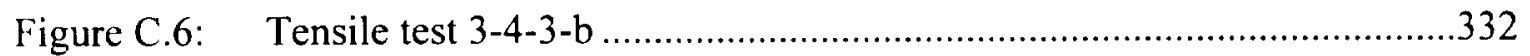

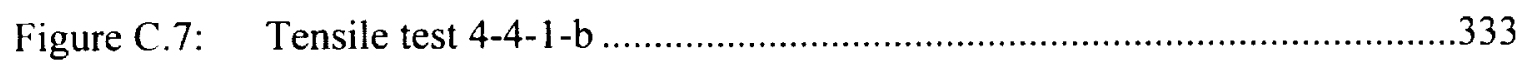

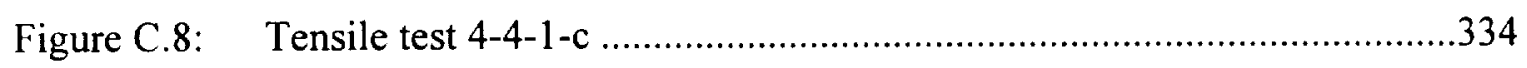

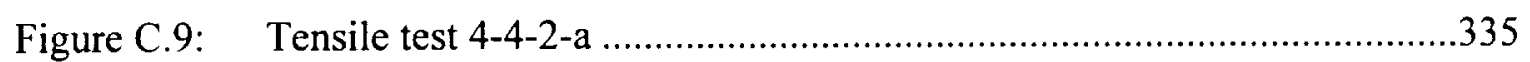

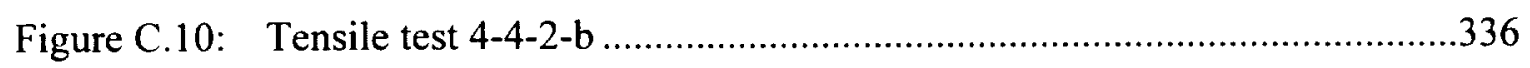

Figure C.11: Tensile test 4-4-3-a _.........................................................................337

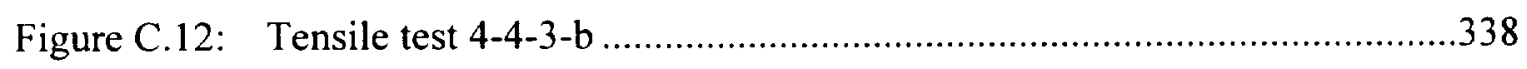

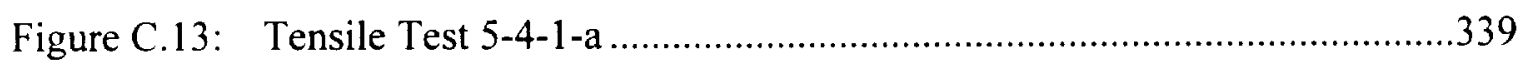

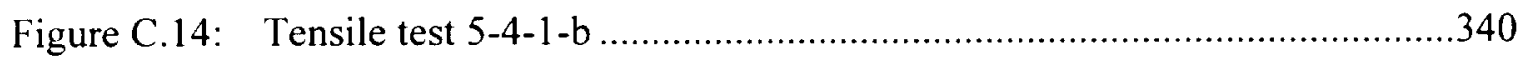

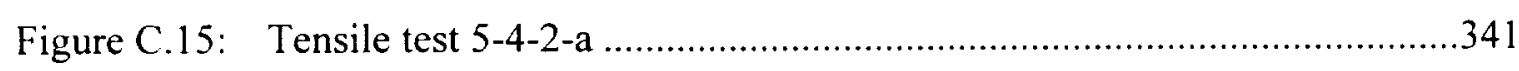

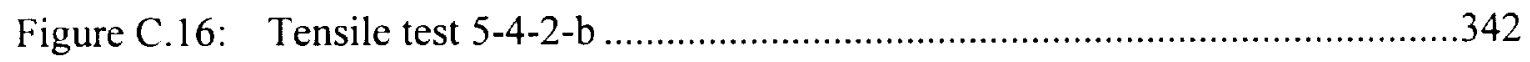

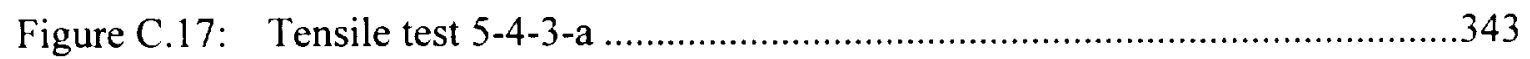

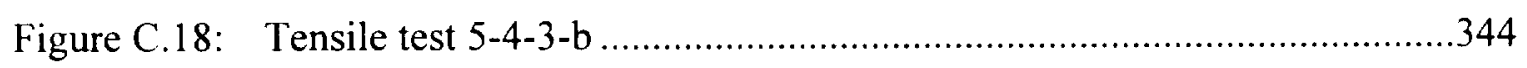

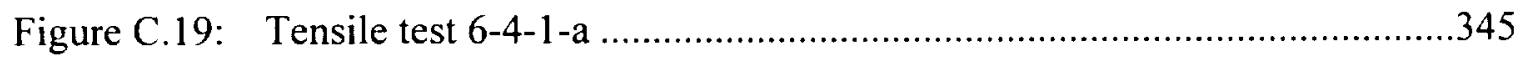

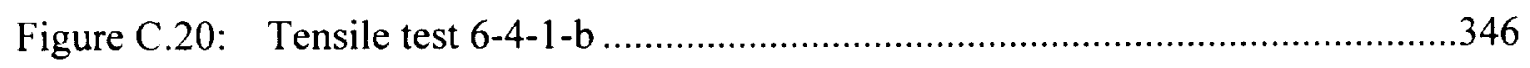

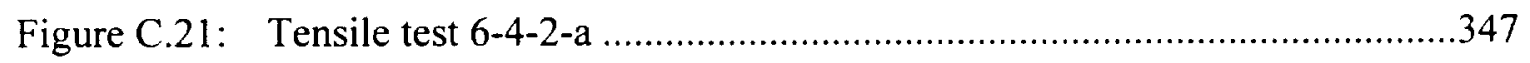

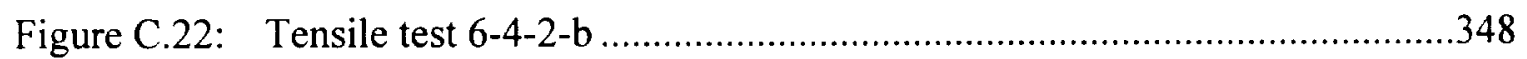

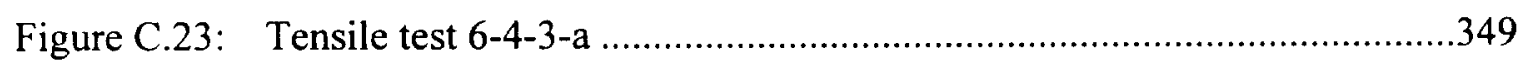

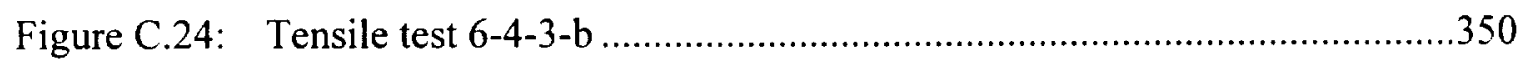

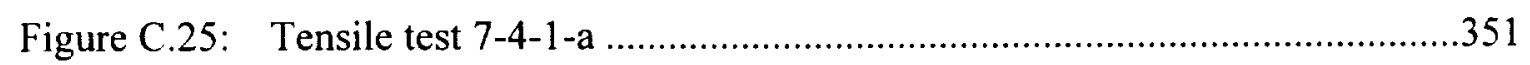

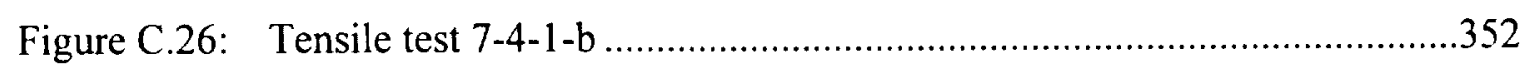

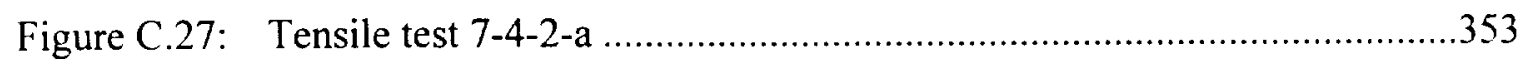




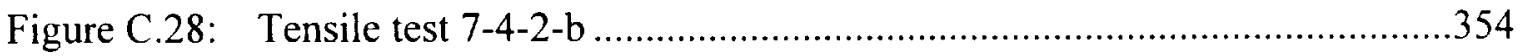

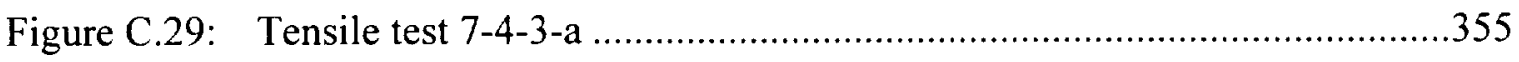

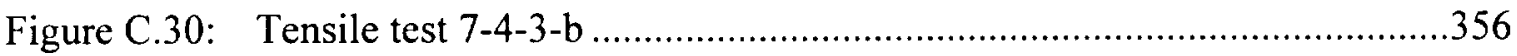

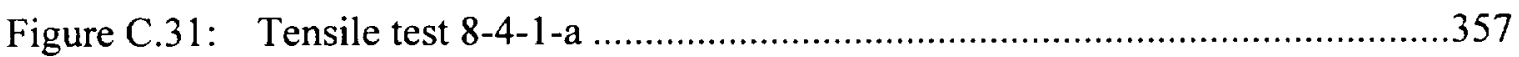

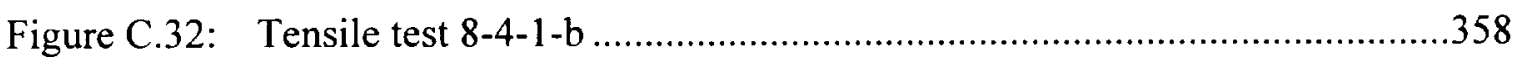

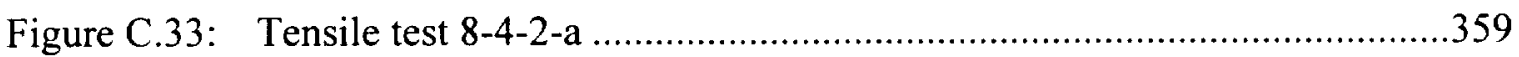

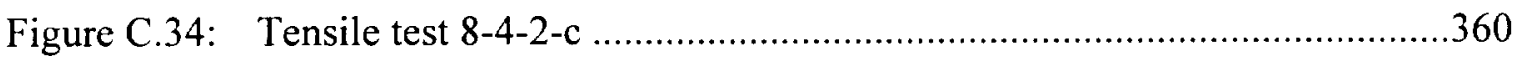

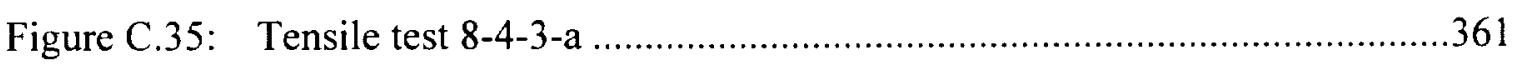

Figure C.36: Tensile test 8-4-3-b ........................................................................320 


\section{LIST OF TABLES}

Table 2.1: $\quad$ Material properties of materials used in this research............................52

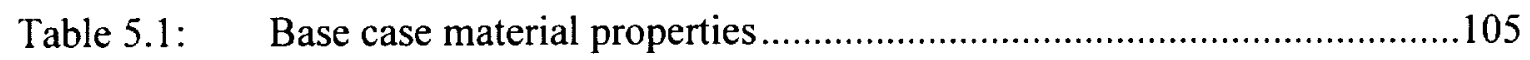

Table 5.2: $\quad$ Sensitivity studies parameter values .............................................. 114

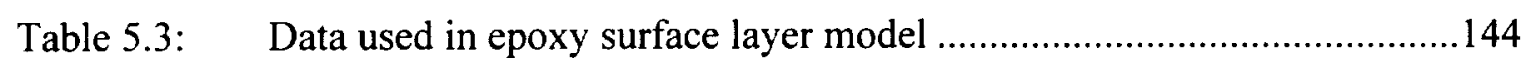

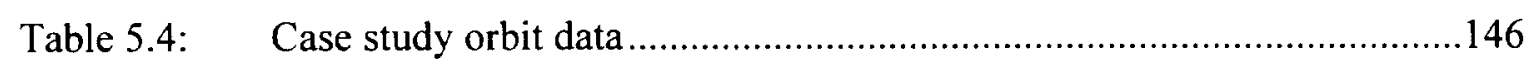

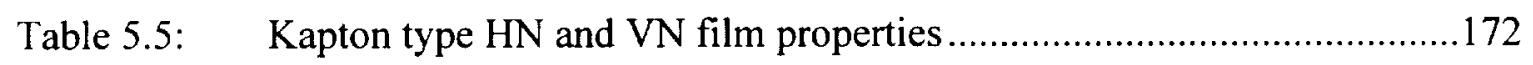

Table 5.6: Kapton type FN film properties ............................................... 172

Table 5.7: Space station test case analysis parameters......................................173

Table 6.1: Layups used for the composite electrical properties measurements .......186

Table 6.2: $\quad$ Electrical conductivity measurement test matrix ............................186

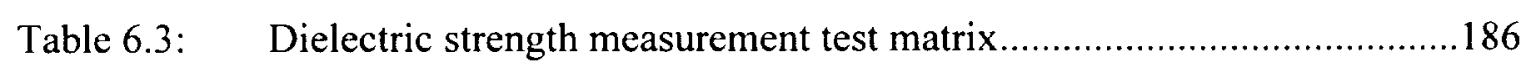

Table 6.4: Conductivity tailorable composite test matrix ....................................

Table 6.5: $\quad$ Rutapox L20/SL resin and hardener mixing ratios used........................196

Table 7.1: Carbon fiber / epoxy resistance and conductivity thickness

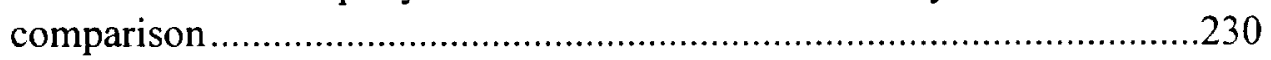

Table 7.2: Carbon fiber / epoxy resistance and conductivity area comparison........231

Table 7.3: Carbon fiber / epoxy resistance and conductivity layup comparison ......231

Table 7.4: Conductivity-tailorable glass fiber / epoxy composite results ...............244

Table 7.5: Glass fiber / epoxy surface layer thickness ..................................24

Table 7.6: $\quad[0]_{4}$ glass fiber / epoxy tensile test results ...................................254

Table 7.7: $\quad$ MCLAM results for $[0]_{4}$ and $[ \pm 45]_{\mathrm{S}}$ glass fiber / epoxy laminates ..........261 
Table C.1: Unidirectional unsanded conductivity resistance data ..........................322

Table C.2: Quasi-isotropic unsanded conductivity resistance data .......................323

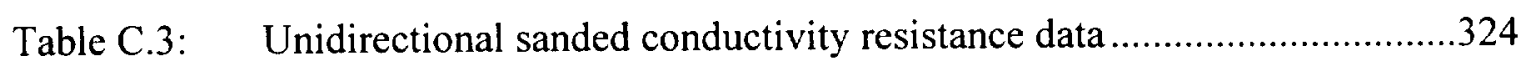

Table C.4: Quasi-isotropic sanded conductivity resistance data ............................325

Table C.5: Dielectric strength breakdown voltage data......................................326 


\begin{tabular}{|c|c|c|}
\hline A & Cross sectional area & $\mathrm{m}^{2}$ \\
\hline$A_{i}$ & Arbitrary constant in solution of $2^{\text {nd }}$ order PDE & $\mathrm{V} / \mathrm{m}$ \\
\hline$B_{i}$ & Arbitrary constant in solution of $2^{\text {nd }}$ order PDE & $\mathrm{V}$ \\
\hline $\mathrm{C}$ & Sample capacitance & $\mathrm{F}$ \\
\hline $\mathrm{C}_{\mathrm{SL}}$ & Capacitance of epoxy surface layer & $\mathrm{F}$ \\
\hline $\mathrm{C}_{1}$ & First integration constant & $\mathrm{V} / \mathrm{m}$ \\
\hline $\mathrm{C}_{2}$ & Second integration constant & $\mathrm{V}$ \\
\hline $\mathrm{C}_{3}$ & Integration constant & $\mathrm{V}$ \\
\hline $\mathrm{dE}_{\mathrm{g}} / \mathrm{dx}$ & Stopping power & $\mathrm{MeV} / \mathrm{m}$ \\
\hline$\dot{\mathrm{D}}$ & Radiation dose rate & $\mathrm{rad} / \mathrm{s}$ \\
\hline $\mathrm{D}_{\mathrm{f}}$ & Fiber diameter & $\mathrm{m}$ \\
\hline e & Charge of an electron & $\mathrm{C}$ \\
\hline E & Electric field & $\mathrm{V} / \mathrm{m}$ \\
\hline $\mathrm{E}_{\mathrm{g}}$ & Energy & $\mathrm{MeV}$ \\
\hline $\mathrm{E}_{\mathrm{o}}$ & Steady-state electric field & $\mathrm{V} / \mathrm{m}$ \\
\hline$E_{\max }$ & Dielectric strength & $\mathrm{V} / \mathrm{m}$ \\
\hline $\mathrm{E}_{\text {mech }}$ & Young's Modulus & $\mathrm{MPa}$ \\
\hline $\mathrm{E}_{\mathrm{r}}$ & Radial steady-state electric field & $\mathrm{V} / \mathrm{m}$ \\
\hline $\mathrm{E}_{\theta}$ & Circumferential steady-state electric field & $\mathrm{V} / \mathrm{m}$ \\
\hline h & Sample thickness & $\mathrm{m}$ \\
\hline
\end{tabular}




\begin{tabular}{|c|c|c|}
\hline$h_{\text {eff }}$ & Dielectric constant effective thickness & $\mathrm{m}$ \\
\hline$h_{\text {ply }}$ & Ply thickness & $\mathrm{m}$ \\
\hline $\mathrm{h}_{\mathrm{SL}}$ & Epoxy surface layer thickness & $\mathrm{m}$ \\
\hline $\mathrm{i}_{\mathrm{in}}$ & Incoming current from the space environment & A \\
\hline $\mathrm{i}_{\mathrm{C}}$ & Current flowing through capacitor & A \\
\hline$i_{R}$ & Current flowing through resistor & A \\
\hline $\mathbf{J}$ & Current density & $\mathrm{A} / \mathrm{m}^{2}$ \\
\hline $\mathrm{k}$ & Boltzmann's constant & $\mathrm{J} / \mathrm{K}$ \\
\hline $\mathrm{k}_{\mathrm{D}}$ & Coefficient of delayed conductivity & $1 / \mathrm{rad}-\Omega-\mathrm{m}$ \\
\hline $\mathrm{k}_{\mathrm{E}}$ & Conductivity scaling factor for high field conductivity & ----- \\
\hline $\mathrm{k}_{\mathrm{R}}$ & Coefficient of radiation induced conductivity & $\mathrm{s} / \Omega$-m-rad \\
\hline 1 & Sample length between loading tabs & $\mathrm{m}$ \\
\hline LET & Linear energy transfer & $\mathrm{MeV}-\mathrm{m}^{2} / \mathrm{kg}$ \\
\hline $\mathrm{m}$ & Mass & $\mathrm{kg}$ \\
\hline $\mathrm{m}_{\text {fill }}$ & Mass of filler powder & $\mathrm{kg}$ \\
\hline $\mathrm{N}_{\mathrm{T}}$ & Total number of plies & ---- \\
\hline $\mathrm{N}_{\theta}$ & Number of plies in the q direction & --.-- \\
\hline $\mathrm{N}_{45}$ & Number of plies oriented at $\pm 45^{\circ}$ to the $\theta$ direction & ---- \\
\hline $\mathrm{P}$ & Load & $\mathrm{N}$ \\
\hline$P_{\text {fail }}$ & Failure load & $\mathrm{N}$ \\
\hline $\mathrm{P}_{\mathrm{i}}(\mathrm{x})$ & Function defined by Rodrigues formula & $\cdots$ \\
\hline QF & Quality factor for particles & ----- \\
\hline
\end{tabular}


Radial distance m

R Sample resistance $\quad \Omega$

$\mathrm{R}_{\mathrm{SL}} \quad$ Resistance of epoxy surface layer $\quad \Omega$

$\mathrm{R}_{\mathrm{f}} \quad$ Fiber radius $\mathrm{m}$

t Time $\quad s$

T Temperature $\quad \mathrm{K}$

V Voltage

$\mathrm{V}_{\mathrm{BD}} \quad$ Breakdown voltage $\quad \mathrm{V}$

$\mathrm{V}_{\mathrm{C}} \quad$ Critical volume fraction

$\mathrm{V}_{\mathrm{f}} \quad$ Fiber volume fraction

$\mathrm{V}_{\mathrm{O}} \quad$ Initial voltage $\mathrm{V}$

Vol Volume $\mathrm{m}^{3}$

$\mathrm{Vol}_{\mathrm{f}} \quad$ Volume of fiber $\quad \mathrm{m}^{3}$

$\mathrm{Vol}_{\text {fill }} \quad$ Volume of tapped filled powder $\mathrm{m}^{3}$

$\mathrm{Vol}_{\mathrm{m}} \quad$ Volume of matrix $\mathrm{m}^{3}$

$\mathrm{x}$ Depth into the material m

X Ratio of sample thickness to effective thickness

$\beta \quad$ Ratio of the ply thickness to fiber diameter

$\begin{array}{lll}\beta_{\mathrm{F}} & \text { Frenkel parameter } & \sqrt{\mathrm{C}}-\sqrt{\mathrm{N}}-\mathrm{m}\end{array}$

$\delta \quad$ Jump distance $\mathrm{m}$

$\Delta \quad$ Radiation induced conductivity exponent

$\Delta \mathrm{l}$ Change in length, stroke $\mathrm{m}$ 


\begin{tabular}{|c|c|c|}
\hline$\varepsilon$ & Permittivity of the material & $\mathrm{C}^{2} / \mathrm{N}-\mathrm{m}^{2}$ \\
\hline$\varepsilon_{\text {max }_{\text {mech }}}$ & Failure strain & $\cdots--$ \\
\hline$\varepsilon_{\text {mech }}$ & Strain & $\cdots--$ \\
\hline$\varepsilon_{0}$ & Permittivity of free space & $\mathrm{C}^{2} / \mathrm{N}-\mathrm{m}^{2}$ \\
\hline$\varepsilon_{\mathrm{x}_{\text {mech }}}$ & Strain perpendicular to direction of applied load & ----- \\
\hline$\varepsilon_{y_{\text {mch }}}$ & Strain parallel to direction of applied load & ----- \\
\hline$\phi$ & Angle & degrees \\
\hline$\Phi$ & Charged particle flux & $\# / \mathrm{m}^{2}-\mathrm{s}$ \\
\hline$\Phi_{\mathrm{p}}$ & Packing fraction & ----- \\
\hline K & Dielectric constant & ---- \\
\hline$\kappa_{\text {actual }}$ & Actual sample dielectric constant & $-\cdots$ \\
\hline$\kappa_{\text {measured }}$ & Measured sample dielectric constant & $-\cdots$ \\
\hline$v$ & Poisson ratio & ---- \\
\hline$\theta$ & Angle & degrees \\
\hline$\rho$ & Charge density & $\# / \mathrm{m}^{3}$ \\
\hline$\rho_{\text {fill }}$ & Theoretical density of filler powder & $\mathrm{kg} / \mathrm{m}^{3}$ \\
\hline$\rho_{\text {in }}$ & Incoming charge density rate & $\# / \mathrm{m}^{3}-\mathrm{s}$ \\
\hline$\rho_{\text {mech }}$ & Density & $\mathrm{kg} / \mathrm{m}^{3}$ \\
\hline$\sigma$ & Electrical conductivity & $1 / \Omega-\mathrm{m}$ \\
\hline$\sigma_{\mathrm{D}}$ & Delayed conductivity & $1 / \Omega-\mathrm{m}$ \\
\hline$\sigma_{\mathrm{E}}$ & High field conductivity & $1 / \Omega-\mathrm{m}$ \\
\hline
\end{tabular}




$\begin{array}{lll}\sigma_{\mathrm{f}} & \text { Conductivity of the fiber } & 1 / \Omega-\mathrm{m} \\ \sigma_{\mathrm{L}} & \text { Conductivity in the longitudinal direction } & 1 / \Omega-\mathrm{m} \\ \sigma_{\mathrm{m}} & \text { Conductivity of the matrix } & 1 / \Omega-\mathrm{m} \\ \sigma_{\text {max }_{\text {nech }}} & \text { Failure stress } & \mathrm{MPa} \\ \sigma_{\text {mech }} & \text { Stress } & \mathrm{MPa} \\ \sigma_{\mathrm{o}} & \text { Dark conductivity } & 1 / \Omega-\mathrm{m} \\ \sigma_{\mathrm{R}} & \text { Radiation induced conductivity } & 1 / \Omega-\mathrm{m} \\ \sigma_{\mathrm{T}} & \text { Conductivity in the transverse direction } & 1 / \Omega-\mathrm{m} \\ \sigma_{\mathrm{thick}} & \text { Conductivity in the through-thickness direction } & 1 / \Omega-\mathrm{m} \\ \sigma_{0} & \text { Conductivity in the fiber direction } & 1 / \Omega-\mathrm{m} \\ \sigma_{1} & \text { Conductivity in the first principal direction } & \mathrm{s} \\ \sigma_{2} & \text { Conductivity in the second principal direction } & 1 / \Omega-\mathrm{m} \\ \tau & \text { RC circuit time constant } & 1 / \Omega-\mathrm{m}\end{array}$




\section{CHAPTER 1}

\section{INTRODUCTION}

The charging of spacecraft, and its possible role in spacecraft anomalies due to electrostatic discharges, has been a well known problem since the beginning of space flight some thirty years ago. Charging is caused by energetic particles in the space environment: electrons, protons and positively charged heavy ions. There are three types of charging: entire vehicle charging, surface charging, and internal charging, also known as deep dielectric charging. Entire vehicle charging is when the entire potential of the spacecraft is raised. Surface charging is when only the potential of the spacecraft surface is raised; however this may also occur locally where only part of the surface has its potential raised due to geometric and material considerations. Deep dielectric charging is like surface charging, except that the potential increase is not on the surface of the spacecraft component but inside the material of the component. The last two types are a concern for composite material structures, and the last type, deep dielectric charging, is the focus of this research.

Most of the work done up until now on charging has been on surface charging. It is caused by low energy electrons (less than $30-50 \mathrm{keV}$ ) which do not penetrate the surface of the external structural material. These particles accumulate on the surface of 
the structure, and can lead to large differential charging between various parts of the spacecraft. This charging can grow large enough to cause an electrostatic discharge, which can lead to surface damage or spacecraft anomalies. These spacecraft anomalies are caused by an induced internal current created by the discharge which can cause electronics damage or cause erroneous commands to be processed by the spacecraft.

Deep dielectric charging occurs when high energy electrons or ions penetrate the surface of, and deposit charge within, a insulating material. If the deposition of incoming charged particles is greater than the charge leakage through the material, a large potential difference can build up in the material and lead to an electrostatic discharge. The discharge can occur within the material or from the interior of the material to one of its surfaces. In either case a current path is created and the material is locally damaged around the discharge site. There seems to be a correlation between these discharges and periods of increased flux, as spacecraft anomalies have been observed when there are more high energy particles in their local space environment.

The goals of this research are to develop a model for the analysis of deep dielectric charging of insulating materials and composite materials, determine relevant composite electrical properties, and to develop technology to alleviate charging problems by changing the properties of the composite. A schematic of the model is shown in Figure 1.1. The model was used to create a Fortran Code, The Composite Deep Dielectric Charging Analyzer (CoDDCA). The code will have as inputs the space environment and material properties. The space environment is given in terms of electron 


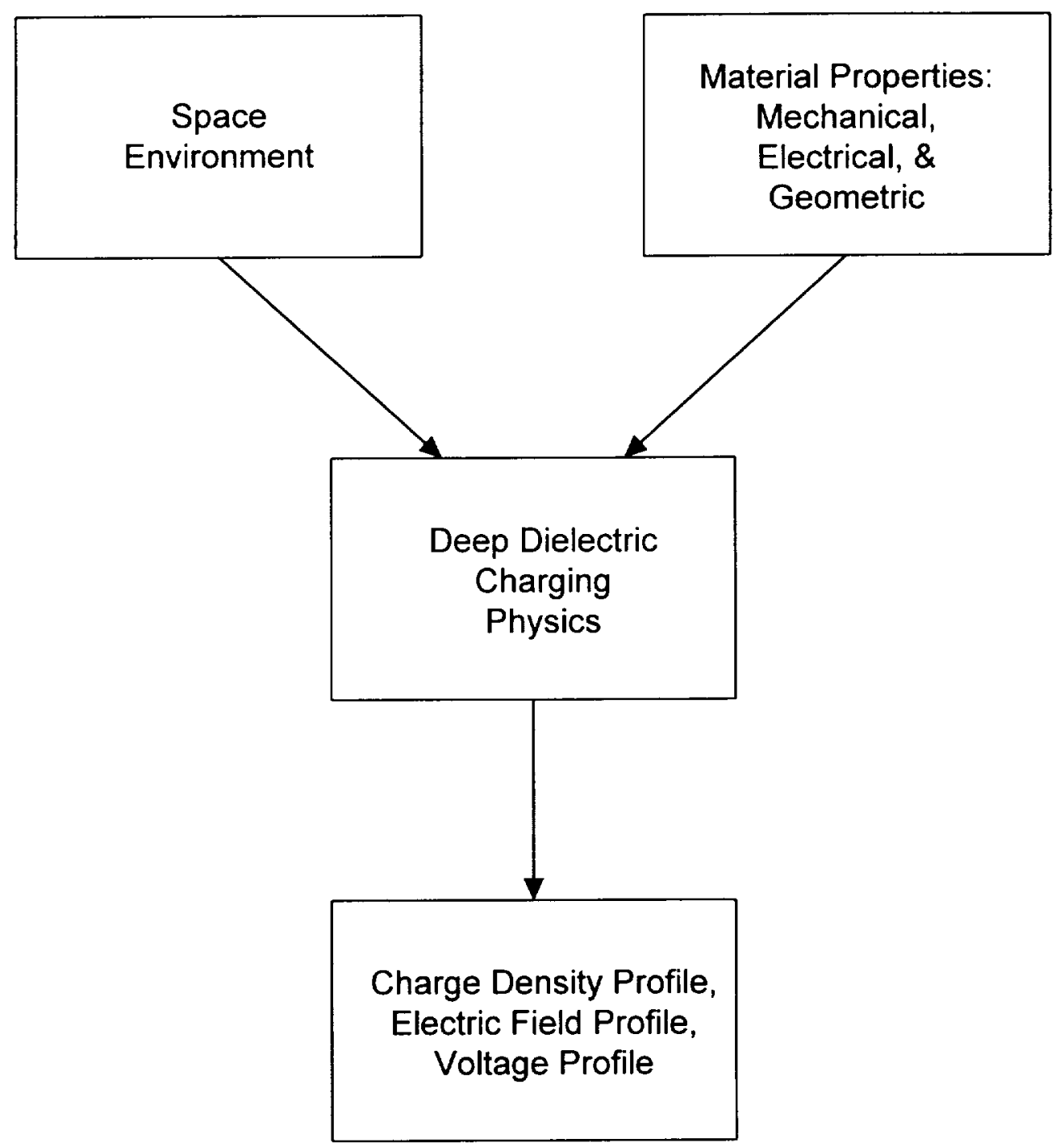

Figure 1.1: Schematic of the deep dielectric charging model 
and proton fluxes versus energy; also geomagnetic substorms and solar particle events may be specified. The material properties are divided up into mechanical, electrical, and geometrical properties. The outputs from the code are given as functions of depth into the material from the surface exposed to the space environment and time. They include the charge density profile, the electric field profile, and the voltage profile.

The model was used to examine the effects of the various parameters input into the model. These sensitivity studies revealed which parameters are important to deep dielectric charging and which play minimal roles. The model was also used to examine specific case study orbits, including orbits where there have been suspected incidents of deep dielectric charging.

Composites are treated as a homogenous material in the above model. Therefore composites on a micro-scale were modeled separately to determine the effects of their inhomogeneity on the electric field. The different arrangements investigated include the effects on the electric field due to a conductive fiber surrounded by an insulative matrix, unlikely fiber arrangements which can create points of electric field intensification, and the effects of an insulative surface layer of pure epoxy often found on top of conductive carbon fiber / epoxy composites.

Experimental work was done to measure the continuum electrical properties of composites. Carbon fiber / epoxy composites were made of various thicknesses, areas, and layups and were used to investigate the through-thickness conductivity and dielectric 
strength. Values typical of previous work were measured, but the conductivity and dielectric strength did not behave as continuum properties; they displayed non-classical thickness and surface layer dependencies.

Another part of the experimental work was to create a conductivity tailorable composite. This work was undertaken because the sensitivity studies showed that the deep dielectric charging problem is dependent on conductivity, and that increasing conductivity can have a significant effect in reducing the likelihood of an electrostatic discharge. Therefore, a composite system was developed that had conductivity that could be tailored without greatly affecting its mechanical properties. A glass fiber / epoxy composite was used, the conductivity was increased by adding carbon black powder to the epoxy. Laminates made from this composite were investigated to determine the effects of the carbon black on the electrical and mechanical properties of the composite. Control of electrical properties over large ranges ( 3 orders of magnitude) without significant effects on mechanical properties, were achieved.

Previous work relevant to the current research is described in Chapter 2. This includes a description of the space environment, analytical work on surface charging and deep dielectric charging, and some general information on composites. The problem statement and approach for the current research is presented in Chapter 3. The analytical methodology which is used in the Composite Deep Dielectric Charging Analyzer (CoDDCA) Code is developed in Chapter 4, as are micro-mechanical modeling of composite electrical properties, and the data reduction equations used in the experimental 
analysis. The results of the code, including the parametric studies and the case studies are presented and discussed in Chapter 5. Chapter 6 describes the test matrices used, the procedures used to manufacture the composite laminates, and the experimental procedures used to measure the mechanical and electrical properties of the composite samples. The results of the experimental analysis are presented and discussed in Chapter 7. Finally, conclusions and recommendations for future work are presented in Chapter 8 . The appendices include a description of the CoDDCA code and how to use it, as well as the code output profiles and the experimental data. 


\section{CHAPTER 2}

\section{BACKGROUND}

This chapter presents background information on the space environment, charging in the space environment, and composite materials. The space environment is broken up into different sources of radiation: the Van Allen radiation belts, galactic cosmic rays, solar particle events and geomagnetic substorms. The discussion of spacecraft charging in the space environment includes both surface charging and deep dielectric charging. Finally, a general description of composites and composite mechanics relevant to this work are given.

\subsection{RADIATION SOURCES}

The main sources of energetic particles in the space environment are trapped radiation of the Van Allen radiation belts, galactic cosmic rays consisting of interplanetary protons and ionized heavy nuclei, and particles associated with solar particle events and substorms. ${ }^{1,2}$ The first two sources are fairly constant, while the third is highly time dependent. The Van Allen belt radiation commonly leads to deep dielectric charging, while radiation from the other sources is more likely to lead to single event phenomena (SEP) in electronic components. This occurs because the belts contain certain types of particle; the particle types determine the phenomena. The are two types 
of single event phenomena, hard and soft, depending on if the damage which occurs is permanent or temporary. Soft single event phenomena are also known as single event upsets (SEU). They result in such problems as a change in stored data or wrong commands. Hard single event phenomena can result in permanent damage such as burnout of power MOSFET's, gate rupture, latchup, or the freezing of bits. The simplest solution to single event phenomena is to use shielding. A major source of shielding is the spacecraft structure. However, not all wires and components can be easily shielded, and shielding can add significantly to the spacecraft mass.

The Sun's activity occurs in an 11 year cycle know as the solar cycle. The activity is quantified by the sunspot number (typically called the $\mathrm{R}$ value), the higher the number the more active the Sun is. Solar minimum defines the beginning of the cycle when the sunspot number is at its lowest. Solar maximum is when the Sun is most active and the sunspot number is at its highest, as shown in Figure 2.1. Each cycle can have maximums and minimums which vary by a factor of 4 from one cycle to the next.

\subsubsection{Van Allen Radiation Belts}

The Van Allen radiation belts consists primarily of energetic electrons and protons, with a smaller percentage of heavy ions like oxygen $\left(0^{+}\right)$. They are trapped by the Earth's magnetic field lines, and thus gyrate about the poles along the magnetic field lines. They form toroidal belts around the Earth as shown in Figure 2.2. There are two belts of high concentrations of particles, the inner belt and the outer belt. The inner belt extends from approximately $1000 \mathrm{~km}$ to $6000 \mathrm{~km}$ in altitude, and is populated by very 


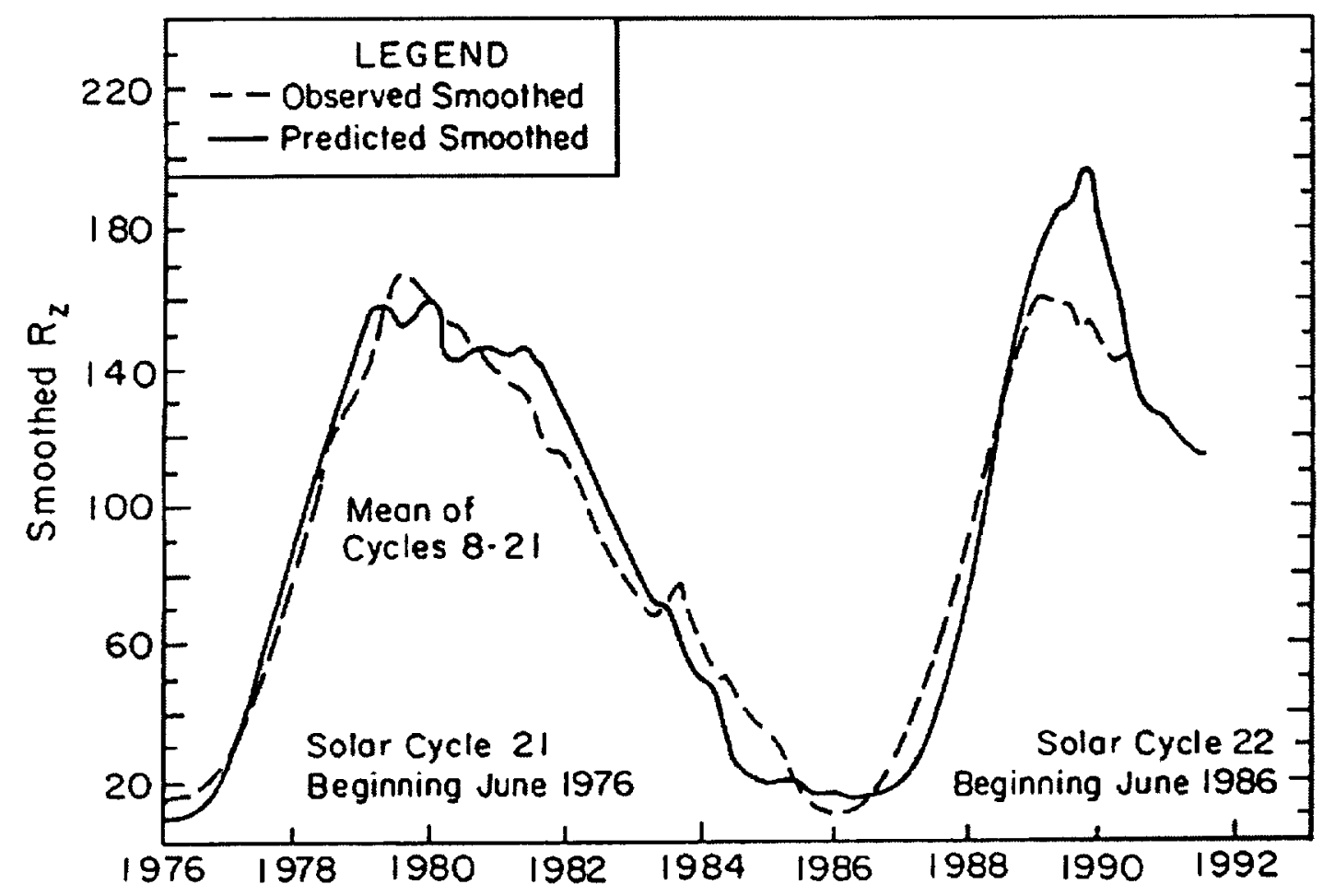

Figure 2.1: Observed and one-year-ahead predicted sunspot numbers ${ }^{3}$ 
Omnidirectional Electron Flux (\#/cm $\left.{ }^{2}-\mathrm{s}\right)$

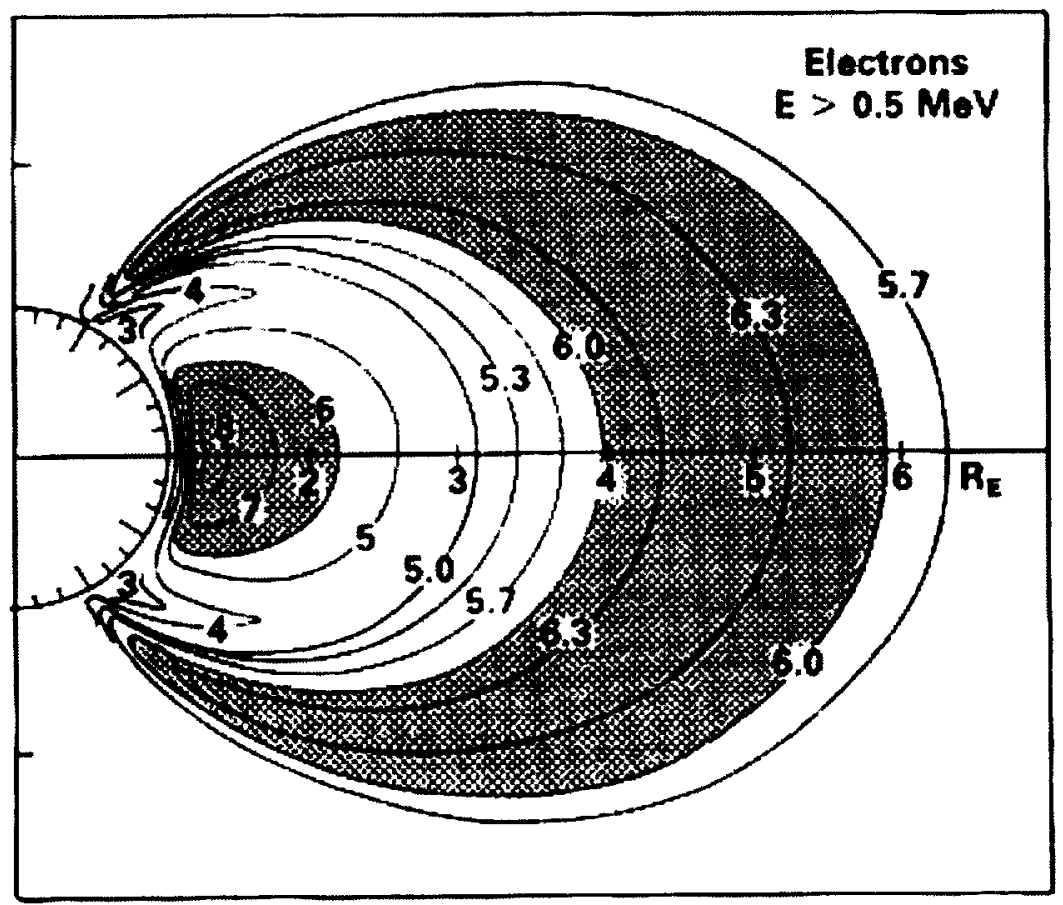

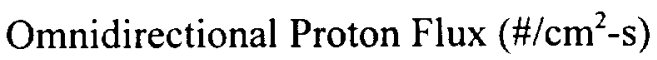

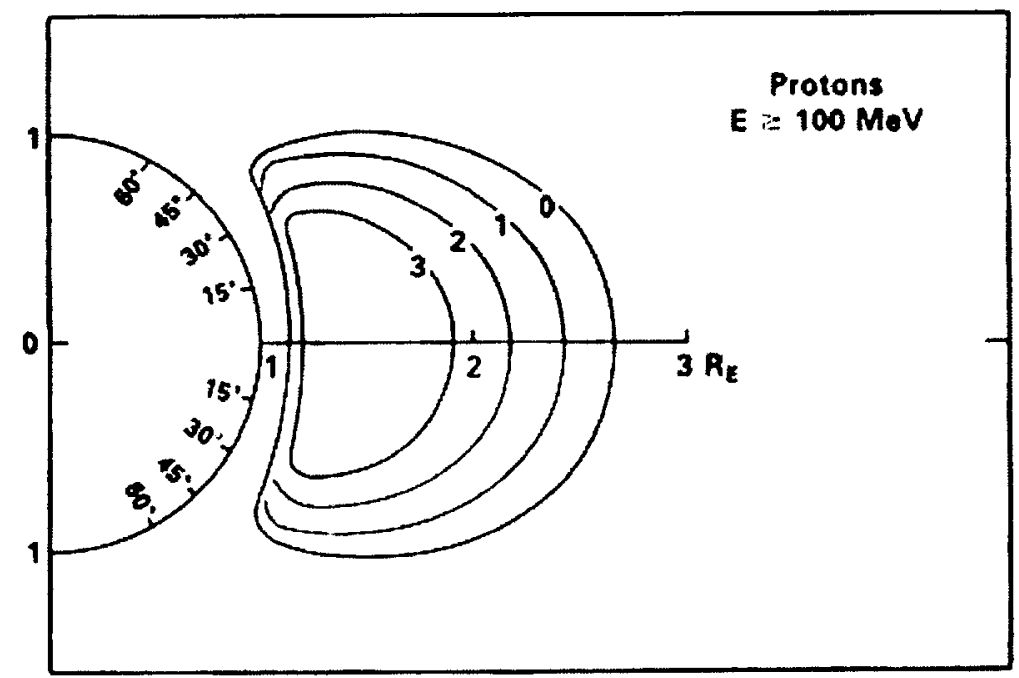

Figure 2.2: Electron and proton Van Allen radiation belts ${ }^{4}$ 
high energy (10's of MeV) protons and high energy (1-10 MeV) electrons. The outer belt extends from approximately $20,000 \mathrm{~km}$ to $32,000 \mathrm{~km}$ in altitude, and is populated primarily by high energy electrons. For this reason, most spacecraft have orbits with altitudes in the hundreds of kilometers, below the inner belt, and at geosynchronous orbit (GEO) which is above the upper belt, at $35,782 \mathrm{~km}$ in altitude. However, the Van Allen radiation belts continue beyond the high concentration belts out to an altitude of 60,000 $\mathrm{km}$, with smaller amounts of particles. It should be noted that when the particles travel near the poles, higher energy particles travel to lower altitudes producing the aurora borealis and aurora austrailialis. Therefore, spacecraft in low altitude polar orbits are subjected to the same density of high energy particles found in much higher equatorial orbits.

\subsubsection{Galactic Cosmic Rays}

Galactic cosmic rays are energetic particles which originate outside our solar system. They are believed to be ejected at high energies from nova and supernova explosions, solar flares from other solar systems, or quasars. The rays consists mainly of protons and Helium nuclei but may also consist of other heavier nuclei. Even though they amount to only a small portion of the total dose a spacecraft receives, galactic cosmic rays can lead to single event upset phenomena due to their high energies. The Van Allen radiation belts shield the Earth from galactic cosmic rays, and thus spacecraft in geosynchronous orbit are more susceptible to single event phenomena than those in lower altitude orbits. 


\section{$\underline{2.1 .3 \text { Solar Particle Events }}$}

Solar particle events are similar to galactic cosmic rays but are ejected from our Sun instead of from other solar systems' suns. The Van Allen radiation belts also protect Earth from solar particle events. These events are fairly infrequent, on average a few per year. They consist mainly of protons which are ejected from the Sun during solar flares. A solar flare is created by a highly concentrated explosive release of high energy radiation. Events may last from a couple of hours to over a week. Typically, the effects last 2 or 3 days. Solar particle events can be a significant contributor to the total dose received by a spacecraft, in addition to creating single event phenomena. During a solar particle event, the flux levels of protons in the Van Allen radiation belts increase by a couple of orders of magnitude. The belts will retain high levels of particles in them until the particles dissipate; typically these high levels last from several hours to several days.

\section{$\underline{2.1 .4 \text { Geomagnetic Substorms }}$}

A geomagnetic substorm occurs when an interplanetary disturbance, a giant magnetic cloud containing hot gas from the Sun's corona which is ejected from the Sun, strikes the Earth's magnetic field. The magnetic cloud collides with, and envelopes, the Earth's magnetic field, it compresses the magnetic field on the day side and stretches it out on the night side, thus causing the geomagnetic substorm. This phenomena is different from the solar particle event mentioned earlier. Substorms can last from several hours to a couple of days, depending on the severity of the storm. There exists a correlation between the geomagnetic activity index and the 11 year sunspot cycle. The geomagnetic activity index has a peak which occurs during the declining phase of the 
sunspot cycle, and a secondary peak near solar maximum. Therefore, spacecraft can be affected by substorms during and for several years after an extreme solar maximum. Substorms consist of three distinct phases: growth, expansion, and recovery. The net result of a substorm is to increase the electron flux seen by spacecraft by 2 to 3 orders of magnitude. ${ }^{5}$

\subsection{SURFACE CHARGING}

During the 1970's, 1980's, and continuing into the 1990's, protection techniques have been developed which have basically taken care of the surface charging problem. ${ }^{6,7}$ Several of these have been published as design guidelines, "Design Guidelines for Assessing and Controlling Spacecraft Charging Effects"8, "Space Environmental Effects on Spacecraft: LEO Materials Selection Guide", "On Orbit Charging: Current TWR Design Requirements"10, and MIL-STD $1541 \mathrm{~A}^{\prime \prime}$. One of the powerful engineering tools used to analyze surface charging is NASA's Charging Analyzer Program (NASCAP). ${ }^{8,12}$ It analyzes the surface charging of a three dimensional spacecraft surface as a function of time, for a given space environment, surface potentials, and material properties. By calculating and locating the regions of high surface voltage gradients, the areas where discharges are likely of occur are identified. Therefore, the materials or geometry of the spacecraft in these regions can be varied to minimize the likelihood of an electrostatic discharge.

The general approach used by all of the above mentioned guidelines, is to select where possible the materials and conductive coating to be used on the surface of the 
spacecraft, as to minimize differential charging of spacecraft parts. Differential charging is minimized when the voltage across the surface is as uniform as possible. If the above mentioned selection is not possible, the approach is then to use alternatives such as special filtering, cabling, or grounding. Once these have been selected, the charging codes are run to simulate the charging effects and to analyze whether the chosen configuration will work. However, since anomalies continue to occur on spacecraft, there must be another source of electrostatic discharges, such as deep dielectric charging. Therefore, an investigation of deep dielectric charging is required.

\subsection{DEEP DIELECTRIC CHARGING}

Vampola $^{13}$ showed that anomalies on spacecraft due to deep dielectric charging are linked to geomagnetic substorms, where the number of high energy electrons increases greatly. He was able to link the anomalies to deep dielectric charging by providing evidence that not all anomalies could be linked to surface charging. At geosynchronous orbit, deep dielectric charging is not affected by the spacecraft's local time, while surface charging is known to occur between pre-midnight (22:00) and dawn (8:00), when the surface potentials become high enough for a discharge to occur. Therefore, between pre-noon (10:00) and dusk (20:00) no discharges should be observed. Since discharges do occur as shown in Figure 2.3, they must be due to deep dielectric charging instead of surface charging.

Several spacecraft failures have been associated with electrostatic discharges

resulting from deep dielectric charging, including the $\$ 300$ million Telesat Canada 


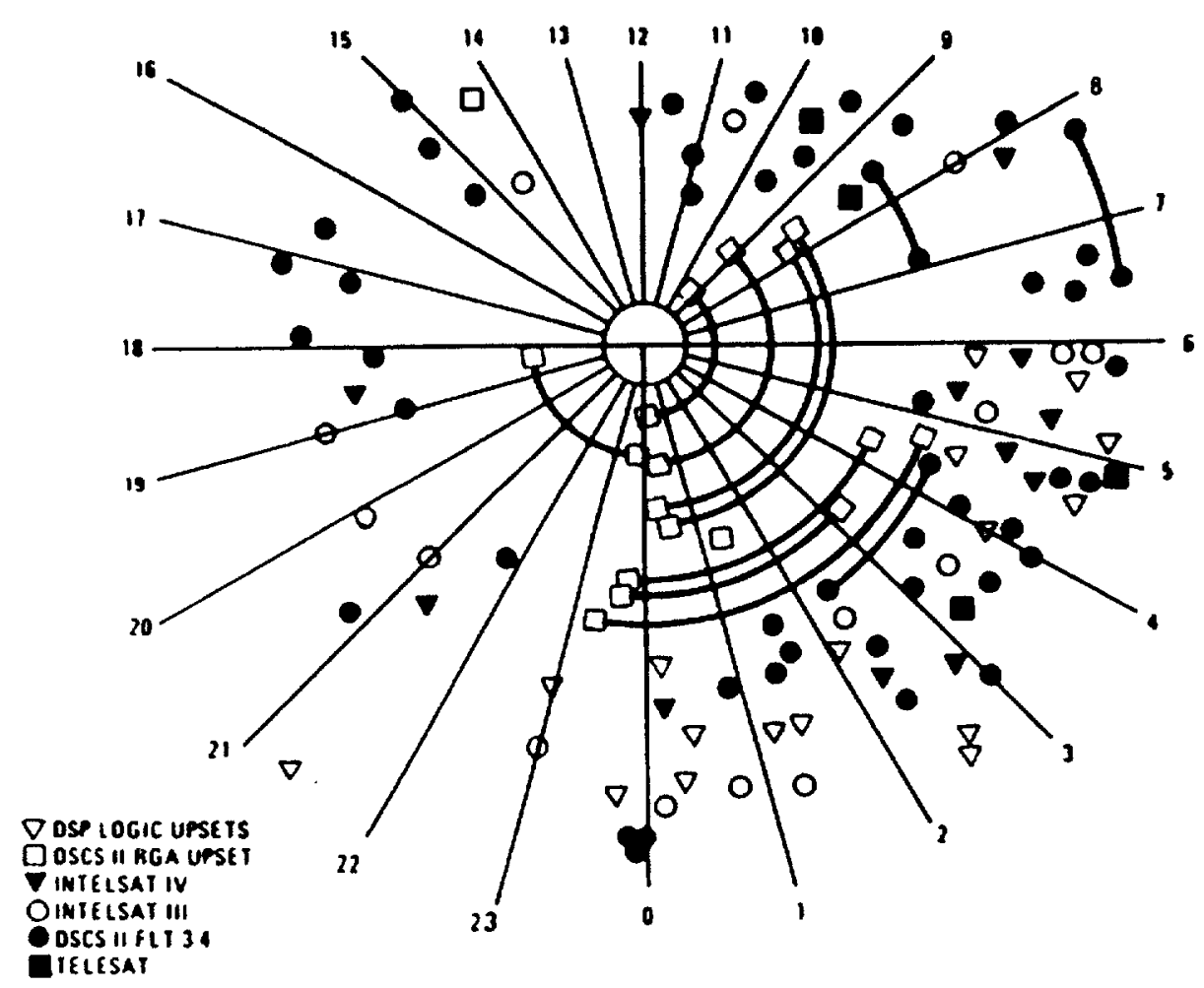

Figure 2.3: A local-time plot of the occurrence of anomalies on various spacecraft ${ }^{13}$ 
communications satellites Anik E1 and E2, Intelsat K, the European Space Agency (ESA) telecommunications spacecraft Olympus, and recently AT\&T's Telstar 401. Both Aniks failed a day apart in January 1994, when they suffered a temporary loss of altitude control due to problems with their momentum wheels. It is believed that a discharge from deep dielectric charging disabled key circuitry in the momentum wheel control systems. Anik E1 was out of service for a few hours, but E2 was out for over 6 months and is now operating with a reduced lifetime on orbit, due to failure of the redundant momentum wheel control system. On the same day as the Anik E1 failure, Intelsat K's momentum wheel control circuitry experienced an operation anomaly which caused it to lose attitude control. Control was reestablished when the backup circuitry was engaged. Analysis of the radiation environment at the time of the failures indicates that the amount of high energy electrons was greatly elevated. In August 1993, Olympus experienced a critical anomaly which lead to its eventual failure and out-of-service status. Telstar 401 experienced a massive power failure in January 1997, rendering the spacecraft completely inoperable. This occurred after an intense geomagnetic substorm. An interesting point to note is that as Intelsat K, both Anik E's, and Telstar 401 were all made by Lockheed Martin, these anomalies may be due to a similar design feature.

Other spacecraft have experienced switchings or anomalies due to electrostatic discharges resulting from deep dielectric charging, including Voyager 1 as it passed by Jupiter in September 1977. There, it experienced a series of power on resets (POR). Each time a reset was executed, the onboard clock was offset. This offset caused a sequence of camera commands to be executed late and almost caused the loss of one of 
the objectives of the photographic mission. The European communications spacecraft ECS-2 and ECS-4 experienced several automatic reconfiguration mode switchings in the early 1990's. Anomalies where also reported on the US Air Force Defense Support Program (DSP) spacecraft, which affected the star-sensor shutter, and also on Meteosat-1. These anomalies occurred on both spacecraft following periods of increased solar activity. Most anomalies are suspected to be due to discharges occurring in insulation around cables exposed to the outside of the spacecraft.

There have also been electrostatic discharges on the Combined Release and Radiation Effects Spacecraft (CRRES). CRRES was sent up to measure outer-zone electrons and internal discharges during 1990 and 1991. ${ }^{14-16}$ The internal discharge experiment (IDM) exposed 16 samples of standard insulating materials with electrodes to the Earth's radiation environment. The samples were composed of two different geometries, cables and printed circuit boards (PCB), and with various connections; grounding, floating, or semi-conducting elements. The samples were shielded with a thin aluminum foil, thus stopping all electrons below $150 \mathrm{keV}$. The orbit used was a high elliptical transfer orbit so that the spacecraft was exposed to all the magnetosphere environment. Over the 14 months of the flight, approximately 4300 spontaneous discharges were recorded from the samples, thus proving that deep dielectric charging did exist and that it is of concern. The results are of the form of the flux above a certain energy level on the days that discharges were recorded. It should also be noted that while some spacecraft have been affected by deep dielectric charging, other spacecraft of 
similar design and in operation at the same time have not been affected. This demonstrates that more research into the issue of deep dielectric charging is required.

A general overview of deep dielectric charging was done by Garrett and Whittlesey ${ }^{17}$, and Soubeyran ${ }^{18}$, who also gives some basic design guidelines to follow to minimize deep dielectric charging. Some early modeling of deep dielectric charging was done by Berkley ${ }^{19}$ and Frederickson ${ }^{20}$. Berkley analyzed the charging of thin polymer films, using multiple electron energy ranges. The model can incorporate both open and short circuit boundary conditions, and can include non-linear transport terms, as well as time-varying radiation induced conductivity. The electron-irradiation parameters, the radiation induced conductivity and the electron deposition profile, are computed using a single-scattering Monte Carlo model of the polymer. The time-dependent chargetransport equations are solved by direct discretization of the partial differential equations. With his code, Berkley was able to study the effects of a time-varying electron beam, conductivity, and the influence of various boundary conditions, using both linear and non-linear transport. The results showed that charging reaches a steady-state quickly, and that the model has excellent qualitative and in many cases quantitative agreement with the experiments he performed using an electron beam to irradiate samples.

Frederickson used basically the same model as Berkley, but improved the calculations to include the dose effects of secondary X-rays and bremsstrahlung radiation, and to include the effect of the electric field on the trajectories of the incident penetrating electrons. A Monte Carlo simulation is used for the electron trajectories of the mono- 
energetic electron beam, and a computerized iteration technique is used for the motion of the space charge and the induced electric fields. Two different boundary conditions are used, both the front and rear surfaces are held at zero potential (grounded), and the front surface allowed to float free. The first boundary condition represents the spacecraft surface in full sun which allows solar photoemission to keep the surface at zero potential. The second represents the spacecraft surface in full shade where without solar photoemission the potential can vary. It was found that the bremsstrahlung and $\mathrm{x}$-rays produced very little effect, and that the space charge electric fields do not significantly alter the trajectories of the incident electrons. Both Berkley's and Frederickson's models work well, but do not simulate the space environment, as they work only for an electron beam of mono-energetic electrons.

Recently, Soubeyran ${ }^{21}$ of Matra Marconi Space developed ESA-DDC 22.23 (European Space Agency Deep Dielectric Charging), a numerical tool to analyze deep dielectric charging in the space environment, for the European Space Agency. The electron and proton transport is calculated using Monte Carlo transport codes to track the path followed by the electrons and protons. This provides the deposited dose and charge within the material for a given energy distribution or mono-energetic particles. Ampere's equation with Ohm's law are transformed into equivalent electrical circuits and sent to a circuit analyzer. The circuit analyzer calculates the induced conductivity, current, electrical potential, and electric field. The code is restricted to 1-D geometries with the upper surface exposed to the space environment and the lower surface grounded to the structure potential. The material between the surfaces can be made up of multiple layers 
of materials, both conducting and insulating. Subsequently, the code has been modified for the analysis of 2-D geometries.

Another method, which was proposed by Whittlesey ${ }^{24}$, is to manufacture the spacecraft and before it is launched, to test it to see if electrostatic discharges occur. Testing is performed using the MIL-STD 1541 sparking device, which simulates the effects of space electrostatic discharges. Thus if discharges occur modification of the spacecraft is required after the spacecraft is designed and assembled. This technique can be effectively used to double-check spacecraft which have been designed to have minimal electrostatic discharges, but is grossly inefficient for finding and fixing problems.

Most previous work on deep dielectric charging has dealt with the charging of spacecraft components such as wire insulation and printed circuit boards, where the electrostatic discharges can lead to anomalies in spacecraft operation. However, with the increasing use of composite materials, which can be dielectrics, deep dielectric charging is becoming a problem with the structure of spacecraft.

\subsection{COMPOSITES}

Composites are starting to replace metals, such as aluminum, as the structure of spacecraft, due primarily to their higher stiffness-to-weight ratios. Composites are made up of multiple layers or plies, which are stacked at various angles to get the desired material properties. These plies are in turn made up of fibers and matrix material which surrounds the fiber. The dominant fibers used are carbon (sometimes referred to as 
graphite), glass, and Kevlar. The dominant matrix materials for space applications are epoxies, cyanates, and poly-ether-ether-keytones (PEEK).

By varying the angles of the plies and the stacking sequence of the plies (refer to Figure 2.4), one can tailor the properties of the laminate or composite structure. The reason changing the ply angle has an effect on the material properties is that the plies are anisotropic. The material properties in the longitudinal direction can be very different from the properties in the transverse and through-thickness directions (refer to Figure 2.5). The ply angle is defined as the angle between the geometric coordinate system and the ply coordinate system. The range of angles is from $+90^{\circ}$ to $-90^{\circ}$. The geometric coordinate system is arbitrarily assigned to a structural direction, for example the length of a solar panel array, and the ply coordinate system is aligned with the fiber direction, as shown in Figures 2.4 and 2.5. Therefore a $0^{\circ}$ ply will have its longitudinal properties aligned with the principal direction of the structure. The laminate properties are based on the ply properties and the ply angles, and can be calculated using Classical Laminated Plate Theory (CLPT). For more information refer to Jones ${ }^{25}$ and Tsai ${ }^{26}$.

In modeling composites in general, and specifically for the charging problem, the homogenized equivalent properties for the composite ply are used instead of the individual properties of the fibers and matrix. This is an acceptable simplification for a first order solution of the problem, as the thickness of the material is much greater than the diameter of the fibers for most material systems of interest. To determine the ply properties, one has to combine the fiber properties with the matrix properties. The ply 


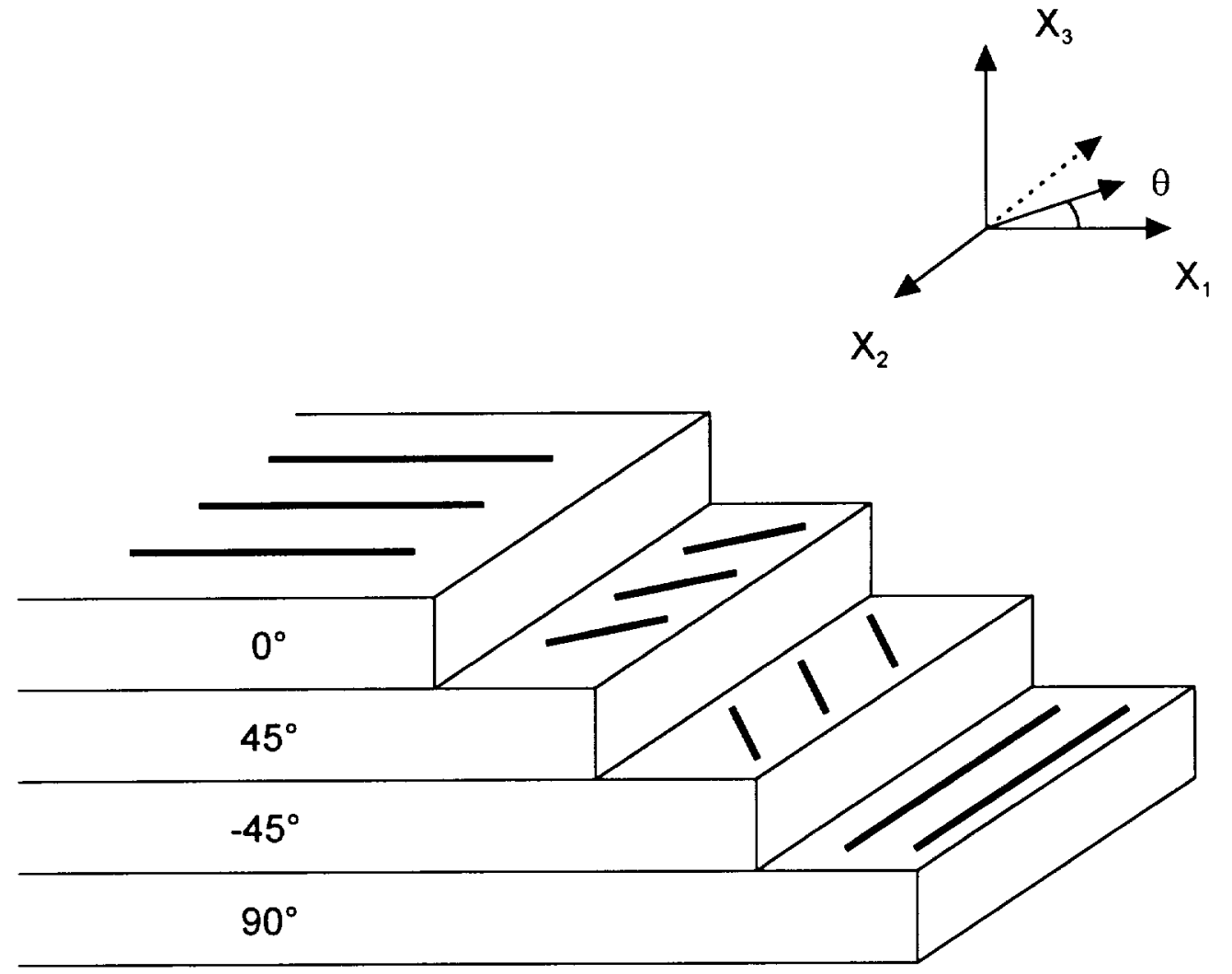

Figure 2.4: Typical composite in the geometric coordinate system 


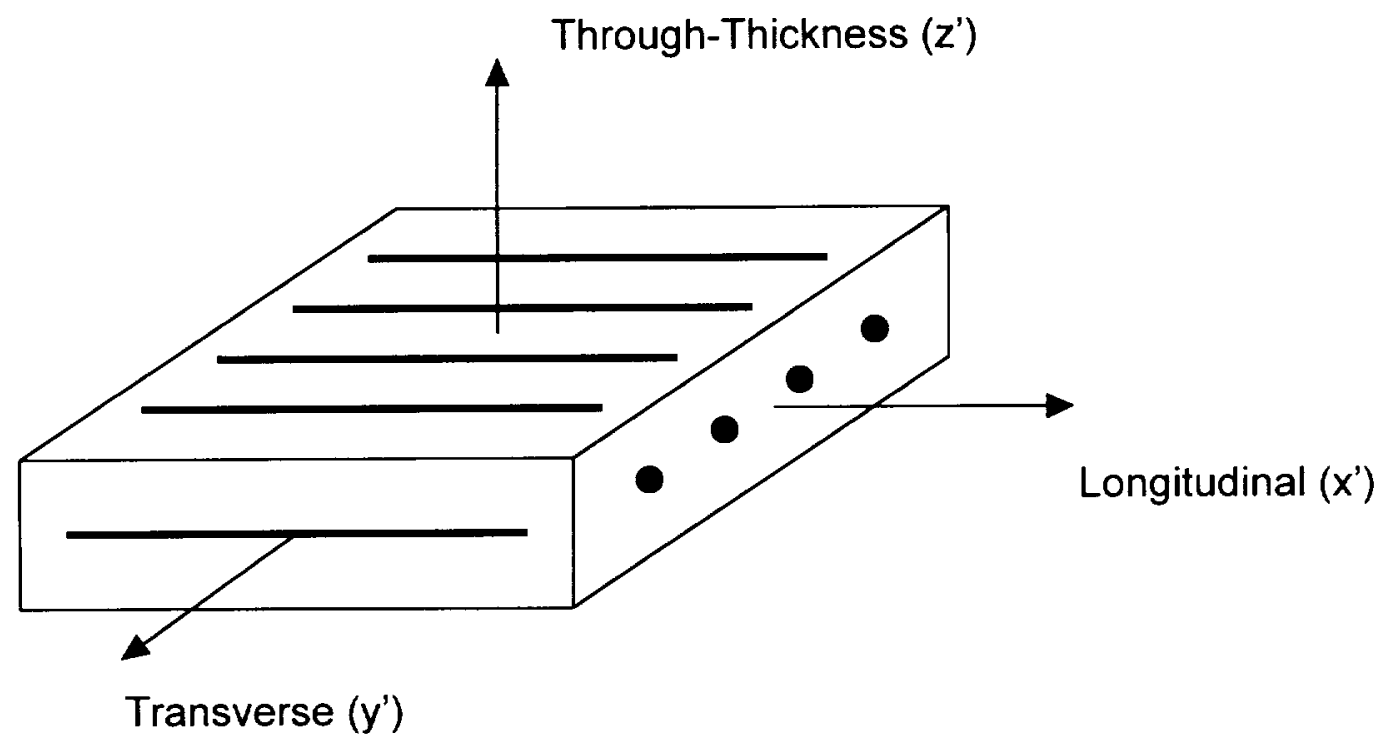

Figure 2.5: Typical ply in the ply coordinate system 
properties are dependent on the volume fraction of fiber used in the composite material, the matrix properties, and the fiber properties. There are many models which are used to determine the ply properties, all of which have modeling limitations. Typically the simplest models are those which are used to determine ply properties in the longitudinal direction. The most basic models are the "rule of mixtures" (ROM) and the "inverse rule of mixtures" (IROM), which result in relations similar to the equations for resistors or springs in series and in parallel. These models are independent of the details of the fiber and matrix geometry. More complicated models are required for the more difficult to model properties, such as the transverse and through-thickness directions properties. These models are can be based on experimental or analytical work, and therefore different researches can develop greatly different models for the same property. More specific details are given in section 4.5 .

However, the details of the fiber and matrix can matter, especially in cases where the properties of the fiber and matrix differ radically. This is the case with electrical properties. Geometry can be important, as in the case of a low resistance percolation path, as shown in Figure 2.6. Another case is the insulative epoxy rich surface layer on composite laminates, as shown in Figure 2.7. These details will be examined on a case by case basis in section 4.4 .

The materials used in this research are carbon fiber/epoxy, glass fiber/epoxy, and Kapton. The epoxy, glass fibers, and Kapton are insulators, while the carbon fibers are conductors. The electrical properties of these materials are tabulated in Table $2.1 .^{27-36}$ 

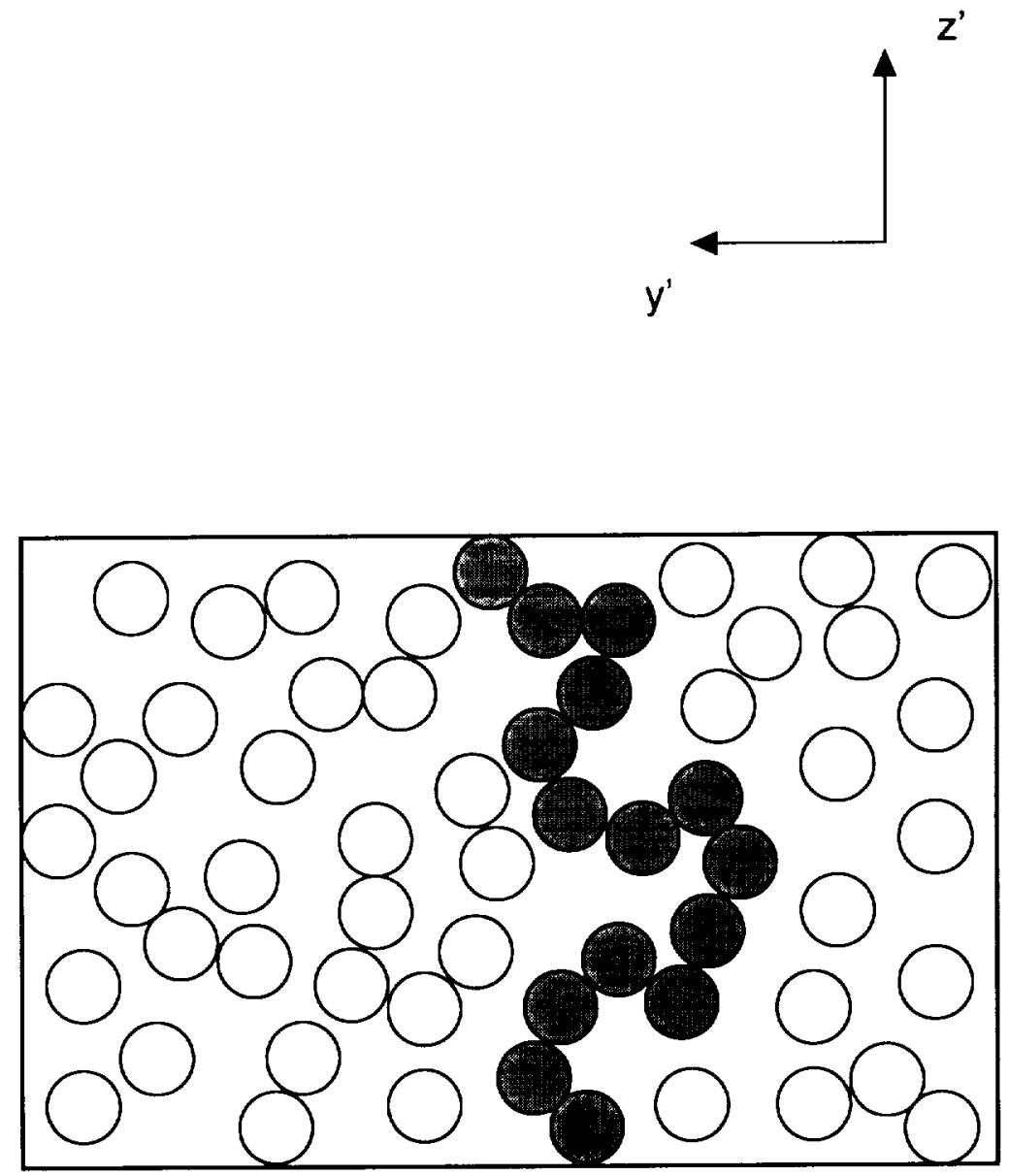

Figure 2.6: Typical composite with a percolation path 


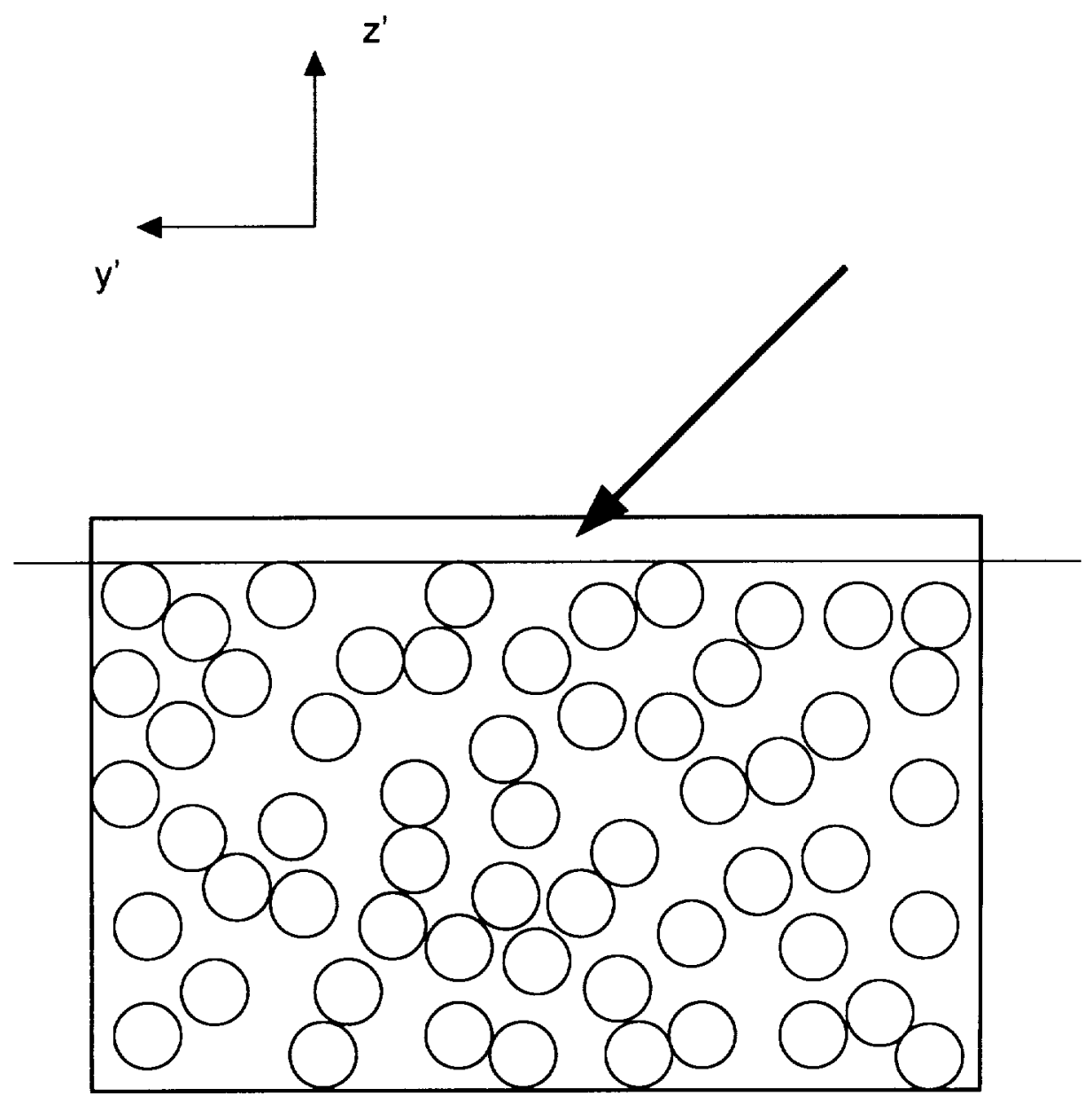

Figure 2.7: Typical composite with matrix rich surface layer 
Table 2.1: Material properties of materials used in this research

\begin{tabular}{||l|c|c|c|c||}
\hline Material & $\begin{array}{c}\text { Density } \\
\left(\mathbf{k g} / \mathbf{m}^{\mathbf{3}}\right)\end{array}$ & $\begin{array}{c}\text { Conductivity } \\
(\mathbf{1} / \mathbf{\Omega}-\mathbf{m})\end{array}$ & $\begin{array}{c}\text { Dielectric } \\
\text { Constant }\end{array}$ & $\begin{array}{c}\text { Dielectric } \\
\text { Strength }(\mathbf{M V} / \mathbf{m})\end{array}$ \\
\hline \hline Carbon Fibers & $1384-2200$ & $2.0 \times 10^{4}--10^{6}$ & ---- & $0.0032-0.0044$ \\
\hline Epoxy & $1052-2187$ & $10^{-3}-10^{-8}$ & $2.78-5.2$ & ---- \\
\hline Glass/Epoxy & $1550-2076$ & $\leq 10^{-10}$ & $4.2-5.68$ & $17.7-21.7$ \\
\hline Carbon/Epoxy Long. & $1577-1700$ & $374-47,600$ & ---- & ---- \\
\hline Carbon/Epoxy Trans. & $1577-1700$ & $1.5-2000$ & ---- & ---- \\
\hline Carbon/Epoxy Thick.. & $1577-1700$ & $0.1-106$ & $-\cdots--$ & --- \\
\hline Kapton & $1420-1670$ & $7 \times 10^{-16}-10^{-15}$ & $2.7-3.5$ & $154-303$ \\
\hline
\end{tabular}




\section{CHAPTER 3}

\section{PROBLEM STATEMENT AND APPROACH}

\subsection{PROBLEM STATEMENT}

In this work, we will develop a model of the deep dielectric charging of composites such that, given the orbit data (apogee height, perigee height, inclination, and solar cycle), the composite electrical properties (conductivity, dielectric constant, and dielectric strength), the density, and the thickness, we can calculate the distribution of charge density, electric field, and voltage with respect to position through the thickness of the composite. We will make suggestions to reduce the probability of electrostatic discharges from occurring due to deep dielectric charging. We will also experimentally measure the continuum electrical properties of carbon fiber / epoxy composites. Finally, a composite material system will be developed with conductivity properties that can be tailored without affecting its mechanical properties.

\subsection{APPROACH}

Previous literature on spacecraft charging is used to develop an understanding of the space environment and the charging phenomena. This understanding is used to develop the analytical models. The analysis has three fundamental goals. The analysis is used to identify key parameters associated with deep dielectric charging and the resulting 
electrostatic discharges, as well as sensitivity of the analysis to these parameters. Secondly, the analysis provides insight into the interaction of the fiber and matrix on a micro-scale. This is required since in the deep dielectric charging model the composite is treated as a homogeneous material, when in fact it is made up of fibers surrounded by matrix. The analysis will also be used to examine specific orbits as case studies, where spacecraft are suspected to have suffered problems due to deep dielectric charging.

The purpose of the experimental program is gain an insight into the parameters which control the through-thickness electrical properties of composites. The critical properties to be investigated are determined from the analytical program; they include the conductivity and the dielectric strength. These properties are initially assumed to be continuum properties. The second purpose on the experimental program is to investigate a conductivity tailorable composite. This is done to aid at reducing the chance of electrostatic discharges occurring, since increasing the conductivity decreases the electric field. Therefore, by increasing the conductivity, the likelihood of an electrostatic discharge is reduced. This material system is to have tailorable electrical properties without changing the mechanical properties significantly.

\subsection{ANALYTICAL TASKS}

The analysis is made up of two parts, the deep dielectric charging analytical model and the modeling of the fiber/matrix micro-scale effects. The deep dielectric charging model comprises several parts. First comes the acquisition of the environmental data from the Environmental Workbench software. In the actual charging model, the 
composite is treated as a homogenous material and the fiber/matrix micro-scale effects are studied separately. The model includes the effects of the space environment on the conductivity of the composite, including radiation induced conductivity and high field conductivity. The model is used to perform sensitivity studies on various parameters to determine which ones affect the likelihood of discharges occurring. These results gave direction to the experimental program, as to which properties should be examined. The model is also used to evaluate the likelihood of discharges occurring on various case study orbits.

The composite is modeled as a homogenous material in the charging model, when in actuality it can be highly anisotropic and locally inhomogeneous. It is made up of fibers surrounded by matrix; therefore various effects of the fiber/matrix micro-scale are modeled. These include the effects of a conductive fiber surrounded by an insulating matrix, unlikely fiber arrangements, and the pure epoxy surface layer inherent on composites.

\subsection{EXPERIMENTAL TASKS}

There are two major experimental tasks. The first task is to get good measurements of through-thickness conductivity and dielectric strength of carbon fiber / epoxy composites. The effects of laminate thickness, surface area, and ply stacking sequence are investigated. The second task is create a conductivity tailorable composite. This task will involve adding conductive carbon black powder to a glass fiber / epoxy composite to control the conductivity. The material properties, both electrical and 
mechanical, are measured and plotted against percentage of carbon black to create preliminary design charts. 


\section{CHAPTER 4}

\section{THEORY}

This chapter presents the theory used in the development of the composite deep dielectric charging analyzer. The geometry being analyzed is presented first, followed by the basic electrostatic equations used. The development of the deep dielectric charging model is then presented. Additional models used to model micromechanical details and composite electrical micromechanics are described. Finally, the equations used to reduce the experimental data are presented.

\subsection{MODEL GEOMETRY}

The geometry of the composite is a 3-D plate with longitudinal, transverse, and through-thickness material properties, as shown in Figures 2.4 and 2.5. However, for the deep dielectric charging model the composite is modeled as a 1-D plate in the throughthickness direction with homogenized material properties, as shown in Figure 4.1. The incoming radiation from the space environment is assumed to be perpendicular to the surface, and uniform across the surface. The front surface of the composite is the surface that is exposed to the space environment, and the back surface is the surface that faces the inside of the spacecraft. The depth ( $\mathrm{x}$ ) is defined as starting at the front surface and ending at the back surface. 

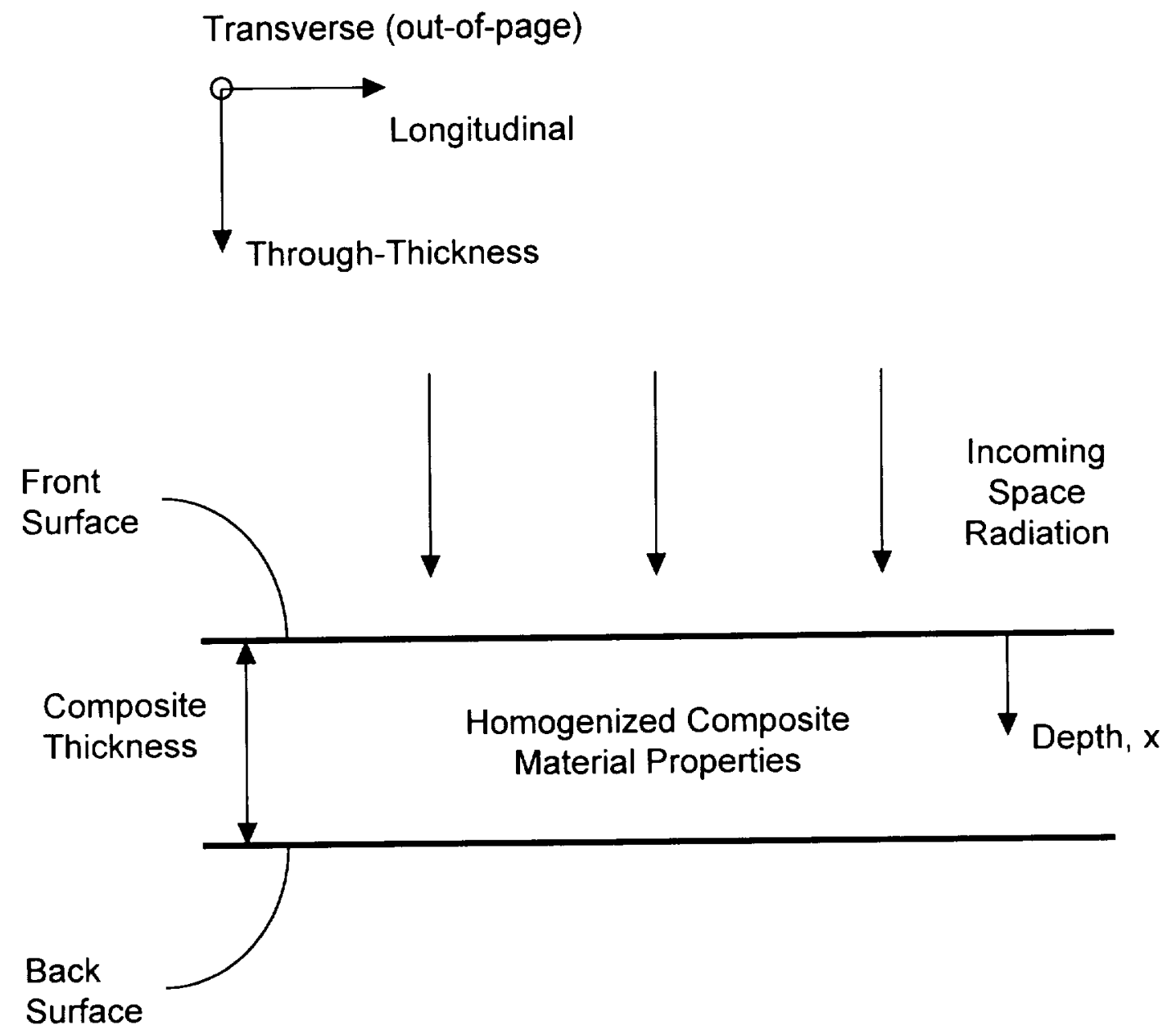

Figure 4.1: Geometry used in the CoDDCA model 


\subsection{BASIC ELECTROSTATIC EQUATIONS}

The equations used in modeling deep dielectric charging are derived in this section. The continuity equation ${ }^{37}$ relates the current density to the charge density. The charge density includes both the incoming charge density rate from the space environment and the charge density in the material.

$$
\nabla \cdot \bar{J}=-\frac{\partial \rho}{\partial t}+\dot{\rho}_{\text {in }}
$$

where $J$ is the current density vector $\left(\mathrm{A} / \mathrm{m}^{2}\right)$, $t$ is the time (s), $\rho$ is the charge density $\left(\# / \mathrm{m}^{3}\right)$, and $\dot{\rho}_{\mathrm{in}}$ is the incoming charge density rate $\left(\# / \mathrm{m}^{3}-\mathrm{s}\right)$ from the space environment. The current density can be represented by:

$$
\overline{\mathrm{J}}=\sigma \overline{\mathrm{E}}
$$

where $\sigma$ is the conductivity $(1 / \Omega-m)$ of the material. It should be noted that the conductivity can be a tensor for composites, and $\mathrm{E}$ is the electric field vector $(\mathrm{V} / \mathrm{m})$. Rewriting equation 1 for the 1-D case, and substituting equation 2 for J:

$$
\frac{\partial \rho}{\partial t}=\dot{\rho}_{\text {in }}-\frac{\partial \sigma E}{\partial x}
$$

where $\mathrm{x}$ is the depth $(\mathrm{m})$ into the material.

Gauss' Law in differential form ${ }^{37}$ relates the electric field to the charge density:

$$
\nabla \cdot \overline{\mathrm{E}}=\frac{1}{\varepsilon} \rho
$$

where $\varepsilon$ is the permittivity $\left(\mathrm{C}^{2} / \mathrm{N}-\mathrm{m}^{2}\right)$ of the material, defined as:

$$
\varepsilon=\kappa \varepsilon_{\mathrm{o}}
$$


where $\kappa$ is the dielectric constant of the material, and $\varepsilon_{\mathrm{o}}$ is the permittivity of free space $\left(\mathrm{C}^{2} / \mathrm{N}-\mathrm{m}^{2}\right)$. Rewriting equation 4 for the 1-D case:

$$
\frac{\partial \mathrm{E}}{\partial \mathrm{x}}=\frac{1}{\varepsilon} \rho
$$

Poisson's equation ${ }^{37}$ relates the voltage to the charge density:

$$
\nabla^{2} \bar{v}=-\frac{1}{\varepsilon} \rho
$$

where $V$ is the voltage $(V)$. Rewriting equation 7 for the 1-D case:

$$
\frac{\partial^{2} \mathrm{~V}}{\partial \mathrm{x}^{2}}=-\frac{1}{\varepsilon} \rho
$$

It can be seen that the voltage and electric field are related by:

$$
\nabla \bar{V}=-\bar{E}
$$

Rewriting equation 9 for the 1-D case:

$$
\frac{\partial V}{\partial x}=-E
$$

In the space environment, the conductivity of a material is not constant, it is affected by the incoming radiation and the electric fields produced within the material. Therefore the conductivity can be expressed as:

$$
\sigma=\sigma_{\mathrm{o}} \mathrm{k}_{\mathrm{E}}+\sigma_{\mathrm{R}}+\sigma_{\mathrm{D}}
$$

where $\sigma_{0}$ is the dark conductivity $(1 / \Omega-m)$ of the material, $k_{E}$ is the conductivity scaling factor for the effect of high electric fields on conductivity, $\sigma_{R}$ is the radiation induced 
conductivity $(1 / \Omega-\mathrm{m})$, and $\sigma_{\mathrm{D}}$ is the delayed conductivity $(1 / \Omega-\mathrm{m})$. The conductivity in materials occurs because atoms have electrons which are free to move through the material. With no electric field applied, the electrons move in random directions, while when a field is applied to the material, the electrons drift in the opposite direction of the field.

\subsubsection{Conductivity In High Electric Fields}

Additional conduction is caused by the generation of charge carriers (electrons) by the ionization of neutral centers (atoms) in the bulk of the material. This ionization is due to a thermal activation process which may be modified by an applied electric field: at low fields there is minimal effects. The conductivity of insulating materials exposed to high electric fields is modeled based on a 'classic dielectric' approach, ${ }^{38}$ as follows:

$$
\sigma_{\mathrm{E}}=\sigma_{\mathrm{o}} \mathrm{k}_{\mathrm{E}}
$$

where $\mathrm{k}_{\mathrm{E}}$ is defined as:

$$
k_{\mathrm{E}}=\left(\frac{2+\cosh \left(\beta_{\mathrm{F}} \sqrt{\mathrm{E}} / 2 \mathrm{kT}\right)}{3}\right)\left(\frac{2 \mathrm{kT}}{\mathrm{eE} \delta} \sinh \left(\frac{\mathrm{e} E \delta}{2 \mathrm{kT}}\right)\right)
$$

where $\mathrm{k}$ is Boltzmann's constant $\left(1.380658 \times 10^{-23} \mathrm{~J} / \mathrm{K}\right), \mathrm{T}$ is temperature $(\mathrm{K})$, e is the charge of an electron $(\mathrm{C}), \delta$ is the jump distance $(\mathrm{m})$, and $\beta_{\mathrm{F}}$ is the Frenkel parameter ${ }^{39}$ $(\sqrt{C}-\sqrt{N}-m)$ defined as:

$$
\beta_{\mathrm{F}}=\left(\frac{\mathrm{e}^{3}}{\pi \varepsilon}\right)^{\frac{1}{2}}
$$

In $k_{\mathrm{E}}$ the first term in brackets is the field dependence of carrier concentration and the second term is the field dependence of carrier mobility. It has been shown ${ }^{38}$ that because 
of small jump distance, only a few Angstrom units, the rise in the carrier mobility may be neglected up to fields of approximately $100 \mathrm{MV} / \mathrm{m}$. Since this value is above the dielectric strength of most materials, the second term will be set to one in the charging model. It should be noted that there is extremely good agreement between this model and measure values of high field conductivity.

\subsubsection{Radiation Induced Conductivity}

When insulators are exposed to radiation, it is known that the conductivity of the material increases. ${ }^{40-42}$ This occurs because the number of additional conducting ionelectron pairs produced in the material is proportional to the energy deposited by incident radiation. This radiation induced conductivity is defined as:

$$
\sigma_{\mathrm{R}}=\mathrm{k}_{\mathrm{R}} \dot{\mathrm{D}}^{\Delta}
$$

where $k_{R}$ is the coefficient of radiation induced conductivity (s/ $\left./ \Omega-m-r a d\right), \dot{D}$ is the radiation dose rate $(\mathrm{rad} / \mathrm{s})$, and $\Delta$ is the radiation induced conductivity exponent which is a material dependent parameter. Both $k_{R}$ and $\Delta$ have been empirically determined for many materials by many investigators. $k_{R}$ is relatively small for most organic insulators, and can be 2 or more orders of magnitude greater for inorganic insulators. It should also be noted that $k_{R}$ for a given material can vary by as much as 2 orders of magnitude between different sources. This could be due to older experimental techniques used in some of the older sources and the use of samples manufactured at different times with different manufacturing procedures. The radiation induced conductivity exponent $(\Delta)$ usually lies between 0.5 and 1.0; with most organic substances it lies around 1.0. 


\subsubsection{Delayed Conductivity}

The delayed conductivity is defined as:

$$
\sigma_{\mathrm{D}}=\mathrm{k}_{\mathrm{D}} \dot{\mathrm{D}} \mathrm{t}
$$

where $k_{D}$ is the coefficient of delayed conductivity $(1 / \mathrm{rad}-\Omega-\mathrm{m})$. Frederickson ${ }^{20}$ found that delayed conductivity is negligibly small, and will therefore be assumed to equal zero and will not be included.

\subsubsection{Conductivity Summary}

The expression for the conductivity that will be used in the charging model is as follows:

$$
\sigma=\sigma_{\mathrm{o}}\left(\frac{2+\cosh \left(\beta_{\mathrm{F}} \sqrt{\mathrm{E}} / 2 \mathrm{kT}\right)}{3}\right)+\mathrm{k}_{\mathrm{R}} \dot{\mathrm{D}}^{\wedge}
$$

\subsection{DEEP DIELECTRIC CHARGING MODEL}

The basic problem is that high energy particles from the space environment penetrate the surface of the composite material, and start losing energy until they stop somewhere within the material. These stopped particles induce an electric field within the material. This electric field causes the particles to move, thus causing a current in the material. This current in turn influences the electric field. The electric field continues to grow until equilibrium is reached between the incoming particles from the space environment $\left(\dot{\mathrm{\rho}}_{\text {in }}\right)$ and the particles leaving due to the current $(\mathrm{J})$. This is also known as the steady state. Another possibility is that the electric field exceeds the dielectric strength of the material before equilibrium is reached, at which point a breakdown and 
subsequent electrostatic discharge occurs. A model of this process will be described. A Fortran code, CoDDCA (Composite Deep Dielectric Charging Analyzer), based on this model, was written. It calculates the electric field, voltage, and charge density as functions of depth into material and time, until one of the two above mentioned possibilities occur. Appendix A contains the user manual for CoDDCA. Magnetic field effects are assumed to be negligible because of the low current density $\left(\leq 10 \mathrm{nA} / \mathrm{cm}^{2}\right)$ and low velocity of charge carriers in insulators $(\leq 105 \mathrm{~m} / \mathrm{s}$ « $\mathrm{c}) .^{18}$

\subsubsection{Environment}

The charged particle environment is acquired from the Environment Workbench (EWB) software, written by the S-Cubed division of Maxwell Laboratories, Inc. ${ }^{43}$ The program runs the electron and proton models AE8 and AP8, and gives empirical omnidirectional flux data at different energy levels for different orbits and solar cycle conditions (maximum or minimum). The output from the Environmental Workbench is unidirectional penetrating flux, EWB converted it internally. The E and $\mathrm{P}$ in the model names AE8 and AP8 refer to electron and proton, and 8 is the version number of the models. These models of the near Earth radiation regime were created by the National Space Science Data Center, from data compiled from many different spacecraft. AE8 gives the fluxes of electrons in the energy range $50 \mathrm{keV}$ to $7 \mathrm{MeV}$, and AP8 gives the fluxes of protons in the energy range $50 \mathrm{keV}$ to $500 \mathrm{MeV}$. The time dependent variations of radiation fluxes such as those due to geomagnetic substorms or short term solar modulations such as solar particle events are not included. 
The depth the particles penetrate into the material is calculated using empirical information on the stopping power of silicon. The stopping power is given as the linear energy transfer (LET) which is a function of energy, as shown in Figure 4.2. The stopping power in silicon can be used, even thought the materials used here are composites, because the linear energy transfer scales with density. This allows it to be used with any material as long as its density is known. Using the particle flux versus energy from EWB and the LET curves, the particle flux versus depth of penetration into the material can be calculated. EWB produces a distribution of particles as a function of energy, as shown in Figures 4.3. The LET curve provides the depth a particle penetrates into a material as a function of energy. These two sets of data are combined graphically to produce a distribution of particle flux as a function of depth into the material, as shown in Figures 4.4. This is accomplished by determining equations for the electron and proton LET, as shown in Figures 4.5, which can be used to convert the EWB energy data into penetrating depth data, using the following equation:

$$
\mathrm{x}=\frac{\mathrm{E}_{\mathrm{g}}}{\operatorname{LET} \rho_{\text {mech }}}
$$

where Eg is the particle energy $(\mathrm{MeV})$, and $\rho_{\text {mech }}$ is the density $\left(\mathrm{kg} / \mathrm{m}^{3}\right)$ of the material. Figures 4.6 show the relationship between the electron and proton energy and the depth the particles penetrate.

Finally, to determine the incoming particle charge density rate, as shown in Figure 4.7, the incoming particles per area (particle flux) must be converted to incoming particles per volume (charge density rate). This is easily accomplished since the flux data 


\section{STOPPING POWERS IN SILICON}

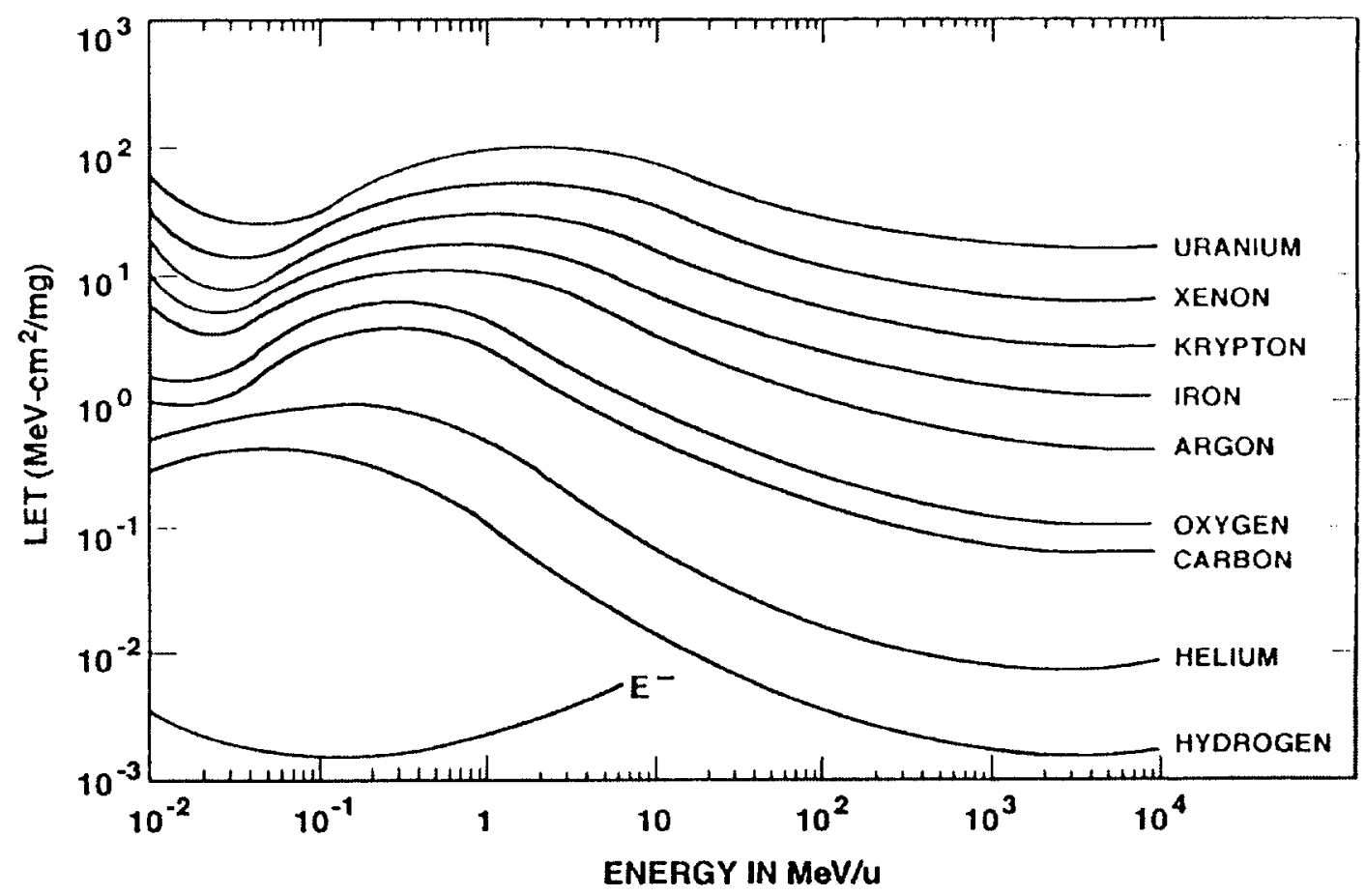

Figure 4.2: $\quad$ Stopping powers in silicon for various particles ${ }^{44}$ 

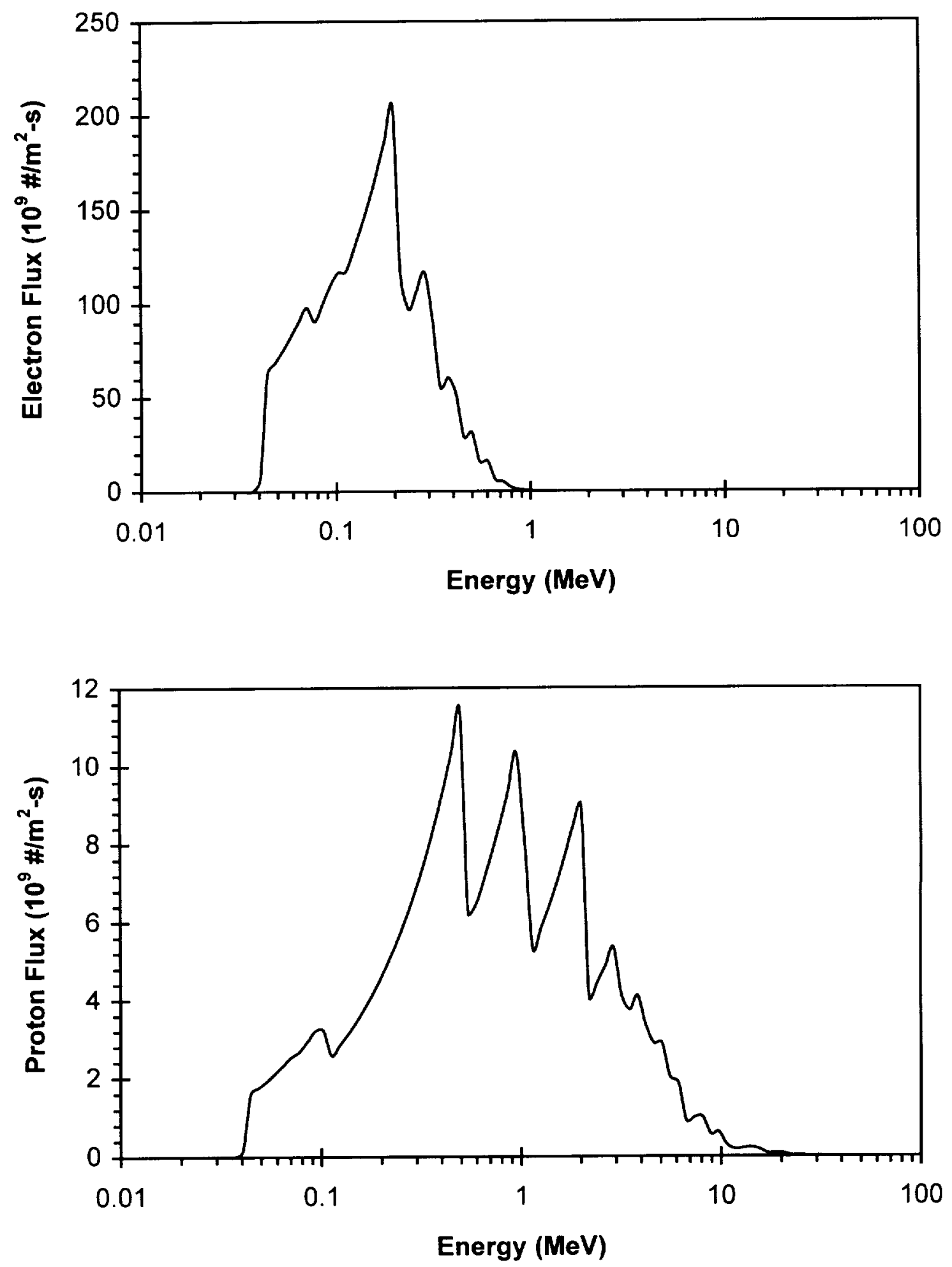

Figure 4.3: Electron and proton fluxes as a function of energy 

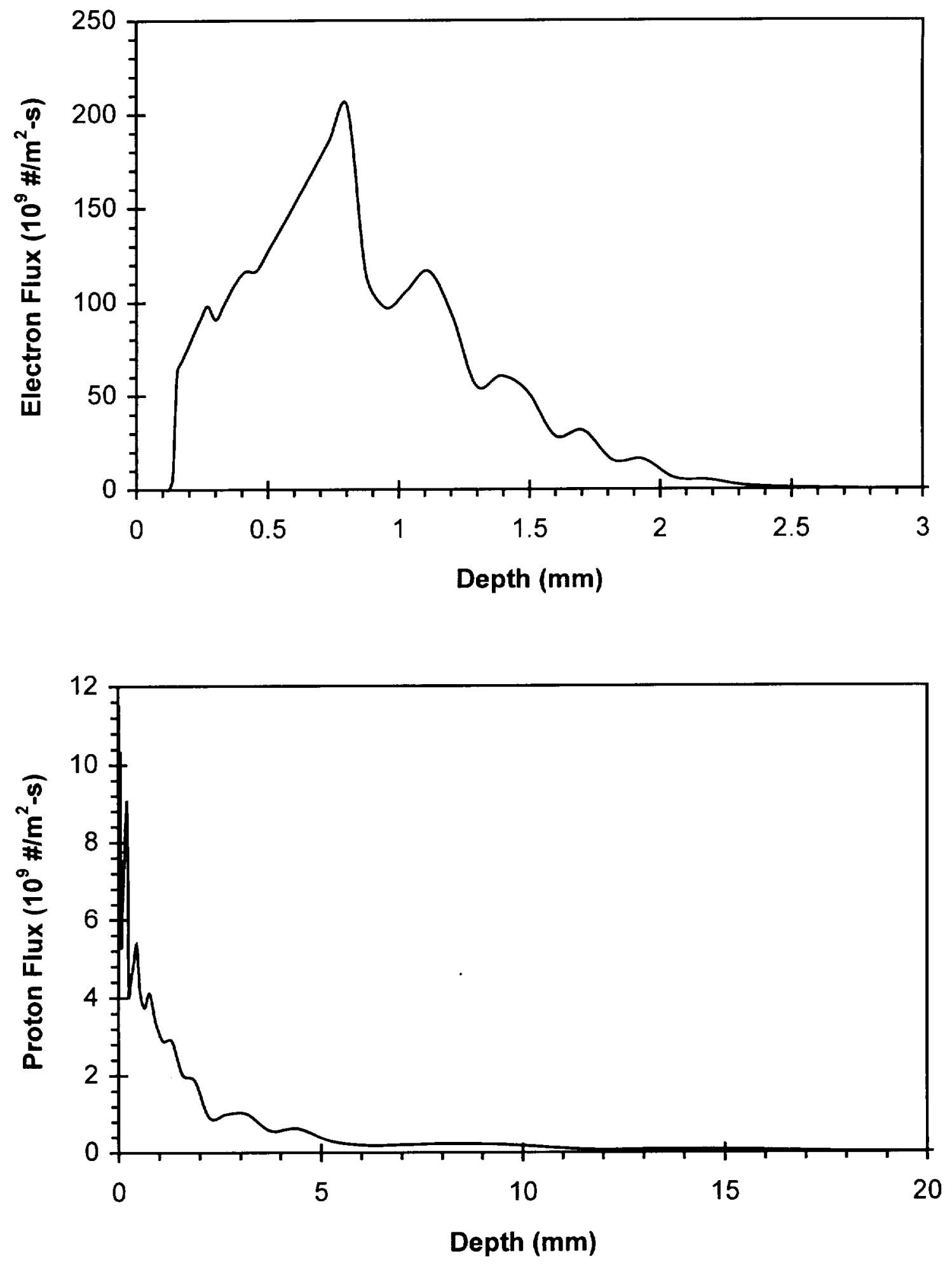

Figure 4.4: Electron and proton fluxes as a function of depth 

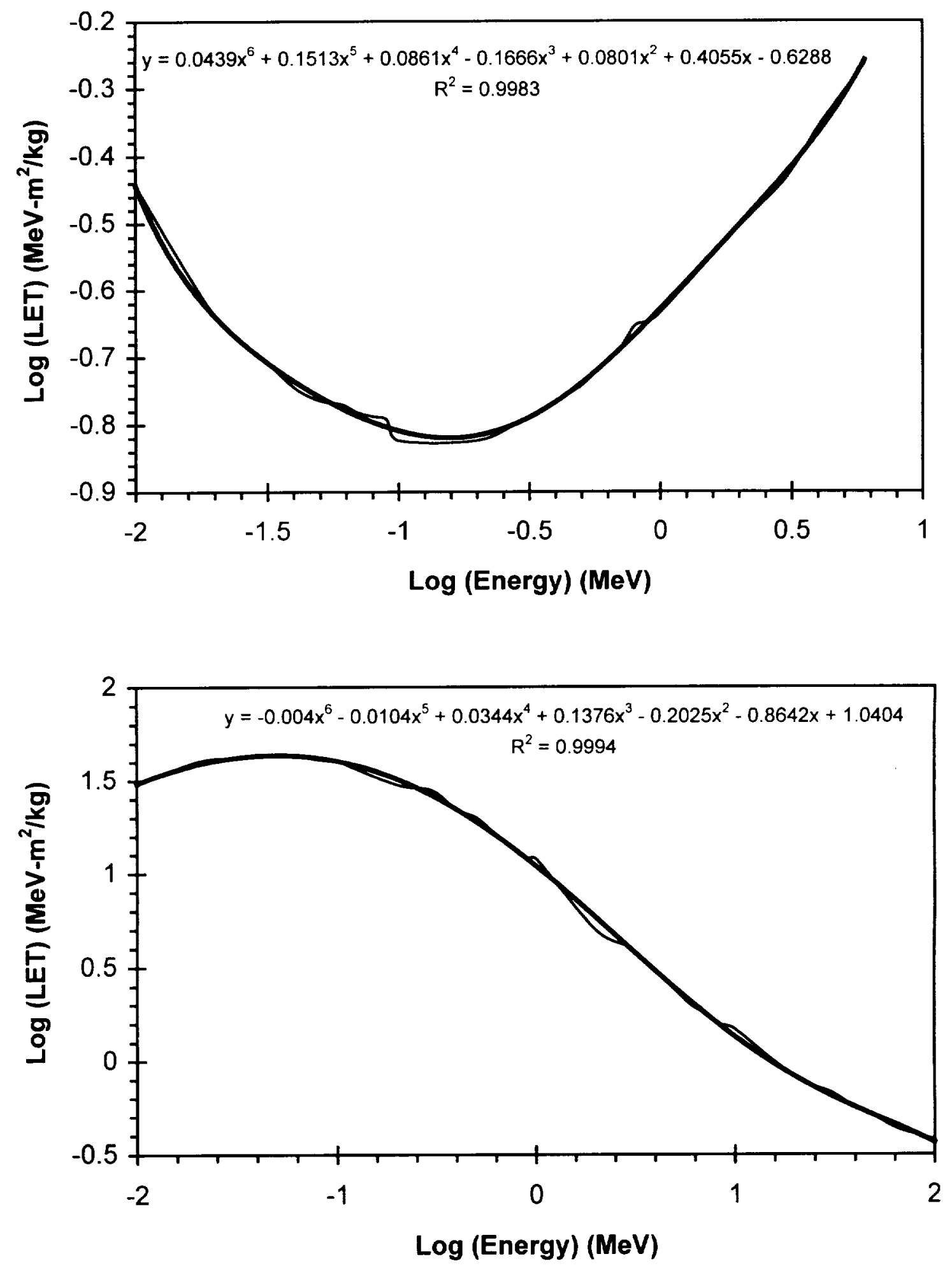

Figure 4.5: Electron and proton LET as a function of energy 

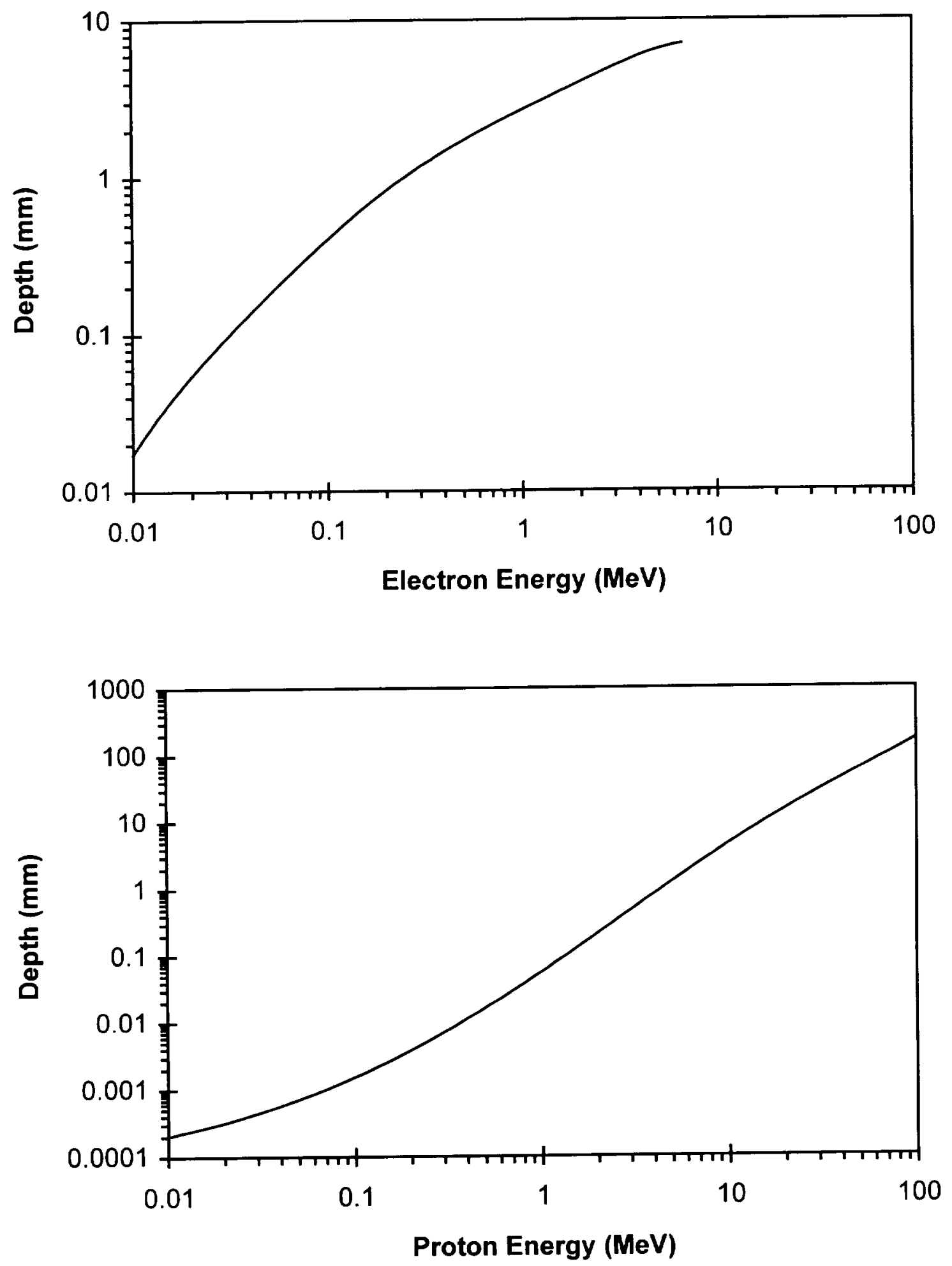

Figure 4.6: Electron and proton penetration depth as a function of energy 

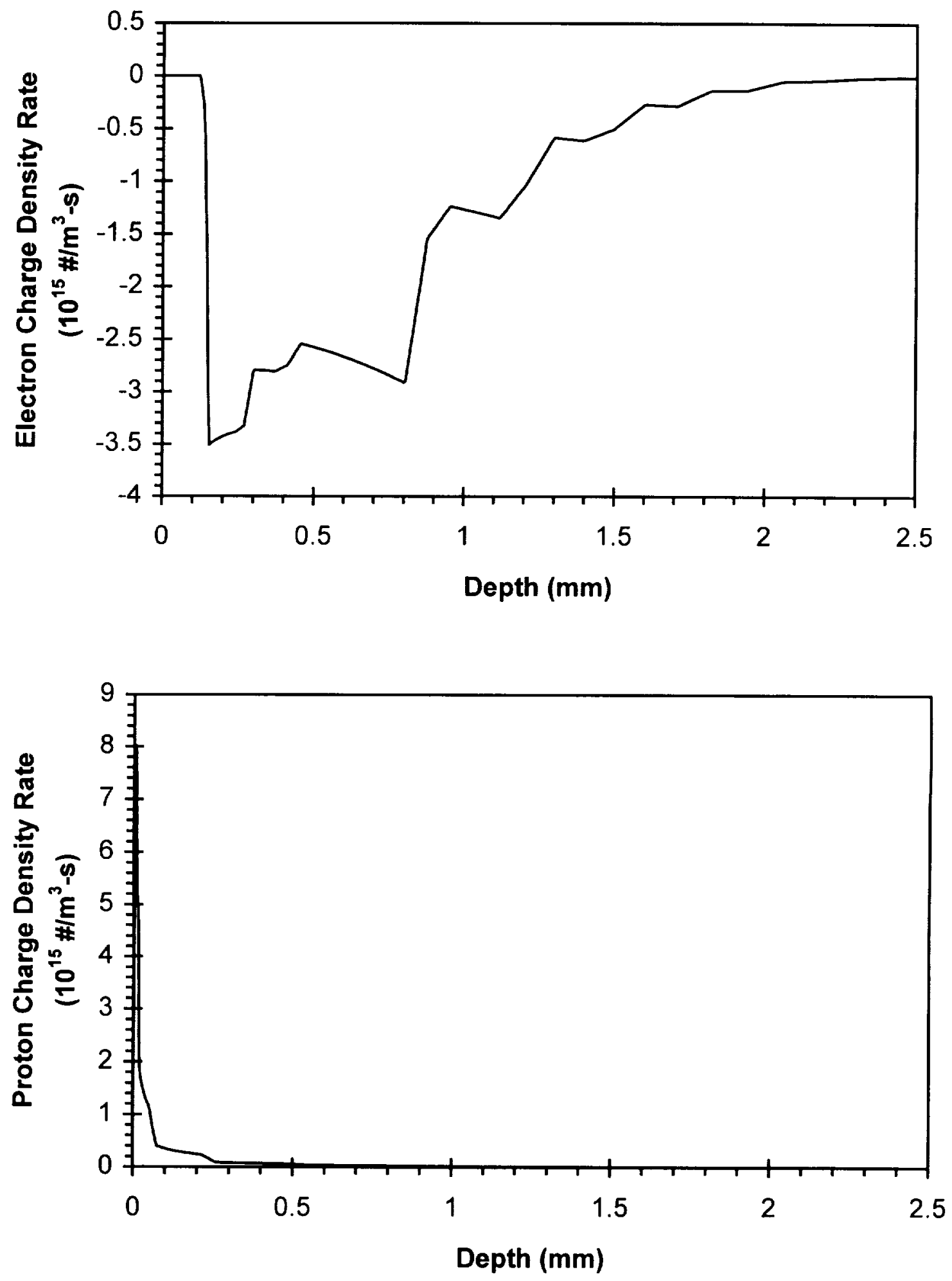

Figure 4.7: Electron and proton charge density rates as a function of depth 
is grouped in discrete bins, each bin is at a different depth in the material. Therefore, by assuming that the flux of particles that penetrate to a certain depth are evenly distributed from midway back to the previous depth to midway forward to the next depth $(\Delta x)$, as shown in Figure 4.8. Thus mathematically the charge density rate of a certain depth bin is equal to the flux associated with that depth bin divided by $\Delta x$. The difference between the electron charge density rate and the proton charge density rate gives the net incoming charge density rate, $\dot{\rho}_{\text {in }}$, as a function of penetrating depth, as shown in Figure 4.9.

This is a simpler approach than what Soubeyran ${ }^{21}$ did with a Monte Carlo deposition model. However, a simpler model is acceptable for our work since the model is primarily being used to find order-of-magnitude estimates of the problem and to give direction to the experimental portion of this research. The added accuracy of the Monte Carlo deposition would not change the general conclusions of our model or the choice of parameters to be examined experimentally. The most likely materials to be susceptible to deep dielectric charging will still be identified as well as which parameters affect the likelihood of an electrostatic discharge. The main difference between the Monte Carlo model and our model is that in our model all particles of a certain energy penetrate to a certain depth, while with the Monte Carlo model there is a distribution as to where the particles of a certain energy penetrate to. This would have the effect of spreading out the 'bins' used in our model and smoothing out the distributions of particles with depth somewhat. 


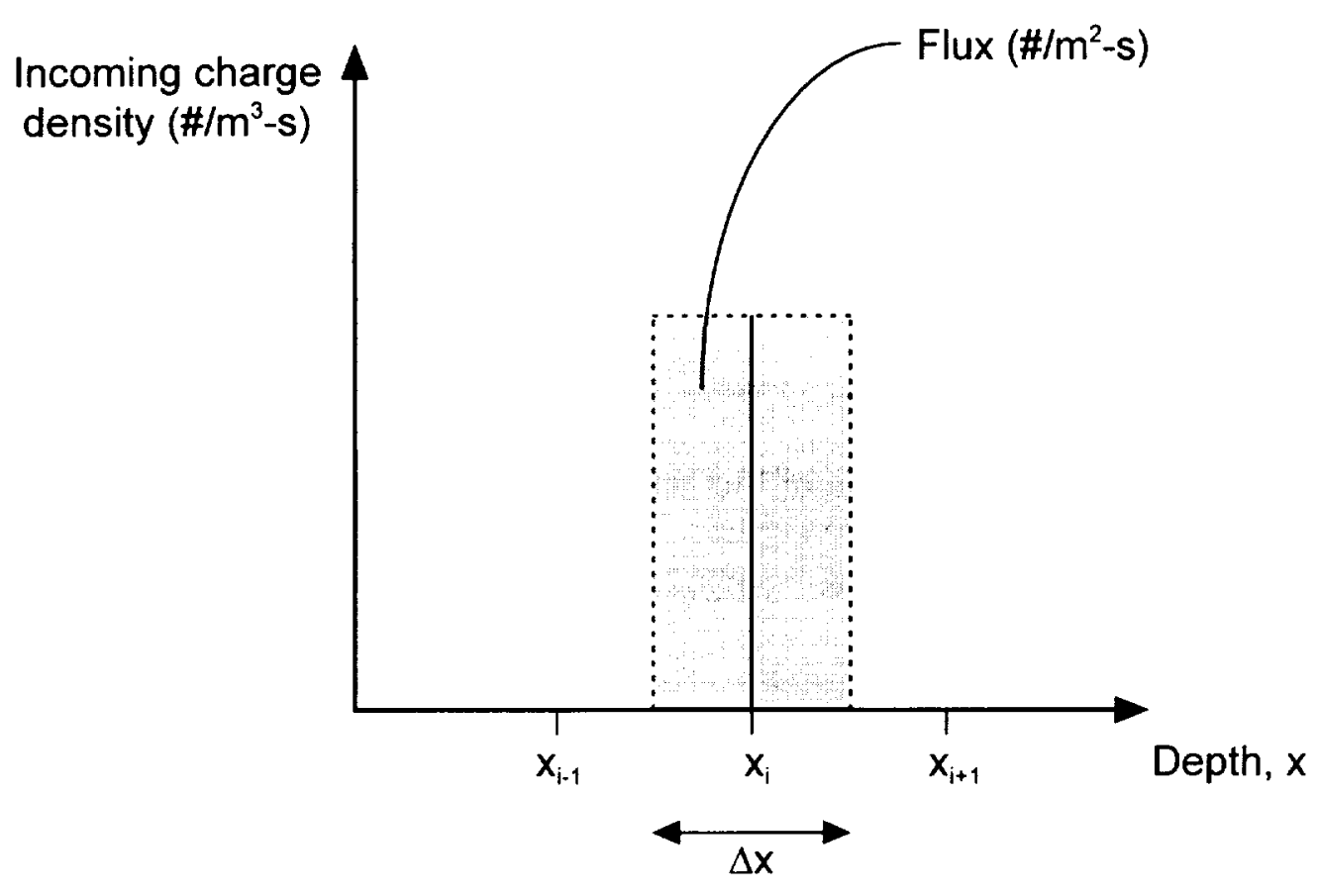

Figure 4.8: Representation of how the particle per area data is converted into particle per volume data 


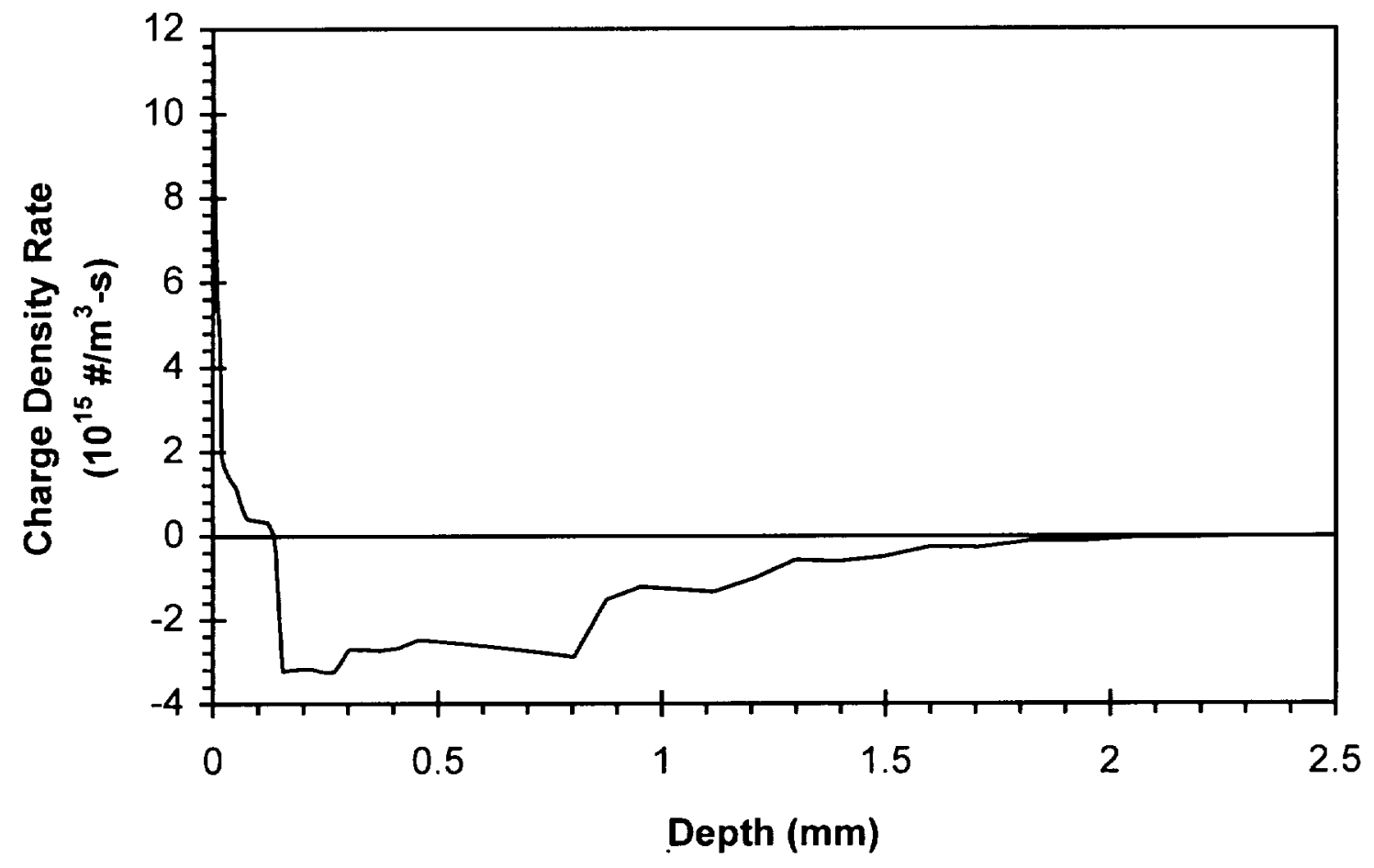

Figure 4.9: Incoming charge density rate as a function of depth 
For certain calculations, such as the radiation induced conductivity, the dose rate is required. The dose rate is defined as: ${ }^{45}$

$$
\dot{\mathrm{D}}=\int_{\mathrm{E}_{\mathrm{g}}} \frac{\Phi\left(\mathrm{E}_{\mathrm{g}}\right) \mathrm{dE}_{\mathrm{g}}\left(\mathrm{dE}_{\mathrm{g}} / \mathrm{dx}\right) \mathrm{QF}\left(\mathrm{E}_{\mathrm{g}}\right)}{\rho_{\text {mech }}}
$$

where $\Phi\left(E_{g}\right)$ is the charged particle flux $\left(\# / \mathrm{m}^{2}-\mathrm{s}\right)$ with kinetic energy between $E_{g}$ and $E_{g}+$ $\mathrm{dE}_{\mathrm{g}}, \mathrm{dE}_{\mathrm{g}} / \mathrm{dx}$ is the stopping power $(\mathrm{MeV} / \mathrm{m})$ of the material, and $\mathrm{QF}\left(\mathrm{E}_{\mathrm{g}}\right)$ is the quality factor for particles of energy $E_{\mathrm{g}}$. The quality factor is defined as 1 for electrons and 10 for protons with energy less than $14 \mathrm{MeV}$. Equation 19 can also be written for numerical data as:

$$
\dot{\mathrm{D}}=\sum_{\mathrm{i}} \frac{\Phi_{\mathrm{i}}\left(\mathrm{dE}_{\mathrm{g}} / \mathrm{dx}\right) \mathrm{QF}_{\mathrm{i}}}{\rho_{\text {mech }}}
$$

where $\mathrm{i}$ represents the parameter at energy i. This equation can be simplified by observing that:

$$
\frac{\mathrm{dE}_{\mathrm{g}} / \mathrm{dx}}{\rho_{\text {mech }}}=\mathrm{LET}
$$

therefore, equation 20 becomes:

$$
\dot{\mathrm{D}}=\sum_{\mathrm{i}} \Phi_{\mathrm{i}} \mathrm{LET}_{\mathrm{i}} \mathrm{QF}_{\mathrm{i}}
$$

\subsubsection{Closed Form Solution}

A closed form solution to the deep dielectric charging problem can be attempted if it is assumed the conductivity of the material is constant in space (the $\mathrm{x}$ direction). This is the case if high field conductivity is neglected. Therefore equation 3 becomes:

$$
\frac{\partial \rho}{\partial t}=\dot{\rho}_{\text {in }}-\sigma \frac{\partial E}{\partial x}
$$


Substituting equation 6 into equation 23:

$$
\frac{\partial \rho}{\partial \mathrm{t}}=\dot{\rho}_{\text {in }}-\frac{\sigma}{\varepsilon} \rho
$$

this equation can be solved for the charge density. Since $\rho$ is a function of $x$ and $t$, and $\dot{\rho}_{\text {in }}$ is only a function of $x$. Re-writing, equation 24 becomes:

$$
\frac{\partial \rho(\mathrm{x}, \mathrm{t})}{\partial \mathrm{t}}+\frac{\sigma}{\varepsilon} \rho(\mathrm{x}, \mathrm{t})=\dot{\rho}_{\text {in }}(\mathrm{x})
$$

Assuming $\rho(x, t)$ is separable:

$$
\rho(x, t)=X(x) T(t)
$$

Substituting equation 26 into equation 25 , and re-arranging:

$$
\frac{\mathrm{dT}}{\mathrm{dt}}+\frac{\sigma}{\varepsilon} \mathrm{T}=\frac{\dot{\rho}_{\text {in }}}{\mathrm{X}}
$$

This differential equation is general first order linear, and can be solved as:

$$
\rho(\mathrm{x}, \mathrm{t})=\frac{\varepsilon}{\sigma} \dot{\rho}_{\text {in }}(\mathrm{x})+\mathrm{X}(\mathrm{x}) \mathrm{e}^{-\frac{\sigma}{\varepsilon} \mathrm{t}}
$$

$X(x)$ can be solved using the initial condition that at $t=0, \rho(x, 0)=0$ :

$$
\mathrm{X}(\mathrm{x})=-\frac{\varepsilon}{\sigma} \dot{\rho}_{\mathrm{in}}
$$

Therefore, equation 28 becomes:

$$
\rho(\mathrm{x}, \mathrm{t})=\frac{\varepsilon}{\sigma} \dot{\rho}_{\text {in }}(\mathrm{x})\left(1-\mathrm{e}^{-\frac{\sigma}{\varepsilon} \mathrm{t}}\right)
$$

Thus the steady state value of the charge density, $\rho(x, \infty)$ is: 


$$
\rho(\mathrm{x}, \infty)=\frac{\varepsilon}{\sigma} \dot{\rho}_{\mathrm{in}}(\mathrm{x})
$$

The time to steady state, defined as when $\rho$ reaches $99 \%$ of its actual steady-state value, is defined as follows:

$$
\mathrm{t}=-\frac{\varepsilon}{\sigma} \ln (0.01)
$$

The electric field is calculated using equation 6 :

$$
E=\frac{1}{\varepsilon} \int \rho d x
$$

Therefore, by integrating equation 30 , the electrical field is:

$$
E=\frac{1}{\sigma}\left(1-\mathrm{e}^{-\frac{\sigma}{\varepsilon} t}\right) \int \dot{\rho}_{\text {in }}(x) d x+C_{1}
$$

This equation is integrated numerically using the trapezoidal rule. The voltage is calculated using equation 10 :

$$
V=-\int E d x
$$

Therefore, by integrating equation 34 , the voltage is:

$$
V=-\frac{1}{\sigma}\left(1-e^{-\frac{\sigma}{\varepsilon}}\right) \iint \dot{\rho}_{\text {in }}(x) d x^{2}+C_{1} x+C_{2}
$$

This equation is also integrated numerically using the trapezoidal rule.

It should be noted that only the incoming charge density rate needs to be integrated, as the rest of the equation is not a function of $\mathrm{x}$. This allows the integrals to be evaluated only once for the electric field and voltage at any desired time. The 
boundary conditions $C_{1}$ and $C_{2}$ can be calculated based on either one electric field and one voltage boundary condition, or two voltage boundary conditions.

\subsubsection{Finite Difference Solution}

If the high field conductivity is not ignored, the material conductivity is not constant in space, and cannot be removed from the differential in equation 3. Therefore, by using the product rule for differentials, equation 3 becomes:

$$
\frac{\partial \rho}{\partial t}=\dot{\rho}_{i n}-\sigma \frac{\partial E}{\partial x}-E \frac{\partial \sigma}{\partial x}
$$

Substituting equation 6 into equation 37 :

$$
\frac{\partial \rho}{\partial \mathrm{t}}=\dot{\rho}_{\mathrm{in}}-\frac{\sigma}{\varepsilon} \rho-\mathrm{E} \frac{\partial \sigma}{\partial \mathrm{x}}
$$

This equation is solved numerically with a central difference in space, forward difference in time routine.

The electric field can be calculated using equation 33 :

$$
E=\frac{1}{\varepsilon} \int \rho \mathrm{dx}+C_{1}
$$

This equation is integrated numerically using the trapezoidal rule. The voltage can be calculated using equation 35 :

$$
V=-\frac{1}{\varepsilon} \iint \rho d x+C_{1} x+C_{2}
$$

This equation is also integrated numerically using the trapezoidal rule. It should be noted that since the charge density changes, these integrals need to be evaluated for every time 
step. The boundary conditions $C_{1}$ and $C_{2}$ can be calculated based on either one electric field and one voltage boundary condition, or two voltage boundary conditions.

Equation 39, equation 40 , and equation 38 are solved sequentially, in a time stepping routine. This continues until either steady state is reached or the electric field exceeds the dielectric strength of the material and an electrostatic discharge occurs.

\subsubsection{The Analytical Model}

To summarize, a tool now exists which can determine the charge density, electric field, and voltage as functions of depth into the composite material and time, in a specified orbital environment, and thus predict if an electrostatic discharge will occur. The program can run either model, and can be run for any orbit by specifying the orbit parameters (the apogee height, perigee height, inclination, and solar cycle condition) in EWB. Substorms and solar particle events can also be user specified, as well as the material parameters. The material is characterized electrically by its conductivity, dielectric constant, dielectric strength, density, and boundary conditions. The structure is characterized by its thickness, temperature, and boundary conditions. There are also options to include radiation induced conductivity and high field conductivity.

\subsection{MICROMECHANICAL DETAILS}

This section deals with the modeling of the interactions between the fibers and matrix of the composite material and their effects on the electric field. The first model examines the electric field in the matrix as a function of the radial distance from a 
conductive fiber, in which the electric field is zero. The second model examines the insulative pure epoxy surface layer of carbon fiber / epoxy composites.

\subsubsection{Micro-scale Model}

At the micro-scale, a concern is that higher electric fields could be possible due to geometric details of the fibers imbedded in the matrix. This model examines the steadystate electric field in an insulative matrix which passes around a conductive fiber, as shown in Figure 4.10. Here $r$ is the radial distance $(m)$ from the center of the fiber, $\theta$ is the angular distance counter-clockwise from the $x$ axis, $R_{f}$ is the radius of the fiber $(m)$, and $E_{o}$ is the steady-state electric field $(\mathrm{V} / \mathrm{m})$ calculated using the composite deep dielectric charging model. The electric field is assumed to be constant far away from the fiber, since the scale of the problem is much smaller than the scale used in the composite deep dielectric charging model.

To solve the problem, Poisson's equation (equation 7) needs to be expressed in radial coordinates:

$$
\frac{1}{\mathrm{r}^{2}} \frac{\partial}{\partial \mathrm{r}}\left(\mathrm{r}^{2} \frac{\partial \mathrm{V}}{\partial \mathrm{r}}\right)+\frac{1}{\mathrm{r}^{2} \sin (\theta)} \frac{\partial}{\partial \theta}\left(\sin (\theta) \frac{\partial \mathrm{V}}{\partial \theta}\right)+\frac{1}{\mathrm{r}^{2} \sin ^{2}(\theta)} \frac{\partial \mathrm{V}}{\partial \phi}=-\frac{1}{\varepsilon} \rho
$$

assuming $\rho=0$ for simplicity, equation 41 becomes Laplace's equation:

$$
\frac{1}{r^{2}} \frac{\partial}{\partial r}\left(r^{2} \frac{\partial V}{\partial r}\right)+\frac{1}{r^{2} \sin (\theta)} \frac{\partial}{\partial \theta}\left(\sin (\theta) \frac{\partial V}{\partial \theta}\right)+\frac{1}{r^{2} \sin ^{2}(\theta)} \frac{\partial V}{\partial \phi}=0
$$

assuming $\mathrm{V}$ is independent of $\phi$, and separable in $\mathrm{r}$ and $\theta$, the solution to equation 42 is: 

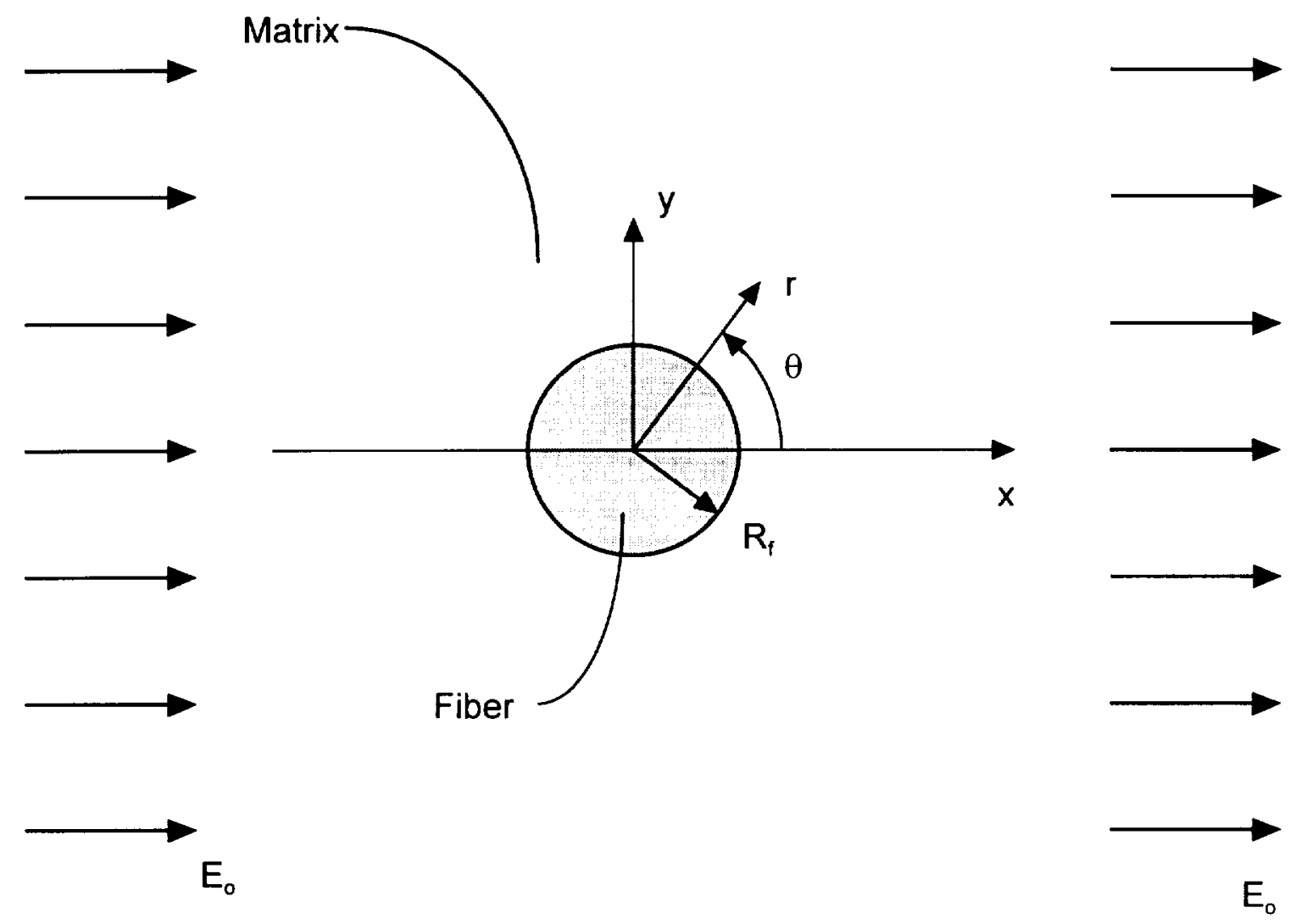

Figure 4.10: Schematic of the fiber-matrix micro-scale model 


$$
V(r, \theta)=\sum_{i=0}^{\infty}\left(A_{i} r^{i}+\frac{B_{i}}{r^{i+1}}\right) P_{i}(\cos (\theta))
$$

where $A_{i}$ and $B_{i}$ are arbitrary constants associated with the solution of $2^{\text {nd }}$ order differential equations, and $\mathrm{P}_{\mathrm{i}}(\mathrm{x})$ is defined by the Rodrigues formula as:

$$
P_{i}(x)=\frac{1}{2^{i} i !}\left(\frac{d}{d x}\right)^{i}\left(x^{2}-1\right)^{i}
$$

The first boundary conditions required to solve for the constants is the voltage is zero at the fiber-matrix interface since the fiber is a conductor. The second boundary condition is that far from the fiber the voltage approaches the far field voltage created by the far field electric field $\left(\mathrm{E}_{\mathrm{o}}\right)$. The far field voltage is equal to the electric field multiplied by the distance from the center of the fiber. The boundary conditions in circular coordinates are:

$$
\begin{array}{ll}
\mathrm{V}=0 & @ \mathrm{r}=\mathrm{R}_{\mathrm{f}} \\
\mathrm{V} \Rightarrow-\mathrm{E}_{\mathrm{o}} \mathrm{r} \cos (\theta) & @ \mathrm{r}>>\mathrm{R}_{\mathrm{f}}
\end{array}
$$

Applying the first boundary condition, and observing that the cosine term cannot be always equal to zero, the second constant is determined in terms of the first constant:

$$
B_{i}=-A_{i} R_{f}^{2 i+1}
$$

Therefore, equation 43 becomes:

$$
V(r, \theta)=\sum_{i=0}^{\infty} A_{i}\left(r^{i}-\frac{R_{f}^{2 i+1}}{r^{i+1}}\right) P_{i}(\cos (\theta))
$$


Applying the second boundary condition, and observing that at $r$ \& $R_{r}$ the second term in the brackets of equation 49 is negligible, the first constant can be solved for by equating like terms. The first constant is solved as:

$$
A_{i}=-E_{0}
$$

Therefore the voltage solution is:

$$
V(r, \theta)=-E_{0}\left(r-\frac{R_{f}{ }^{3}}{r^{2}}\right) \cos (\theta)
$$

To calculate the electric field equation 9 is used in radial coordinates:

$$
E(r, \theta)=-\left[\frac{\partial V}{\partial r} \hat{r}+\frac{1}{r} \frac{\partial V}{\partial \theta} \hat{\theta}\right]
$$

Therefore the electric field is:

$$
E(r, \theta)=E_{o}\left[\cos (\theta)\left(1+2 \frac{R_{f}^{3}}{r^{3}}\right) \hat{r}-\sin (\theta)\left(1-\frac{R_{f}^{3}}{r^{3}}\right) \hat{\theta}\right]
$$

The magnitude of the electric field is:

$$
E(r, \theta)=E_{o}\left(\cos ^{2}(\theta)\left(1+4 \frac{R_{f}^{3}}{r^{3}}+4 \frac{R_{f}^{6}}{r^{6}}\right)+\sin ^{2}(\theta)\left(1-2 \frac{R_{f}^{3}}{r^{3}}+\frac{R_{f}^{6}}{r^{6}}\right)\right)^{\frac{1}{2}}
$$

\subsubsection{Epoxy Surface Layer}

Composite laminates are made up of a fairly even distribution of fibers and matrix, however at the top and bottom of the laminate there exists a surface layer comprised of mainly epoxy, as shown in Figure 4.11. This surface layer is highly insulative, and can lead to problems in conductive composites such as carbon fiber / 


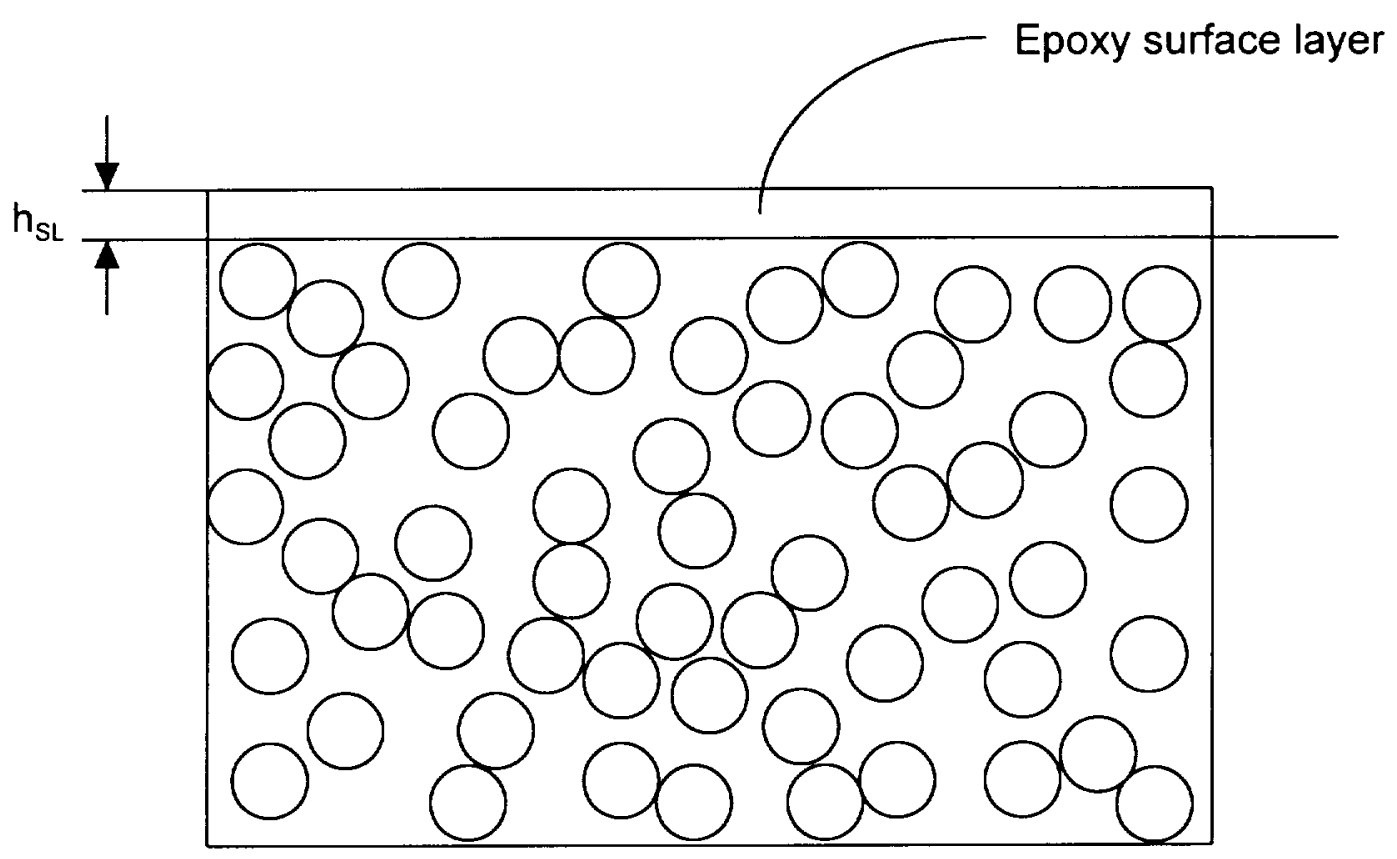

Figure 4.11: Schematic of the pure epoxy surface layer 
epoxy. Problems occur because while the bulk composite is conductive, right at the surface it is insulative, resulting in a large (many orders of magnitude, as shown in Table 2.1) jump in the conductivity. Therefore, the time to steady state also jumps by many orders of magnitude.

To calculate the electric field in the surface layer, the layer is modeled as a resistor and capacitor in parallel, as shown in Figure 4.12. Here $\mathrm{C}_{\mathrm{SL}}$ is the capacitance $(\mathrm{F})$ of the surface layer, $\mathrm{R}_{\mathrm{SL}}$ is the resistance $(\Omega)$ of the surface layer, $\mathrm{h}_{\mathrm{SL}}$ is the thickness $(\mathrm{m})$ of the surface layer, $\mathrm{V}$ is the voltage (V) across the surface layer, $\mathrm{i}_{\mathrm{in}}$ is the incoming current (A) from the space environment, $i_{C}$ is the current (A) flowing through the capacitor, and $i_{R}$ is the current (A) flowing through the resistor. The capacitance of the surface layer can be related to its dielectric constant by:

$$
\mathrm{C}_{\mathrm{SL}}=\frac{\varepsilon \mathrm{A}}{\mathrm{h}_{\mathrm{SL}}}
$$

where $A$ is the cross sectional area $\left(\mathrm{m}^{2}\right)$. The resistance can be related to the conductivity of the surface layer by:

$$
\mathrm{R}_{\mathrm{SL}}=\frac{\mathrm{h}_{\mathrm{SL}}}{\sigma \mathrm{A}}
$$

The electric field across the surface layer is defined as:

$$
\mathrm{E}=\frac{\mathrm{V}}{\mathrm{h}_{\mathrm{SL}}}
$$



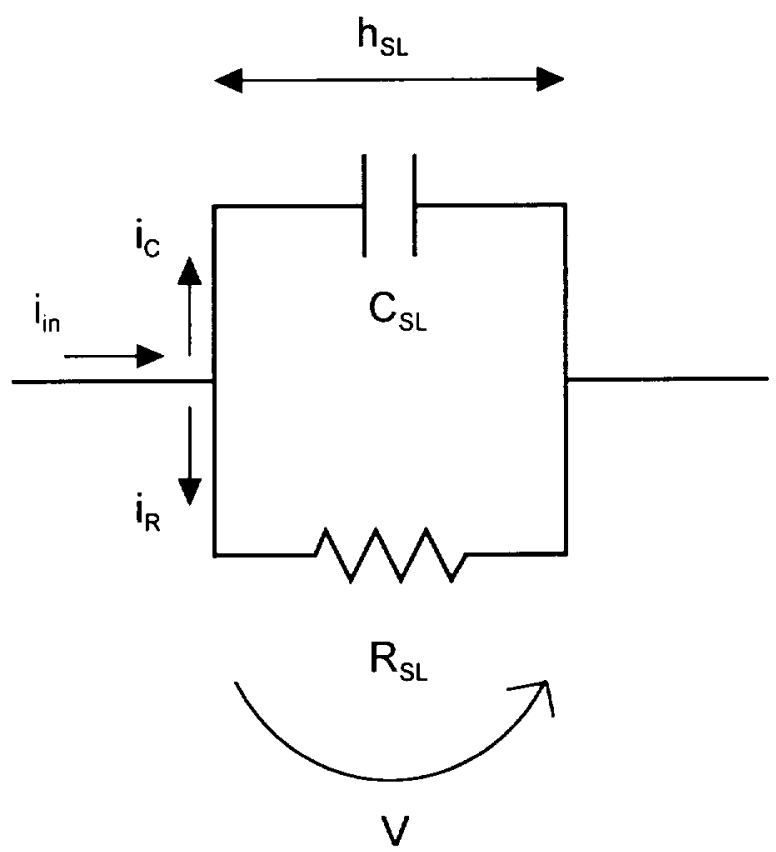

Figure 4.12: Schematic of the epoxy surface layer model 
Using Kirchhoff's conservation of charge rule, the current in the model can be expressed as:

$$
\mathrm{i}_{\mathrm{in}}=\mathrm{i}_{\mathrm{C}}+\mathrm{i}_{\mathrm{R}}
$$

the incoming current can be related to the incoming charge density from the space environment at the front surface by:

$$
\mathrm{i}_{\text {in }}=\dot{\rho}_{\text {in }} \mathrm{h}_{\mathrm{SL}} \mathrm{A}
$$

Using the equations for the current flowing through a capacitor, the current flowing through a resistor, and equation 59 , equation 58 becomes:

$$
\dot{\rho}_{\mathrm{in}} \mathrm{h}_{\mathrm{SL}} \mathrm{A}=\mathrm{C}_{\mathrm{sL}} \frac{\mathrm{dV}}{\mathrm{dt}}+\frac{1}{\mathrm{R}_{\mathrm{SL}}} \mathrm{V}
$$

Substituting equations 55 and 56 into equation 60 :

$$
\dot{\rho}_{\mathrm{in}} \mathrm{h}_{\mathrm{SL}} \mathrm{A}=\frac{\varepsilon \mathrm{A}}{\mathrm{h}_{\mathrm{SL}}} \frac{\mathrm{dV}}{\mathrm{dt}}+\frac{\sigma \mathrm{A}}{\mathrm{h}_{\mathrm{SL}}} \mathrm{V}
$$

Re-arranging and simplifying, equation 61 becomes:

$$
\frac{\mathrm{dV}}{\mathrm{dt}}+\frac{\sigma}{\varepsilon} \mathrm{V}=\dot{\rho}_{\mathrm{in}} \frac{\mathrm{h}_{\mathrm{SL}}{ }^{2}}{\varepsilon}
$$

This differential equation is general first order linear, and can be solved as:

$$
\mathrm{V}=\dot{\rho}_{\mathrm{in}} \frac{\mathrm{h}_{\mathrm{SL}}{ }^{2}}{\sigma}+\mathrm{C}_{3} \mathrm{e}^{-\frac{\sigma}{\varepsilon}}
$$

where $\mathrm{C}_{3}$ can be solved for with the initial condition that the voltage is zero at time equal zero. Therefore equation 63 becomes: 


$$
V=\dot{\rho}_{\text {in }} \frac{h_{S L}{ }^{2}}{\sigma}\left(1-e^{-\frac{\sigma}{\varepsilon} t}\right)
$$

Substituting equation 64 into equation 57, the electric field across the surface layer is:

$$
E=\dot{\rho}_{\text {in }} \frac{h_{S L}}{\sigma}\left(1-e^{-\frac{\sigma}{\varepsilon}}\right)
$$

Therefore, the steady-state electric field is:

$$
\mathrm{E}=\dot{\rho}_{\mathrm{in}} \frac{\mathrm{h}_{\mathrm{SL}}}{\sigma}
$$

The time to steady state, defined once again as when E reaches $99 \%$ of its actual steadystate value, is defined as:

$$
\mathrm{t}=-\frac{\varepsilon}{\sigma} \ln (0.01)
$$

It should be noted that this is the same equation as for the steady-state time for the composite deep dielectric charging model closed form solution (equation 32).

\subsection{COMPOSITE MICROMECHANICS}

\subsubsection{Continuous Fiber Composites}

This section will give an overview of the micromechanics of composite electrical properties. As referred to earlier, micromechanics is the determination of ply properties based on the constituent properties of the fiber and matrix. Micromechanics is used to homogenize material properties; this may or may not be a good idea depending on the usage of the material property. A general overview of the topic is given by Kaddour et al. ${ }^{34}$ An important definition in micromechanics is the fiber volume fraction, defined as: 


$$
\mathrm{V}_{\mathrm{f}}=\frac{\mathrm{Vol}_{\mathrm{f}}}{\mathrm{Vol}_{\mathrm{f}}+\mathrm{Vol}_{\mathrm{m}}}
$$

where $V_{f}$ is the fiber volume fraction, $V_{0 l}$ is the volume of fibers $\left(\mathrm{m}^{3}\right)$ in the composite, and $\mathrm{Vol}_{\mathrm{m}}$ is the volume $\left(\mathrm{m}^{3}\right)$ of matrix in the composite.

The composite conductivity in the longitudinal direction $\left(\sigma_{\mathrm{L}}\right)$ is based on a simple rule of mixtures as: ${ }^{46}$

$$
\sigma_{L}=\sigma_{f} V_{f}+\sigma_{m}\left(1-V_{f}\right)
$$

where $\sigma_{\mathrm{f}}$ is the conductivity $(1 / \Omega-\mathrm{m})$ of the fiber, and $\sigma_{\mathrm{m}}$ is the conductivity $(1 / \Omega-\mathrm{m})$ of the matrix.

The composite conductivity in the transverse direction $\left(\sigma_{\mathrm{T}}\right)$ is more complicated. One model expresses it as: ${ }^{46}$

$$
\frac{\sigma_{\mathrm{T}}-\sigma_{\mathrm{m}}}{\sigma_{\mathrm{T}}}=2 \mathrm{~V}_{\mathrm{f}} \frac{\sigma_{\mathrm{f}}-\sigma_{\mathrm{m}}}{\sigma_{\mathrm{T}}+\sigma_{\mathrm{f}}}
$$

The equations given above for the longitudinal and transverse conductivity were derived analytically using a continuum approach. The self consistent model assumes that the path of each fiber can be divided into a series of short straight segments. Each segment is treated as a long thin rod for the purpose of calculating the average electric field within a fiber. The presence of the other fibers is taken into account by assuming the segment is embedded directly in a matrix having the bulk properties of the sample. It should be noted that equations 69 and 70 can also be used to calculated the dielectric constant of the lamina because of the identical form of the conduction laws controlling both properties. 
Therefore the conductivity variables can be replaced with dielectric constant variables in the given equations.

To calculate the in-plane electrical properties (dielectric constant and conductivity) of a plain weave fabric composite, refer to Chou and Ning ${ }^{47}$. For an angled ply, the conductivity in the principal directions are expressed as: ${ }^{48}$

$$
\begin{aligned}
& \sigma_{1}=\sigma_{\mathrm{L}} \cos ^{2}(\theta)+\sigma_{\mathrm{T}} \sin ^{2}(\theta) \\
& \sigma_{2}=\sigma_{\mathrm{L}} \sin ^{2}(\theta)+\sigma_{\mathrm{T}} \cos ^{2}(\theta)
\end{aligned}
$$

where $\theta$ is the ply angle (refer to Figure 2.4).

Volpe $^{49}$ suggested an empirical expression for estimating the conductivity of laminates made of the family of $[0 / \pm 45 / 90]$ plies, in the fiber directions as:

$$
\sigma_{\theta}=\frac{\left(N_{\theta}+\frac{1}{2} N_{45}\right)}{N_{T}} \sigma_{L}
$$

where $N_{\theta}$ is the number of plies in the $\theta$ direction, $N_{45}$ is the number of plies oriented at \pm 45 degrees to the $\theta$ direction, $\mathrm{N}_{\mathrm{T}}$ is the total number of plies, and $\sigma_{\mathrm{L}}$ is the longitudinal conductivity of a unidirectional laminate. This expression assumes that the laminate is an electrically homogenous material, the conductivity is not a function of thickness or stacking sequence, the plies perpendicular to the $\theta$ direction are negligible, and that each $\pm 45^{\circ}$ oriented ply with respect to the $\theta$ direction is equivalent to a single ply in the $\theta$ direction. This expression was verified with actual tests with good agreement. 
The conductivity in the through-thickness direction is theoretically the same as the transverse conductivity. An additional model, appropriate to high fiber conductivity to matrix conductivity ratios, is based on the longitudinal conductivity, and approximated by the following expression: $:^{50}$

$$
\sigma_{\text {thick }}=V_{f}^{\beta} \sigma_{L}
$$

where $\beta$ is defined as:

$$
\beta=\frac{h_{p l y}}{D_{f}}
$$

where $h_{p l y}$ is the thickness $(m)$ of the ply, and $D_{f}$ is the diameter $(m)$ of the fibers. Equation 74 assumes that the conductivity is not a continuum property, and that the matrix is an insulator and its conductivity is zero. This equation is significantly different from the equations presented above for the longitudinal and transverse conductivity. The main reason for this difference is that the equation for the through-thickness conductivity was developed using an empirical approach with measured experimental data which showed that the conductivity depends mainly on the fiber conductivity and the amount of fiber contact (i.e. percolation). It should be noted that equation 74 underestimates the experimental results of through-thickness conductivity, especially when used on thick plies (more than 10 fiber diameters thick).

Percolation occurs when sufficient fibers exist in the composite as to create a path of touching fibers from the top surface of the composite to the bottom surface (refer to Figure 2.6). Percolation theory predicts that below a certain fiber volume fraction, the transverse conductivity is negligible compared to the longitudinal conductivity, as there 
are not sufficient fibers to link up together to create a percolation path. Thus the conductivity changes by several orders of magnitude after crossing the percolation threshold. This critical volume fraction is defined theoretically as 0.45 for unidirectional laminates. ${ }^{51}$ Joy et $\mathrm{al}^{51-53}$ found that transverse ply conductivity decreases with increasing thickness up to a thickness of 150 fiber diameters, above which it was considered to be of infinite thickness. The higher conductivity at small thicknesses is due to additional conduction paths. Therefore, there is theoretically a critical ply thickness, above which the transverse conductivity becomes negligible as compared to the longitudinal conductivity for a certain fiber volume fraction.

It is interesting to note the widely varying results from different micromechanics models when the conductivity of the fibers is much greater than the conductivity of the matrix, as in carbon fiber / epoxy composites. If the assumption that the conductivity of the matrix is effectively equal to zero is extended to the previous models, equation 69 becomes:

$$
\sigma_{L}=\sigma_{f} V_{f}
$$

and equation 70 becomes:

$$
\sigma_{\mathrm{T}}=\left(2 \mathrm{~V}_{\mathrm{f}}-1\right) \sigma_{\mathrm{f}}
$$

This is a "weak" percolation based model which shows that the transverse conductivity becomes zero below a critical volume fraction $\left(V_{f}\right)$ of 0.5 , and rises linearly after that. Finally combining equation 74 and 76 gives:

$$
\sigma_{\text {thick }}=\mathrm{V}_{\mathrm{f}}^{\beta+1} \sigma_{\mathrm{f}}
$$


which is a "stronger" percolation based model. These three models are plotted in Figure 4.13 , the fiber conductivity used is $1,000,0001 / \Omega-\mathrm{m}$, the fiber diameter used is $5 \mu \mathrm{m}$, and the ply thickness used is $0.125 \mathrm{~mm}$.

\subsubsection{Particulate Filled Composites}

In particulate filled composites, particles are used as the reinforcing medium instead of continuous length fibers. The particulates can be short chopped fibers, flakes and microballoons, or powders. One of the main uses of these composites in to create conductive composites out of insulative matrices.

Percolation phenomena in particulate filled composites occurs when sufficient conductive filler is added to an insulating polymer matrix, such that the composite transforms from an insulator to a conductor. This occurs because there is sufficient filler to create a continuous linkage of filler particles from one edge to another, as shown in Figure 4.14. The critical volume fraction, $V_{c}$, is when the change occurs.

The critical volume fraction can be approximated by the packing fraction of the filler powder: ${ }^{54}$

$$
\mathrm{V}_{\mathrm{C}} \approx \phi_{\mathrm{P}}-0.05
$$

where the packing fraction $\left(\phi_{\mathrm{P}}\right)$ can be calculated using:

$$
\phi_{\mathrm{P}}=\frac{\mathrm{m}_{\text {fill }}}{\operatorname{Vol}_{\text {fill }} \rho_{\text {fill }}}
$$




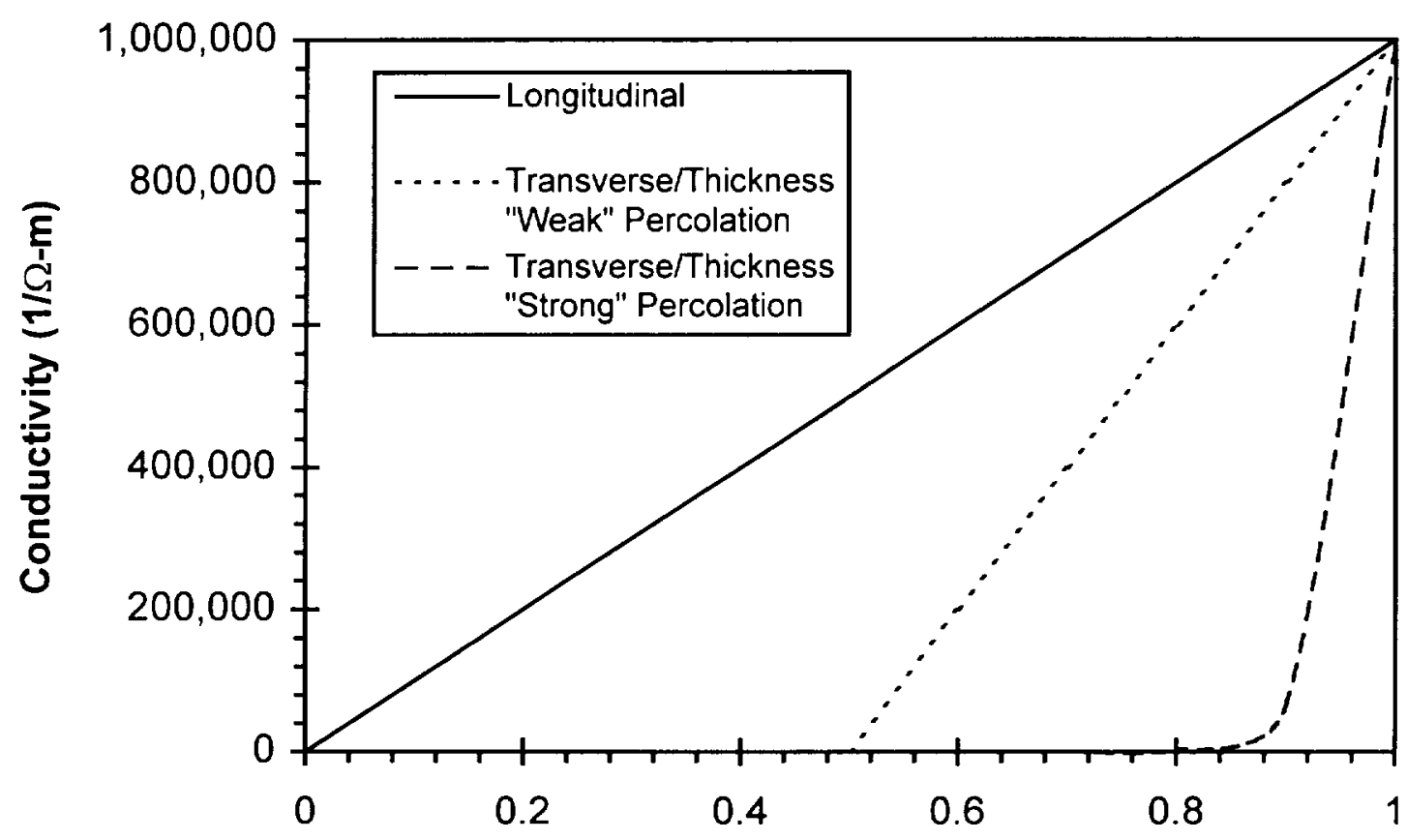

Fiber Volume Fraction

Figure 4.13: Various conductivity micromechanical models 


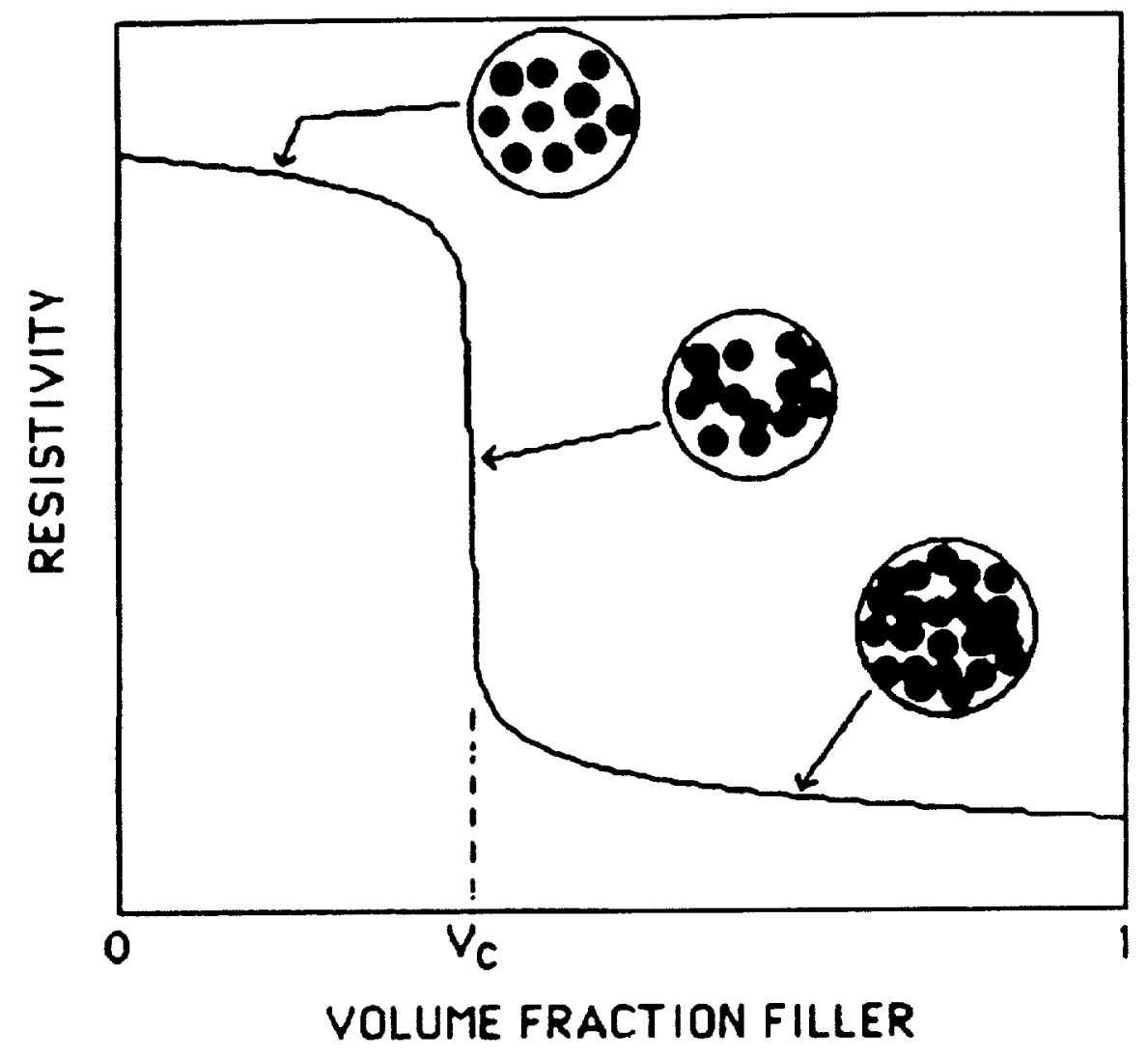

Figure 4.14: Critical fiber volume fraction in conductive composites ${ }^{54}$ 
where $\mathrm{m}_{\mathrm{fill}}$ is the measured mass $(\mathrm{kg})$ of the filler powder, $\mathrm{Vol}_{\text {fill }}$ is the volume $\left(\mathrm{m}^{3}\right)$ of tapped filler powder, and $\rho_{\text {fill }}$ is the theoretical density $\left(\mathrm{kg} / \mathrm{m}^{3}\right)$ of the filler powder.

Therefore the rules of particle packing can be applied to the critical volume fraction, allowing particle filled composites to be thought of as conductive skeletons. Fine particles pack less densely than larger particles, and thus result in lower critical volume fractions. This occurs due to an increase in surface area, lower particle mass, and a greater significance of the weak short range attractive/repulsive forces. Therefore, the critical volume fraction increases with particle size. Also, the more spherical the powder, the less interparticle friction and the higher the critical volume fraction. Fiber and flake fillers do not pack densely due to extensive bridging, and thus their packing fraction and critical volume fraction decrease as the aspect ratio of the filler increases.

In particulate composites, the electrical conductivity is not only a function of the constituent conductivities and the filler volume fraction, but also of the shape or aspect ratio of the particulates, and the relative particle arrangement.

\subsection{DATA REDUCTION EQUATIONS}

This section will present the equations which will be used to reduce the data from the experimental analysis. The geometry of the electrical test sample is as shown in Figure 4.15 , where $\mathrm{A}$ is the cross-sectional area $\left(\mathrm{m}^{2}\right)$, and $\mathrm{h}$ is the thickness $(\mathrm{m})$. The 


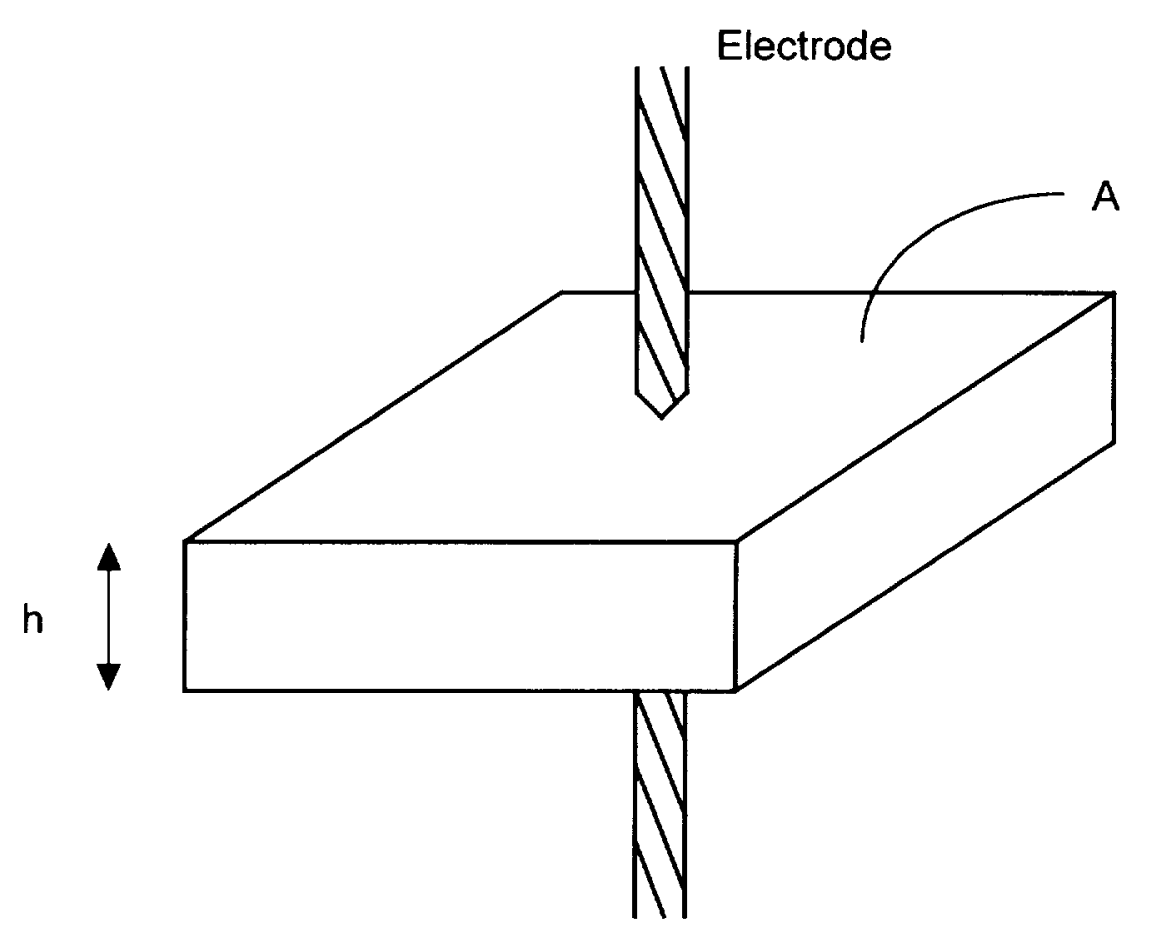

Figure 4.15: Geometry of the electrical test sample 
geometry of the mechanical test sample is as shown in Figure 4.16, where 1 is the length $(\mathrm{m})$, and $\mathrm{A}$ is the cross-sectional area $\left(\mathrm{m}^{2}\right)$.

\subsubsection{Conductivity}

The conductivity $(1 / \Omega-m)$ of the sample is calculated as:

$$
\sigma=\frac{h}{R A}
$$

where $\mathrm{R}$ is the resistance $(\Omega)$ across the sample.

The high resistance samples act as RC circuit, as shown in Figure 4.17, where $\mathrm{C}$ is the capacitance (F) of the sample, and R is the resistance $(\Omega)$ of the sample. The voltage in the circuit as the capacitor discharges is given by:

$$
V(t)=V_{0} e^{-\frac{t}{t}}
$$

where $\mathrm{V}(\mathrm{t})$ is the voltage $(\mathrm{V})$ at time $t, \mathrm{~V}_{\mathrm{o}}$ is the initial voltage $(\mathrm{V})$, and $\tau$ is the $\mathrm{RC}$ circuit time constant (s), defined as:

$$
\tau=\mathrm{RC}
$$

To calculate the conductivity of the surface layer, equation 81 is used replacing the sample thickness $(\mathrm{h})$ with the surface layer thickness $\left(\mathrm{h}_{\mathrm{SL}}\right)$ :

$$
\sigma=\frac{\mathrm{h}_{\mathrm{SL}}}{\mathrm{RA}}
$$




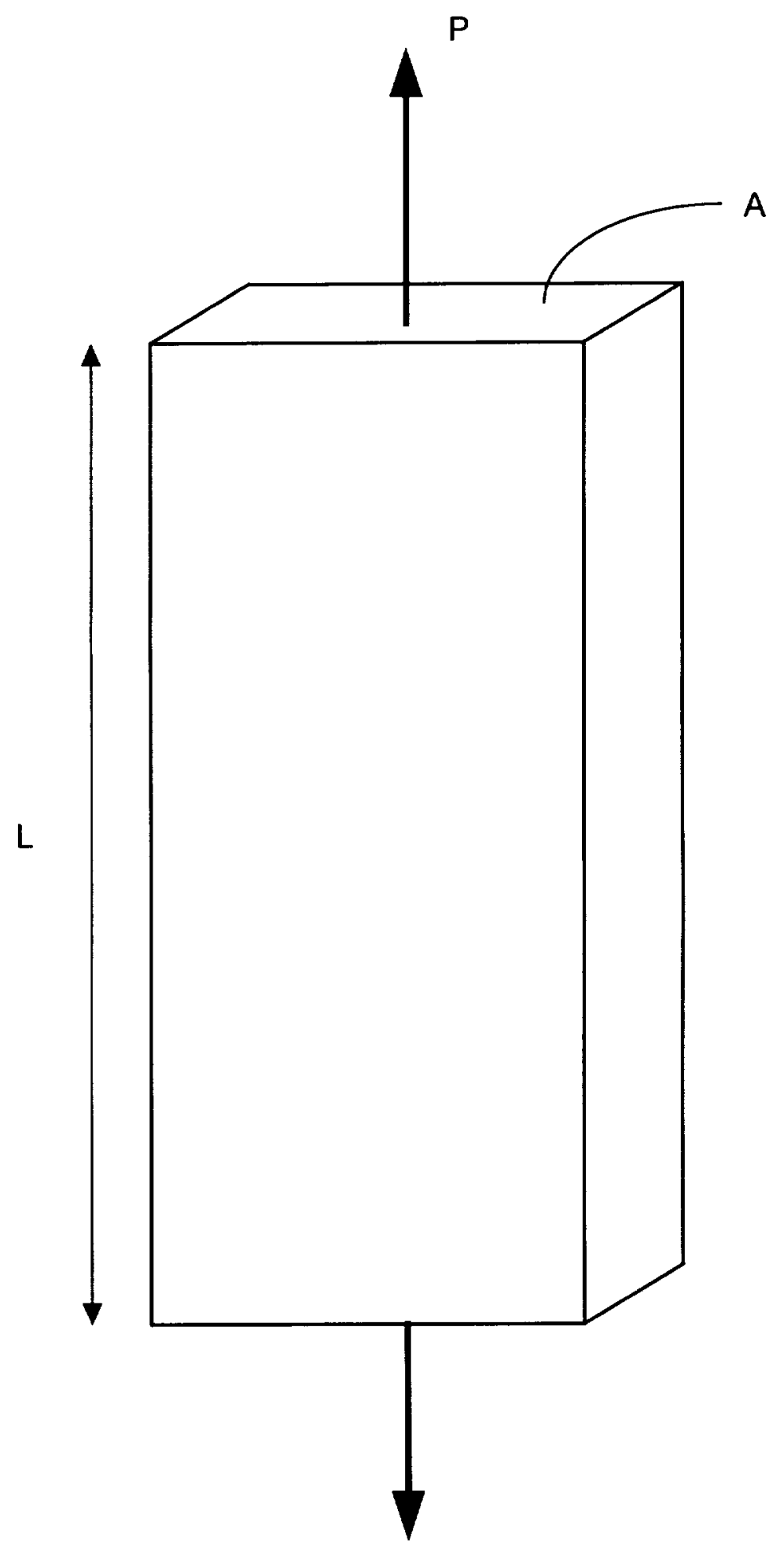

Figure 4.16: Geometry of the tensile test specimen 


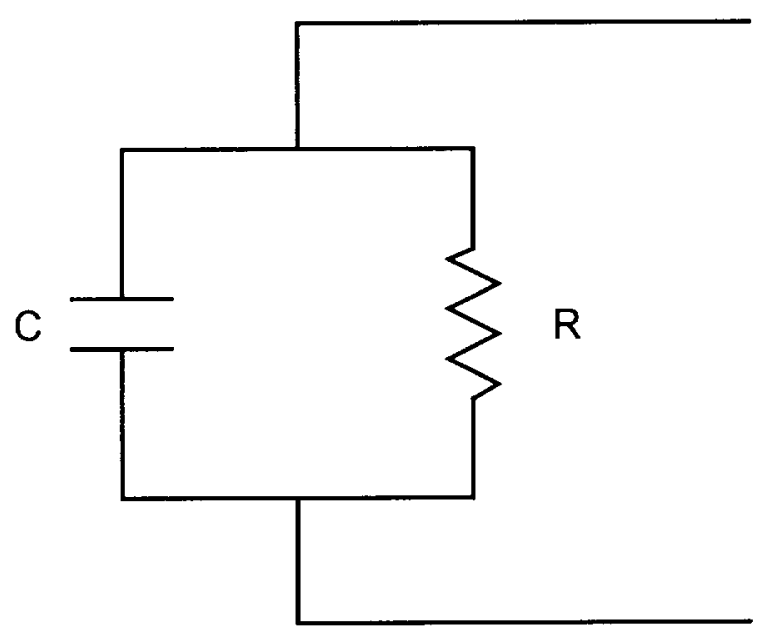

Figure 4.17: RC circuit model of high resistance conductivity samples 


\subsubsection{Dielectric Constant}

The dielectric constant of the sample is defined as:

$$
\kappa=\frac{C h}{\varepsilon_{0} A}
$$

\subsubsection{Dielectric Strength}

The dielectric strength $(\mathrm{V} / \mathrm{m})$ of the sample is defined as:

$$
E_{\max }=\frac{V_{B D}}{h}
$$

where $V_{B D}$ is the voltage applied to breakdown the material (V). To calculate the dielectric strength of the surface layer, equation 86 is used replacing the sample thickness (h) with the surface layer thickness $\left(\mathrm{h}_{\mathrm{SL}}\right)$ :

$$
\mathrm{E}_{\max }=\frac{\mathrm{V}_{\mathrm{BD}}}{\mathrm{h}_{\mathrm{SL}}}
$$

\subsubsection{Density}

The density $\left(\mathrm{kg} / \mathrm{m}^{3}\right)$ is defined as:

$$
\rho_{\text {mech }}=\frac{\mathrm{m}}{\mathrm{Vol}}
$$

where $\mathrm{m}$ is the mass $(\mathrm{kg})$ of the sample, and Vol is the volume $\left(\mathrm{m}^{3}\right)$ of the sample.

\section{$\underline{4.6 .5}$ Young's Modulus}

The Young's modulus (MPa) is defined as:

$$
\mathrm{E}_{\text {mech }}=\frac{\sigma_{\text {mech }}}{\varepsilon_{\text {mech }}}=\frac{P}{A \varepsilon_{\text {mech }}}
$$


where $\sigma_{\text {mech }}$ is the stress $(\mathrm{MPa})$ applied to the material, $\varepsilon_{\text {mech }}$ is the strain due to the applied stress, and $\mathrm{P}$ is the load $(\mathrm{N})$ applied to the material.

\subsubsection{Poisson Ratio}

The Poisson ratio is defined as:

$$
v=-\frac{\varepsilon_{\mathrm{y}_{\text {mech }}}}{\varepsilon_{\mathrm{x}_{\text {mech }}}}
$$

where $\varepsilon_{y_{\text {meen }}}$ is the strain perpendicular to the direction of the applied load, and $\varepsilon_{x_{\text {mech }}}$ is the strain in the direction of the applied load.

\subsubsection{Failure Stress}

The failure stress $(\mathrm{MPa})$ is the maximum stress in the material before failure, it is defined as:

$$
\sigma_{\text {max }_{\text {mcch }}}=\frac{P_{\text {fail }}}{A}
$$

where $P_{\text {fail }}$ is the load $(N)$ applied to the sample at failure.

\subsubsection{Failure Strain}

The failure strain is the strain when the material fails, it is defined as:

$$
\varepsilon_{\text {max }_{\text {mach }}}=\frac{\Delta l}{1}
$$

where $\Delta \mathrm{l}$ is the change in length, stroke, $(\mathrm{m})$ of the sample at failure. 


\section{CHAPTER 5}

\section{ANALYTICAL RESULTS AND DISCUSSION}

This chapter presents the result from the deep dielectric charging model and the fiber-matrix interaction models. A base case was used with the deep dielectric charging model, from which all other runs are just modifications. A summary of the results are presented here. The plots from individual code runs can be found in Appendix B.

\subsection{DEEP DIELECTRIC CHARGING MODEL}

\subsubsection{Base Case Results}

In this section, results are presented for a typical case, the base case from which all other code runs are variations. The orbit used has an altitude of $7,000 \mathrm{~km}$ (approximately one earth radii), an inclination of $0^{\circ}$, and was set during the solar cycle condition of solar maximum. This orbit is not a particularly practical orbit. It was chosen for two reasons. It is in the middle of the Van Allen belts (refer to Figure 2.2), where the highest concentrations of electrons are located, and geostationary spacecraft must fly through this region to get to geosynchronous orbit. The orbit environment is shown in Figure 4.3, it has a total electron flux of $2.8 \times 10^{8} \# / \mathrm{cm}^{2}-\mathrm{s}$ with a total dose rate 
of $7.15 \mathrm{rad} / \mathrm{s}$, and a total proton flux of $2.9 \times 10^{7} \# / \mathrm{cm}^{2}-\mathrm{s}$ with a total dose rate of 771 $\mathrm{rad} / \mathrm{s}$.

The material properties used in the base case are those for a typical organic polymer, and are shown in Table 5.1. The base case was run using both the closed form solution and the finite difference solution, without including radiation induced conductivity and the high field conductivity. The two boundary conditions used were an electric field and voltage of zero on the back surface. The electric field of zero on the back surface represents the back surface being a conductively grounded backplane. The results shown in Figures 5.1 through 5.3 for the closed form solution and Figures 5.4 through 5.6 for the finite difference solution, show that both solutions give similar results with the finite difference solution taking slightly longer to reach steady state than the closed form solution. This is due to the definition of when the finite difference solution has converged - in this case when the residual is less than 0.1 . The residual is defined as the summation of the difference between the incoming charge density rate and the current density for every node. If a larger residual was chosen for when steady state is reached, the time to steady state would be lower. This validates both models since they give the same result distributions, with a slight difference in the time to steady state. The results show that it took an average of 1.3 seconds to reach steady state, with a maximum electric field of approximately $4400 \mathrm{~V} / \mathrm{m}$ and a maximum voltage of approximately 3 volts. The electric field result is several orders of magnitude below the breakdown value of 15 $\mathrm{MV} / \mathrm{m}$, therefore an electrostatic discharge will not occur. 
Table 5.1: $\quad$ Base case material properties

\begin{tabular}{||l|c||}
\hline \hline Property & Value \\
\hline \hline Density, $\rho_{\text {mech }}$ & $1600 \mathrm{~kg} / \mathrm{m}^{3}$ \\
\hline Thickness, $\mathrm{h}$ & $2.5 \mathrm{~mm}$ \\
\hline Dielectric Constant, $\kappa$ & 3.0 \\
\hline Electrical Conductivity, $\sigma$ & $1 \times 10^{-10} 1 / \Omega-\mathrm{m}$ \\
\hline Dielectric Strength, $\mathrm{E}_{\max }$ & $15 \times 10^{6} \mathrm{~V} / \mathrm{m}$ \\
\hline $\begin{array}{l}\text { Radiation induced conductivity } \\
\text { exponent, } \Delta\end{array}$ & 1.0 \\
\hline $\begin{array}{l}\text { Coefficient of radiation induced } \\
\text { conductivity, } \mathrm{k}_{\mathrm{R}}\end{array}$ & $1.1 \times 10^{-14} \mathrm{~s} / \Omega-\mathrm{m}-\mathrm{rad}$ \\
\hline Temperature, $\mathrm{T}$ & $120^{\circ} \mathrm{C}$ \\
\hline
\end{tabular}




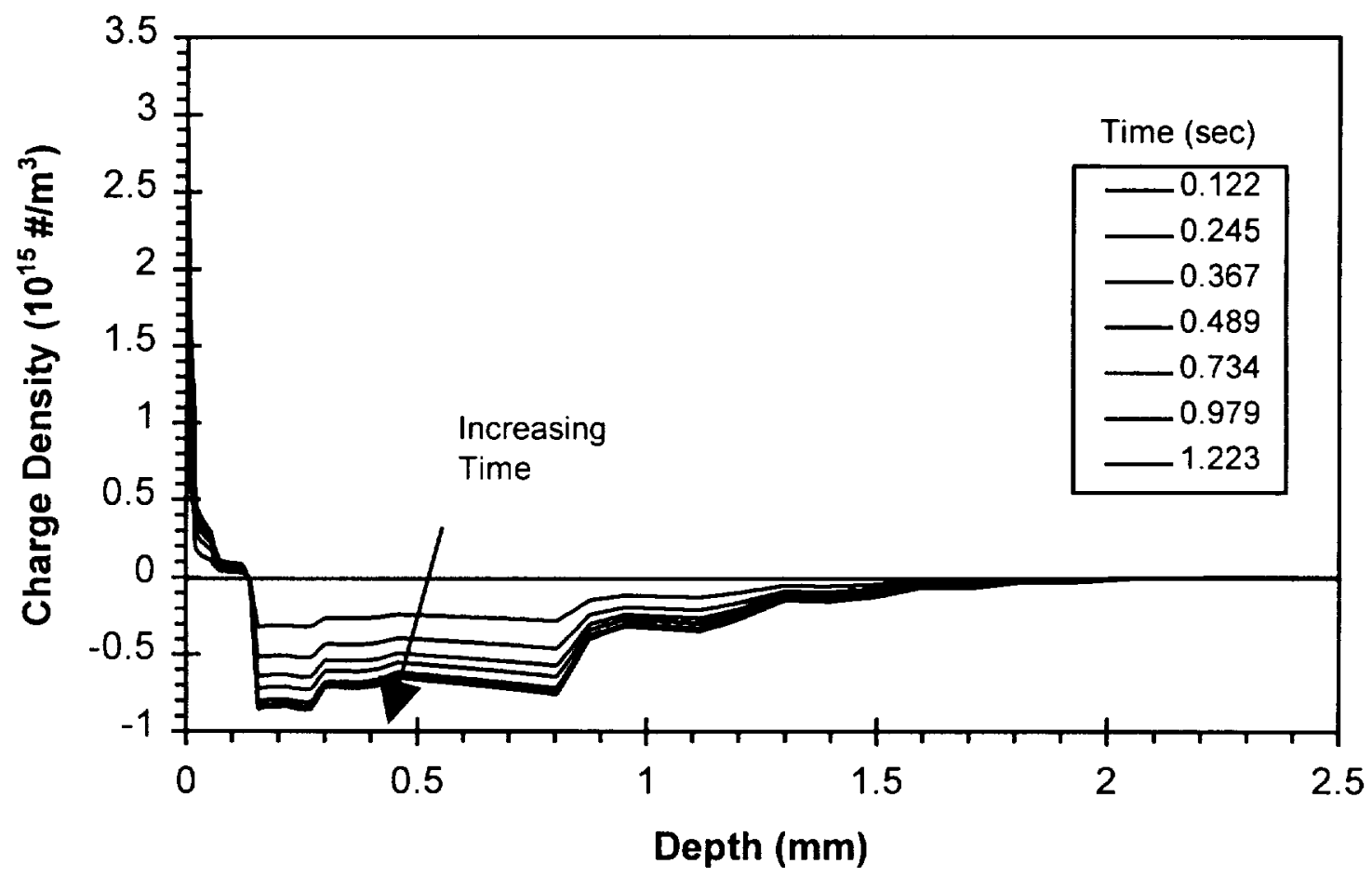

Figure 5.1: Closed form base case charge density 


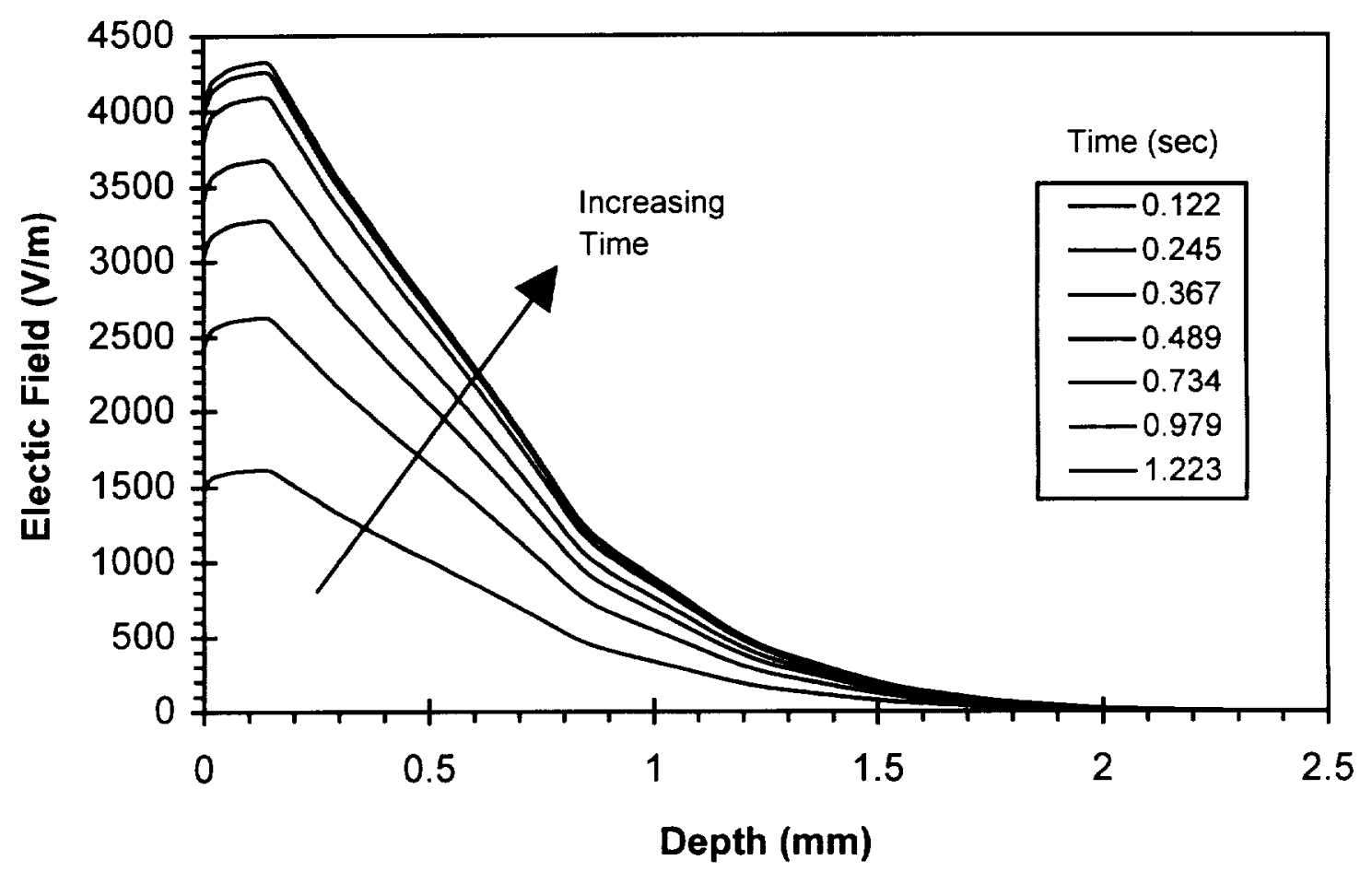

Figure 5.2: Closed form solution base case electric field 


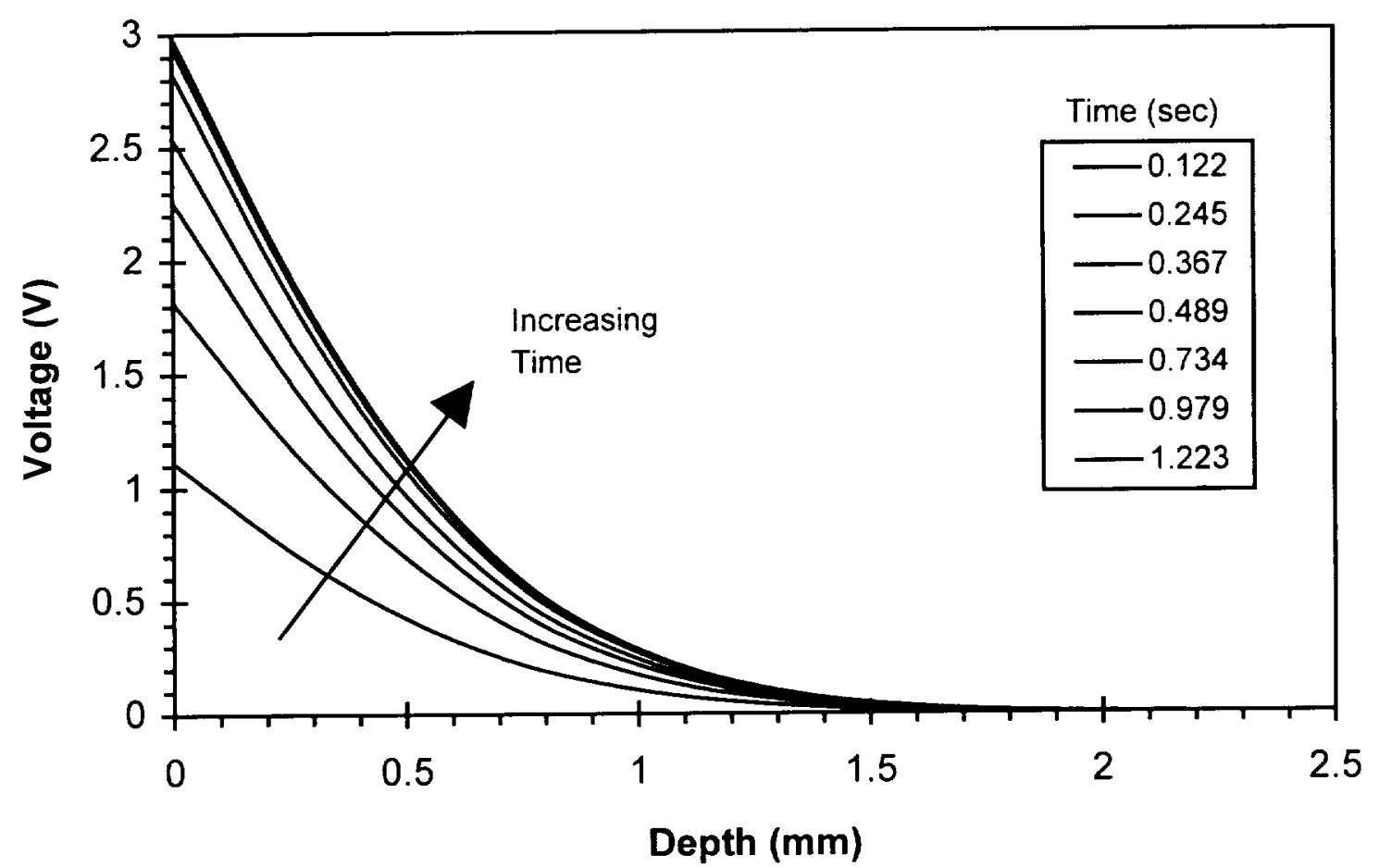

Figure 5.3: Closed form solution base case voltage 


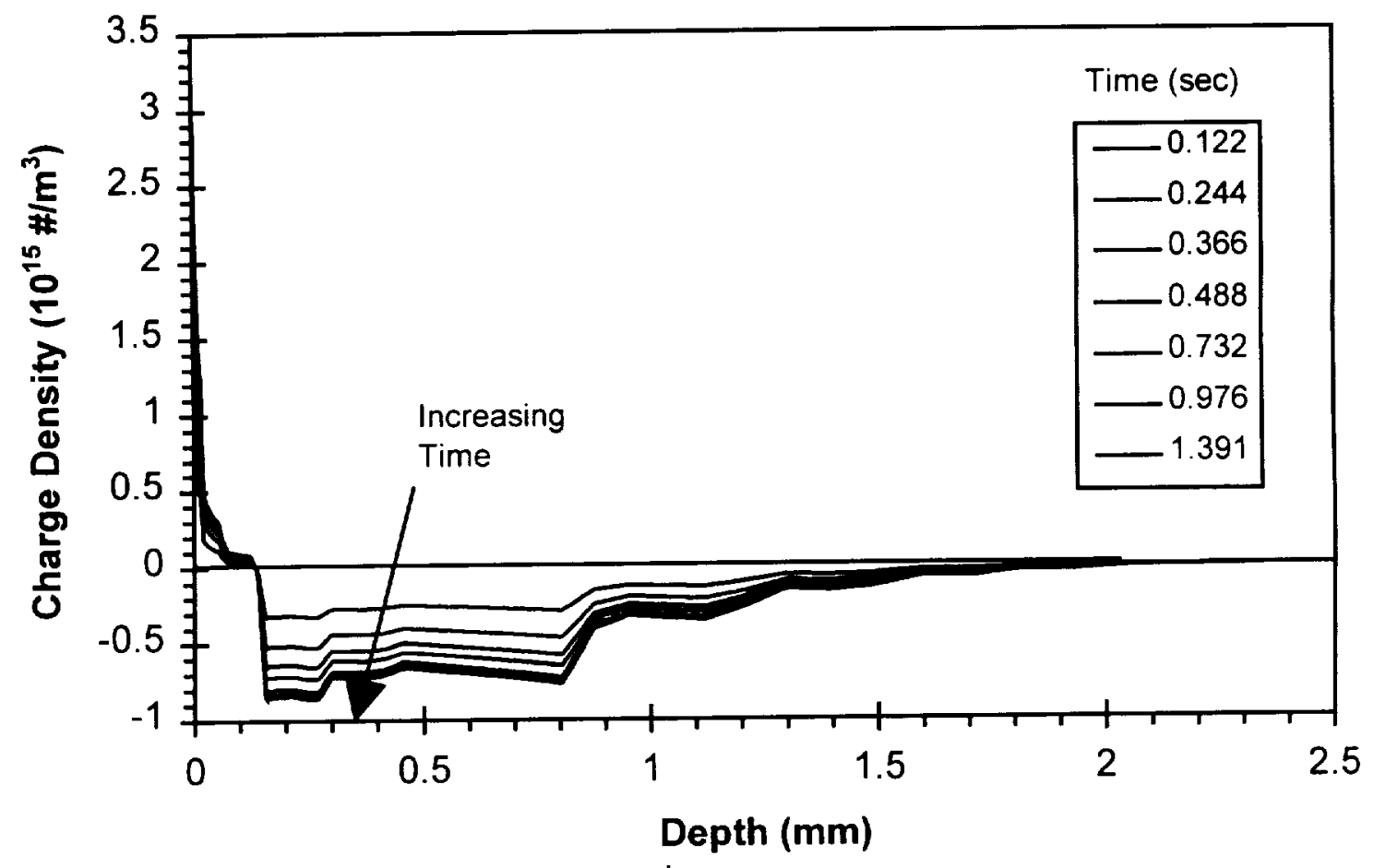

Figure 5.4: Finite difference solution base case charge density 


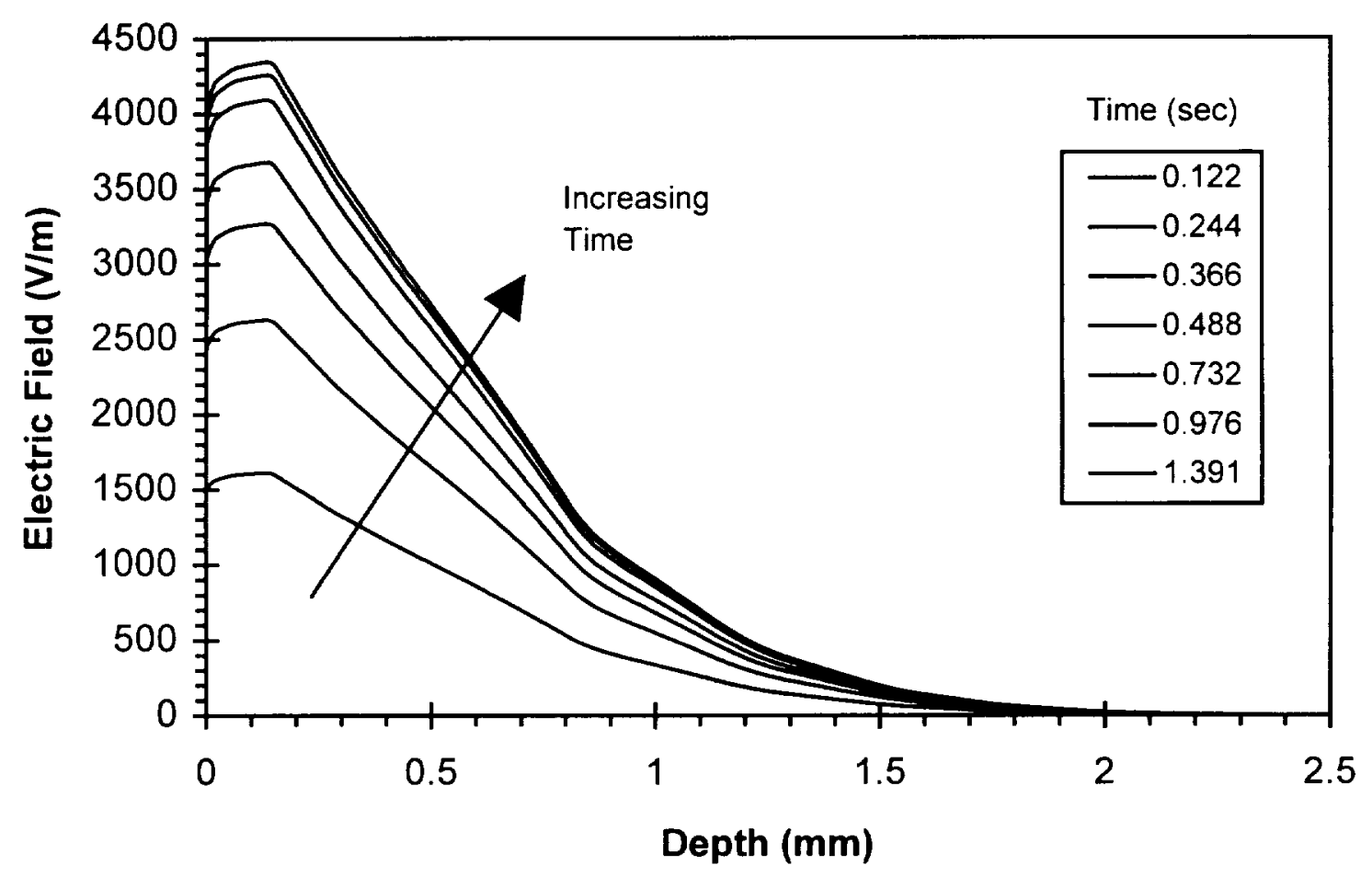

Figure 5.5: Finite difference solution base case electric field 


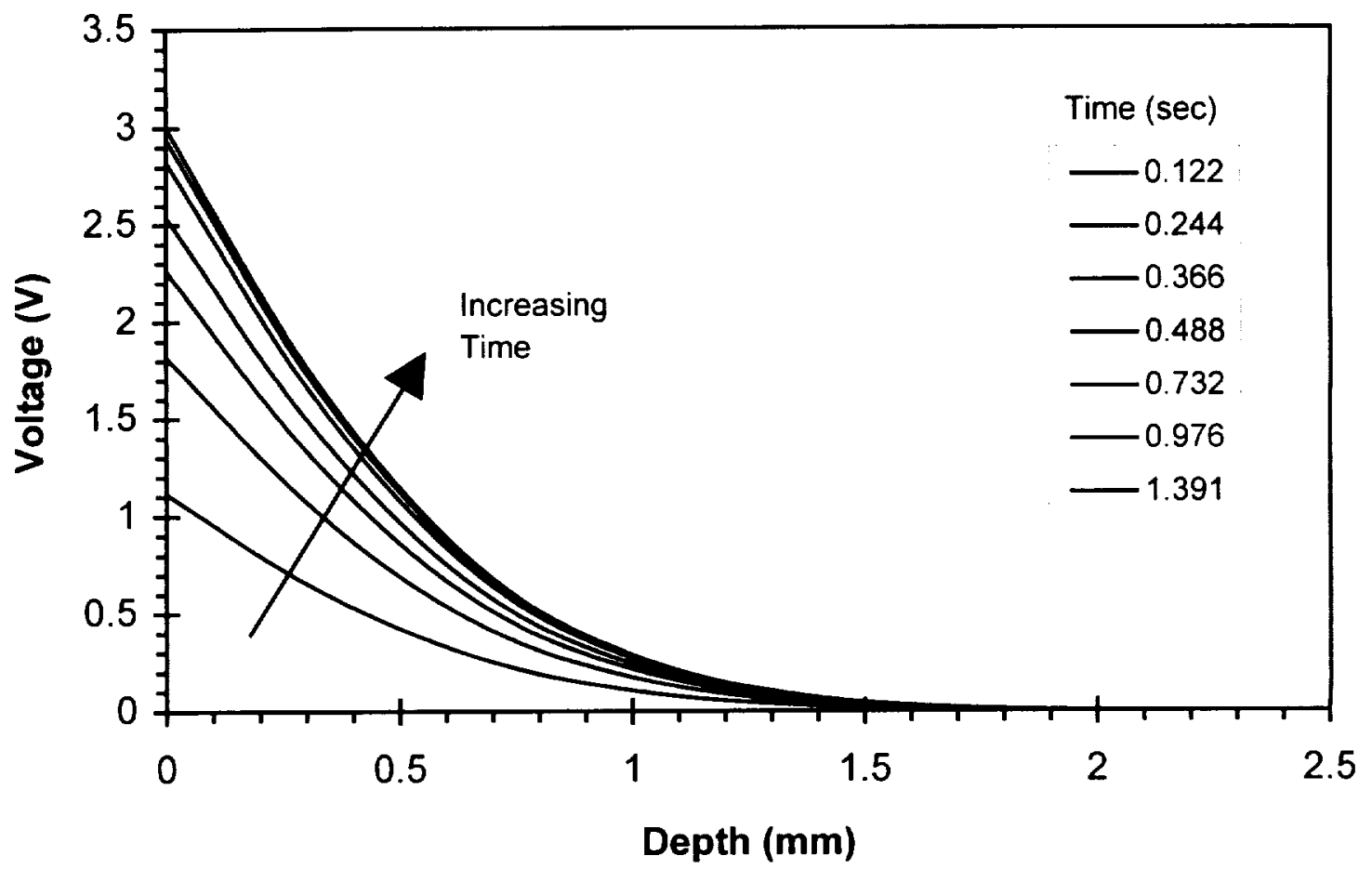

Figure 5.6: Finite difference solution base case voltage 


\subsubsection{Base Case With High Field Conductivity}

The base case was run including high field conductivity. The temperature used was $120^{\circ} \mathrm{C}$, as indicated in Table 5.1 . The temperature was chosen arbitrarily within the possible range of spacecraft temperatures. The analysis showed that the high field conductivity scaling factor has the same form as the electric field profile, as shown in Figure 5.7. However, the variations are quite small and the scaling factor can be approximated as 1 . Thus the results showed that there is no appreciable change to the shape of the profiles, and the steady-state charge density, electric field, and voltages were unchanged. It should also be noted that the time to steady state did not change.

Since the high field conductivity had no effect for the given steady-state electric fields of the base case, sensitivity studies were performed on the high field conductivity equation to determine the effects of dielectric constant and temperature. The range of values used are shown in Table 5.2. The dielectric constant was varied through the range of possible values for all materials, from 1 to 9 , as shown in Figure 5.8. The temperature was varied through the possible range of temperatures seen by spacecraft in Earth orbit, from -200 to $150^{\circ} \mathrm{C}$, as shown in Figure 5.9. The results show that there is no effect due to high field conductivity until an electric field of at least $100,000 \mathrm{~V} / \mathrm{m}$, after which the high field conductivity scaling factor increases rapidly. Also the high field conductivity scaling factor decreases significantly with increasing dielectric constant and temperature. 


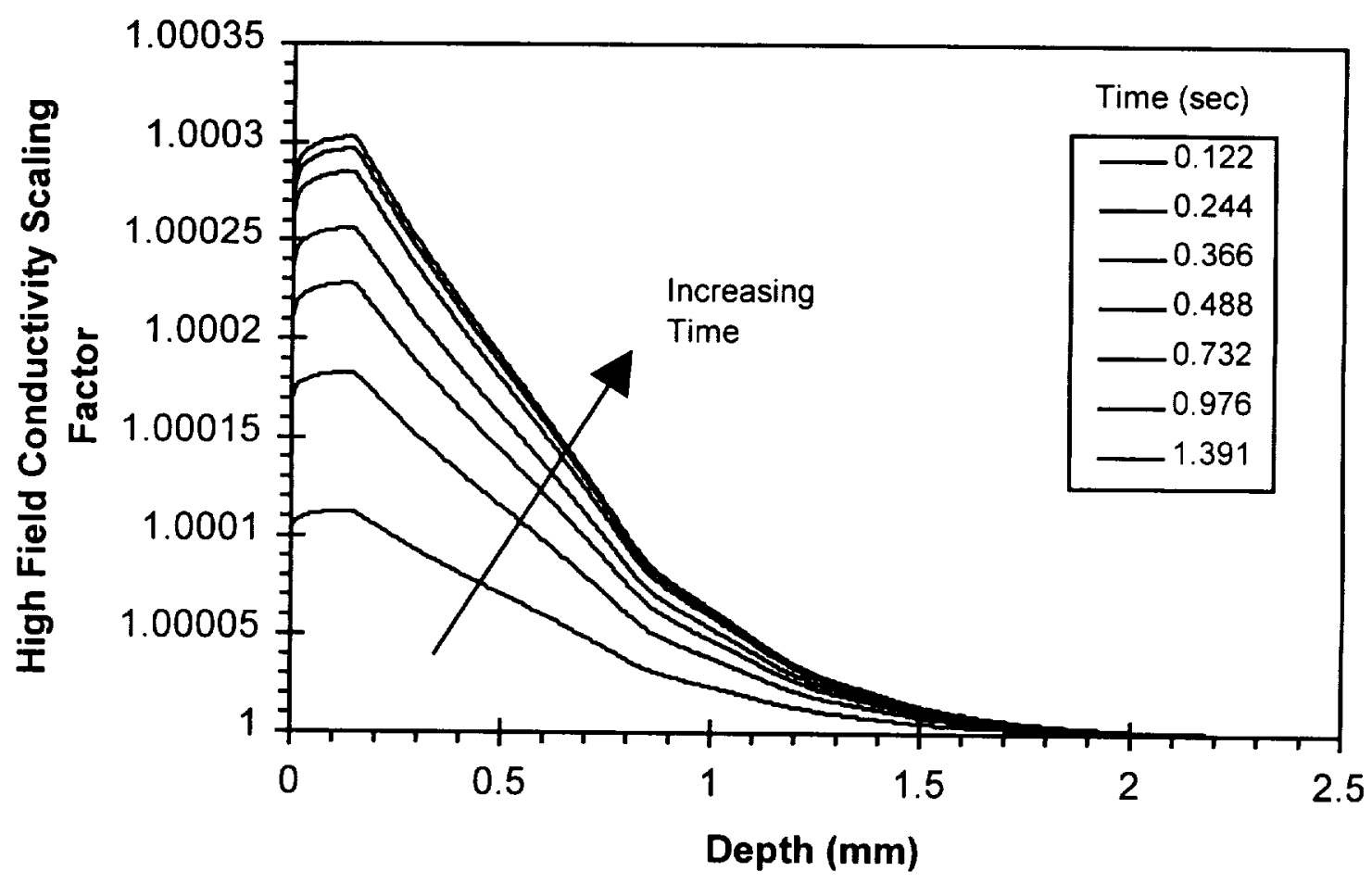

Figure 5.7: High field conductivity scaling factor 
Table 5.2: $\quad$ Sensitivity studies parameter values

\begin{tabular}{||l|c||}
\hline \hline Property & Range \\
\hline \hline Conductivity, $\sigma$ & $10^{-8}$ to $10^{-12} 1 / \Omega-\mathrm{m}$ \\
\hline Dielectric Constant, $\kappa$ & 1.0 to 9.0 \\
\hline Thickness, $\mathrm{h}$ & 0.01 to $5.0 \mathrm{~mm}$ \\
\hline Density, $\rho_{\text {mech }}$ & 600 to $2400 \mathrm{~kg} / \mathrm{m}^{3}$ \\
\hline Temperature, $\mathrm{T}$ & -200 to $150^{\circ} \mathrm{C}$ \\
\hline $\begin{array}{l}\text { Radiation Induced Conductivity } \\
\text { Exponent }\end{array}$ & 0.5 to 1.0 \\
\hline $\begin{array}{l}\text { Coefficient of Radiation Induced } \\
\text { Conductivity }\end{array}$ & $10^{-15}$ to $1.1 \times 10^{-14} \mathrm{~s} / \Omega$-m-rad \\
\hline Solar Cycle Condition & Minimum \& Maximum \\
\hline Substorm Intensity & 1 to 1000 \\
\hline Solar Paticle Event Intensity & 1 to 1000 \\
\hline
\end{tabular}




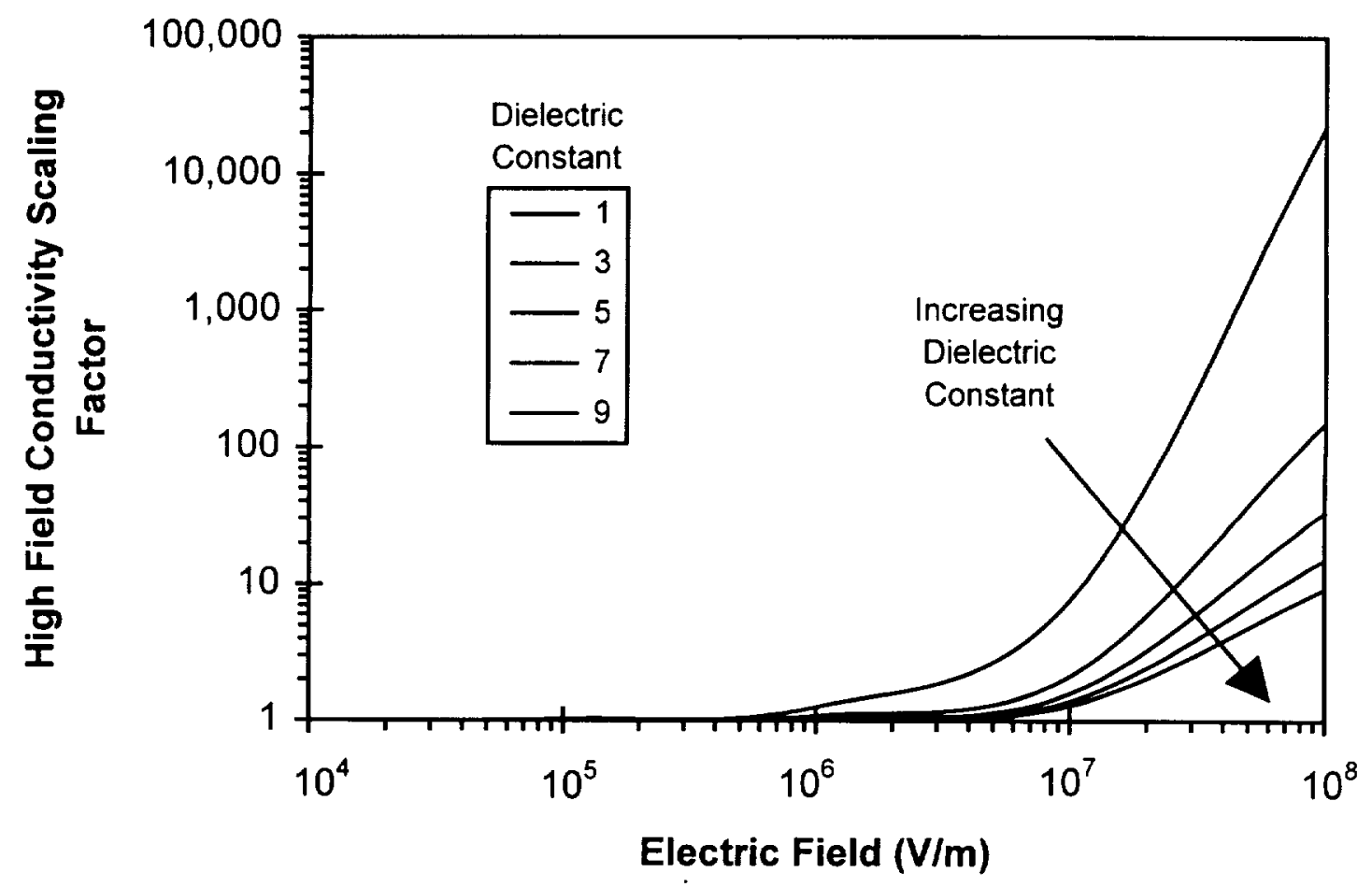

Figure 5.8: High field conductivity scaling factor dielectric strength sensitivity 


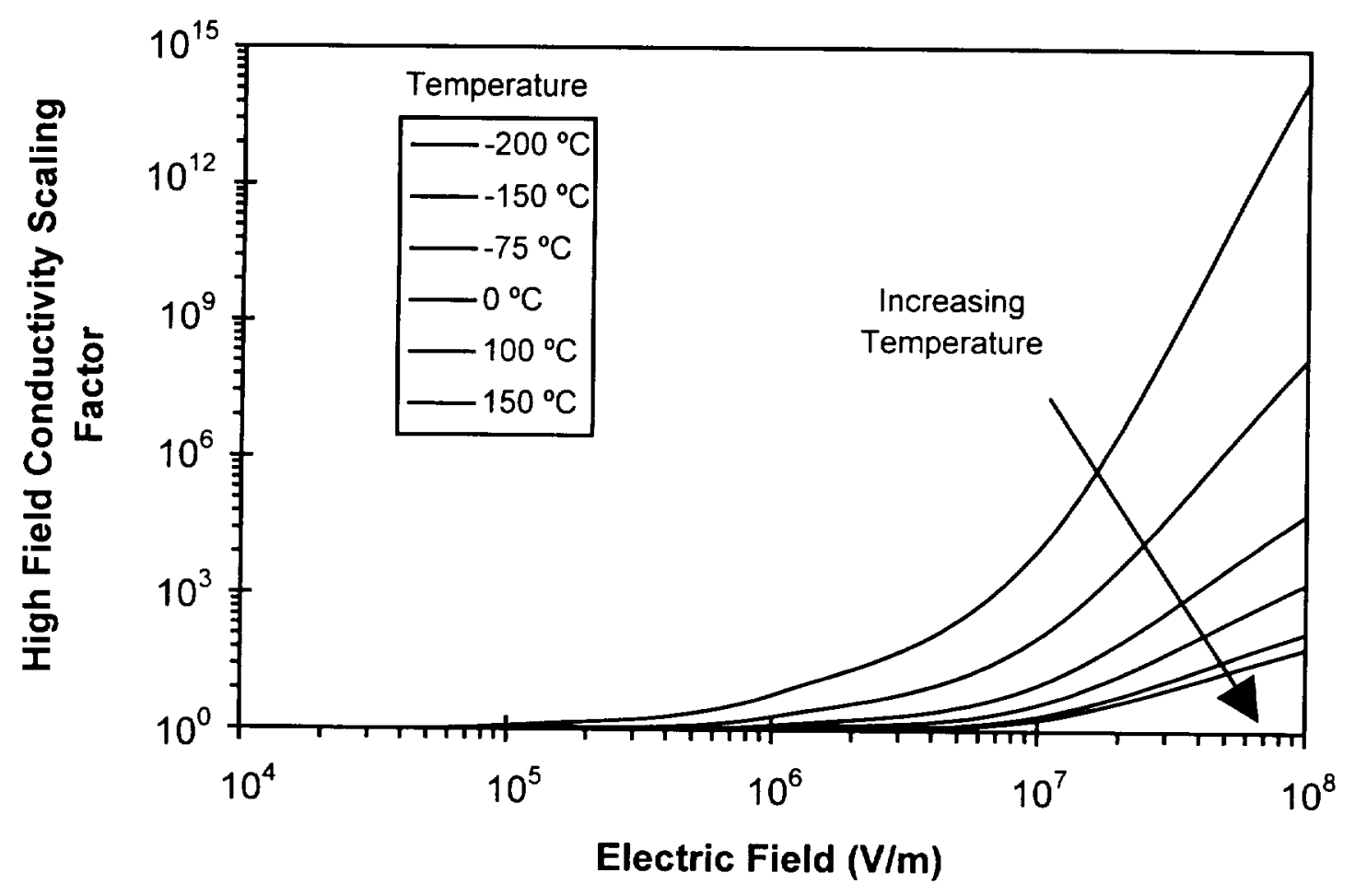

Figure 5.9: High field conductivity scaling factor temperature sensitivity 


\subsubsection{Base Case with Radiation Induced Conductivity}

The base case was run including radiation induced conductivity, the coefficient of radiation induced conductivity $(\kappa)$ and the radiation induced conductivity exponent $(\Delta)$ where selected (refer to Table 5.1) to produce the worst case possible (the highest possible radiation induced conductivity within the range of values for polymers and composites). The results showed no change to the shape of the profiles, and a slight decrease in the steady-state charge density, electric field, and voltage as compared to the base case. The reason for the slight decrease is that the radiation induced conductivity is $9.2 \times 10^{-12} 1 / \Omega-\mathrm{m}$ which is an order of magnitude less than the dark conductivity of the material. Thus when it is added to the dark conductivity, the conductivity increases slightly to $1.09 \times 10^{-10} 1 / \Omega-\mathrm{m}$. Therefore since the conductivity increased, the time to steady state also decreases slightly from 1.22 seconds to 1.12 seconds. It should be noted that even though the dark conductivity of the material could decrease below the value of the radiation induced conductivity, the value obtained for the radiation induced conductivity is a worst case value and in reality it may even be lower. Also, the effect of radiation induced conductivity is to decrease the value of the electric field and thus helps in preventing electrostatic discharges from occurring, so it is conservative to ignore it.

To observe the effects of the coefficient of radiation induced conductivity and the radiation induced conductivity exponent, sensitivity studies were performed on the radiation induced conductivity equation. The range of values used are shown in Table 5.2. The radiation induced conductivity exponent was varied through all possible values, 0.5 to 1.0 , and the coefficient of radiation induced conductivity was varied through the 
range of possible values for composites and polymers, from $10^{-15}$ to $1.1 \times 10^{-14} \mathrm{~s} / \Omega$-m-rad. The results, as shown in Figures 5.10 and 5.11, show that the radiation induced conductivity increases with dose rate, the radiation induced conductivity exponent, $\Delta$, and the coefficient of radiation induced conductivity, $\kappa$.

\subsection{SENSITIVITY STUDIES}

To understand the effects of the various parameters input into the deep dielectric charging model, sensitivity studies were performed on the material properties: the conductivity, dielectric constant, thickness, and material density. Sensitivity studies were also performed on the space environment: solar cycle condition, geomagnetic substorms, and solar particle events. The range of values used in each sensitivity study is shown in Table 5.2. No sensitivities were performed on the dielectric strength even though it is one of the input material parameters. This is because the only role the dielectric strength plays is to determine when an electrostatic discharge will occur. Therefore changing the dielectric strength will only have an effect if it is decreased below the maximum steadystate electric field, thus allowing an electrostatic discharge to occur.

\subsubsection{Conductivity}

The conductivity was varied within the range of insulating composites and polymers, from $10^{-12}$ to $10^{-8} 1 / \Omega-\mathrm{m}$, as shown in Figure 5.12. The result show that both the maximum steady-state electric field and the time to steady state scale inversely with conductivity. Also there was no change in the shape of the charge density, electric field, and voltage distributions, they were just decreased by the inverse of the conductivity. 


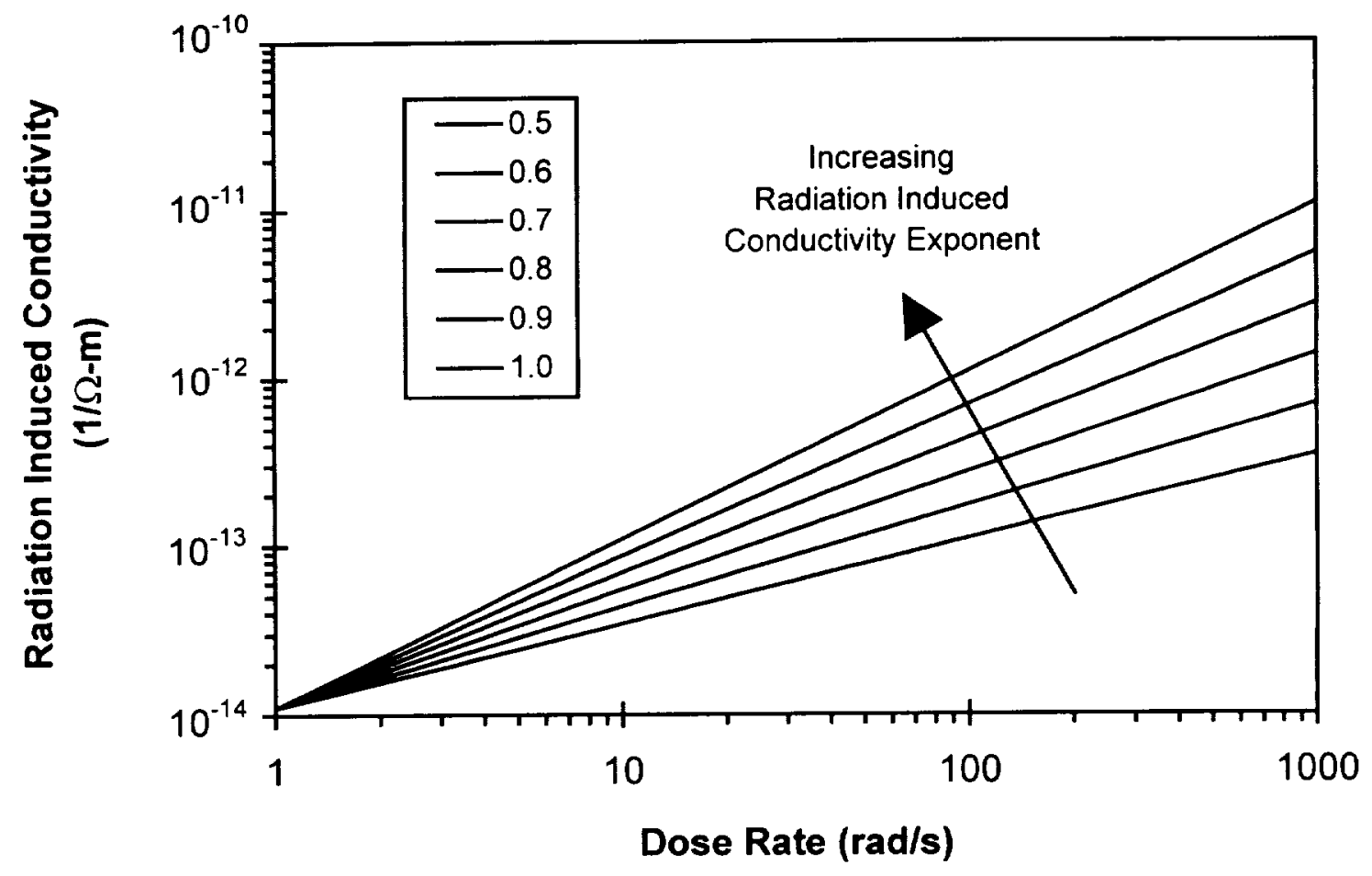

Figure 5.10: Radiation induced conductivity exponent sensitivity 


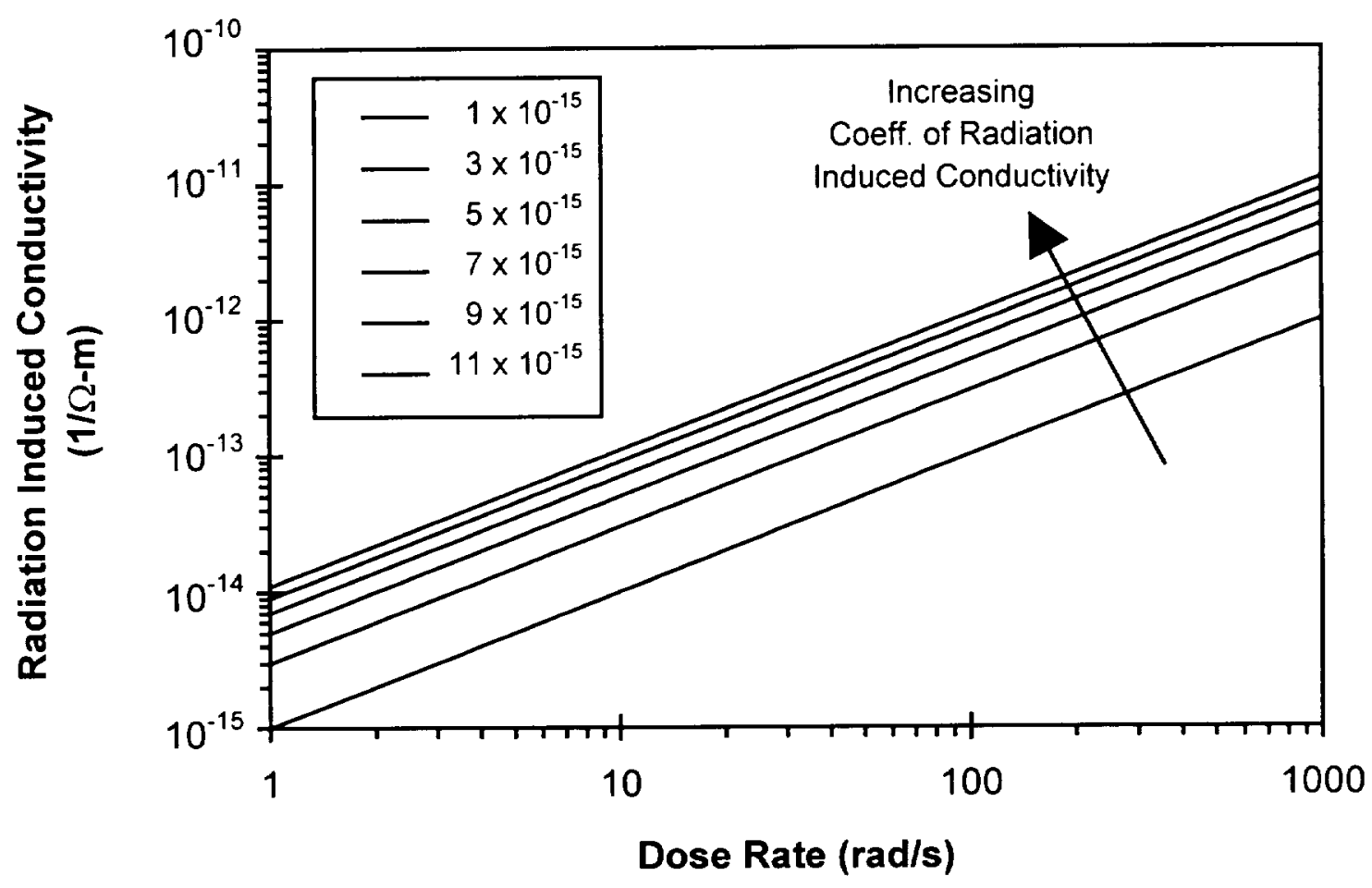

Figure 5.11: Coefficient of radiation induced conductivity sensitivity 


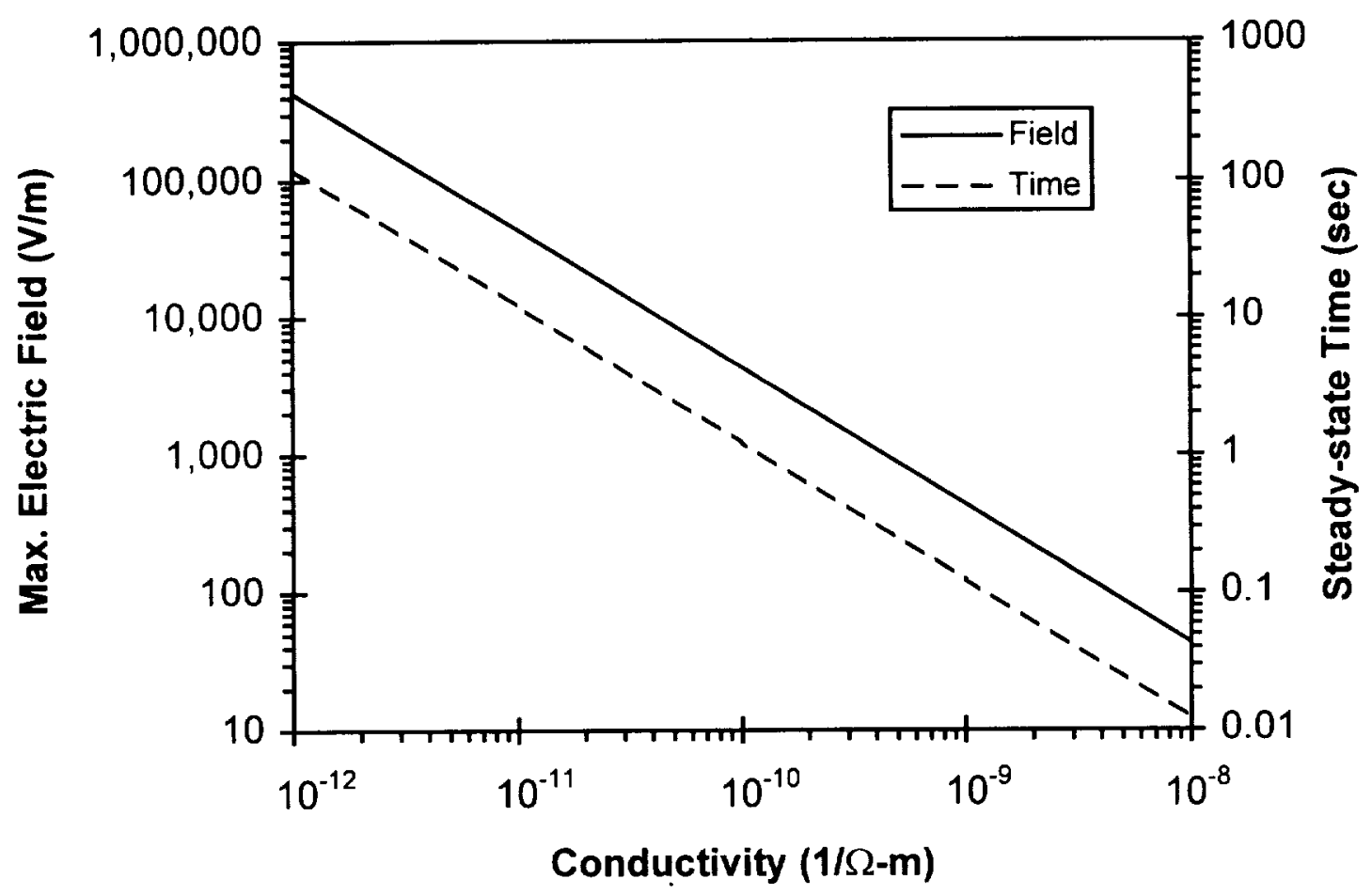

Figure 5.12: Conductivity sensitivity 
These results are expected, as can be seen in equations $31,32,34$, and 36 where the conductivity is in the denominator.

\subsubsection{Dielectric Constant}

The dielectric constant was varied within the range of possible values for materials, from 1 to 9 , as shown in Figure 5.13. The results show that the maximum steady-state electric field is unchanged with increasing dielectric constant, and the time to steady state increases linearly with dielectric constant. Also there was a slight change in the shape of the charge density distribution, and no change at all to the electric field and voltage distribution. These results are expected, as can be seen in equations 31 and 32 where the dielectric constant is in the numerator, and in equations 34 and 36 which show that the electric field and voltage are not a function of the dielectric constant.

\subsubsection{Thickness}

The thickness was varied within the range of possible structural thicknesses, from 0.01 to $5.0 \mathrm{~mm}$, as shown in Figure 5.14. The results show that the maximum steadystate electric field increases with thickness until a constant value is obtained after which the electric field does not change with increasing thickness. The time to steady state is unchanged with increase in thickness. There were also changes to the charge density, electric field, and voltage distributions. These results occur because at small thicknesses there are less particles in the material, as the higher energy particles have enough energy to pass through the material. While as the thickness increases, more particles are stopped in the material increasing the electric field. This continues until the material is thick 


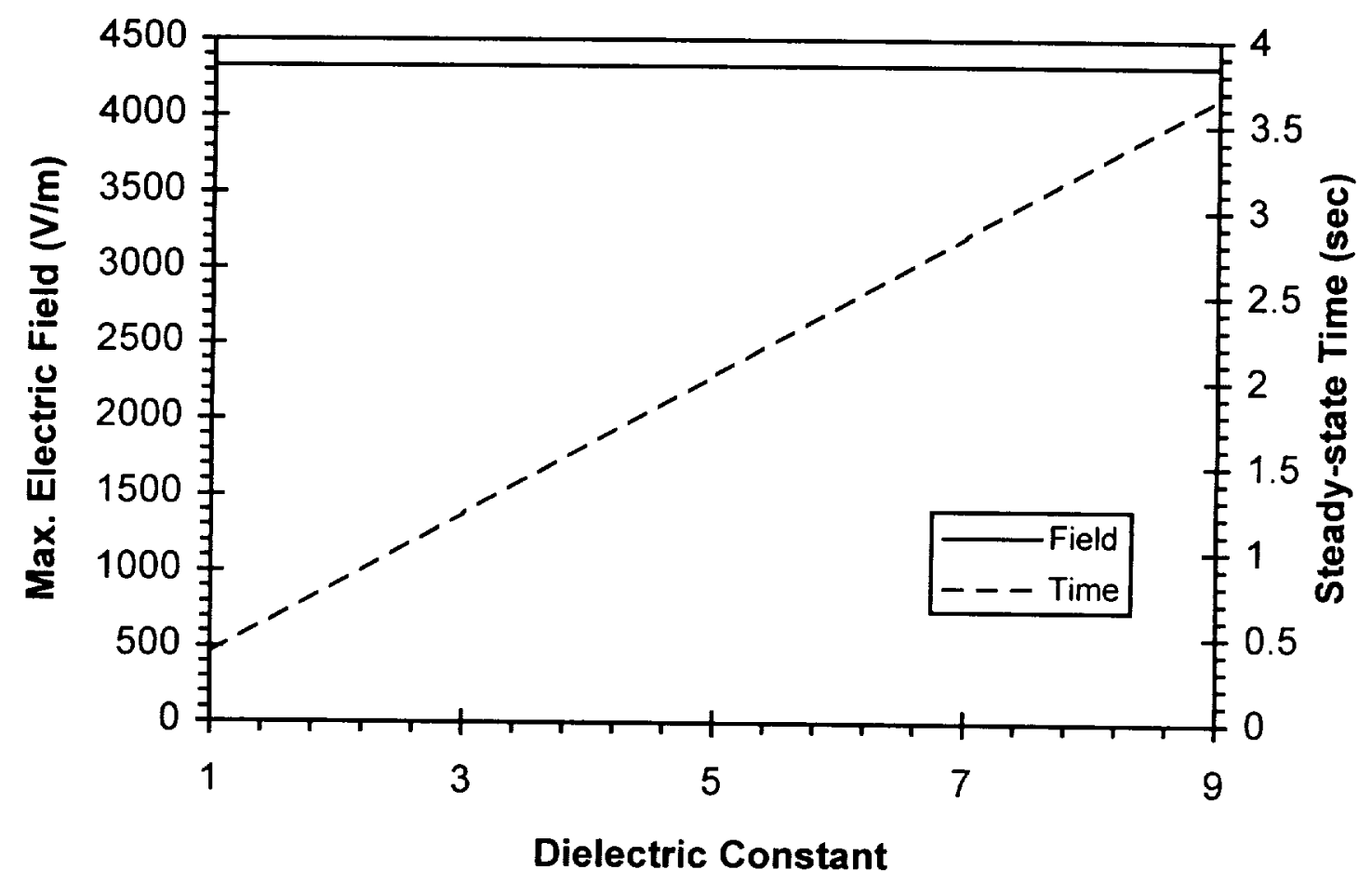

Figure 5.13: Dielectric constant sensitivity 


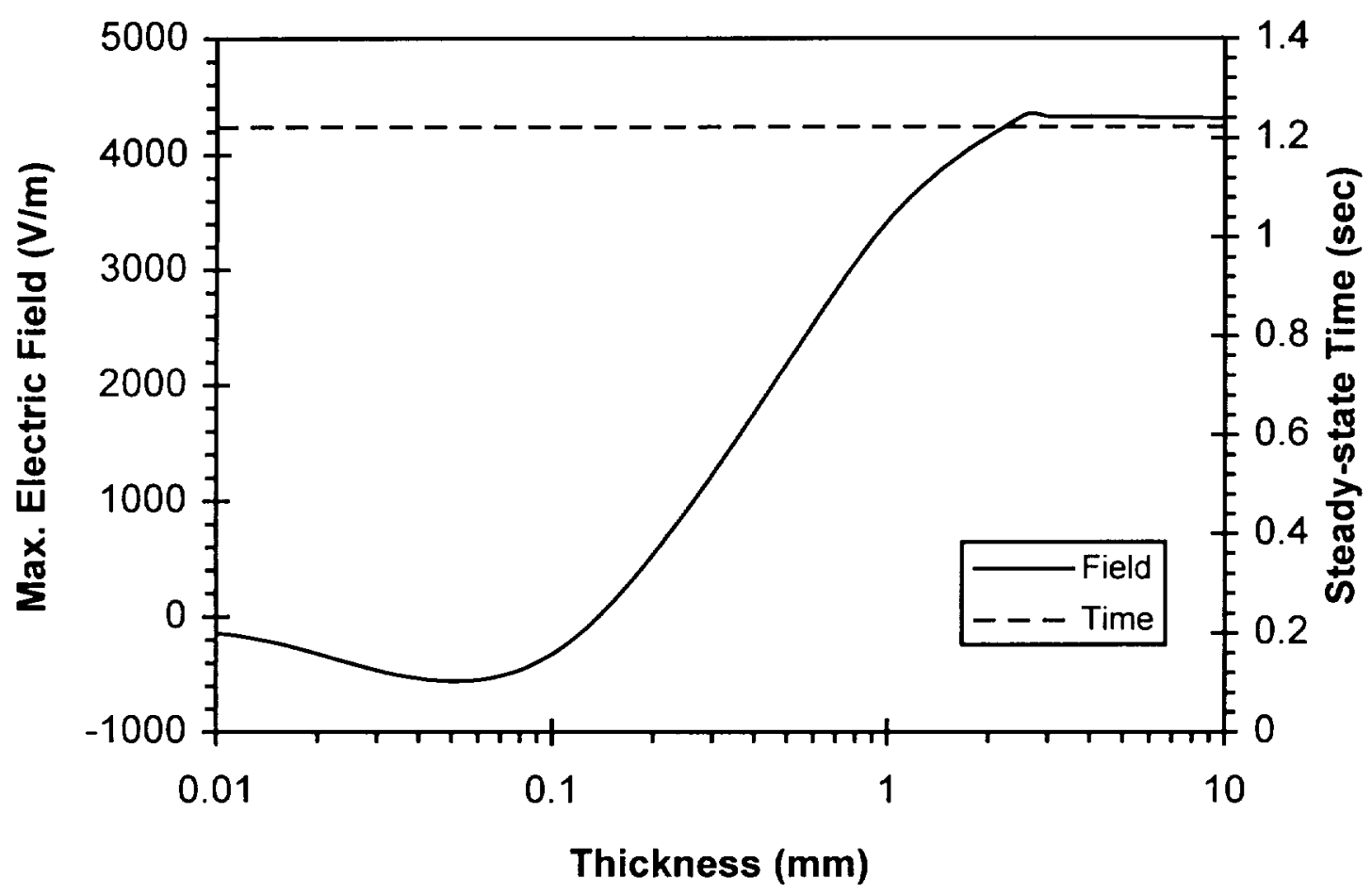

Figure 5.14: Thickness sensitivity 
enough to stop the majority of the particles, and thus the maximum electric field does not increase with any further increase in thickness.

\subsubsection{Density}

The density was varied within the range of possible densities for composites and polymers, from 600 to $2400 \mathrm{~kg} / \mathrm{m}^{3}$, as shown in Figure 5.15 . The results show that the maximum steady-state electric field increases with density until it reaches a constant value. The time to steady state is unchanged with increase in density. Also, the charge density, electric field, and voltage distributions change with density. The explanation for the electric field trend is similar to that for the thickness sensitivities, since the density of the material controls how far a particle penetrates for a given energy, as shown in Figure 5.16. Therefore, at low densities the higher energy particles pass through the material, thus resulting in a lower electric field. As the density increases, more particles are stopped by the material and the maximum electric field increases, until the density is such that the majority of the particles are stopped and the maximum electric field is unaffected by further increases in density, as shown in Figure 5.17 .

\subsubsection{Solar Cycle Condition}

The base case was run with the environment of the same orbit but at the solar cycle condition of solar minimum. As can be seen in Figures 5.18, the solar minimum condition does not affect the shape of the flux profiles, it reduces the electron flux by about 3.5 times and does not affect the proton flux. This results in the decrease of the negative charge density, the maximum electric field, and the maximum voltage by approximately the same amount, as shown in Figures 5.19 through 5.21. However, since 


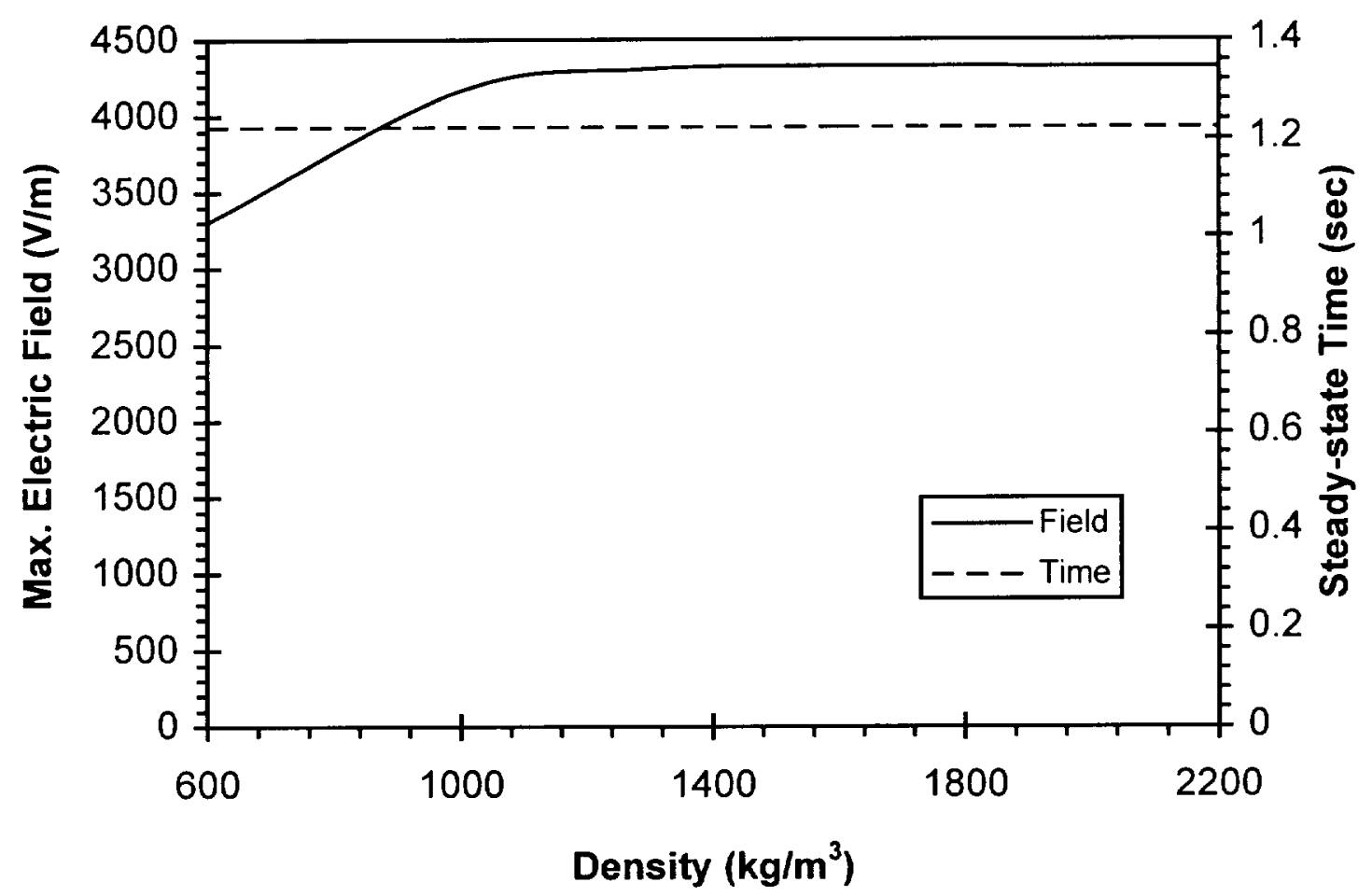

Figure 5.15: Density sensitivity 

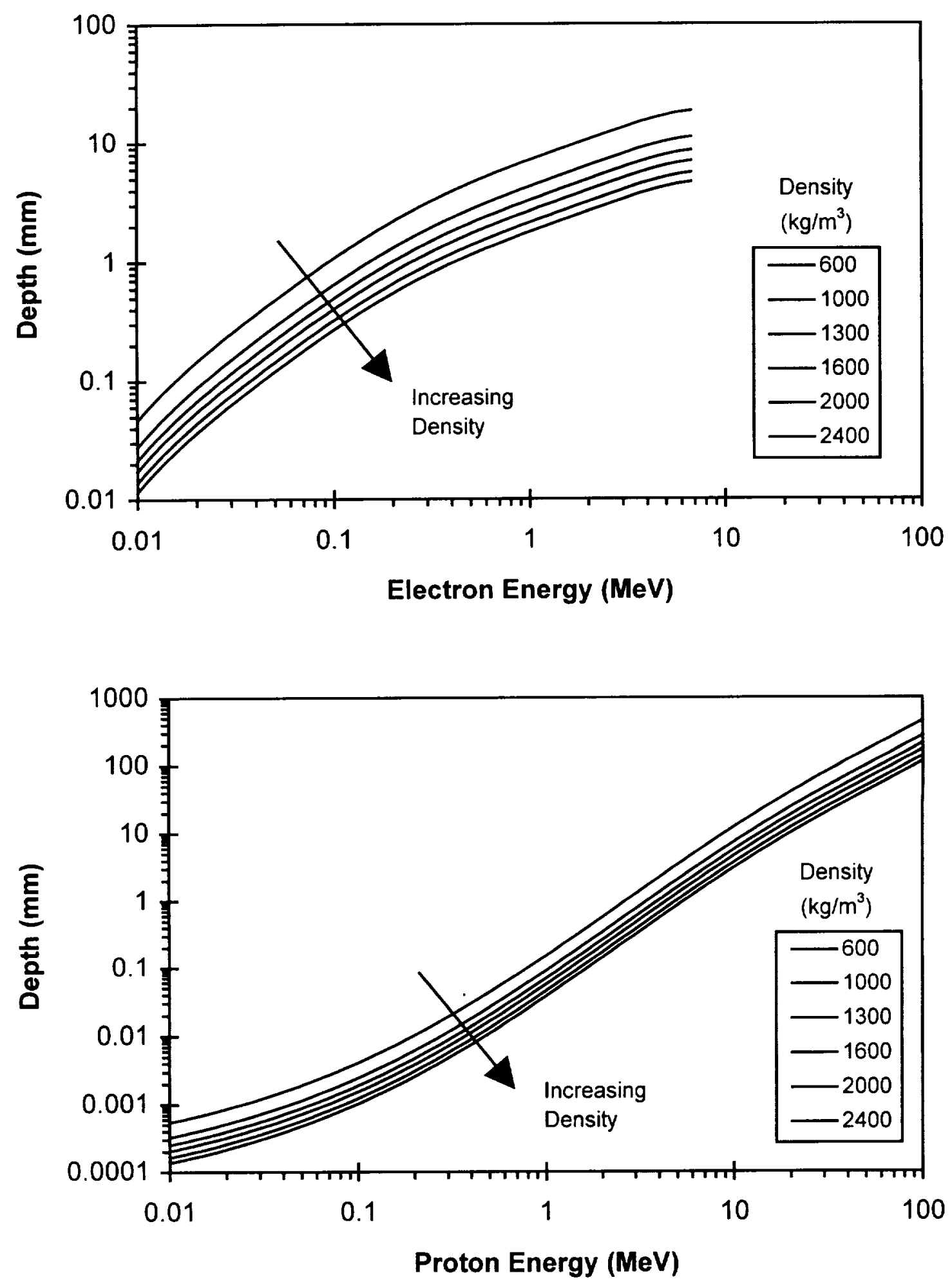

Figure 5.16: Electron and proton penetration depth density sensitivity 


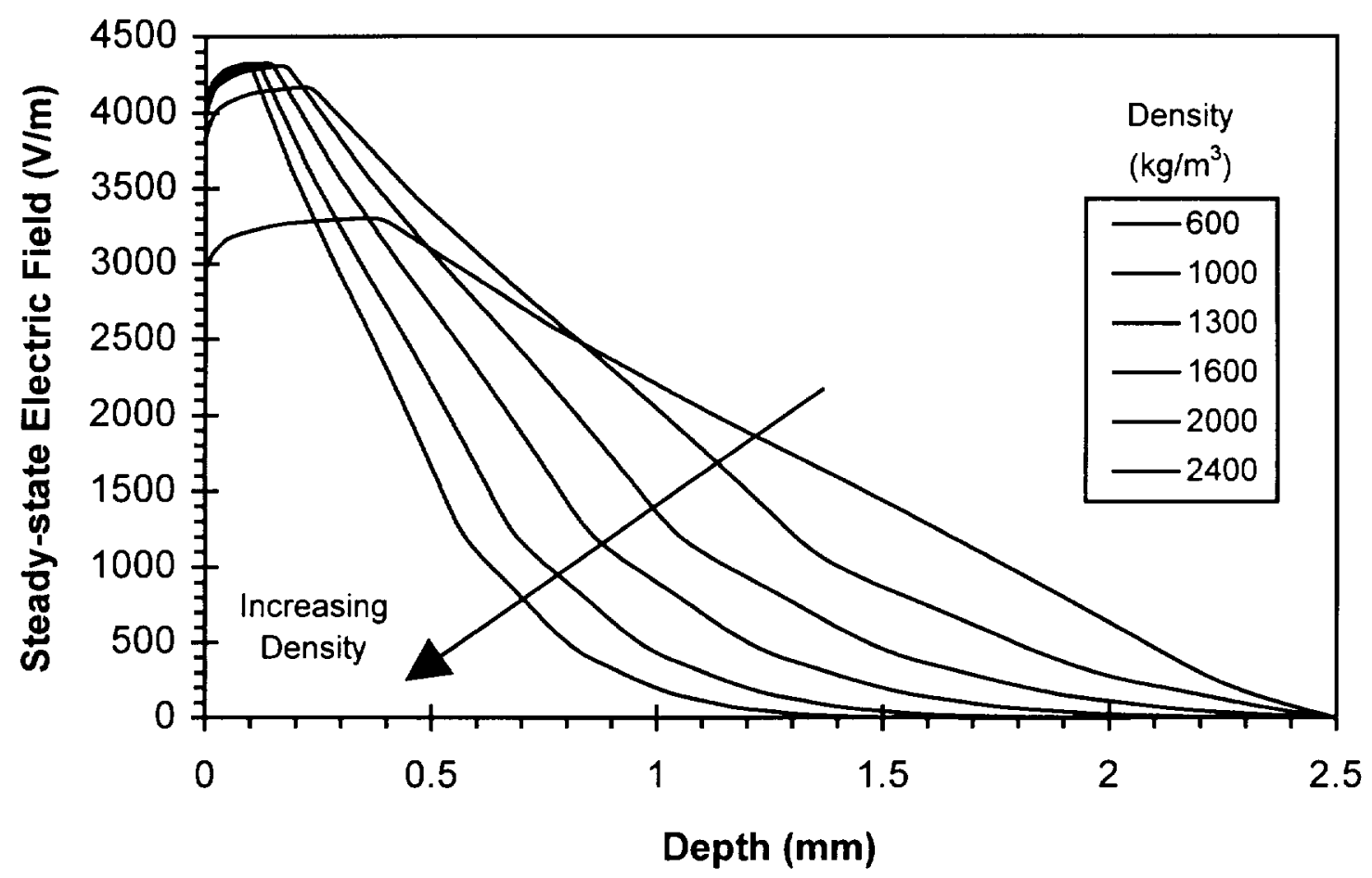

Figure 5.17: Steady-state electric field for various densities 

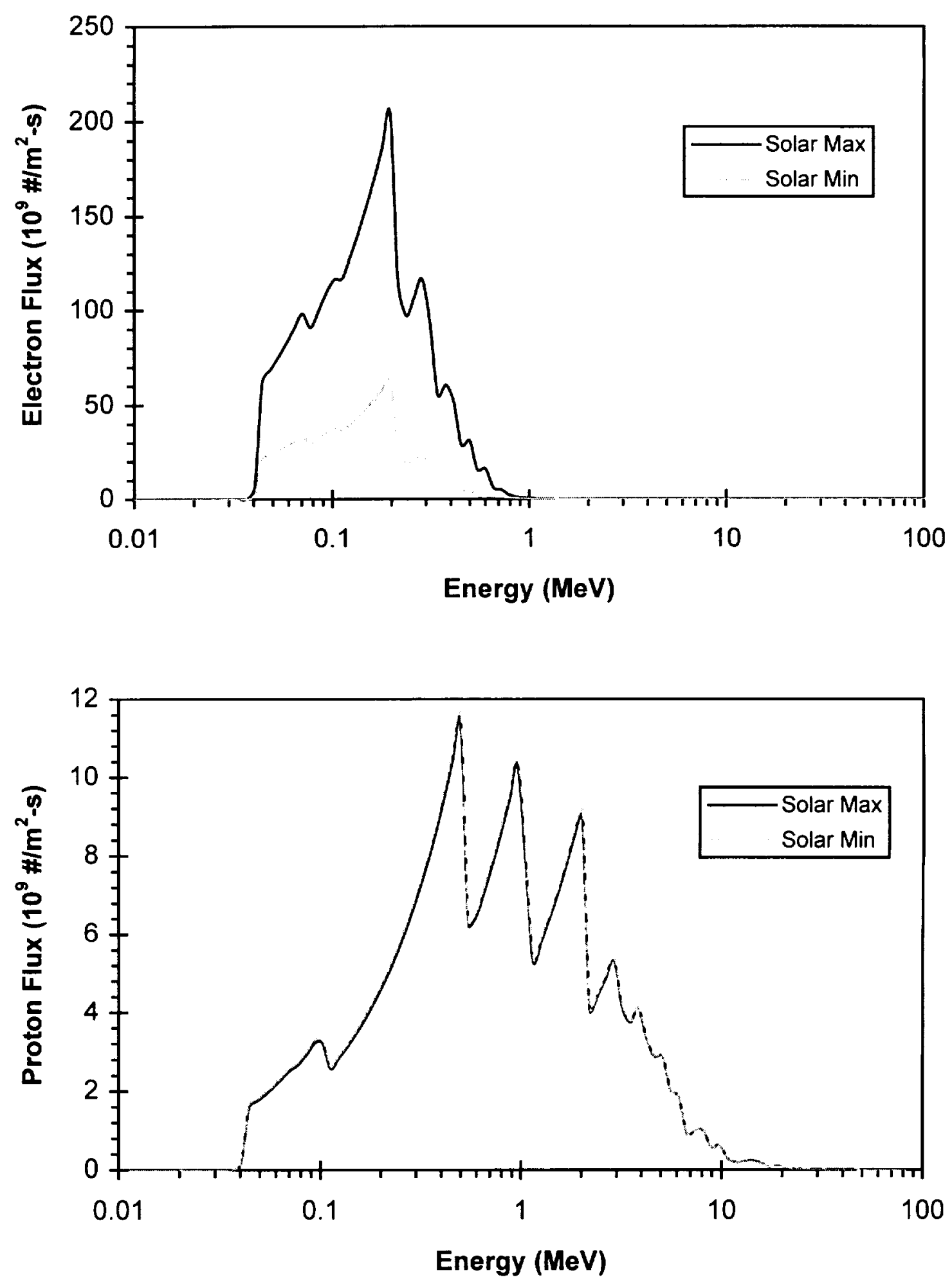

Figure 5.18: Base case orbit environment 


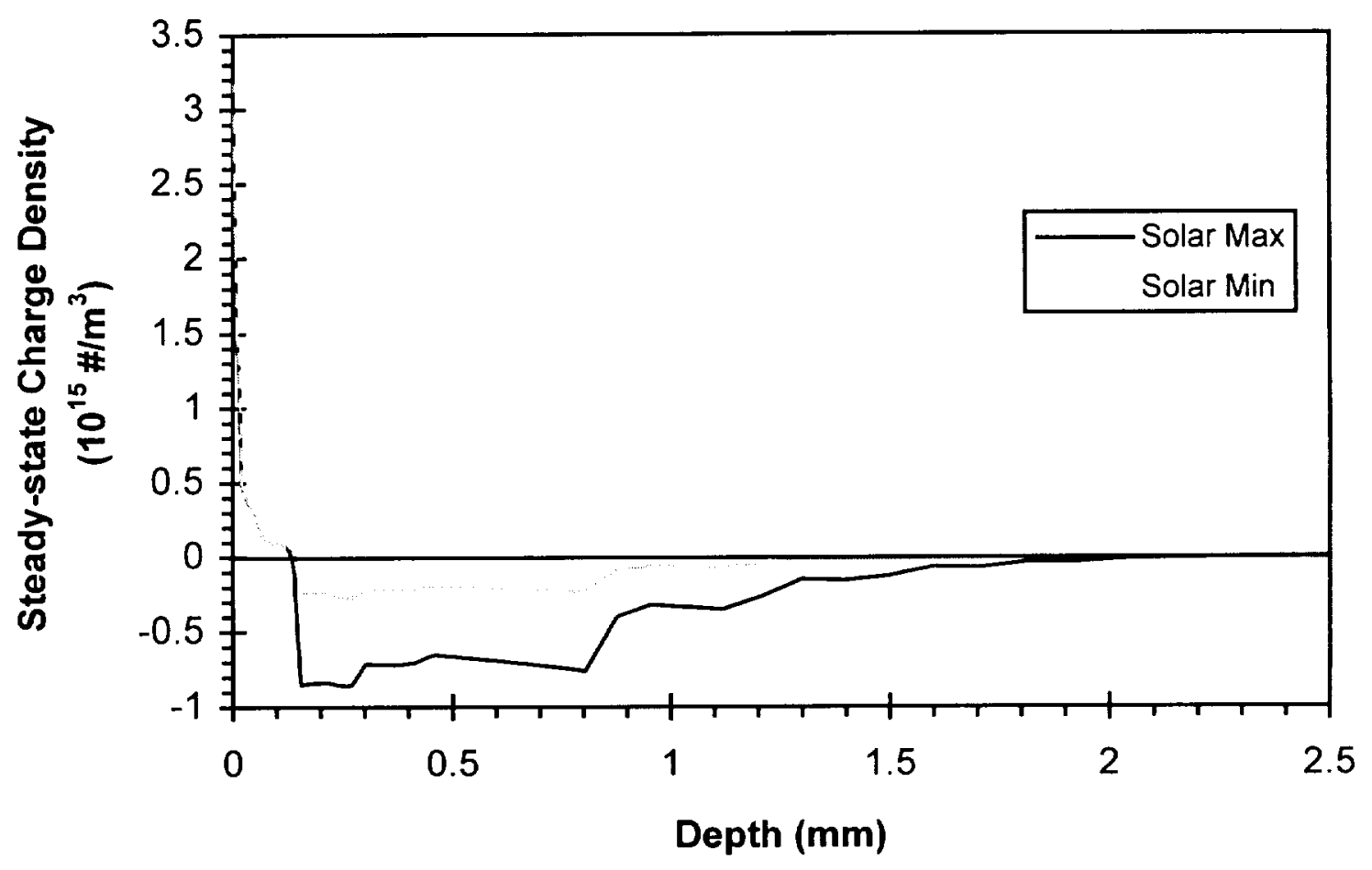

Figure 5.19: Solar condition charge density sensitivity 


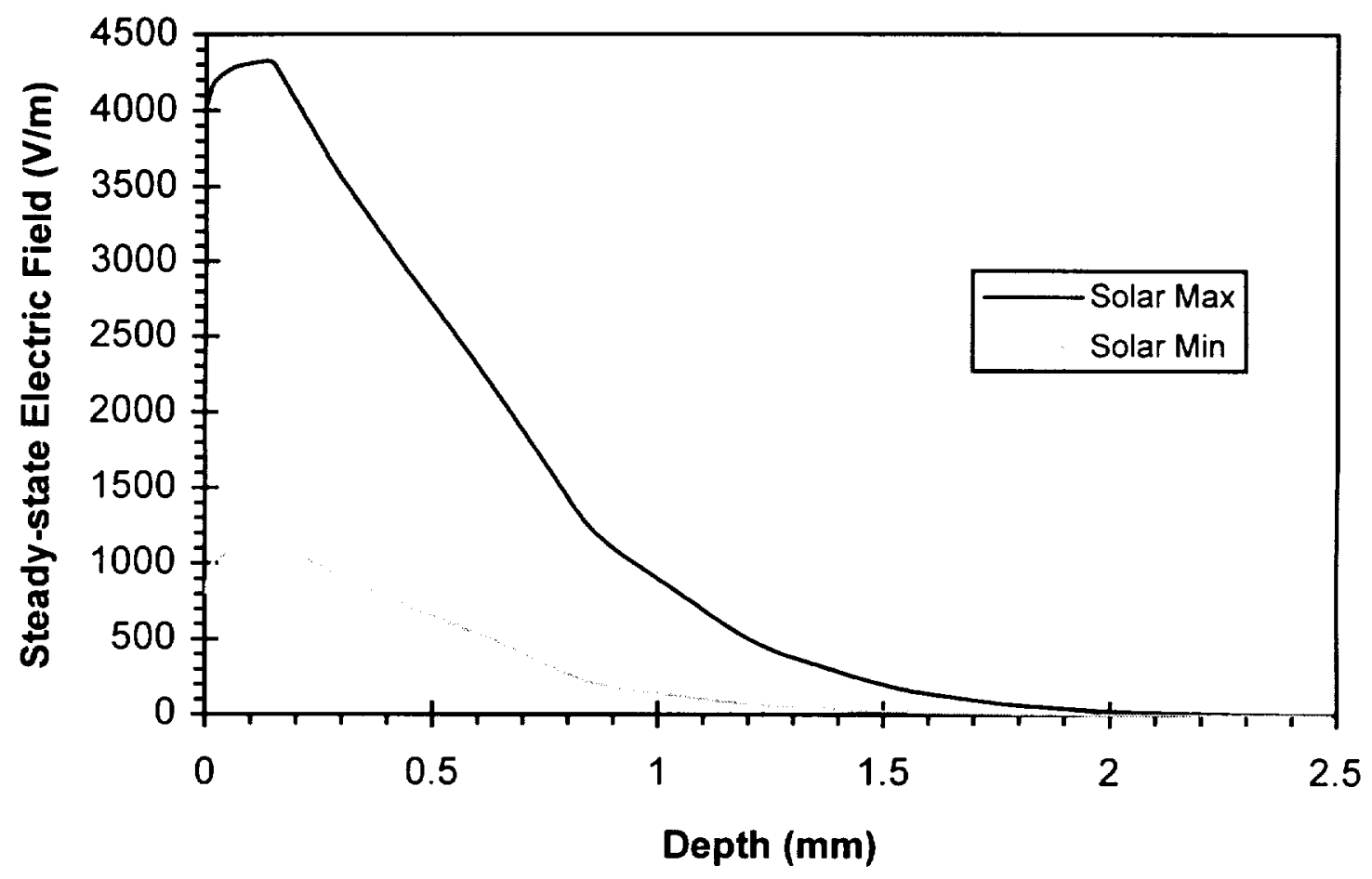

Figure 5.20: Solar condition electric field sensitivity 


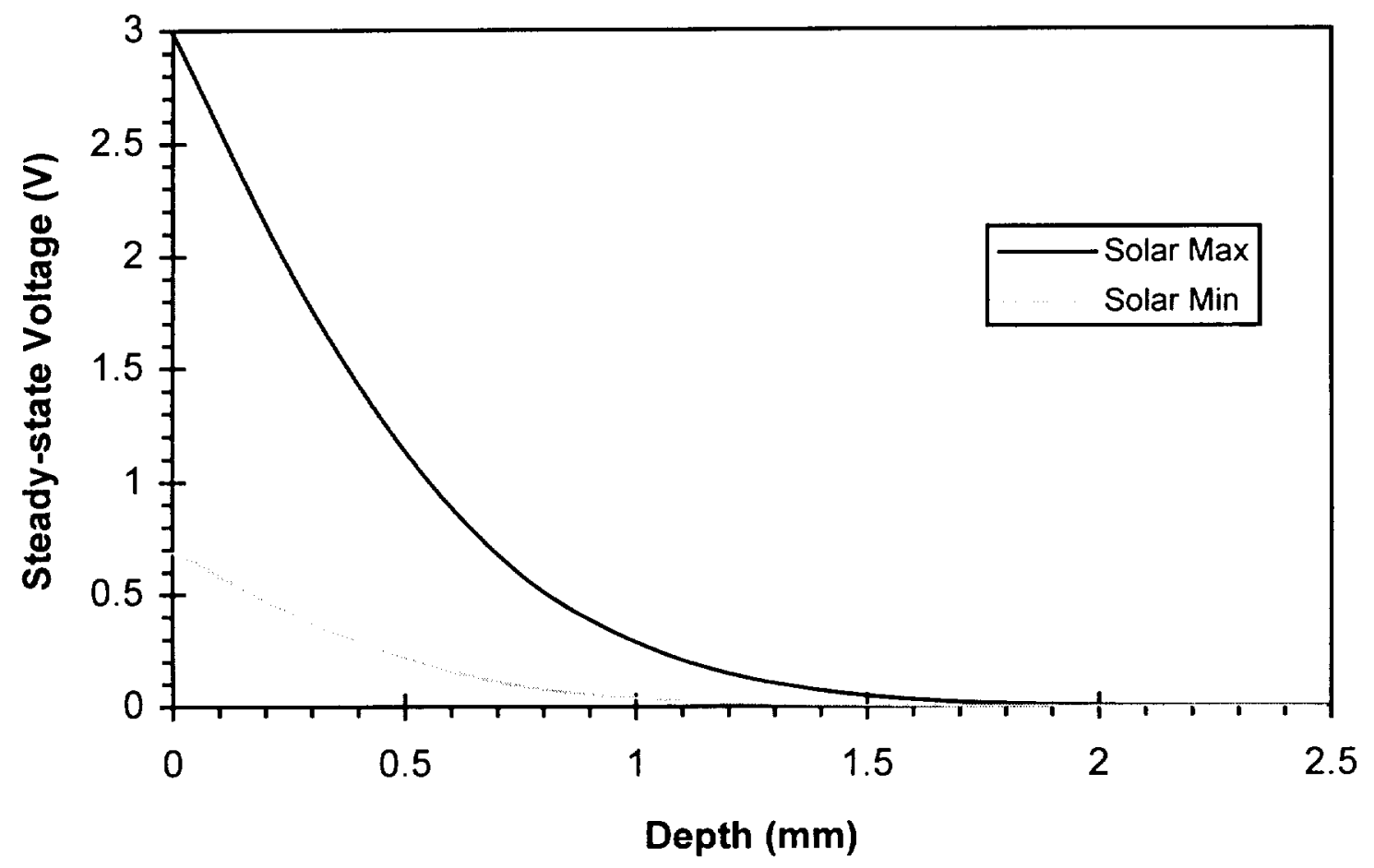

Figure 5.21: Solar condition voltage sensitivity 
the proton flux was not affected, most of the positive charge density was not affected. Thus there is no change to shape of the electric field near the front surface (Depth $(x)=0$ $\mathrm{mm})$. It should also be noted that the time to steady state did not change between solar maximum and solar minimum; this is because the time to steady state is only dependent on the conductivity and the dielectric constant of the material.

\subsubsection{Geomagnetic Substorm}

As mentioned earlier, geomagnetic substorms increase the amount of electron flux in orbit. Therefore the substorm intensity factor is used to scale the incoming electron flux. The intensity factor was incremented from 1 to 1000 , representing no substorms up to a worst case substorm, as shown in Figure 5.22. The results show that the maximum steady-state electric field increased linearly with the substorm intensity factor, while the

time to steady state remained unchanged. Also, the charge density, electric field, and voltage distribution changed with the substorm intensity factor, as while the number of electrons was increasing the number of protons remained constant. Thus as the intensity factor increased, the effect of the protons becomes more and more negligible. This is shown in Figure 5.23, where the increase in electric field due to protons near the front surface (refer to Figure 5.2) is unobservable.

\subsubsection{Solar Particle Event}

As mentioned earlier, solar particle events increase the proton flux in orbit. Therefore the solar particle event intensity factor is used to scale the incoming proton flux. The intensity factor was incremented from 1 to 1000 , representing no solar particle event up to a worst case solar particle event, as shown in Figure 5.24. The results show 


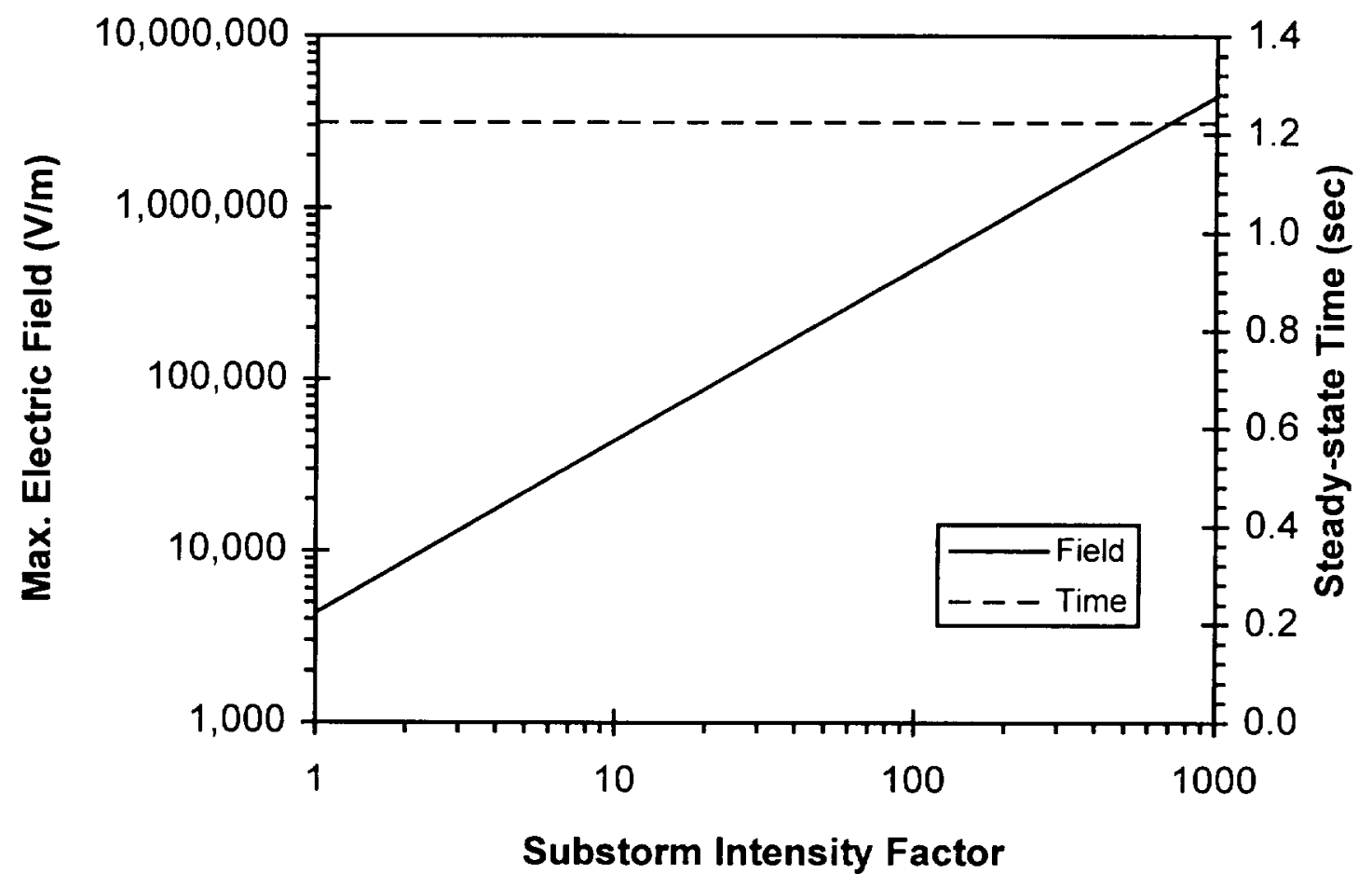

Figure 5.22: Geomagnetic substorm sensitivity 


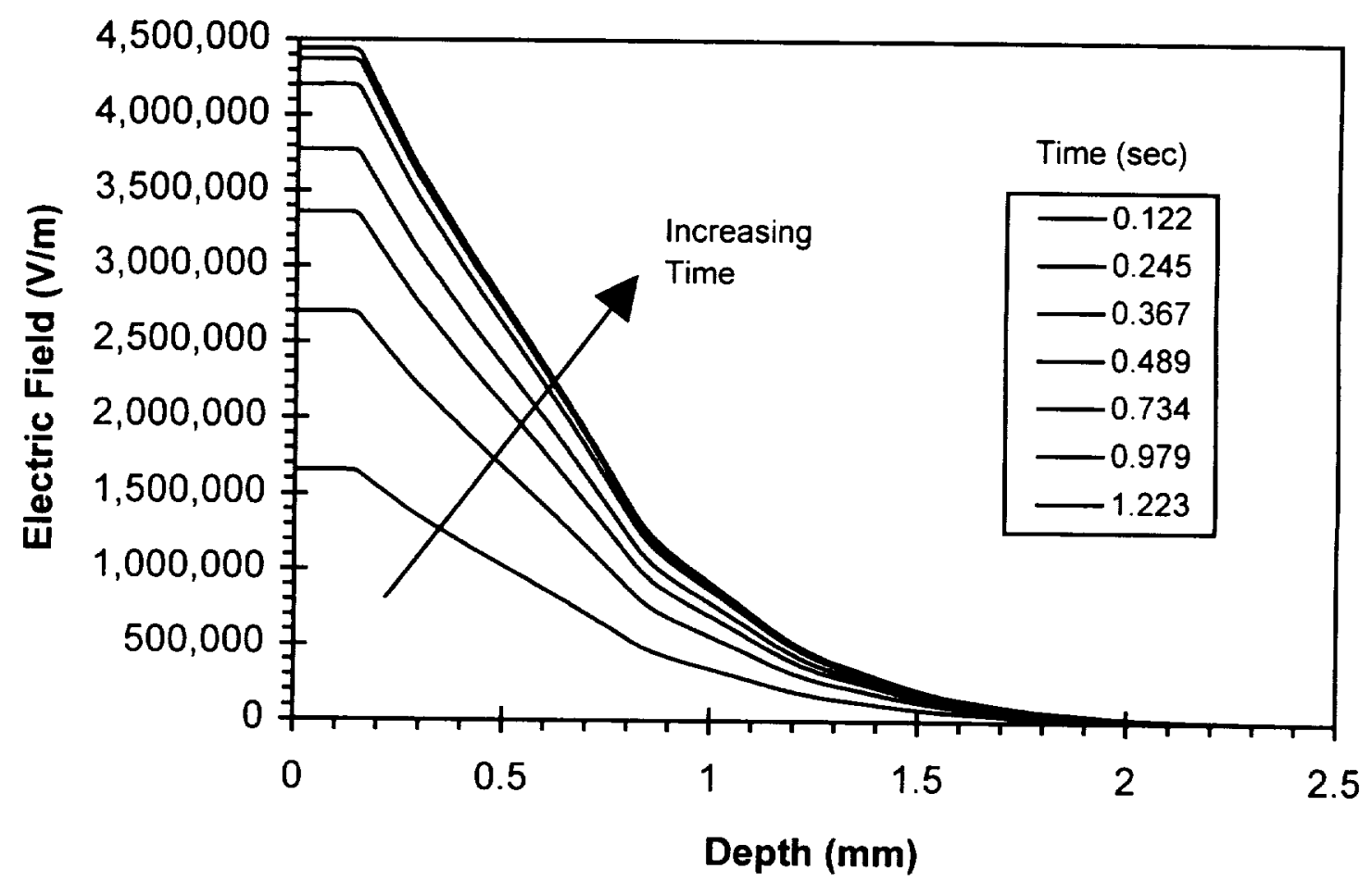

Figure 5.23: Electric field of base case with a substorm intensity of 1000 


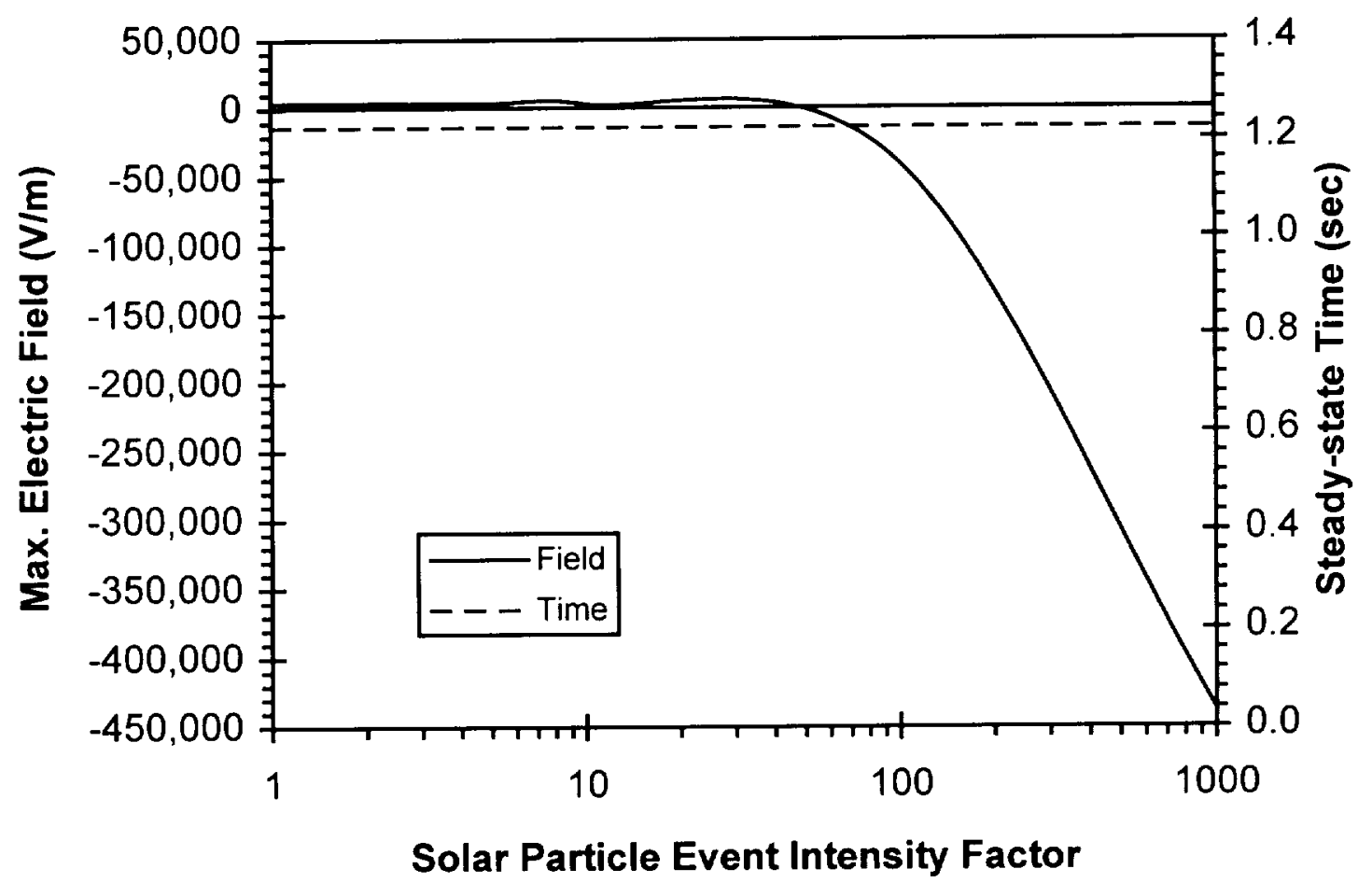

Figure 5.24: Solar particle event sensitivity 
that the maximum steady-state electric field remains constant until the intensity factor is high enough to start increasing the magnitude of the (negative) electric field. The time to steady state remains unchanged with increasing intensity factor. Also, the charge density, electric field, and voltage distributions changed with solar particle event intensity factor, as while the number of protons increased, the number of electrons remained constant. The reason for the initial constant electric field is the boundary condition of zero electric field on the back surface. With no solar particle event, the protons cause an increase in electric field of approximately $400 \mathrm{~V} / \mathrm{m}$, while the electrons cause a decrease in electric field of approximately $4400 \mathrm{~V} / \mathrm{m}$. Therefore since the electrons penetrate further than the protons, to obtain an electric field of zero on the rear surface, there must be an electric field of approximately $4000 \mathrm{~V} / \mathrm{m}$ on the front surface. As the proton flux increases, it creates a greater increase in electric field, however until the increase is greater than approximately $4000 \mathrm{~V} / \mathrm{m}$, the only change is to decrease the electric field on the front surface, the maximum electric field remains the same. As the intensity factor increases, the influence of the electrons becomes more and more negligible, as shown in Figure 5.25 , where the decrease in electric field due to the electrons is unperceivable.

\subsubsection{Summary}

The time to steady state depends inversely on the conductivity and linearly on the dielectric constant. The steady-state electric field is inversely dependent on the conductivity and dependent on the amount of particles present in the material. The amount of particles present in the material is controlled by the sample thickness, material density, and the substorm and solar particle event intensity factors. The shape of the 


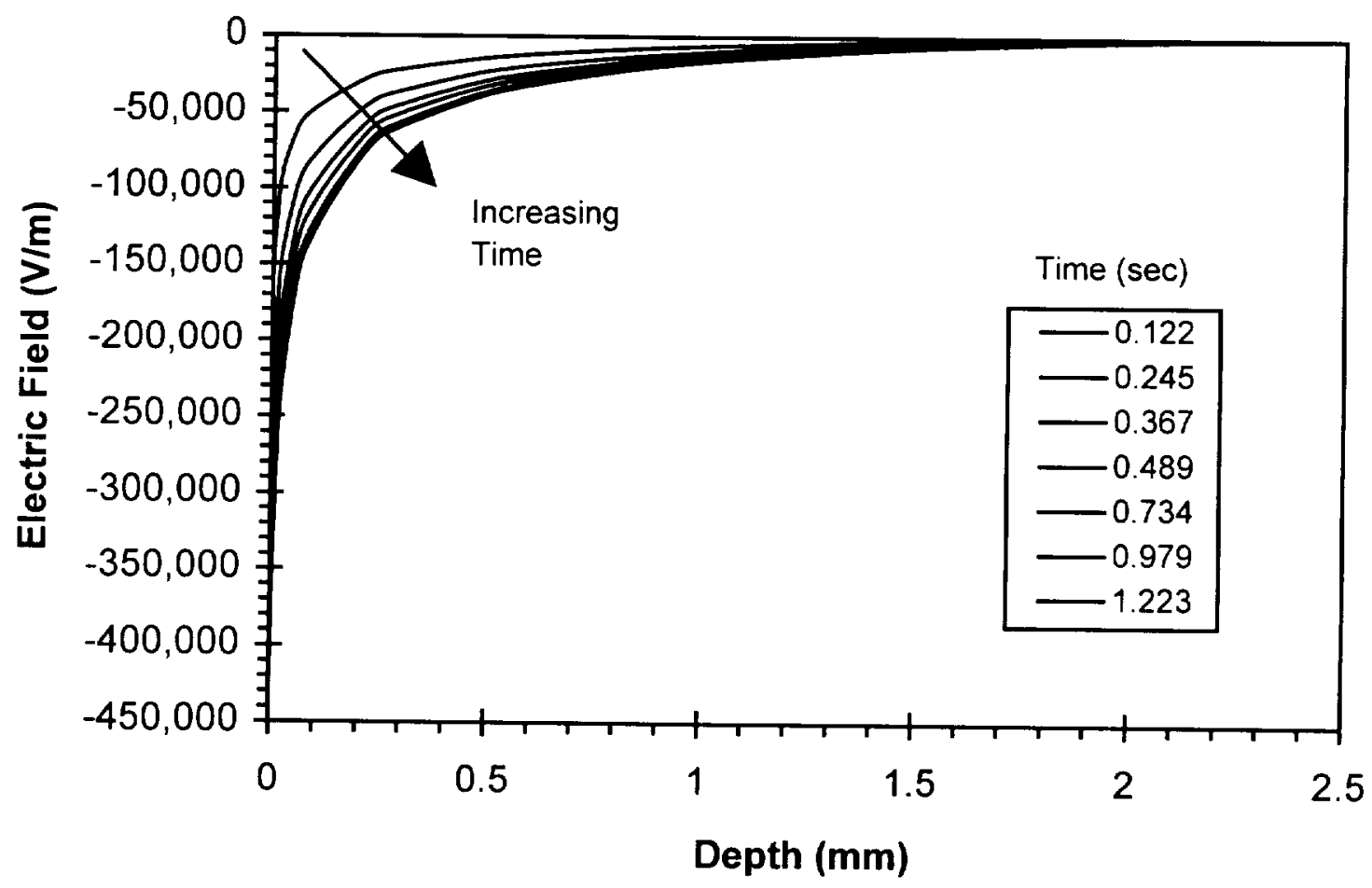

Figure 5.25: Electric field of base case with a solar particle event intensity of 1000 
charge density, electric field, and voltage distributions are dependent on the location of particles and on the relative amounts of protons and electrons. The location of particles is controlled by the sample thickness and the material density, and the relative amounts of particles is controlled by the substorm intensity factor and the solar particle event intensity factor.

\subsection{MICROMECHANICAL DETAILS}

This section presents the results of the modeling of the interactions between fibers and matrix on the electric field. The two models examined are the micro-scale model of a fiber surrounded by matrix, and the insulative pure epoxy surface layer of carbon fiber / epoxy composites.

\subsubsection{Micro-scale Model}

At the micro-scale, a concern is that higher fields can be possible due to geometric details of the fibers imbedded in matrix. The micromechanics of the fiber surrounded by epoxy has been looked at, to see if a substantial increase in the electric field can occur as the electric field in the epoxy matrix approaches the highly conductive carbon fibers.

The data used in this model comes from the base case used with the deep dielectric charging model. The far field electric field used is the maximum steady-state electric field from the base case of $4326 \mathrm{~V} / \mathrm{m}$. The far field electric field is approximately 6 to 8 times the fiber radius, this represents a worst case matrix rich zone. For ease of display the results, as shown in Figure 5.26, are plotted as electric field magnification 


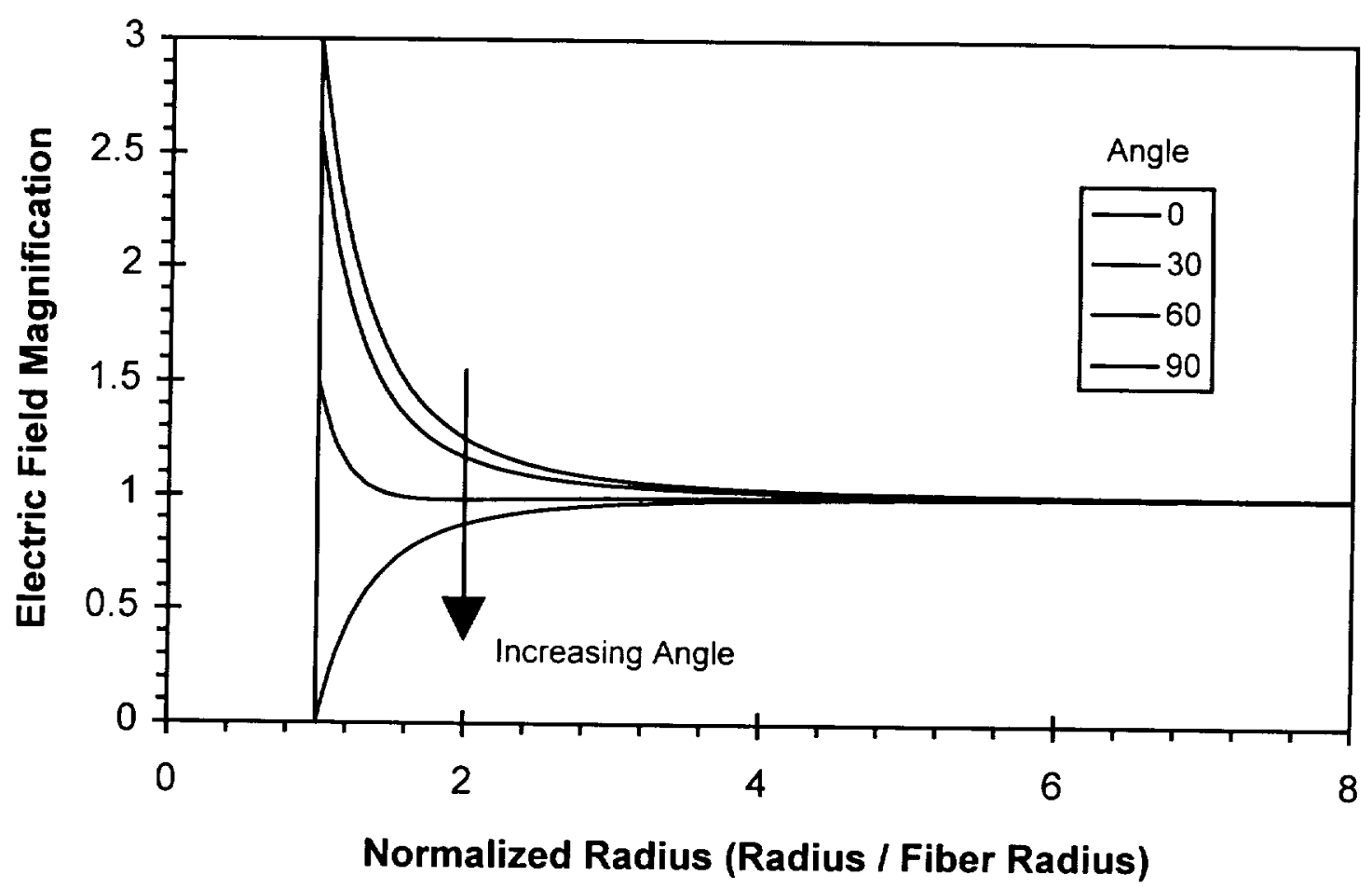

Figure 5.26: Electric field magnification around a fiber 
versus normalized radius. The electric field magnification is the calculated electric field divided by the far field electric field, and the normalized radius is the radius divided by the fiber radius.

Since the magnitude of the electric field is used the results are quadrant symmetric, the results are identical above and below the $\mathrm{x}$ axis and to the left and right of the $y$ axis. The results show that the electric field increases in the matrix as the field approaches the fiber parallel to the far field, with the maximum occurring at the fiber/matrix interface. When the field approaches the fiber perpendicular to the far field direction the electric field decreases, and reaches zero at the fiber/matrix interface. Thus the greatest possible increase is approximately a factor of 3 . It should be noted that this solution is an approximation to the problem as the charge density is assumed to be zero. Also, the typical distance between fibers for a 0.6 volume fraction carbon fiber / epoxy composite is approximately 1.3 times the fiber radius, thus invalidating the far field electric field assumption.

Another related problem is that unlikely arrangements of multiple fibers may create severe distortions in the electric fields, as shown in Figure 5.27, possibly resulting in increases as high as 50 times. $^{55}$ It should be noted that the electric field is small enough that even an increase of 50 times would not be sufficient to create an electrostatic discharge in the base case. However, in other cases where the electric field is close to the dielectric strength of the material an increase of 50 times could cause an electrostatic discharge to occur. 


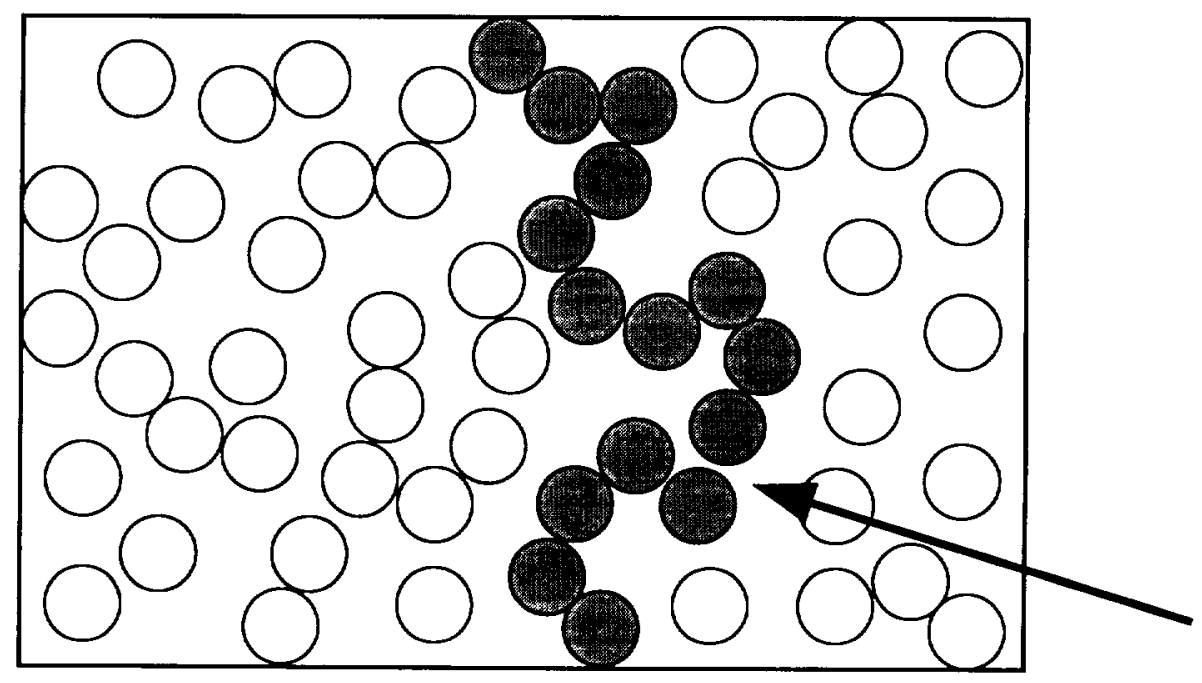

Figure 5.27: Schematic of an unlikely fiber arrangement which can lead to high field magnification 


\subsubsection{Epoxy Surface Layer}

A related problem is that of the electric field in carbon fiber/epoxy with an epoxy rich surface layer. This layer, approximately $20 \mu \mathrm{m}$ thick, has a much lower (several orders of magnitude) electrical conductivity than the bulk composite. The analysis was accomplished by modifying the deep dielectric charging analyzer to include two different conductivities, the dark conductivity of the bulk composite and the surface layer conductivity.

The results showed that the electric field in the bulk composite reached steady state as it would have in the case of only carbon fiber / epoxy with no epoxy surface layer, a maximum steady-state electric field of $4.33 \times 10^{-6} \mathrm{~V} / \mathrm{m}$ and time to steady state of $1.22 \times 10^{-9} \mathrm{sec}$. However, the electric field in the surface layer kept increasing. To solve for the electric field and steady-state time in the surface layer, the R-C circuit model is used (refer to Section 4.4.2), with the data shown in Table 5.3. A simple R-C circuit analysis can be used since there is such a difference in the time scales of the bulk composite and the epoxy surface layer. The results show that the time to steady state is $1.22 \mathrm{sec}$ and the steady-state electric field across the surface layer is $473 \mathrm{~V} / \mathrm{m}$. These values are very close to the values that would have been obtained if the entire sample was made of epoxy, i.e. the base case described earlier. 
Table 5.3: Data used in epoxy surface layer model

\begin{tabular}{||l|c|}
\hline Property & Value \\
\hline \hline Surface layer conductivity, $\sigma$ & $10^{-10} 1 / \Omega-\mathrm{m}$ \\
\hline Bulk composite conductivity, $\sigma$ & $0.11 / \Omega-\mathrm{m}$ \\
\hline Dielectric constant, $\kappa$ & 3 \\
\hline Surface layer thickness, $\mathrm{h}_{\mathrm{SL}}$ & $25 \mu \mathrm{m}$ \\
\hline Incoming charge density, $\rho_{\mathrm{in}}$ & $1.18 \times 10^{16} \mathrm{\#} / \mathrm{m}^{3}-\mathrm{s}$ \\
\hline
\end{tabular}




\subsection{CASE STUDIES}

Case studies were performed for the base case material configuration on actual orbits used by spacecraft where deep dielectric charging may be of concern. These orbits include geosynchronous (GEO) orbit, the Global Positioning System (GPS) orbit, a low altitude retrograde or polar orbit, the Molniya orbit, and the space shuttle parking orbit. The environment data was once again obtained from the Environmental Workbench software for both solar maximum and solar minimum solar cycle conditions. The orbit parameters used are shown in Table $5.4 .^{56}$

\subsubsection{The Geosynchronous Orbit}

According to the Environmental Workbench, at geosynchronous orbit there is no distinction between solar maximum and solar minimum. The electron and proton fluxes can be seen in Figures 5.28. While the electron flux distribution looks similar to the base case, the proton flux does not have the high energy (greater than $1 \mathrm{MeV}$ ) particles. The results, as shown in Figures 5.29 through 5.31, are very similar to the base case except that they are slightly less than an order of magnitude smaller. Also, the electric field and voltage distributions penetrate deeper into the material before becoming negligible.

\subsubsection{Global Positioning System Orbit}

The Global Positioning System orbit environment, as shown in Figures 5.32, has approximately half the electron flux at solar minimum as compared to solar maximum, and the electron flux has higher energy particles, up to $3 \mathrm{MeV}$. Also, the proton flux is unchanged between solar maximum and solar minimum, and once again it does not have the higher energy particles like the base case does. The results, as shown in Figures 5.33 
Table 5.4: Case study orbit data

\begin{tabular}{||l|c|c|c||}
\hline Orbit & Apogee Altitude & Perigee Altitude & Inclination \\
\hline \hline Geosynchronous & $35,782 \mathrm{~km}$ & $35,782 \mathrm{~km}$ & $0^{\circ}$ \\
\hline GPS & $20,222 \mathrm{~km}$ & $20,222 \mathrm{~km}$ & $60^{\circ}$ \\
\hline Polar & $322 \mathrm{~km}$ & $322 \mathrm{~km}$ & $98^{\circ}$ \\
\hline Molniya & $40,172 \mathrm{~km}$ & $272 \mathrm{~km}$ & $63.4^{\circ}$ \\
\hline Shuttle Parking & $322 \mathrm{~km}$ & $322 \mathrm{~km}$ & $28.4^{\circ}$ \\
\hline
\end{tabular}



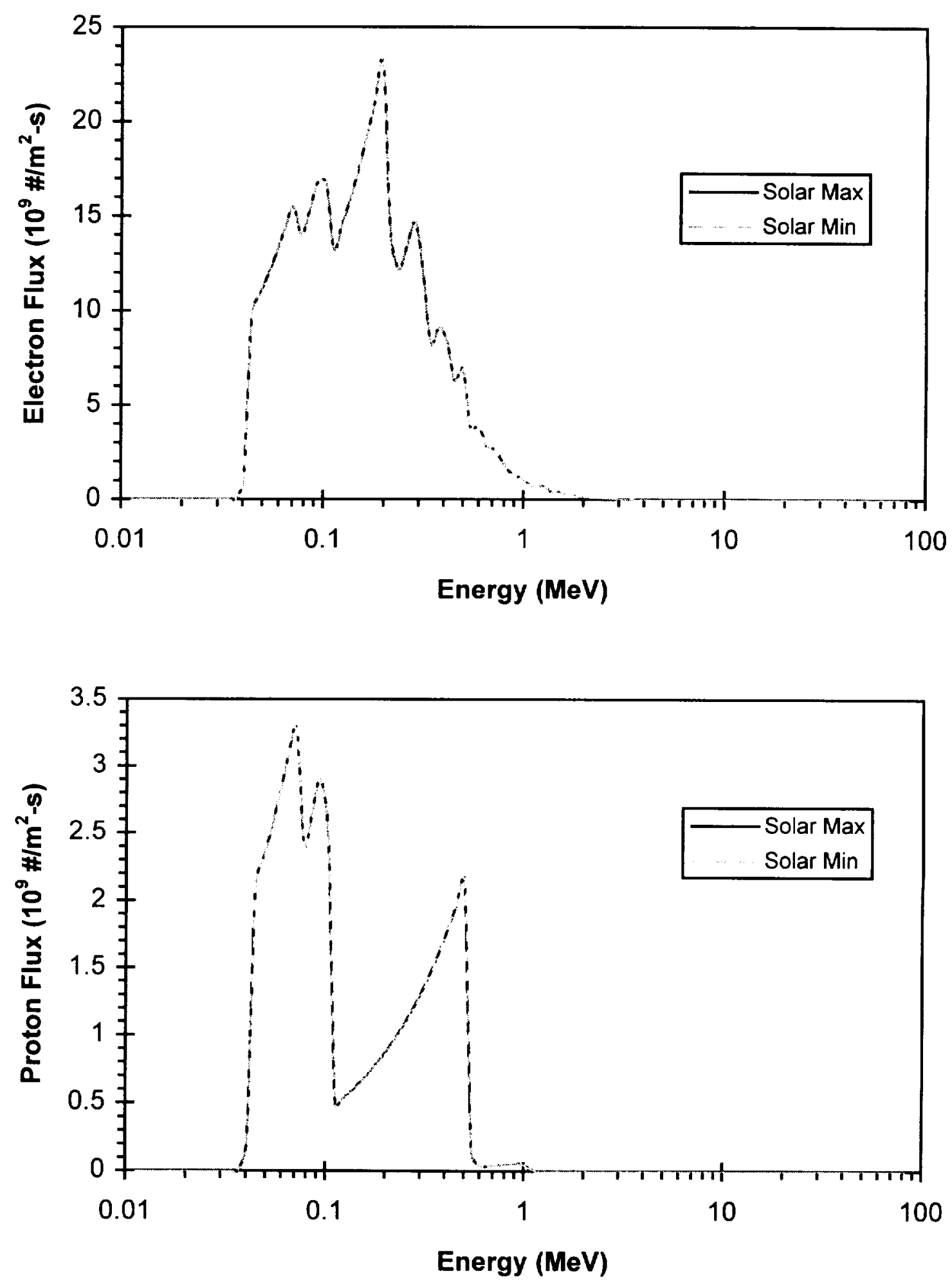

Figure 5.28: Geosynchronous orbit environment 


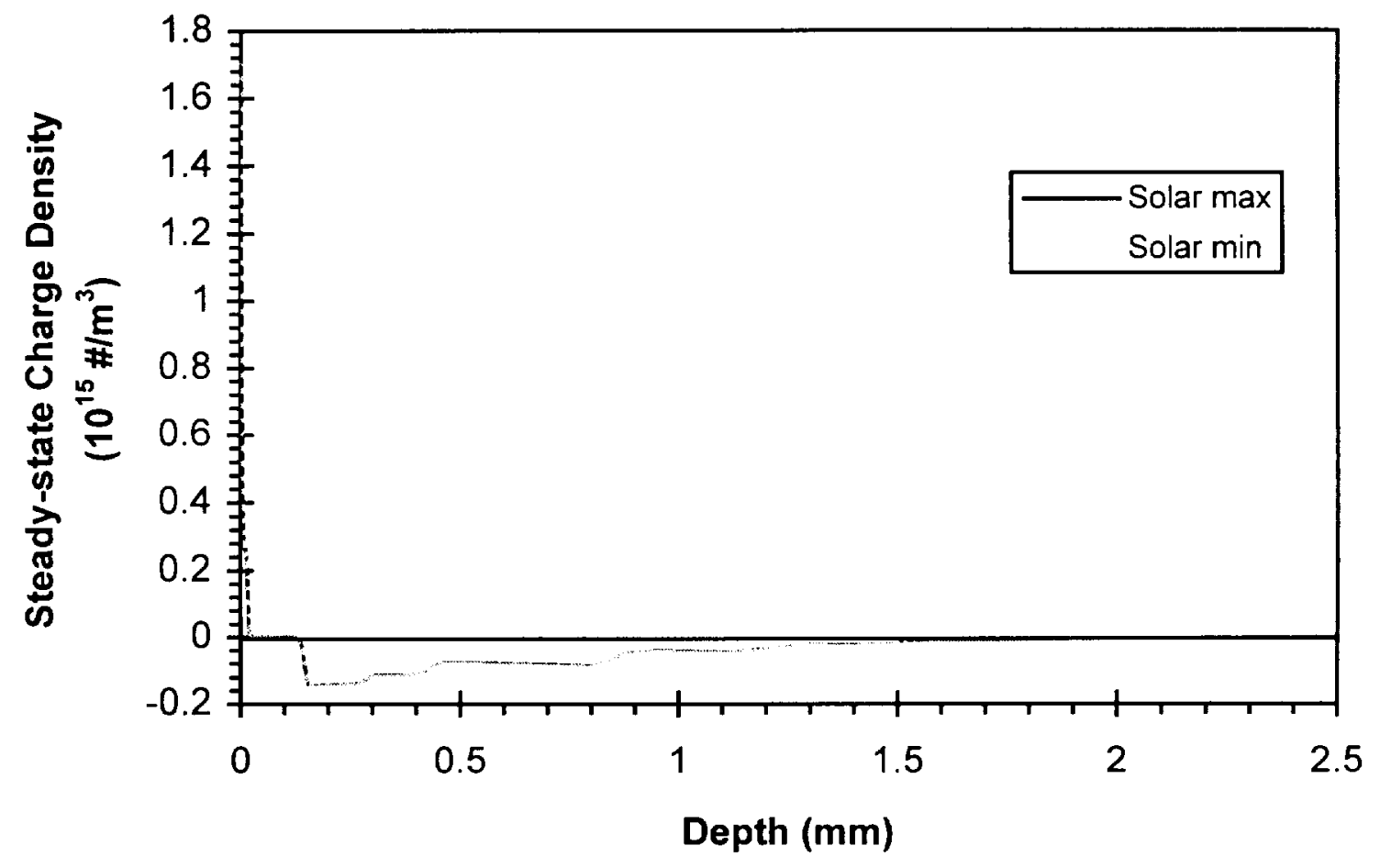

Figure 5.29: Geosynchronous orbit charge density distribution 


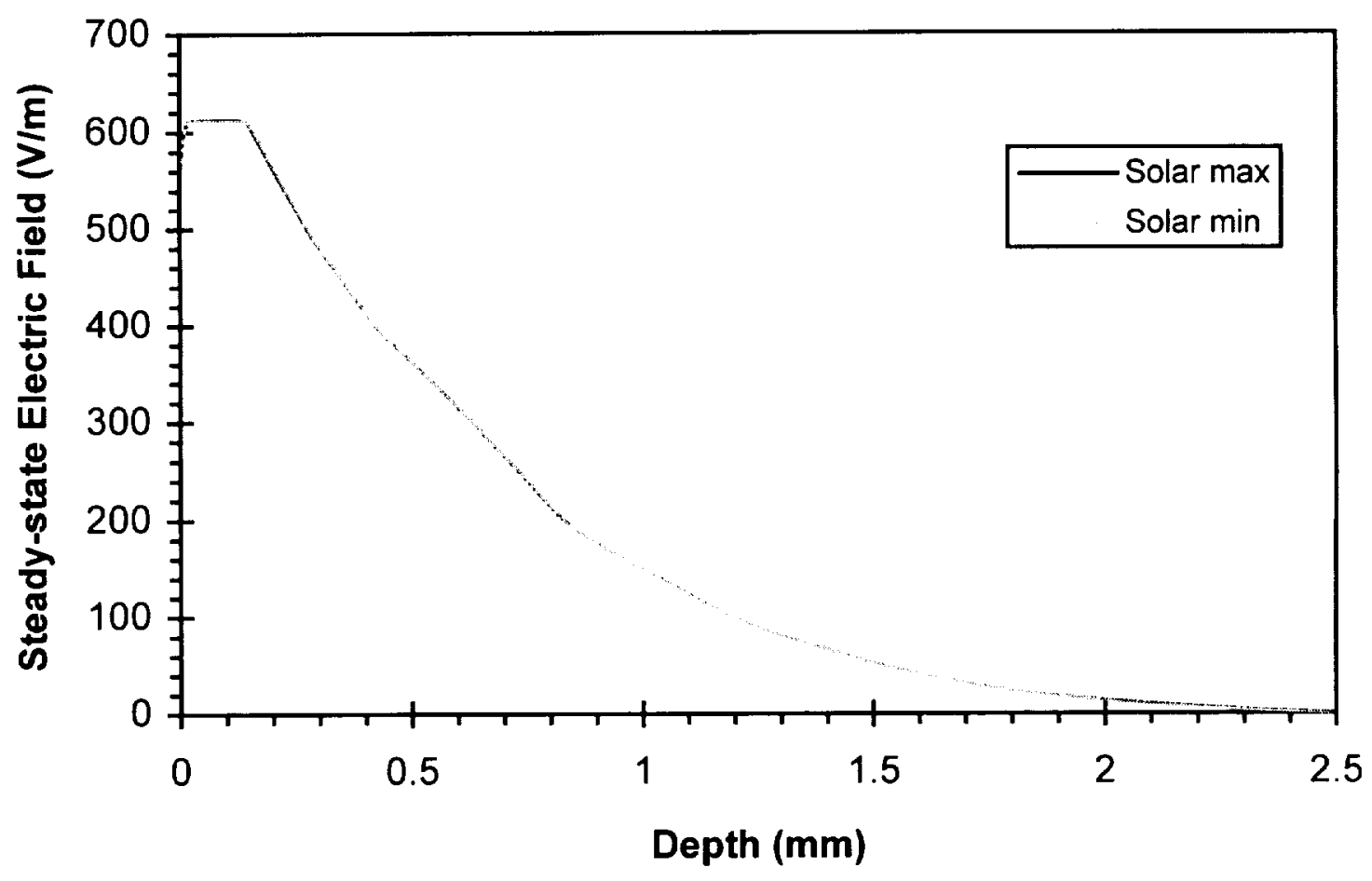

Figure 5.30: Geosynchronous orbit electric field distribution 


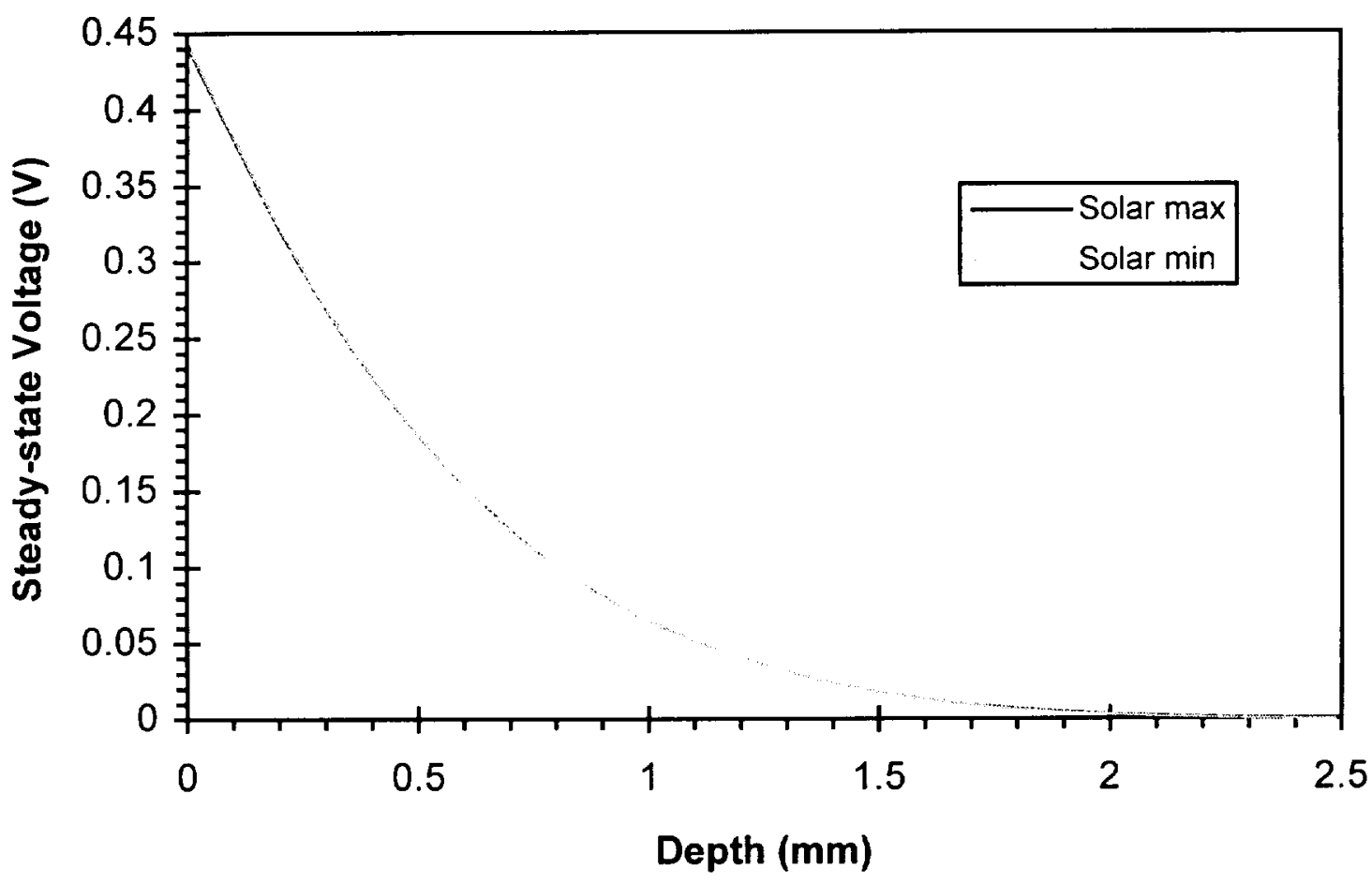

Figure 5.31: Geosynchronous orbit voltage distribution 

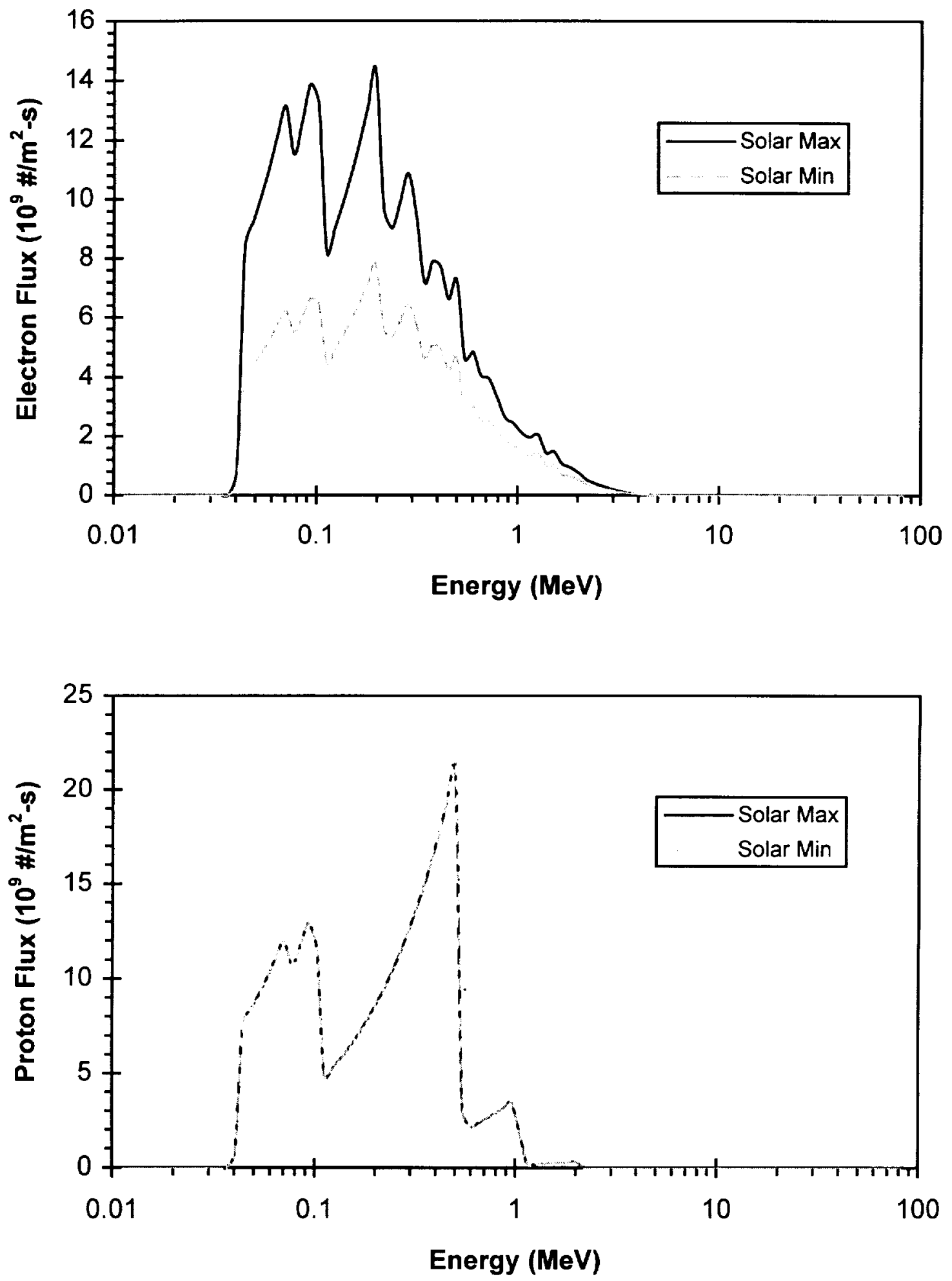

Figure 5.32: Global Positioning System orbit environment 


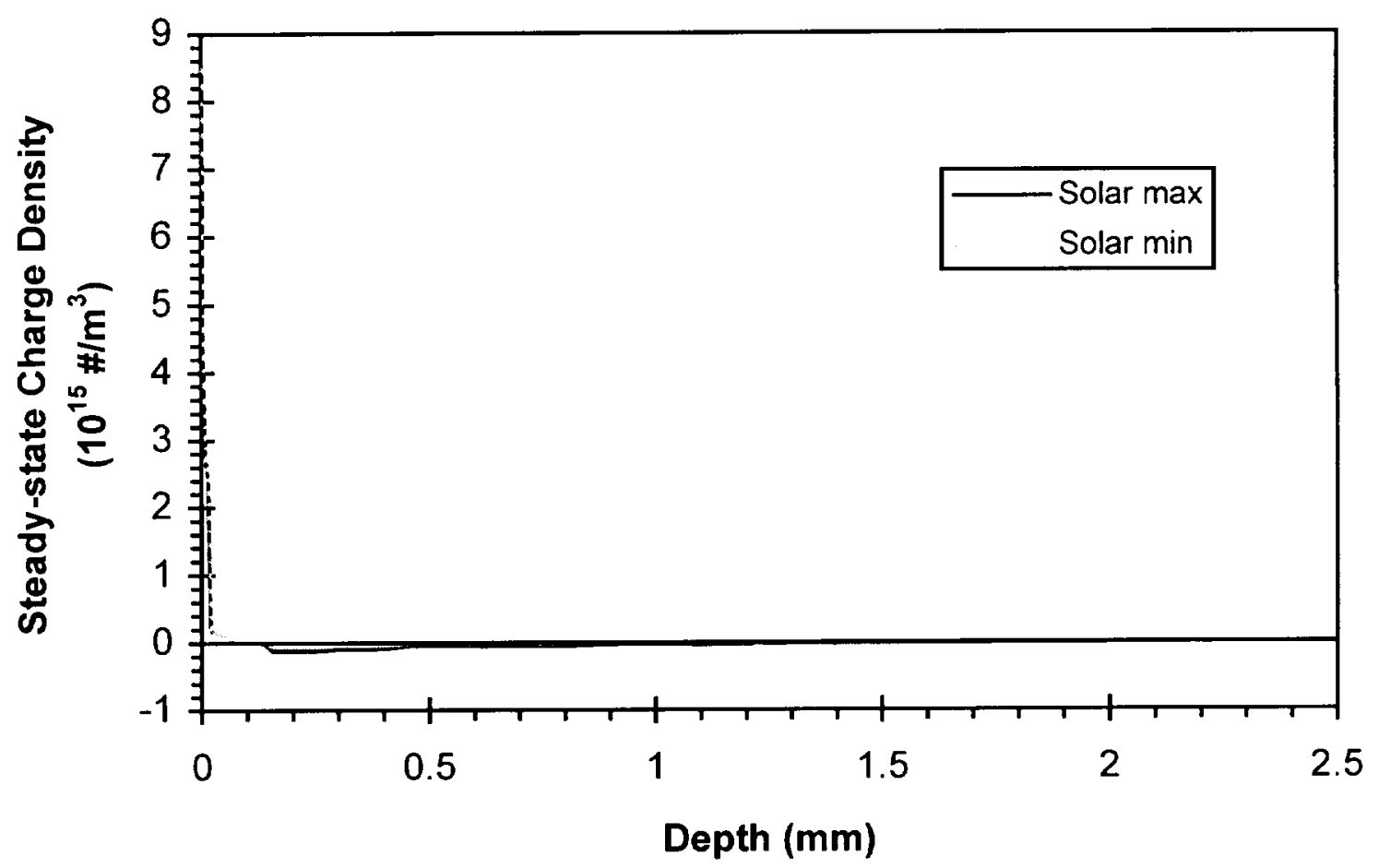

Figure 5.33: Global Positioning System orbit charge density distribution 


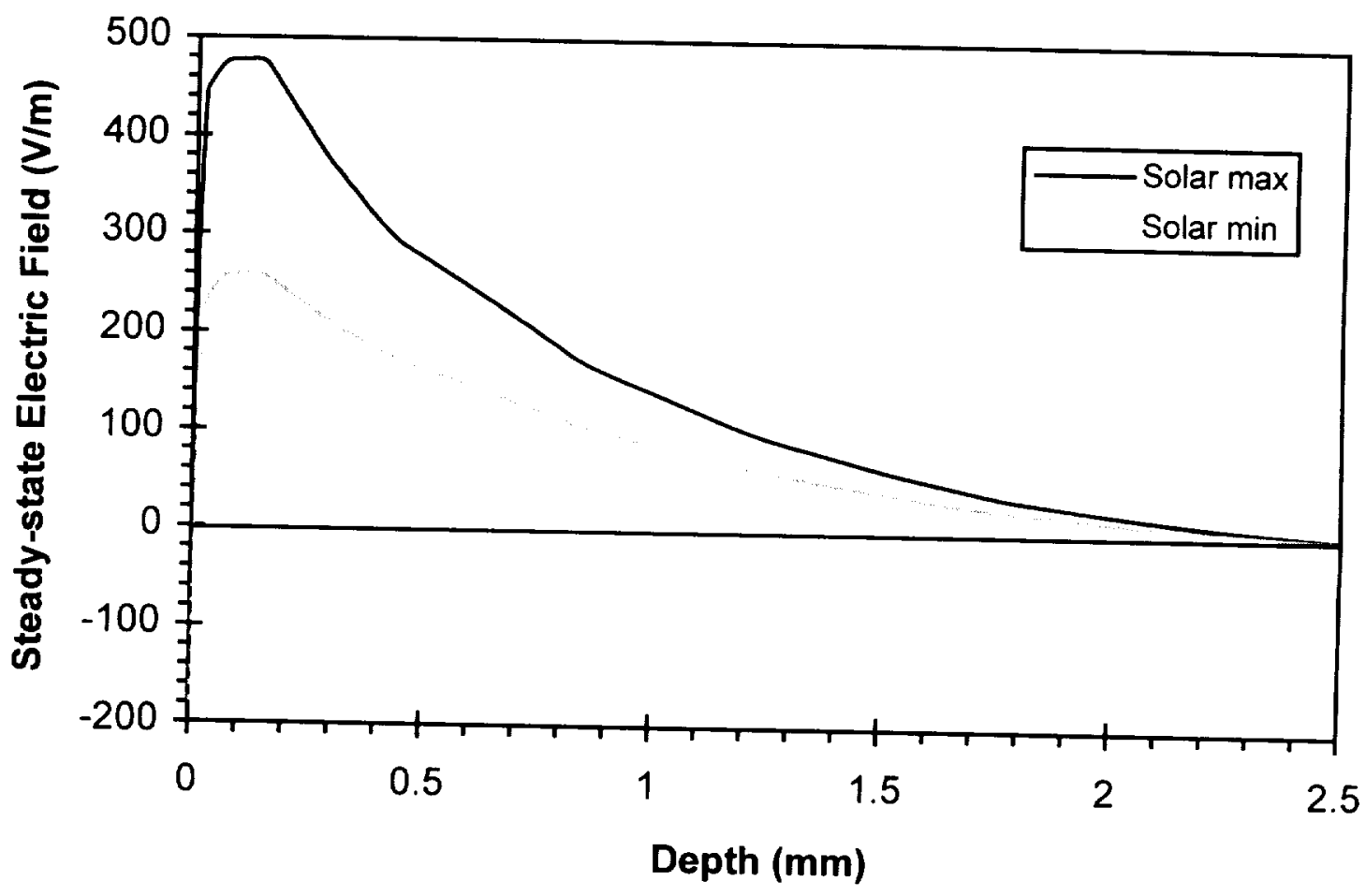

Figure 5.34: Global Positioning System orbit electric field distribution 


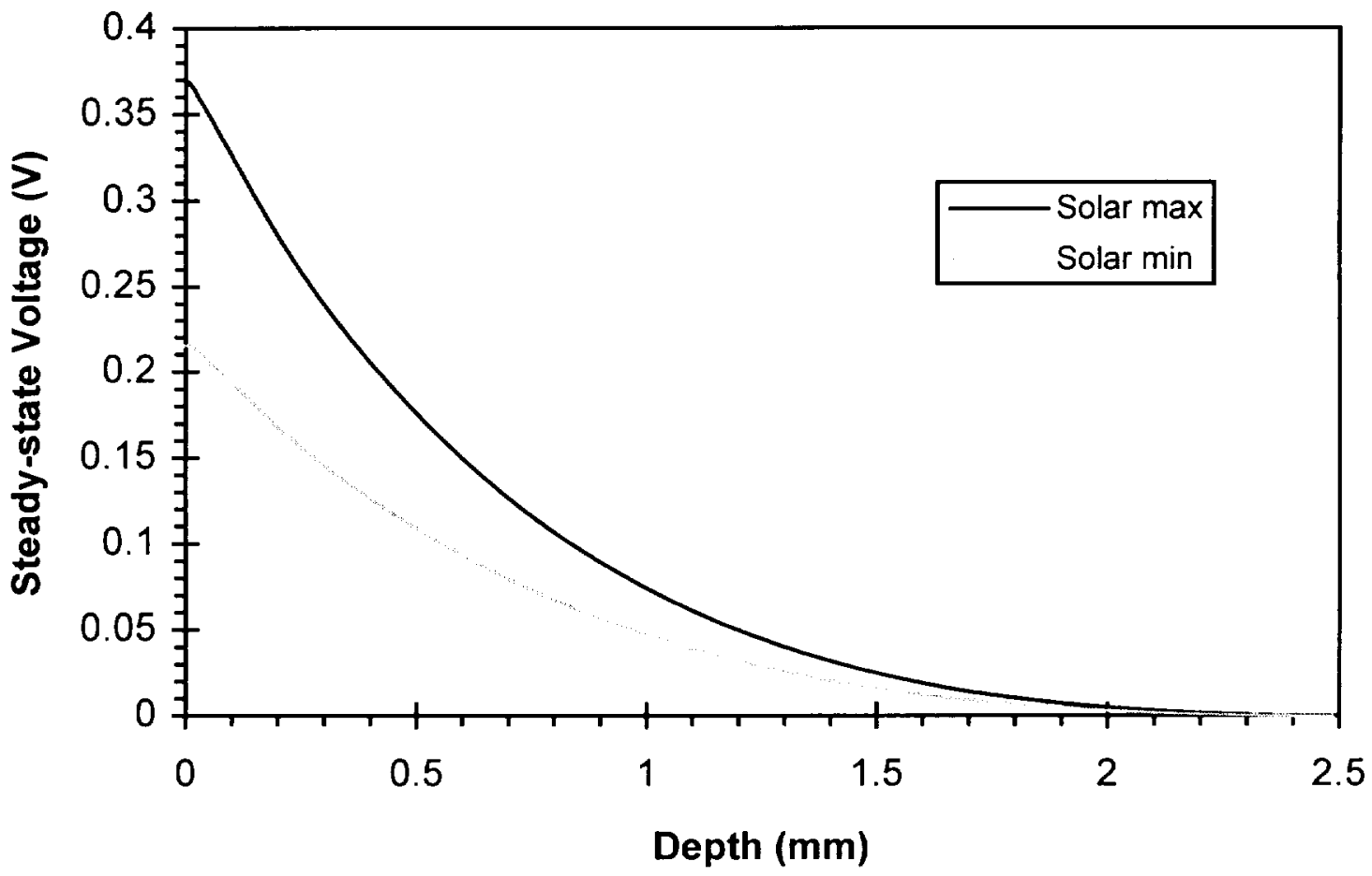

Figure 5.35: Global Positioning System orbit voltage distribution 
through 5.35, show that the charge density distributions for the two solar conditions are very similar to each other. Also, electric field and voltage distributions once again penetrate deeper into the material as described in the geosynchronous orbit case. It is interesting to note is that the ramp up portion of the electric field distribution for the solar minimum case is the same as that for the solar maximum case except that it is shifted down by approximately $200 \mathrm{~V} / \mathrm{m}$.

\subsubsection{The Low Altitude Retrograde Orbit}

The low altitude retrograde orbit environment, as shown in Figures 5.36, has a typical electron flux profile with a few high energy particles, and the solar maximum values are approximately 2.5 times greater than the solar minimum values. The proton flux is more interesting; the solar minimum values are greater than the solar maximum values, as expected for low altitude orbits. Also, the distributions are dissimilar; there are proportionally more high energy particles at solar minimum than at solar maximum. Another interesting fact is there is approximately 3 orders of magnitude more electrons than protons, compared to the previous orbits where there is only at best a 1 order of magnitude difference.

The results, as shown in Figures 5.37 through 5.39, show that even though there are more protons at solar minimum, the steady-state charge density, electric field, and voltage distributions are still larger at solar maximum. This occurs because although there are more protons at solar minimum, they are still 3 orders of magnitude less than the

number of electrons. This can be noticed in the charge density distribution where the 

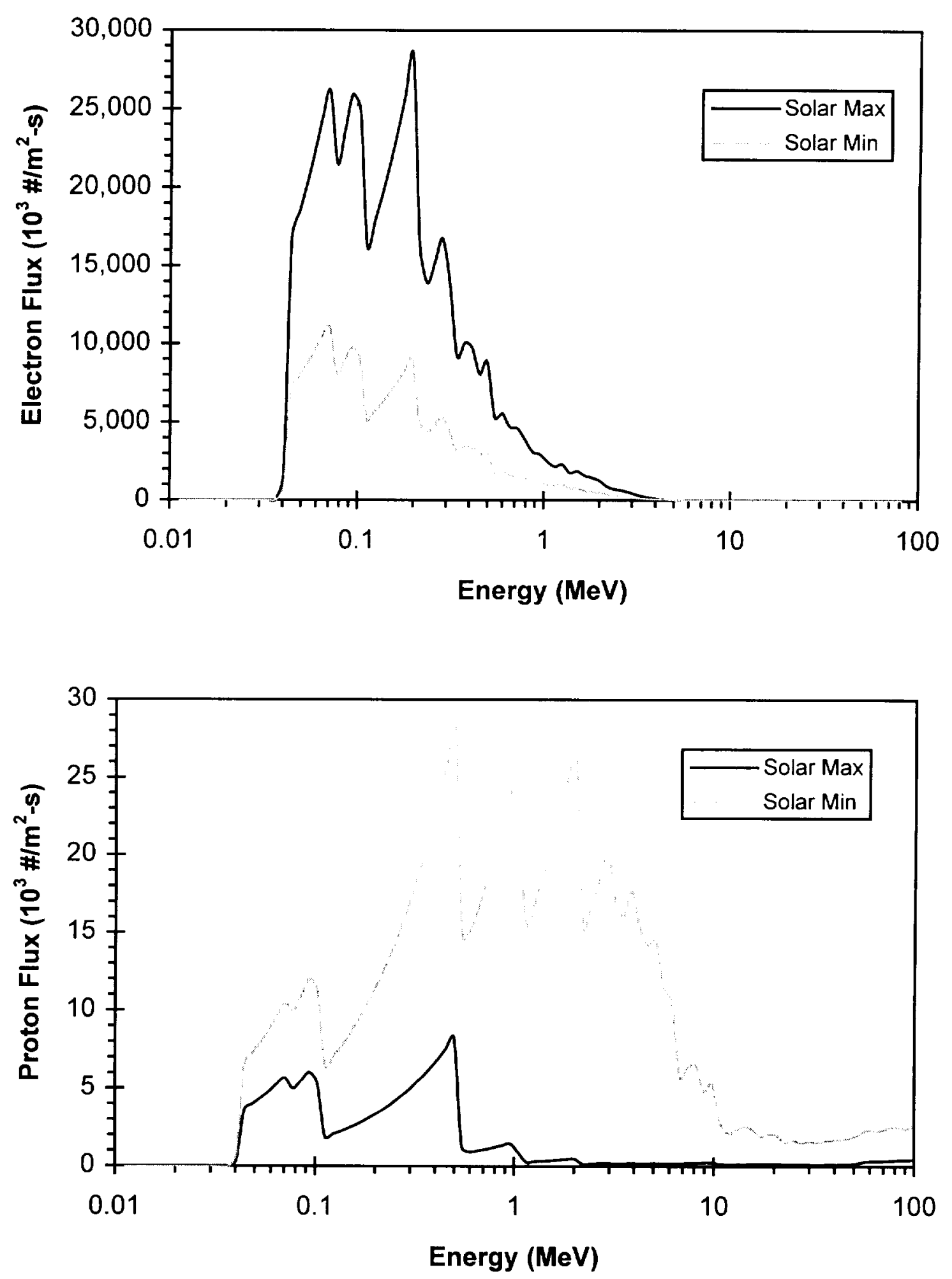

Figure 5.36: Low altitude retrograde orbit environment 


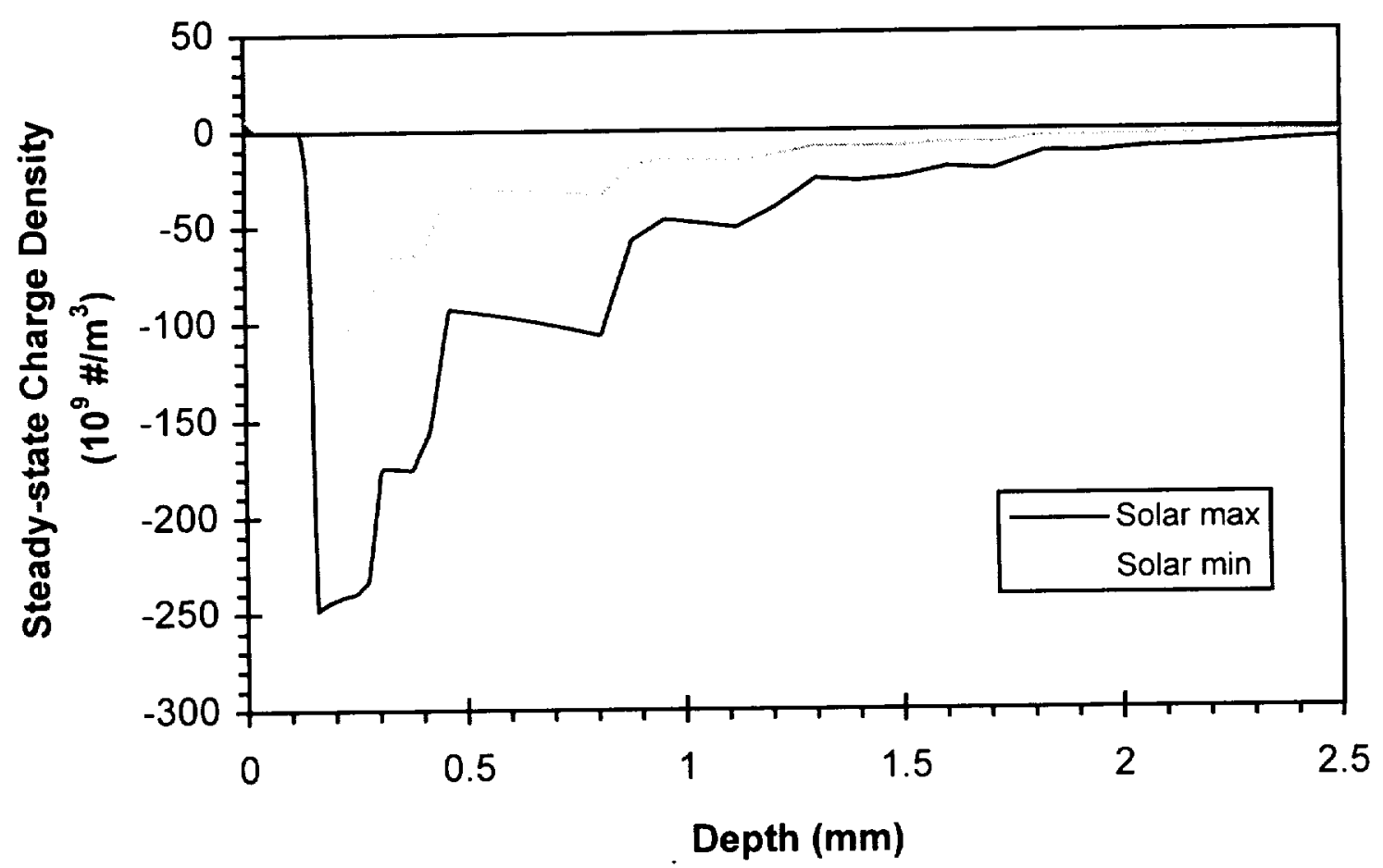

Figure 5.37: Low altitude retrograde orbit charge density distribution 


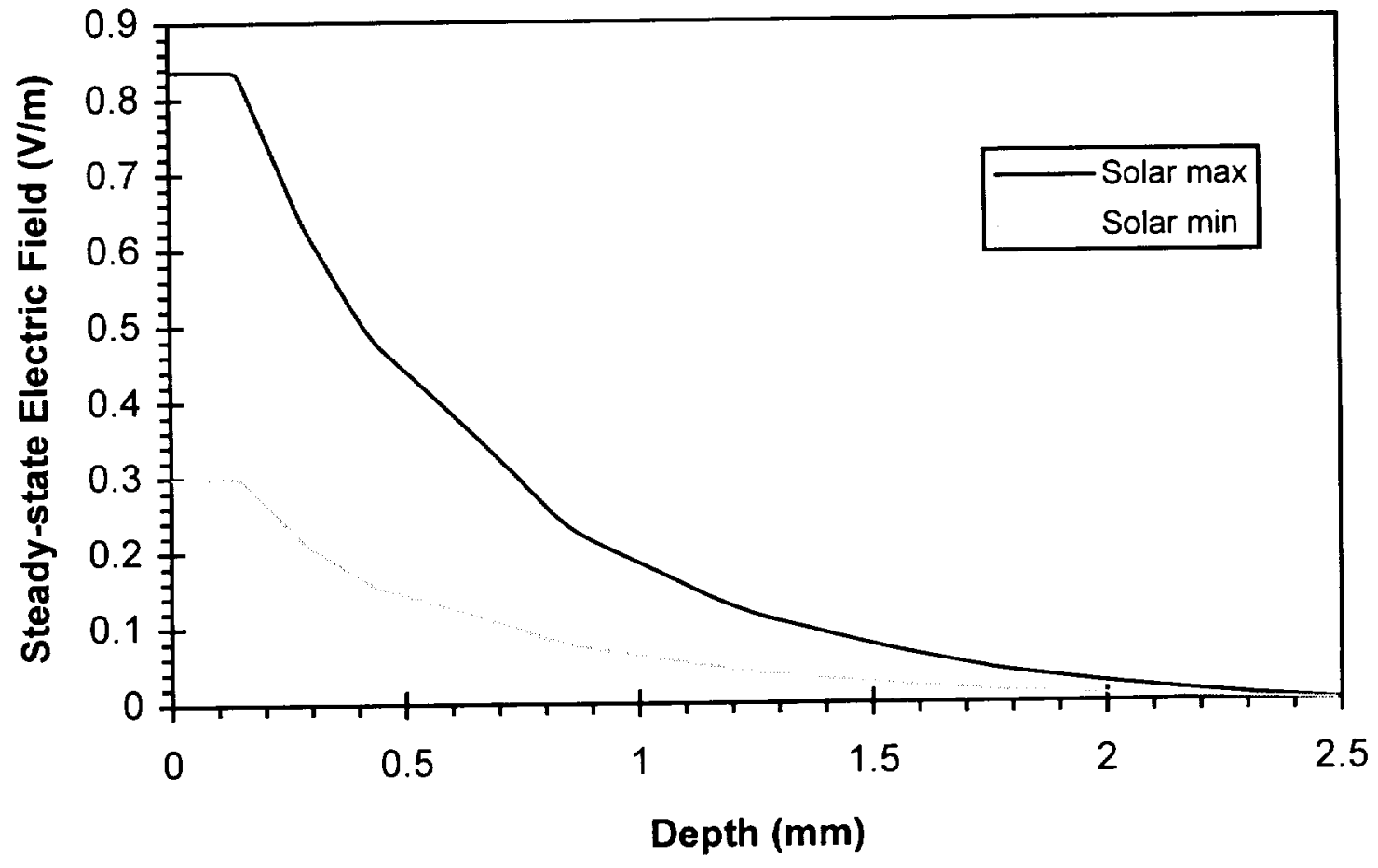

Figure 5.38: Low altitude retrograde orbit electric field distribution 


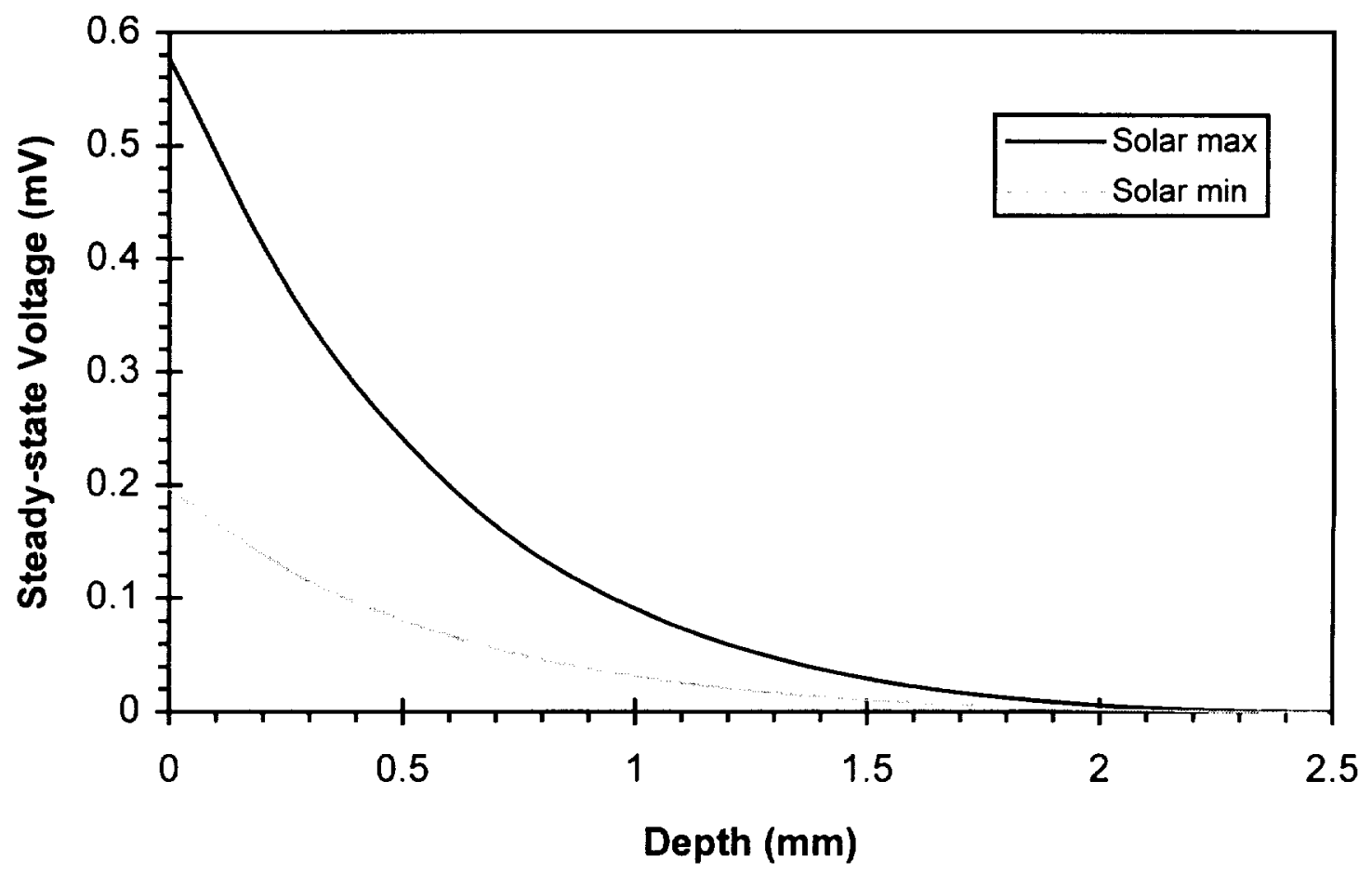

Figure 5.39: Low altitude retrograde orbit voltage distribution 
positive charge density near the front surface due to the protons is essentially zero. It is also the reason for the constant electric field near the front surface. As in previous case studies where there are high energy electrons (greater than $1 \mathrm{MeV}$ ) the distributions are none zero through almost all of the thickness. Also, because of the lower fluxes present in low altitude orbits all the maximum values are significantly lower than previous cases.

\subsubsection{The Molniya Orbit}

The Molniya orbit environment, as shown in Figures 5.40, has no exceptional features. The proton flux is within an order of magnitude of the electron flux. The electron flux has 3 times the particles at solar maximum as compared to solar minimum. The proton flux is the same for solar maximum and solar minimum, and has a relatively small amount of high energy particles. The results, as shown in Figures 5.41 through 5.43 , exhibit the typical behavior seen earlier, the steady-state charge density, electric field, and voltage distributions are greater at solar maximum than at solar minimum by approximately a factor of 3 representing the difference in electron flux. Once again, as in the case studies with few high energy electrons the electric field and voltage distributions penetrate deeper into the material before becoming negligible.

\subsubsection{The Space Shuttle Parking Orbit}

The space shuttle parking orbit environment, as shown in Figures 5.44, has a typical electron flux distribution, the solar maximum values are approximately 2 times greater than the solar minimum values, and no high energy particles. The only difference to the base case environment is that the flux levels are much smaller, as would be expected in a low altitude orbit. The proton flux distributions are very different from 

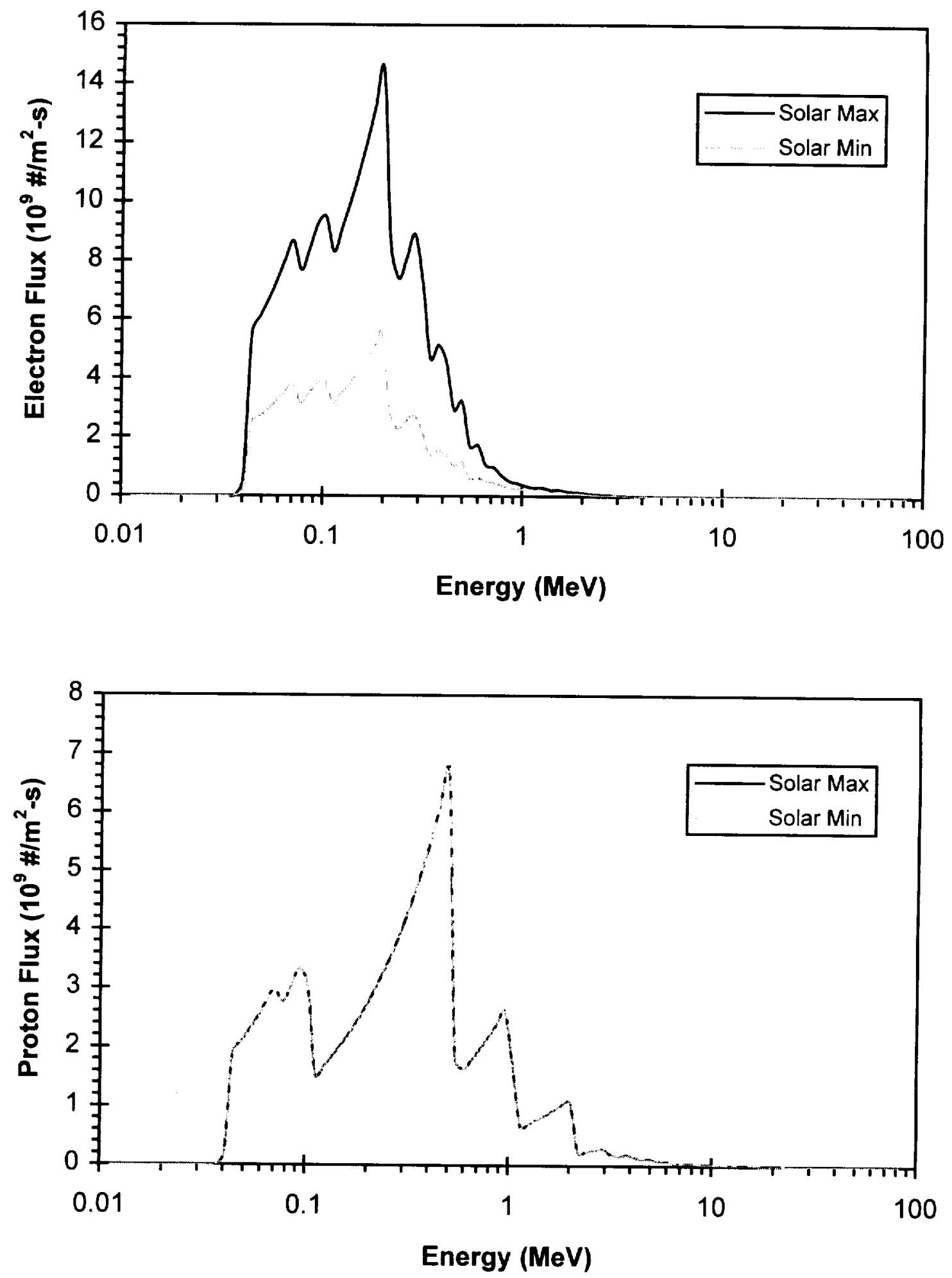

Figure 5.40: Molniya orbit environment 


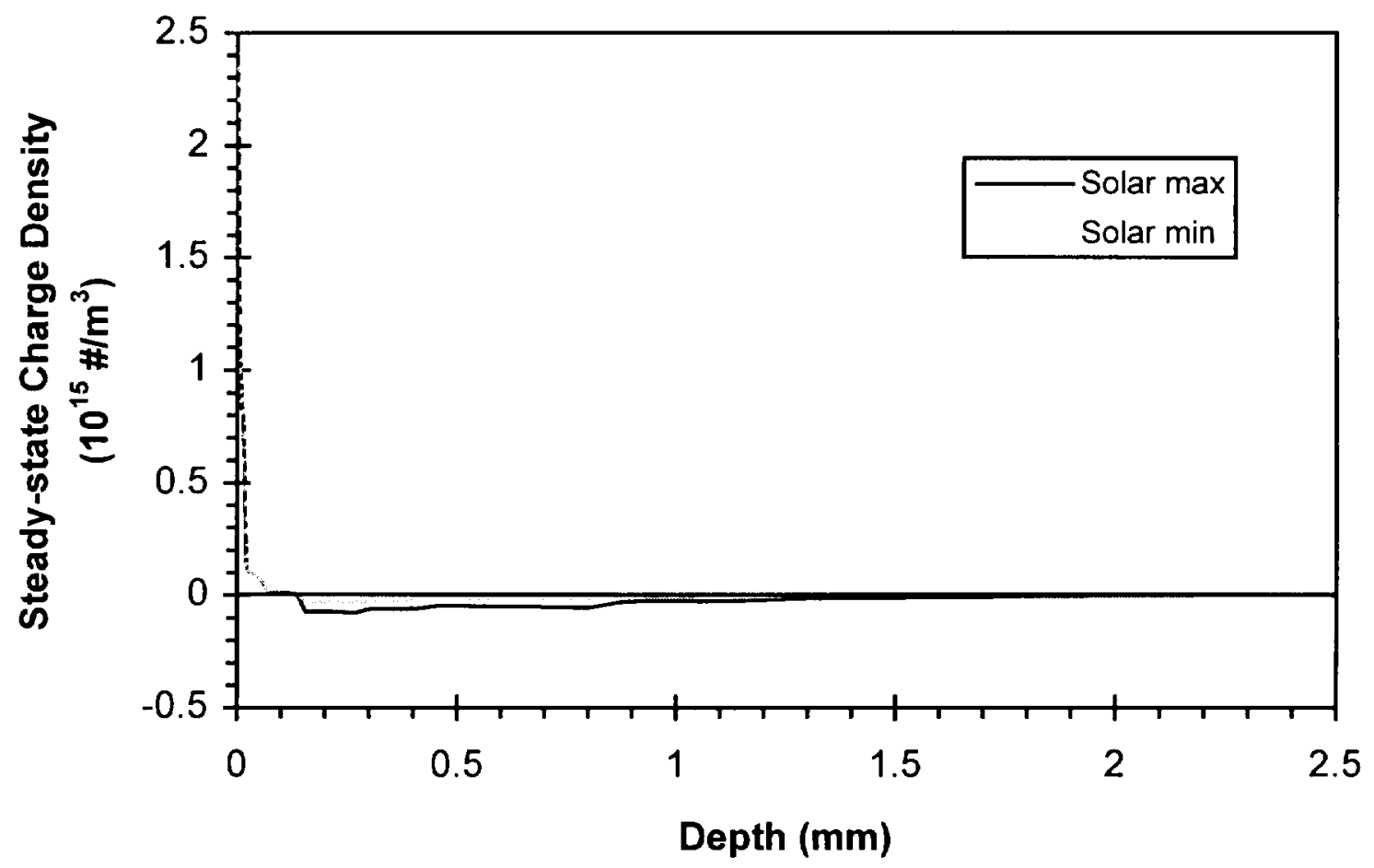

Figure 5.41: Molniya orbit charge density distribution 


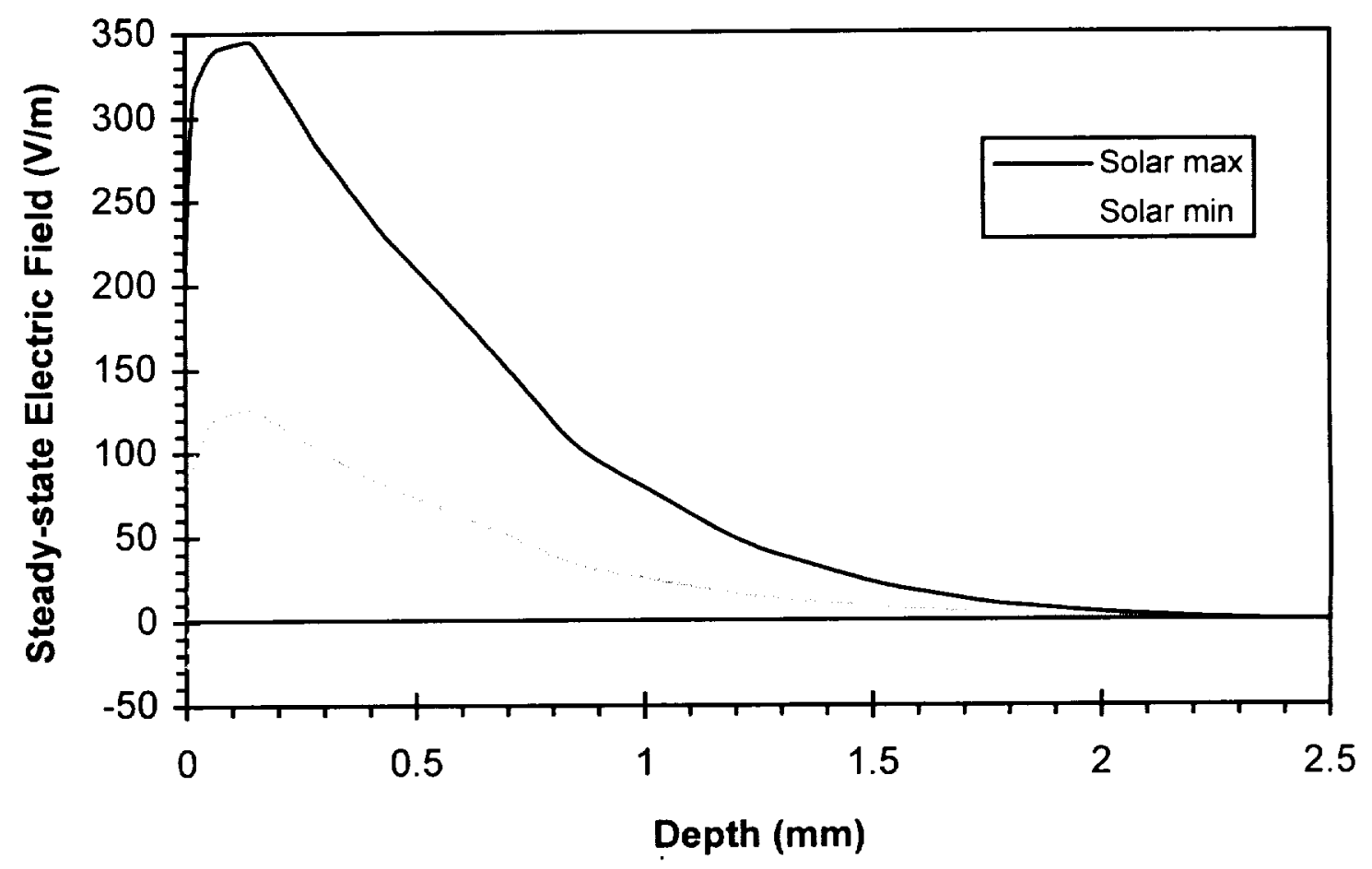

Figure 5.42: Molniya orbit electric field distribution 


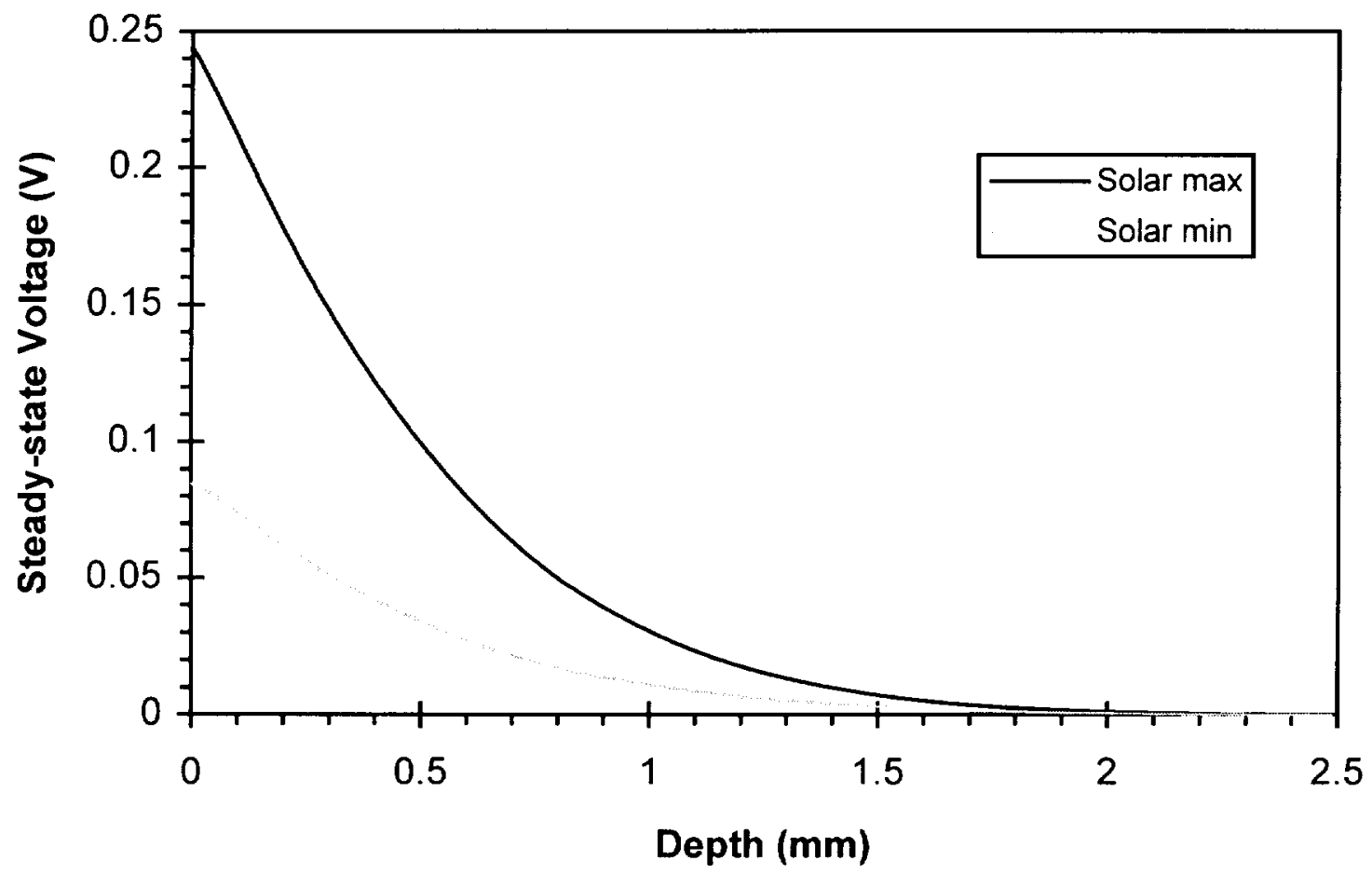

Figure 5.43: Molniya orbit voltage distribution 

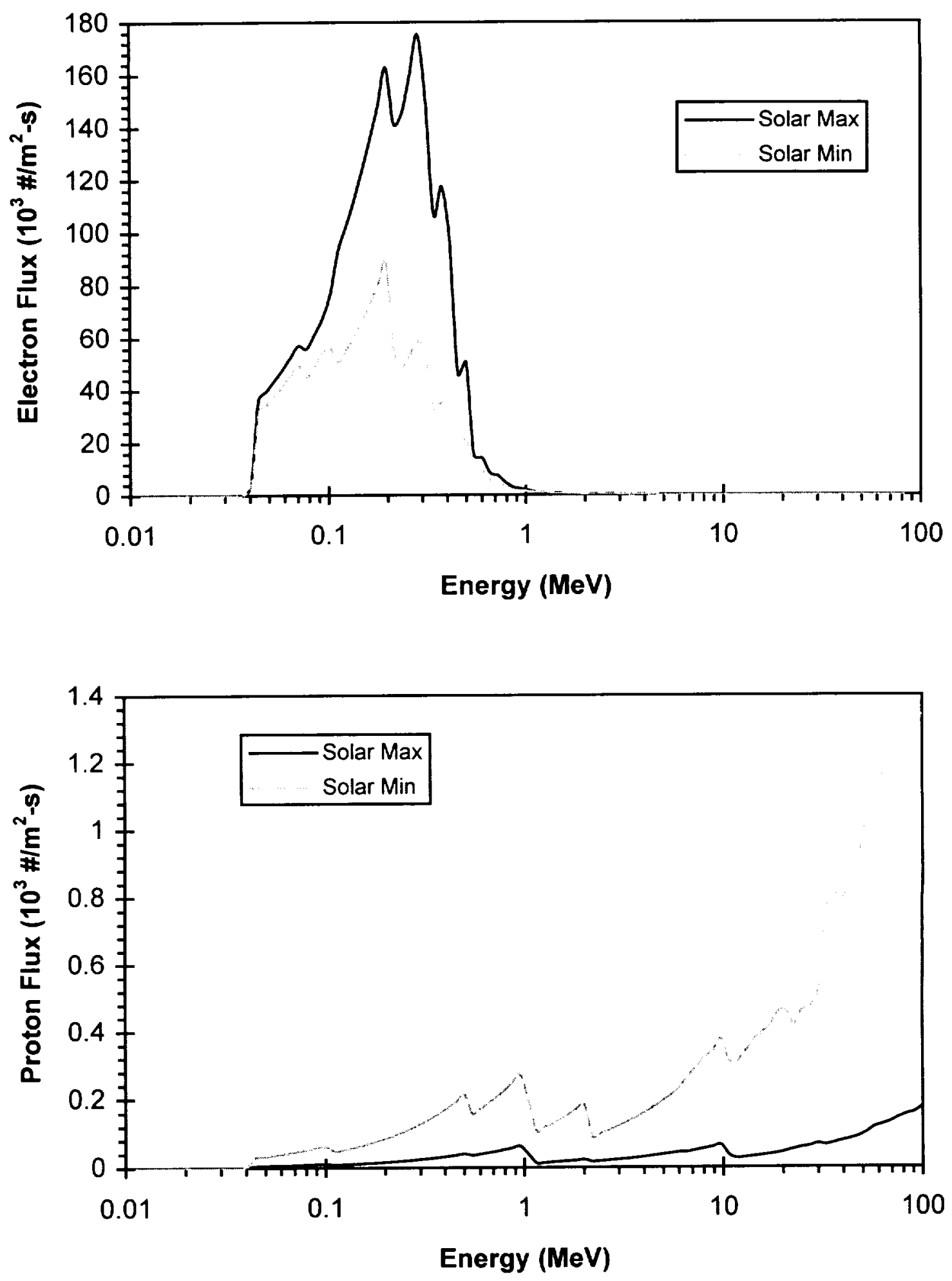

Figure 5.44: Space shuttle parking orbit environment 
anything seen earlier. The distribution is not contained within the usual energy boundaries of 0.01 to $100 \mathrm{MeV}$. However, this is not a concern since as shown in Figure 4.6, particles of these energies will penetrate over $100 \mathrm{~mm}$ of the base case material, and our sample material is only $2.5 \mathrm{~mm}$ thick. Therefore, these missing extremely high energy particles will just pass through the material. Also, as expected with low altitude orbits, the proton flux at solar minimum is much greater than at solar maximum. As with the low altitude retrograde orbit, the electron flux is several orders of magnitude greater than the proton flux.

The results, as shown in Figure 5.45 through 5.47, show than once again since there are many more electrons than protons, the fact that there are more protons at solar minimum than maximum has no consequence. The steady-state charge density, electric field, and voltage distributions are greater for solar maximum than solar minimum. The fact that there are far greater amounts of electrons than protons can also be seen in charge density distribution where the positive portion near the front surface, due to the protons, is almost zero. It can also be seen in the electric field distribution where near the front surface it is constant. It should also be noted that since there are no high energy electrons, the electric field and voltage distribution reach essentially zero before the back surface of the material. Also, since the flux levels are very low in low altitude orbits, the maximum values of the results are also very low, as compared to the base case. 


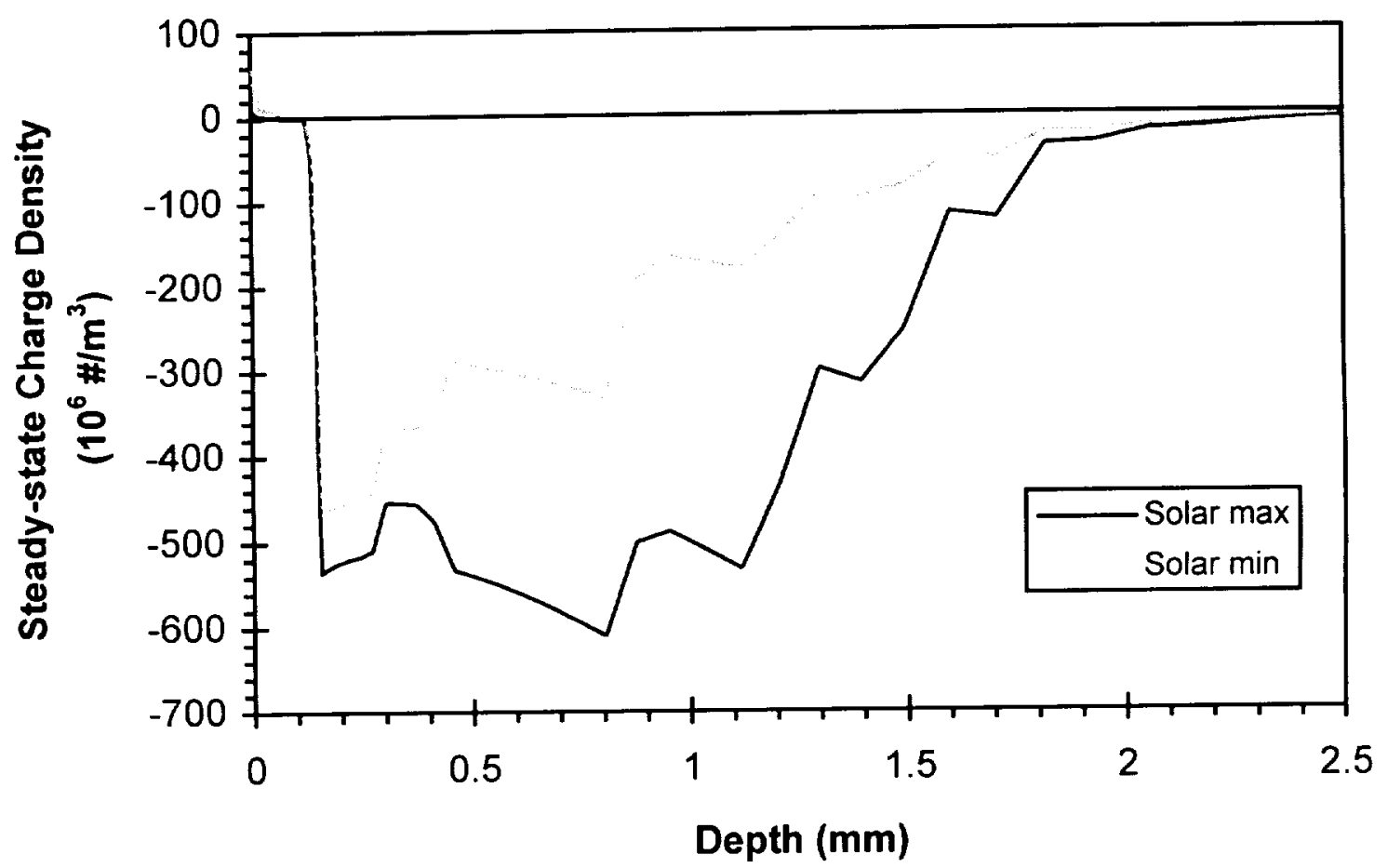

Figure 5.45: Space shuttle parking orbit charge density distribution 


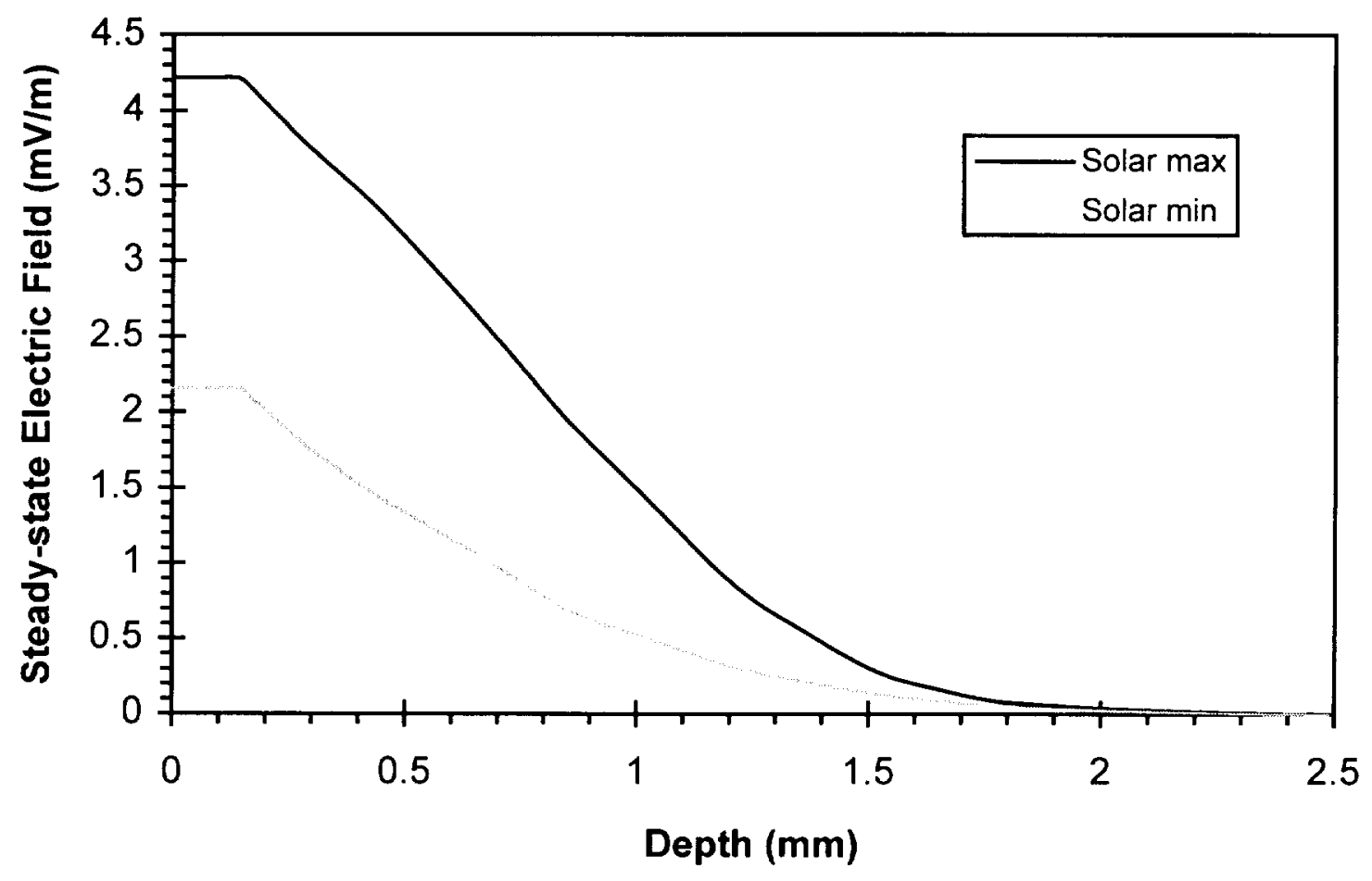

Figure 5.46: Space shuttle parking orbit electric field distribution 


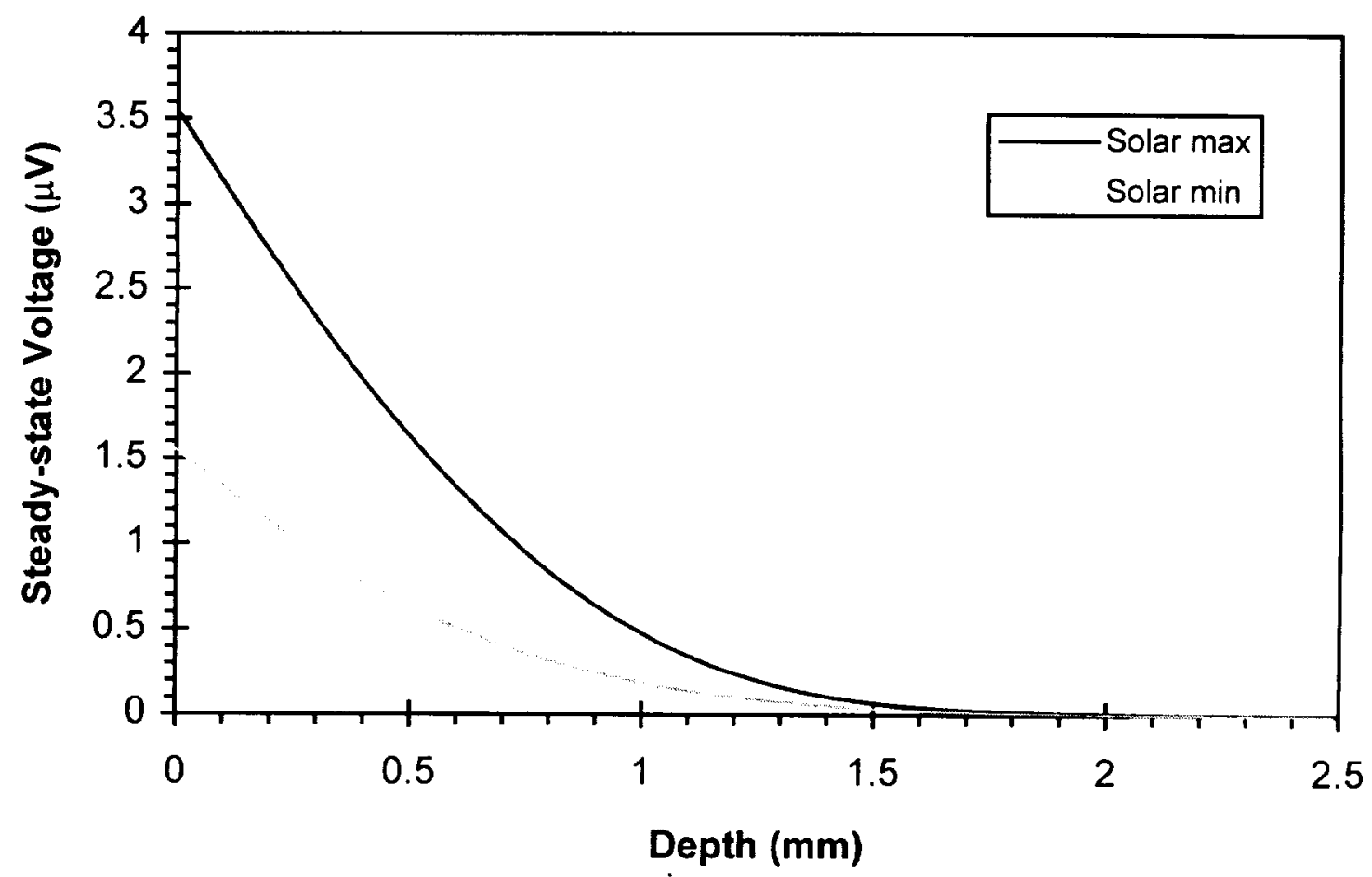

Figure 5.47: Space shuttle parking orbit voltage distribution 


\section{$\underline{5.4 .6}$ Summary}

Even though all the case studies showed that the electric field was not large enough to create an electrostatic discharge in the base case material, this does not mean that deep dielectric charging is not a concern. An extremely high intensity geomagnetic substorm or solar particle event could increase the flux levels sufficiently to create an electrostatic discharge. Also, other materials with either a lower conductivity or lower dielectric strength could also be susceptible to electrostatic discharges due to deep dielectric charging. The worst orbits are the geosynchronous orbit, the Global Positioning System orbit, and the Molniya orbit. These all produced electric fields in the 100 's of volts per meter for the base case material.

\subsection{INTERNATIONAL SPACE STATION}

This section deals with an actual problem. There was a concern that the Kapton layer covering the main power bus of the International Space Station would be affected by deep dielectric charging. The power bus is used to connect electrically the solar cell wing to the space station.

The basic problem is that during operation there is a differential of 160 volts across the Kapton Layer. The space environment is assumed to be at plasma ground (i.e. 0 volts) and the power bus is carrying 160 volts from the solar cells to the space station. The space station orbit used had a constant semi-major axis of $6771 \mathrm{~km}$, this is equivalent to an apogee and perigee altitude of $393 \mathrm{~km}$, with an inclination of $51.6^{\circ} .{ }^{57}$ Since the 
exact type of Kapton being used was unknown, an analysis was performed on three types of Kapton, type HN, VN, and FN films. ${ }^{58}$ The properties used are summarized in Tables 5.5 and 5.6 .

The composite deep dielectric charging analyzer was run on each of the different types of Kapton listed in the above mentioned tables to determine which type would produce the worst results, the type that was most likely to undergo an electrostatic discharge. Once these were done, the worst case material type was analyzed to determine the effect of changing various parameters such as the solar cycle condition, including radiation induced conductivity, including high field conductivity, a negative voltage applied through the power bus, and no voltage applied through the power bus. Table 5.7 shows the settings used during each test case.

The space station orbit environment data, as shown in Figures 5.48, has typical flux distributions for low altitude orbits. However, it should be noted that unlike the low altitude cases investigated earlier, the higher proton flux during solar minimum had a noticeable effect on the results. This is because the charging in entirely dominated by the protons as all the electrons pass straight through the Kapton film, due to its small thickness.

The results, as shown in Figures 5.49 through 5.51 for Test Case No. 1, show that in each case where there is a voltage applied through the power bus, the problem is dominated by the voltage difference applied across the Kapton film. The voltage varies 
Table 5.5: Kapton type HN and VN film properties

\begin{tabular}{||l|c|c|c|c||}
\hline \hline Property & 100VN, HN & 200VN, HN & 300VN, HN & 500VN, HN \\
\hline \hline Density $\left(\mathrm{kg} / \mathrm{m}^{3}\right)$ & 1420 & 1420 & 1420 & 1420 \\
\hline Thickness $(\mu \mathrm{m})$ & 25 & 50 & 75 & 125 \\
\hline Dielectric Strength $(\mathrm{V} / \mathrm{m})$ & $303 \times 10^{6}$ & $240 \times 10^{6}$ & $205 \times 10^{6}$ & $154 \times 10^{6}$ \\
\hline Dielectric Constant & 3.4 & 3.4 & 3.5 & 3.5 \\
\hline Resistivity $(\Omega-\mathrm{m})$ & $1.5 \times 10^{15}$ & $1.5 \times 10^{15}$ & $1.4 \times 10^{15}$ & $1.0 \times 10^{15}$ \\
\hline
\end{tabular}

Table 5.6: Kapton type FN film properties

\begin{tabular}{||l|c|c|c||}
\hline Property & 120 FN 616 & 150 FN 019 & 250 FN 029 \\
\hline \hline Density $\left(\mathrm{kg} / \mathrm{m}^{3}\right)$ & 1530 & 1670 & 1570 \\
\hline Thickness $(\mu \mathrm{m})$ & 30 & 38 & 63 \\
\hline Dielectric Strength $(\mathrm{V} / \mathrm{m})$ & $272 \times 10^{6}$ & $197 \times 10^{6}$ & $197 \times 10^{6}$ \\
\hline Dielectric Constant & 3.1 & 2.7 & 3.0 \\
\hline Resistivity $(\Omega-\mathrm{m}) @ 23^{\circ} \mathrm{C}$ & $1.4 \times 10^{15}$ & $2.3 \times 10^{15}$ & $1.9 \times 10^{15}$ \\
\hline
\end{tabular}


Table 5.7: Space station test case analysis parameters

\begin{tabular}{||l|c|c|c|c|c||}
\hline \hline Test Case & Material & B.C. & Solar Cycle & R.I.C. & H.F.C \\
\hline \hline 1 & $100 \mathrm{VN}, 100 \mathrm{HN}$ & $0,160 \mathrm{~V}$ & $\max$ & no & no \\
\hline 2 & $200 \mathrm{VN}, 200 \mathrm{HN}$ & $0,160 \mathrm{~V}$ & $\max$ & no & no \\
\hline 3 & $300 \mathrm{VN}, 300 \mathrm{HN}$ & $0,160 \mathrm{~V}$ & $\max$ & no & no \\
\hline 4 & $500 \mathrm{VN}, 500 \mathrm{HN}$ & $0,160 \mathrm{~V}$ & $\max$ & no & no \\
\hline 5 & $120 \mathrm{FN} 616$ & $0,160 \mathrm{~V}$ & $\max$ & no & no \\
\hline 6 & $150 \mathrm{FN} 019$ & $0,160 \mathrm{~V}$ & $\max$ & no & no \\
\hline 7 & $250 \mathrm{FN} 029$ & $0,160 \mathrm{~V}$ & $\max$ & no & no \\
\hline 8 & $100 \mathrm{VN}, 100 \mathrm{HN}$ & $0,160 \mathrm{~V}$ & $\max$ & yes & no \\
\hline 9 & $101 \mathrm{VN}, 100 \mathrm{HN}$ & $0,160 \mathrm{~V}$ & $\max$ & no & yes \\
\hline 10 & $100 \mathrm{VN}, 100 \mathrm{HN}$ & $0,-160 \mathrm{~V}$ & $\max$ & no & no \\
\hline 11 & $100 \mathrm{VN}, 100 \mathrm{HN}$ & $0,0 \mathrm{~V}$ & $\max$ & no & no \\
\hline 12 & $100 \mathrm{VN}, 100 \mathrm{HN}$ & $0,160 \mathrm{~V}$ & $\min$ & no & no \\
\hline \hline
\end{tabular}

B.C.: Voltage boundary conditions

R.I.C.: Radiation induced conductivity

H.F.C.: High field conductivity 

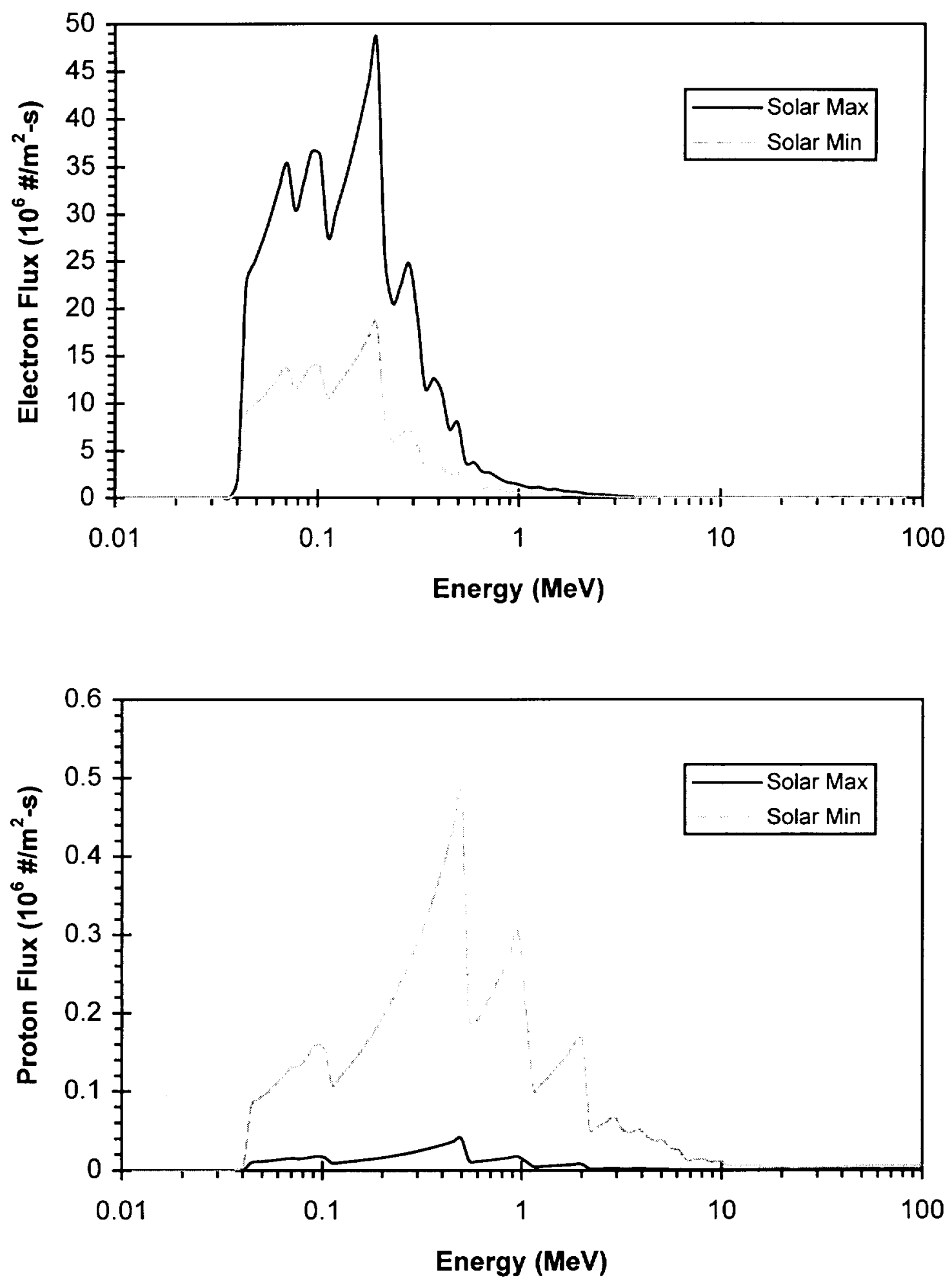

Figure 5.48: Space station orbit environment 


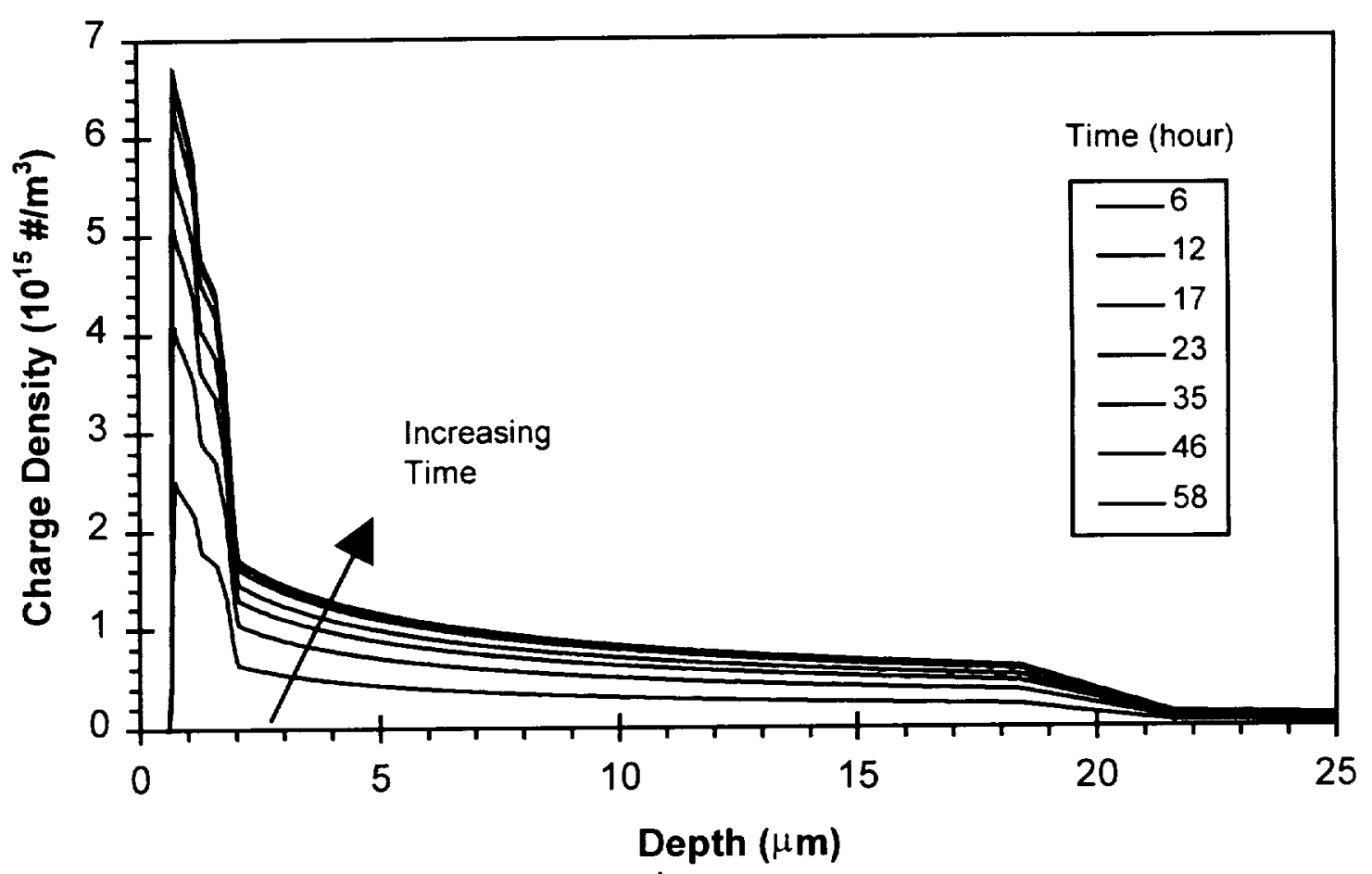

Figure 5.49: Space station Test Case No. 1 charge density distribution 


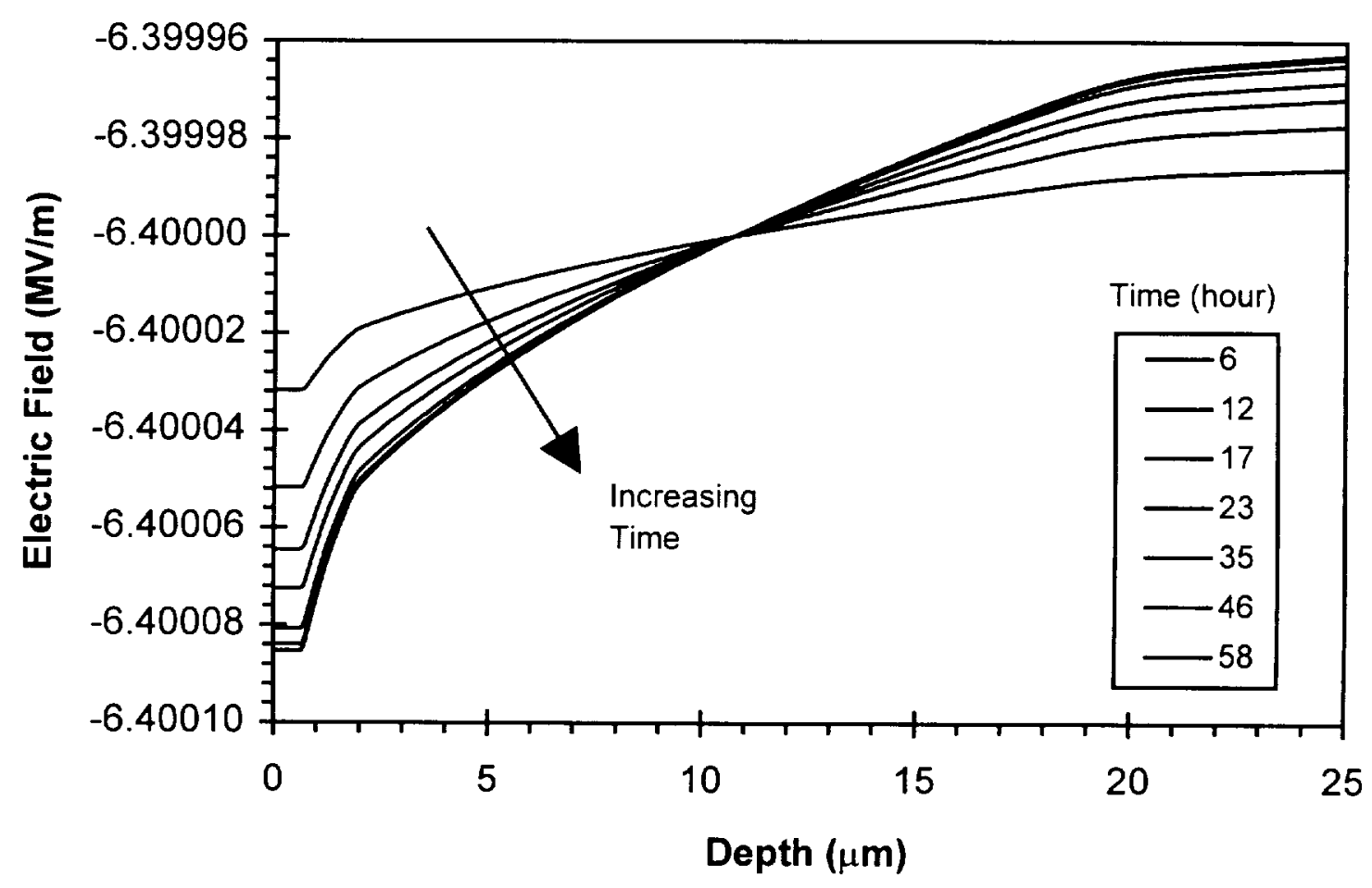

Figure 5.50: Space station Test Case No. 1 electric field distribution 


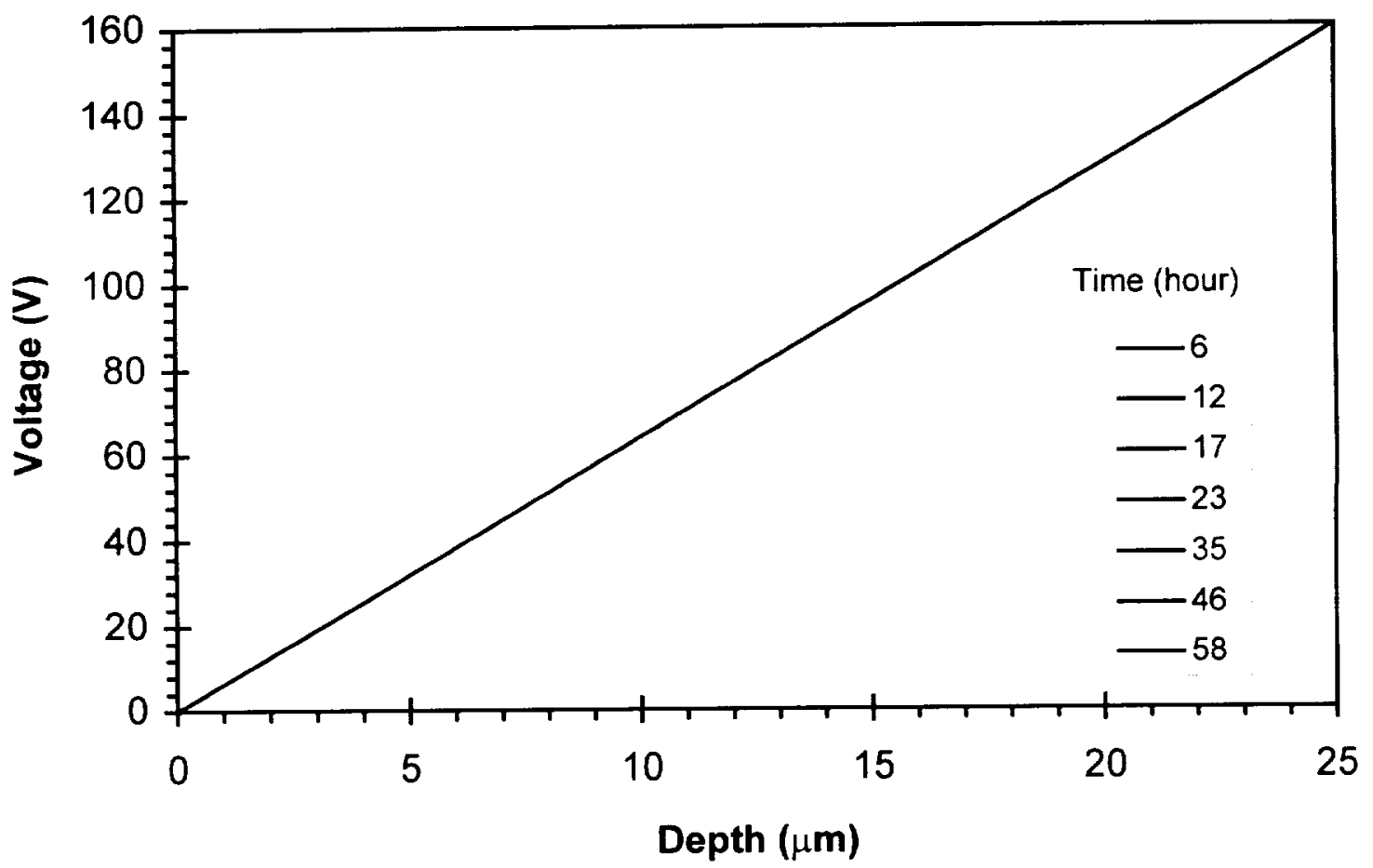

Figure 5.51: Space station Test Case No. 1 voltage distribution 
linearly from $0 \mathrm{~V}$ at the front surface to $160 \mathrm{~V}$ at the rear surface. This results in the electric field being simply the change in voltage divided by the film thickness. The only effect of the incoming radiation is to perturb the electric field about the voltage induced field value. However, since the amount of incoming particles is so small compared to the base case, the perturbation is only approximately $100 \mathrm{~V} / \mathrm{m}$, which is insignificant against the voltage induced electric field in the millions of volts per meter. The constant portion of the electric field distribution near the front surface occurs because there are no particles deposited in the film there, as even the smallest energy protons penetrate at least $0.5 \mu \mathrm{m}$.

When the solar cycle was changed to solar minimum, as shown in Figures 5.52 and 5.53 , the only observable change to the results was the charge density and the radiation induced electric field perturbation increased by approximately an order of magnitude. This occurred because, as mentioned earlier, during solar minimum there is approximately an order of magnitude more protons. The effect of including the radiation induced conductivity, using the base case radiation induced conductivity parameter values, was to slightly decrease the electric field perturbation and the charge density, this occurred since the conductivity only increased from $6.67 \times 10^{-16} 1 / \Omega-\mathrm{m}$ to $7.20 \times 10^{-16}$ $1 / \Omega-\mathrm{m}$. The same result was seen for the high field conductivity using a temperature $120^{\circ} \mathrm{C}$, for the same reason, the conductivity increased by a factor 1.48 from $6.67 \times 10^{-16}$ $1 / \Omega-\mathrm{m}$ to $9.85 \times 10^{-16} 1 / \Omega-\mathrm{m}$. By applying a negative voltage of $-160 \mathrm{~V}$ through the power bus, the only effect was to make the voltage induced electric field become positive of the same magnitude. The no voltage through the power bus case showed the effect of 


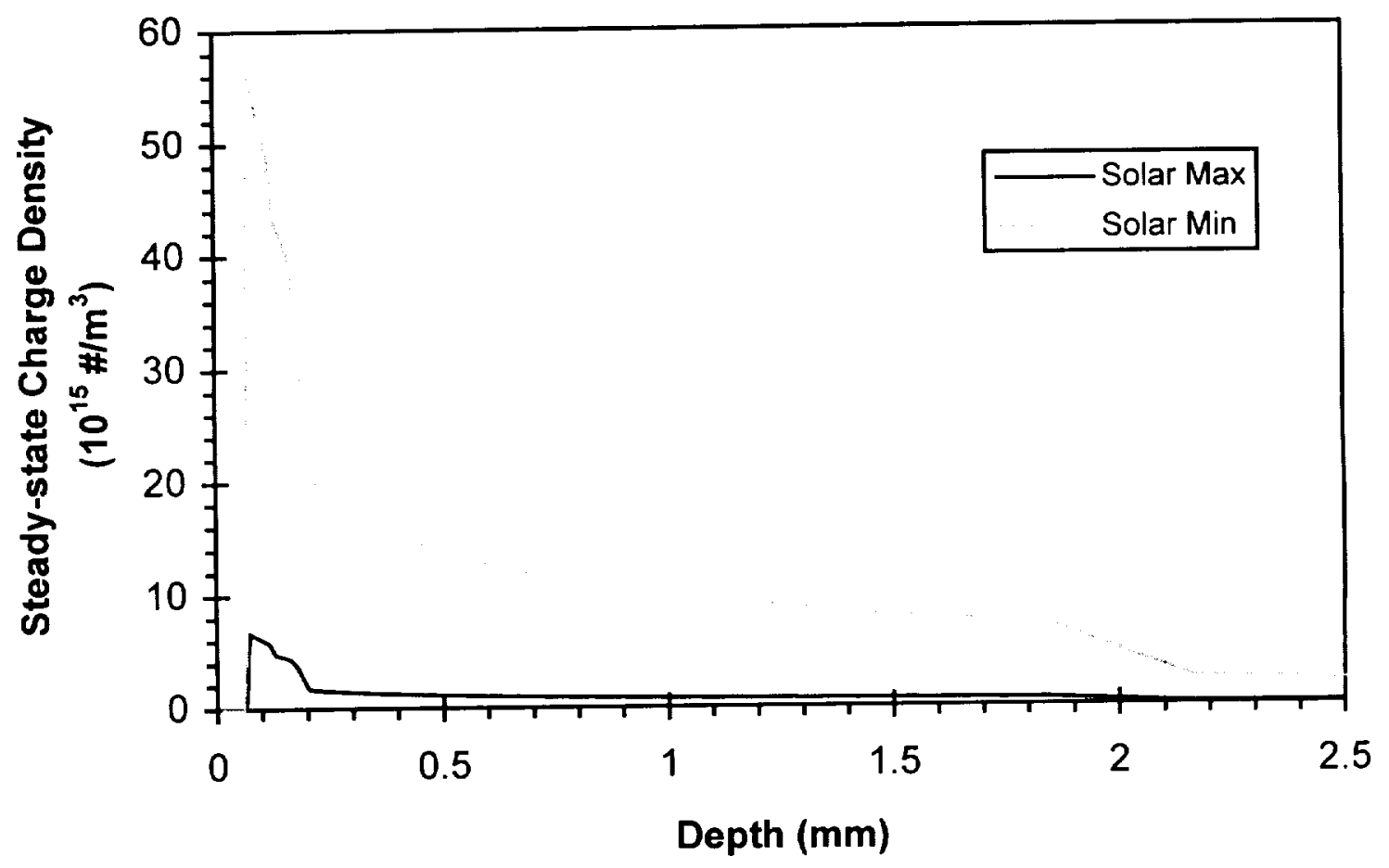

Figure 5.52: Space station charge density distribution 


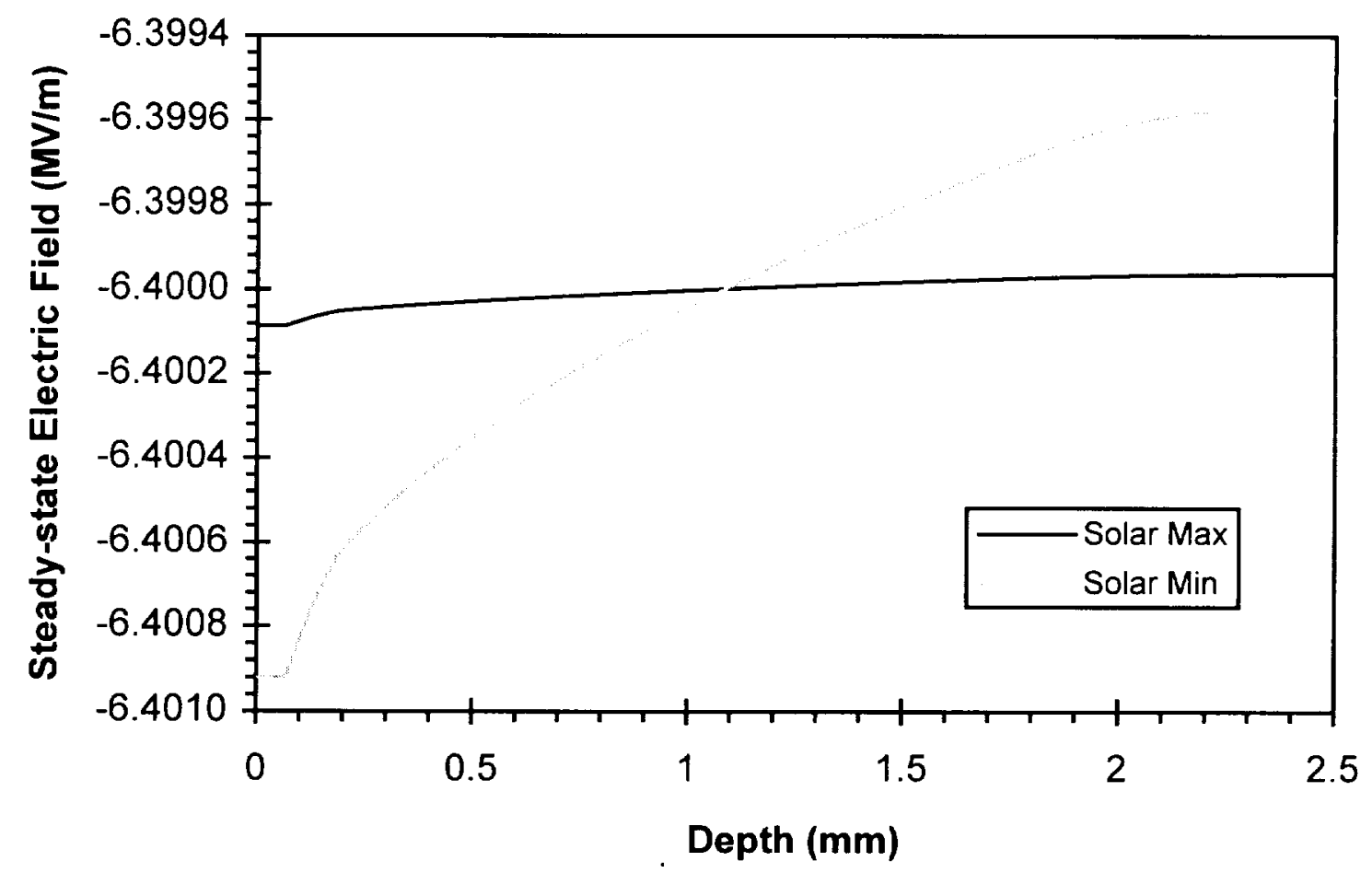

Figure 5.53: Space station electric field distribution 
only the radiation induced electric field, as shown in Figure 5.54. In this case the voltage created a parabolic shape, starting and finishing at $0 \mathrm{~V}$, as shown in Figure 5.55. 


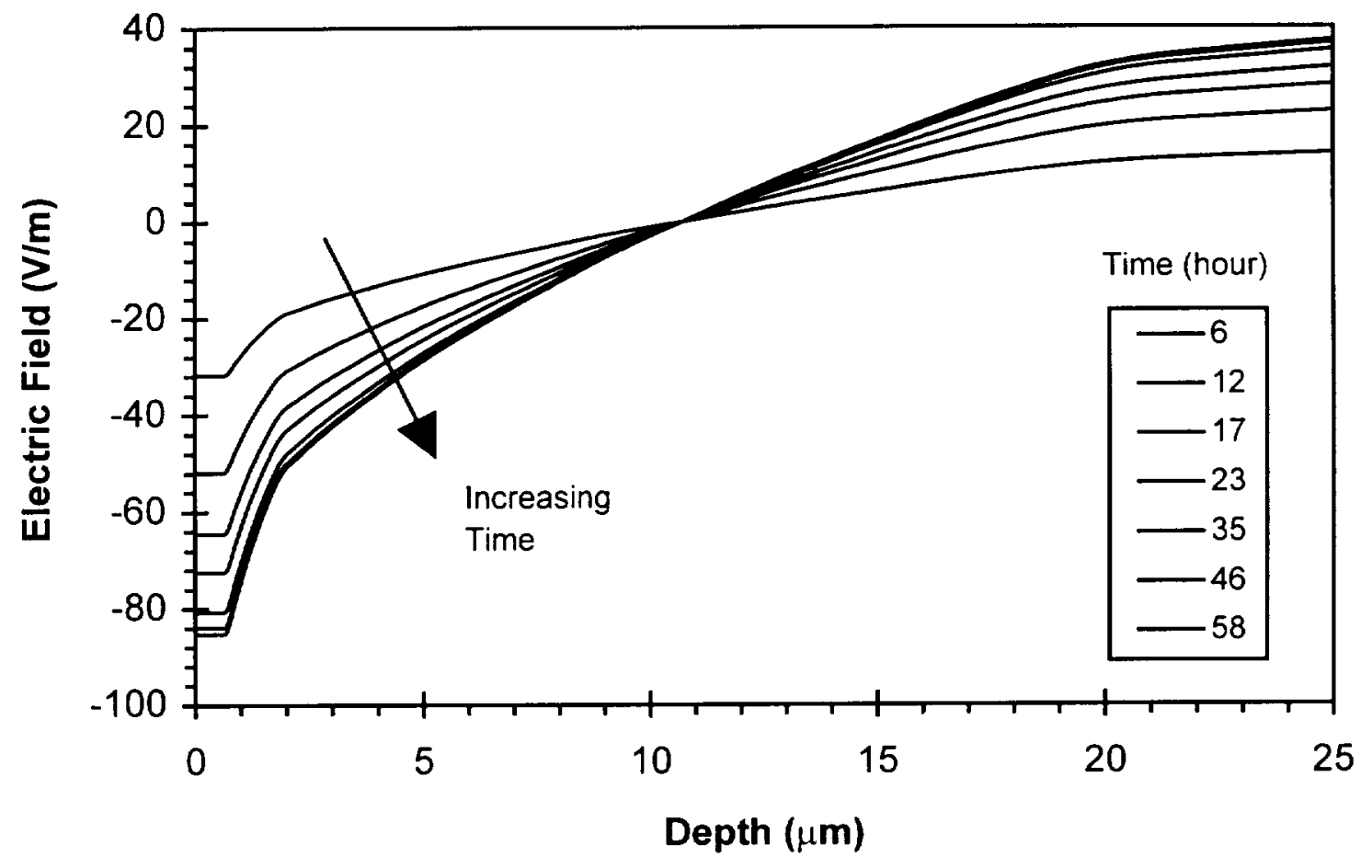

Figure 5.54: Space station power off electric field distribution 


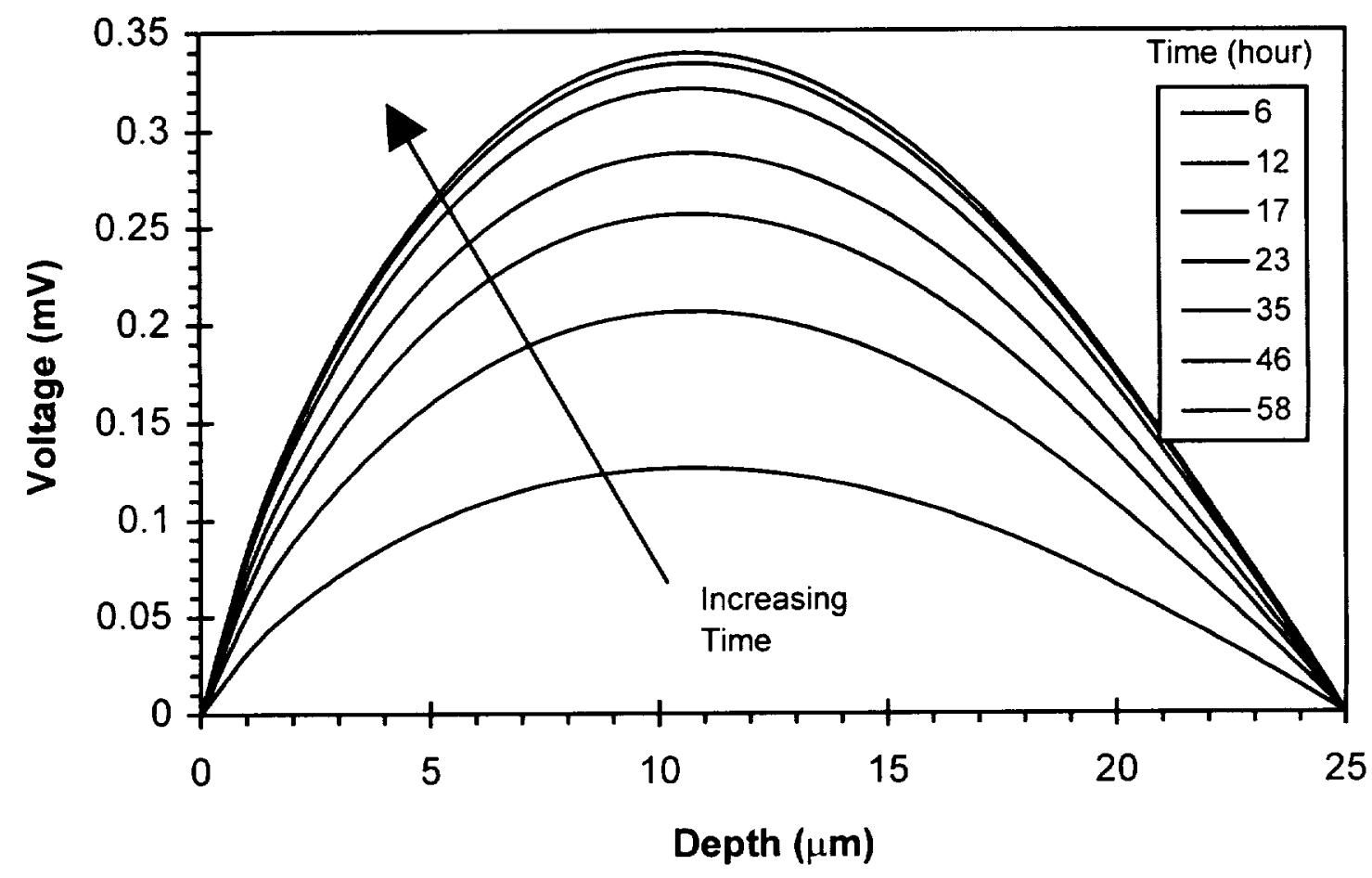

Figure 5.55: Space station power off voltage distribution 


\section{CHAPTER 6}

\section{EXPERIMENTAL PROCEDURE}

This chapter presents the experimental procedures used in this research. The test matrices are explained, followed by information on the manufacturing process used to produce the composite samples. Finally the measurement techniques used are described.

\subsection{TEST MATRICES}

There are two separate experimental efforts in this research. The first part is the investigation of the conductivity and the dielectric strength of a carbon fiber / epoxy composite. The second part is an investigation into creating a conductivity tailorable composite material, using a fiberglass / epoxy composite. The two material systems used in this investigation are: Hercules AS4/3501-6 tape prepreg, and a woven fabric fiberglass / epoxy wet layup. The first material system, AS4/3501-6, is a unidirectional prepreg and has a first generation brittle (low strain-to-failure) 3501-6 matrix, which has been widely used in the aerospace industry. The second system is comprised of a unidirectional woven fiberglass cloth type 1543-38 prepared in a wet layup with Rutapox L20/SL resin. This system is being used in the aircraft industry by Grob Aerospace of Germany in their general aviation aircraft. 


\subsubsection{Composite Electrical Properties}

The through-thickness electrical properties of composites made of carbon fiber / epoxy prepreg were investigated. The composite parameters that were looked at were the laminate thickness, the laminate stacking sequence, and the sample cross sectional area, as shown in Table 6.1. Three different thicknesses were used, 4 plies, 8 plies, and 32 plies, corresponding to approximately $0.5 \mathrm{~mm}, 1.0 \mathrm{~mm}$, and $3.8 \mathrm{~mm}$ thick, respectively. Two different stacking sequences were used, unidirectional and quasi-isotropic. With 4 plies a quasi-isotropic laminate cannot be produced, therefore $[ \pm 45]_{\mathrm{S}}$ was used as an approximation. Therefore six different laminates need to be manufactured. The cross sectional areas used for the conductivity samples are $25.4 \mathrm{~mm} \times 25.4 \mathrm{~mm}$ (1" x 1"), 25.4 $\mathrm{mm} \times 50.8 \mathrm{~mm}(1 " \times 2 ")$, and $50.8 \mathrm{~mm} \times 50.8 \mathrm{~mm}\left(2^{\prime \prime} \times 2 "\right)$, corresponding to approximately $645 \mathrm{~mm}^{2}, 1290 \mathrm{~mm}^{2}$, and $2580 \mathrm{~mm}^{2}$. All the dielectric strength samples were $25.4 \mathrm{~mm} \times 25.4 \mathrm{~mm}$.

One $152.4 \mathrm{~mm} \times 177.8 \mathrm{~mm}\left(6^{\prime \prime} \times 7^{\prime \prime}\right)$ laminate of each layup was manufactured, and 3 samples of each geometry were used. Six different laminates, and 3 different sample areas were required for the conductivity investigation, resulting in 18 different sample types and a total of 54 samples, as shown in Table 6.2. For the dielectric strength investigation, six different laminates and samples with only one area were required, resulting in 6 different sample types and a total of 18 samples, as shown in Table 6.3. Each sample was measured 3 times for electrical properties. The thickness was measured in 5 different locations, and the area was calculated by measuring the length and width in 3 different places, as shown in Figure 6.1. 
Table 6.1: Layups used for the composite electrical properties measurements

\begin{tabular}{||c|c|c||}
\hline \hline \multirow{2}{*}{ Thickness } & \multicolumn{2}{|c|}{ Stacking Sequence } \\
& Unidirectional & Quasi-Isotropic \\
\hline \hline 4 Plies & {$[0]_{4}$} & {$[ \pm 45]_{\mathrm{S}}$} \\
\hline 8 Plies & {$[0]_{8}$} & {$[0 / \pm 45 / 90]_{\mathrm{S}}$} \\
\hline 32 Plies & {$[0]_{32}$} & {$[0 / \pm 45 / 90]_{4 \mathrm{~S}}$} \\
\hline
\end{tabular}

Table 6.2: Electrical conductivity measurement test matrix

\begin{tabular}{|c|c|c|c|c|c|c|c|}
\hline \multirow[t]{2}{*}{ Area } & \multicolumn{3}{|c|}{ Unidirectional } & \multicolumn{3}{|c|}{ Quasi-Isotropic } & \multirow[t]{2}{*}{ Total } \\
\hline & {$[0]_{4}$} & {$[0]_{8}$} & {$[0]_{16}$} & {$[ \pm 45]_{\mathrm{S}}$} & {$[0 / \pm 45 / 90]_{S}$} & {$[0 / \pm 45 / 90]_{4 \mathrm{~S}}$} & \\
\hline $1 " \times 1 "$ & 3 & 3 & 3 & 3 & 3 & 3 & 18 \\
\hline $1 " \times 2 "$ & 3 & 3 & 3 & 3 & 3 & 3 & 18 \\
\hline $2 " \times 2 "$ & 3 & 3 & 3 & 3 & 3 & 3 & 18 \\
\hline Total & 9 & 9 & 9 & 9 & 9 & 9 & 54 \\
\hline
\end{tabular}

Table 6.3: Dielectric strength measurement test matrix

\begin{tabular}{|c|c|c|c|c|c|c|c|}
\hline \multirow[t]{2}{*}{ Area } & \multicolumn{3}{|c|}{ Unidirectional } & \multicolumn{3}{|c|}{ Quasi-Isotropic } & \multirow[t]{2}{*}{ Total } \\
\hline & {$[0]_{4}$} & {$[0]_{8}$} & {$[0]_{16}$} & {$[ \pm 45]_{\mathrm{S}}$} & {$[0 / \pm 45 / 90]_{S}$} & {$[0 / \pm 45 / 90]_{4 S}$} & \\
\hline $1 " \times 1 "$ & 3 & 3 & 3 & 3 & 3 & 3 & 18 \\
\hline
\end{tabular}




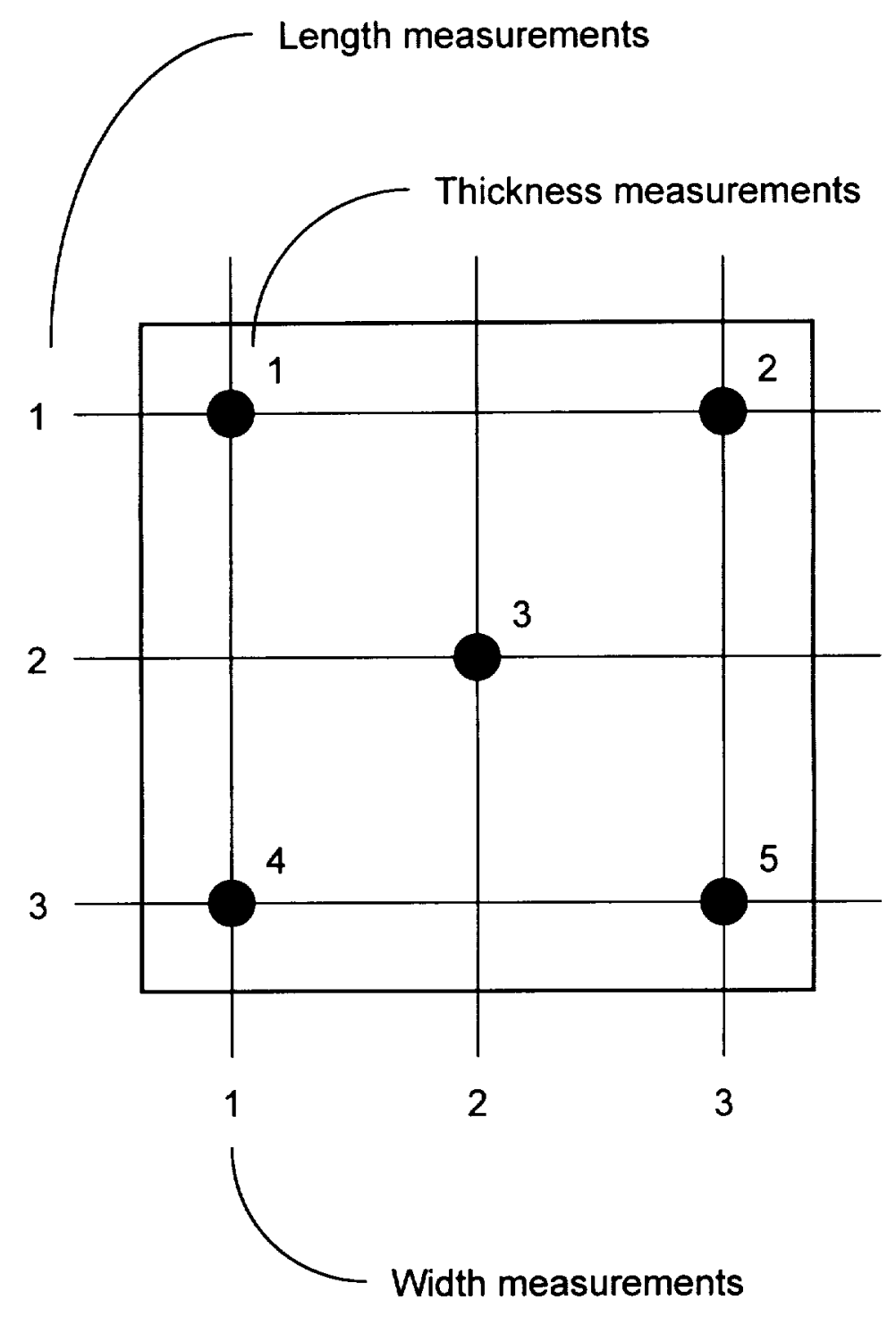

Figure 6.1: Measurement locations for electrical property samples 


\subsubsection{Conductivity Tailorable Composite}

To investigate the development of a conductivity tailorable composite, a glass fiber / epoxy wet layup procedure was used. Carbon black was added to the epoxy in 4 different percentage by mass: $5,10,15$, and 20 percent. The carbon black used was Columbian Conductex Ultra High Conductivity carbon black. The carbon black filled epoxy resins are identified by Penn Color as RD $38663-50$ for the 5\%, RD $38662-50$ for the $10 \%$, RD $38661-50$ for the $15 \%$, and $\mathrm{RD} 38660-50$ for the $20 \%$. The electrical properties measured included the conductivity, dielectric constant, and dielectric strength, and the mechanical properties measured included the density, Young's modulus, Poisson ratio, failure stress, and failure strain. Three $304.8 \mathrm{~mm} \times 355.6 \mathrm{~mm}(12 " \times 14 ")$ laminates of each percentage were manufactured, and 2 samples were taken from each laminate. Therefore 6 samples of each percentage of carbon black were manufactured, for a total of 30 samples for each test. As shown in Table 6.4, the test matrix is fully populated. The size of the samples used for the electrical properties are $25.4 \mathrm{~mm} \times 25.4$ $\mathrm{mm}(1 " \mathrm{x} 1 ")$. The density was measured using the conductivity samples. The size of the tensile test specimen used were $50.8 \mathrm{~mm}$ x $355.6 \mathrm{~mm} \mathrm{(2"} \mathrm{\times} \mathrm{14")} \mathrm{with} 50.8 \mathrm{~mm} \times 76.2$ mm (2" $\times 3 ")$ tapered glass loading tabs at each end on both sides. Each sample was tested once. The geometry of the electrical property samples were measured as shown in Figure 6.1. For the tensile specimen the thickness was measured 9 times, the width 3 times, and the length between tabs twice, as shown in Figure 6.2. 
Table 6.4: Conductivity tailorable composite test matrix

\begin{tabular}{|l|c|c|c|c|c||c||}
\hline \hline \multirow{2}{*}{ Property } & \multicolumn{5}{|c|}{ Percent Carbon Black By Mass } & Total \\
& $\mathbf{0 \%}$ & $\mathbf{5 \%}$ & $\mathbf{1 0 \%}$ & $\mathbf{1 5 \%}$ & $\mathbf{2 0 \%}$ & \\
\hline \hline Conductivity & 6 & 6 & 6 & 6 & 6 & 30 \\
\hline Dielectric Constant & 6 & 6 & 6 & 6 & 6 & 30 \\
\hline Dielectric Strength & 6 & 6 & 6 & 6 & 6 & 30 \\
\hline Density & 6 & 6 & 6 & 6 & 6 & 30 \\
\hline Young's Modulus & 6 & 6 & 6 & 6 & 6 & 30 \\
\hline Poisson Ratio & 6 & 6 & 6 & 6 & 6 & 30 \\
\hline Failure Stress & 6 & 6 & 6 & 6 & 6 & 30 \\
\hline Failure Strain & 6 & 6 & 6 & 6 & 6 & 30 \\
\hline \hline Total & 48 & 48 & 48 & 48 & 48 & 240 \\
\hline
\end{tabular}




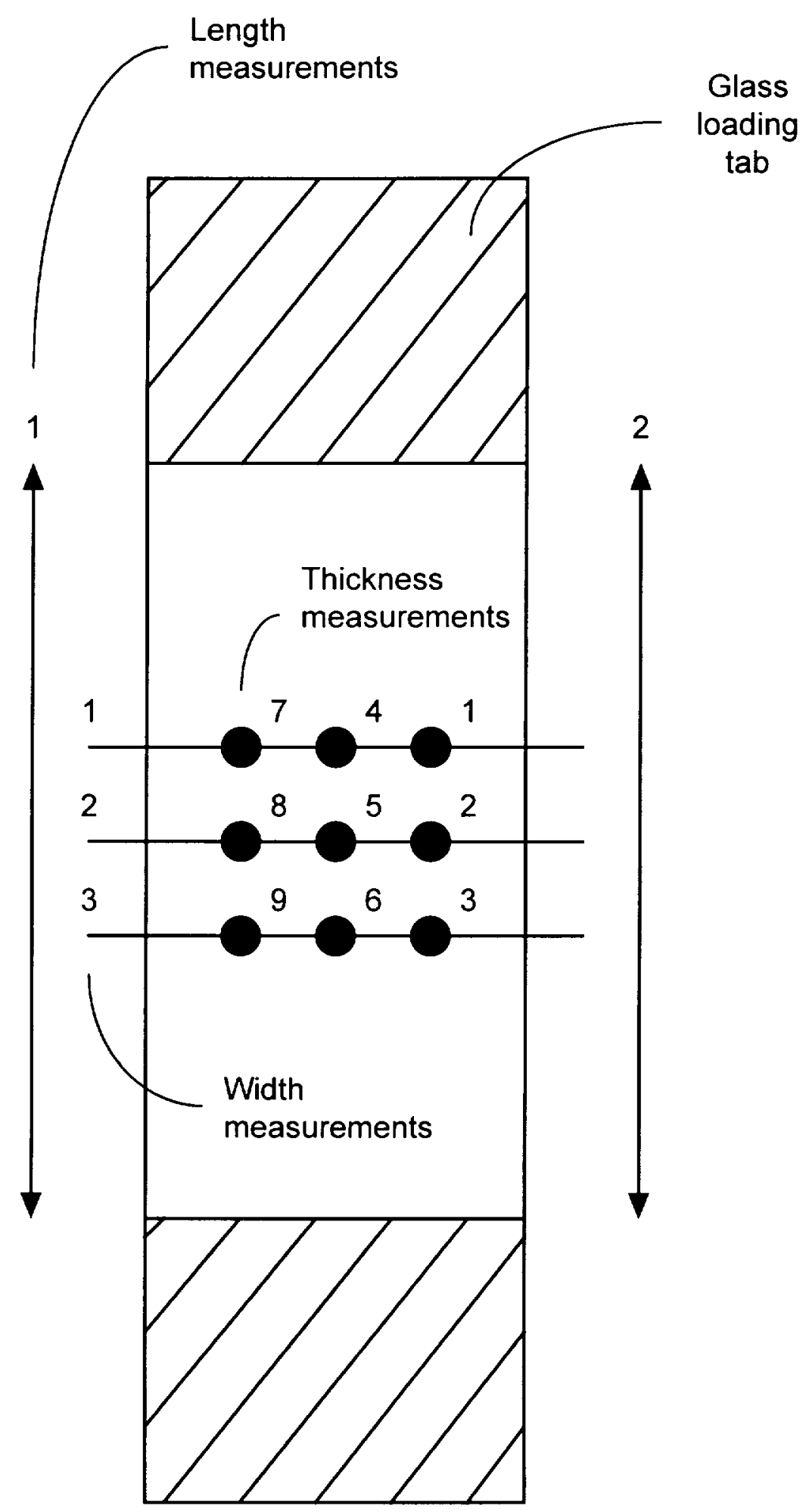

Figure 6.2: Measurement locations for tensile test specimen 


\subsection{MANUFACTURING PROCEDURES}

The manufacturing procedure used for the AS4/3501-6 laminates is the TELAC net resin cure, ${ }^{59}$ and is summarized here. Procedures for manufacturing the glass fiber / epoxy laminates was documented by Ed Wolf ${ }^{60}$ and the manufacturer, and are also presented here. Two different layup techniques were used in this investigation: a prepreg layup for the carbon fiber / epoxy material, and a wet layup for the glass fiber / epoxy system. Latex gloves were worn whenever the raw materials were handled directly, and care was taken to avoid unnecessary contact with the uncured materials at all times.

\subsubsection{Carbon Fiber / Epoxy Prepreg Layup}

The carbon fiber / epoxy prepreg tape came in the form of rolls $305 \mathrm{~mm}$ wide. The rolls of prepreg were stored in sealed bags in a freezer at a temperature lower than $-18^{\circ} \mathrm{C}$. In preparation for layup, the prepreg was taken out of the freezer and allowed to sit at room temperature in the sealed bag for at least 1 hour, or until the material was no longer cold to the touch. If the bag had been opened while the material was still cold, unwanted moisture could have condensed on it. The prepreg was then unrolled and the appropriate plies were cut using Teflon coated aluminum patterns and a sharp utility knife. The plies were cut in such a way that any seams within a ply were parallel to the fiber direction. Once cutting was completed, the prepreg roll was resealed and placed back in the freezer.

To make the smaller-than-standard laminates, $152.4 \mathrm{~mm} \times 177.8 \mathrm{~mm}\left(6^{\prime \prime} \times 7 "\right)$, the prepreg was cut using the standard $304.8 \mathrm{~mm} \times 355.6 \mathrm{~mm}\left(12^{\prime \prime} \times 14^{\prime \prime}\right)$ patterns. 
Following this, each ply was cut into 4 pieces using small patterns. The individual plies were stacked in the proper sequence using an L-shaped aluminum jig to aid in alignment. The corner of the laminate which was situated directly in the corner of the aluminum jig was assumed to have the most accurate ply stacking and was marked for future reference. Between each application of a ply, the paper backing was removed from the laminate. When the laminate was completed, it was trimmed with a sharp utility knife and an aluminum pattern to its nominal $152 \mathrm{~mm}$ wide by $178 \mathrm{~mm}$ long size, and florocarbon release film was placed on both the top and bottom surfaces. If the laminates were to be cured within 24 hours, they were sealed in a vacuum bag and left out at room temperature. Otherwise, they were sealed in a bag and put into the freezer.

In preparation for the cure, an aluminum cure plate was sprayed with Mold Wiz ${ }^{\circledR}$ release agent and covered with a sheet of nonporous Teflon. On top on this, three layers of cork dam were used to make six $152 \mathrm{~mm}$ by $178 \mathrm{~mm}\left(6^{\prime \prime} \mathrm{x} 7 "\right)$ rectangular vessels for the laminates, followed by another cork dam made of one layer of cork, this second dam was used to create a step down from the 3 layer dam height. A sheet of nonporous Teflon was placed in the bottom of the vessel, to line the bottom and sides of the vessel. This was followed by the prepreg laminate with florocarbon release film on both sides, and a sheet of porous Teflon. The location of the "good" corner was marked. The aluminum top plate, sprayed with Frekote 700 , and wrapped in nonporous Teflon, was then placed on top of the laminate. The assembly was then covered with a single sheet of porous Teflon, several layers of glass breather, and finally, the vacuum bag, which was sealed to 
the caul plate with vacuum tape. The cure plate was now ready for the autoclave. Figure 6.3 shows a schematic of the cure layup.

\subsubsection{Glass Fiber / Epoxy Wet Layup}

The glass fiber / epoxy system requires a wet layup procedure. The raw materials used in the preparation of this system were a 1 meter wide roll of woven unidirectional (the transverse weave is just used to keep the cloth together, it has no strength) glass cloth type 1543-38, a drum of Rutapox L20 epoxy resin and another smaller container of liquid SL hardener for the resin. The Columbian Conductex Ultra High Conductivity carbon black was added directly into the L20 epoxy resin by Penn Color Inc. Therefore the percent mass of carbon black is based on the mass of the resin only. The resin and hardener were mixed in the correct proportion. This mixture was then added to the dry fabric plies, producing a layup ready to be cured in the autoclave under vacuum.

The wet layup was performed in the following manner. First, the necessary plies of glass fabric were cut from the roll with sharp scissors. This was accomplished by tracing out the plies to be cut using a felt pen and a specially designed Teflon coated aluminum pattern. While cutting the fabric, some shearing of the fabric occurred; this was straightened out when the plies were place on the curing plate, by carefully flattening out all the wrinkles in the fabric. The glass plies to be used in each laminate were carefully weighed. The appropriate mass of liquid matrix was prepared by mixing the resin and hardener in a 100 to 34 mass ratio to give a total mass of matrix equal to the total mass of the fabric plies. The layup calls for the combining of equal masses of 


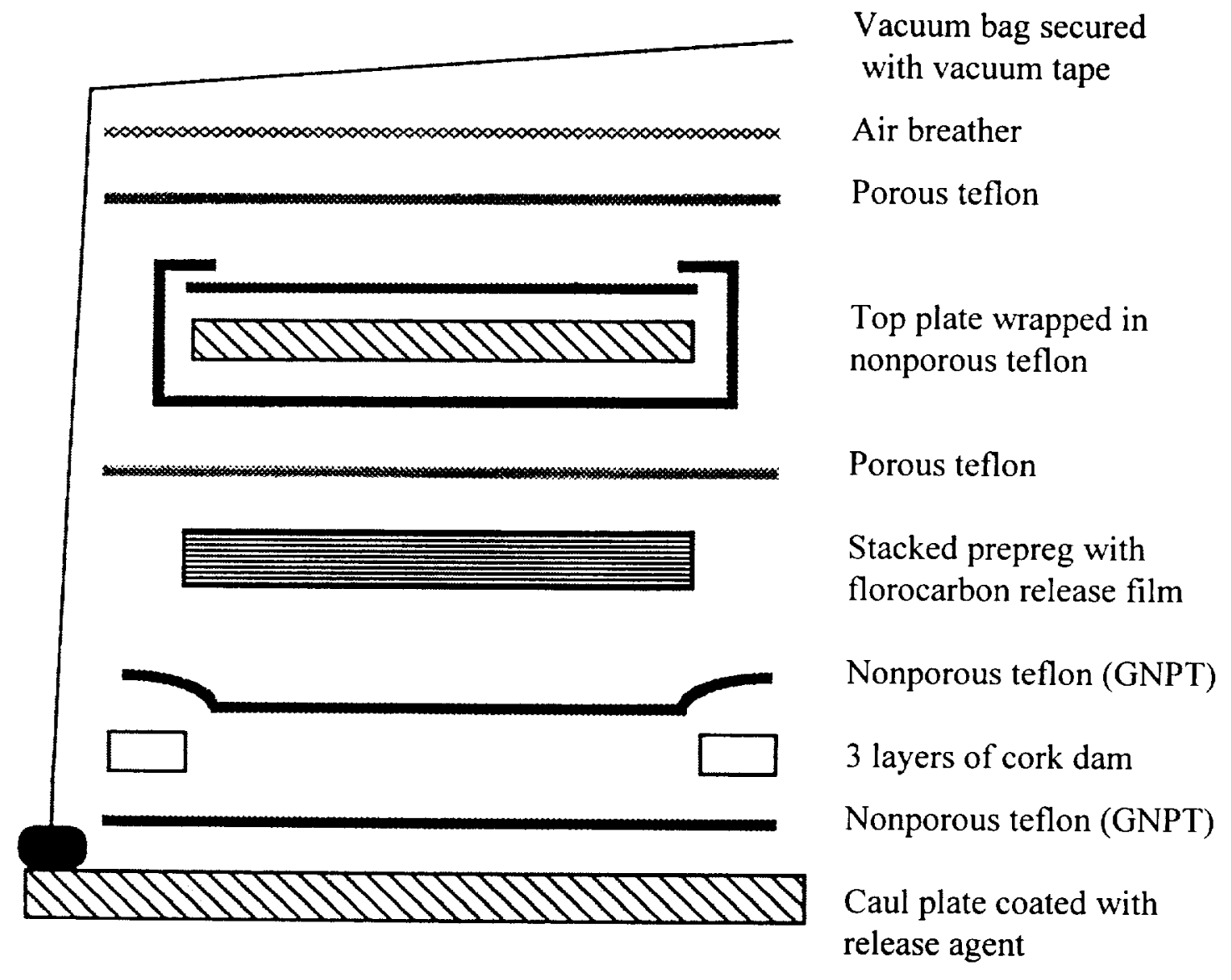

Figure 6.3: Schematic of AS4/3501-6 cure layup 
matrix and fibers. For the carbon black filled resin, the total mass of filled resin used was increased to keep the mass of actual resin without carbon black the same, as shown in Table 6.5. Once mixed together the epoxy was well stirred to ensure complete mixing of the resin and hardener.

In preparation for the wet layup and cure, the cure plate was sprayed with Mold Wiz $B$ release agent and covered with a sheet of nonporous Teflon. On top on this, three layers of cork dam were used to make three $305 \mathrm{~mm}$ by $356 \mathrm{~mm}$ (12" x 14") rectangular vessels for the laminates, followed by another cork dam made of one layer of cork, this second dam was used to create a step down from the 3 layer dam height. A sheet of nonporous Teflon was placed in the bottom of the vessel, to line the bottom and sides of the vessel, followed by a sheet of peel-ply and then the layers of dry fabric. After the last ply of fabric was placed in the vessel, the matrix was poured on top and allowed to soak into the plies. The matrix was spread across the top ply using a half inch acid brush, starting at the center of the laminate and working outward radially toward the edges. By working from the center outward, the amount of trapped air was minimized. Care was taken not to disturb the alignment of the fibers while spreading the matrix with the brush.

When wetted, the layup changed appearance from white in color to transparent. However, this only occurred with the pure epoxy as the epoxy with carbon black is black in color. With the higher percentages of carbon black, the epoxy did not soak through the plies very well. In these cases it was necessary to proceed to the next step without waiting for the epoxy to soak through the plies completely. When no areas of white were 
Table 6.5: $\quad$ Rutapox L20/SL resin and hardener mixing ratios used

\begin{tabular}{|c|c|c|c||}
\hline Carbon Black & Resin & Resin Mass & Hardener Mass \\
\hline \hline $0 \%$ & $100 \%$ & $92.16 \mathrm{~g}$ & $31.34 \mathrm{~g}$ \\
\hline $5 \%$ & $95 \%$ & $97.01 \mathrm{~g}$ & $31.34 \mathrm{~g}$ \\
\hline $10 \%$ & $90 \%$ & $102.40 \mathrm{~g}$ & $31.34 \mathrm{~g}$ \\
\hline $15 \%$ & $85 \%$ & $108.42 \mathrm{~g}$ & $31.34 \mathrm{~g}$ \\
\hline $20 \%$ & $80 \%$ & $115.67 \mathrm{~g}$ & $31.34 \mathrm{~g}$ \\
\hline
\end{tabular}


visible, any remaining matrix was spread evenly over the laminate, and a sheet of peelply was smoothed on top, followed by a sheet of porous Teflon. The aluminum top plate, sprayed with Frekote 700 and wrapped in nonporous Teflon, came next. Sheets of bleeder paper were rolled into dams and added around the cork vessels and the vacuum ports to absorb any extra liquid epoxy and to prevent it from flowing into the vacuum system. The assembly was then covered with a single sheet of porous Teflon, several layers of glass breather, and finally, the vacuum bag, which was sealed to the caul plate with vacuum tape. The cure plate was now ready for the autoclave. Figure 6.4 shows a schematic of the cure layup, and Figure 6.5 shows a typical cure plate with the bleeder paper rolls around the laminate vessels to absorb the excess epoxy.

\subsubsection{AS4/3501-6 Cure}

The AS4/3501-6 material was cured according to the TELAC net cure procedure, in the TELAC autoclave. The TELAC autoclave is a $0.91 \mathrm{~m}(3 \mathrm{ft})$ diameter Baron Blakeshee Inc. model BAC 35 autoclave, as shown in Figure 6.6, it has a $1.68 \mathrm{~m}(5.5 \mathrm{ft})$ length, a maximum temperature capability of $427^{\circ} \mathrm{C}\left(800^{\circ} \mathrm{F}\right)$, a maximum pressure capability of $1.72 \mathrm{MPa}(250 \mathrm{psi})$, and a maximum vacuum capability of $762 \mathrm{~mm} \mathrm{Hg}$ (30 in $\mathrm{Hg}$ ). Six laminates were cured at one time in the autoclave. The position of the laminates on the cure plate is shown in Figure 6.7.

A vacuum check was performed on the cure plate before it was rolled into the autoclave. This was done by applying a vacuum of $762 \mathrm{~mm} \mathrm{Hg} \mathrm{(30} \mathrm{in} \mathrm{Hg})$ and then shutting off the vacuum source. The vacuum seal was considered satisfactory if less than 


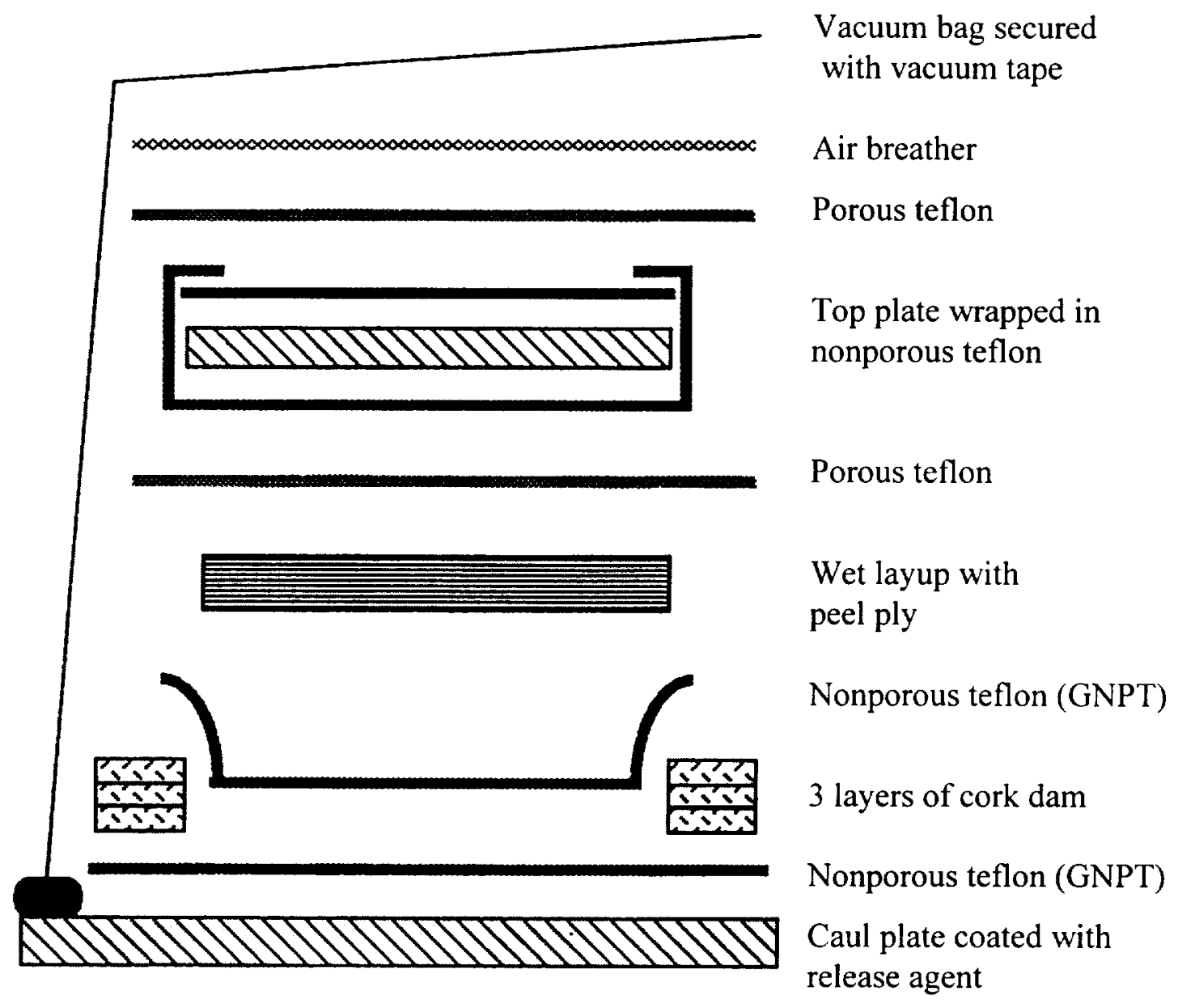

Figure 6.4: Schematic of glass fiber / epoxy cure layup 


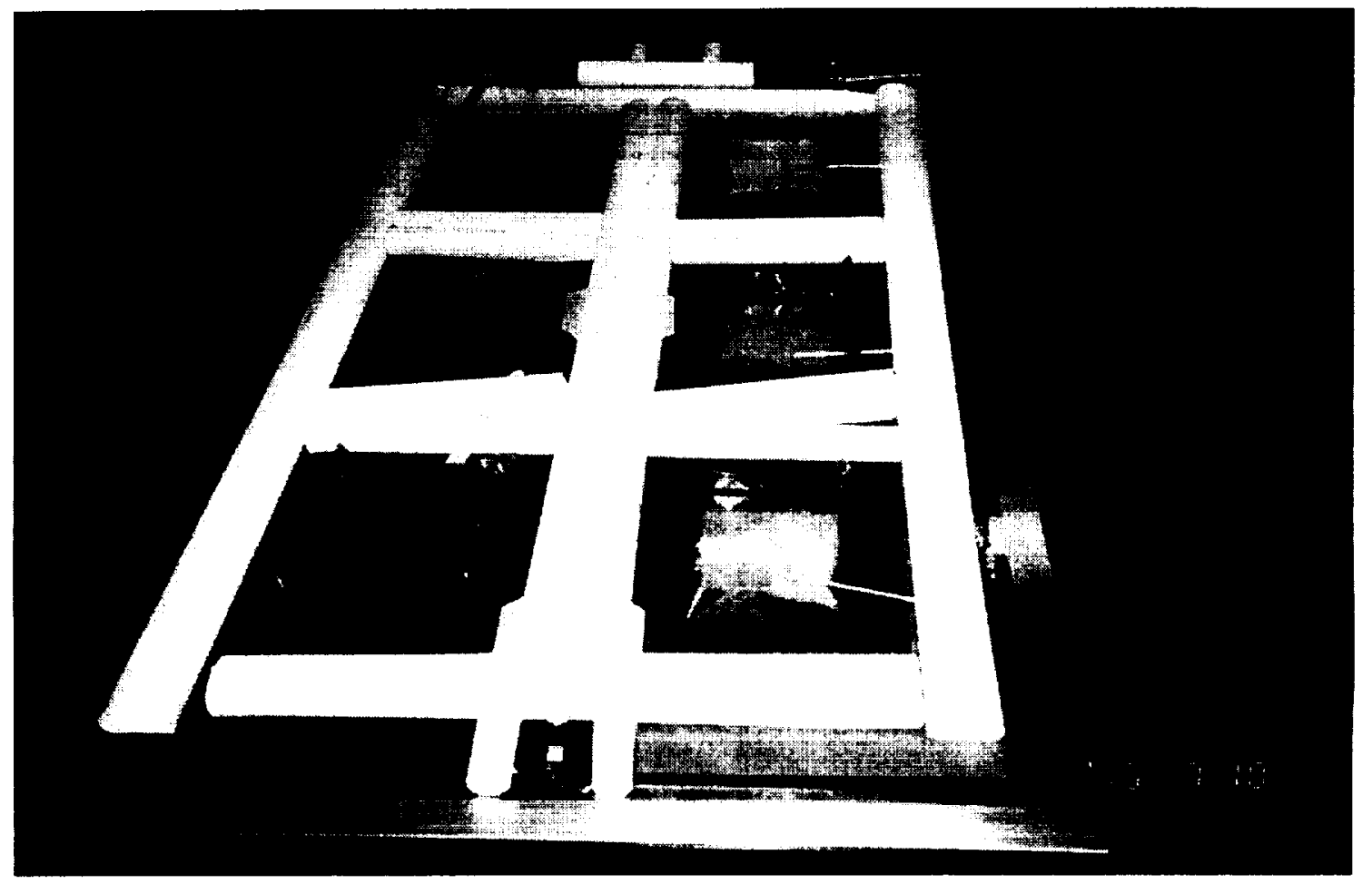

Figure 6.5: Typical cure plate with bleeder paper rolls around laminate vessels 


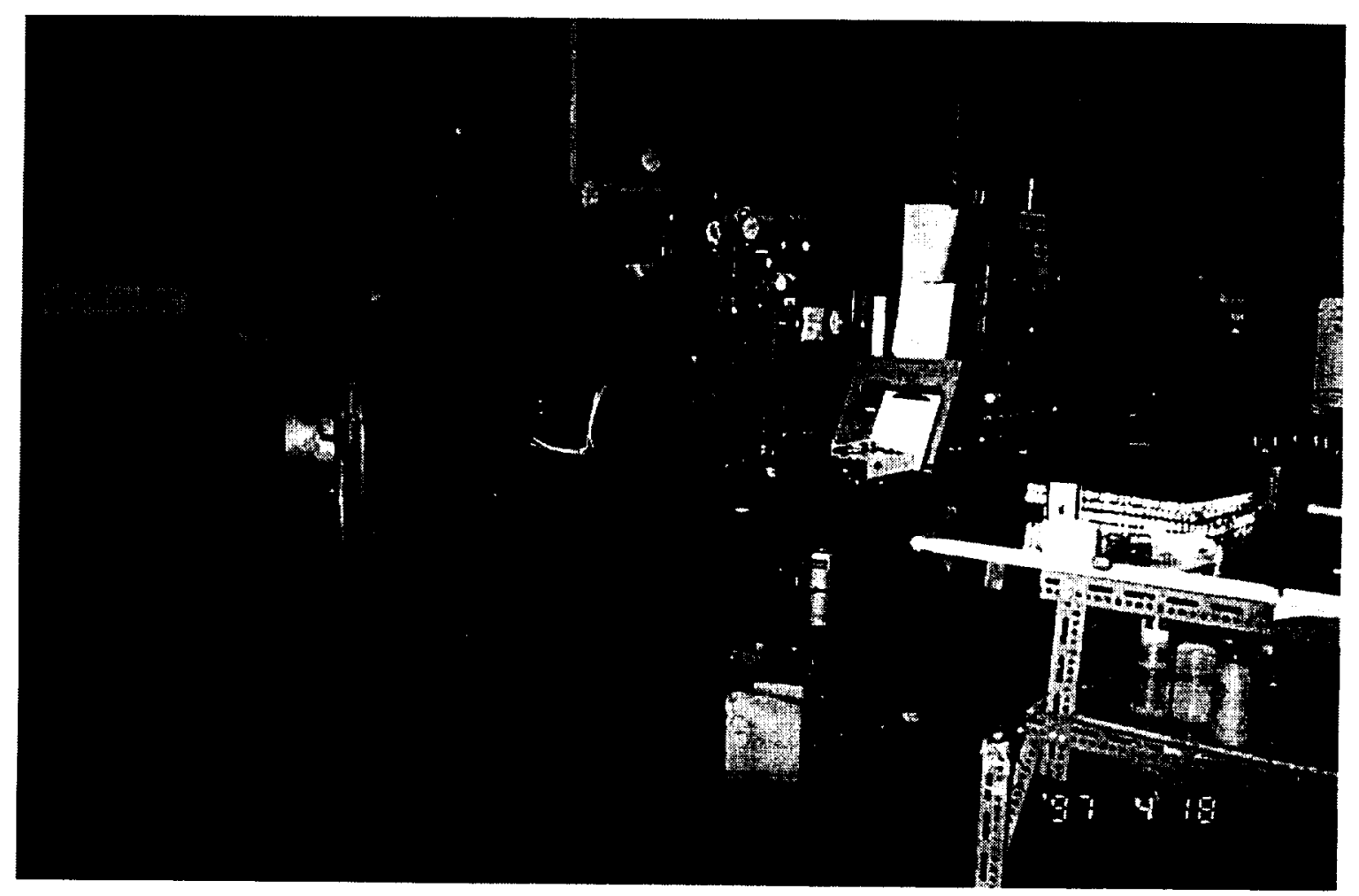

Figure 6.6: TELAC's Baron Blackshee autoclave 


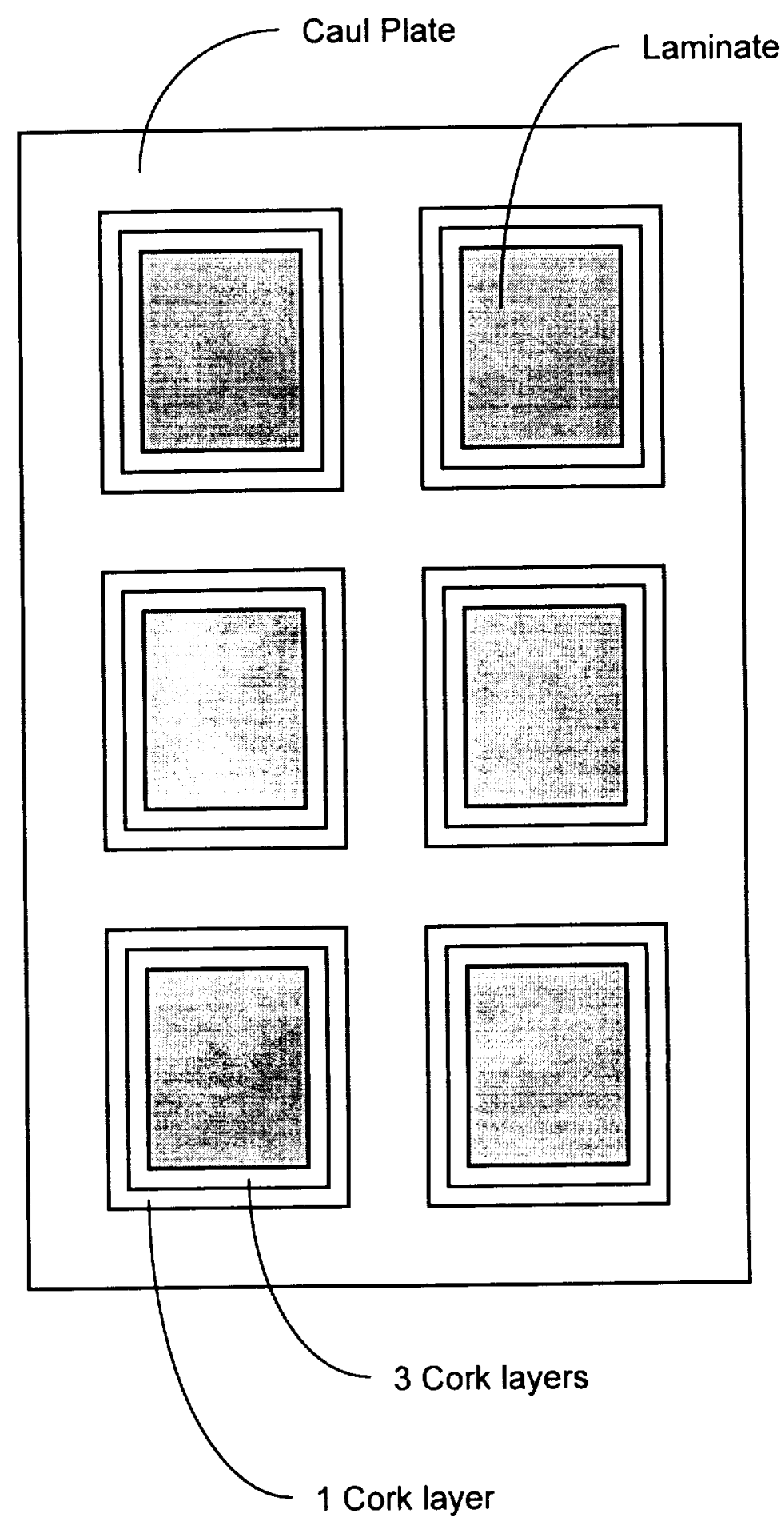

Figure 6.7: Illustration of the cure plate used for the AS4/3501-6 cure (top view) 
$127 \mathrm{~mm} \mathrm{Hg} \mathrm{(5} \mathrm{in} \mathrm{Hg}$ ) was lost in 5 minutes. Otherwise, attempts were made at trying to find and repair leaks in the vacuum bag, or the entire bag was replaced. Once a successful vacuum check was performed, the cure plate was rolled into the autoclave and the vacuum was rechecked.

In the autoclave, a vacuum of $762 \mathrm{~mm} \mathrm{Hg}(30 \mathrm{in} \mathrm{Hg}$ ) was applied to the cure plate. The autoclave pressure was raised to a gage pressure of $0.59 \mathrm{MPa}(85 \mathrm{psi})$ and held. Once the pressure was achieved, the autoclave temperature was raised at a rate of 1 to $3{ }^{\circ} \mathrm{C}$ per minute until $116^{\circ} \mathrm{C}\left(240{ }^{\circ} \mathrm{F}\right)$ was reached. This temperature was held for 1 hour, following which the temperature was raised at the same rate to $177^{\circ} \mathrm{C}\left(350^{\circ} \mathrm{F}\right)$ and held for 2 hours. Finally the temperature was decreased at a rate 3 to $5{ }^{\circ} \mathrm{C}$ per minute to $80^{\circ} \mathrm{C}\left(176^{\circ} \mathrm{F}\right)$ at which point the pressure was released. The temperature, pressure, and vacuum cycles are shown in Figure 6.8. The laminates were postcured in an oven at 177 ${ }^{\circ} \mathrm{C}\left(350^{\circ} \mathrm{F}\right)$ for 8 hours with no applied pressure or vacuum.

\subsubsection{Glass Fiber / Epoxy Cure}

The glass fiber / epoxy was cured according to the manufacture's specification in the TELAC autoclave, refer to section 6.2 .3 for description of the autoclave. Three laminates were cured at one time in the autoclave. The position of the laminates on the cure plate is shown in Figure 6.9.

After a successful vacuum check, refer to section 6.2 .3 for explanation, a vacuum of $762 \mathrm{~mm} \mathrm{Hg} \mathrm{(30} \mathrm{in} \mathrm{Hg}$ ) was applied to the cure plate in the autoclave and the 

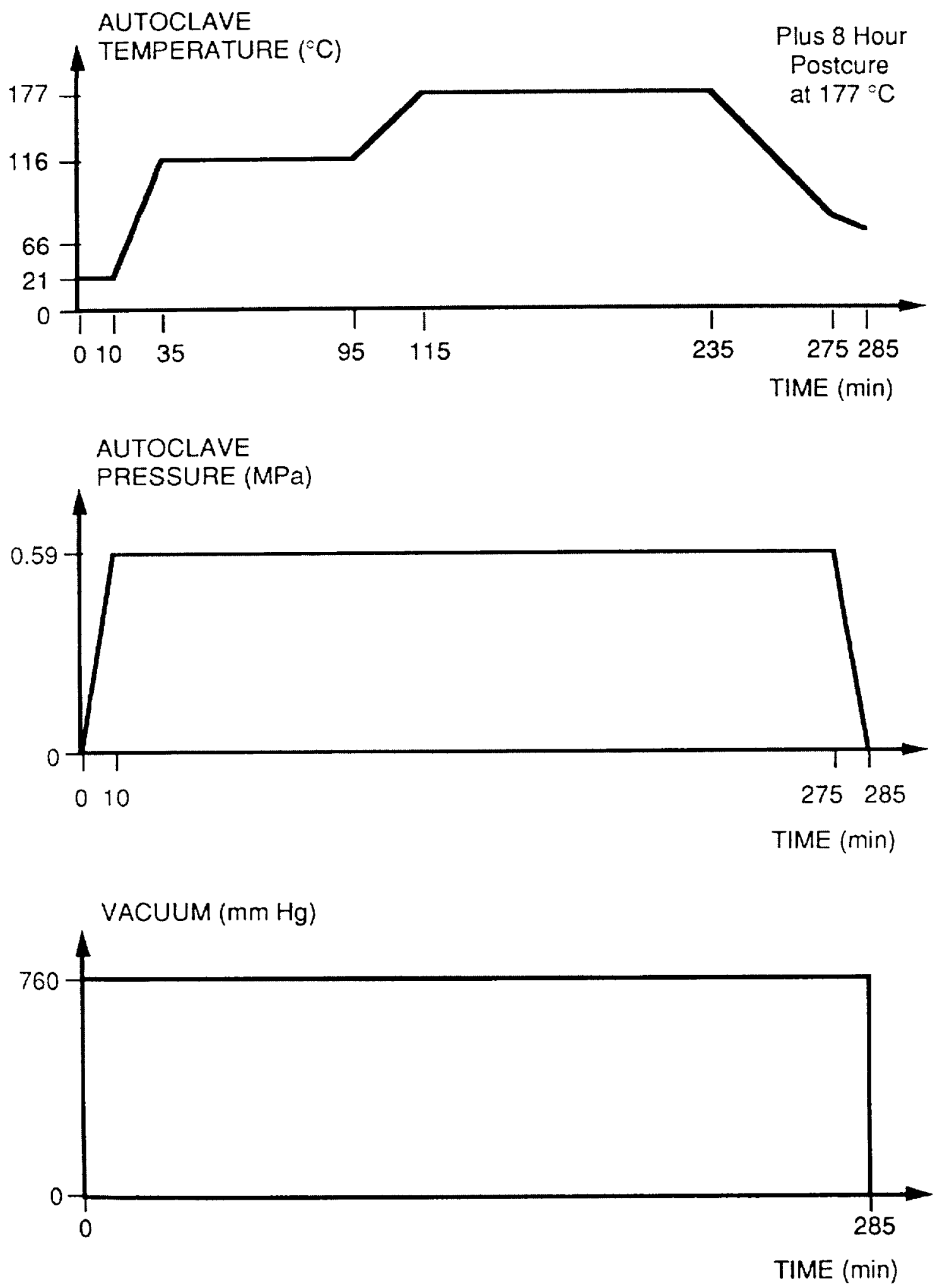

Figure 6.8: AS4/3501-6 cure cycle 


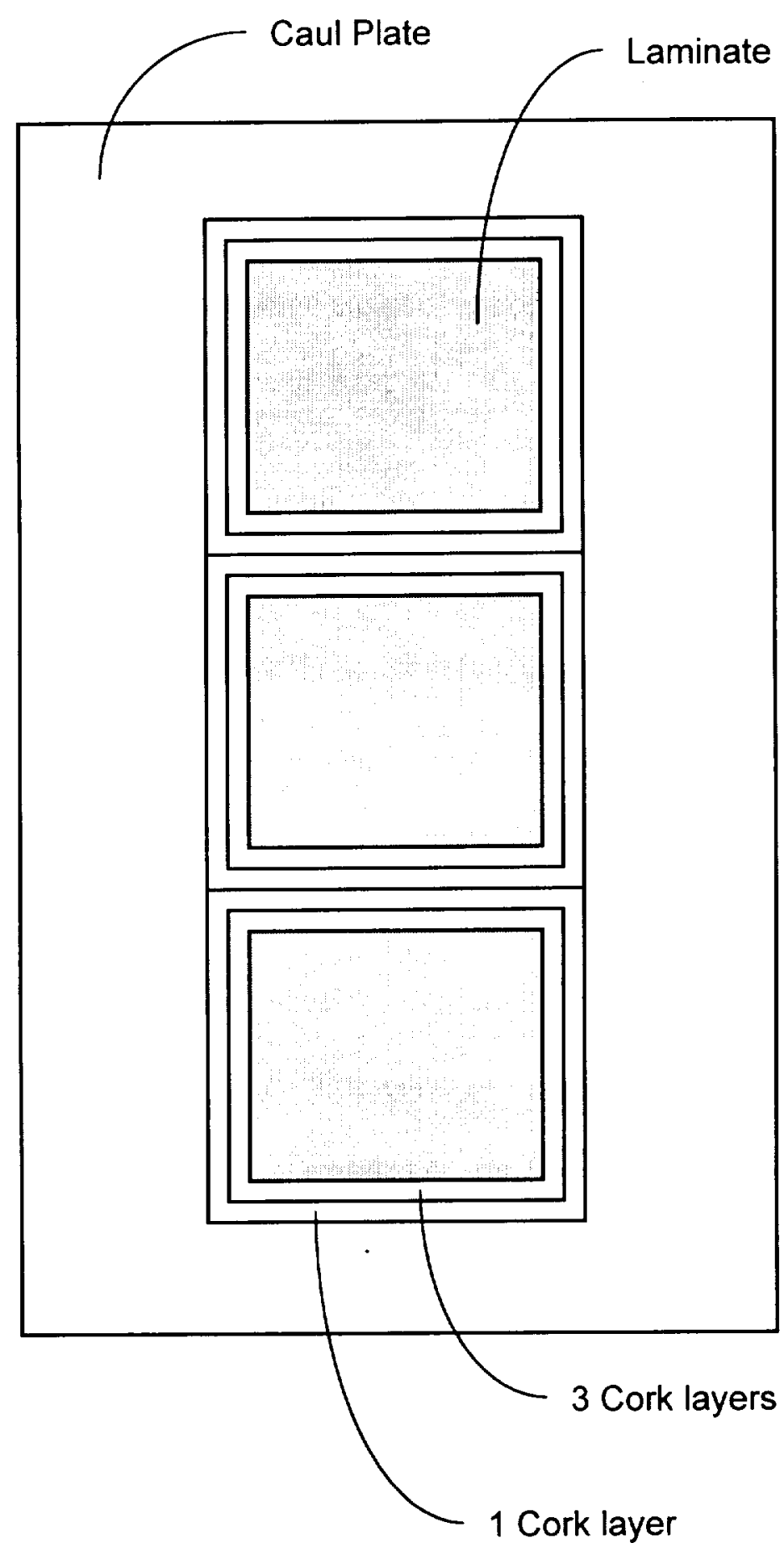

Figure 6.9: Illustration of the cure plate used for the Glass Fiber / Epoxy cure (top view) 
temperature was raised to $60^{\circ} \mathrm{C}\left(140^{\circ} \mathrm{F}\right)$ at a rate of 5 to $6^{\circ} \mathrm{C}$ per minute. No external pressure was applied to the laminates. This temperature was held for 8 hours, after which the vacuum was released and the laminates were removed from the autoclave. The temperature, pressure, and vacuum cycles are shown in Figure 6.10. The laminates were postcured in an oven at $80^{\circ} \mathrm{C}\left(176^{\circ} \mathrm{F}\right)$ for 15 hours with no applied pressure or vacuum.

\subsubsection{Final Preparation}

All laminates were milled into coupons with a Van Norman model 22-L milling machine mounted with a water-cooled 220 grit diamond cutting wheel. The diameter of the cutting wheel is $254 \mathrm{~mm}$ and a spindle rate of $1100 \mathrm{rpm}$ was used. The milling table feed rate was $279 \mathrm{~mm}$ per minute. First, the edges of the laminate were cleaned by trimming approximately $3 \mathrm{~mm}$ (1/8 in) from each side. For the AS4/3501-6 samples, two $25.4 \mathrm{~mm}(1 \mathrm{in})$ wide strips were cut off the shorter side, making sure to start with the "good" corner side, after which a $50.8 \mathrm{~mm}$ ( 2 in) wide strip was cut off. The strips were then cut into the required sized samples, three $50.8 \mathrm{~mm}$ by $50.8 \mathrm{~mm}$, three $25.4 \mathrm{~mm}$ by $50.8 \mathrm{~mm}$, and six $25.4 \mathrm{~mm}$ by $25.4 \mathrm{~mm}$. As shown in Figure $6.11,9$ conductivity and 3 dielectric strength samples were cut. For the glass fiber / epoxy samples, three $50.8 \mathrm{~mm}$ ( 2 in) wide strips were cut off the shorter side, followed by a $25.4 \mathrm{~mm}$ ( 1 in) wide strip. The first $50.8 \mathrm{~mm}$ strip was not used, in an effort to eliminate curing defects located near the edge of the laminates. The next two were used for tensile tests. The $25.4 \mathrm{~mm}$ wide strip was cut into six $25.4 \mathrm{~mm}$ by $25.4 \mathrm{~mm}$ samples. The first 2 from the edge were discarded for edge defects, the next 2 samples were used for the conductivity measurement, and the remaining 2 were used for the dielectric strength measurement, as 

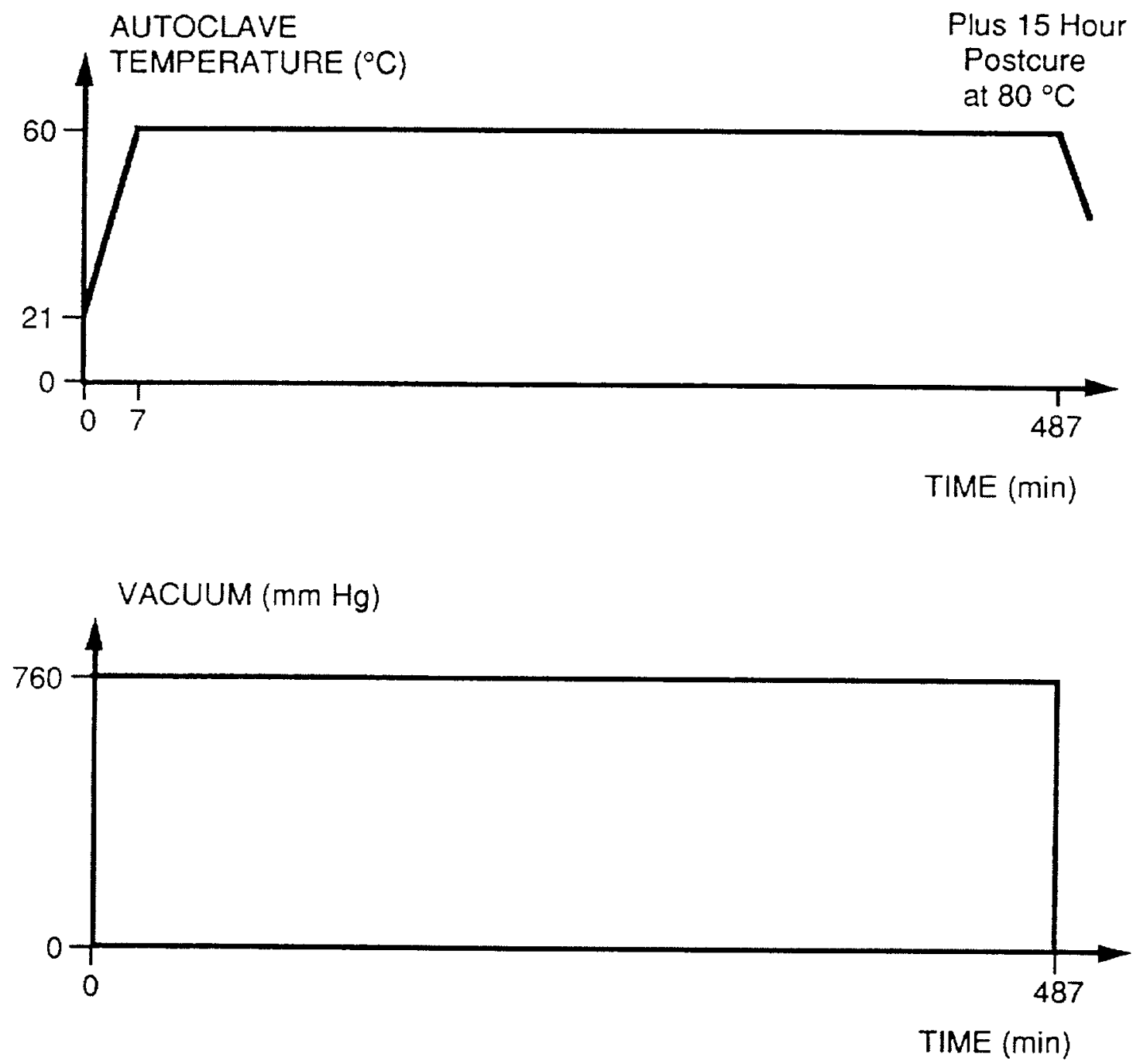

Figure 6.10: Glass fiber / epoxy cure cycle 


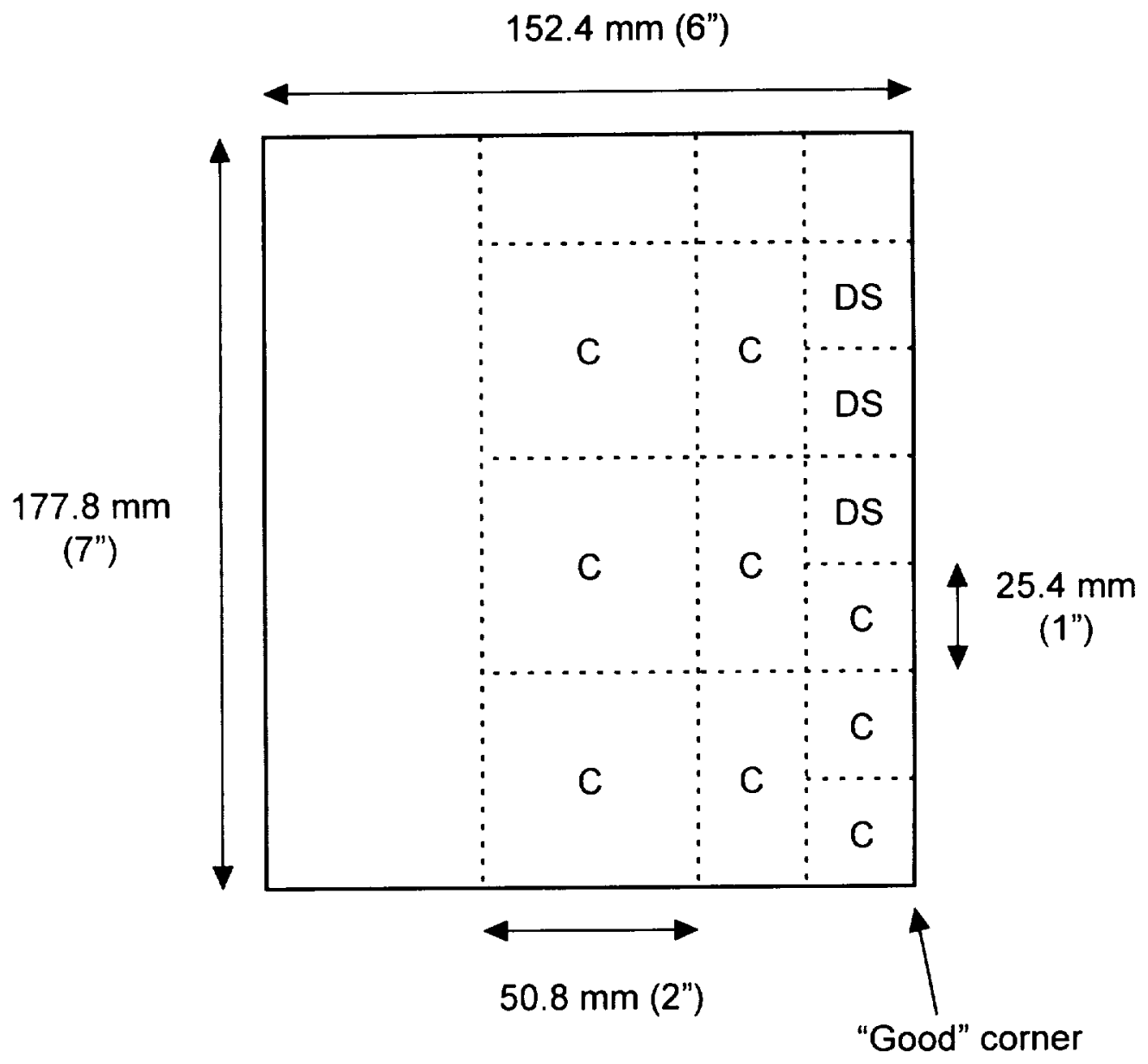

C: Conductivity sample

DS: Dielectric Strength sample

Figure 6.11: Schematic of the cutting patterns for the AS4/3501-6 laminates 
shown in Figure 6.12. The same cutting process was used to manufacture the $50.8 \mathrm{~mm} \times$ $76.2 \mathrm{~mm}\left(2^{\prime \prime} \times 3^{\prime \prime}\right)$ glass loading tabs, from sheets of purchased fiberglass. Once the glass tabs were cut, one edge was tapered to approximately $45^{\circ}$ on a belt sander.

\subsection{CONDUCTIVITY MEASUREMENT}

ASTM D257-93 Standards summarizes the issues related to measurement of DC resistivity in insulating materials. ${ }^{61}$ To measure the conductivity of the samples, the current had to be uniformly distributed across the surface of the samples. Therefore the application of a conductive coating was required. After experimenting with various coatings, including gold sputtering, vapor deposited silver, and conductive epoxy with various curing processes, it was decided to use conductive epoxy with an aluminum foil surface. To prevent moisture from penetrating all the samples, they were stored in an airtight jar with desiccant.

\subsubsection{Conductive Epoxy Cure}

The conductive epoxy used was Epo-tek 410LV two part silver epoxy, manufactured by Epoxy Technology. The resin and the hardener were mixed at a specific ratio by mass of 15.0 resin to 2.1 hardener. The resin was thoroughly mixed before being used to ensure an even distribution of the silver. Once the two parts were thoroughly mixed together, it was applied to the surface of the conductivity samples with a half inch acid brush. The sample was then placed on a piece of aluminum foil, and a second piece of aluminum foil was place on top of the sample. A weight was placed on top of the samples to ensure an even distribution of the epoxy. The samples were then cured in an 


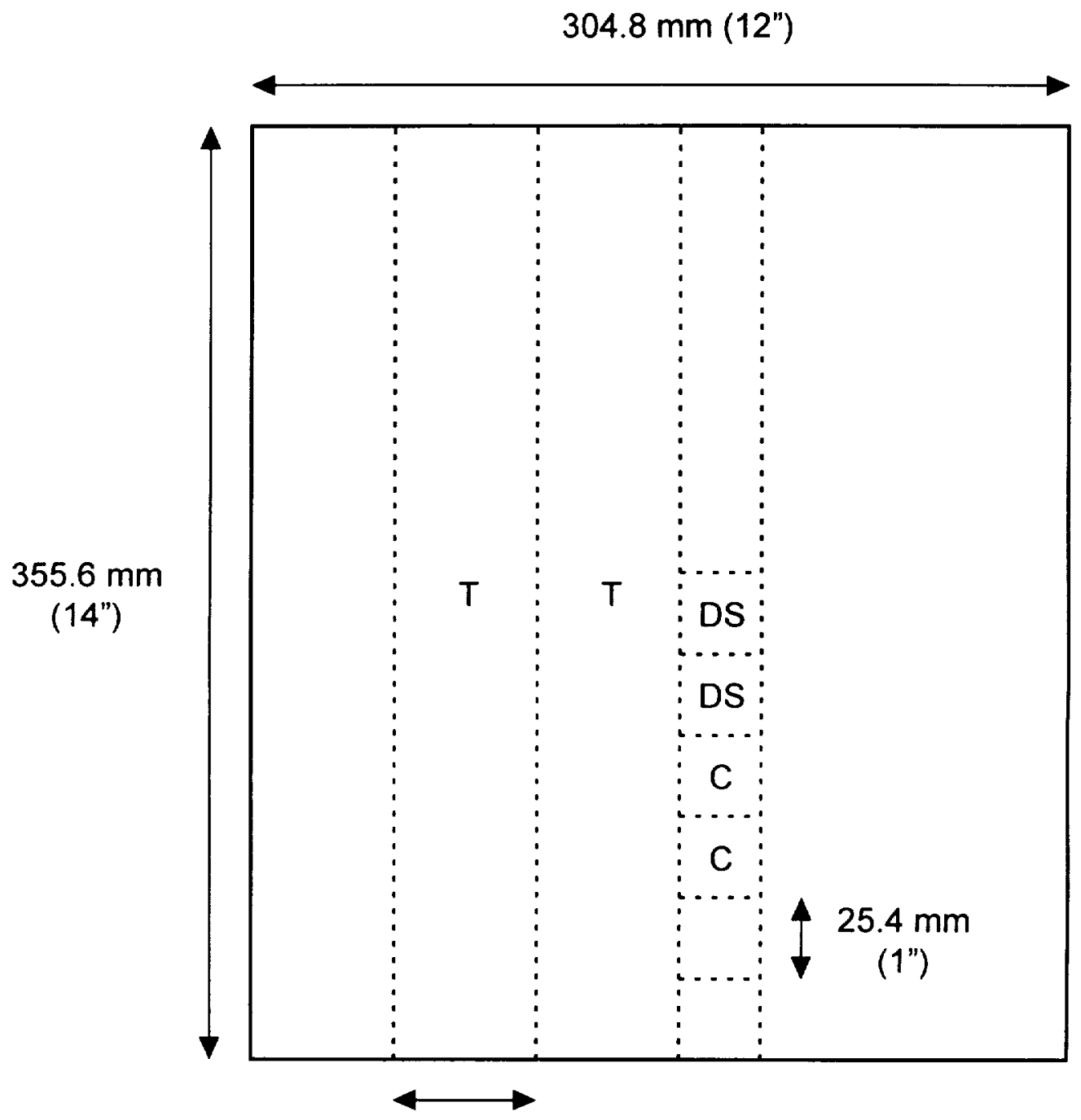

$50.8 \mathrm{~mm}(2 ")$

\section{C: Conductivity samples}

DS: Dielectric strength samples

$\mathrm{T}$ : Tensile test samples

Figure 6.12: Schematic of the cutting patterns for the glass fiber / epoxy laminates 
oven. The AS4/3501-6 samples were cured for 1 hour at $100^{\circ} \mathrm{C}\left(212^{\circ} \mathrm{F}\right)$, and because of the lower cure temperature of the glass fiber / epoxy, it was cured for 4 hours at $60{ }^{\circ} \mathrm{C}$ $\left(140^{\circ} \mathrm{F}\right)$. The edges of the samples were then sanded to remove any epoxy the may have ended up on the sides of the sample creating a conductive path between the two surfaces.

\subsubsection{Surface Layer Removal}

After the first set on conductivity measurements, it was postulated that the epoxy surface layer was affecting the measurement. Therefore the conductivity samples were sanded to remove the surface layer. The sanding process was done with a hand-held orbital sander, the sample were held to the table with double-sided tape. Once the sanding was complete, the sample thicknesses were re-measured and a new conductive epoxy layer was applied.

\subsubsection{Carbon Fiber / Epoxy Measurement}

The conductivity of the carbon fiber / epoxy samples was determined by measuring the resistance across the sample and calculating the conductivity base on equation 81. The resistance was measured using the "direct method" of measurement. This refers to the application of a known voltage, and measuring the resulting current. The instrument used was a Keithley 6517 High Resistance Electrometer with an applied voltage of 0.05 Volts. It was borrowed from the Adaptive Materials and Structures Laboratory (AMSL). The sample was placed between to electrodes in a sample holder made of Lexan, a highly resistive material, as shown in Figure 6.13. The sample holder was placed in a shielded enclosure, and a two probe technique specified by the manufacturer was used to reduce signal noise. Figure 6.14 shows the test setup used. 


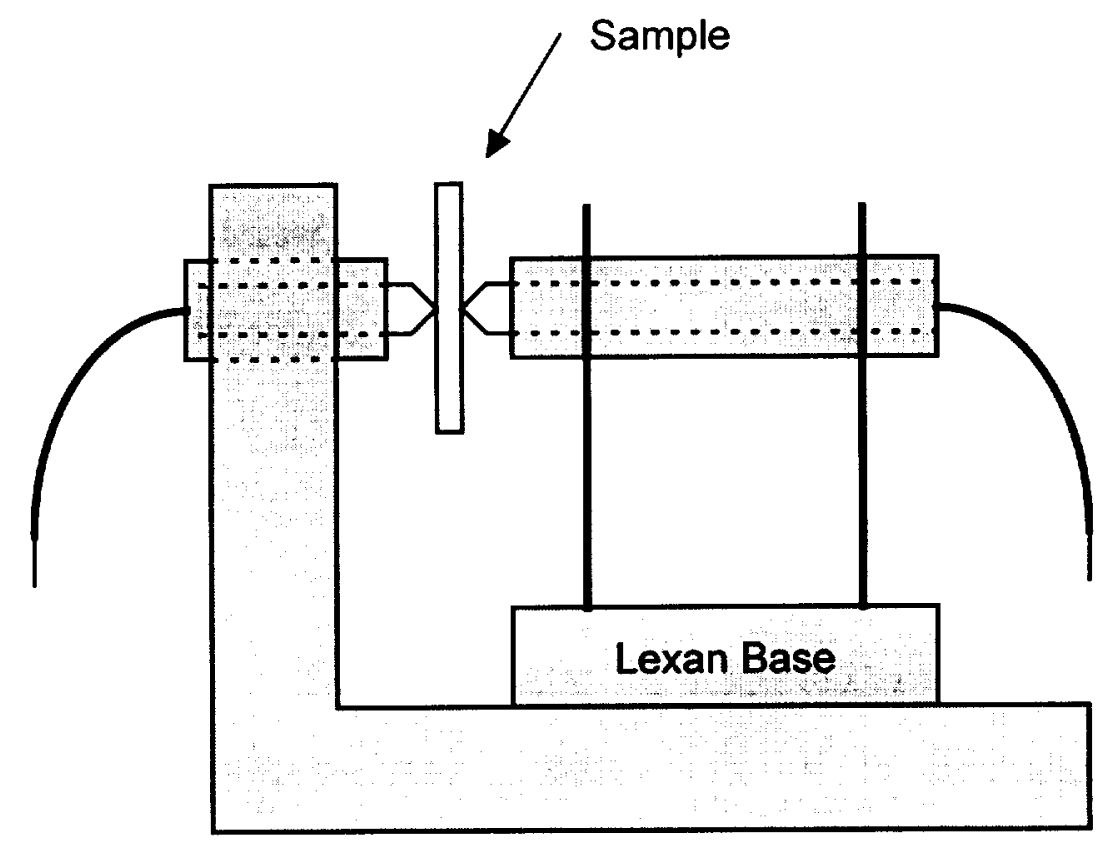

Figure 6.13: Schematic of the conductivity sample holder 


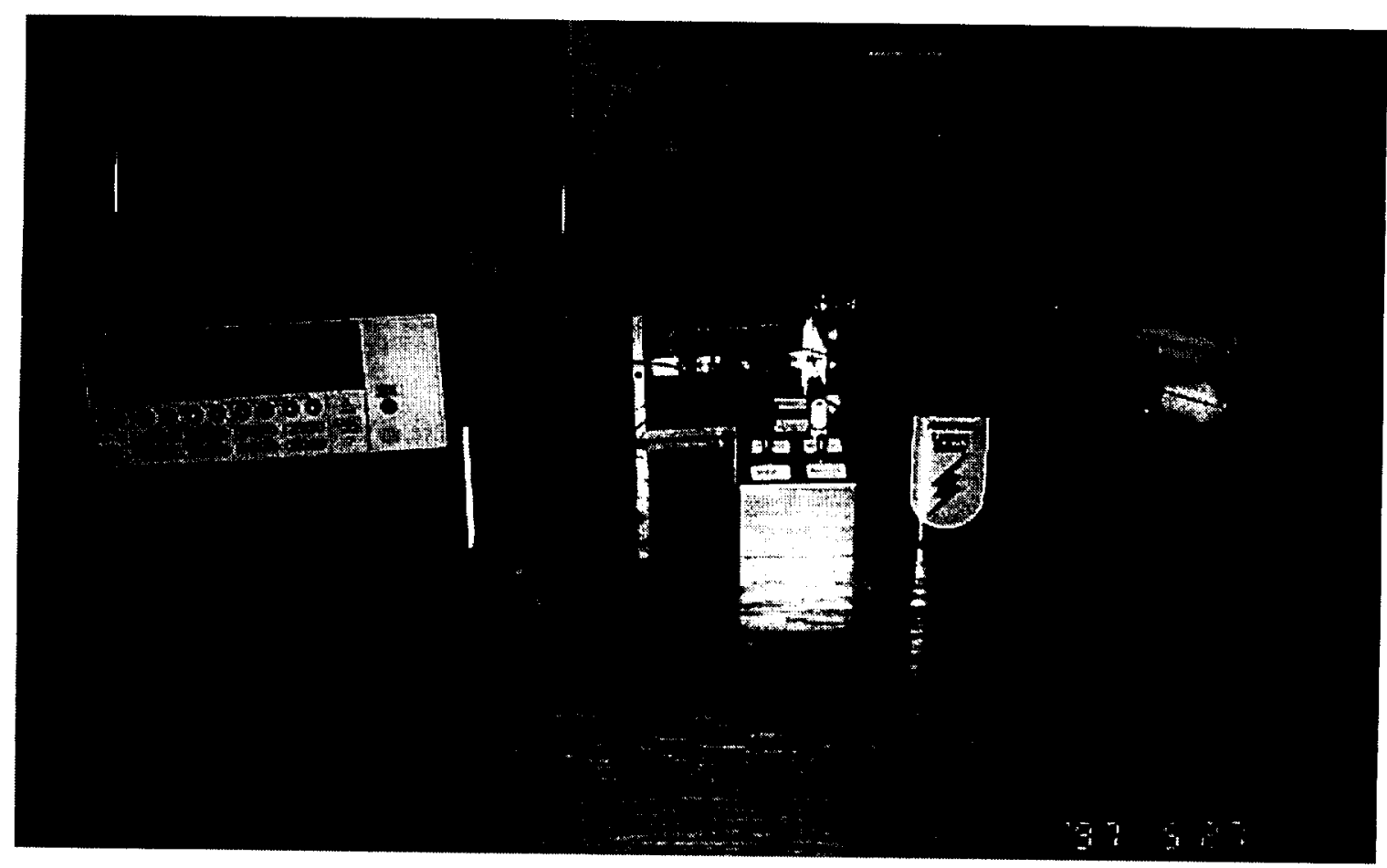

Figure 6.14: Experimental setup used for the conductivity measurements 
Note the batteries and switch-box assembly are used for the glass fiber / epoxy measurements only.

\subsubsection{Glass Fiber / Epoxy Measurement}

The glass fiber / epoxy samples required a different procedure because of their high resistance. When direct measurements were tried with these samples, the conductive coatings on the surfaces made the sample act like a capacitor - the resistance reading kept increasing as the current able to flow decreased. To get the resistance of the sample, the sample was assumed to be modelable as a resistor and capacitor in parallel, and the RC circuit discharge constant was measured (refer to section 4.6.1). By using two 6 volt batteries in series, 12 volts was applied across the sample. The voltage source was removed, and 500 voltage and time data points were recorded by the Keithley 6517 electrometer. The experimental setup can be seen in Figure 6.14. Once the data was collected a curve was fit to the data, thus giving the time constant from which the conductivity was calculated using equations 83 and 81 .

\subsection{DIELECTRIC CONSTANT MEASUREMENT}

The dielectric constant was calculated by measuring the capacitance of the sample. The conductivity samples were used since they were already coated with conductive epoxy and they were not damaged by the conductivity measurements. The samples were placed in the above mentioned Lexan sample holder, and the capacitance was measured using a Continental Specialties Corporation model 3001 Capacitance 
Meter borrowed from AMSL. Figure 6.15 shows the experimental setup used. The dielectric constant was calculated using equation 85 .

\subsection{DIELECTRIC STRENGTH MEASUREMENT}

ASTM D149-94 Standards summarizes the issues related to measurement of dielectric strength of insulating materials. ${ }^{62}$ The testing procedure described here was developed by Aaron Bent of AMSL. ${ }^{63}$ The testing was performed using a Philips model PM5138 Function Generator, which can output a DC source voltage from 0 to 1.0 in 0.01 increments. The two voltage amplifiers used were a TREK model 663A H.V. power supply and model $662 \pm 10,000$ volt amplifier and a Kepco Bipolar Operational Power Supply/Amplifier model BOP 1000M, which supplies 1000 volts. This test setup was also borrowed from AMSL. When using the Kepco amplifier the voltage increment was 10 volts, while when using the TREK amplifier the voltage increment was 100 volts. Figure 6.16 shows the experimental setup used. The samples were placed in a silicone oil test fixture, as shown in Figure 6.17. Silicone oil surrounded the specimen to prevent flashover and partial discharges, as per ASTM D149-94. The oil was kept at room temperature. The sample was held between two 0.25 " diameter hemispherical electrodes (electrode type \#5 in ASTM D149-94), which were connected to the high voltage amplifier. Hemispherical electrodes make contact with a discrete point of the sample, in contrast to electroded samples which would result in measurement of the "weakest link" dielectric strength of the entire sample. To prevent moisture absorption, the samples were stored in an air-tight jar with desiccant before testing. 


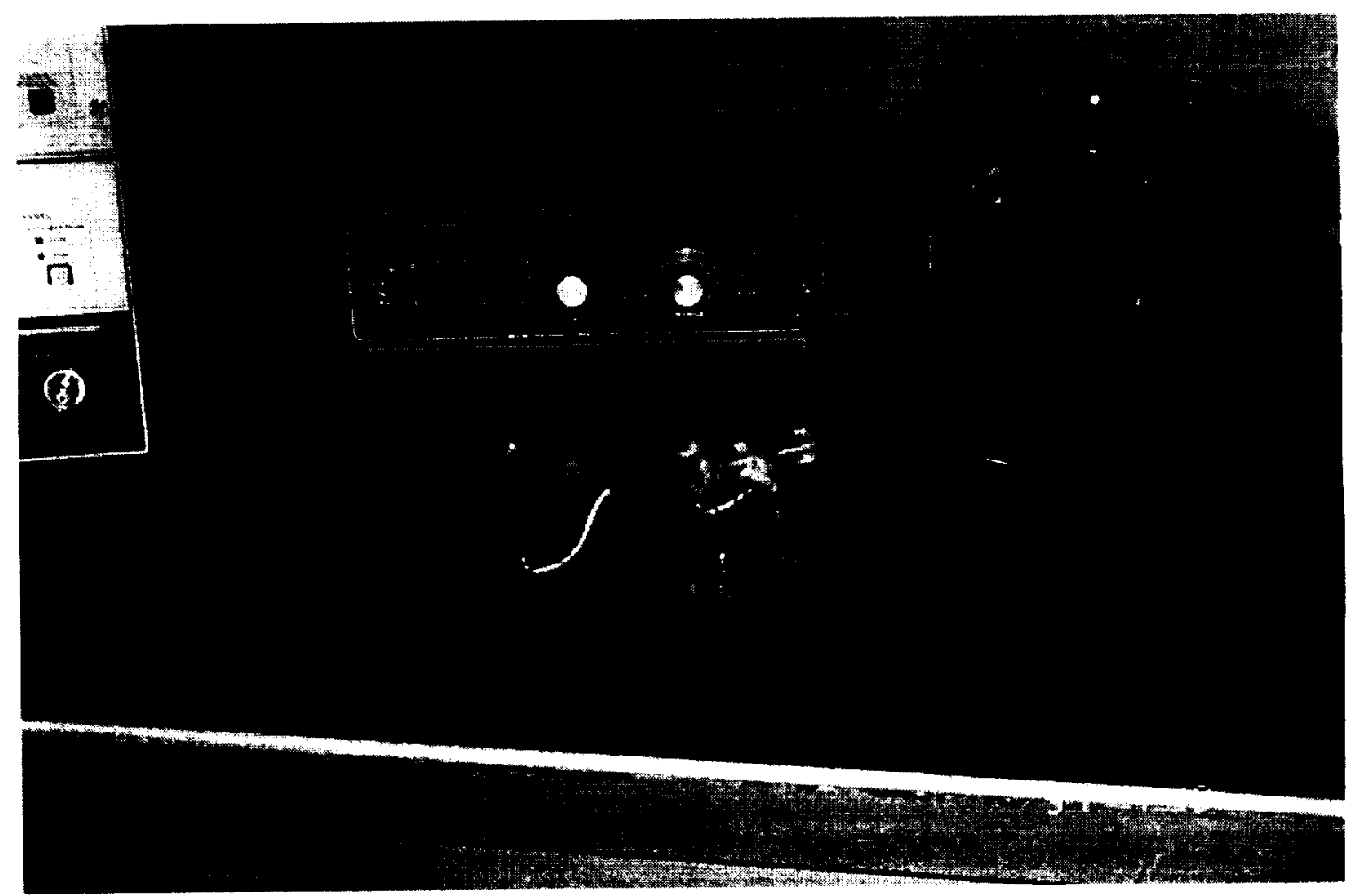

Figure 6.15: Experimental setup used to measure the dielectric constant 


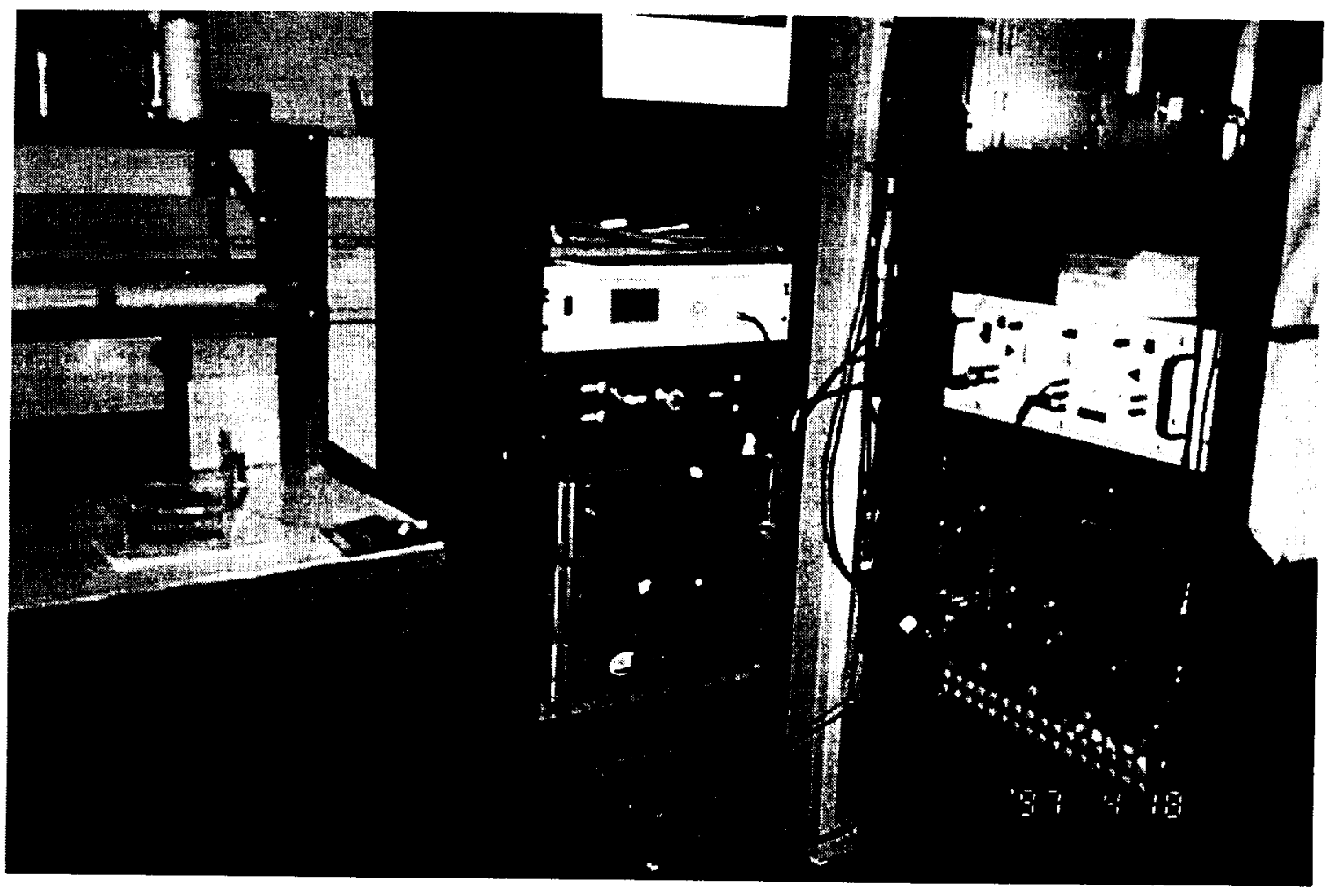

Figure 6.16: Experimental setup used to measure the dielectric strength 


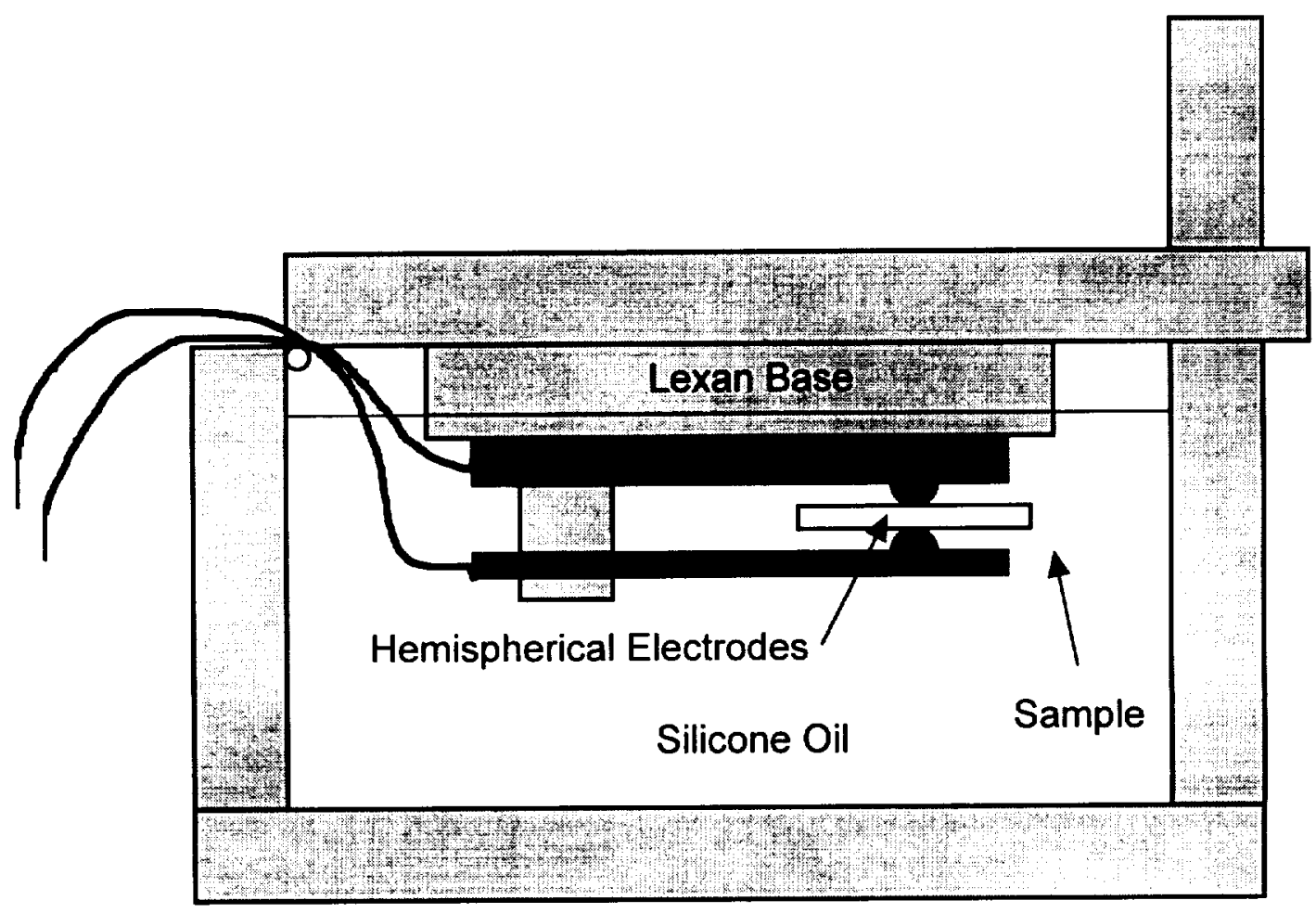

Figure 6.17: Schematic of the dielectric strength sample holder 
The Method B, Step-by-Step testing method of ASTM D149-94 was used in the application of voltage. A DC voltage was applied to the specimen, in single increments of the voltage generator. At each time step, the voltage was held for 5 seconds soak time, and then immediately dialed to the next voltage. The starting voltage for each sample was approximately $50 \%$ of the breakdown voltage. For a test to be valid at least 5 increments need to be made before breakdown. The recorded breakdown voltage is the highest level reached where the sample survived for the entire 5 second duration. Breakdown occurs when the voltage is sufficiently high to allow a current path through the sample material. The test was first done using the Kepco amplifier. If breakdown did not occur by the 1000 volt maximum of the amplifier, the TREK amplifier was then used. The dielectric strength was calculated using equation 86 .

\subsection{DENSITY MEASUREMENT}

The density measurement was performed on the conductivity samples before the conductive epoxy surface layer was applied, since the area and thickness of the samples was already measured. The sample mass was then measured using an OHaus model TS4K0 digital balance, and the density was calculated using equation 88 .

\subsection{TENSILE TESTS}

The tensile test was performed to determine the Young's modulus, Poisson ratio, failure stress, and failure stain. The testing apparatus used was a 110,000 lbs MTS model 311.21 testing machine with an Instron model 8500 Plus Controller, and a Macintosh IIx for data acquisition. Figure 6.18 shows the experimental setup used. The tests were 


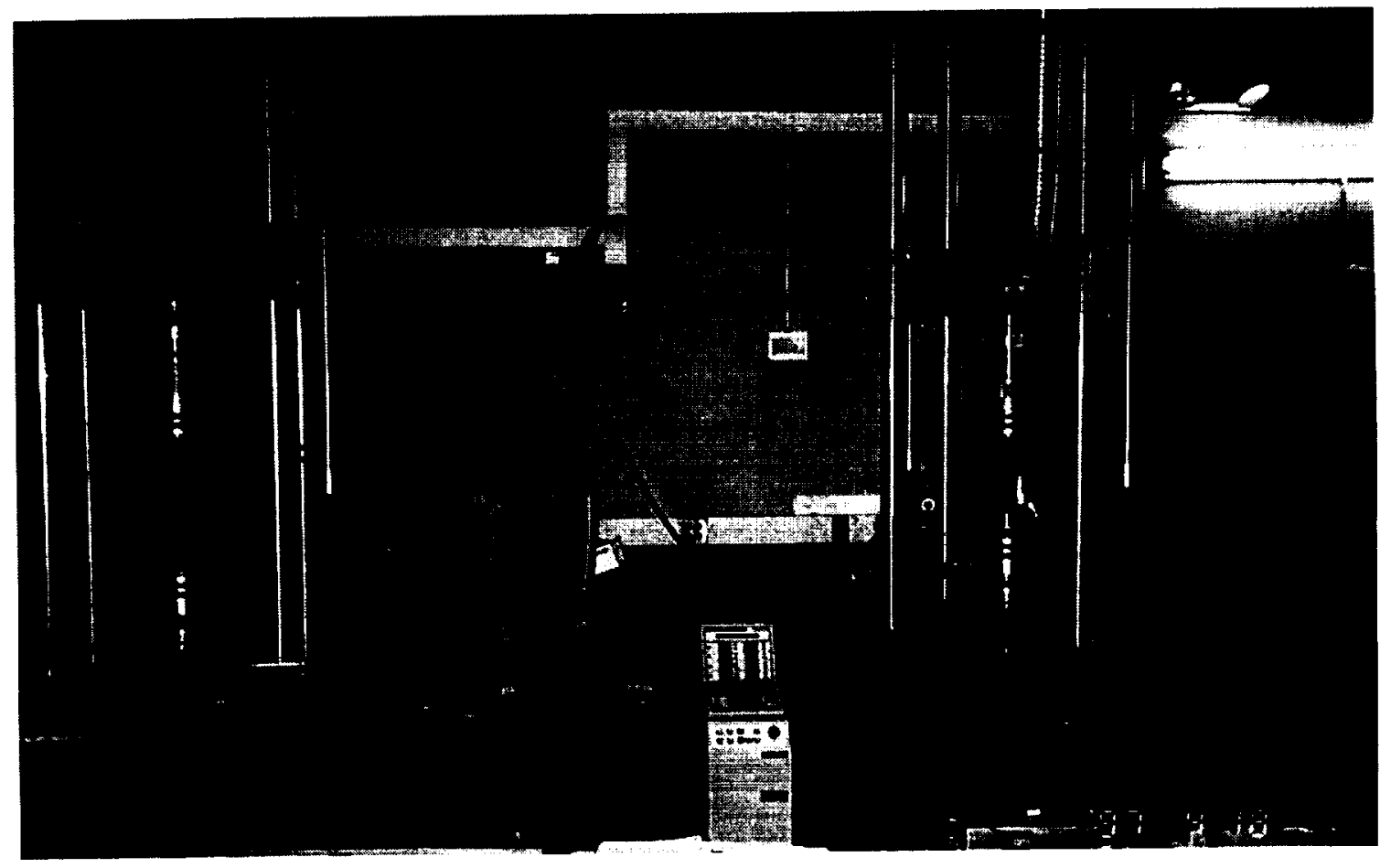

Figure 6.18: Experimental setup used to perform tensile tests 
performed using stroke control with a rate of $0.02 \mathrm{in} / \mathrm{sec}$, and a gripping pressure of 500 psi. The load range used was $\pm 10,000 \mathrm{lbs}$, and the stroke range used was \pm 1 ". The data acquisition was done using the "TELAC Data Acquisition" Labview program using a sampling frequency of $2 \mathrm{~Hz}$. The procedure followed is documented in the "TELAC Manufacturing Course Class Notes". ${ }^{59}$ All the tensile test data can be found in Appendix C.

Prior to testing, the samples were prepared by bonding tapered glass loading tabs to each end of the samples on both sides. The tabs were bonded using Epoxi-Patch 0151 Clear, a 2 part epoxy which cures at room temperature in 48 hours with steel weights placed on the tabs to hold them in place. Following the tab application, 2 Measurements Group EA-06-125AD-120 strain gages were applied to the tensile specimen using a MBond 200 strain gage adhesive kit. More detailed directions are given in the "TELAC Manufacturing Course Class Notes". ${ }^{59}$ The gages were placed near the center of the sample, one in the longitudinal direction and the other in the transverse direction, as shown in Figure 6.19.

\subsubsection{Young's Modulus Measurement}

The Young's modulus was calculated by plotting the applied stress versus the longitudinal stain and graphically measuring the slope of the linear portion of the curve, as shown in Figure 6.20. The Young's modulus was calculated using equation 89. 


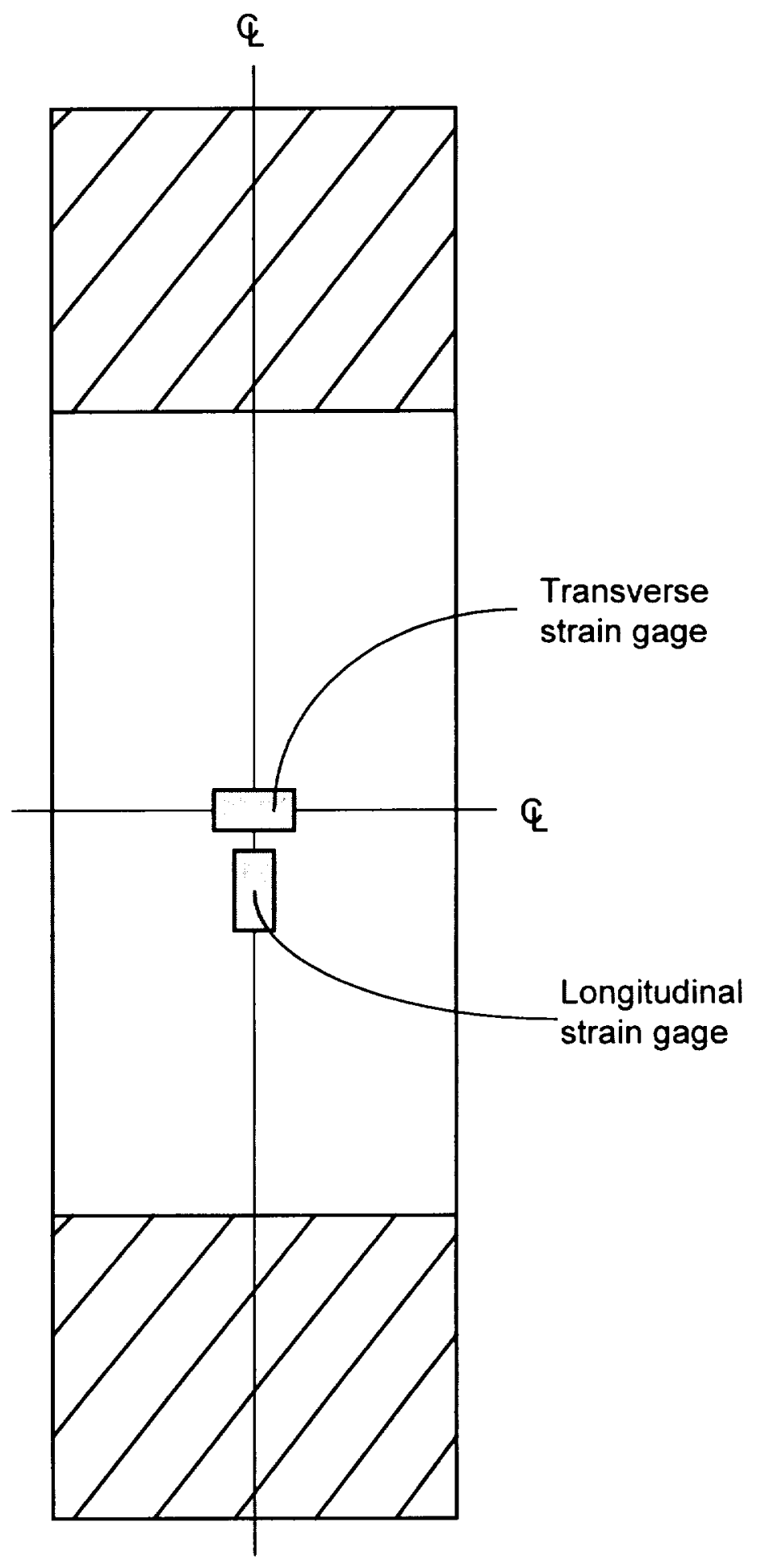

Figure 6.19: Illustration of the location of the strain gages on the tensile test sample 


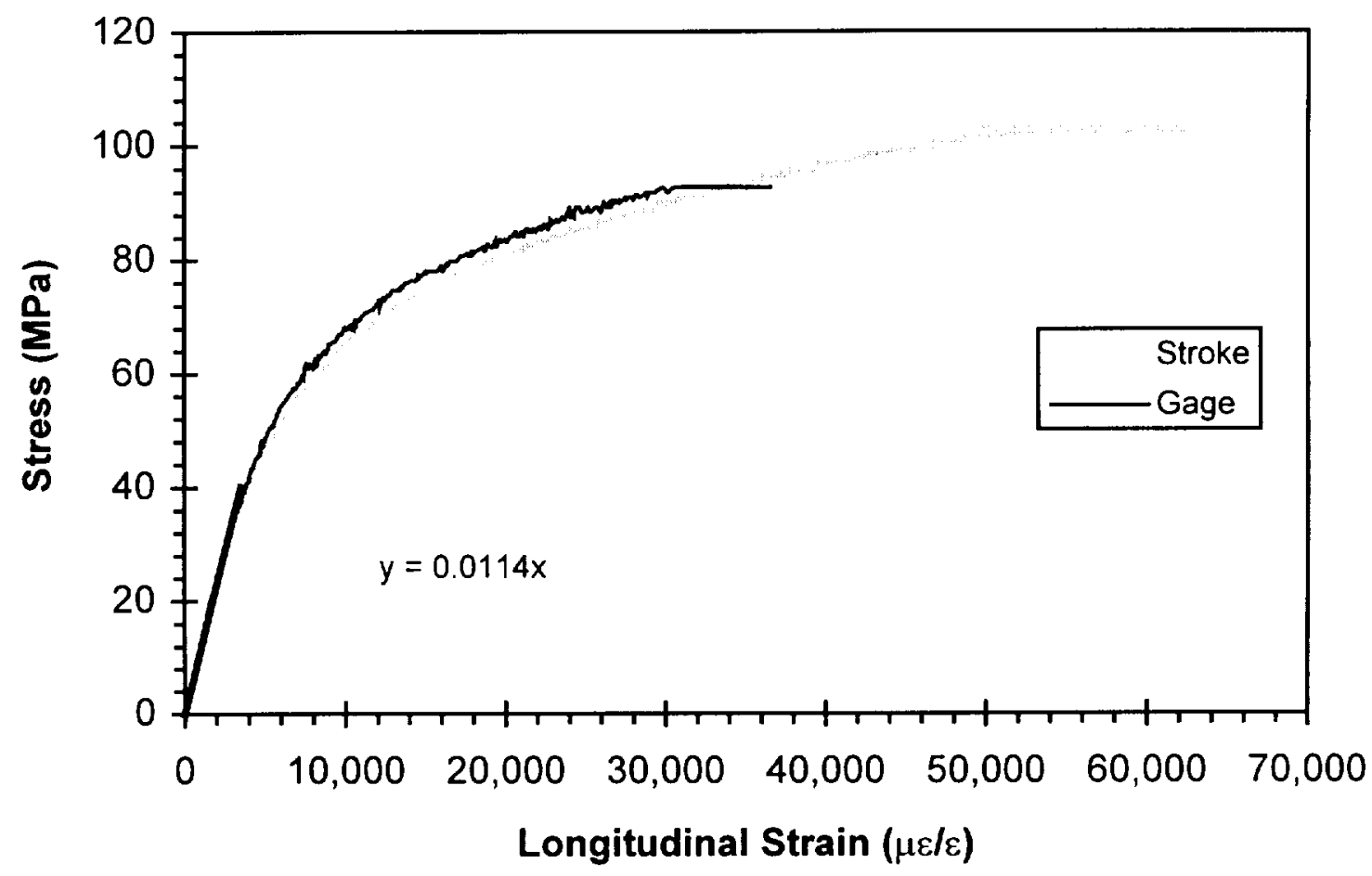

Figure 6.20: Typical stress strain curve 


\subsubsection{Poisson Ratio Measurement}

The Poisson ratio was calculated by plotting the transverse strain versus the longitudinal strain and graphically measuring the slope of the linear portion of the curve, as shown in Figure 6.21. The Poisson ratio was calculated using equation 90.

\subsubsection{Failure Stress Measurement}

The failure stress was calculated from the maximum load applied to the sample. The load can be converted into stress using equation 91 .

\subsubsection{Failure Strain Measurement}

The failure strain is the strain in the material at the maximum load. Unfortunately, by the time the sample failed the strain gages had long since stopped functioning. Therefore, the strain was calculated from the stroke using equation 92 . This value can typically be inaccurate, as using stroke as a measurement of strain is a gross approximation. However, in some cases, as shown in Figure 6.20, there can be quite good agreement between the strain gage strain and the stroke strain.

\subsection{MICROSCOPY}

Microscopy was performed on the carbon fiber/ epoxy samples and the glass fiber / epoxy samples to measure the thickness of the pure epoxy surface layer and to look for percolation paths. The carbon black particles were not visible under either the optical microscope or the scanning electron microscope. In preparation for the microscopy the samples were potted in Buehler EPO-Kwick two part epoxy, which cures at room temperature in 24 hours. Once the epoxy had cured, the samples were polished using 


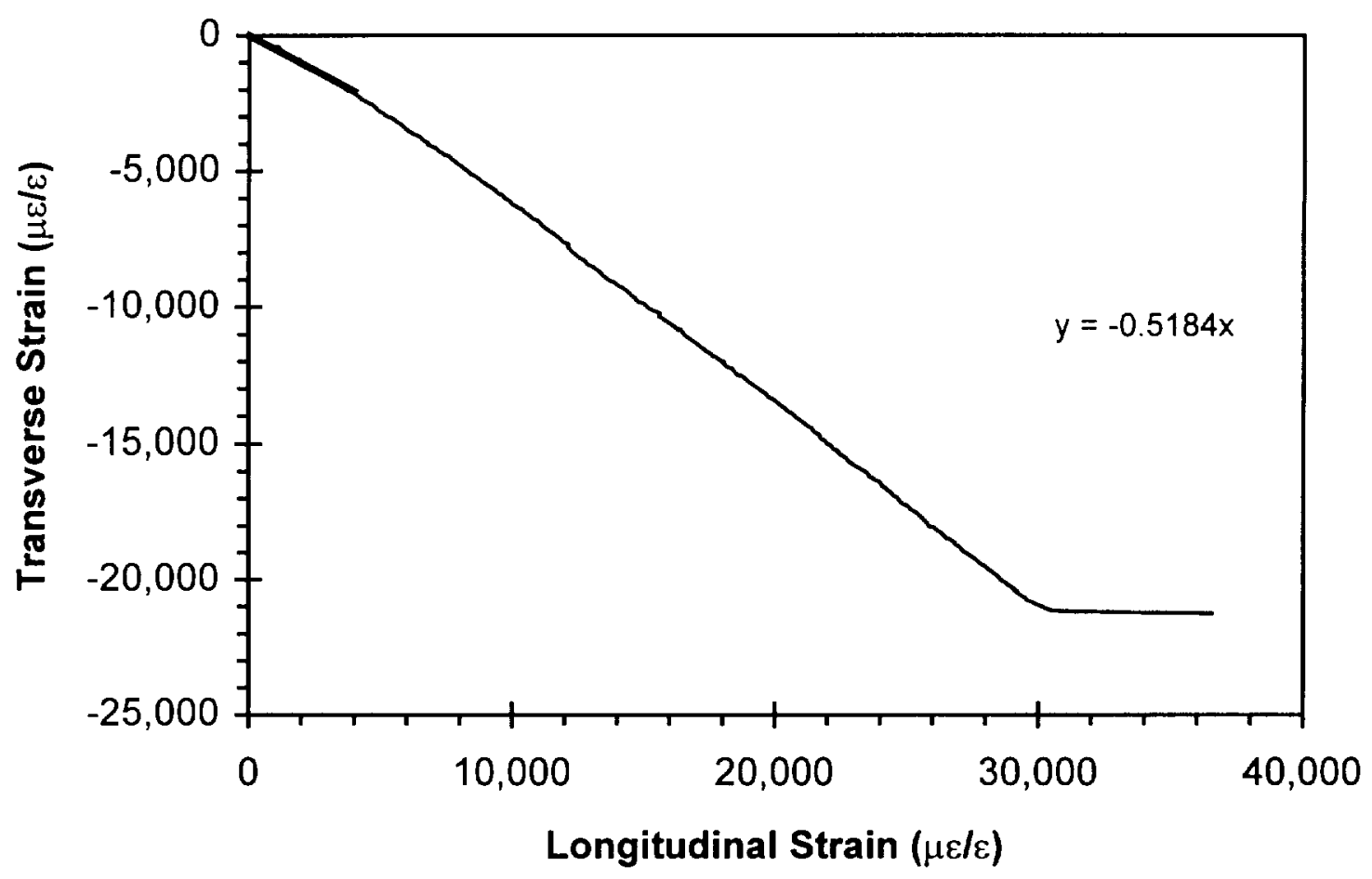

Figure 6.21: Typical transverse versus longitudinal strain curve 
diamond grit polish on a Buehler Ecomet IV polisher with an Euromet I power head. The samples were examined using an Olympus model BH-2 optical microscope with a maximum magnification of 750 times. Micro-graphs were taken of the samples, from which the surface layer thickness was measured several times and averaged. 


\section{CHAPTER 7}

\section{EXPERIMENTAL RESULTS AND DISCUSSION}

This section presents the results from the experimental portion of this research. The through-thickness electrical properties of carbon fiber / epoxy are discussed first. Then the development of a conductivity-tailorable glass fiber / epoxy composite is discussed, and the change in the electrical and mechanical properties due to adding conductive carbon black to the composite are presented. Some typical results will be presented here, while all the results can be found in Appendix C.

\subsection{CARBON FIBER / EPOXY ELECTRICAL PROPERTIES}

The data is shown in Figures 7.1 through 7.3, and Tables 7.1 through 7.3. The same formatting is used on all three figures. The sample electrical resistances are plotted versus sample area, and the sample breakdown voltages are plotted versus sample thickness. The mean of each group of data, (with each thickness and layup), is shown. The error bars represent one standard deviation. The unidirectional samples are displayed with open symbols, and the quasi-isotropic samples are displayed with filled-in symbols. The thickness of the samples is represented by increasing size of the symbol; a triangle for the 4 ply samples, a circle for the 8 ply samples, and a square for the 32 ply samples. 


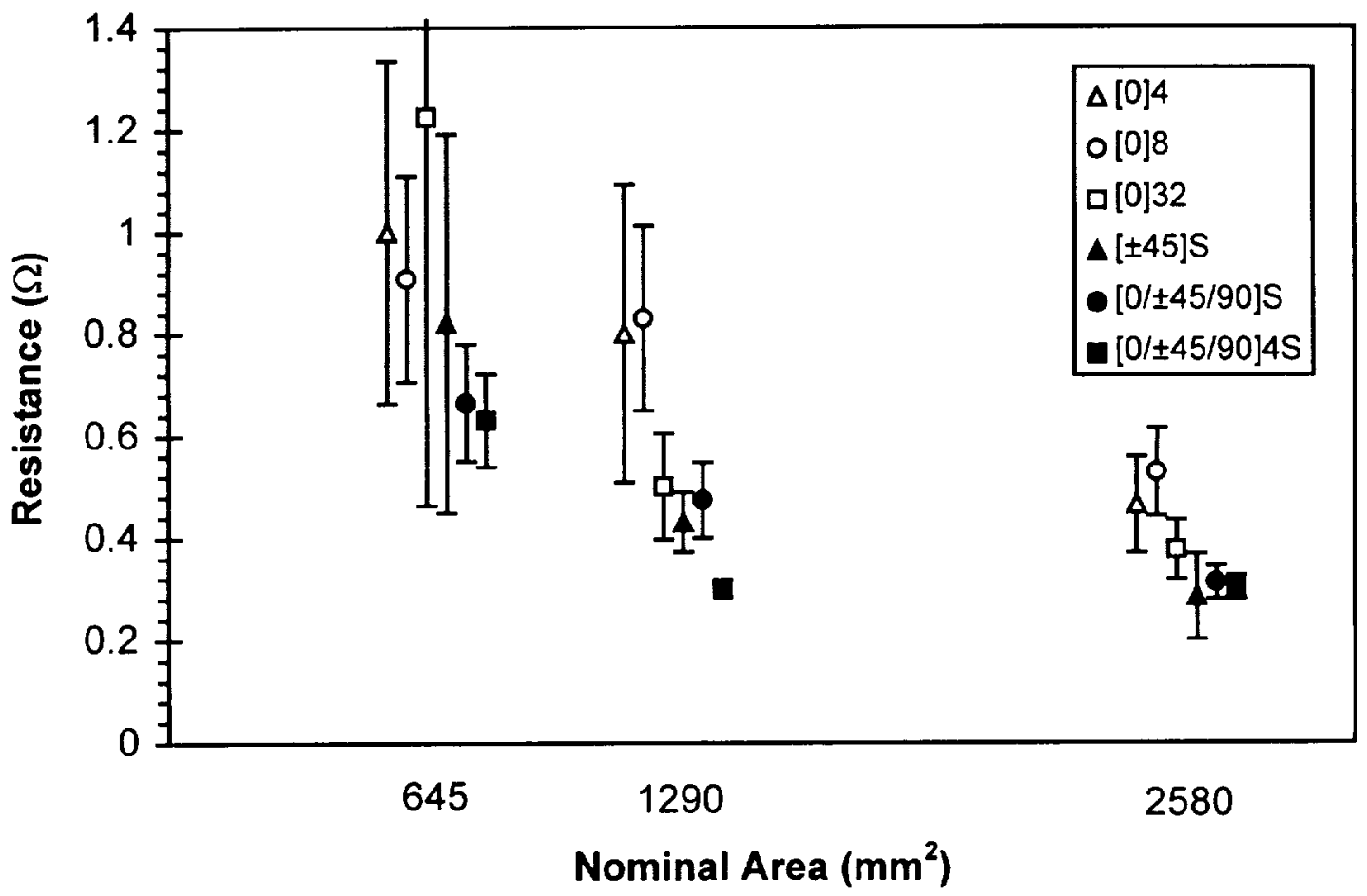

Figure 7.1: Unsanded carbon fiber / epoxy resistance data 


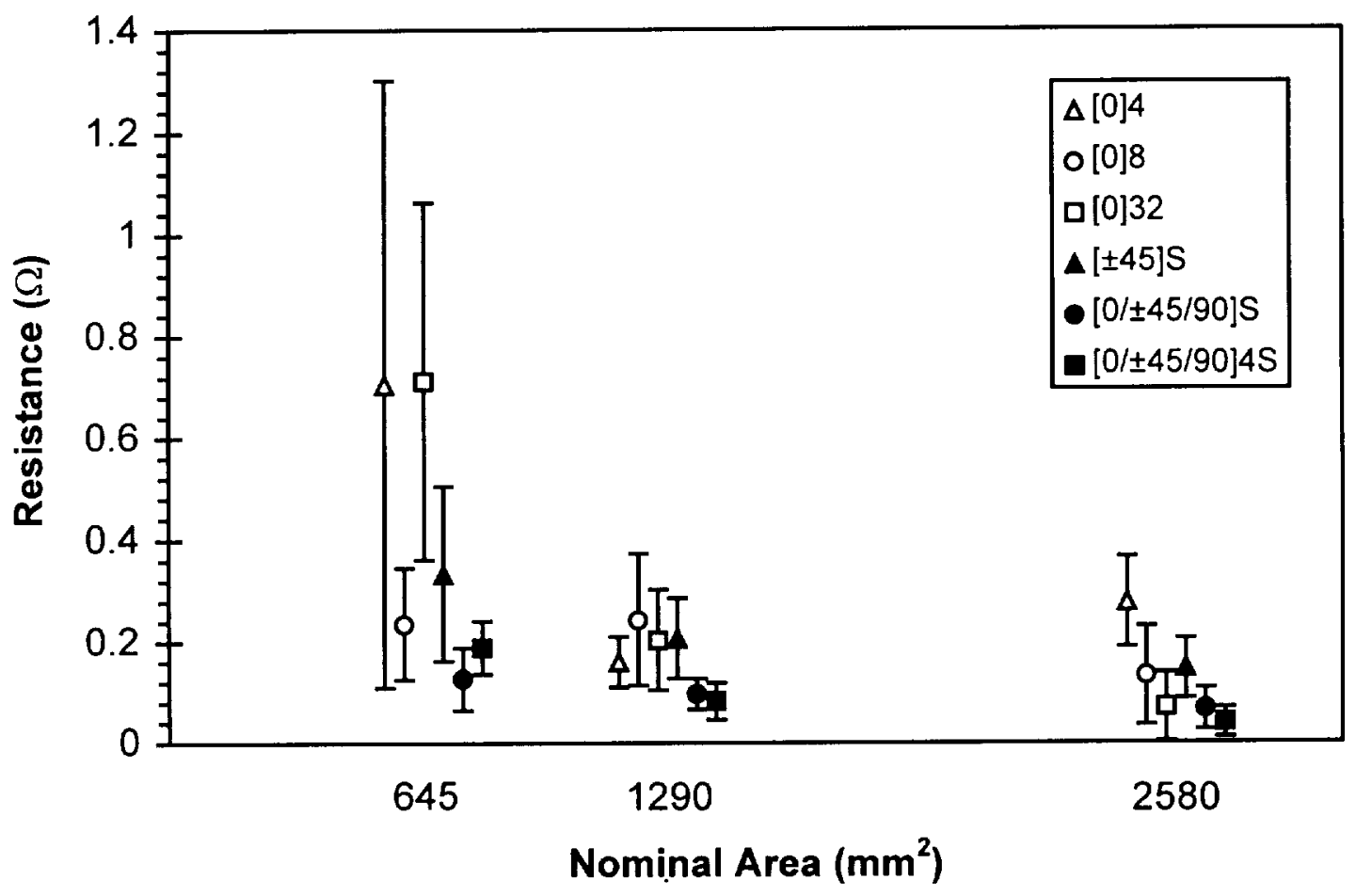

Figure 7.2: $\quad$ Sanded carbon fiber / epoxy resistance data 


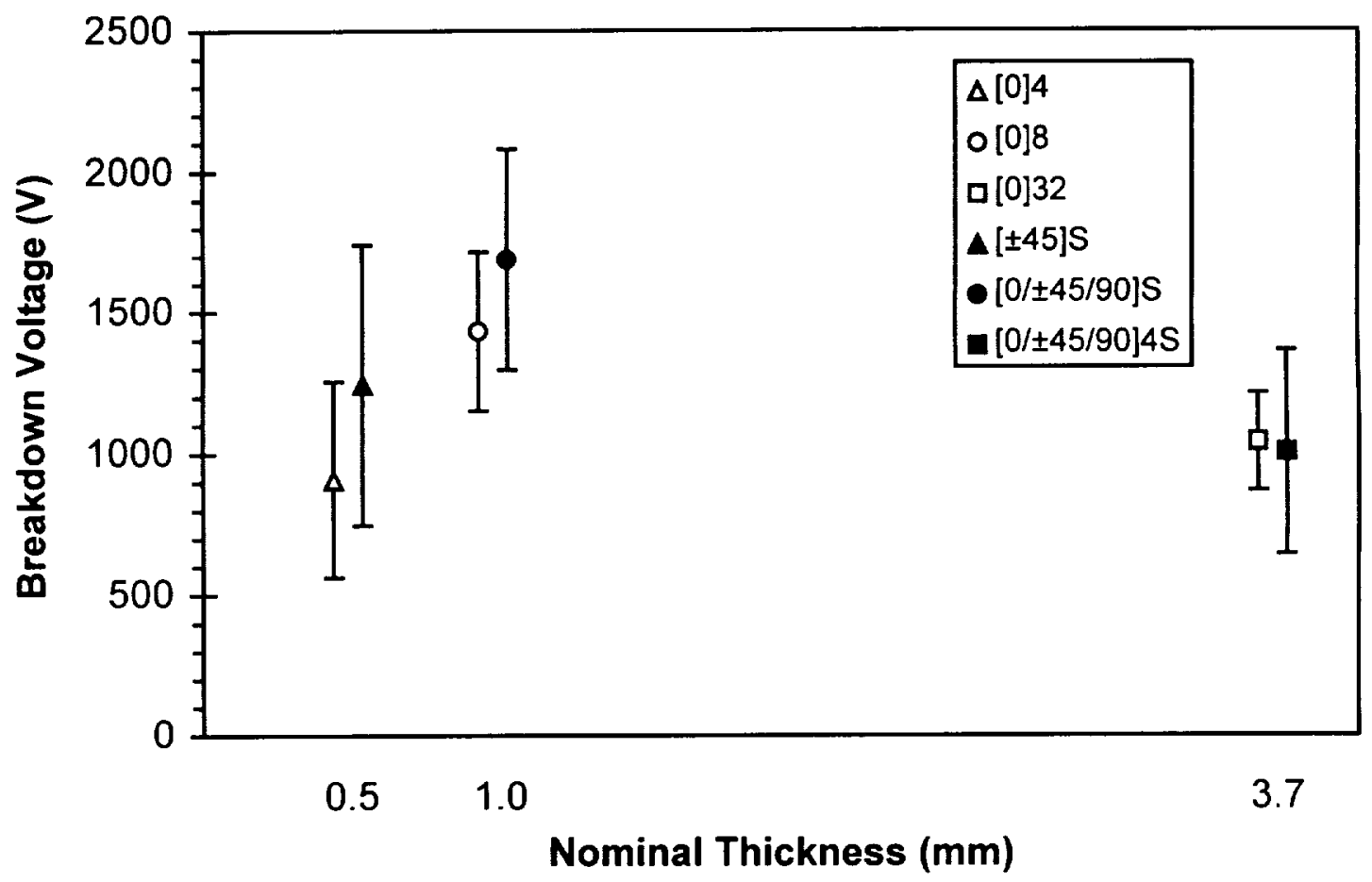

Figure 7.3: Unsanded carbon fiber / epoxy breakdown voltage data 
Table 7.1: Carbon fiber/ epoxy resistance and conductivity thickness comparison

\begin{tabular}{||l|c|c|c|c|c||}
\hline \hline Test Case & Thickness & \multicolumn{2}{|c|}{ Resistance $(\Omega)$} & \multicolumn{2}{c||}{ Conductivity $(\mathbf{1} / \Omega$-m) } \\
& $(\mathbf{m m})$ & Average & S.D. & Average & S.D. \\
\hline \hline \multirow{3}{*}{ Unsanded } & 0.52 & 0.634 & 0.279 & 0.817 & 0.273 \\
\cline { 2 - 6 } & 0.99 & 0.620 & 0.226 & 1.476 & 0.605 \\
\cline { 2 - 6 } & 3.75 & 0.556 & 0.351 & 6.791 & 2.485 \\
\hline \hline \multirow{3}{*}{ Sanded } & 0.38 & 0.305 & 0.209 & 1.587 & 0.705 \\
\cline { 2 - 6 } & 0.84 & 0.150 & 0.073 & 7.071 & 3.722 \\
\cline { 2 - 6 } & 3.63 & 0.216 & 0.252 & 33.727 & 17.345 \\
\hline \hline \multirow{3}{*}{ Lurface } & 0.52 & 0.305 & 0.209 & 0.015 & 0.005 \\
\cline { 2 - 6 } & 0.99 & 0.150 & 0.073 & 0.014 & 0.006 \\
\cline { 2 - 6 } & 3.75 & 0.216 & 0.252 & 0.017 & 0.007 \\
\hline \hline
\end{tabular}

S.D.: Standard deviation 
Table 7.2: Carbon fiber / epoxy resistance and conductivity area comparison

\begin{tabular}{||l|c|c|c|c|c||}
\hline \hline Test Case & Area & \multicolumn{2}{|c|}{ Resistance $(\Omega)$} & \multicolumn{2}{c||}{ Conductivity (1/ $\Omega$-m) } \\
& $\left(\mathbf{m m}^{\mathbf{2}}\right)$ & Average & S.D. & Average & S.D. \\
\hline \hline \multirow{3}{*}{ Unsanded } & 634 & 0.875 & 0.221 & 3.723 & 3.514 \\
\cline { 2 - 6 } & 1269 & 0.557 & 0.213 & 3.372 & 3.795 \\
\cline { 2 - 6 } & 2558 & 0.378 & 0.099 & 1.990 & 1.897 \\
\hline \hline \multirow{3}{*}{ Sanded } & 634 & 0.384 & 0.261 & 11.449 & 12.472 \\
\cline { 2 - 6 } & 1269 & 0.165 & 0.065 & 13.079 & 17.580 \\
\cline { 2 - 6 } & 2558 & 0.122 & 0.087 & 17.848 & 23.104 \\
\hline \hline \multirow{2}{*}{\begin{tabular}{l} 
Lurface \\
\cline { 2 - 6 }
\end{tabular}} & 634 & 0.875 & 0.221 & 0.020 & 0.005 \\
\cline { 2 - 6 } & 1269 & 0.557 & 0.213 & 0.016 & 0.006 \\
\cline { 2 - 6 } & 2558 & 0.378 & 0.099 & 0.011 & 0.003 \\
\hline \hline
\end{tabular}

S.D.: Standard deviation

Table 7.3: Carbon fiber / epoxy resistance and conductivity layup comparison

\begin{tabular}{|c|c|c|c|c|c|}
\hline \multirow[t]{2}{*}{ Test Case } & \multirow[t]{2}{*}{ Layup } & \multicolumn{2}{|c|}{ Resistance $(\Omega)$} & \multicolumn{2}{|c|}{ Conductivity $(1 / \Omega-m)$} \\
\hline & & Average & S.D. & Average & S.D. \\
\hline \multirow[t]{2}{*}{ Unsanded } & Unidirectional & 0.737 & 0.286 & 2.417 & 2.392 \\
\hline & Quasi-isotropic & 0.469 & 0.195 & 3.639 & 3.697 \\
\hline \multirow[t]{2}{*}{ Sanded } & Unidirectional & 0.304 & 0.238 & 10.611 & 16.319 \\
\hline & Quasi-isotropic & 0.143 & 0.090 & 17.639 & 18.614 \\
\hline \multirow{2}{*}{$\begin{array}{l}\text { Surface } \\
\text { Layer }\end{array}$} & Unidirectional & 0.737 & 0.286 & 0.012 & 0.004 \\
\hline & Quasi-isotropic & 0.469 & 0.195 & 0.019 & 0.005 \\
\hline
\end{tabular}

S.D.: Standard deviation 
For clarity, the horizontal placement of the data has been shifted slightly so the $\mathrm{x}$-axis variation within each area or thickness group is not meaningful. The data in the tables is the average over all samples with the specified parameter (thickness, area, and stacking sequence or layup), and S.D. represents the standard deviation of all these samples.

\subsubsection{Through-Thickness Conductivity}

The raw data shows high scatter. As shown in Figure 7.1, there are no observable thickness trends. The resistance decreases with increasing area, but it is unclear if there is the expected (inverse) area dependency. Also, there seems to be a stacking sequence trend; the resistance of the quasi-isotropic samples are lower than that of the unidirectional samples. These resistance values are all low, and all close to the noise floor of the electrometer of approximately $0.1 \Omega$.

Figure 7.2 shows the resistance of the samples after the epoxy rich surface layer has been removed. Comparison of Figures 7.1 and 7.2 shows that the resistance of the samples decreased after sanding, as would be expected since the bulk composite conductivity is much greater than the pure epoxy conductivity (refer to Table 2.1). There is still a fair amount of scatter in the data. The sanded resistances are for the most part in or near the noise floor of the electrometer. The data still seems to have no apparent thickness trend. There seems to be the expected trend of decreasing resistance with increasing area. Also, the stacking sequence trend observed in the unsanded samples is less clear. 
When the resistance data is interpreted as conductivity, there are non-classical thickness and surface area dependencies. If conductivity is a true material property, all data from the various samples with different thickness, areas, and stacking sequences would reduce (using equation 81) to the same value of conductivity. Instead, there seems to be some decrease in conductivity with area, and a distinct increase in conductivity with thickness. The range of values of conductivity fit within the range, 0.1 to $1061 / \Omega-m$, of through-thickness conductivities for carbon fiber / epoxy composites previously reported (refer to Table 2.1). When the resistance of the sanded samples is interpreted as conductivity, there seems to be no area dependency, but there is still a non-classical thickness dependency. Most of the sanded conductivity values are close to the upper previously reported value of $1061 / \Omega-\mathrm{m}$.

As noted in section 4.6.1, it is possible that the resistance of the sample is dominated by low resistance surface layers. The apparent thickness of these were measured, and the unsanded resistance data was interpreted as the conductivity of the surface layer. The apparent epoxy surface layer thickness was measured using microscopy on samples from the same laminate as the conductivity samples. The thickness of the surface layer was measured 9 times on each surface and averaged. For the unidirectional laminates it was approximately $9.0 \mu \mathrm{m}$, and for the quasi-isotropic laminates it was approximately $10.0 \mu \mathrm{m}$. Results are shown in Tables 7.1 through 7.3. A reasonably consistent set of conductivities results. There is a slight non-classical area dependency, but no thickness dependency. The values of the conductivity computed in 
this way are above the range of reasonable values for the conductivity of epoxy by approximately one order of magnitude, indicating that the surface layer is not a uniform insulator.

It can be seen that the measurement of the through-thickness conductivity of carbon fiber / epoxy composites is difficult to achieve accurately. The measured values of the conductivity are within the range of previously reported values. However, there are non-classical area and thickness dependencies, and a high degree of scatter. The through-thickness conductivity appears to be influenced by both the layup and the presence of an epoxy surface layer. The conductivity does not appear to behave as a continuum property; it is more likely dominated by percolation effects, in which the properties of the lowest resistance percolation path are being measured, which will vary from sample to sample.

\subsubsection{Through-Thickness Dielectric Strength}

The through-thickness dielectric strength was measured using samples with no applied conductive surface layer. When the raw breakdown voltage was looked at there was no observable trend with thickness. The data seemed to be scattered around a value of approximately 1500 volts, as shown in Figure 7.3. It was postulated that the epoxy surface layer might again be dominating the data, so the surface layers were sanded off as done above with the conductivity samples. When the sanded samples were tested, no breakdown voltage could be measured as there was a current flow at the lowest possible voltage increment. 
When the breakdown voltage data is interpreted as a material property (dielectric strength), there is a non-classical thickness dependency. Instead of being a constant value with some scatter, there seems to be a decrease in dielectric strength with thickness. The range of data however is within the previously reported range for carbon fiber / epoxy composites. When the breakdown voltage data is interpreted as the dielectric strength of the epoxy surface layer (using equation 87), more consistent results are obtained. The dielectric strength of the surface layer measured in this way shows a fair bit of scatter around a constant value of approximately $150 \mathrm{MV} / \mathrm{m}$, which is within the range of previously reported values for organic polymers.

The through-thickness dielectric strength of carbon fiber / epoxy composites seems to be epoxy surface layer dominated. The measured breakdown voltages make sense if interpreted as the dielectric strength of the epoxy surface layer. As with the conductivity, it may not be meaningful to assign a continuum value to the dielectric strength of carbon fiber/ epoxy composites.

\subsubsection{Summary}

The through-thickness electrical properties, the conductivity and the dielectric strength, of carbon fiber / epoxy composites are dominated by the epoxy surface layer. This effect is observed in carbon fiber / epoxy composites because of the large difference in the conductivity of the bulk composite and the surface layer. Even when this layer is sanded off, there is still a high degree of scatter and non-classical thickness dependencies in the resistance data, making the determination of a bulk conductivity difficult. 


\subsection{CONDUCTIVITY TAILORABLE GLASS FIBER / EPOXY}

The results of tests on the conductivity-tailorable glass fiber / epoxy composites were as desired-the electrical properties were increased significantly while the mechanical properties displayed minimal change. Figures 7.4 through 7.10 represent the effects of adding conductive carbon black on the material properties of the composite. The actual values can be found in Table 7.4, where the listed property is the average of the six samples measured, and S.D. is the standard deviation of these six samples.

Some manufacturing difficulties were encountered during the cure process. The main reason for these was that the higher percentages of carbon black made the epoxy very viscous. This resulted in the epoxy not flowing well during the cure process, which resulted in thicker laminates, as shown in Figure 7.11. This increase in thickness was created when the extra epoxy on the top surface of the laminate did not flow out around the top cure plate during the curing process. The surface layer was measured using microscopy and the results can be seen in Figure 7.12, and Table 7.5. The total surface layer thickness of the carbon black filled laminates was greater than that of the pure glass fiber / epoxy laminate. In particular, the surface layer thickness for the $15 \%$ and $20 \%$ carbon black laminates increased significantly. Micrographs of the samples are shown in Figure 7.13. The black circles represent voids in the potting epoxy.

A side effect of this surface layer was to cause the laminates to bend when they cooled from the cure temperature to room temperature, as shown in Figure 7.14. The reason for this effect was that the surface layer caused the laminate to appear to be 


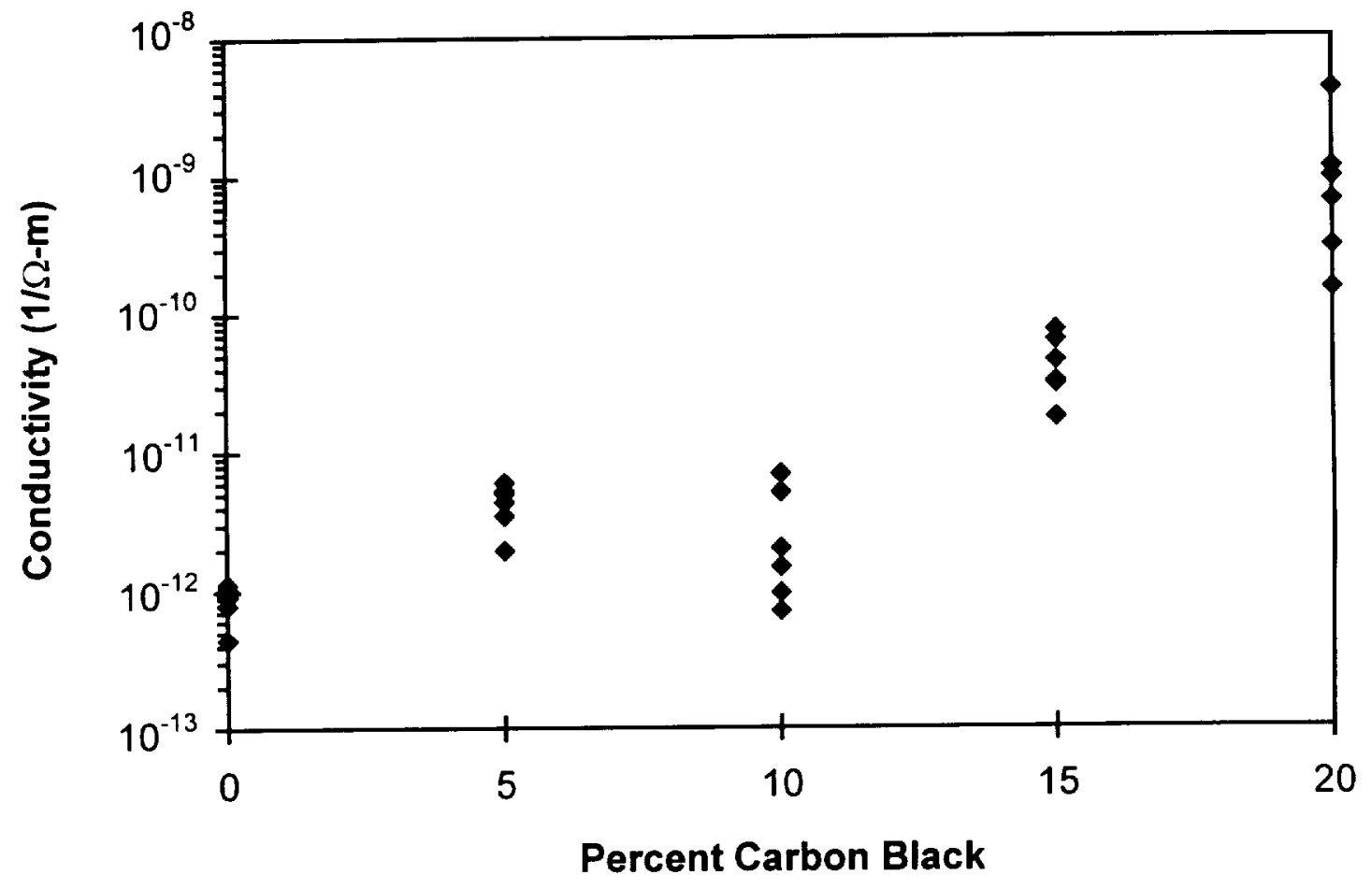

Figure 7.4: Glass fiber / epoxy conductivity 


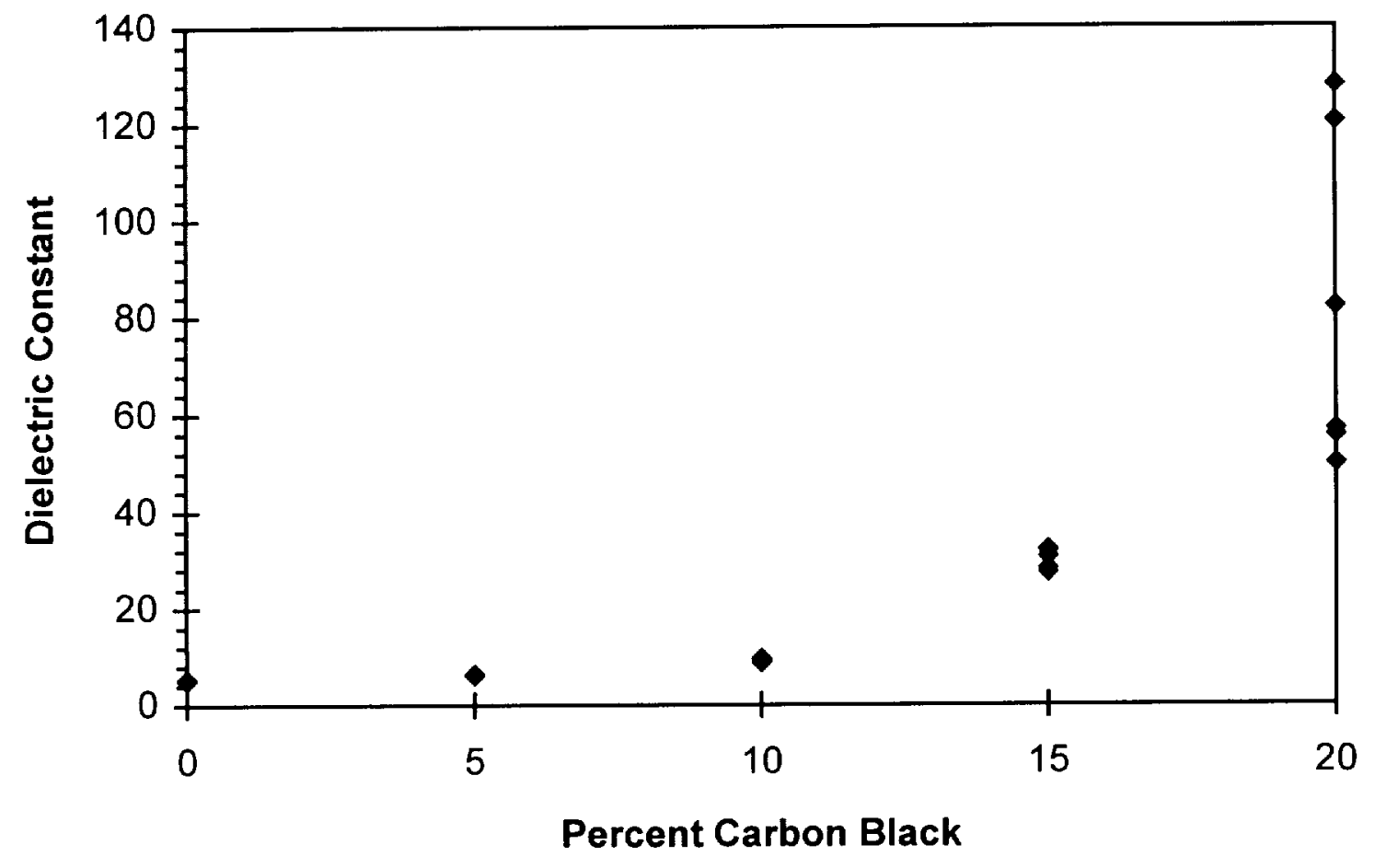

Figure 7.5: Glass fiber / epoxy dielectric constant 


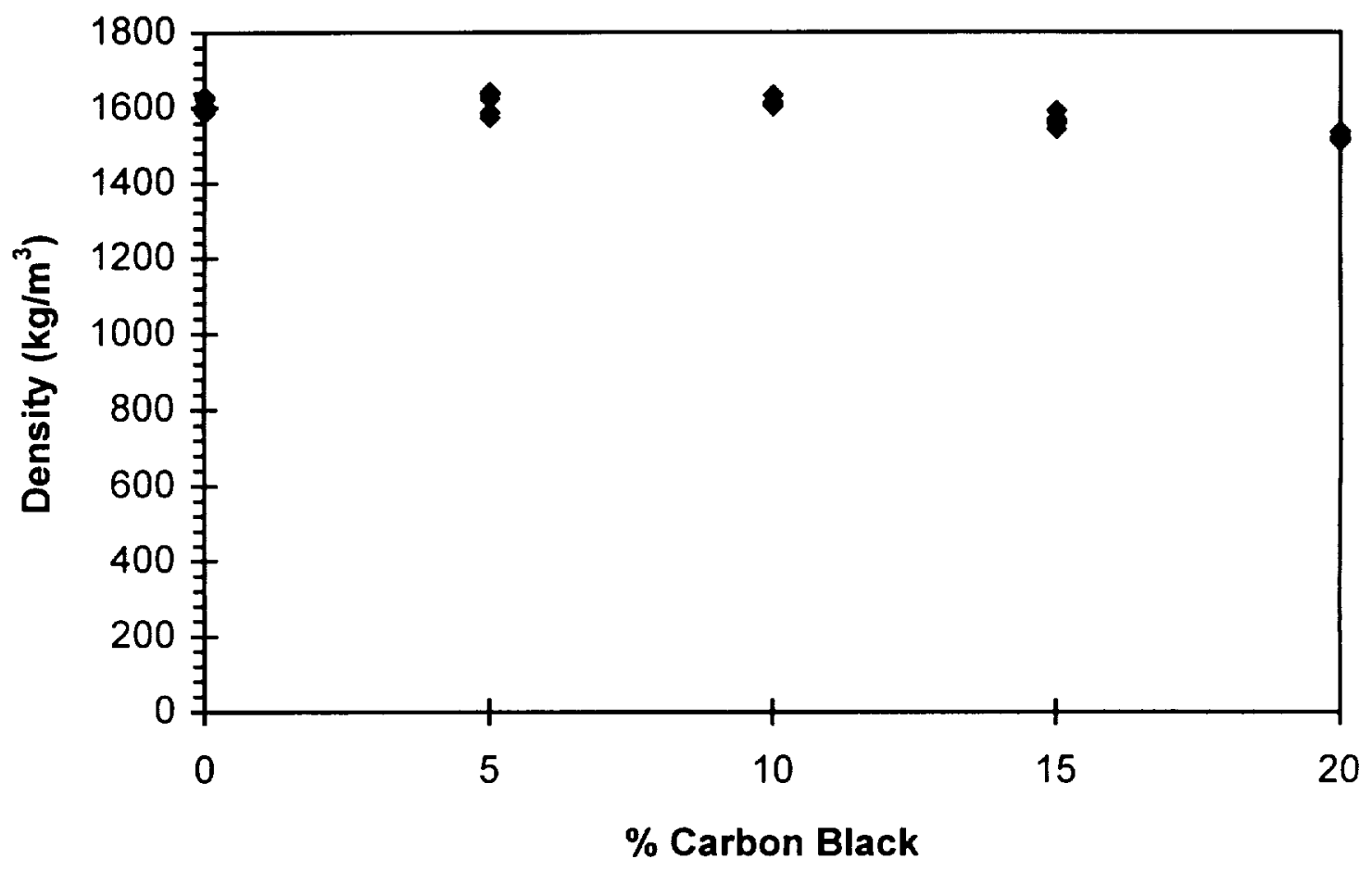

Figure 7.6: Glass fiber / epoxy density 


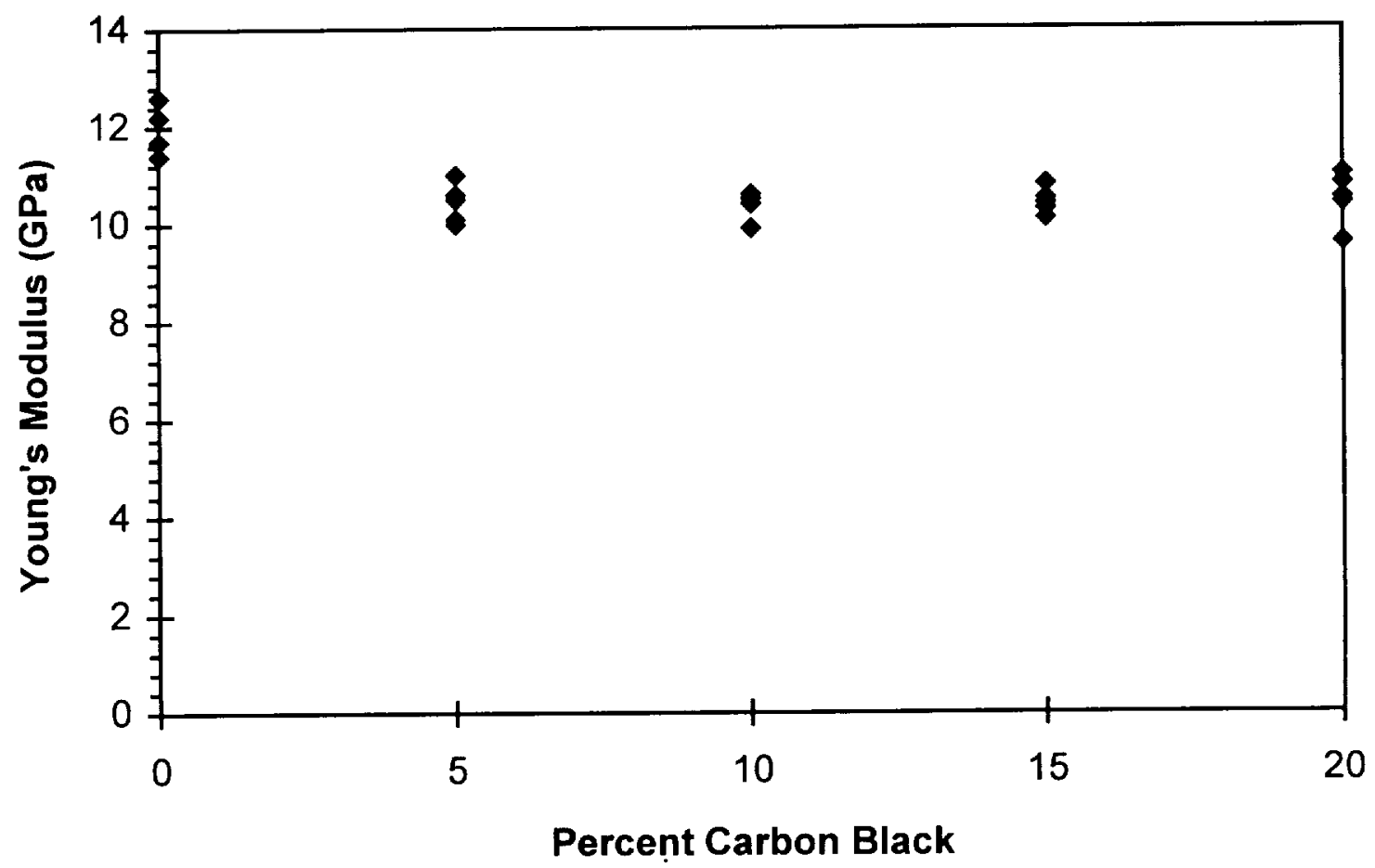

Figure 7.7: Glass fiber / epoxy Young's modulus 


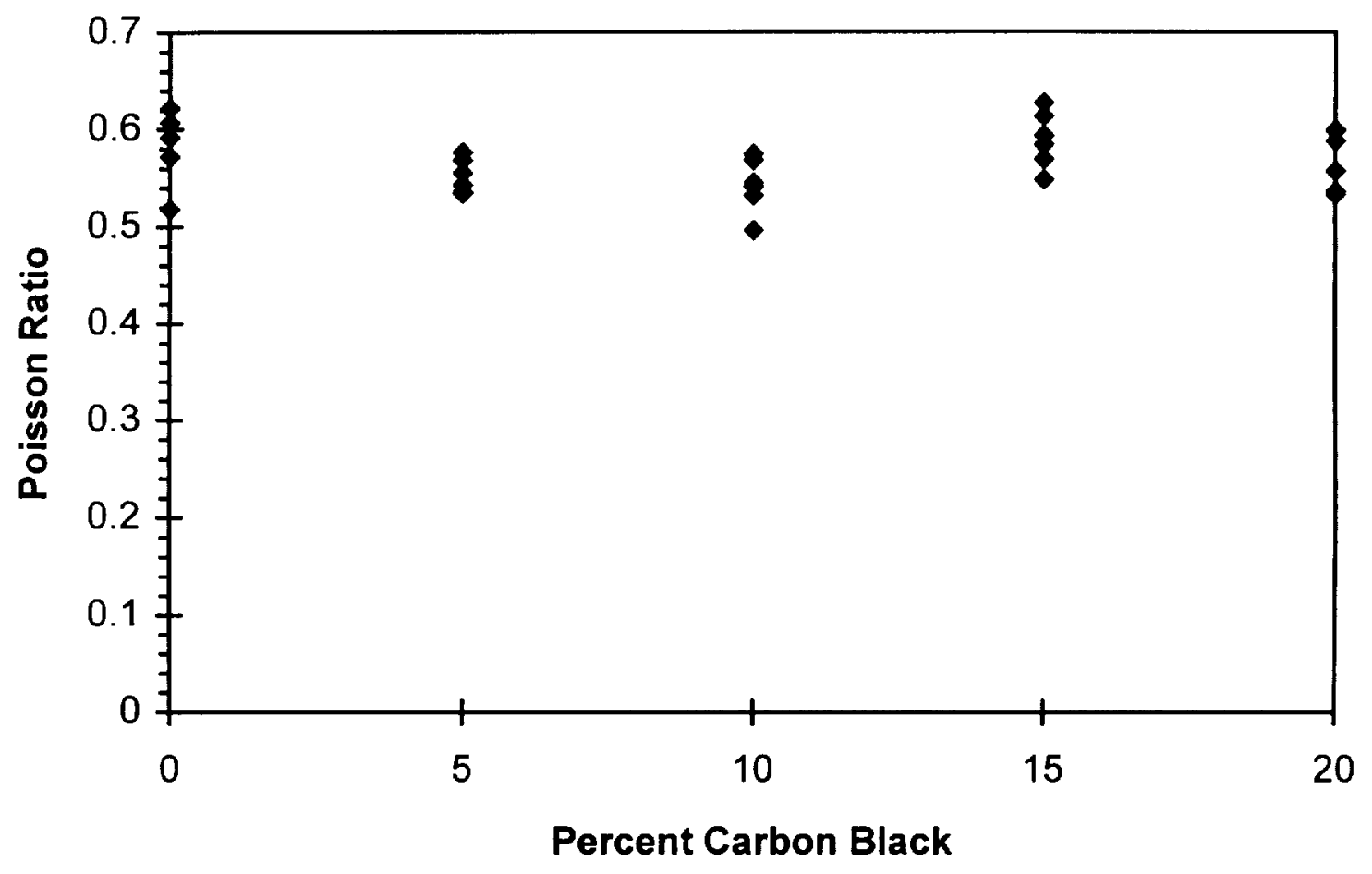

Figure 7.8: Glass fiber/ epoxy Poisson ratio 


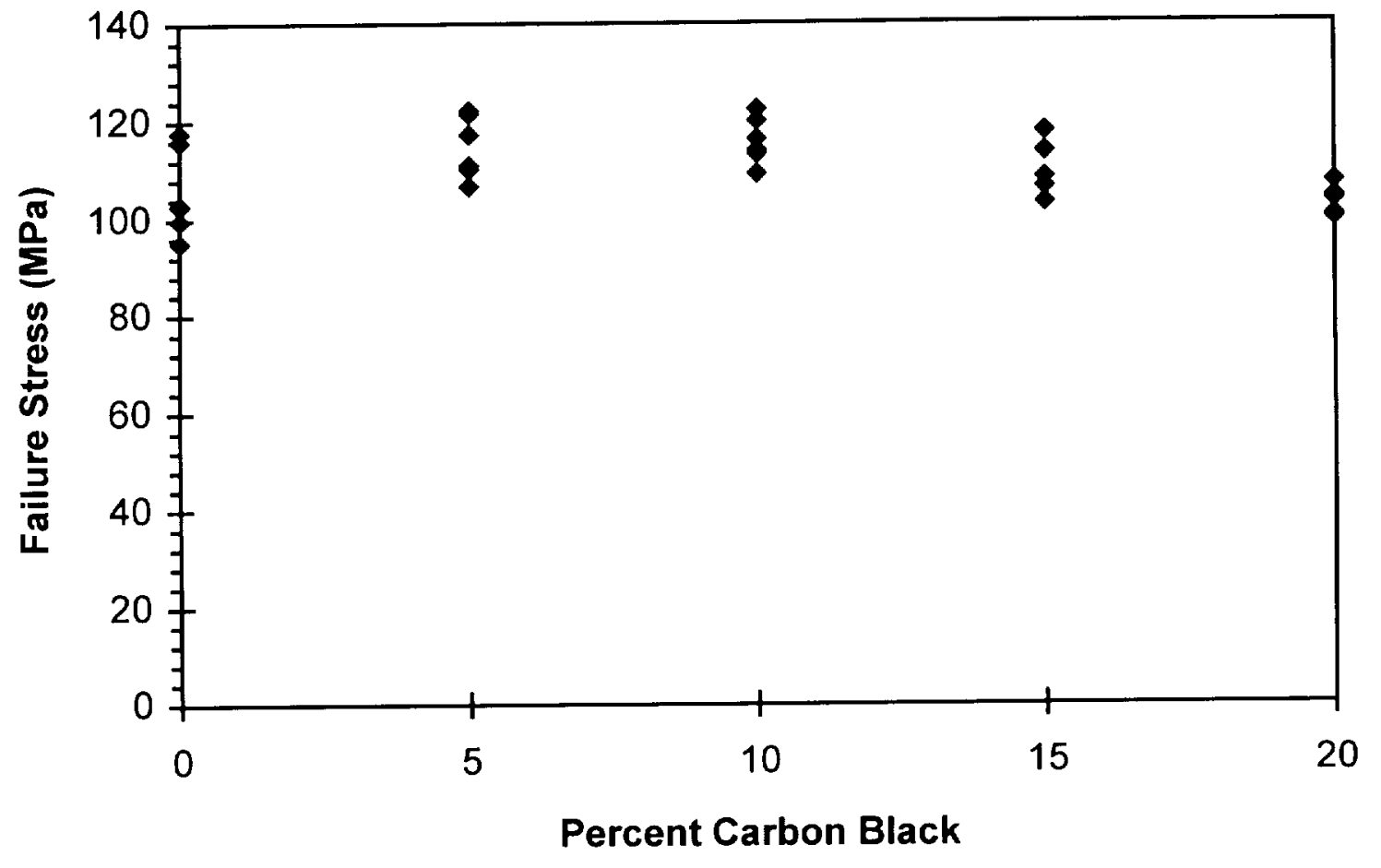

Figure 7.9: Glass fiber / epoxy failure stress 


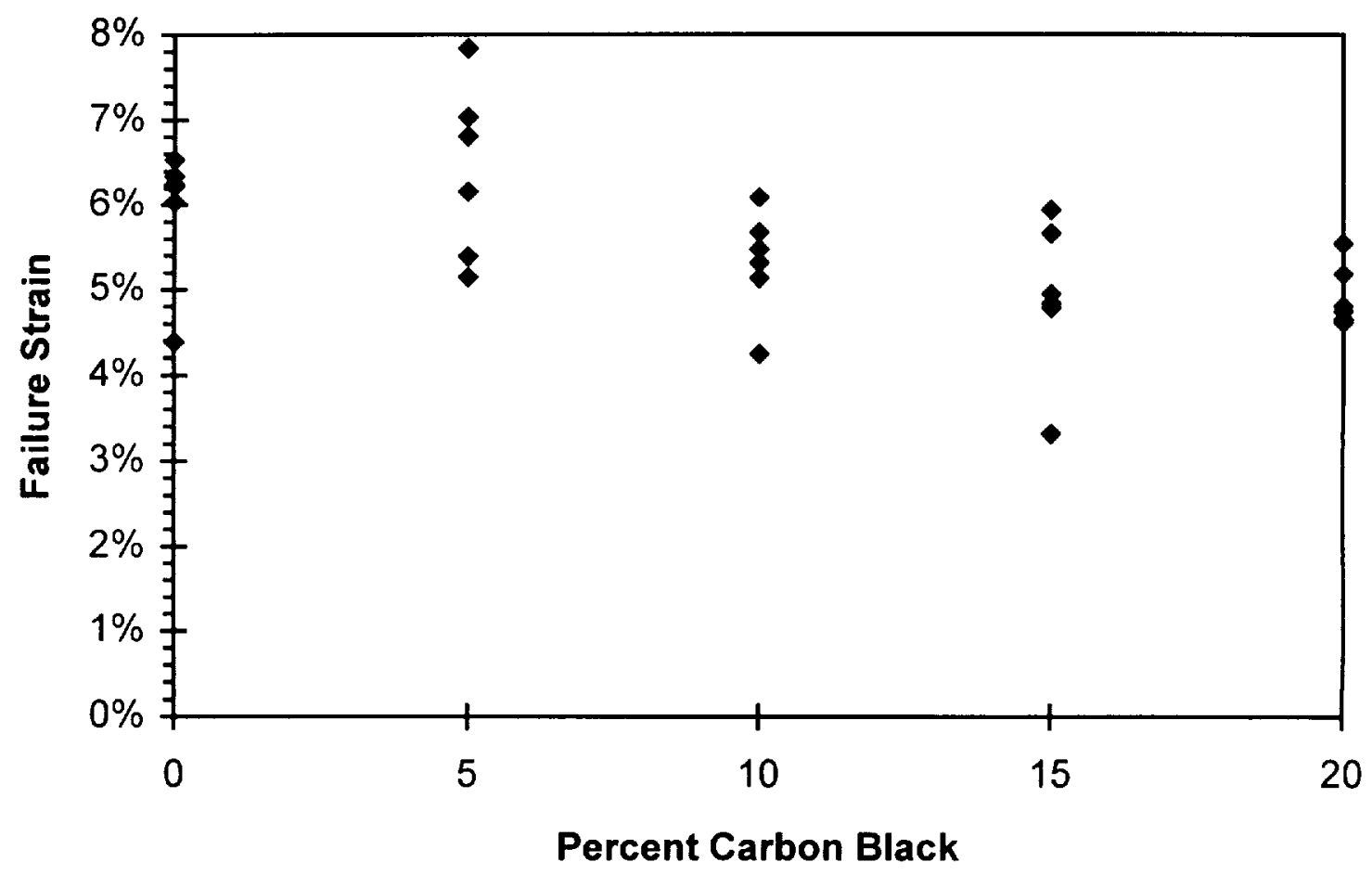

Figure 7.10: Glass fiber / epoxy failure strain 
Table 7.4: Conductivity-tailorable glass fiber / epoxy composite results

\begin{tabular}{||l|c|c|c|c|c||}
\hline \hline \multirow{2}{*}{ Property } & \multicolumn{5}{|c|}{ Percentage Carbon Black By Mass } \\
& $\mathbf{0 \%}$ & $\mathbf{5 \%}$ & $\mathbf{1 0 \%}$ & $\mathbf{1 5 \%}$ & $\mathbf{2 0 \%}$ \\
\hline \hline Conductivity $(1 / \Omega-\mathrm{m})$ & $8.82 \times 10^{-13}$ & $4.37 \times 10^{-12}$ & $2.86 \times 10^{-12}$ & $4.40 \times 10^{-11}$ & $1.23 \times 10^{-9}$ \\
S.D. & $2.45 \times 10^{-13}$ & $1.45 \times 10^{-12}$ & $2.54 \times 10^{-12}$ & $2.19 \times 10^{-11}$ & $1.49 \times 10^{-9}$ \\
\hline Dielectric Constant & 5.30 & 6.33 & 9.20 & 29.81 & 82.23 \\
S.D. & 0.12 & 0.16 & 0.36 & 2.08 & 54.53 \\
\hline Density $\left(\mathrm{kg} / \mathrm{m}^{3}\right)$ & 1607 & 1618 & 1618 & 1565 & 1523 \\
S.D. & 18 & 30 & 12 & 16 & 10 \\
\hline Young's Modulus $(\mathrm{GPa})$ & 11.92 & 10.47 & 10.40 & 10.43 & 10.47 \\
S.D. & 0.49 & 0.37 & 0.25 & 0.23 & 0.48 \\
\hline Poisson Ratio & 0.572 & 0.552 & 0.543 & 0.589 & 0.569 \\
S.D. & 0.045 & 0.017 & 0.028 & 0.029 & 0.030 \\
\hline Failure Stress $(\mathrm{MPa})$ & 105.8 & 114.9 & 115.9 & 110.4 & 103.1 \\
S.D. & 9.1 & 6.5 & 4.8 & 5.4 & 2.7 \\
\hline Failure Strain $(\mu \varepsilon / \varepsilon)$ & 59590 & 63940 & 53200 & 49100 & 49250 \\
S.D. & 7890 & 10270 & 6190 & 9120 & 3660 \\
\hline
\end{tabular}

S.D.: Standard deviation 


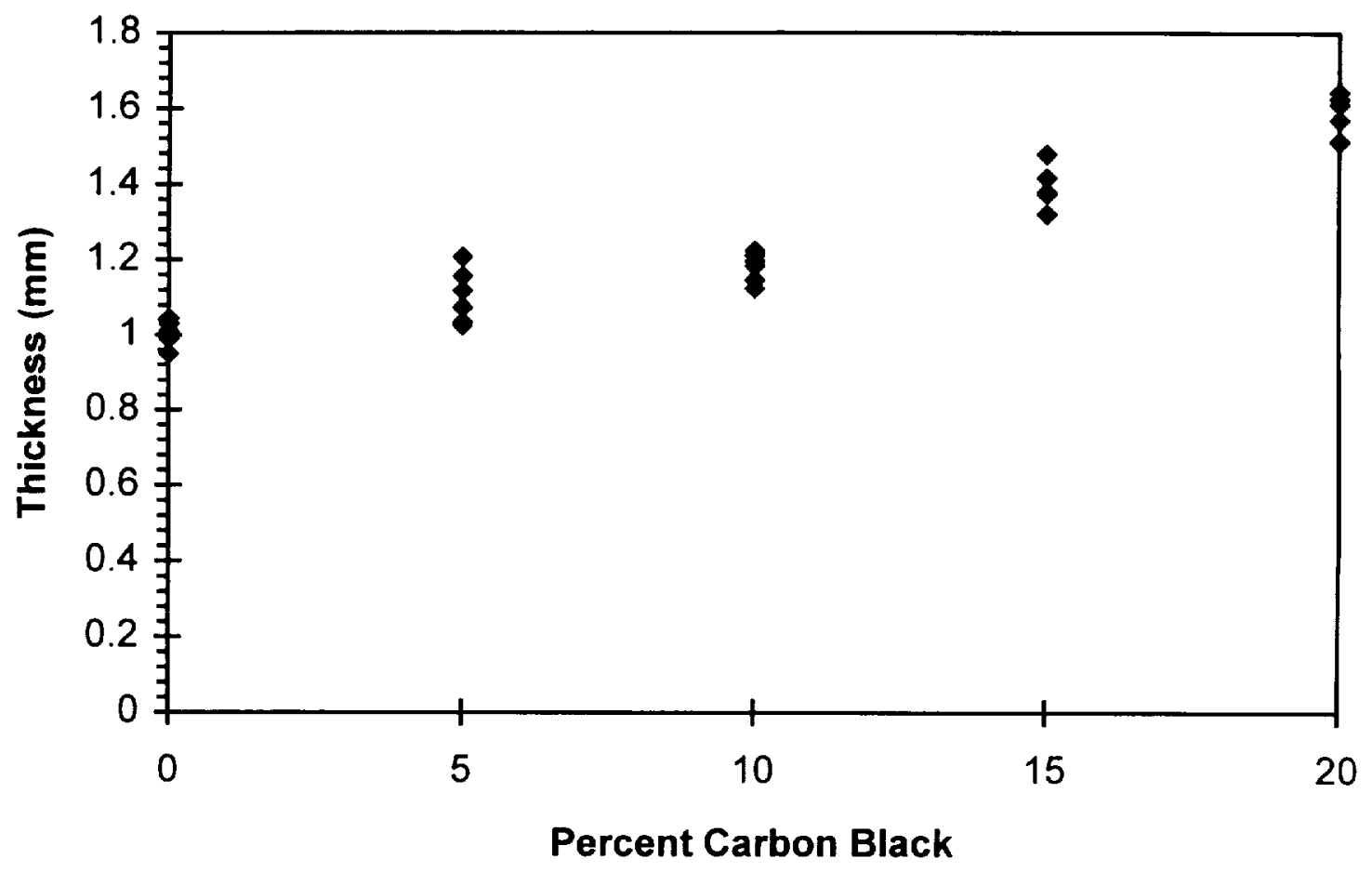

Figure 7.11: Glass fiber / epoxy thickness 


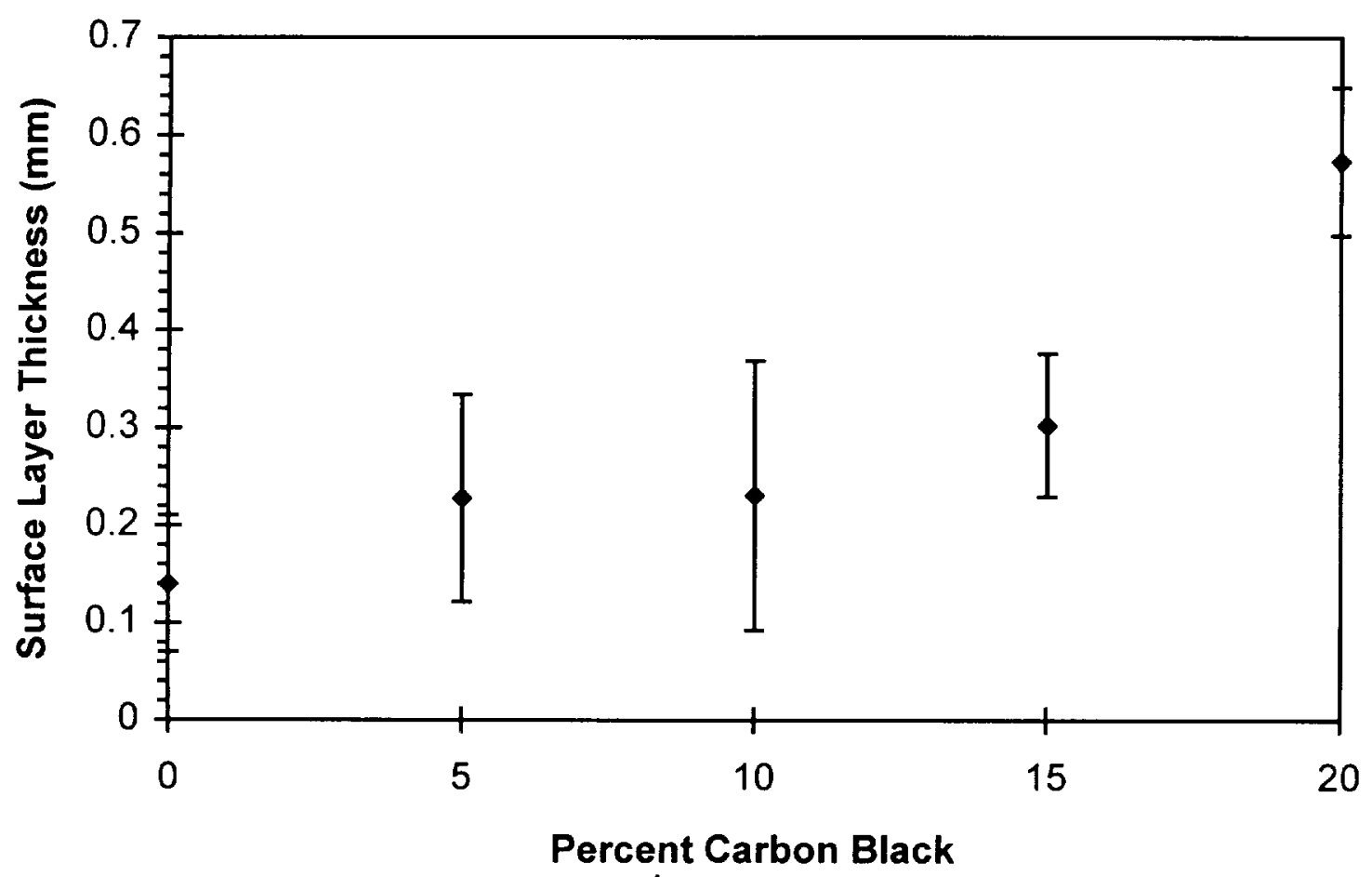

Figure 7.12: Glass fiber / epoxy surface layer thickness 
Table 7.5: Glass fiber / epoxy surface layer thickness

\begin{tabular}{||l|c|c|c|c|c||}
\hline Surface Layer & \multicolumn{5}{|c||}{ Percentage Carbon Black By Mass } \\
Thickness (mm) & $\mathbf{0 \%}$ & $\mathbf{5 \%}$ & $\mathbf{1 0 \%}$ & $\mathbf{1 5 \%}$ & $\mathbf{2 0 \%}$ \\
\hline \hline Upper Surface & 0.075 & 0.131 & 0.129 & 0.224 & 0.486 \\
S.D. & 0.029 & 0.053 & 0.082 & 0.027 & 0.046 \\
\hline Lower Surface & 0.065 & 0.097 & 0.102 & 0.079 & 0.087 \\
S.D. & 0.041 & 0.053 & 0.057 & 0.046 & 0.030 \\
\hline
\end{tabular}

S.D.: Standard deviation 


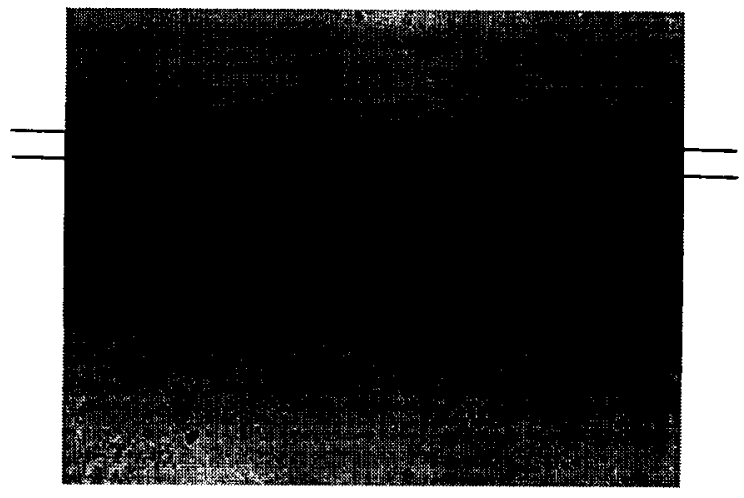

$0 \%$ Carbon Black

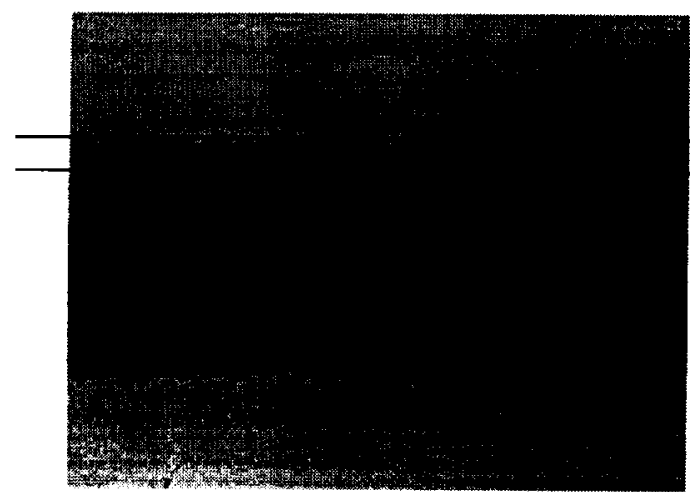

$10 \%$ Carbon Black

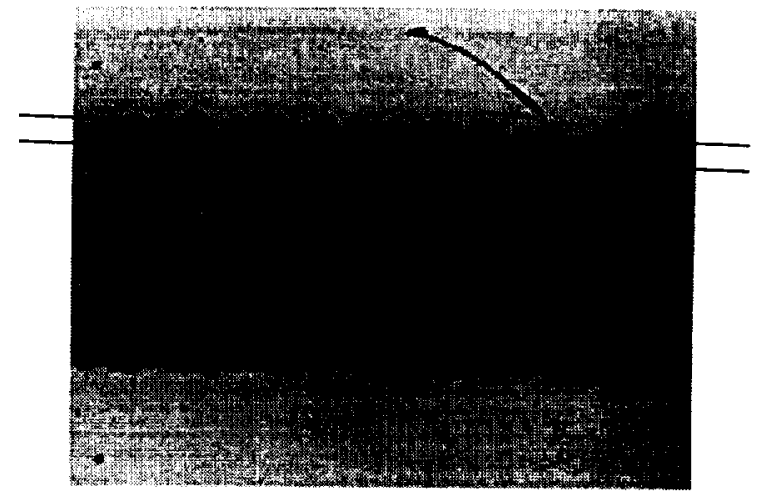

5\% Carbon Black

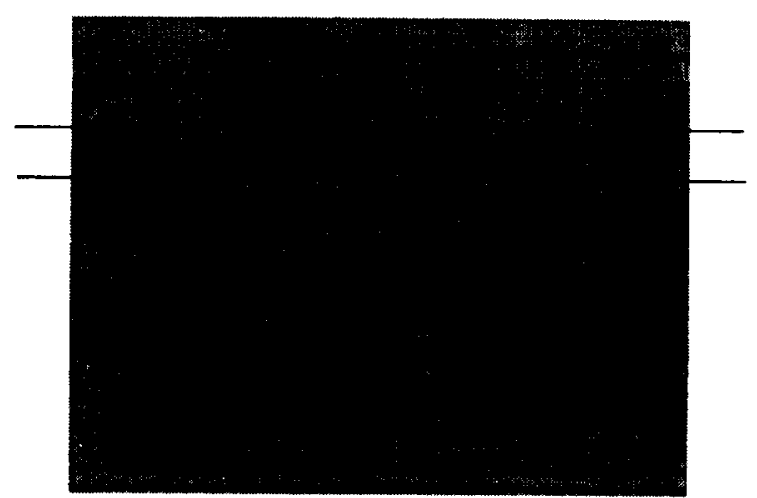

$15 \%$ Carbon Black

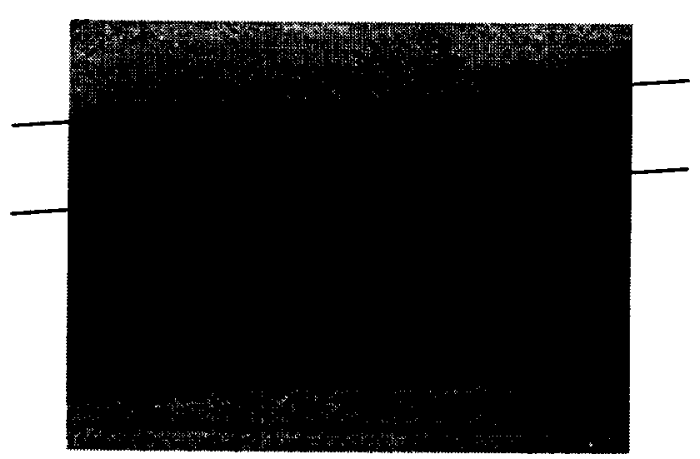

$20 \%$ Carbon Black

Figure 7.13: Micro-graphs of the surface layer of the conductivity-tailorable glass fiber / epoxy composite 


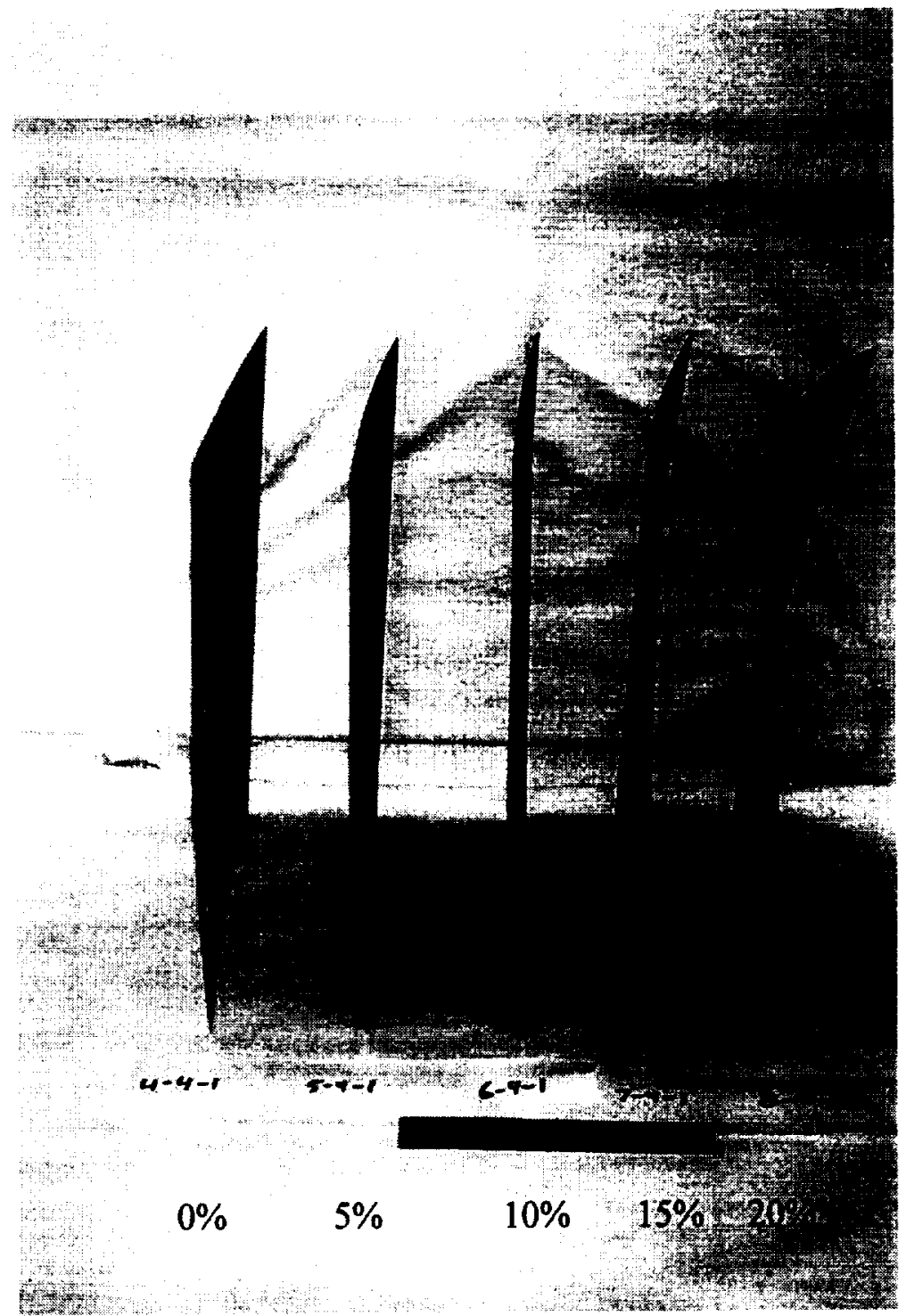

Figure 7.14: Glass fiber / epoxy laminates 
unsymmetric; therefore thermal bending strains were induced in the laminate as it cooled. This curvature had no effect on the mechanical properties since only a small load was required to straighten out the laminate.

\subsubsection{Conductivity}

The conductivity, as shown in Figure 7.4, increased with the addition of carbon black to the epoxy resin. It increased by up to 3 orders of magnitude with the $20 \%$ carbon black samples. The conductivity seems to be constant until approximately $10 \%$ carbon black, at which point there is a rapid increase. This is consistent with percolation theory, where there is no significant increase in properties until there are sufficient particles to create percolation paths from one edge of the sample to the other, as shown in

Figure 4.14. Therefore it would appear that the percolation limit for this carbon black system is around $10 \%$ carbon black by mass of epoxy resin.

\subsubsection{Dielectric Constant}

The dielectric constant, as shown in Figure 7.5, also displayed the same trend as the conductivity, remaining fairly constant up to $10 \%$ carbon black then increasing rapidly. Exponential increases were observed by Bent ${ }^{64}$, who examined up to $5 \%$ carbon black samples, and by Yacubowicz ${ }^{65}$, who observed dielectric constants in the hundreds with a lower percolation limit. Both the large variation in the dielectric constant at $20 \%$ carbon black and the very high values may be due to unusual percolation paths, as shown in Figure 7.15. These paths would have the same effect as reducing the apparent thickness of the sample used in the calculation of the dielectric constant from the measured capacitance (refer to equation 85). If this geometry existed, the dielectric 


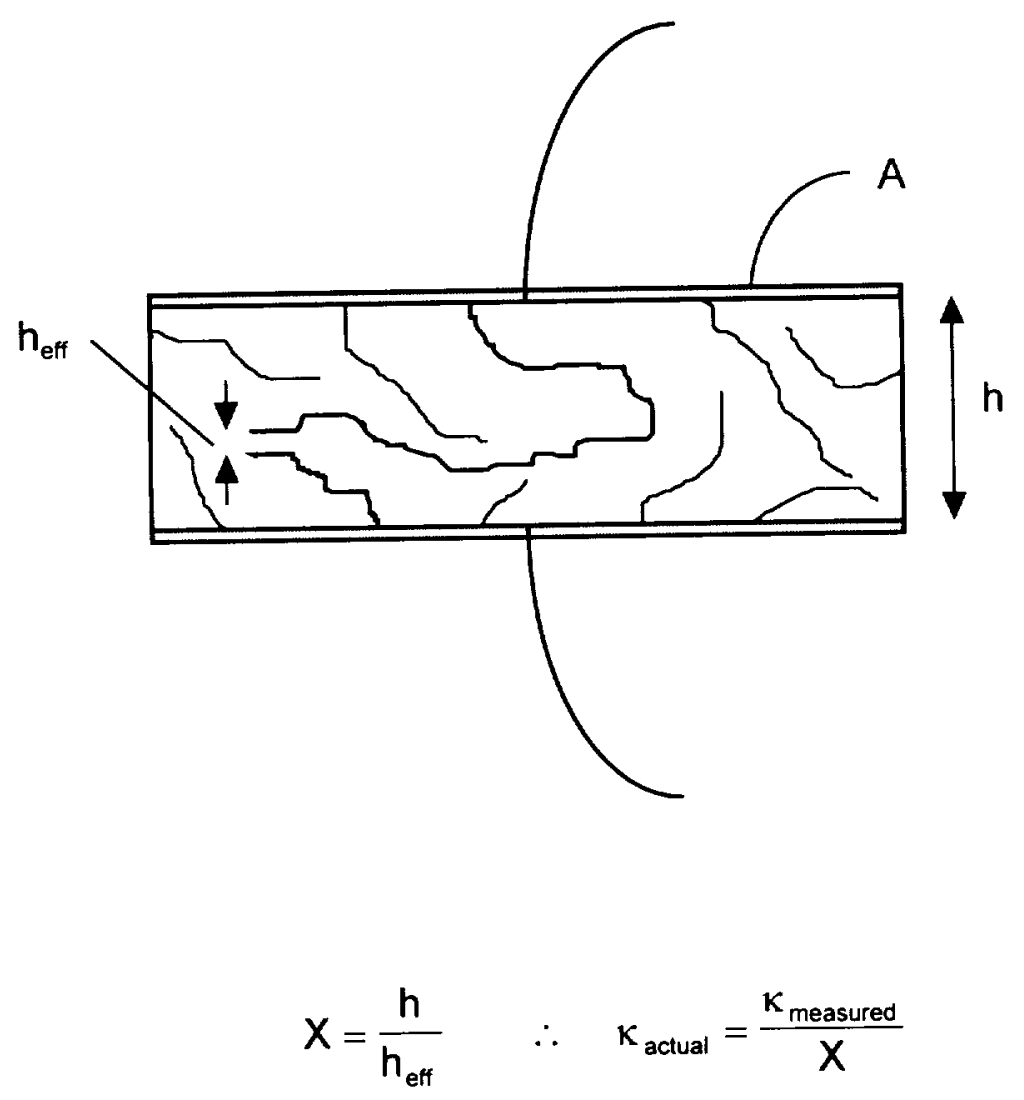

Figure 7.15: Schematic of unusual percolation paths which can lead to high dielectric constant 
constant of the material ( $\left.\kappa_{\text {actual }}\right)$ should be calculated using the effective thickness $\left(h_{\text {eff }}\right)$. However, the dielectric constant $\left(\kappa_{\text {measured }}\right)$ is actually calculated using the sample thickness $(\mathrm{h})$; this results in the measured dielectric constant being magnified by the ratio of the sample thickness over the effective thickness $\left(\mathrm{h} / \mathrm{h}_{\mathrm{eff}}\right)$. Therefore, very big effective values for the dielectric constant of the sample are possible. This variation also shows the randomness of carbon aggregation that can radically affect the electrical properties, even with extremely careful dispersion during manufacturing of the samples, and careful measurement techniques.

\subsubsection{Dielectric Strength}

When the dielectric strength was measured using samples with no added conductive surface layer, no data was obtainable as none of the samples had broken down when the maximum voltage, 10,000 volts, was applied across the samples. Therefore, the dielectric strength of all samples was greater than $10.5 \mathrm{MV} / \mathrm{m}$. This result makes sense since the published values for the dielectric strength of glass fiber / epoxy composites is between 17.7 and $21.7 \mathrm{MV} / \mathrm{m}$.

\subsubsection{Density}

Theoretically the density should increase linearly with increasing percentage of carbon black. This trend is not seen in Figure 7.6, probably due to the high viscousness of the epoxy, which limited its ability to soak completely through the glass fiber bundles, resulting in voids. Also as mentioned earlier, the increase in surface layer thickness is also affecting the density measurements. 


\subsubsection{Tensile Tests}

Tensile tests were performed on the laminates to determine the Young's modulus, Poisson ratio, failure stress, and failure strain. To verify that epoxy was still usable, an initial set of tests were performed on unidirectional glass fiber / epoxy laminates. The results are presented in Table 7.6, and they agreed with data measured by Ed Wolf when he used the same system. It should be noted that all the failures initiated near one of ends close to the loading tabs, as shown in Figures 7.16 through 7.21. This indicates that the data is not a very good absolute strength measurement. It is used here for comparison with the carbon black filled data. The data was also verified using MCLAM, an in-house CLPT code using a Tsai-Wu failure criterion, with typical glass fiber / epoxy material properties. The results are shown in Table 7.7. There was excellent agreement for the Young's modulus, Poisson ratio, and failure stress of the $[ \pm 45]_{\mathrm{S}}$ laminates. There was also good agreement for the Young's modulus and Poisson ratio of the $[0]_{4}$ laminates. The failure stress measured was $50 \%$ of the calculated failure stress, due to the splitting failure mode initiated near the loading tabs (refer to Figure 7.16).

The Young's modulus, as shown in Figure 7.7, decreases slightly with increasing percentage of carbon black. The Poisson ratio, as shown in Figure 7.8, remained fairly constant around 0.56 with a little scatter above and below this value. The failure stress, as shown in Figure 7.9, remains fairly constant, slightly increasing with increasing percentage of carbon black then slightly decreasing. The failure strain, as shown in Figure 7.10, decreases linearly with increasing percentage of carbon black. Even though there is a significant decrease in the failure strain from just over $6 \%$ strain to $5 \%$ strain, 
Table 7.6: $\quad[0]_{4}$ glass fiber/epoxy tensile test results

\begin{tabular}{|l|c|c||}
\hline Property & Average & S.D. \\
\hline \hline Young's Modulus $(\mathrm{GPa})$ & 49.62 & 1.23 \\
\hline Poisson Ratio & 0.216 & 0.018 \\
\hline Failure Stress $(\mathrm{MPa})$ & 554.4 & 23.9 \\
\hline Failure Strain $(\mu \varepsilon / \varepsilon)$ & 22134 & 1091 \\
\hline
\end{tabular}

S.D.: Standard deviation 


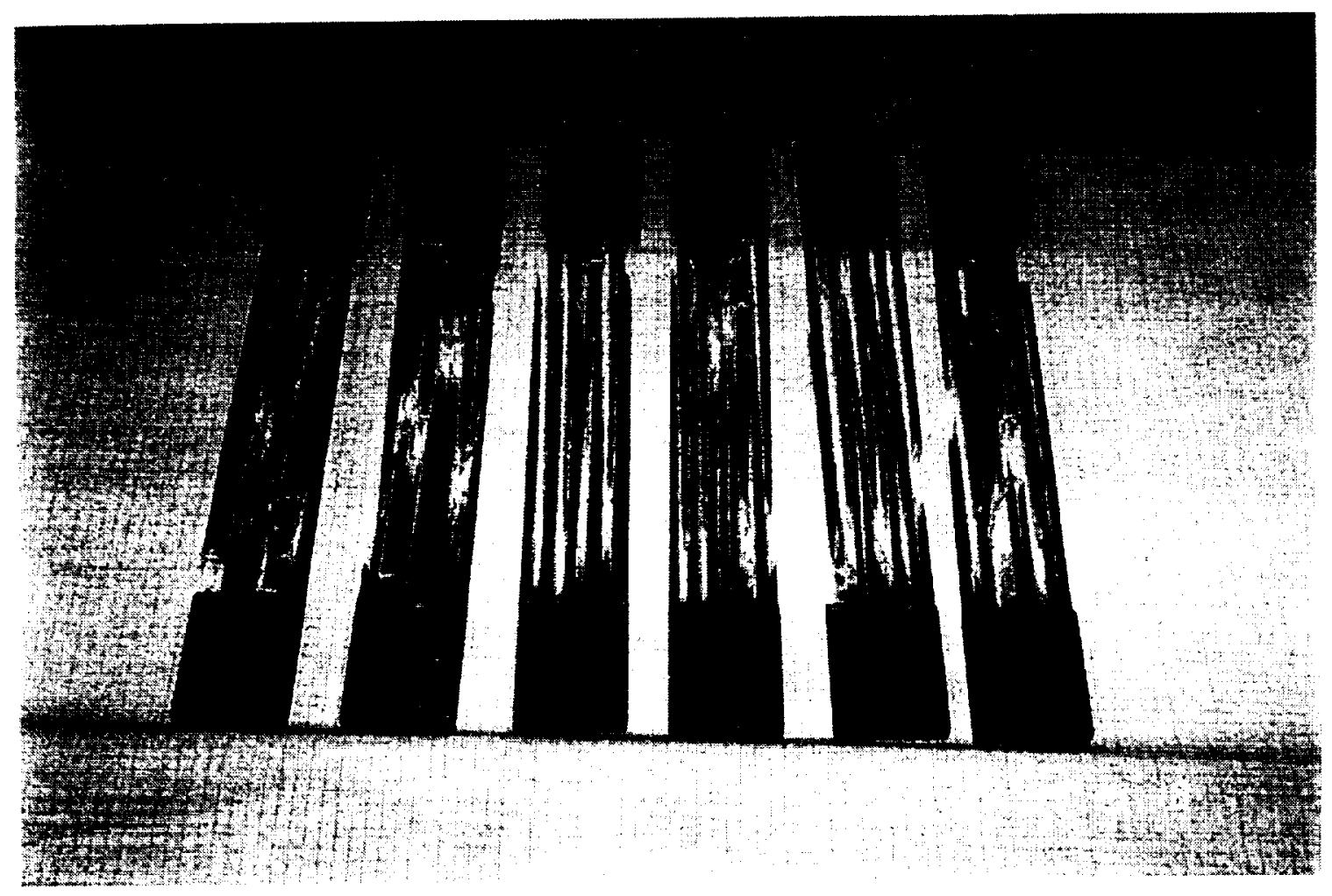

Figure 7.16: Broken $[0]_{4}$ glass fiber / epoxy tensile specimens 


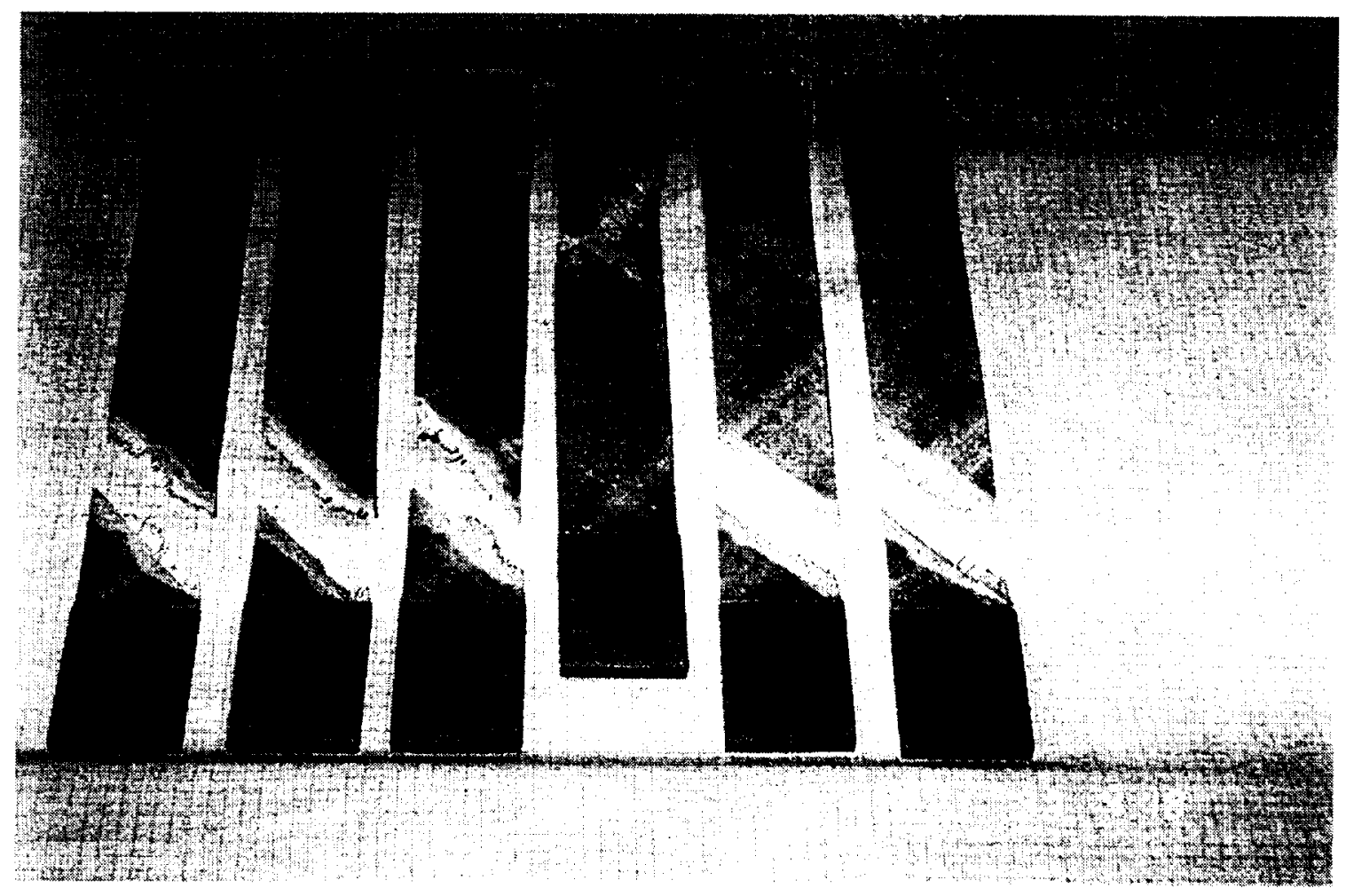

Figure 7.17: Broken $0 \%$ carbon black tensile specimens 


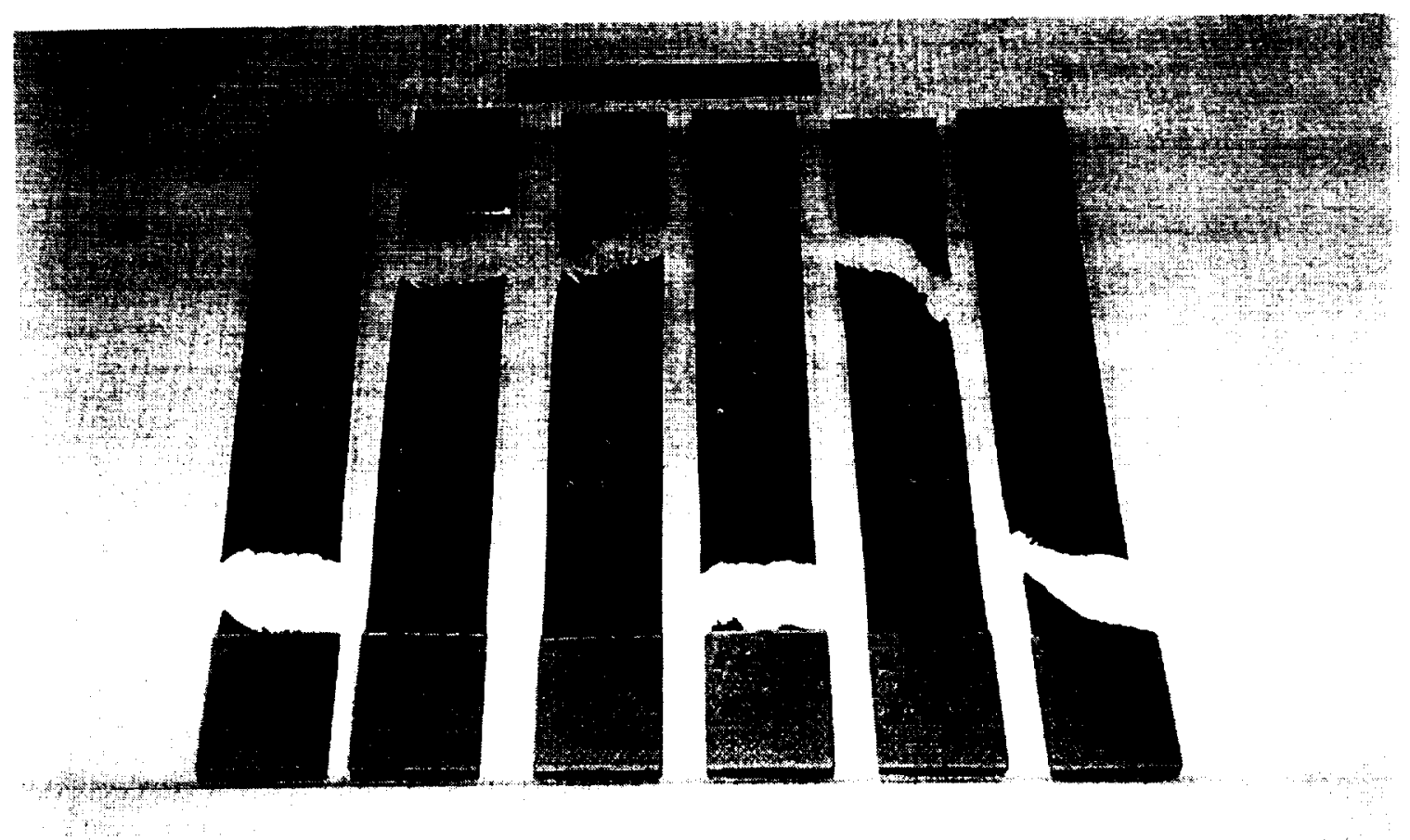

Figure 7.18: Broken $5 \%$ carbon black tensile specimens 


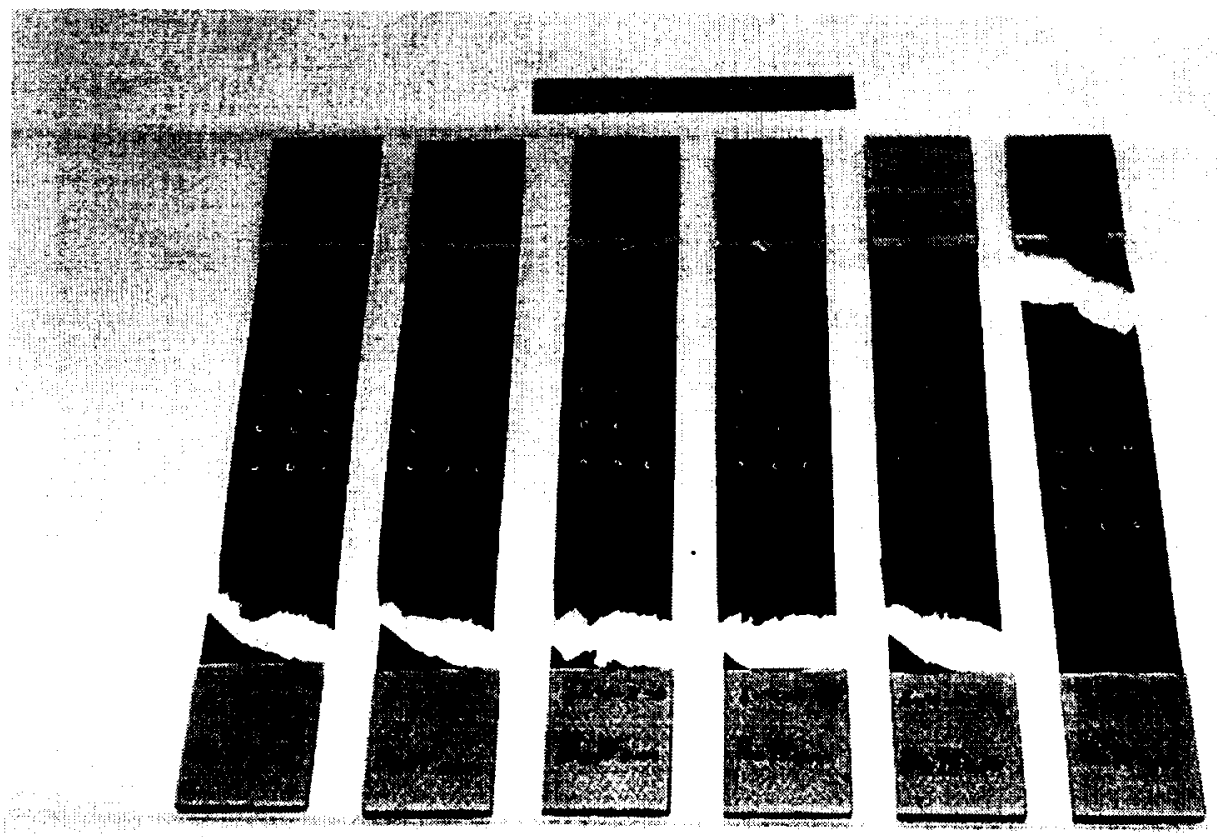

Figure 7.19: Broken $10 \%$ carbon black tensile specimens 


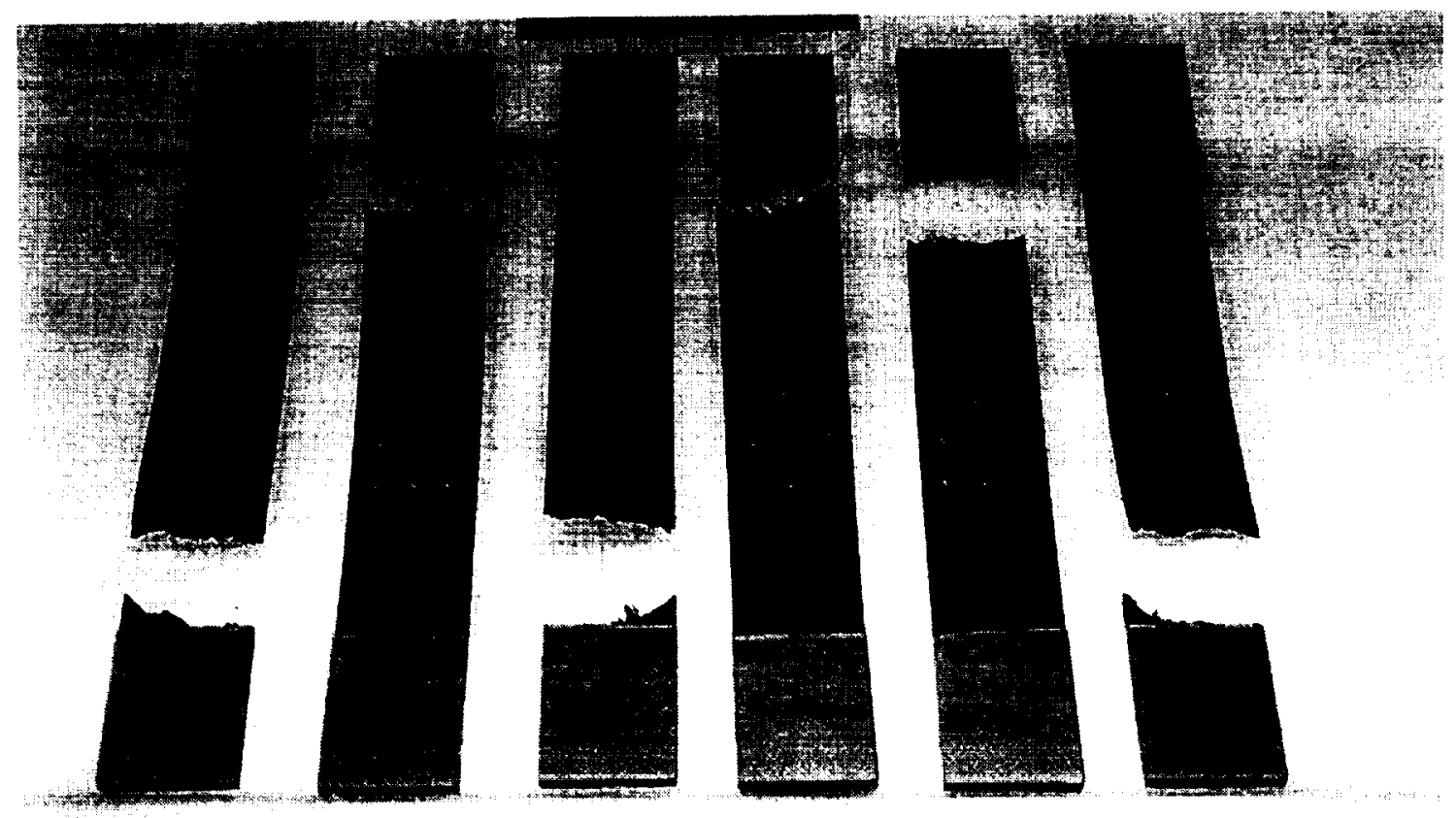

Figure 7.20: Broken $15 \%$ carbon black tensile specimens 


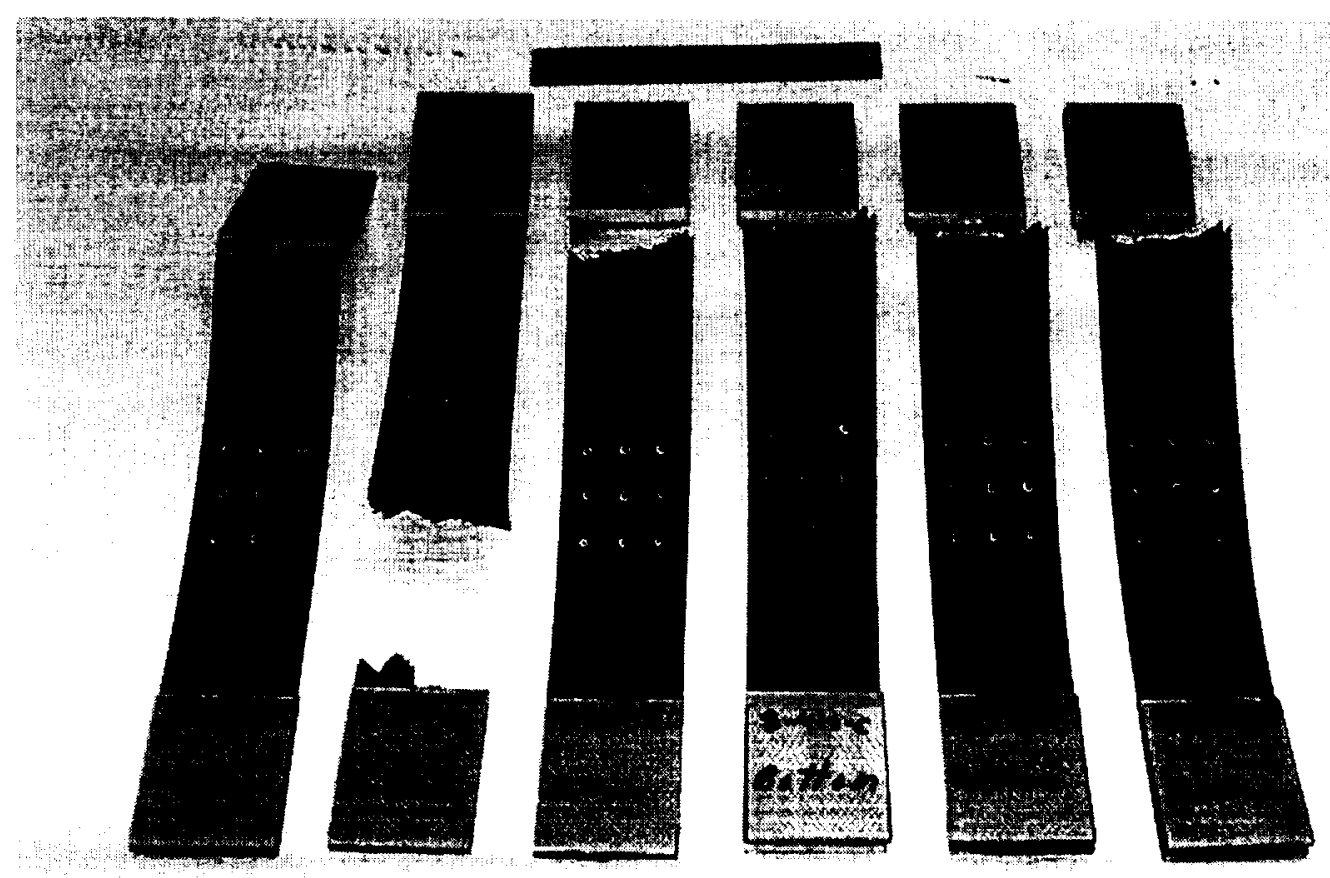

Figure 7.21: Broken 20\% carbon black tensile specimens 
Table 7.7: $\quad$ MCLAM results for $[0]_{4}$ and $[ \pm 45]_{\mathrm{S}}$ glass fiber / epoxy laminates

\begin{tabular}{|l|c|c||}
\hline Property & {$[\mathbf{0}]_{\mathbf{4}}$} & {$[ \pm \mathbf{4 5}]_{\mathbf{S}}$} \\
\hline \hline Young's Modulus (GPa) & 38.59 & 12.55 \\
\hline Poisson Ratio & 0.260 & 0.516 \\
\hline Failure Stress $(\mathrm{MPa})$ & 1061.79 & 99.91 \\
\hline
\end{tabular}


these values are well above any acceptable amount of strain that should be seen in an actual aerospace structure. It should also be noted that the high values of failure strain was calculated from the stroke data since at failure the strain gages had long since stopped working. This value can typically be inaccurate, as using stroke as a measurement of strain is a gross approximation, as with the $[0]_{\mathrm{S}}$ laminates (refer to Figure C.1). However, in some cases there can be quite good agreement between the strain gage strain and the stroke strain, as with the $[ \pm 45]_{\mathrm{S}}$ laminates which had acceptable to excellent agreement (refer to Figure C.29).

\subsubsection{Summary}

With the addition of conductive carbon black into the insulative matrix of a composite, the conductivity can be increased significantly, as was desired. The dielectric constant was also increased. Due to testing limitations no trends could be found for the dielectric strength, however the value is greater than $10.5 \mathrm{MV} / \mathrm{m}$ in all cases. The increase in electrical properties had little effect on the mechanical properties of the composite. They remained fairly constant across all values of carbon black added. The only problem with the addition of carbon black to the matrix of the composite was the manufacturing difficulties caused by the increase in the epoxy viscosity. This problem could be overcome by using a less viscous epoxy, or by developing a manufacturing technique which would allow the epoxy to flow out of the composite during the curing process. Possible manufacturing technique variations include heating the epoxy before mixing so that it is less viscous, using pressure during the cure to press the top cure plate down into the laminate and thus force the extra epoxy out, or modifying the cure cycle to 
include a hold time at the flow temperature of the epoxy (as is done with AS4/3501-6 cure cycle) instead of ramping straight up to the cure temperature. 


\section{CHAPTER 8}

\section{CONCLUSIONS}

A tool now exists which can determine the charge density, electric field, and voltage as a function of depth into a composite material, in a specified orbital environment, and thus predict if an electrostatic discharge will occur. The program, CoDDCA, can be run for any orbit and material system, by specifying the relevant parameters. The program can also include geomagnetic substorms and solar particle events of various intensities specified by the user. The space environment is determined using the Environmental Workbench software by specifying the orbit parameters (the apogee height, perigee height, inclination and solar cycle condition).

For conducting materials, such as carbon fiber / epoxy, there is no apparent problem with deep dielectric charging, however, peculiarities of the composite such as surface layers can be a concern. For insulating materials, such as glass fiber / epoxy, polymers, and Kevlar, there is no electrostatic discharge concern with average day-to-day flux levels. Problems can arise during geomagnetic substorms and solar particle events, where flux levels increase by several orders of magnitude. There is also a concern for electrostatic discharges if materials which have extremely low conductivities are used. The conductivity enhancements, such as high field conductivity and radiation induced 
conductivity, have a minimal effect on the deep dielectric charging analysis and in any case only help to reduce the likelihood of an electrostatic discharge. The micromechanical details between the fiber and matrix on the electric field are in the most part negligible, with a maximum electric field magnification of 3 times. Unlikely fiber arrangements in percolation paths can lead to electric field increases on the order of 50 times. Another related problem is that of the electric field in the insulative surface layer of a conductive composite. The results show that there can be a large electric field in the surface layer even if the bulk composite is not charged significantly, due to the large difference in conductivities between these two areas.

Parametric studies showed several relationships exist between the deep dielectric charging parameters and the material properties. The time to steady state is proportional to the dielectric constant and inversely proportional to the conductivity. The electric field is inversely proportional to the conductivity and proportional to the amount of particles present in the space environment. The charge density, electric field, and voltage distributions are affected by the location of particles in the material and the relative amounts of electrons and protons.

From the case study orbits, the worst orbit was the base case orbit, a $7000 \mathrm{~km}$ altitude circular orbit. A similar environment could be expected in a geosynchronous transfer orbit. Next came the geosynchronous orbit, the Global Positioning System orbit, and the Molniya orbit. These all produced an order of magnitude smaller electric fields in the base case material, as compared to the base case orbit. The space shuttle parking orbit 
and the low altitude retrograde or polar orbit produced negligible electric fields due to the small numbers of high energy particles at low altitudes.

A complete set of tests were done on the through-thickness electrical properties of a carbon fiber / epoxy composite. The results were incompatible with the idea that the electrical properties were continuum material properties on the scale of the specimen. The actual numerical values were within the range of previously measured values $(0.1$ to $1061 / \Omega-\mathrm{m}$ for the through-thickness conductivity), however the thickness, area, and stacking sequence dependencies were either non-classical or indiscernible. The throughthickness electrical properties appear to be affected by the surface layer, since the conductivity increased and the dielectric strength goes away when the surface layer is sanded off. The other factor affecting the through-thickness electrical properties is percolation effects. Therefore, when dealing with electrical properties of composites one has to be wary of previously published numbers, and attention must be paid to surface effects.

A conductivity tailorable composite can be manufactured using a glass fiber / epoxy composite with conductive carbon black added to the epoxy resin. The conductivity can be increased by 3 orders of magnitude (from $10^{-12} 1 / \Omega-\mathrm{m}$ to $10^{-9} 1 / \Omega-\mathrm{m}$ ) with minimal change to the mechanical properties of the composite. The only drawback to using high percentages of carbon black in the epoxy resin is the increased manufacturing difficulties due to the high viscosity of the epoxy. This tailorable system 
may be very useful if a material used in the space environment needs to be an insulator, but deep dielectric charging also needs to be minimized. 


\section{REFERENCES}

1. Vaughan, W.W., Niehuss, K.O., and Alexander, M.B., "Spacecraft Environments Interactions: Solar Activity and Effects on Spacecraft", NASA Reference Publication 1396, November 1996.

2. Garrett, H.B., and Hastings, D., "Space Radiation Models", AIAA 94-0590, 32nd Aerospace Sciences Meeting \& Exhibit, Reno, NV, January 1994.

3. Hastings, Daniel, and Garrett, Henry, "Spacecraft Environmental Interactions", The Press Syndicate of the University of Cambridge, New York, NY, USA, 1996, pg. 47.

4. Larson, Wiley J., and Wertz, James R., "Space Mission Analysis and Design", 2nd Ed., Microcosm, Inc., Torrance, California, and Kluwer Academic Publishers, Dordrecht / Boston / London, USA, 1993, pp. 200-201.

5. Osepian, A., Vlaskov, V., Ranta, H., Ranta, A., "High-Latitude Plasma Densities In The Middle Atmosphere and Characteristics of Precipitating Electrons During an Auroral Absorption Substorm", Advanced Space Research, Vol. 13, No. 4, 1993, pp. (4)99-(4)104.

6. Tribble, A.C., "The Space Environment and its Impact on Spacecraft Design", AIAA 93-0491, 31 st Aerospace Sciences Meeting, Reno, NV, January 1993.

7. James, Bonnie F., Norton, O.W., and Alexander, Margaret B., "The Natural Space Environment: Effects of Spacecraft", NASA Reference Publication 135, November 1994.

8. Purvis, Carolyn K., Garrett, Henry B., Whittlesey, A.C., and Stevens, N. John, "Design Guidelines for Assessing and Controlling Spacecraft Charging Effects", NASA Technical Paper 2361, September 1984.

9. Silverman, Edward M., "Space Environment Effects on Spacecraft: LEO Materials Selection Guide", NASA Contractor Report 4661 Part 1, August 1995.

10. Stillwell, R.P., Stevens, N.J., and Underwood, C.S., "On Orbit Charging: Current TWR Design Requirements", AIAA 92-1100, 1992 Aerospace Design Conference, Irvine, CA, February 1992.

11. Anon., "Electromagnetic Compatibility Requirements for Space Systems", MILSTD 1541A, United States Air Force, US Government Printing Office, December 1987. 
12. Stannard, P.R., Katz, I., Gedeon, L., Roche, J.C., Rubin, A.G., and Tautz, M.F., "Validation of the NASCAP Model Using Spaceflight Data", AIAA 82-0269, AIAA 20th Aerospace Sciences Meeting, Orlando, FL, January 1982.

13. Vampola, A.L., "Thick Dielectric Charging on High-Altitude Spacecraft", Journal of Electrostatics, Vol. 20, 1987, pp. 21-30.

14. Wrenn, Gordon L., "Conclusive Evidence for Internal Dielectric Charging Anomalies on Geosynchronous Communications Spacecraft", Journal of Spacecraft and Rockets, Vol. 32, No. 3, May-June 1995, pp. 514-520.

15. Frederickson, A.R., Holeman, E.G., and Mullen, E.G., "Characteristics of Spontaneous Electrical Discharging of Various Insulators in Space Radiation", IEEE Transactions of Nuclear Science, Vol. 39, No. 6, December 1992, pp. 17731782.

16. Frederickson, A.R., Mullen, E.G., Brautigam, D.H., Kerns, K.J., Robinson Jr., P.A., and Holeman, E.G., "Radiation-Induced Insulator Discharge Pulses in The CRRES Internal Discharge Monitor Satellite Experiment", IEEE Transactions on Nuclear Science, Vol. 38, No. 6, December 1991, pp. 1614-1621.

17. Garrett, H.B., and Whittlesey, A.C., "Spacecraft Charging, An Update", AIAA 96-0143, 34th Aerospace Sciences Meeting \& Exhibit, Reno, NV, January 1996.

18. Soubeyran, Amaury, "Internal and Bulk Charging: Analysis of Risks and Protection Means", Space Environment: Prevention of Risks Related To Spacecraft Charging, Toulouse, France, June 1996, pp. 191-205.

19. Berkley, David A., "Computer Simulation of Charge Dynamics in ElectronIrradiated Polymer Foils", Journal of Applied Physics, Vol. 50, No. 5, May 1979, pp. 3447-3453.

20. Frederickson, A.R., and Woolf, S., "Electric Fields in keV Electron Irradiated Polymers", IEEE Transactions on Nuclear Science, Vol. NS-29, No. 6, December 1982, pp. 2004-2011.

21. Soubeyran, A., Estienne, J.P., Borde, J., and Drolshagen, G., "Numerical Simulation of Bulk Charging", unpublished paper, pp. 1-13.

22. Soubeyran, A., "ESA-DDC Demonstration Manual", Matra Espace, June 1992.

23. Soubeyran, A., "Technical Note 1, Extended Capabilities", Matra Marconi Space France, March 1994. 
24. Whittlesey, A., "System Electrostatic Discharge Testing For Space Charging Caused ESD Events", Proceedings of the 14th Aerospace Testing Seminar, Manhattan Beach, CA, March 1993, pp. 1-4.

25. Jones, Robert M., "Mechanics of Composite Materials", Taylor \& Francis, Hemisphere Publishing Corporation, USA, 1975.

26. Tsai, Stephen W., "Theory of Composite Design", Thick Composites, A division of ILT Corporation, Dayton, Ohio, USA, 1992.

27. Lodge, K.J., "The Electrical Properties of Joints in Carbon Fibre Composites", Composites, Vol. 13, No. 3, 1982, pp. 305-310.

28. Owston, C.N., "Electrical Properties of Single Carbon Fibres", Journal of Physics D: Applied Physics, Vol. 3, 1970, pp. 1615-1626.

29. Headifen, G.R., and Fahrenthold, E.P., "Mechanical and Electrical Properties of Glass and Carbon Fiber Reinforced Composites", Composite Material Technology, 13th Annual Energy-source Technology Conference and Exhibit, New Orleans, LA, January 1990.

30. Whitesides, George M., and Weisbecker, Carl S., "Measurements of The Conductivity of Individual $10 \mathrm{~nm}$ Carbon Nanotudes", Materials Research Society Symposium Proceedings, Vol. 349, 1994, pp. 263-268.

31. Goeje, M.P., and Wapenaar, K.E.D., "Non-Destructive Inspection of Carbon Fibre-Reinforced Plastics Using Eddy Current Methods", Composites, Vol. 23, No. 3, May 1992, pp. 147-157.

32. Vernon, Susan N., "Parametric Eddy Current Defect Depth Model and Its Application to Graphite Epoxy", Non-Destructive Testing International, Vol. 22, No. 3, June 1989, pp. 139-148.

33. Lee, Stuart M. Editor, "International Encyclopedia of Composites", Volume 3, VCH Publishers, New York, NY, USA, 1990, pp. 150-153.

34. Kaddour, A.S., Al-Salehi, F.A.R., Al-Hassani, S.T.S., and Hinton, M.J., "Electrical Resistance Measurement Technique For Detecting Failures In CFRP Materials At High Strain Rates", Composite Science and Technology, Vol. 51, No. 3, 1994, pp. 377-385.

35. Prakash, R., and Owston, C.N., "Eddy-Current Method For The Determination of Lay-up Order In Cross-Plied CFRP Laminates", Composites, Vol. 7, No. 2, April 1976, pp.88-92. 
36. Manufacturing Chemists' Association, "Technical Data on Plastics", Manufacturing Chemists' Association, Inc., Washington, DC, February 1957, pg. 43.

37. Griffiths, David J., "Introduction To Electrodynamics", 2nd Ed., Prentice Hall, Englewood Cliffs, NJ, USA, 1989.

38. Adamec, V., and Calderwood, J.H., "Electrical Conduction in Dielectrics At High Fields", Journal of Physics D: Applied Physics, Vol. 8, 1975, pp. 551-560.

39. Frenkel, J., "On Pre-Breakdown Phenomena In Insulators and Electronic SemiConductors", Physical Review, Vol. 54, October 1938, pp. 647-648.

40. Frederickson, A.R., "Radiation Induced Currents and Conductivity In Dielectrics", IEEE Transactions on Nuclear Science, Vol. NS-24, No. 6, December 1977, pp. 2532-2539.

41. Frederickson, A.R., "Radiation Induced Dielectric Charging", In H.B. Garrett and C.P. Pike (eds.), Space Systems and Their Interactions With The Earth's Space Environment, Vol. 71, American Institute of Aeronautics and Astronautics, Washington, DC, 1980, pp. 386-412.

42. Frankevich, E.L., and Yakovlev, B.S., "Radiation-Induced Conductivity In Organic Solids", International Journal Radiation Physics and Chemistry, Vol. 6, 1974, pp. 281-296.

43. Davis, V.A., Gardner, B.M., McGeary, C.F., Ramos, D.A., Rankin, T.V., and Wilcox, K.G., "EWB User's Reference Manual", Version 3.0, S-Cubed division of Maxwell Laboratories, Inc., La Jolla, CA, USA, 1994.

44. Hastings, Daniel, and Garrett, Henry, "Spacecraft Environmental Interactions", The Press Syndicate of the University of Cambridge, New York, NY, USA, 1996, pg. 213.

45. Tsoulfanidis, Nicholas, "Measurement and Detection of Radiation", 2nd Ed., Taylor \& Francis, Washington, DC, USA, 1995.

46. Davies, W.E.A., "The Dielectric Constant of Fibre Composites", Journal of Physics D: Applied Physics, Vol. 7, 1974, pp. 120-130.

47. Chou, Tsu-Wei, and Ning, Qiong-Gong, "Analytical Characterization of Thermal and Dielectric Properties of Woven Fabric Composites", Proceedings of ICCM10, Whistler, B.C., Canada, August 1995, pp. IV-465 - IV-471. 
48. Knibbs, R.H., and Morris, J.B., "The effects of Fibre Orientation On The Physical Properties of Composites", Composites, Vol. 5, 1974, pp. 209-218.

49. Volpe, Vincent, "Estimation of Electrical Conductivity and Electromagnetic Shielding Characteristics of Graphite/Epoxy Laminates", Journal of Composite Materials, Vol. 14, July 1980, pp. 189-198.

50. Gajda, W.J., "A Fundamental Study of The Electromagnetic Properties of Advanced Composite Materials", Report RADC-TR-78-158, A059029, 1978.

51. Joy, Thomas, and Strieder, William, "Percolation In a Thin Ply of Unidirectional Composite", Journal of Composite Materials, Vol. 13, January 1979, pp. 72-78.

52. Joy, Thomas, Ajmera, Pratul, and Strieder, William, "Effect of Thickness on Single Ply Percolation and Conductivity", Journal of Composite Materials, Vol. 14, April 1980, pp. 130-141.

53. Li, Peter, Strieder, William, and Joy, Thomas, "Random Lattice Electrical Conductivity Calculations For a Graphite/Epoxy Ply of Finite Thickness", Journal of Composite Materials, Vol. 16, January 1982, pp. 53-64.

54. Ruschau, G.R., and Newnham, R.E., "Critical Volume Fractions in Conductive Composites", Journal of Composite Materials, Vol. 26, No. 18, 1992, pp. 27272735.

55. Bent, Aaron, "Active Fiber Composites for Structural Actuation", Ph.D. Thesis, Massachusetts Institute of Technology, Cambridge, MA, January 1997.

56. Larson, Wiley J., and Wertz, James R., "Space Mission Analysis and Design", 2nd Ed., Microcosm, Inc., Torrance, California, and Kluwer Academic Publishers, Dordrecht / Boston / London, USA, 1993, pp. 136-137, 142.

57. International Space Station Report JSC-36186, Revision A, pg. 4-22.

58. DuPont, "Kapton Polyimide Film, Summary of Properties".

59. Lagace, Paul A., Beaumont, Matthew, Brewer, John C., and Varnerin, Carl F., "TELAC Manufacturing Course Notes", Technology Laboratory for Advanced Composites, Massachusetts Institute of Technology, Cambridge, MA, September 1991.

60. Wolf, Edward, "Impact Damage Mechanisms In Several Laminated Material Systems", Masters of Science Thesis, Massachusetts Institute of Technology, Cambridge, MA, June 1992, pp. 36-46. 
61. ASTM, "D257-93 - Standard Test Methods for DC Resistance or Conductance of Insulating Materials", Annual Book of ASTM Standards, Vol. 10.01, ASTM, Philadelphia, PA, 1993, pp. 108-124.

62. ASTM, "D149-94 - Standard Test Method for Dielectric Breakdown Voltage and Dielectric Strength of Solid Electrical Insulating Materials at Commercial Power Frequencies", Annual Book of ASTM Standards, Vol. 10.01, ASTM, Philadelphia, PA, 1994, pp. 19-30.

63. Bent, Aaron, "Active Fiber Composites for Structural Actuation", Ph.D. Thesis, Massachusetts Institute of Technology, Cambridge, MA, January 1997, pp. 74-76.

64. Bent, Aaron, "Active Fiber Composites for Structural Actuation", Ph.D. Thesis, Massachusetts Institute of Technology, Cambridge, MA, January 1997, pp. 70-71.

65. Yacubowicz, J., and Narkis, M., "Dielectric Behavior of Carbon Black Filled Polymer Composites", Polymer Engineering and Science, Vol. 26, No. 22, December 1986, pp. 1568-1573. 


\section{APPENDIX A \\ FORTRAN SOURCE CODE AND USAGE}

\section{INTRODUCTION}

CoDDCA is an easy-to-use computer code which will calculate the charge density, electric field, and voltage distributions through the thickness of the material, as a function of time. All the equations used in this code are given in Chapter 4 . The code can solve the problem in closed form or using a central difference finite difference time stepping routine.

\section{INPUTS}

The inputs required are the material properties requested by the code and entered by the user (use D to represent a power of 10 , hence $1.0 \mathrm{D} 5=1.0 \times 10^{5}$ ), the electric field or voltage boundary conditions, and the environment data. The environmental data is calculated by the Environmental Workbench software. There are two files, one for the electrons and one for the protons. The first column is the energy of the particles in $\mathrm{MeV}$, and the second column is the particle fluence in $\# / \mathrm{cm}^{2}$ for 1 year. There should be 100 data entries. 


\section{ENVIRONMENTAL WORKBENCH DATA}

This section explains how to get the required environment data from EWB. Once EWB is running, to define the desired orbit select 'Orbit' from the 'System Definition' menu; this will open the orbit definition window. Enter the Apogee height, Perigee height, and inclination of the desired orbit, and set the mission duration to 365 days. When done press the 'Update Form' button. To close the window press the 'Dismiss' button. Next open the trapped electron and proton fluences windows. This is done by selecting 'Trapped Electron Fluence' and 'Trapped Proton Fluence' from the 'Environments' menu. The only thing that need to be done in these windows is to set the solar cycle condition to either ' 1 ' for solar maximum or ' 2 ' for solar minimum, followed by pressing the 'Update Form' button. To generate the actual data files, the XY Plot generator windows needs to be opened. This is accomplished by selecting 'XY Plots' from the 'Param-Studies' menu. For simplicity the instructions will be given only for generating the electron fluence data file; the same procedure is followed for the protons. To generate the data, select one of the 'Max Energy Range' cells in the 'Trapped Electron Fluence' window, then press the 'Select' button in the 'Primary Independent Variable' section of the 'XY Plot Specification' window. This sets the first column of the output to be the electron energy. In the same section of the 'XY Plot Specification' window, set the minimum value to 0.01 , the maximum value to 100 , the number of points to 100 , and select a logarithmic spacing. The dependent variable needs to be set; this is done by selecting the 'Fluence' cell to the right of the 'Max Energy Range' cell selected earlier, in the 'Trapped Electron Fluence' window. Now press the 'Select' button in the 'Dependent Variable' section of the 'XY Plot Specification' window to set the second column of the 
output as the electron fluence. Press the 'Calculate' button in the 'XY Plot Specification' window to calculate the data. The data can be viewed by pressing the 'Display' button in the 'XY Plots' window. To save the data press the 'File Table' button in the 'XY Plots' window, and enter a name for the file when asked.

\section{SAMPLE INPUT}

The following is an example of an EWB output file. The output file is in Arial font and comments on the file are to the right of the file and in "Times New Roman" font and contained within quotation marks. For convenience the data has been truncated by removing the middle rows.

$\begin{array}{lll}\begin{array}{l}\text { Energy Column } \\ {[\mathrm{MeV}]}\end{array} & \begin{array}{l}\text { Fluence Column } \\ {[\# / \mathrm{cm} 2]}\end{array} & \\ \text { En_Max_(MEV) } & \text { Ele_Fluence_ } & \text { Row 1, Column heading } \\ & & \\ 9.9999998 \mathrm{E}-03 & 0.0000000 \mathrm{E}+00 & \text { Row } 2 \\ 1.0974987 \mathrm{E}-02 & 0.0000000 \mathrm{E}+00 & \text { Row 3 } \\ 1.2045036 \mathrm{E}-02 & 0.0000000 \mathrm{E}+00 & \text { Row } 4 \\ 1.3219412 \mathrm{E}-02 & 0.0000000 \mathrm{E}+00 & \text { Row } 5 \\ : & \vdots & \vdots \\ \vdots & \vdots & \vdots \\ : & \vdots & \text { Row } 98 \\ 7.5646576 \mathrm{E}+01 & 8.8328767 \mathrm{E}+15 & \text { Row } 99 \\ 8.3022034 \mathrm{E}+01 & 8.8328767 \mathrm{E}+15 & \text { Row } 100 \\ 9.1116585 \mathrm{E}+01 & 8.8328767 \mathrm{E}+15 & \text { Row } 101 \\ 1.0000034 \mathrm{E}+02 & 8.8328767 \mathrm{E}+15 & \end{array}$




\section{SAMPLE SESSION}

The following section is a sample session using the base case data inputs. Everything displayed on the screen are in Arial font, the user inputs are bold, and comments added for explanation are in "Time New Roman" font and contain within quotation marks.

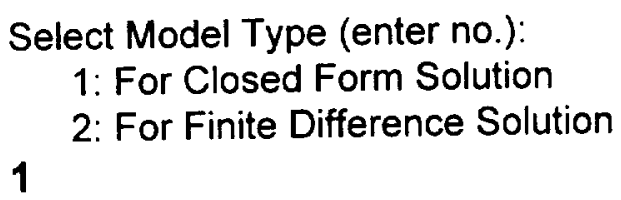

Enter value of boundary condition $1(\mathrm{~V} / \mathrm{m})$
ODO
D sets number to double precision

Enter value of boundary condition $2(\mathrm{~V})$

ODO

Enter EWB electron data filename

e-07k-max

Filenames up to 25 characters are accepted

Enter EWB proton data filename

p-07k-max

Enter the material dark conductivity (1/ohm-m)

1D-10

Enter the dielectric constant

3DO

Enter the dielectric strength $(\mathrm{V} / \mathrm{m})$

$15 D 6$

Enter the material density $(\mathrm{kg} / \mathrm{m} 3)$

1600DO 
Enter the material thickness ( $\mathrm{m}$ )

2.5D-3

Include radiation induced conductivity $(y / n)$ ? Upper or lower case is acceptable n

Include substorms $(\mathrm{y} / \mathrm{n})$ ?

n

Include solar particle events $(y / n) ?$

n

Calculating

\section{OUTPUTS}

The code outputs 2 or 3 ASCII files depending on the user options selected. Two files are always created: 'Incoming Environment.txt' which contains space environment data, and 'CoDDCA Output.txt' which contains the charge density, electric field, and voltage distributions. The third file, 'Conductivity.txt', is only created if high field conductivity is selected. It contains the conductivity and high field conductivity scaling factor distributions. The distributions are output as a set of tables, the first column is the depth and the following columns are the distributions at various times (one time per column). The times are in the first row of the table. The data the columns are separated by tabs.

\section{SAMPLE OUTPUT}

The section contain examples of the output files. As above the output file is in Arial font and comments are to the right and in Times New Roman font, enclosed by 
quotation marks. The tabs between columns is represented using ». Also for clarity only a portion of the output files are present, this is accomplished by omitting the middle rows.

The first file is 'Incoming Environment.txt'. The first 3 rows are used for pertinent data used in the run. The first column of the first table is the energy bins selected by EWB, the second column in the depth an electron penetrates the material for the given energy in column 1 , the third column is the electron flux, which is simply the EWB fluence divided by 365 days $\left(3.153 \times 10^{7}\right.$ seconds). The fourth and fifth columns are the same as the second and third columns but for protons. Following this table of data a second table is displayed containing the incoming charge density rate data. The first column is the depth, the second column in the incoming charge density rate due to the electrons, the third column is the incoming charge density rate due to the protons, and the last column is the total incoming charge density rate.

'Incoming Environment.txt'

\begin{tabular}{|c|c|c|c|c|c|}
\hline Electron data file & & e & & & Row 1 \\
\hline Proton data file & & $p$ & & & Row 2 \\
\hline Density $(\mathrm{kg} / \mathrm{m} 3)$ & & 1600.000000000 & 000000 & & $\begin{array}{l}\text { Row } 3 \\
\text { Row } 4\end{array}$ \\
\hline Energy (MeV) & E Depth $(\mathrm{m})$ & E Flux (\#/m2-s) & P Depth $(m)$ & P Flux (\#/m2-s) & Row 5 \\
\hline $.99999998 E-02$ & $.17253611 \mathrm{E}-04$. & $.00000000 E+00$ & 20449374E-06 . & $.00000000 E+00$ & Row 6 \\
\hline $.10974987 \mathrm{E}-01$ & $.20653364 \mathrm{E}-04$ & $.00000000 E+00$ & $.21585172 \mathrm{E}-06$ & $.00000000 E+00$ & Row 7 \\
\hline $12045036 \mathrm{E}-01$ & $.24469699 E-04$ & $.00000000 E+00$ & $.22839133 E-06$. & $.00000000 E+00$ & Row 8 \\
\hline $.13219412 \mathrm{E}-01$ & $.28734697 \mathrm{E}-04$ & $.00000000 E+00$ & $.24222959 E-06$. & $.00000000 \mathrm{E}+00$ & Row 9 \\
\hline : & : & & & & $:$ \\
\hline : & : & : & : & : & $:$ \\
\hline $.75646576 \mathrm{E}+02$ & $10000000 E+04$ & $.00000000 E+00$ & $11244411 E+00$ & $83095465 E+06$ & \\
\hline $.83022034 E+02$ & $.10000000 E+04$ & $.00000000 E+00$ & $.12894112 E+00$ & $0.68189026 E+06$ & Row 103 \\
\hline $.91116585 E+02$ & $.10000000 E+04$ & $4.00000000 E+00$ & $.14808055 E+00$ & $.48842372 E+06$ & Row 104 \\
\hline $.10000034 \mathrm{E}+03$ & $.10000000 E+04$ & $4.00000000 E+00$ & $.17040497 E+00$ & 0.55502696E+06 & Row 105 \\
\hline
\end{tabular}




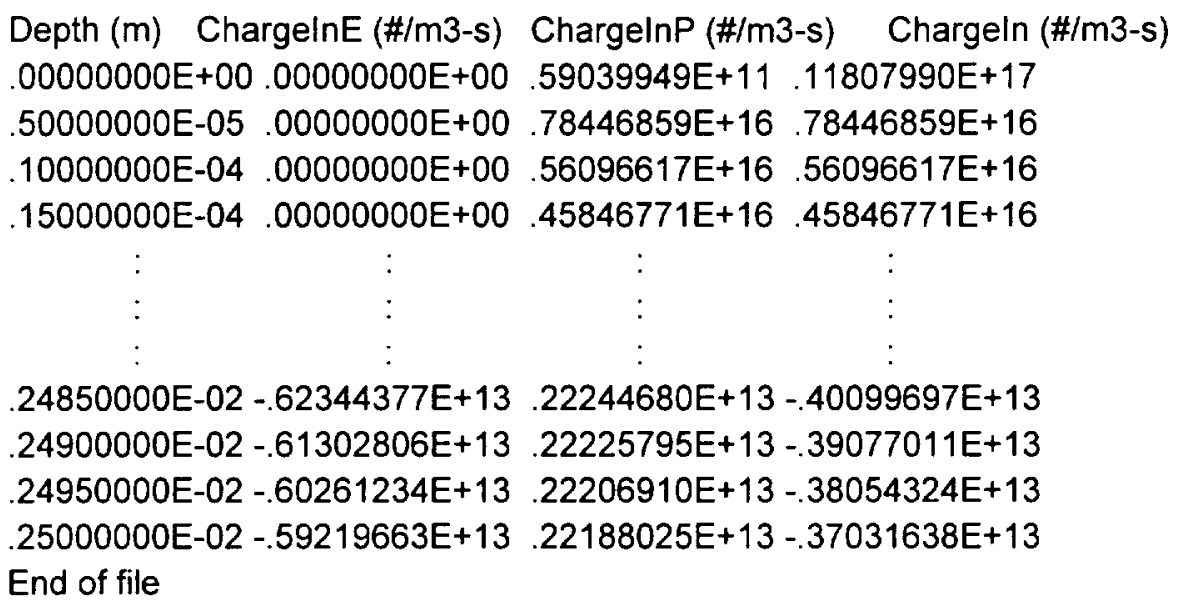

Row 106

Row 107

Row 108

Row 109

Row 110

Row 111

Row 605

Row 606

Row 607

Row 608

Row 609

The second file is 'CoDDCA Output.txt'. The first 25 rows are used for pertinent data used in the run, the actual number of rows used depends on the options selected. The following three tables are of the same format, the first one contains the charge density distribution, the second table contains the electric field distribution, and the last table contains the voltage distribution. The first column in the depth, and the number of columns used for the distribution is always 10 for the closed form solution, but it can vary for the finite difference solution.

$\begin{array}{lll}\text { 'CoDDCA Output.txt' } & \\ \text { Closed Form Solution } & & \text { Row 1 } \\ \text { Conductivity (1/ohm-m) } & 1.000000000000000 \mathrm{E}-010 & \text { Row 2 } \\ \text { Dielectric Constant } & 3.000000000000000 & \text { Row 3 } \\ \text { Dielectric Strength }(\mathrm{V} / \mathrm{m}) & 1.500000000000000 \mathrm{E}+007 & \text { Row 4 } \\ \text { Thickness }(\mathrm{m}) & 2.500000000000000 \mathrm{E}-003 & \text { Row 5 } \\ \text { Density }(\mathrm{kg} / \mathrm{m} 3) & 1600.000000000000000 & \text { Row 6 } \\ \text { Delta }(\mathrm{m}) & 5.000000000000000 \mathrm{E}-006 & \text { Row 7 } \\ \text { Surface Thickness }(\mathrm{m}) & 5.000000000000000 \mathrm{E}-006 & \text { Row 8 } \\ \text { Electron data file } & \mathrm{e}-07 \mathrm{k} \text {-max } & \text { Row 9 } \\ \text { Proton data file } & \mathrm{p}-07 \mathrm{k} \text {-max } & \text { Row 10 } \\ \text { Time Constant }(\mathrm{sec}) & 2.656256339999999 \mathrm{E}-001 & \text { Row } 11 \\ \text { Steady-state time }(\mathrm{sec}) & 1.223251250330985 & \text { Row } 12\end{array}$


Other information goes here depending on options selected

Row 16

Row 17

Row 18

Row 19

Row 20

Row 21

Row 22

Row 23

Row 24

Row 25

Row 26

Steady-state reached

\begin{tabular}{|c|c|c|c|c|}
\hline Column 1 & Column 2 & Column 3 & Column 11 & Row 28 \\
\hline Charge Density & $(\# / \mathrm{m} 3)$ at times (se & indicated at the $t$ & of the column & Row 29 \\
\hline Depth $(m)$ & $.12232513 E+00$ & $.24465025 E+00$ & $\cdots .12232513 E+01$ & Row 30 \\
\hline $.00000000 E+00$ & $.11575041 E+16$ & $.18878397 E+16$ & $.31051397 \mathrm{E}+16$ & Row 31 \\
\hline $.50000000 E-05$ & $.76899251 E+15$ & $.12541940 \mathrm{E}+16$ & $.20629122 E+16$ & Row 32 \\
\hline $10000000 E-04$ & $.54989937 E+15$ & $.89686242 E+15$ & . $.14751693 E+16$ & Row 33 \\
\hline
\end{tabular}

$\begin{array}{lllll}.24850000 \mathrm{E}-02 & -.39308606 \mathrm{E}+12 & -.64110659 \mathrm{E}+12 & \cdots & -.10544992 \mathrm{E}+13 \\ .24900000 \mathrm{E}-02 & -.38306095 \mathrm{E}+12 & -.62475607 \mathrm{E}+12 & \cdots & -.10276057 \mathrm{E}+13 \\ .24950000 \mathrm{E}-02 & -.37303585 \mathrm{E}+12 & -.60840555 \mathrm{E}+12 & \cdots & -.10007122 \mathrm{E}+13 \\ .25000000 \mathrm{E}-02 & -.36301074 \mathrm{E}+12 & -.59205503 \mathrm{E}+12 & \cdots & -.97381869 \mathrm{E}+12\end{array}$

Electric Field $(\mathrm{V} / \mathrm{m})$ at times (sec) indicated at the top of the column

Depth $(\mathrm{m}) \quad .12232513 \mathrm{E}+00$

$.00000000 E+00 \quad .14909883 E+04$

$.50000000 \mathrm{E}-05 \quad .15200387 \mathrm{E}+04$

$.10000000 \mathrm{E}-04 \quad .15399268 \mathrm{E}+04$

$.15000000 \mathrm{E}-04 \quad .15549959 \mathrm{E}+04$
$.24465025 \mathrm{E}+00$

$.24317382 E+04$

$.24791182 E+04$

$.25115549 E+04$

$.25361321 \mathrm{E}+04$
$.12232513 E+01$ .39997500E+04 $40776812 E+04$ $.41310333 E+04$ $.41714581 \mathrm{E}+04$

Row 528

Row 529

Row 530

Row 531

Row 532

Row 533

Row 534

Row 535

Row 536

Row 537

Row 538

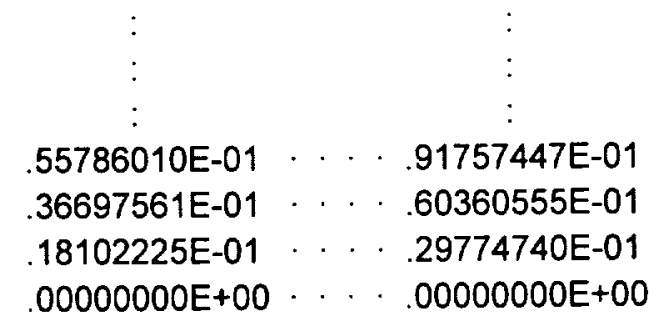

$\begin{array}{ll}.24850000 \mathrm{E}-02 & .34204457 \mathrm{E}-01 \\ .24900000 \mathrm{E}-02 & .22500626 \mathrm{E}-01 \\ .24950000 \mathrm{E}-02 & .11099140 \mathrm{E}-01 \\ .25000000 \mathrm{E}-02 & .00000000 \mathrm{E}+00\end{array}$

$.55786010 \mathrm{E}-01 \cdots .91757447 \mathrm{E}-01$

Row 1032

Row 1033

Row 1034

Row 1035

Row 1036

Row 1037

Voltage (V) at times (sec) indicated at the top of the column

\begin{abstract}
Depth $(\mathrm{m})$ $.12232513 \mathrm{E}+00$

$.00000000 \mathrm{E}+00 \quad .11141258 \mathrm{E}+01$

$.50000000 \mathrm{E}-05 \quad .11065982 \mathrm{E}+01$

$.10000000 E-04 \quad .10989483 E+01$

$.15000000 \mathrm{E}-04 \quad .10912110 \mathrm{E}+01$
\end{abstract}

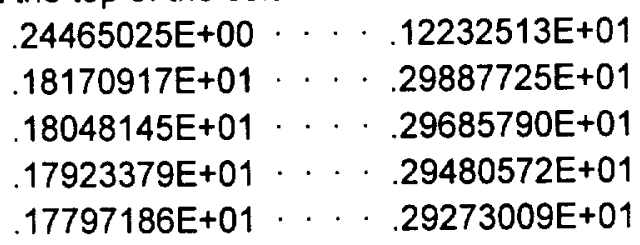

Row 1038

Row 1039

Row 1040

Row 1041

Row 1042 


\section{$.24850000 \mathrm{E}-02 \quad .25350997 \mathrm{E}-06$ \\ $.24900000 \mathrm{E}-02 \quad .11174727 \mathrm{E}-06$ \\ $.24950000 \mathrm{E}-02 \quad .27747850 \mathrm{E}-07$ \\ 25000000E-02 .00000000E+00 \\ End of file}

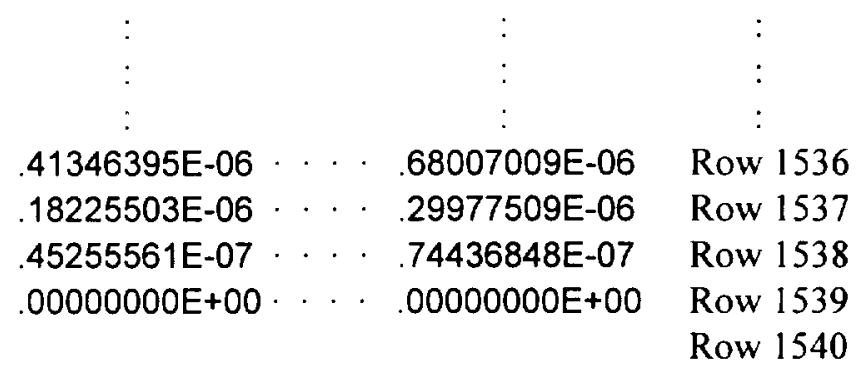

The third file is 'Conductivity.txt'. The first 4 rows are used for pertinent data used in the run, the actual number of rows used depends on the options selected. The following two tables are of the same format, the first one contains the high field conductivity scaling factor distribution, and the second table contains the conductivity distribution. The first column is the depth, and the number of column used for the distributions can vary since this file can only be created when the finite difference solution is used.

'Conductivity.txt'

\begin{tabular}{|c|c|c|c|c|}
\hline \multicolumn{2}{|c|}{ Conductivity (1/ohm-m) } & \multicolumn{2}{|c|}{$1.000000000000000 \mathrm{E}-010$} & Row 1 \\
\hline \multicolumn{2}{|c|}{ Dielectric Constant } & \multicolumn{2}{|c|}{3.000000000000000} & Row 2 \\
\hline \multicolumn{2}{|c|}{ Temperature (degree $\mathrm{C}$ ) } & \multicolumn{2}{|c|}{120.000000000000000} & Row 3 \\
\hline Column 1 & Column? & Column 3 & Column 8 & Row 4 \\
\hline \multicolumn{4}{|c|}{ High Field Conductivity Scaling Factor } & Row 6 \\
\hline Depth $(m)$ & $12200000 E+00$ & $.24400000 E+00$ & $.79300000 E+00$ & Row 7 \\
\hline $.00000000 E+00$ & $.10001039 E+01$ & $.10001694 E+01$ & . . $10002675 E+01$ & Row 8 \\
\hline $.50000000 E-05$ & $.10001059 E+01$ & $.10001727 \mathrm{E}+01$ & ·. $10002727 E+01$ & Row 9 \\
\hline $10000000 \mathrm{E}-04$ & $10001073 E+01$ & $.10001750 \mathrm{E}+01$ & ·. $10002762 E+01$ & Row 10 \\
\hline $15000000 E-04$ & $.10001083 E+01$ & $.10001767 E+01$ & . . 10002789E+01 & Row 11 \\
\hline & : & : & : $\quad$ & : \\
\hline : & : & : & : & : \\
\hline : & : & : & : & : \\
\hline $.24850000 E-02$ & $.10000000 E+01$ & $.10000000 E+01$ & $\cdot .10000000 \mathrm{E}+01$ & Row 505 \\
\hline 24900000 E-02 & $.10000000 \mathrm{E}+01$ & $.10000000 \mathrm{E}+01$ & $.10000000 \mathrm{E}+01$ & Row 506 \\
\hline 24950000 E-02 & $.10000000 E+01$ & $.10000000 \mathrm{E}+01$ & $.10000000 \mathrm{E}+01$ & Row 507 \\
\hline $25000000 \mathrm{E}-02$ & $.10000000 E+01$ & $.10000000 \mathrm{E}+01$ & $.10000000 E+01$ & Row 508 \\
\hline
\end{tabular}




\begin{tabular}{|c|c|c|c|c|}
\hline \multirow{2}{*}{\multicolumn{4}{|c|}{ Conductivity (1/ohm-m) }} & Row 509 \\
\hline & & & & Row 510 \\
\hline Depth $(m)$ & $.12200000 \mathrm{E}+00$ & $.24400000 E+00$ & $\therefore .79300000 \mathrm{E}+00$ & Row 511 \\
\hline $00000000 E+00$ & $.10001039 E-09$ & $.10001694 \mathrm{E}-09$ & $\cdots .10002675 E-09$ & Row 512 \\
\hline $50000000 \mathrm{E}-05$ & $.10001059 E-09$ & $.10001727 \mathrm{E}-09$ & $\cdots .10002727 \mathrm{E}-09$ & Row 513 \\
\hline $10000000 \mathrm{E}-04$ & $.10001073 E-09$ & $.10001750 \mathrm{E}-09$ & $\cdots .10002762 \mathrm{E}-09$ & Row 514 \\
\hline $15000000 E-04$ & $.10001083 E-09$ & $10001767 \mathrm{E}-09$ & $\cdots .10002789 \mathrm{E}-09$ & Row 515 \\
\hline : & : & $:$ & : & $:$ \\
\hline$:$ & : & : & $:$ & $:$ \\
\hline $\begin{array}{c}: \\
4850000 \text { E-02 }\end{array}$ & $:$ & : & & : \\
\hline $\begin{array}{l}24850000 \text { E-02 } \\
24900000 E-02\end{array}$ & $.10000000 \mathrm{E}-09$ & $.10000000 E-09$ & . . . 10000000E-09 & Row 1009 \\
\hline $\begin{array}{l}24900000 \text { E-02 } \\
24950000 E-02\end{array}$ & .10000000 E-09 & $.10000000 \mathrm{E}-09$ & . . 10000000E-09 & Row 1010 \\
\hline $.24950000 \mathrm{E}-02$ & .10000000 E-09 & .10000000 E-09 & $\cdots .10000000$ E-09 & Row 1011 \\
\hline $\begin{array}{l}.25000000 \mathrm{E}-02 \\
\text { End of file }\end{array}$ & .10000000 E-09 & $.10000000 \mathrm{E}-09$ & $\cdots .10000000 \mathrm{E}-09$ & Row 1012 \\
\hline d of file & & & & Row 1013 \\
\hline
\end{tabular}

\section{SOURCE CODE}

! Program CoDDCA, Composite Deep Dielectric Charging Analyzer

! () 1997 Steven A. Czepiela

! Massachusetts Institute of Technology

! 77 Massachusetts Avenue

! Cambridge MA 02139

! Permission to use, copy, and modify this software and its documentation for internal purposes ! only without fee is hereby granted provided that the above copyright notice and this permission ! appear on all copies of the code and supporting documentation. For any use of this software, in ! original or modified form, including but not limited to, adaptation as the basis of a commercial ! software or hardware product, or distribution in whole or part, specific prior permission and/or the ! appropriate license must be obtained from MIT. This software is provided "as is" without any ! warranties whatsoever, either expressed or implied, including but not limited to the implied ! warranties of merchantibility and fitness for a particular purpose. This software is a research ! program and MIT does not represent that it is free of error or bugs or suitable for any particular ! task.

! This program calculates the electric field in a composite material, caused by radiation ! using either a closed form solution for the time or a finite difference solution.

! The initial data comes from the Environmental Workbench software (EWB), and ! is read by the program, the first column is energy [MeV], and the second column is ! fluence [\#/cm2], this data is for 1 year. Radiation induced conductivity and high ! field conductivity may be included. As well as substorms and solar particle events, ! which are modeled by using a scaling factor to increase the amount of incoming ! fluence. The program outputs the electric field, voltage, and charge density every $x$ ! seconds, for an approximate output of 10 different times, in the file called 'CoDDCA ! Output.txt'. The program also outputs the incoming particle data in the file called ! 'Incoming Environment.txt'. If high field conductivity is selected, a third output ! file is created containing the conductivity distribution for the same time intervals ! as mentioned above, in the file called 'Conductivity.txt'. The code terminates when ! the electric field exceeds the dielectric strength of the material, or when the 
! electric field reaches steady state. The surface (Surface) is considered to be $1 / 500$

! of the thickness.

! Variable Definitions

\begin{tabular}{|c|c|}
\hline $\begin{array}{l}\text { ! Again } \\
\text { ! Bad }\end{array}$ & $\begin{array}{l}\text { Logical variable if iterating again on finite difference routine } \\
\text { Logical variable if invalid user input }\end{array}$ \\
\hline ! BC1 & Boundary condition 1 \\
\hline ! BC2 & Boundary condition 2 \\
\hline ! ВСТype & Boundary Condition option selection \\
\hline ! Beta & Frenkel parameter $[\mathrm{C}-\mathrm{N}-\mathrm{m} 2]^{\wedge} 0.5$ \\
\hline ! Break & Logical variable if breakdown has occurred \\
\hline ! BreakTime & Time breakdown occurred [s] \\
\hline ! C1 & First integration constant \\
\hline ! C2 & Second integration constant \\
\hline ! Charge1(501) & Charge density 1 through material [\#/m3] \\
\hline ! Charge2(501) & Charge density 2 through material [\#/m3] \\
\hline ! ChargeE(100) & Incoming electron charge density rate [\#e/m3-s] \\
\hline ! Chargeln(501) & Net incoming particle charge density rate [\#/m3-s] \\
\hline ! ChargelnE(501) & Incoming electron charge density rate [\#e/m3-s] \\
\hline ! ChargelnP(501) & Incoming proton charge density rate [\#p/m3-s] \\
\hline ! ChargeP(100) & Incoming proton charge density rate [\#p/m3-s] \\
\hline ! Charges $(501,20)$ & All charge densities through material $[\# / m 3]$ \\
\hline ! Conduc & Conductivity of the material [1/ohm-m] \\
\hline ! Count & Number of iterations in finite difference solution \\
\hline ! Count2 & Number of iterations in FD solution since the last data output \\
\hline ! C_e & Charge of one electron $[\mathrm{C}]$ \\
\hline ! $\mathrm{C}_{-}^{-} \mathrm{K}$ & Conversion from Celsius to Kelvin \\
\hline ! DārkCon & Dark conductivity of material [1/ohm-m] \\
\hline ! Delta & Radiation induced conductivity exponent \\
\hline DeltaX & Space step $[\mathrm{m}]$ \\
\hline ! DeltaT & Time step [s] \\
\hline ! Density & Density of material [ $\mathrm{kg} / \mathrm{m} 3]$ \\
\hline Depth(501) & Depth into material [m] \\
\hline DepthE(100) & Depth electrons penetrate into material [m] \\
\hline DepthP(100) & Depth protons penetrate into material [m] \\
\hline Dielec & Permittivity of the material [C2/N-m2] \\
\hline DieStr & Dielectric Strength of the material [V/m] \\
\hline Dose & Net dose rate $[\mathrm{rad} / \mathrm{s}]$ \\
\hline DoseE & Electron dose rate $[\mathrm{rad} / \mathrm{s}]$ \\
\hline DoseP & Proton dose rate $[\mathrm{rad} / \mathrm{s}]$ \\
\hline Energy (100) & Energy of particles from EWB [MeV] \\
\hline Error & Value below which the residual is small enough, ie steady-state \\
\hline FDCon(501) & Conductivity [1/ohm-m] \\
\hline FDCons $(501,20)$ & All conductivities [1/omh-m] \\
\hline Field(501) & Electric field through material [V/m] \\
\hline Fields $(501,20)$ & All electric fields through material $[\mathrm{V} / \mathrm{m}]$ \\
\hline FileE & EWB electron fluence filename \\
\hline FileP & EWB proton fluence filename \\
\hline FluenceE(100) & Electron fluence from EWB [\#e/cm2] \\
\hline FluenceP(100) & Proton fluence from EWB [\#p/cm2] \\
\hline FluxE(100) & Electron flux [\#e/m2-s] \\
\hline FluxP(100) & Proton flux [\#p/m2-s] \\
\hline
\end{tabular}


! HFC

! HFCon(501)

! HFCons $(501,20)$

!I, J

! Integral1(501)

! Integral2(501)

! J_MeV

! k

! KRIC

! LETE $(100)$

! LETP(100)

! MDie

! N1

! N2

! Ocount

! Option

! Permittivity

! PCount

! Pie

! QE

! QP

! RadCon

! Rad_Jkg

! Residual

! RIC

! sec_yr

! SPE

! SPEFactor

! SS

! SSTime

! SSFactor

! Surface

! Temp

! Thickness

1 Time

! Times(20)

! TimeConst

! TimeFactor

! Tmp1

! Tmp2

! TmpC

! Voltage(501)

! Voltages $(501,20)$
Logical variable if high field conductivity is included

High field conductivity scaling factor

All high field conductivity scaling factors

Do loop counters

First integration of charge density [V/m]

Second integration of charge density [V]

Conversion factor from joules to mega electron volt $[\mathrm{J} / \mathrm{MeV}]$

Boltzmann's constant [ $\mathrm{J} / \mathrm{K}]$

Coefficient of radiation induced conductivity [s/ohm-m-rad]

Electron LET [MeV-m2/kg]

Proton LET [MeV-m2/kg]

Dielectric constant of material

Number of EWB data points

Number of points used in the space direction (thickness direction)

Counter for the number of outputs

Closed form or finite difference option selection

Permittivity of free space [C2/N-m2]

Number of iterations to be performed before data send to file

Pie, 3.1415...

Electron quality factor

Proton quality factor

Radiation induced conductivity [1/ohm-m]

Conversion factor from Rad to $\mathrm{J} / \mathrm{kg}$ [Rad- $\mathrm{kg} / \mathrm{J}]$

Finite difference residual

Logical variable if radiation induced conductivity is included

Conversion factor from seconds to a year [s/yr]

Logical variable if solar particle events are included

Scaling factor for solar particle events

Logical variable if substorms are included

Time to steady-state

Scaling factor for substorms

Thickness of surface layer [m]

Temperature $[\mathrm{K}]$

Thickness of material [m]

Time [s]

All output times [s]

Charging time constant [1/s]

Scaling factor based on time in closed form solution

Temporary Variable 1

Temporary Variable 2

Character temporary variable

Voltage distribution through material [V]

All voltage distributions through material $[\mathrm{V}]$

\section{! The Main Program}

Implicit Logical (A-Z)

Integer N1, N2, I, J, BCType, Option, Count, Count2, PCount, OCount

Double Precision DeltaX, SSTime, Conduc, Dielec, C_e, MDie, Density, DieStr, Tmp1, \& sec_yr, Tmp2, Energy(100), FluenceE(100), FluenceP(100), FluxE(100), \& FluxP(100), DepthE(100), DepthP(100), ChargeE(100), ChargeP(100), \& Chargeln(501), Thickness, Charge1(501), Depth(501), Field(501), Permittivity, \& TimeConst, Time, TimeFactor, J_MeV, Rad_Jkg, QE, QP, DoseE, DoseP, Dose, \& LETE(100), LETP(100), RadCon, DarkCon, Delta, KRIC, Surface, Voltage(501), \& 
$\mathrm{BC} 1, \mathrm{BC} 2$, Integral1(501), Integral2(501), C1, C2, BreakTime, ChargelnE(501), \& ChargelnP(501), Temp, k, Beta, C_K, Pie, DeltaT, Residual, Error, HFCon(501), \& FDCon(501), Charge2(501), SSFactor, SPEFactor, Charges $(501,20)$, \&

Fields $(501,20)$, Voltages $(501,20)$, Times(20), HFCons $(501,20)$, FDCons $(501,20)$ Character FileE *25, FileP *25, TmpC *1

! Set Constants

$\mathrm{N} 1=100$

$\mathrm{N} 2=500$

Permittivity $=8.8541878 \mathrm{D}-12$

$\mathrm{QP}=10$

$\mathrm{QE}=1$

Pie $=3.14159266 \mathrm{DO}$

Error $=0.1 \mathrm{DO}$

$k=1.380658 D-23$

C_e $=1.60219 \mathrm{D}-19$

sec_yr $=3.153 \mathrm{D} 7$

J_MeV $=1.602 \mathrm{D}-13$

Rad_Jkg $=100$

$\mathrm{cm} 2 \mathrm{~m} 2=10000$

C_K $=273.1500$
! Size of input files for electron \& proton fluences ! Number of depth steps, size of output files ! Permittivity of free space [C2/N-m2] ! Quality factor for protons, used in Dose calculation ! Quality factor for electrons, used in Dose calculation ! Pie

! Value at which the residual is small enough

! Boltzmann's constant [J/K]

! Number of coulombs per electron [C/\#]

! Number of seconds per year [sec/yr]

! Number of joules per MeV [J/MeV]

! Number of rads per $\mathrm{J} / \mathrm{kg}[\mathrm{rad}-\mathrm{kg} / \mathrm{J}]$

! Number of $\mathrm{cm} 2$ per $\mathrm{m} 2$ [cm2/m2]

! Conversion Factor from Celcius to Kelvin

! Set I/O formats

15 Format (E15.8, A1, E15.8, A1, E15.8, A1, E15.8, A1, E15.8)

25 Format (E15.8, A1, E15.8, A1, E15.8, A1, E15.8)

35 Format ('Depth (m)', 20(A1, E15.8))

45 Format (E15.8, 20(A1, E15.8))

! Get user input

Bad $=$. True.

Do While (Bad)

Print *, 'Select Model Type (enter no.):"

Print *., 1: For Closed Form Solution'

Print *, ' 2: For Finite Difference Solution'

Read *, Option

If (Option $<=2$ ) Then

Bad $=$. False.

Else

Print *

Print *, 'Invalid type entered, enter number between 1 and 2'

Print *

End If

End Do

Print *

$\mathrm{Bad}=$. True.

Do While (Bad)

Print *, 'Select type of boundary conditions (enter no.)'

Print *, 'Type Boundary Condition'

Print *, ' $1 \quad E(0) \quad V(0)^{\prime}$

Print *, ' $2 \quad E(0) \quad V(L)^{\prime}$

Print *, ' $3 \quad E(L) \quad V(0)^{\prime}$

Print *, ' $4 \quad E(L) \quad V(L) '$

Print ${ }^{*},{ }^{\prime} 5 \quad \mathrm{~V}(0) \quad \mathrm{V}(\mathrm{L})$ ' 


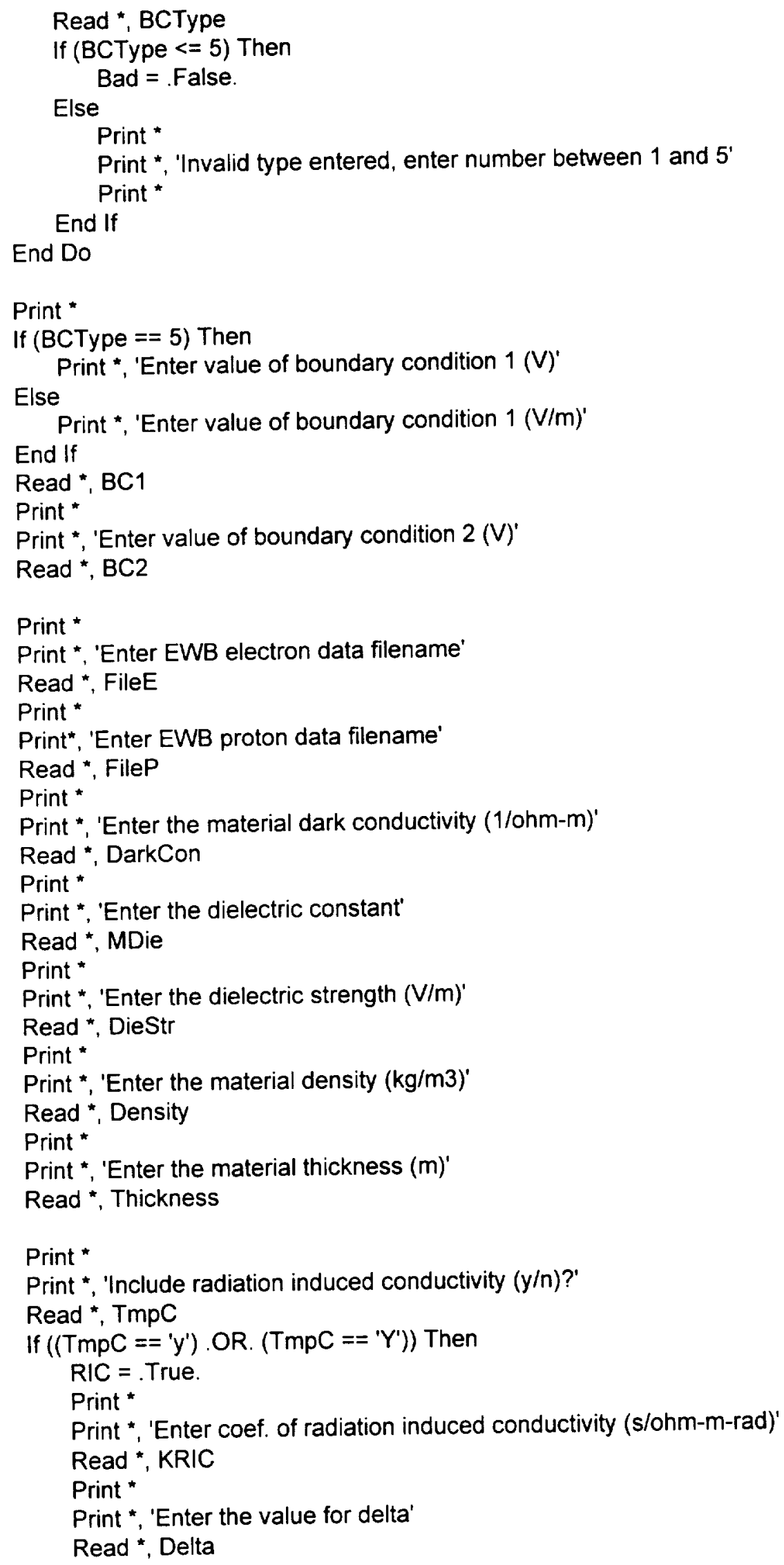




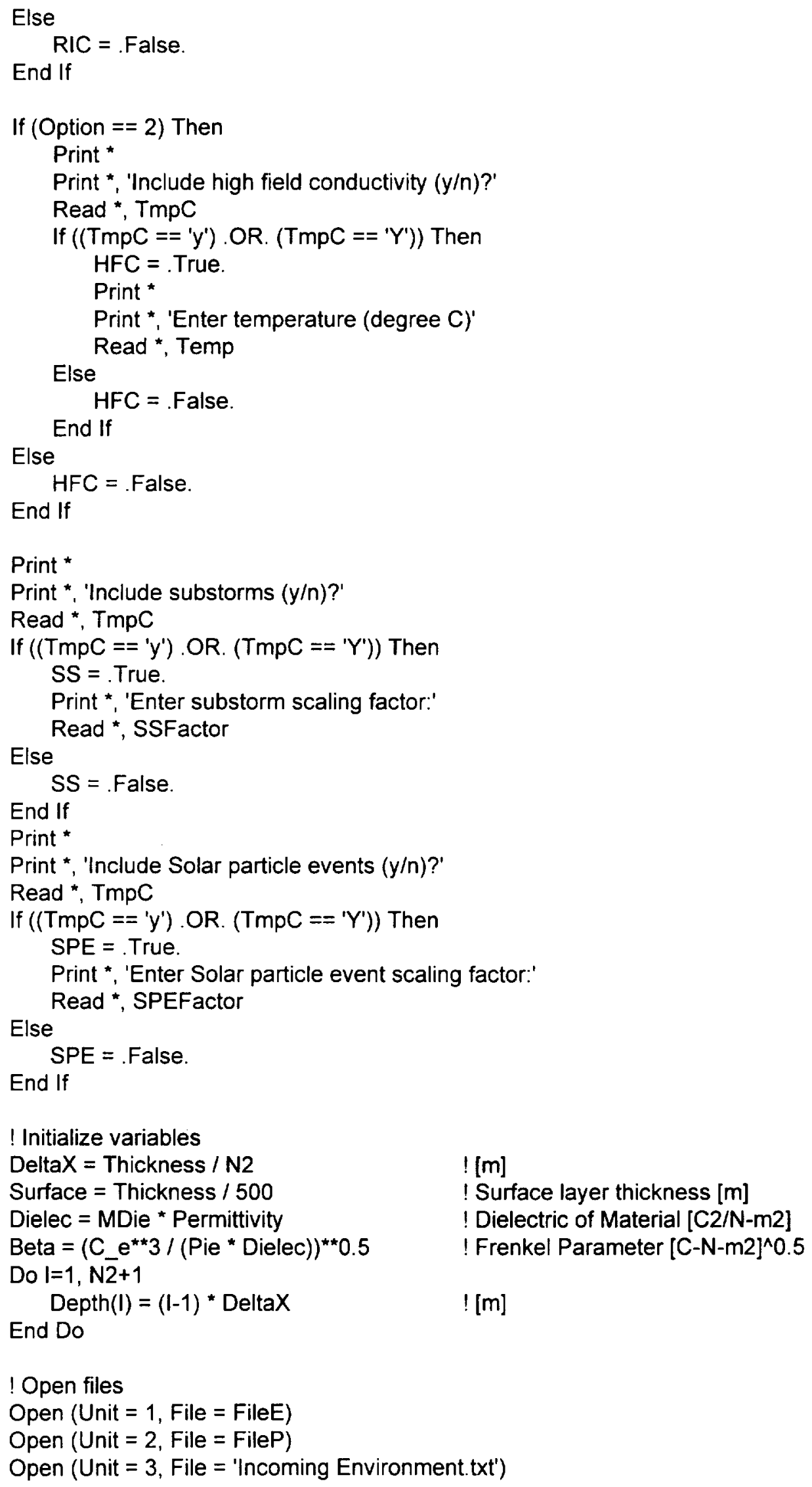




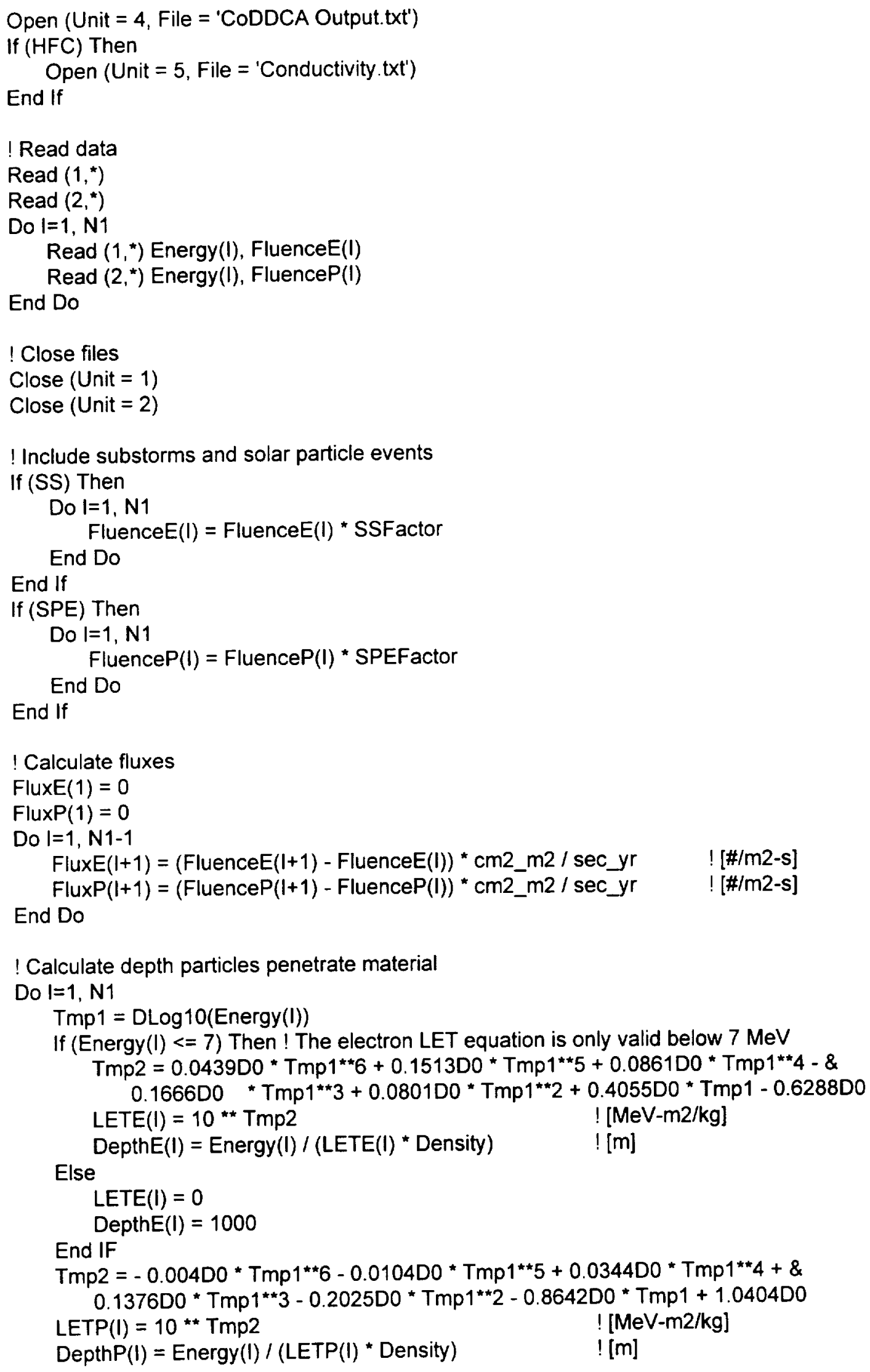


! Calculate dose rate, radiation induced conductivity, net material conductivity

If (RIC) Then

DoseE $=0$

DoseP $=0$

Do I $=1, N 1$

DoseE = DoseE + FluxE(I) * LETE(I) * QE * J_MeV * Rad_Jkg ! [rad/s]

End Do

DoseP $=$ DoseP + FluxP(I) * LETP(I) * QP * J_MeV * Rad_Jkg ! [rad/s]

Dose $=$ DoseE + DoseP

RadCon = KRIC * Dose**Delta ! [1/ohm-m]

Conduc $=$ DarkCon + RadCon $\quad ![1 / 0 h m-m]$

Else

End If

Conduc $=$ DarkCon

! Initialize more variables

TimeConst $=$ Dielec $/$ Conduc

SSTime $=-1$ * TimeConst * DLog(0.01D0) ! [sec]

Temp $=$ Temp + C_K

! [K]

! Get more user input

If (Option $==2$ ) Then

Print *

Print *, 'The estimated time to steady-state is in seconds: ', SSTime

Print *, 'Enter the time increment (seconds): '

Read *, DeltaT

PCount $=\operatorname{Nint}($ SSTime $/(10 *$ DeltaT $))$

End If

! Set-up output files

Write $\left(3,{ }^{*}\right)$ 'Electron data file', Char(9), Char(9), FileE

Write $(3, *)$ 'Proton data file', $\operatorname{Char}(9), \operatorname{Char}(9)$, FileP

Write $\left(3,{ }^{*}\right)$ 'Density $(\mathrm{kg} / \mathrm{m} 3)$ ', $\operatorname{Char}(9)$, Char(9), Density

Write $\left(3,{ }^{*}\right)$

Write $\left(3,{ }^{*}\right)$ 'Energy (MeV)', Char(9), 'E Depth (m)', Char(9), 'E Flux (\#/m2-s)', Char(9), \&

'P Depth (m)', Char(9), 'P Flux (\#/m2-s)'

Do I $=1, \mathrm{~N} 1$

Write $(3,15)$ Energy $(I)$, Char(9), DepthE(I), Char(9), FluxE(I), Char(9), DepthP(I), Char(9), \& FluxP(I)

End Do

If (Option = = 1) Then

Write $\left(4,{ }^{*}\right)$ 'Closed Form Solution'

Else

End If

Write $\left(4,{ }^{*}\right)$ 'Finite Difference Solution'

Write $\left(4,{ }^{*}\right)$ 'Conductivity (1/ohm-m)', Char(9), Char(9), Char(9), Char(9), DarkCon

Write $\left(4,{ }^{\star}\right)$ 'Dielectric Constant', $\operatorname{Char}(9), \operatorname{Char}(9), \operatorname{Char}(9), \operatorname{Char}(9)$, MDie

Write $\left(4,{ }^{*}\right)$ 'Dielectric Strength $(\mathrm{V} / \mathrm{m})$ ', Char(9), Char(9), Char(9), Char(9), DieStr

Write $(4, *)$ 'Thickness (m)', Char(9), Char(9), Char(9), Char(9), Thickness

Write $\left(4,{ }^{\star}\right)$ 'Density $(\mathrm{kg} / \mathrm{m} 3)^{\prime}$, Char(9), Char $(9)$, Char(9), Char(9), Density

Write $\left(4,{ }^{*}\right)$ 'Delta $x(\mathrm{~m})$ ', Char(9), Char(9), Char(9), Char(9), DeltaX

Write $(4, *)$ 'Surface Thickness (m)', Char(9), Char(9), Char(9), Char(9), Surface

Write $\left(4,{ }^{*}\right)$ 'Electron data file', Char(9), Char(9), Char(9), Char(9), FileE 
Write $(4, *)$ 'Proton data file', $\operatorname{Char}(9), \operatorname{Char}(9), \operatorname{Char}(9), \operatorname{Char}(9)$, FileP

Write (4,*) 'Time Constant (sec)', Char(9), Char(9), Char(9), Char(9), TimeConst

Write $\left(4,{ }^{*}\right)$ 'Steady-state time (sec)', Char(9), Char(9), Char(9), Char(9), SSTime

Write $\left(4,{ }^{*}\right)$ 'Rad. Conductivity', Char(9), Char(9), Char(9), Char(9), RIC

If $(\mathrm{RIC})$ Then

Write $(4, *)$ 'Delta', Char(9), Char(9), Char(9), Char(9), Delta

Write $\left(4,{ }^{*}\right)$ 'Coef. Rad. Conductivity (s/ohm-m-rad)', Char(9), Char(9), Char(9), Char(9), KRIC

Write $(4, *)$ 'Electron dose rate (rad/s)', Char(9), Char(9), Char(9), Char(9), DoseE

Write $\left(4,{ }^{*}\right)$ 'Proton dose rate (rad/s)', $\operatorname{Char}(9), \operatorname{Char}(9), \operatorname{Char}(9)$, Char(9), DoseP

Write $\left(4{ }^{\star}\right)$ 'Total dose rate (rad/s)', Char(9), Char(9), Char(9), Char(9), Dose

Write $(4, *)$ 'Radiation induced conductivity (1/ohm-m)', Char(9), Char(9), Char(9), Char(9), \&

RadCon

End If

If (Option $==2$ ) Then

Write $\left(4,{ }^{*}\right)$ 'Time step (sec)', Char(9), Char(9), Char(9), Char(9), DeltaT

Write $\left(4,{ }^{*}\right)$ 'High Field Conductivity', Char(9), Char(9), Char(9), Char(9), HFC

If (HFC) Then

Write (4,*) 'Temperature (degree C)', Char(9), Char(9), Char(9), Char(9), Temp - C_K

Write $(4, *)$ 'Frenkel Parameter (C-N-m2)^0.5', Char(9), Char(9), Char(9), Char(9), Beta

End if End If

If (SS) Then

End If

Write $(4, *)$ 'Substorm scaling factor', Char(9), Char(9), Char(9), Char(9), SSFactor

If (SPE) Then

Write $\left(4,{ }^{*}\right)$ 'Solar Particle Event scaling factor', Char(9), Char(9), Char(9), Char(9), SPEFactor

End If

If (.NOT. RIC) Then

Write $\left(4,{ }^{*}\right)$

Write $\left(4,{ }^{*}\right)$

Write $\left(4,{ }^{*}\right)$

Write $(4, *)$

Write $(4, *)$

Write $(4, *)$

End If

If (Option / = 2) Then

Write $(4, *)$

Write $(4, *)$

End If

If (.NOT. HFC) Then

Write $\left(4,{ }^{*}\right)$

Write $\left(4,{ }^{*}\right)$

End If

If (.NOT. SS) Then

Write $(4, *)$

End If

If (.NOT. SPE) Then

Write $\left(4,{ }^{*}\right)$

End If

If (HFC) Then

Write $\left(5,{ }^{*}\right)$ 'Conductivity (1/ohm-m)', Char(9), Char(9), Char(9), DarkCon

Write $(5, *)$ 'Dielectric Constant', Char(9), Char(9), Char(9), MDie

Write $(5, *)$ 'Temperature (degree C)', Char(9), Char(9), Char(9), Temp - C_K

If (RIC) Then 
Write $(5, *)$ 'Radiation induced conductivity (1/ohm-m)', Char(9), Char(9), Char(9), \& Else Char(9), RadCon

Write $\left(5,{ }^{*}\right)$

End If End If

! Calculate charge density

ChargeE(1) $=-1$ * FluxE(1) / (DepthE(1) + (DepthE(2) - DepthE(1))/2) ! [\#/m3-s]

ChargeP(1) $=$ FluxP(1) $/(\operatorname{Depth} P(1)+(\operatorname{DepthP}(2)-\operatorname{Depth} P(1)) / 2)$

! [\#/m3-s]

Do $I=2, N 1-1$

If (DepthE $(I)==1000)$ Then

$$
\text { ChargeE }(I)=0
$$

Else If (DepthE $(1+1)==1000)$ Then

Else

ChargeE $(I)=-1$ * FluxE(I) / (DepthE(I) - Depth(I-1)) ! [\#/m3-s]

End If

ChargeE $(I)=-1$ * FluxE $(I) /((\operatorname{DepthE}(I+1)-\operatorname{DepthE}(I-1)) / 2) \quad ![\# / m 3-\mathrm{s}]$

ChargeP $(I)=$ FluxP(I) $/((\operatorname{DepthP}(I+1)-\operatorname{DepthP}(I-1)) / 2) \quad ![\# / m 3-\mathrm{s}]$

End Do

If $($ DepthE $(\mathrm{N} 1)==1000)$ Then

ChargeE $(\mathrm{N} 1)=0$

Else

End If

ChargeE(N1) $=-1$ * FluxE(N1) /((DepthE(N1) - DepthE(N1-1))/2) ![\#/m3-s]

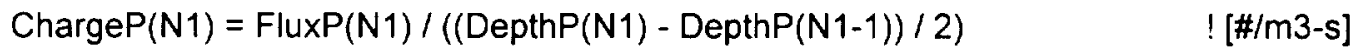

! Calculate surface charge density

Tmp1 $=0$

Tmp2 = 0

Do $l=1, N 1$

If (DepthE(I)< Surface) Then

Tmp1 = Tmp1 + FluxE(I)

End If

! [\#/m2-s]

If (DepthP(I) < Surface) Then

Tmp2 $=$ Tmp2 + FluxP(I) $\quad ![/ m 2-s]$

End If

End Do

Tmp1 = -1 * Tmp1

ChargelnE(1) $=$ Tmp1

Chargeln $\mathrm{P}(1)=\mathrm{Tmp} 2$

Chargeln(1) $=($ Tmp1 + Tmp2 $) /$ Surface $\quad ![\# / m 3-s]$

! Bin charge densities

Do $1=2, N 2+1$

Tmp $1=0.0$

Do $J=1, N 1-1$

If ((DepthE $(\mathrm{J})<\operatorname{Depth}(\mathrm{I}))$.And. (DepthE $(\mathrm{J}+1)>\operatorname{Depth}(\mathrm{I})))$ Then

Call Interp (Depth(I), DepthE(J), DepthE(J+1), ChargeE(J), ChargeE $(J+1)$, Tmp1)

Else If $(\operatorname{Depth} E(J)==$ Depth $(I))$ Then End If

Tmp1 = ChargeE $(\mathrm{J}) \quad$ ! [\#/m3-s]

End Do

Tmp2 $=0$ 


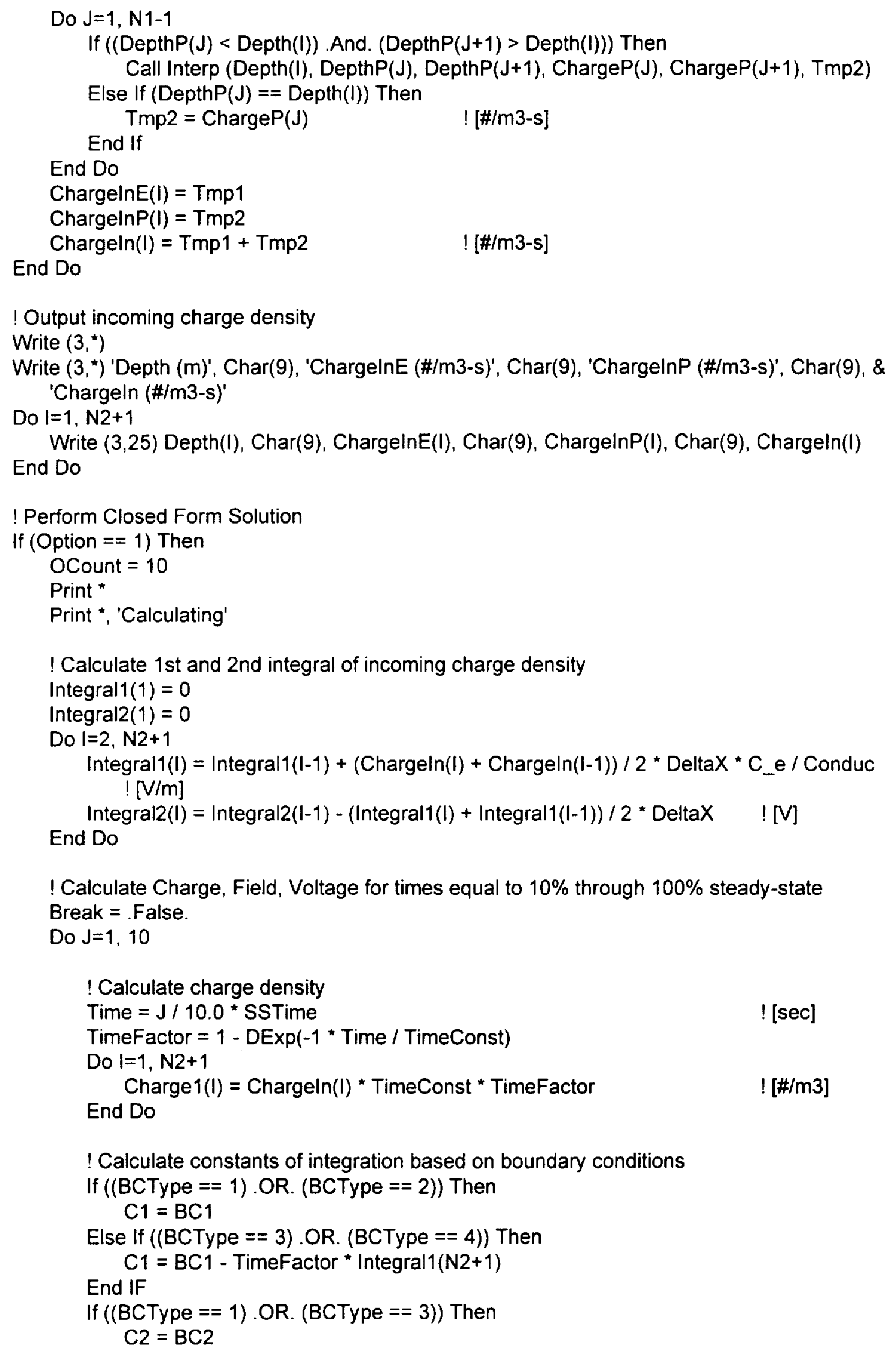

! Calculate Charge, Field, Voltage for times equal to $10 \%$ through $100 \%$ steady-state Break $=$. False.

Do $\mathrm{J}=1,10$

! Calculate charge density

Time $=\mathrm{J} / 10.0^{*}$ SSTime

TimeFactor $=1-\operatorname{DExp}(-1 *$ Time $/$ TimeConst $)$

Do $1=1, N 2+1$

End Do

Charge $1(I)=$ Chargeln $(I) *$ TimeConst * TimeFactor

! [sec]

! [\#/m3]

! Calculate constants of integration based on boundary conditions

If $((B C T y p e==1) \cdot$ OR. $(B C$ Type $==2))$ Then

$$
\mathrm{C} 1=\mathrm{BC} 1
$$

Else If $((B C T y p e==3)$.OR. (BCType $==4))$ Then

End IF

$\mathrm{C} 1=\mathrm{BC} 1-$ TimeFactor * Integral1(N2+1)

If $((B C T y p e==1) \cdot$ OR. $(B C T y p e==3))$ Then

$\mathrm{C} 2=\mathrm{BC} 2$ 


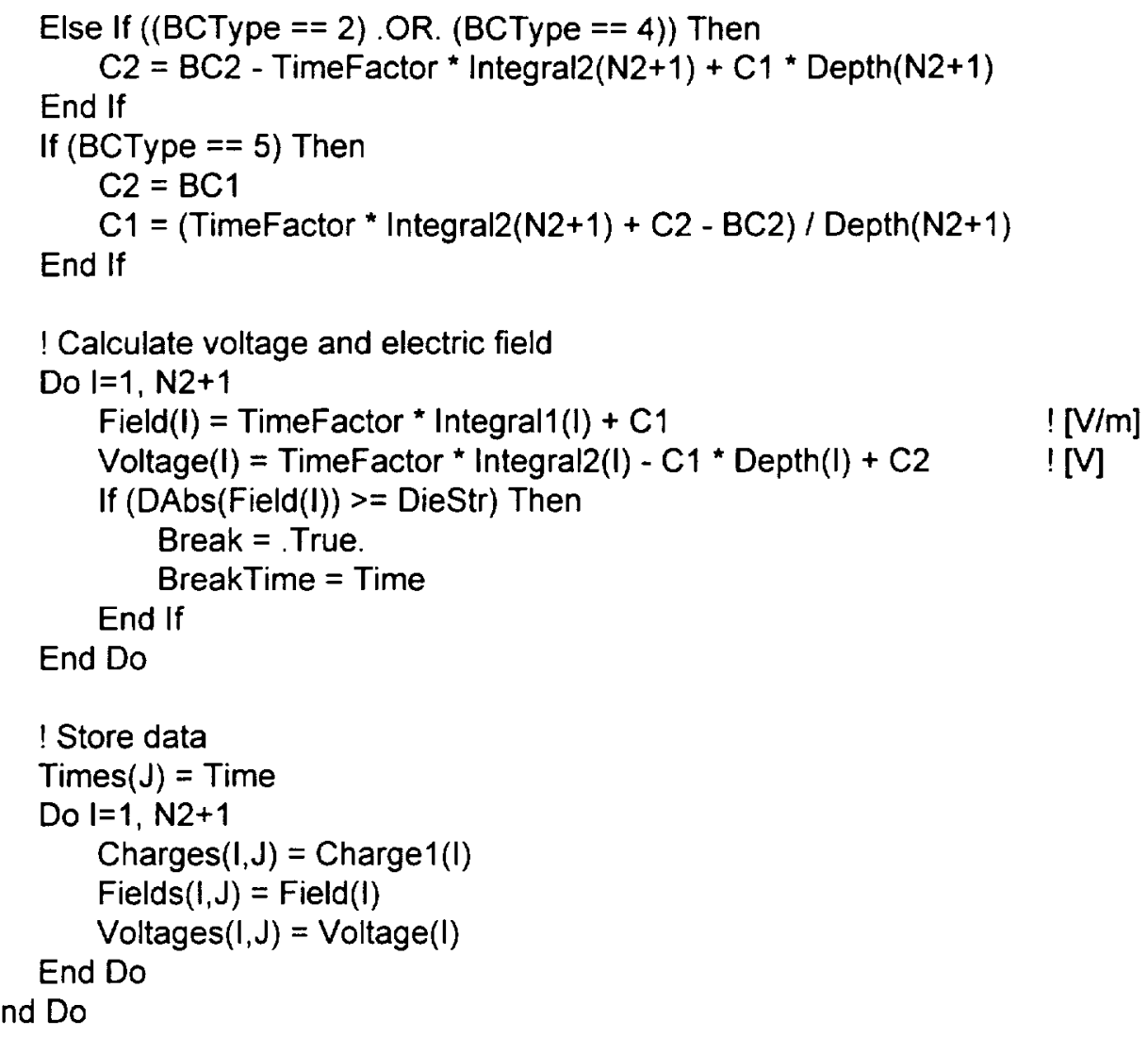

\section{! Perform Finite Difference Solution}

Else If (Option $==2$ ) Then

! First charge density is the input charge density

Do $1=1, N 2+1$

End Do

Charge1 $(I)=$ Chargeln $(I) *$ DeltaT ! [\#/m3]

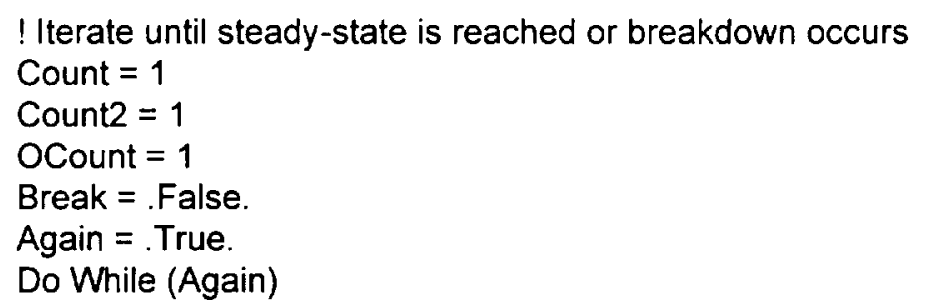

! Calculate 1 st and 2nd integral of charge density

Integral1(1) $=0$

Integral2(1) $=0$

Do $1=2, N 2+1$

Integral1 $(I)=$ Integral1 $(I-1)+($ Charge $1(I)+$ Charge1(I-1)) $/ 2 *$ DeltaX *C_e / Dielec ! $[\mathrm{V} / \mathrm{m}]$

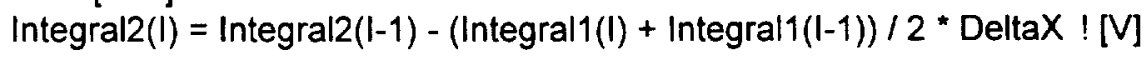
End Do

! Calculate constants of integration based on boundary conditions

If $((B C$ Type $==1)$. OR.$(B C T y p e==2))$ Then 


$$
\mathrm{C} 1=\mathrm{BC} 1
$$

Else If $((B C T y p e==3)$. OR. $(B C T y p e==4))$ Then

End IF

$$
\mathrm{C} 1=\mathrm{BC} 1-\text { Integral1 }(\mathrm{N} 2+1)
$$

If $((B C$ Type $==1) \cdot$ OR. $(B C$ Type $==3))$ Then

$$
\mathrm{C} 2=\mathrm{BC} 2
$$

Else If $((B C T y p e==2)$.OR. $(B C T y p e==4))$ Then

\section{End if}

$$
\mathrm{C} 2=\mathrm{BC} 2-\text { Integral2(N2+1) + C1 * Depth(N2+1) }
$$

If (BCType $==5$ ) Then

End If

$$
\begin{aligned}
& \mathrm{C} 2=\mathrm{BC} 1 \\
& \mathrm{C} 1=(\text { Integral2 }(\mathrm{N} 2+1)+\mathrm{C} 2-\mathrm{BC} 2) / \operatorname{Depth}(\mathrm{N} 2+1)
\end{aligned}
$$

! Calculate voltage and electric field

Do I $=1, N 2+1$

Field $(I)=$ Integral $1(I)+C 1$

Voltage $(I)=$ Integral $2(I)-C 1 * \operatorname{Depth}(I)+C 2$

If $($ DAbs (Field $(I))>=$ DieStr $)$ Then

Break $=$. True.

End If

Again $=$.False .

End Do

! Calculate high field conductivity

If (HFC) Then

Do I $=1, \mathrm{~N} 2+1$

$$
\begin{aligned}
& \text { HFCon }(1)=(2+\text { DCosh }(\text { Beta * DSqrt(DABS }(\text { Field }(I))) /(2 \text { * } k \text { *Temp }))) / 3 \\
& \text { If }(\text { RIC }) \text { Then }
\end{aligned}
$$

$$
\text { Else } \mathrm{FDCon}(I)=\text { DarkCon * HFCon }(I)
$$$$
\text { FDCon }(I)=\text { DarkCon * HFCon }(I)+\text { RadCon }
$$

\section{End Do}

\section{End If}

! Calculate charge density

If (HFC) Then

Charge2 $(1)=$ Charge $1(1)+($ Chargeln $(1)-($ FDCon $(1) /$ Dielec * \& Charge 1(1) $)-($ Field $(1) *(($ FDCon $(2)-$ FDCon(1)) $/($ DeltaX $)))) *$ DeltaT

Charge2 $(\mathrm{N} 2+1)=$ Charge1 $(\mathrm{N} 2+1)+($ Chargeln $(\mathrm{N} 2+1)-($ FDCon $(\mathrm{N} 2+1) /$ Dielec * \& Charge1(N2+1)) $-($ Field $(\mathrm{N} 2+1) *((\mathrm{FDCon}(\mathrm{N} 2+1)-\mathrm{FDCon}(\mathrm{N} 2)) /($ DeltaX $)))) *$ \& DeltaT

Do I $=2, \mathrm{~N} 2$

Charge2 $(I)=$ Charge $1(I)+($ Chargeln $(I)-($ FDCon $(I) /$ Dielec * Charge1(I)) $-\&$

End Do

$($ Field $(I) *(($ FDCon $(I+1)-$ FDCon $(I-1)) /(2 *$ DeltaX $)))) *$ DeltaT

Else

Do $1=1, N 2+1$

End Do

Charge2 $(I)=$ Charge $1(I)+($ Charge $\ln (I)-($ Conduc $/$ Dielec * Charge1(I)) * DeltaT

End If

! Calculate Residual 


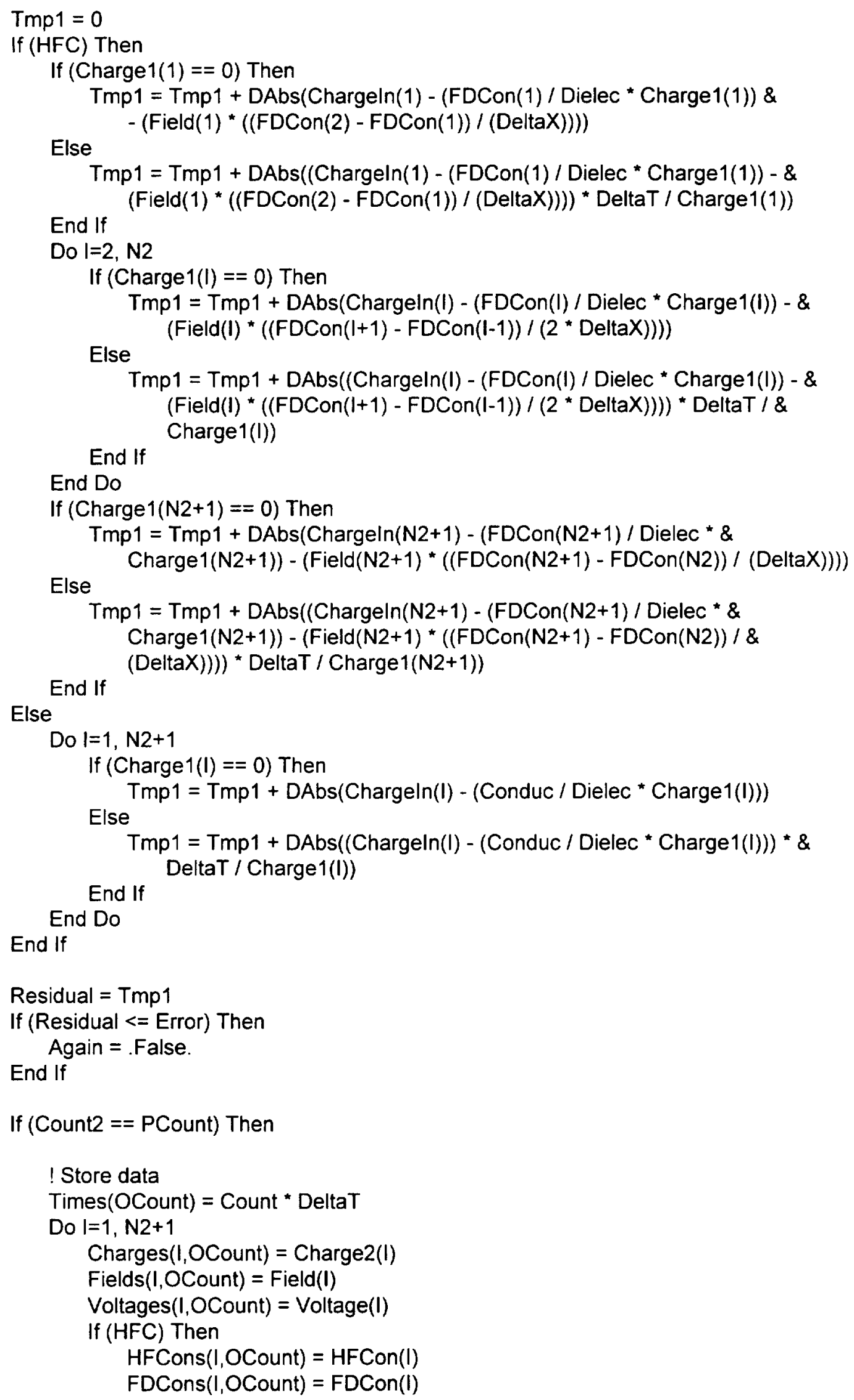




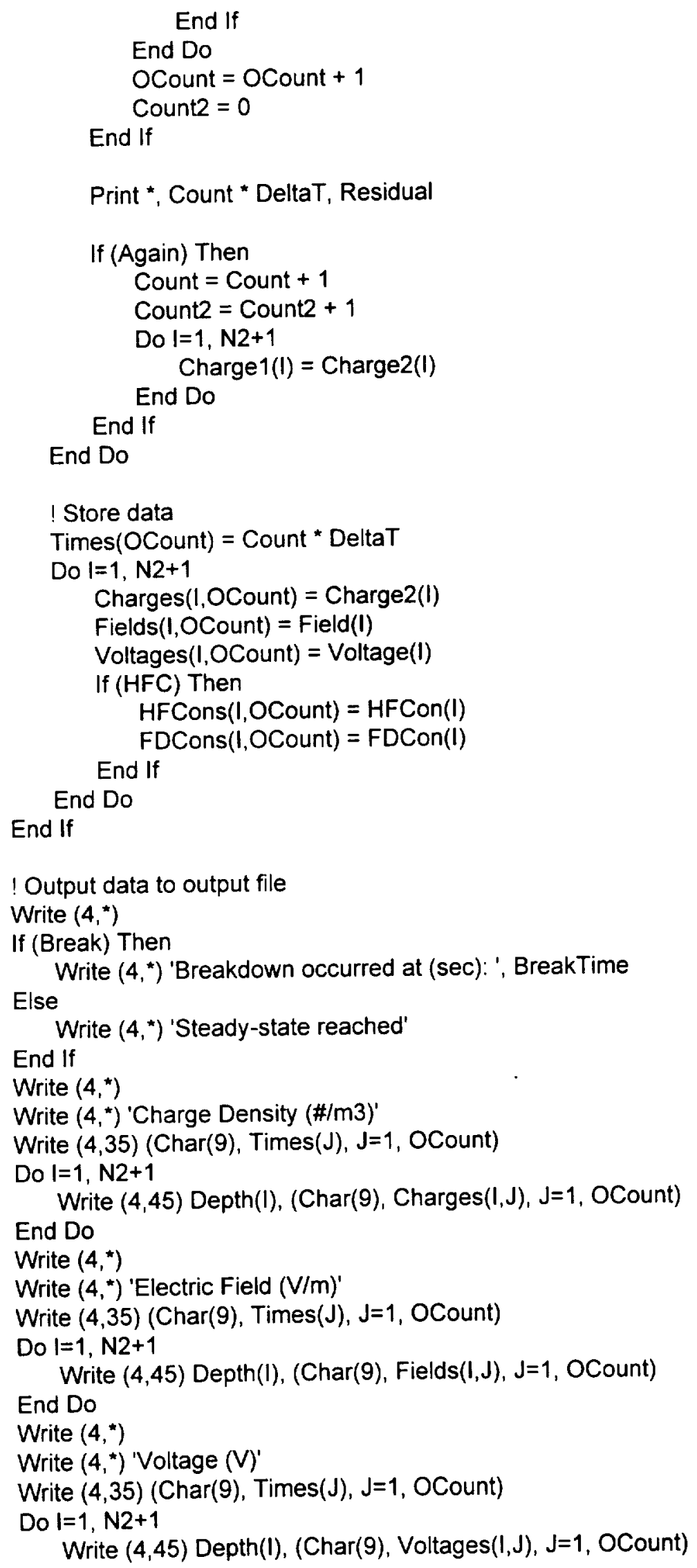

! Output data to output file 


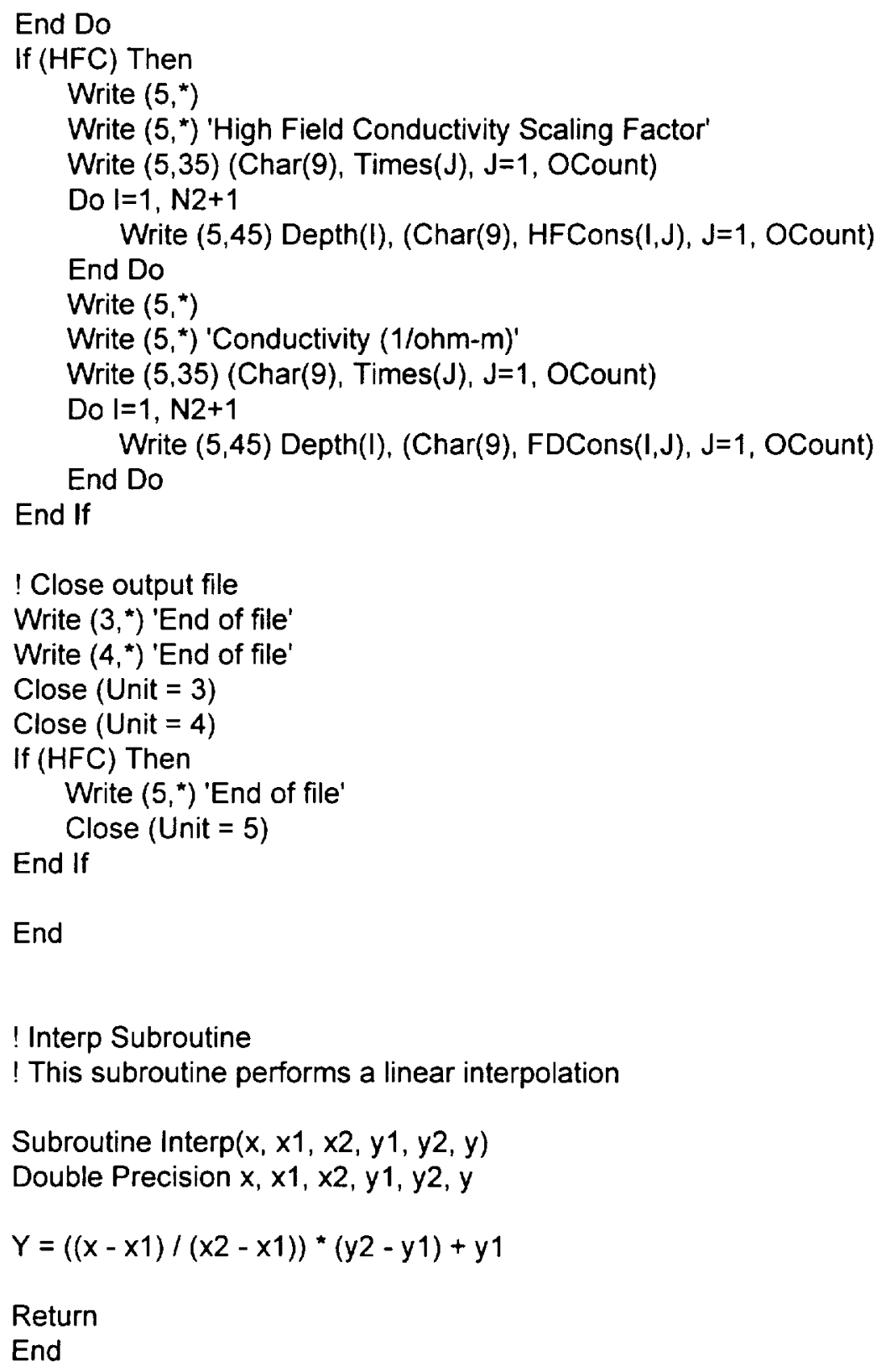

End 


\section{APPENDIX B}

\section{CODE RUN PLOTS}

This appendix contains the output plots from CoDDCA for high field conductivity, radiation induced conductivity, and specific cases where the results differ significantly from the base case. The cases that were significantly different are those from the sensitivity studies of the thickness, density, geomagnetic substorms, and solar particle events. The plots included are the charge density distribution, electric field distribution, and voltage distribution as functions of depth into the material and time.

The data used for inputs can be found by referring to Chapter 5 , and Tables 5.1, 5.2, 5.4, 5.5, 5.6, and 5.7. 


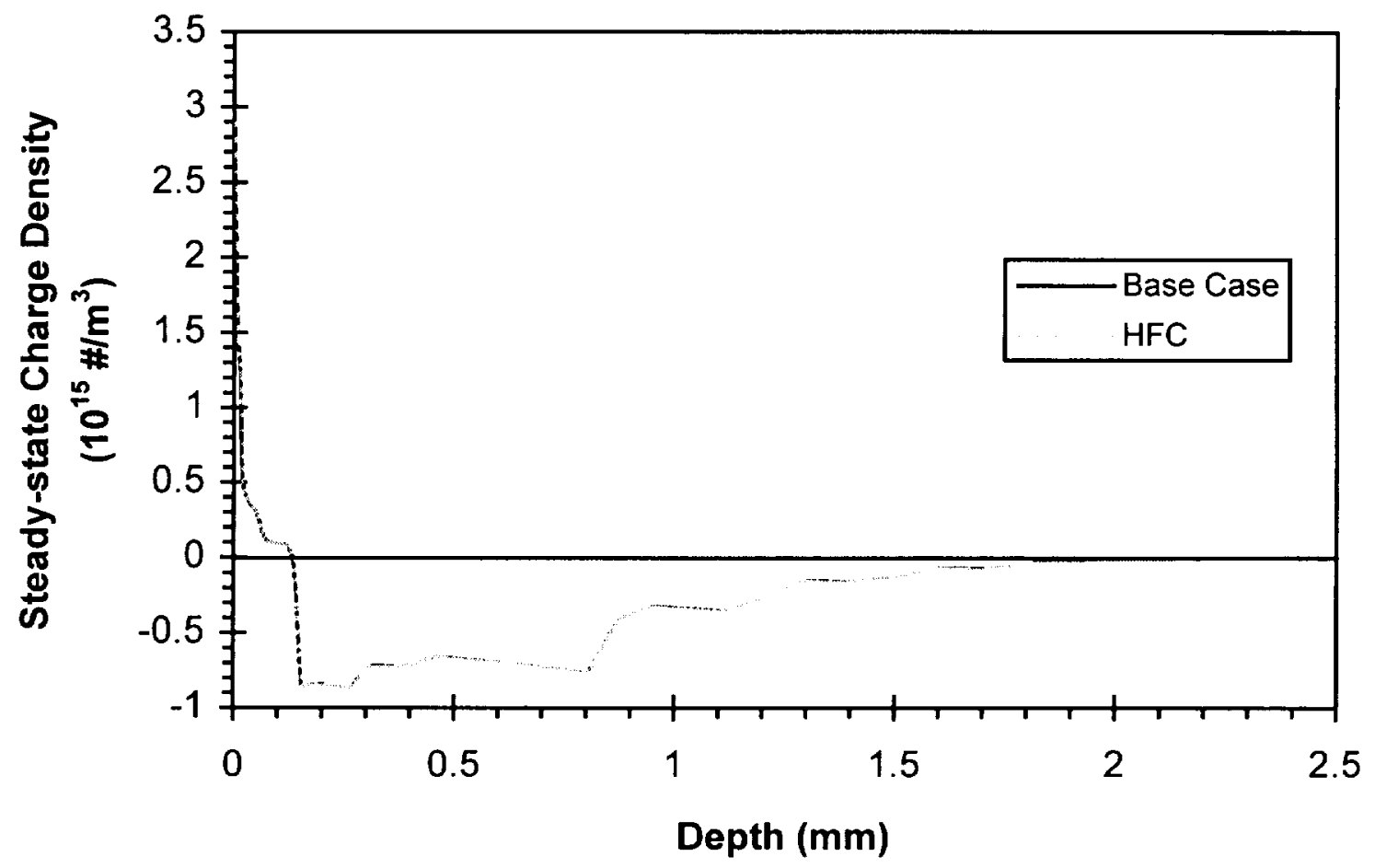

Figure B.1: High field conductivity charge density comparison with base case 


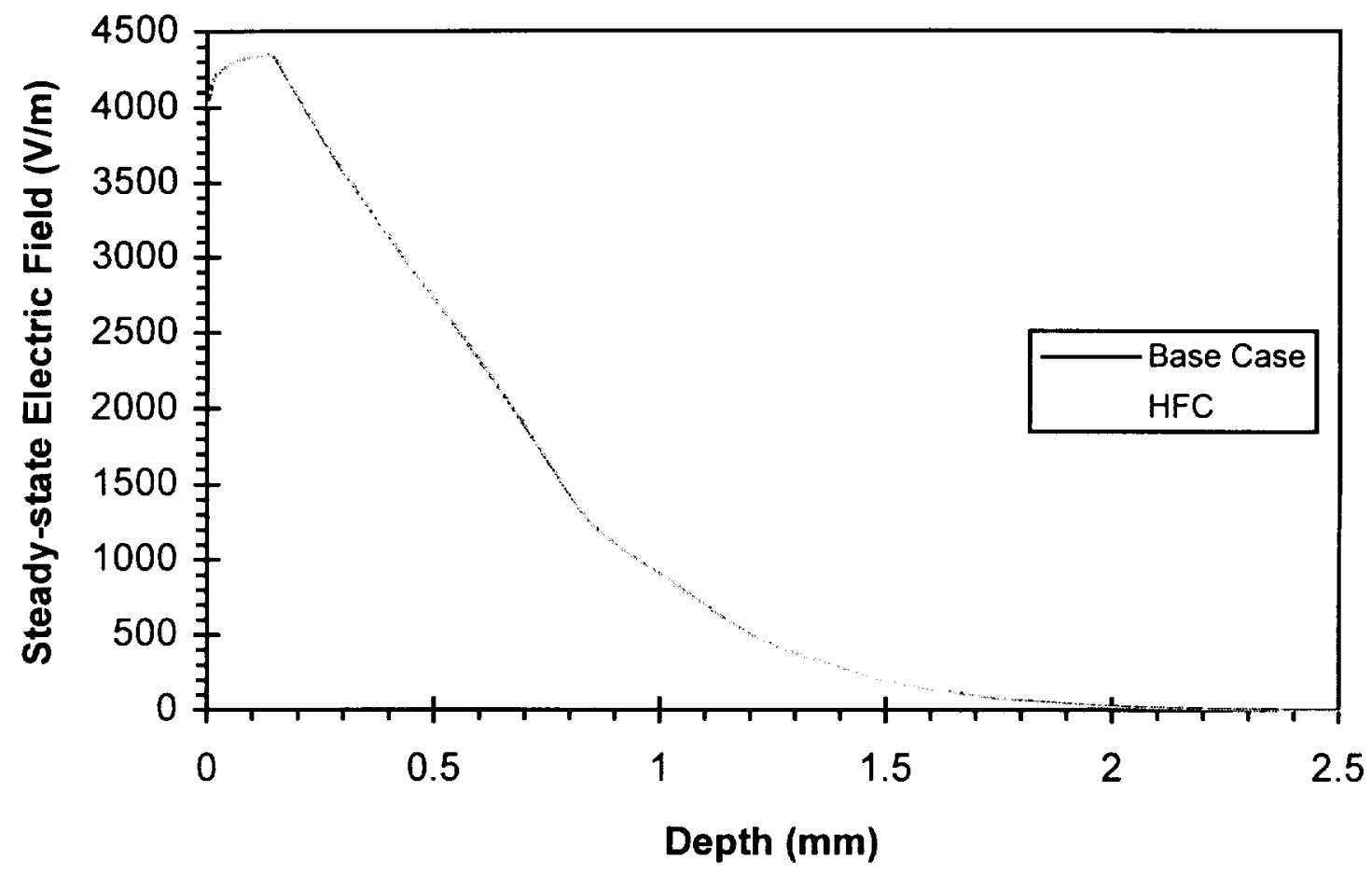

Figure B.2: High field conductivity electric field comparison with base case 


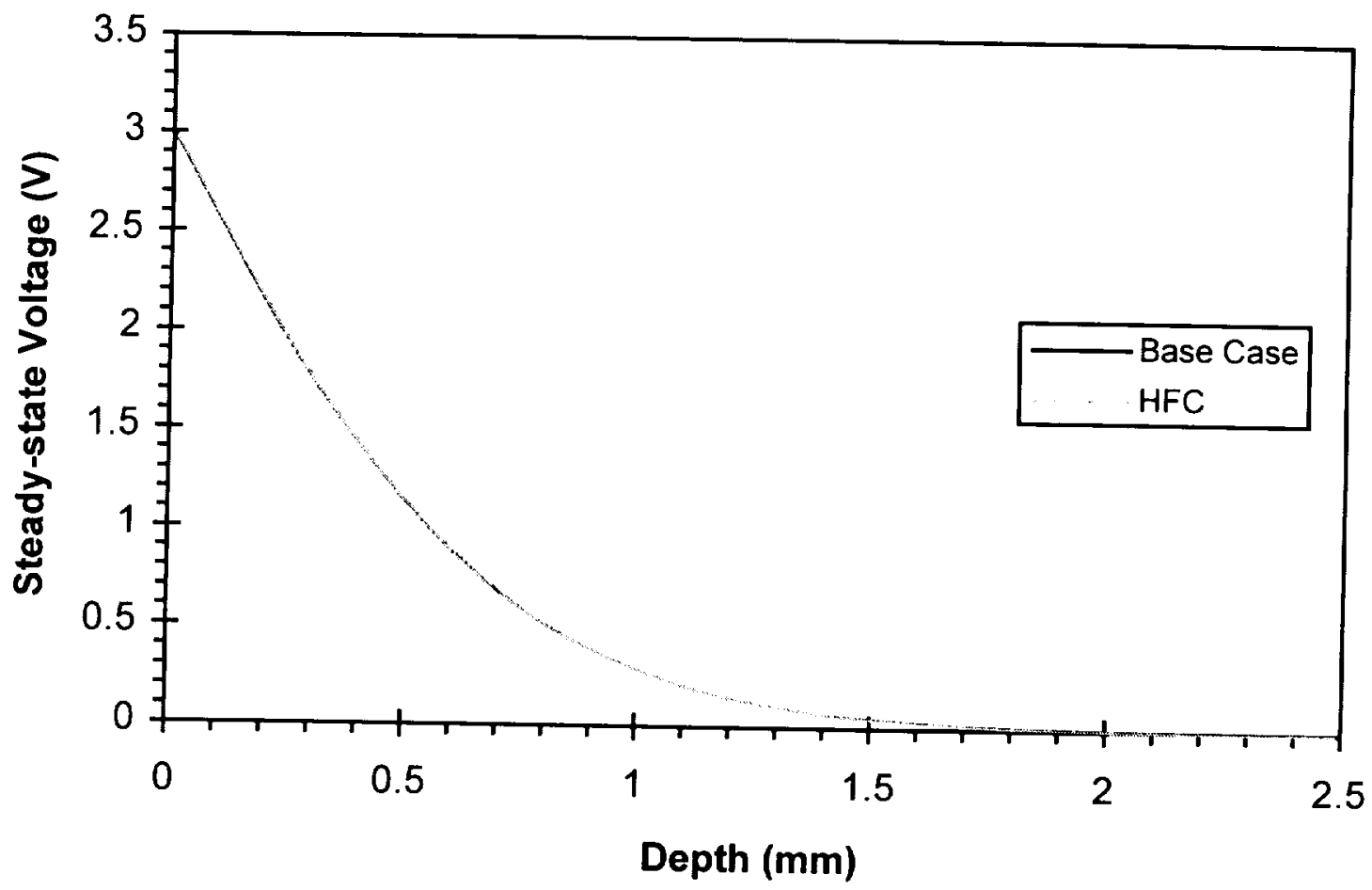

Figure B.3: High field conductivity voltage comparison with base case 


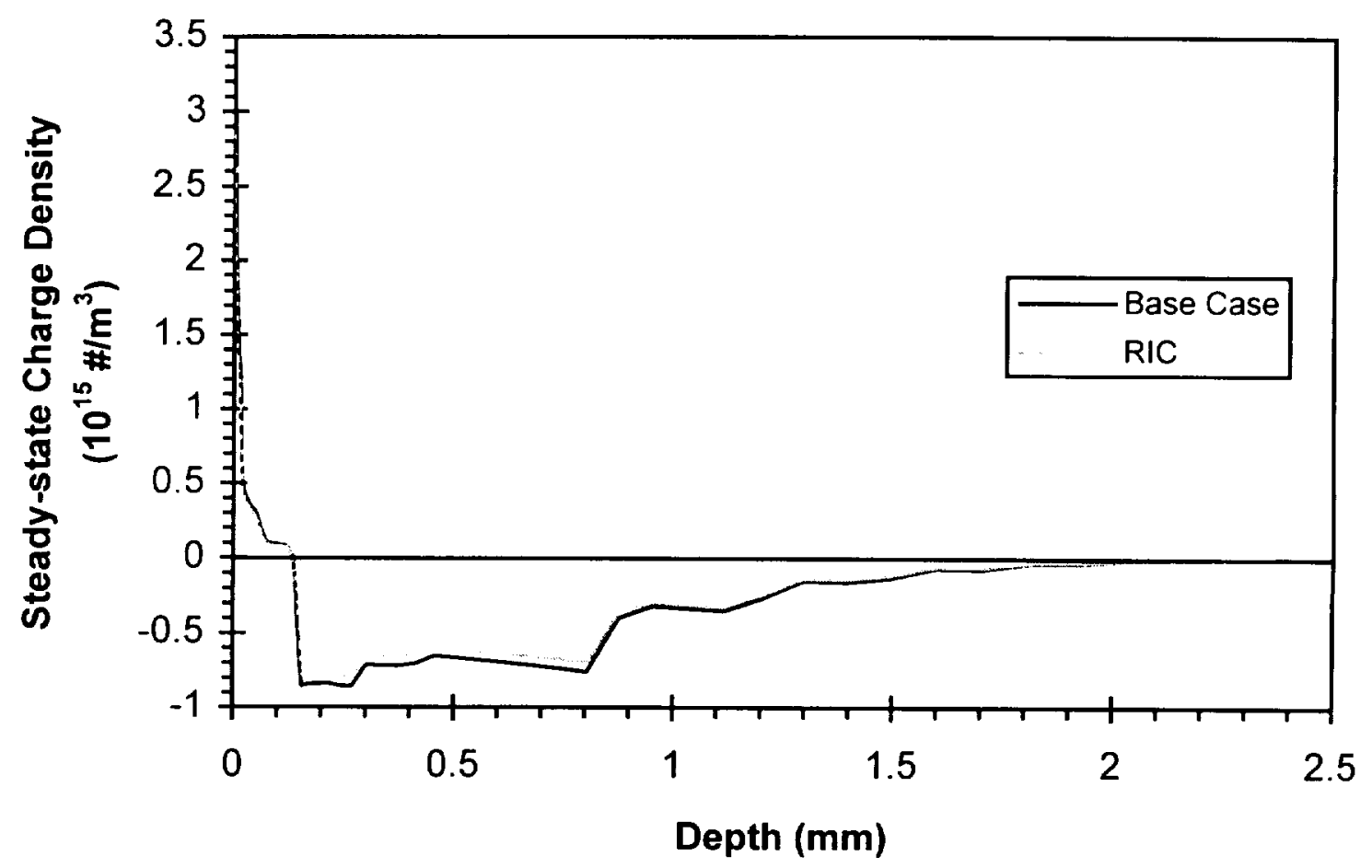

Figure B.4: Radiation induced conductivity charge density comparison with base case 


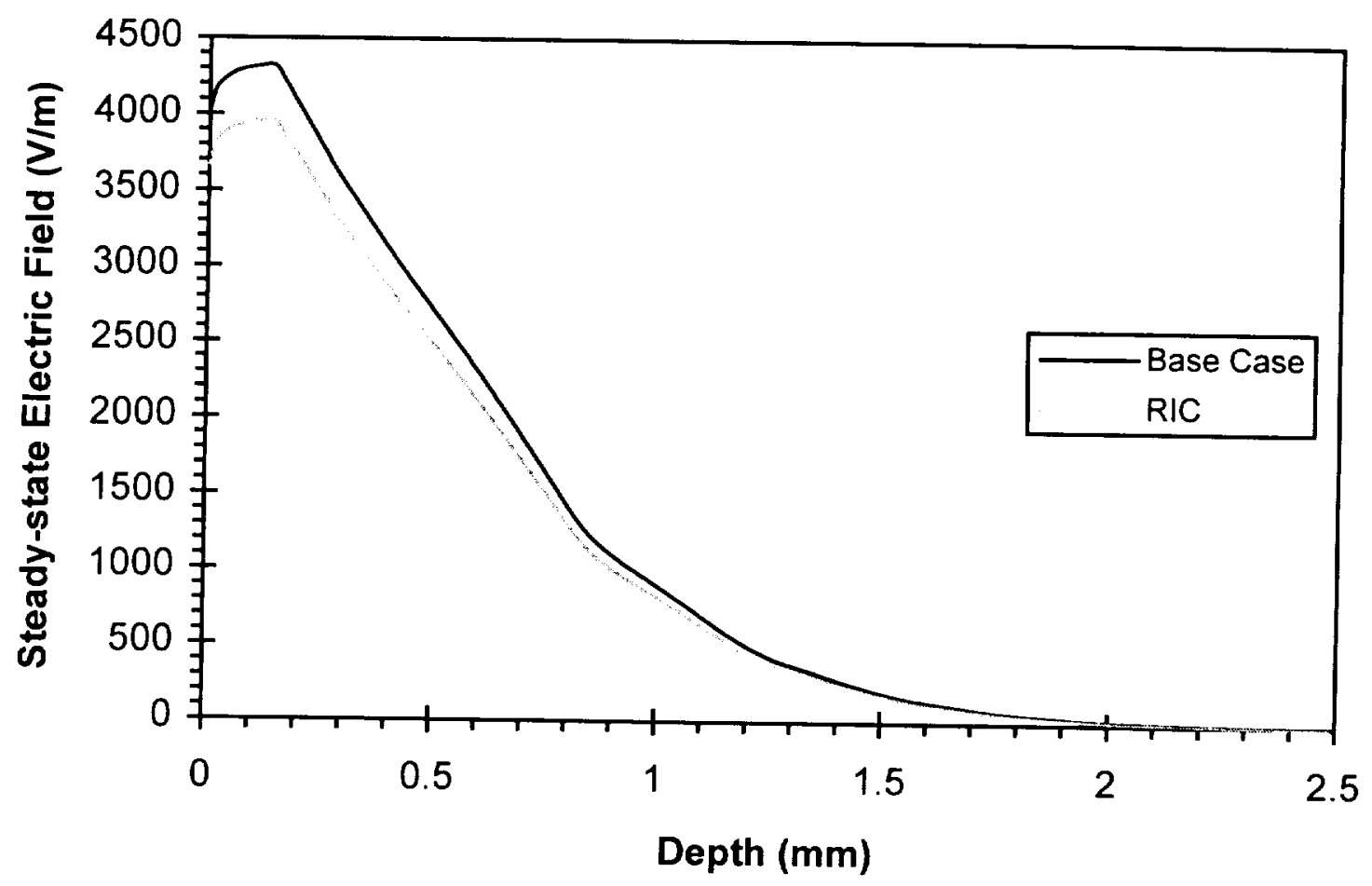

Figure B.5: Radiation induced conductivity electric field comparison with base case 


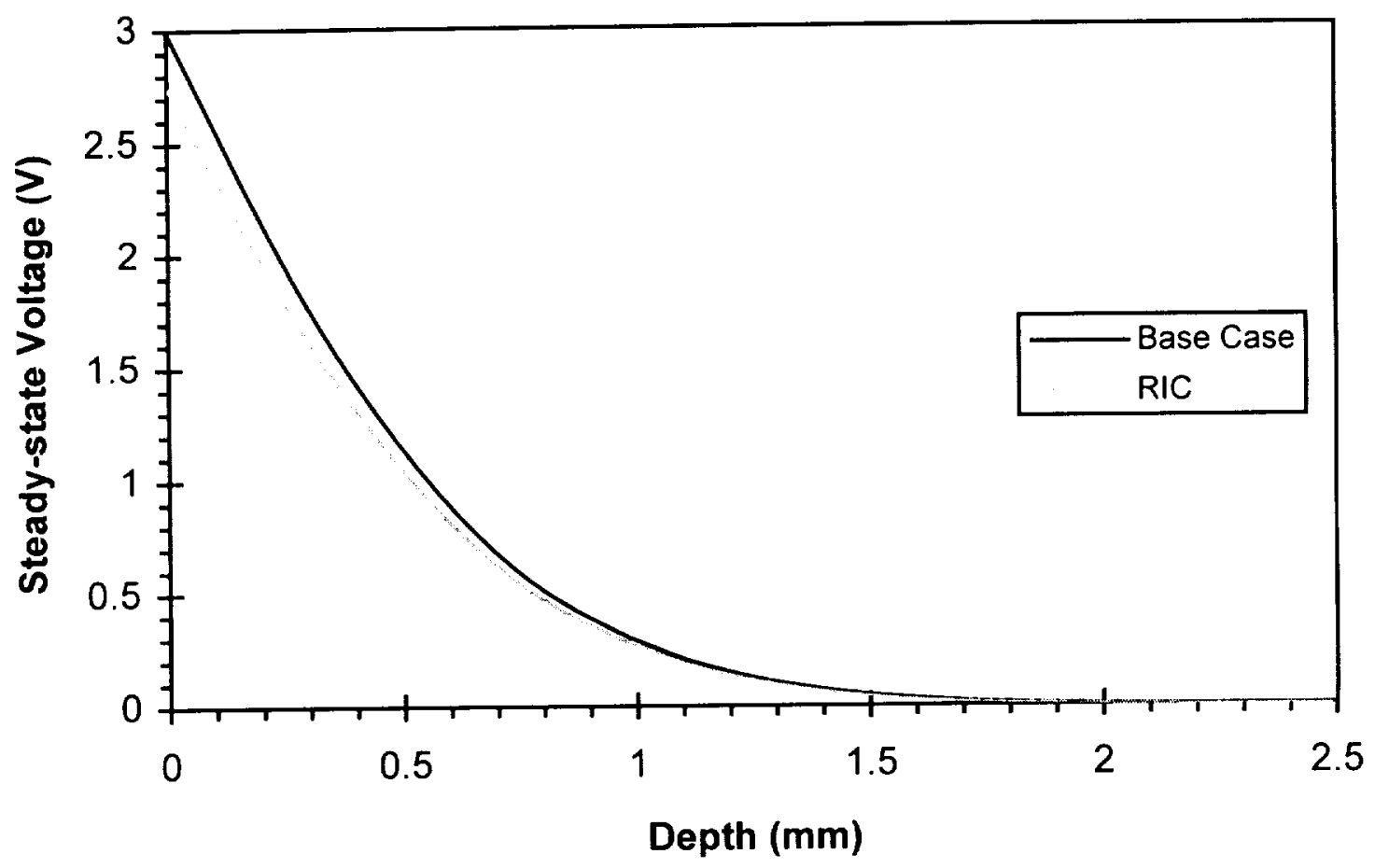

Figure B.6: Radiation induced conductivity voltage comparison with base case 


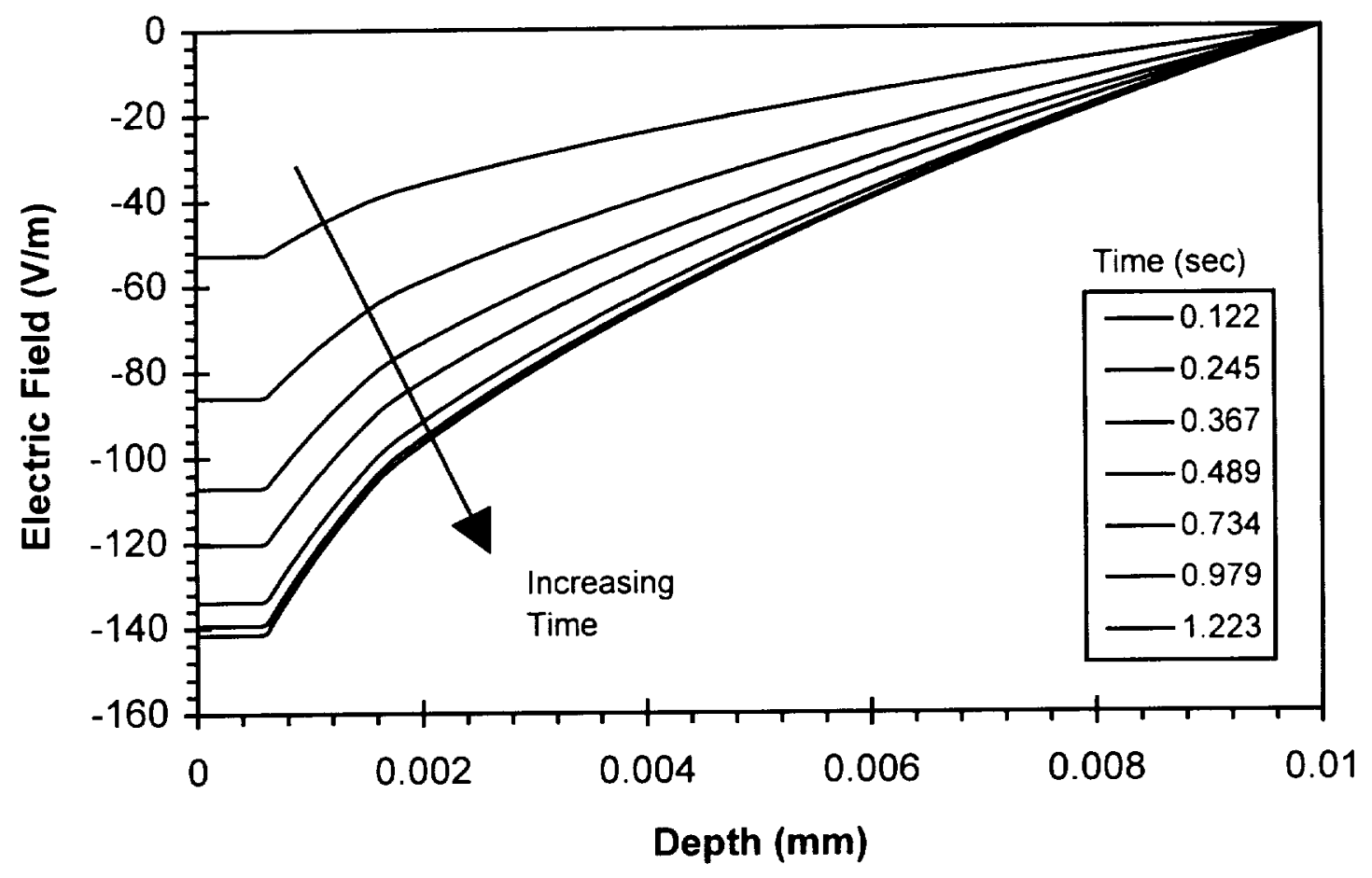

Figure B.7: Electric field of base case with thickness of $0.01 \mathrm{~mm}$ 


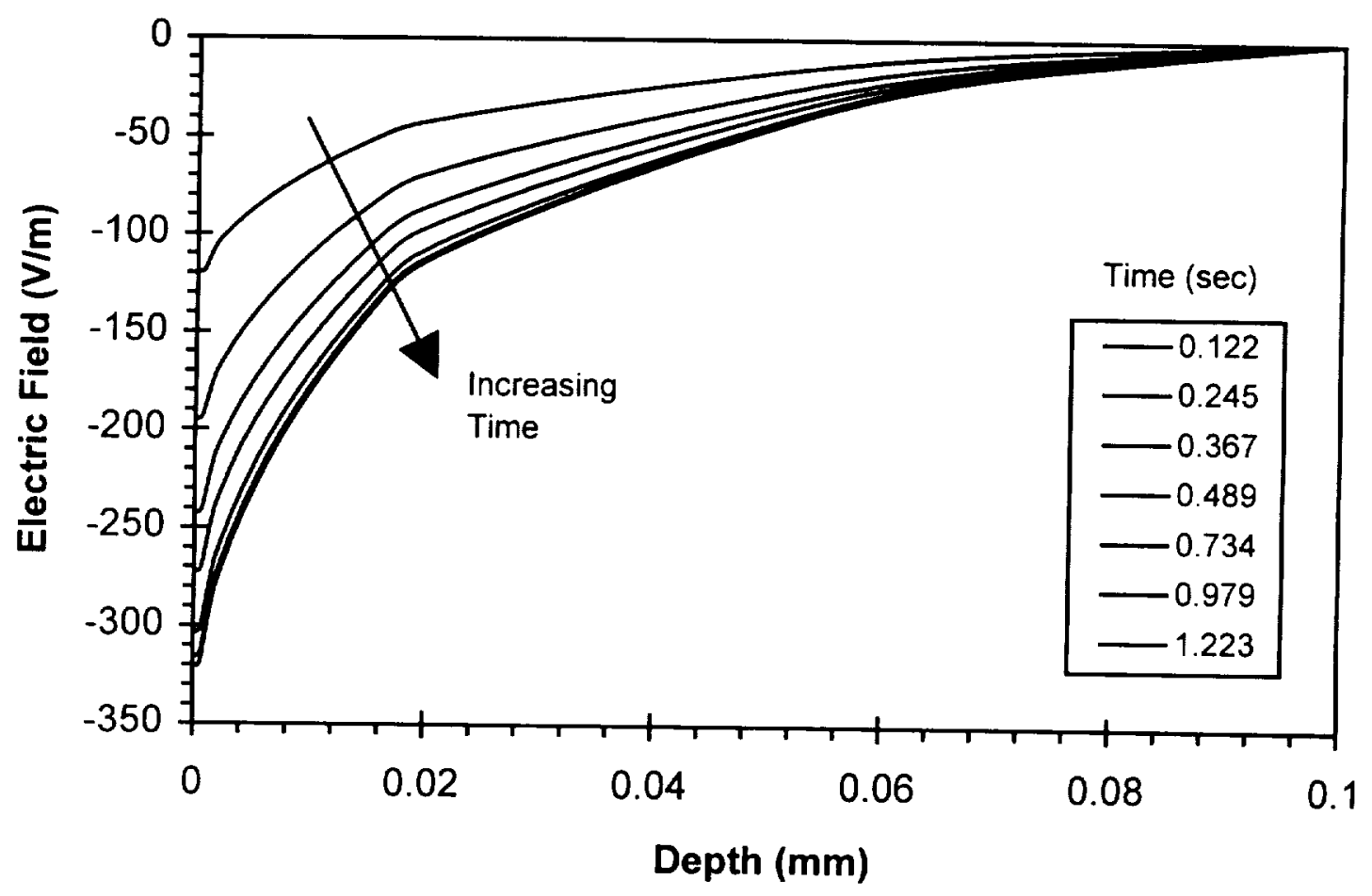

Figure B.8: Electric field of base case with thickness of $0.1 \mathrm{~mm}$ 


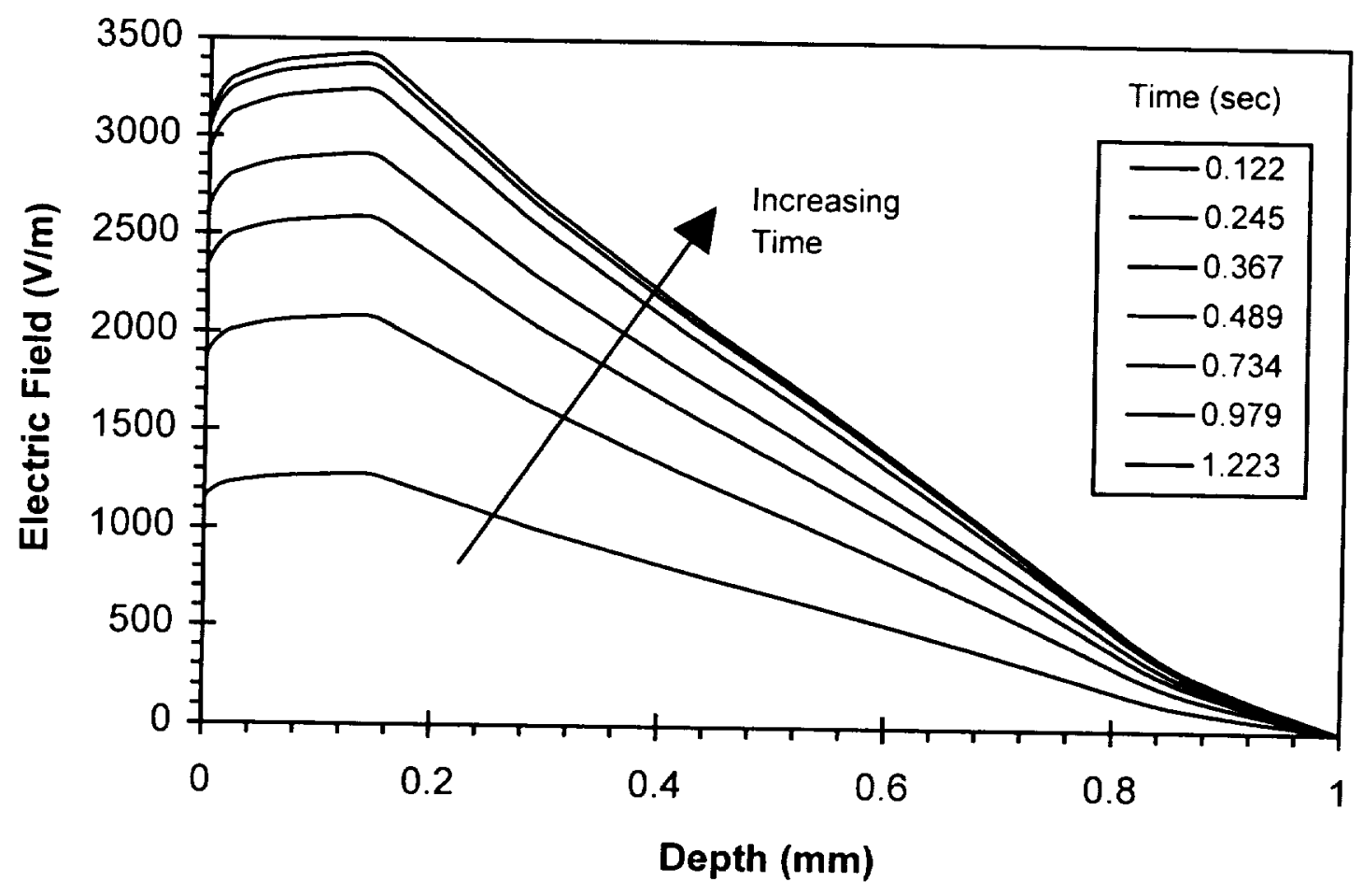

Figure B.9: Electric field of base case with thickness of $1 \mathrm{~mm}$ 


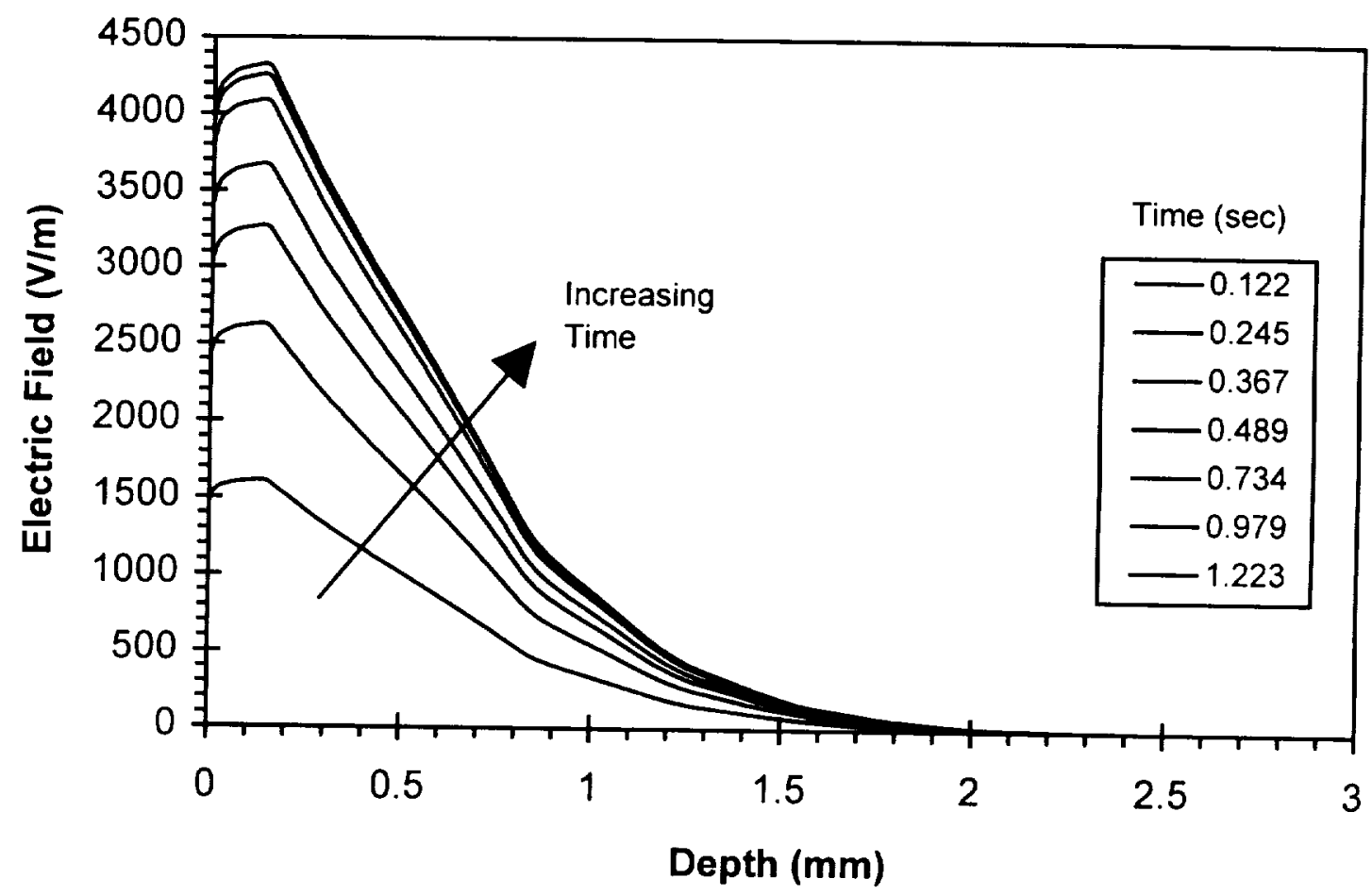

Figure B.10: Electric field of base case with thickness of $3 \mathrm{~mm}$ 


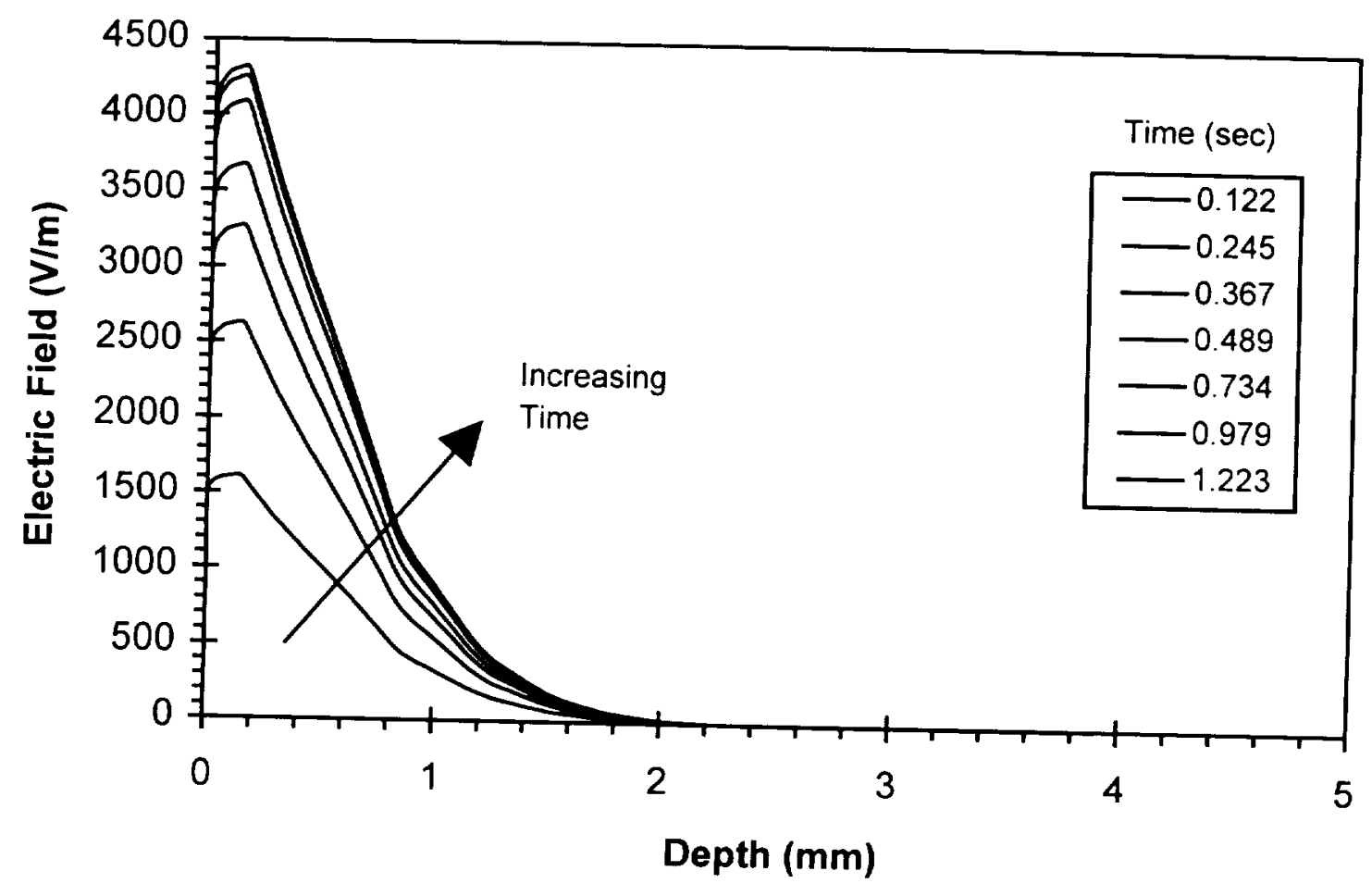

Figure B.11: Electric field of base case with thickness of $5 \mathrm{~mm}$ 


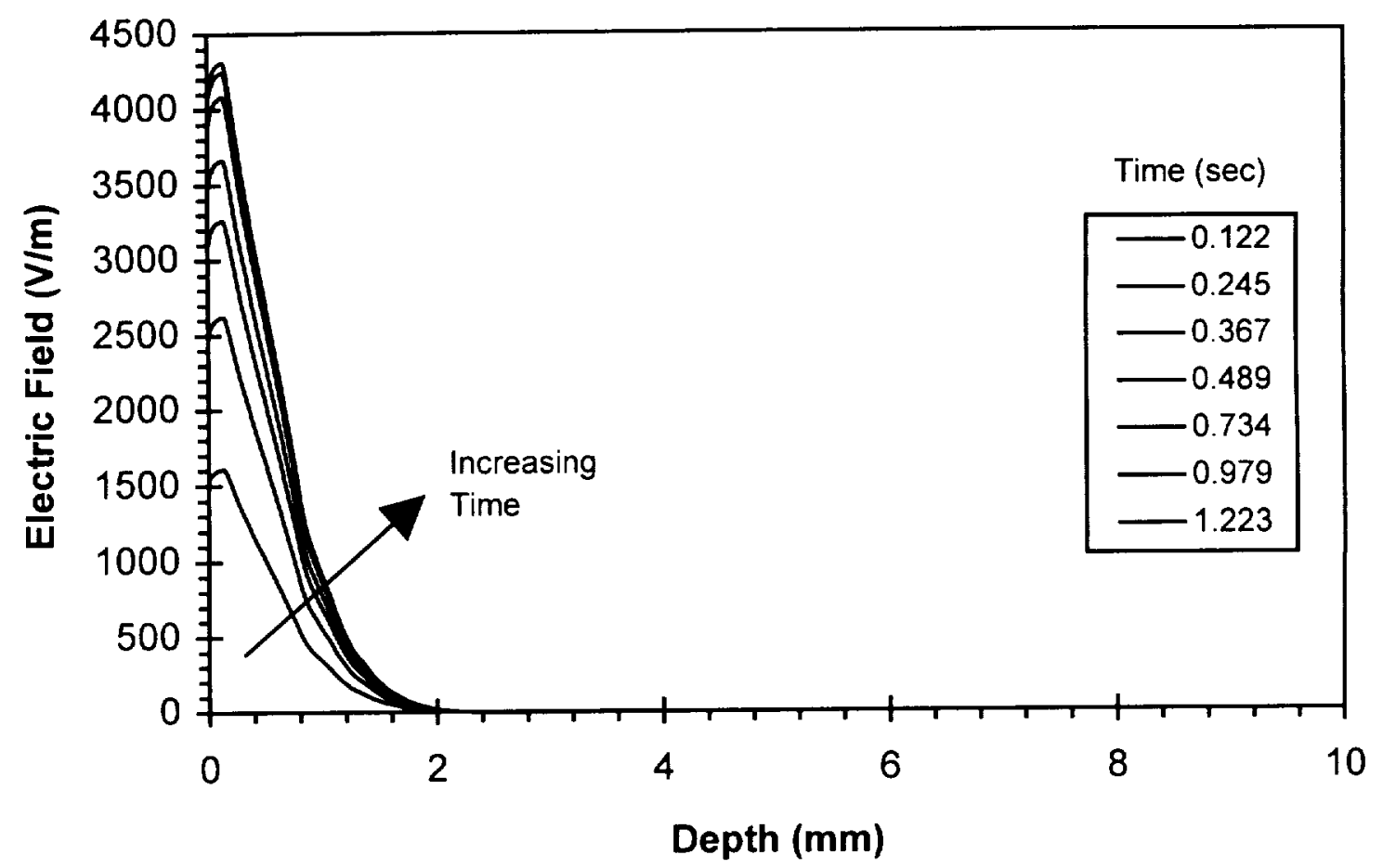

Figure B.12: Electric field of base case with thickness of $10 \mathrm{~mm}$ 


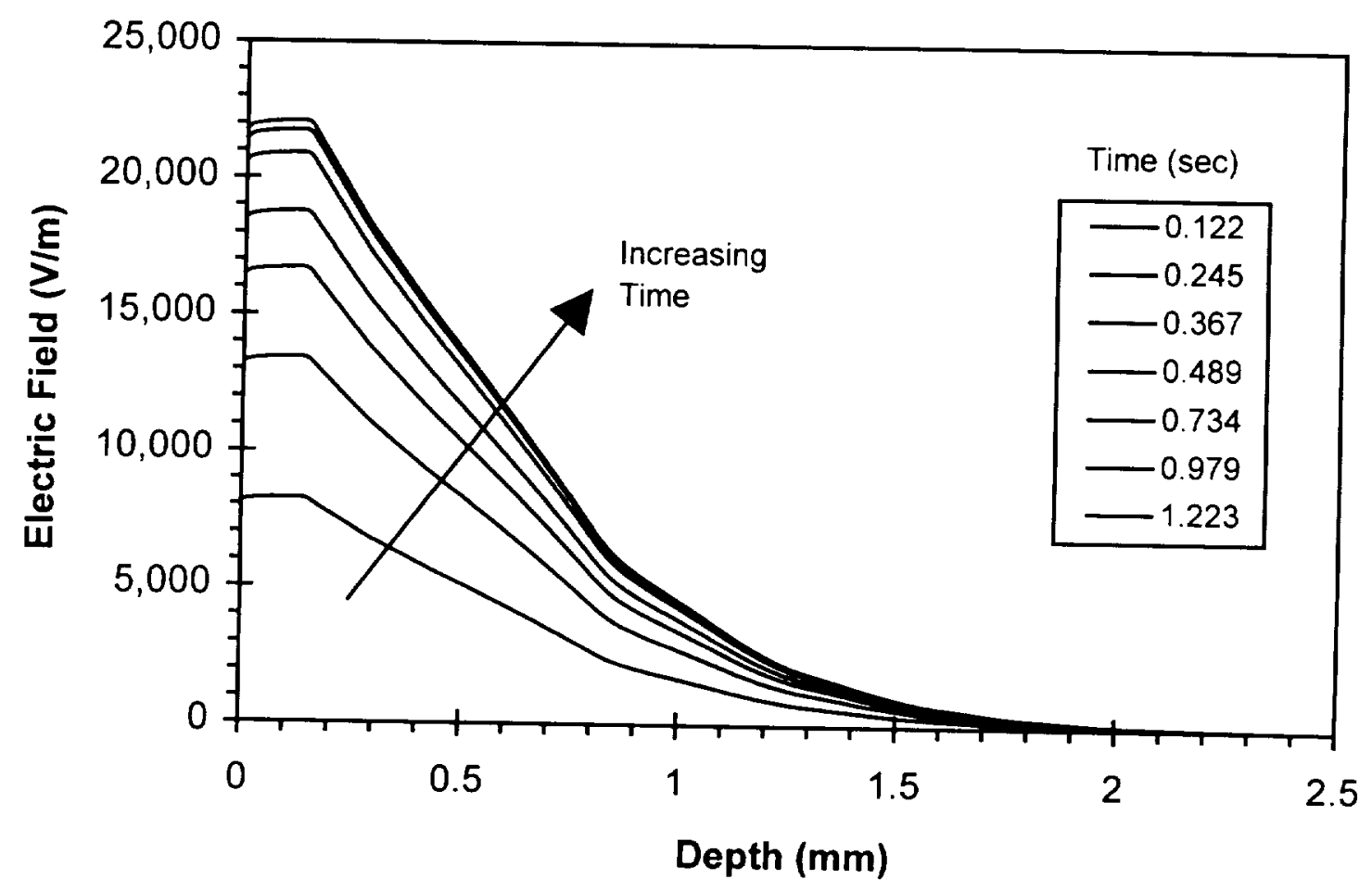

Figure B.13: Electric field of base case with a substorm intensity of 5 


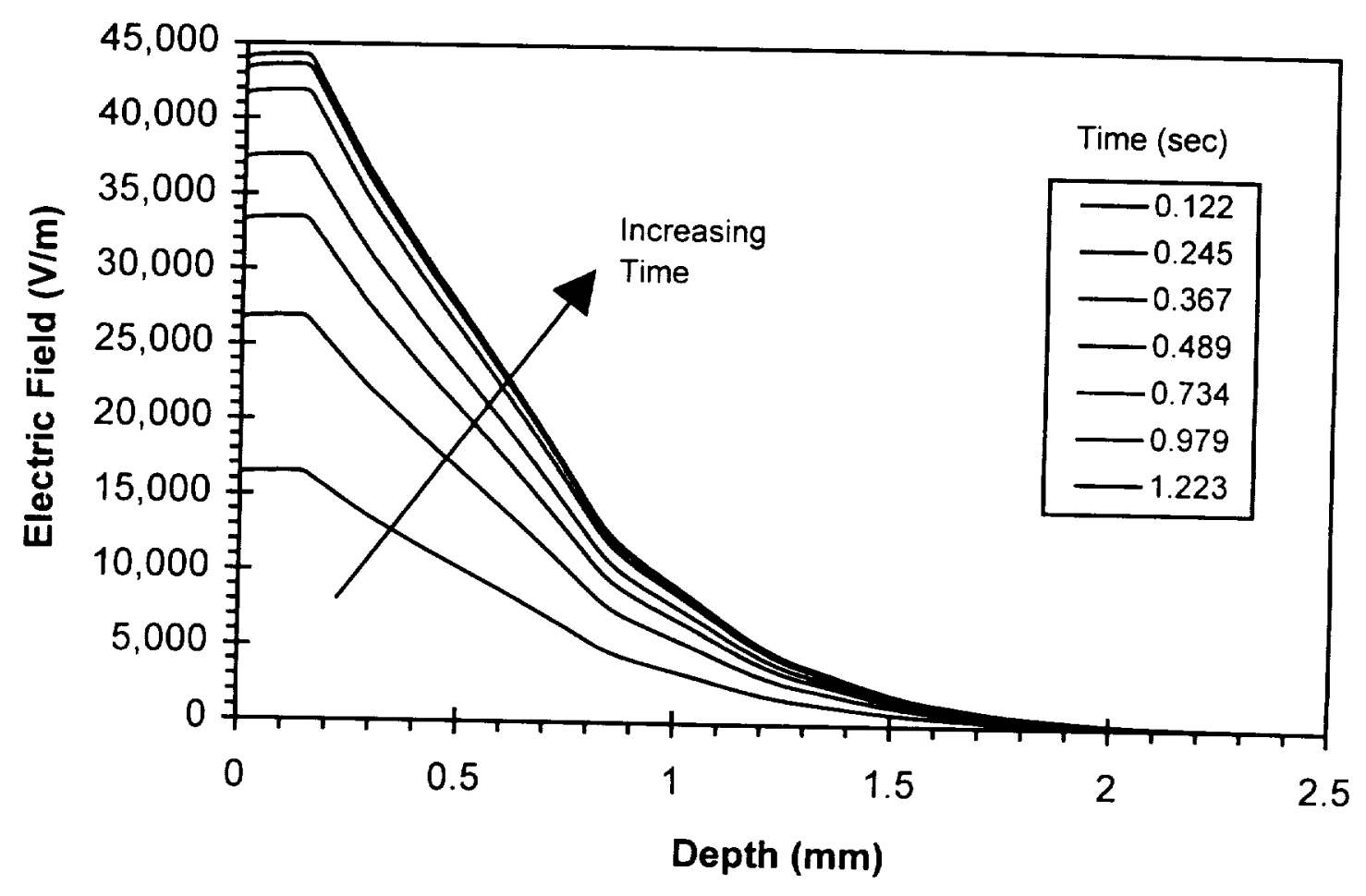

Figure B.14: Electric field of base case with a substorm intensity of 10 


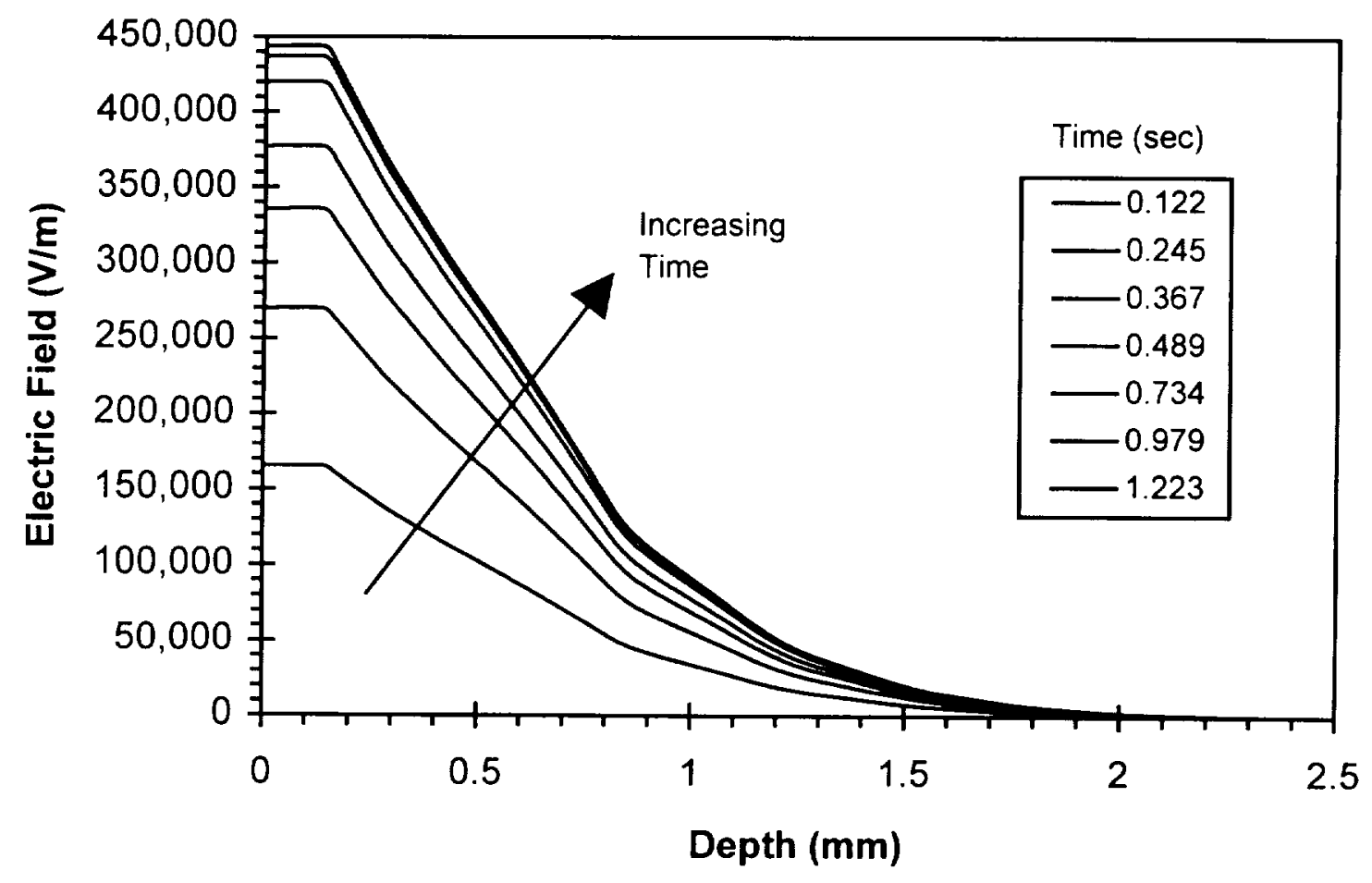

Figure B.15: Electric field of base case with a substorm intensity of 100 


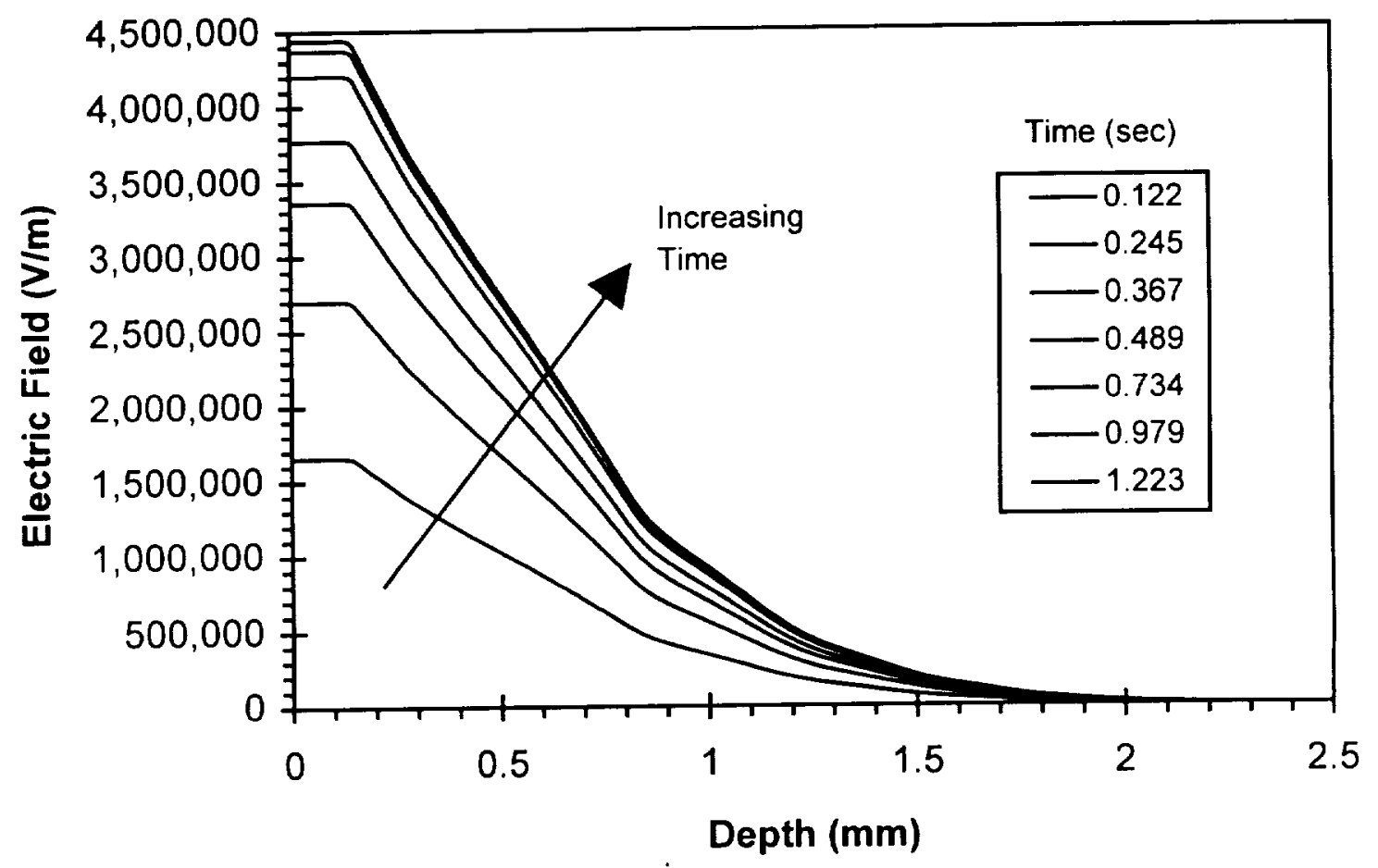

Figure B.16: Electric field of base case with a substorm intensity of 1000 


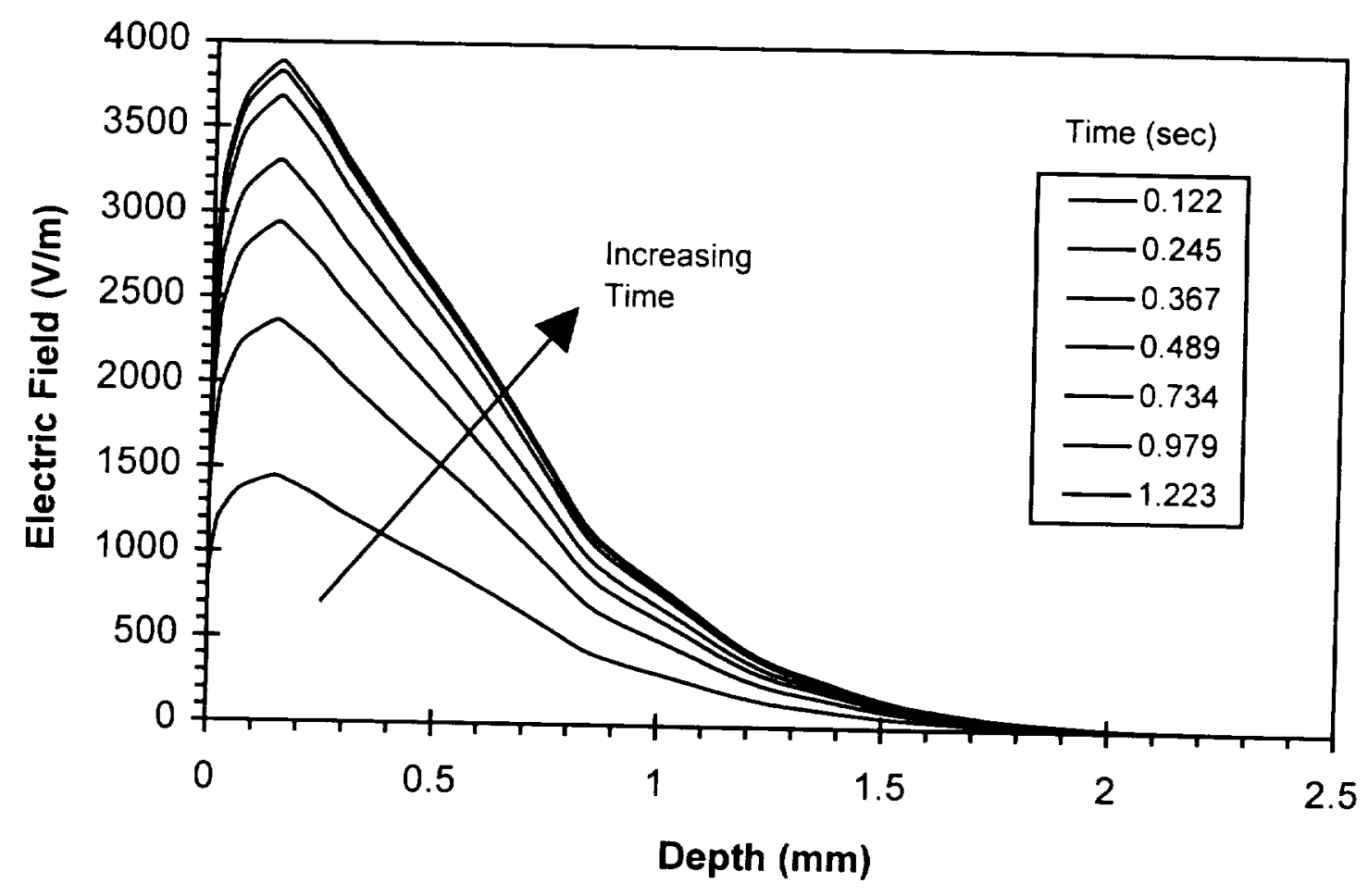

Figure B.17: Electric field of base case with a solar particle event intensity of 5 


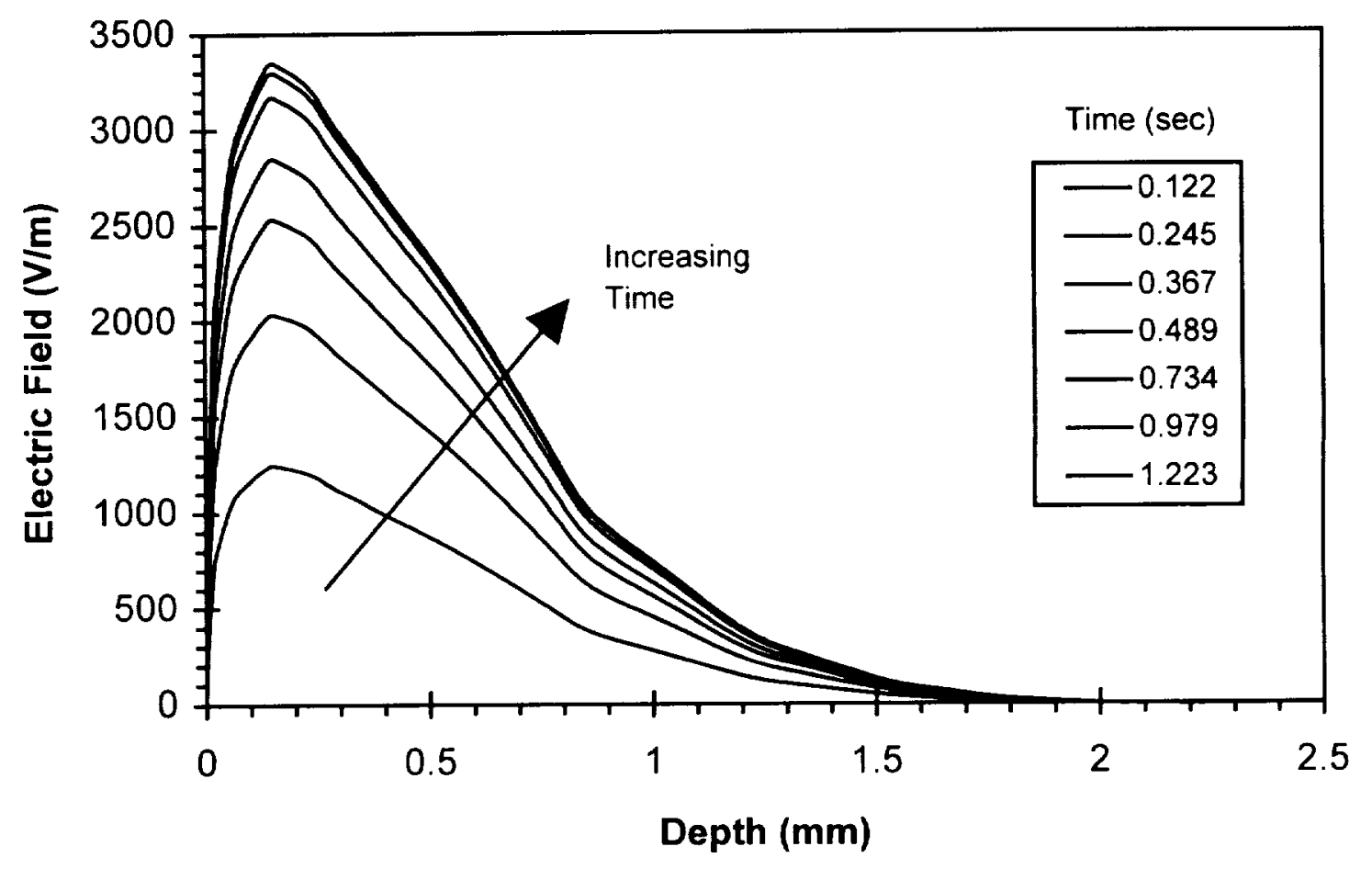

Figure B.18: Electric field of base case with a solar particle event intensity of 10 


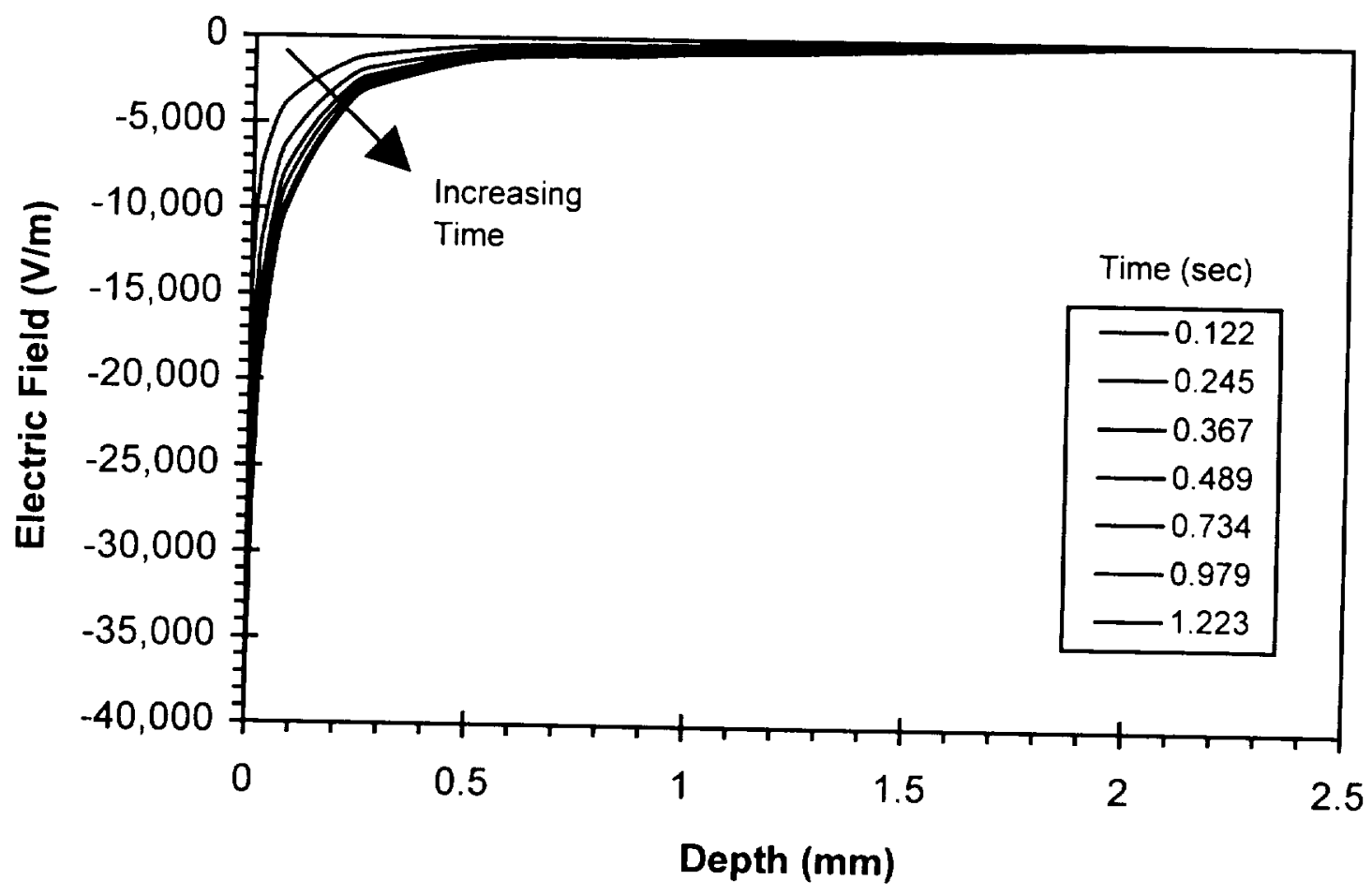

Figure B.19: Electric field of base case with a solar particle intensity of 100 


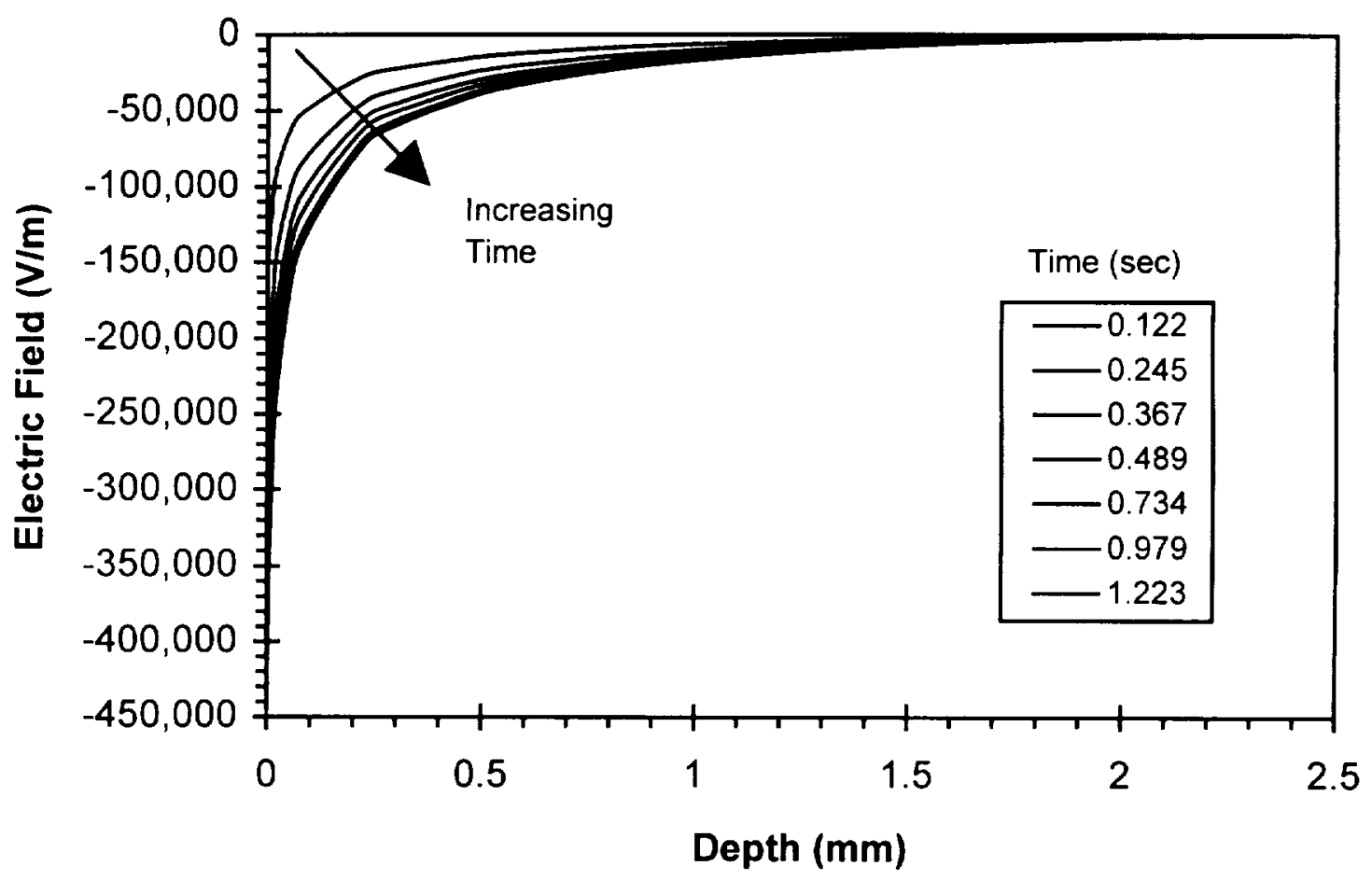

Figure B.20: Electric field of base case with a solar particle event intensity of 1000 


\section{APPENDIX C \\ EXPERIMENTAL DATA}

This appendix contain the conductivity and dielectric strength measurements, and the stress-strain and transverse versus longitudinal strain plots from the tensile tests. The slope of the linear portion of stress-strain curve is the Young's modulus in terapascals, 'Stroke' represents strain calculated from the stroke and 'Gage' represents strain obtained from a strain gage. The slope of the linear portion of the transverse versus longitudinal strain curve is the negative of the Poisson ratio.

The numbering system used for conductivity measurement samples is 'R-\#-\#-\#'; the ' $R$ ' stands for resistance measurements, the first number is the layup number (' 1 ' is for $[0]_{4}, ' 2$ ' is for $[0]_{8}, ' 3$ ' is for $[0]_{32},{ }^{\prime} 4$ ' is for $[ \pm 45]_{S}, ' 5$ ' is for $[0 / \pm 45 / 90]_{S}$, and ' 6 ' is for $\left.[0 / \pm 45 / 90]_{4 \mathrm{~S}}\right)$, the second number is the sample area number (' 1 ' is for $645 \mathrm{~mm}^{2}\left(1 \mathrm{in}^{2}\right)$, ' 2 ' is for $1290 \mathrm{~mm}^{2}\left(2 \mathrm{in}^{2}\right)$, and ' 3 ' is for $\left.2580 \mathrm{~mm}^{2}\left(4 \mathrm{in}^{2}\right)\right)$, and the last number is the sample number in the specified test group.

The numbering system used for the dielectric strength measurement samples is 'B-\#-\#'; the 'B' stands for breakdown voltage measurements, the first number is the 
layup number defined above, and the second number is the sample number for the specified test group.

The numbering system used for the tensile test specimen is '\#-4-\#-x'; the first number is the cure set number (' 3 ' is for $[0]_{4} 0 \%$ carbon black, the rest of the numbers are for $[ \pm 45]_{\mathrm{S}}$ layups, ' 4 ' is for $0 \%$ carbon black, ' 5 ' is for $5 \%$ carbon black, ' 6 ' is for $10 \%$ carbon black, ' 7 ' is for $15 \%$ carbon black, and ' 8 ' is for $20 \%$ carbon black), the ' 4 ' represent that the layup contains 4 plies, the third number represents the laminate number ( 3 laminates of the same layup were cured in one cure set), and the last letter represents the sample designator. 
Table C.1: Unidirectional unsanded conductivity resistance data

\begin{tabular}{||l|c|c|c|c|c|c||}
\hline Sample & \multicolumn{2}{|c|}{ Thickness $\mathbf{( m m}$ ) } & \multicolumn{2}{c|}{ Area $\left(\mathbf{m m}^{2}\right)$} & \multicolumn{2}{c|}{ Resistance $(\Omega)$} \\
& Average & SD & Average & SD & Average & SD \\
\hline R-1-1-1 & 0.52 & 0.007 & 630.5 & 3.03 & 0.71 & 0.049 \\
\hline R-1-1-2 & 0.52 & 0.005 & 624.0 & 1.47 & 1.43 & 0.046 \\
\hline R-1-1-3 & 0.52 & 0.005 & 621.3 & 3.58 & 0.86 & 0.115 \\
\hline R-1-2-1 & 0.52 & 0.007 & 1240.9 & 3.88 & 0.90 & 0.199 \\
\hline R-1-2-2 & 0.52 & 0.007 & 1240.7 & 5.47 & 1.05 & 0.010 \\
\hline R-1-2-3 & 0.52 & 0.008 & 1230.0 & 0.94 & 0.45 & 0.087 \\
\hline R-1-3-1 & 0.50 & 0.011 & 2541.3 & 23.77 & 0.48 & 0.010 \\
\hline R-1-3-2 & 0.51 & 0.011 & 2540.6 & 5.26 & 0.47 & 0.182 \\
\hline R-1-3-3 & 0.51 & 0.008 & 2541.3 & 16.66 & 0.45 & 0.035 \\
\hline \hline R-2-1-1 & 0.99 & 0.008 & 644.4 & 4.72 & 0.70 & 0.093 \\
\hline R-2-1-2 & 1.00 & 0.005 & 632.9 & 13.72 & 0.94 & 0.176 \\
\hline R-2-1-3 & 0.96 & 0.023 & 643.6 & 1.41 & 1.09 & 0.059 \\
\hline R-2-2-1 & 0.96 & 0.019 & 1277.9 & 1.10 & 0.98 & 0.046 \\
\hline R-2-2-2 & 1.01 & 0.009 & 1270.6 & 1.14 & 0.81 & 0.212 \\
\hline R-2-2-3 & 1.00 & 0.004 & 1276.1 & 0.99 & 0.70 & 0.156 \\
\hline R-2-3-1 & 0.99 & 0.016 & 2567.9 & 4.33 & 0.58 & 0.040 \\
\hline R-2-3-2 & 0.97 & 0.025 & 2585.7 & 3.84 & 0.58 & 0.040 \\
\hline R-2-3-3 & 0.97 & 0.036 & 2577.9 & 4.72 & 0.43 & 0.078 \\
\hline \hline R-3-1-1 & 3.71 & 0.005 & 645.7 & 1.91 & 0.55 & 0.051 \\
\hline R-3-1-2 & 3.67 & 0.013 & 647.0 & 0.38 & 2.22 & 0.012 \\
\hline R-3-1-3 & 3.67 & 0.013 & 641.7 & 2.16 & 0.90 & 0.055 \\
\hline R-3-2-1 & 3.71 & 0.013 & 1285.7 & 5.55 & 0.40 & 0.023 \\
\hline R-3-2-2 & 3.70 & 0.020 & 1281.2 & 2.33 & 0.47 & 0.040 \\
\hline R-3-2-3 & 3.73 & 0.005 & 1287.7 & 4.57 & 0.63 & 0.010 \\
\hline R-3-3-1 & 3.64 & 0.047 & 2583.0 & 7.06 & 0.40 & 0.052 \\
\hline R-3-3-2 & 3.66 & 0.041 & 2574.2 & 14.09 & 0.34 & 0.078 \\
\hline R-3-3-3 & 3.69 & 0.029 & 2585.0 & 1.80 & 0.39 & 0.046 \\
\hline
\end{tabular}


Table C.2: Quasi-isotropic unsanded conductivity resistance data

\begin{tabular}{||l|c|c|c|c|c|c||}
\hline Sample & \multicolumn{2}{|c|}{ Thickness $\mathbf{( m m )}$} & \multicolumn{2}{c|}{ Area $\left(\mathrm{mm}^{2}\right)$} & \multicolumn{2}{c|}{ Resistance $(\Omega)$} \\
& Average & SD & Average & SD & Average & SD \\
\hline \hline R-4-1-1 & 0.54 & 0.007 & 624.8 & 5.63 & 1.30 & 0.141 \\
\hline R-4-1-2 & 0.52 & 0.009 & 627.5 & 2.71 & 0.55 & 0.051 \\
\hline R-4-1-3 & 0.53 & 0.005 & 609.1 & 1.25 & 0.61 & 0.096 \\
\hline R-4-2-1 & 0.53 & 0.008 & 1269.9 & 0.96 & 0.42 & 0.021 \\
\hline R-4-2-2 & 0.52 & 0.016 & 1271.6 & 2.80 & 0.46 & 0.010 \\
\hline R-4-2-3 & 0.54 & 0.005 & 1267.9 & 2.93 & 0.42 & 0.105 \\
\hline R-4-3-1 & 0.52 & 0.009 & 2558.5 & 3.25 & 0.34 & 0.139 \\
\hline R-4-3-2 & 0.51 & 0.011 & 2560.3 & 6.14 & 0.26 & 0.017 \\
\hline R-4-3-3 & 0.52 & 0.007 & 2549.6 & 2.95 & 0.27 & 0.006 \\
\hline \hline R-5-1-1 & 0.98 & 0.011 & 625.5 & 0.72 & 0.67 & 0.065 \\
\hline R-5-1-2 & 0.94 & 0.015 & 626.3 & 1.77 & 0.75 & 0.159 \\
\hline R-5-1-3 & 1.00 & 0.013 & 627.0 & 1.74 & 0.58 & 0.051 \\
\hline R-5-2-1 & 1.01 & 0.025 & 1269.6 & 1.18 & 0.39 & 0.049 \\
\hline R-5-2-2 & 1.03 & 0.008 & 1272.3 & 0.66 & 0.54 & 0.020 \\
\hline R-5-2-3 & 0.99 & 0.028 & 1267.9 & 8.68 & 0.50 & 0.021 \\
\hline R-5-3-1 & 0.99 & 0.033 & 2539.3 & 25.02 & 0.33 & 0.040 \\
\hline R-5-3-2 & 0.97 & 0.027 & 2565.1 & 3.05 & 0.32 & 0.031 \\
\hline R-5-3-3 & 1.01 & 0.011 & 2556.2 & 2.74 & 0.29 & 0.006 \\
\hline \hline R-6-1-1 & 3.83 & 0.021 & 647.8 & 3.46 & 0.66 & 0.087 \\
\hline R-6-1-2 & 3.84 & 0.011 & 648.3 & 4.05 & 0.70 & 0.015 \\
\hline R-6-1-3 & 3.88 & 0.007 & 648.3 & 5.28 & 0.53 & 0.026 \\
\hline R-6-2-1 & 3.88 & 0.008 & 1272.0 & 2.39 & 0.28 & 0.010 \\
\hline R-6-2-2 & 3.78 & 0.075 & 1276.4 & 3.39 & 0.31 & 0.006 \\
\hline R-6-2-3 & 3.79 & 0.068 & 1282.1 & 4.59 & 0.31 & 0.000 \\
\hline R-6-3-1 & 3.74 & 0.072 & 2513.0 & 4.60 & 0.31 & 0.021 \\
\hline R-6-3-2 & 3.82 & 0.033 & 2542.3 & 3.92 & 0.32 & 0.015 \\
\hline R-6-3-3 & 3.73 & 0.069 & 2567.1 & 5.85 & 0.28 & 0.000 \\
\hline
\end{tabular}


Table C.3: Unidirectional sanded conductivity resistance data

\begin{tabular}{|c|c|c|c|c|c|c|}
\hline \multirow[t]{2}{*}{ Sample } & \multicolumn{2}{|c|}{ Thickness (mm) } & \multicolumn{2}{|c|}{ Area $\left(\mathrm{mm}^{2}\right)$} & \multicolumn{2}{|c|}{ Resistance $(\Omega)$} \\
\hline & Average & SD & Average & SD & Average & SD \\
\hline$R-1-1-1$ & 0.39 & 0.031 & 630.5 & 3.03 & 1.49 & 0.179 \\
\hline$R-1-1-2$ & 0.37 & 0.050 & 624.0 & 1.47 & 0.33 & 0.067 \\
\hline R-1-1-3 & 0.34 & 0.048 & 621.3 & 3.58 & 0.30 & 0.110 \\
\hline $\mathrm{R}-1-2-1$ & 0.39 & 0.029 & 1240.9 & 3.88 & 0.16 & 0.062 \\
\hline$R-1-2-2$ & 0.41 & 0.046 & 1240.7 & 5.47 & 0.15 & 0.029 \\
\hline$R-1-2-3$ & 0.34 & 0.045 & 1230.0 & 0.94 & 0.17 & 0.068 \\
\hline$R-1-3-1$ & 0.29 & 0.054 & 2541.3 & 23.77 & 0.24 & 0.120 \\
\hline $\mathrm{R}-1-3-2$ & 0.36 & 0.051 & 2540.6 & 5.26 & 0.30 & 0.108 \\
\hline$R-1-3-3$ & 0.34 & 0.055 & 2541.3 & 16.66 & 0.30 & 0.035 \\
\hline$R-2-1-1$ & 0.78 & 0.048 & 644.4 & 4.72 & 0.20 & 0.078 \\
\hline$R-2-1-2$ & 0.80 & 0.017 & 632.9 & 13.72 & 0.36 & 0.086 \\
\hline$R-2-1-3$ & 0.77 & 0.020 & 643.6 & 1.41 & 0.15 & 0.020 \\
\hline$R-2-2-1$ & 0.82 & 0.028 & 1277.9 & 1.10 & 0.32 & 0.156 \\
\hline$R-2-2-2$ & 0.82 & 0.045 & 1270.6 & 1.14 & 0.28 & 0.080 \\
\hline$R-2-2-3$ & 0.78 & 0.045 & 1276.1 & 0.99 & 0.14 & 0.099 \\
\hline R-2-3-1 & 0.85 & 0.041 & 2567.9 & 4.33 & 0.17 & 0.107 \\
\hline R-2-3-2 & 0.84 & 0.053 & 2585.7 & 3.84 & 0.16 & 0.119 \\
\hline $\mathrm{R}-2-3-3$ & 0.82 & 0.054 & 2577.9 & 4.72 & 0.07 & 0.055 \\
\hline R-3-1-1 & 3.41 & 0.179 & 645.7 & 1.91 & 0.92 & 0.284 \\
\hline R-3-1-2 & 3.56 & 0.042 & 647.0 & 0.38 & 0.81 & 0.425 \\
\hline R-3-1-3 & 3.57 & 0.031 & 641.7 & 2.16 & 0.41 & 0.099 \\
\hline$R-3-2-1$ & 3.58 & 0.064 & 1285.7 & 5.55 & 0.25 & 0.155 \\
\hline$R-3-2-2$ & 3.56 & 0.052 & 1281.2 & 2.33 & 0.20 & 0.085 \\
\hline$R-3-2-3$ & 3.60 & 0.047 & 1287.7 & 4.57 & 0.15 & 0.031 \\
\hline R-3-3-1 & 3.55 & 0.062 & 2583.0 & 7.06 & 0.14 & 0.070 \\
\hline$R-3-3-2$ & 3.56 & 0.040 & 2574.2 & 14.09 & 0.01 & 0.006 \\
\hline R-3-3-3 & 3.56 & 0.036 & 2585.0 & 1.80 & 0.06 & 0.031 \\
\hline
\end{tabular}


Table C.4: Quasi-isotropic sanded conductivity resistance data

\begin{tabular}{||l|c|c|c|c|c|c||}
\hline Sample & \multicolumn{2}{|c|}{ Thickness $(\mathbf{m m})$} & \multicolumn{2}{c|}{ Area $\left(\mathrm{mm}^{2}\right)$} & \multicolumn{2}{c|}{ Resistance $(\Omega)$} \\
& Average & SD & Average & SD & Average & SD \\
\hline \hline R-4-1-1 & 0.45 & 0.023 & 624.8 & 5.63 & 0.38 & 0.085 \\
\hline R-4-1-2 & 0.41 & 0.022 & 627.5 & 2.71 & 0.41 & 0.275 \\
\hline R-4-1-3 & 0.44 & 0.016 & 609.1 & 1.25 & 0.21 & 0.035 \\
\hline R-4-2-1 & 0.38 & 0.029 & 1269.9 & 0.96 & 0.25 & 0.095 \\
\hline R-4-2-2 & 0.38 & 0.047 & 1271.6 & 2.80 & 0.23 & 0.057 \\
\hline R-4-2-3 & 0.39 & 0.015 & 1267.9 & 2.93 & 0.13 & 0.031 \\
\hline R-4-3-1 & 0.41 & 0.048 & 2558.5 & 3.25 & 0.11 & 0.015 \\
\hline R-4-3-2 & 0.39 & 0.029 & 2560.3 & 6.14 & 0.18 & 0.099 \\
\hline R-4-3-3 & 0.42 & 0.024 & 2549.6 & 2.95 & 0.15 & 0.035 \\
\hline \hline R-5-1-1 & 0.89 & 0.012 & 625.5 & 0.72 & 0.14 & 0.087 \\
\hline R-5-1-2 & 0.81 & 0.042 & 626.3 & 1.77 & 0.17 & 0.006 \\
\hline R-5-1-3 & 0.90 & 0.021 & 627.0 & 1.74 & 0.07 & 0.025 \\
\hline R-5-2-1 & 0.87 & 0.029 & 1269.6 & 1.18 & 0.07 & 0.010 \\
\hline R-5-2-2 & 0.88 & 0.040 & 1272.3 & 0.66 & 0.09 & 0.026 \\
\hline R-5-2-3 & 0.91 & 0.022 & 1267.9 & 8.68 & 0.13 & 0.010 \\
\hline R-5-3-1 & 0.87 & 0.044 & 2539.3 & 25.02 & 0.09 & 0.059 \\
\hline R-5-3-2 & 0.80 & 0.070 & 2565.1 & 3.05 & 0.03 & 0.010 \\
\hline R-5-3-3 & 0.87 & 0.048 & 2556.2 & 2.74 & 0.08 & 0.006 \\
\hline \hline R-6-1-1 & 3.72 & 0.023 & 647.8 & 3.46 & 0.22 & 0.021 \\
\hline R-6-1-2 & 3.76 & 0.018 & 648.3 & 4.05 & 0.20 & 0.021 \\
\hline R-6-1-3 & 3.80 & 0.025 & 648.3 & 5.28 & 0.15 & 0.075 \\
\hline R-6-2-1 & 3.79 & 0.042 & 1272.0 & 2.39 & 0.04 & 0.012 \\
\hline R-6-2-2 & 3.68 & 0.061 & 1276.4 & 3.39 & 0.10 & 0.012 \\
\hline R-6-2-3 & 3.69 & 0.054 & 1282.1 & 4.59 & 0.11 & 0.021 \\
\hline R-6-3-1 & 3.61 & 0.073 & 2513.0 & 4.60 & 0.07 & 0.031 \\
\hline R-6-3-2 & 3.73 & 0.037 & 2542.3 & 3.92 & 0.02 & 0.015 \\
\hline R-6-3-3 & 3.64 & 0.072 & 2567.1 & 5.85 & 0.04 & 0.021 \\
\hline
\end{tabular}


Table C.5: Dielectric strength breakdown voltage data

\begin{tabular}{||l|c|c|c|c||}
\hline \hline Sample & \multicolumn{2}{|c|}{ Thickness (mm) } & \multicolumn{2}{c||}{ Breakdown Voltage (V) } \\
& Average & SD & Average & SD \\
\hline \hline B-1-1 & 0.51 & 0.013 & 1100 & 446.8 \\
\hline B-1-2 & 0.51 & 0.013 & 810 & 424.6 \\
\hline B-1-3 & 0.50 & 0.013 & 817 & 127.0 \\
\hline \hline B-2-1 & 1.01 & 0.000 & 1533 & 57.7 \\
\hline B-2-2 & 1.01 & 0.004 & 1400 & 264.6 \\
\hline B-2-3 & 0.94 & 0.025 & 1367 & 472.6 \\
\hline \hline B-3-1 & 3.70 & 0.011 & 1000 & 264.6 \\
\hline B-3-2 & 3.71 & 0.005 & 1100 & 100.0 \\
\hline B-3-3 & 3.68 & 0.015 & 1020 & 170.9 \\
\hline \hline B-4-1 & 0.54 & 0.004 & 830 & 270.0 \\
\hline B-4-2 & 0.53 & 0.004 & 1200 & 173.2 \\
\hline B-4-3 & 0.55 & 0.005 & 1700 & 556.8 \\
\hline \hline B-5-1 & 0.96 & 0.020 & 1767 & 378.6 \\
\hline B-5-2 & 1.01 & 0.007 & 1767 & 503.3 \\
\hline B-5-3 & 1.02 & 0.005 & 1533 & 404.1 \\
\hline \hline B-6-1 & 3.73 & 0.033 & 1157 & 567.0 \\
\hline B-6-2 & 3.71 & 0.033 & 953 & 331.3 \\
\hline B-6-3 & 3.80 & 0.023 & 897 & 179.5 \\
\hline \hline
\end{tabular}



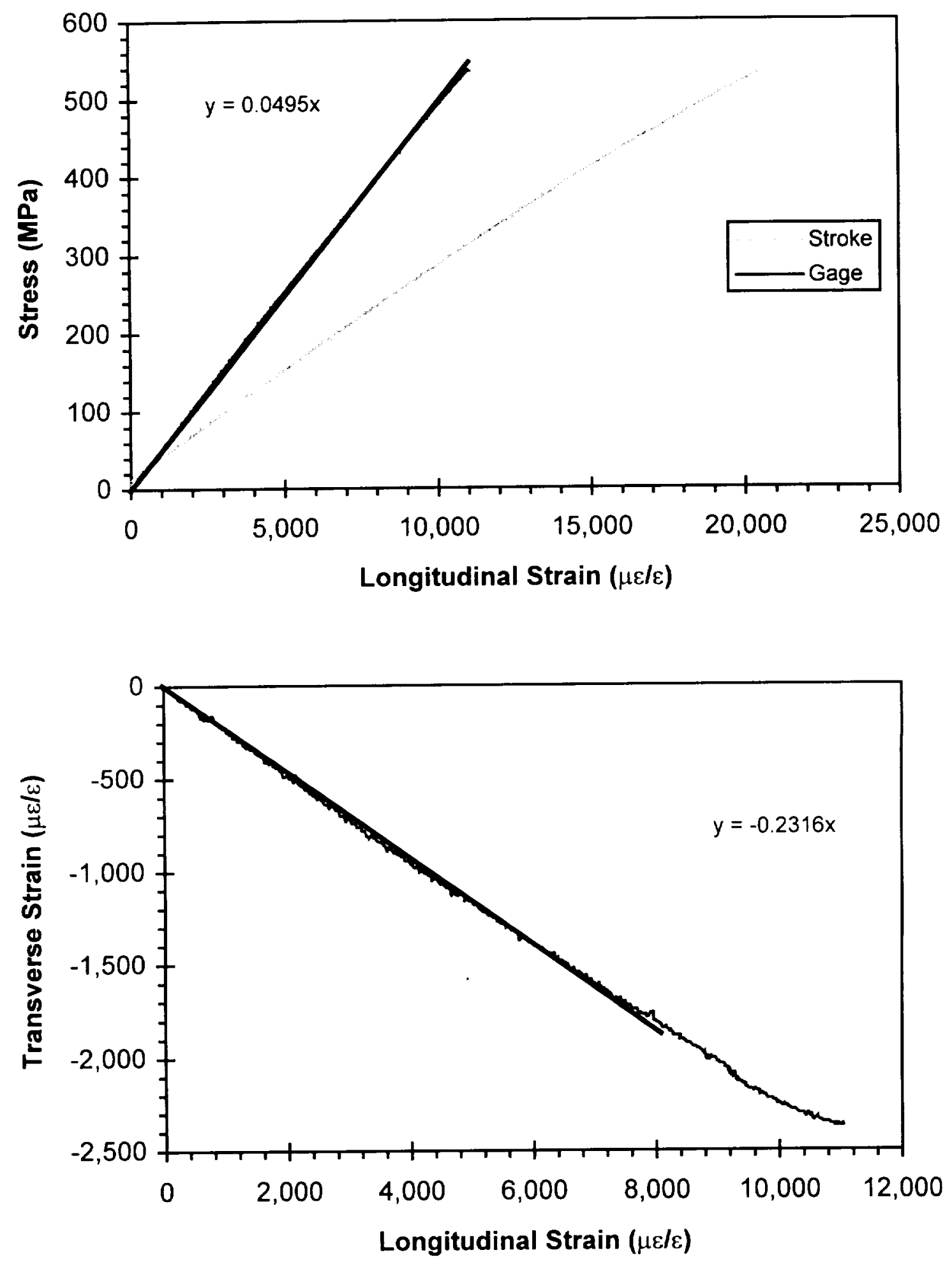

Figure C.1: Tensile test 3-4-1-a 

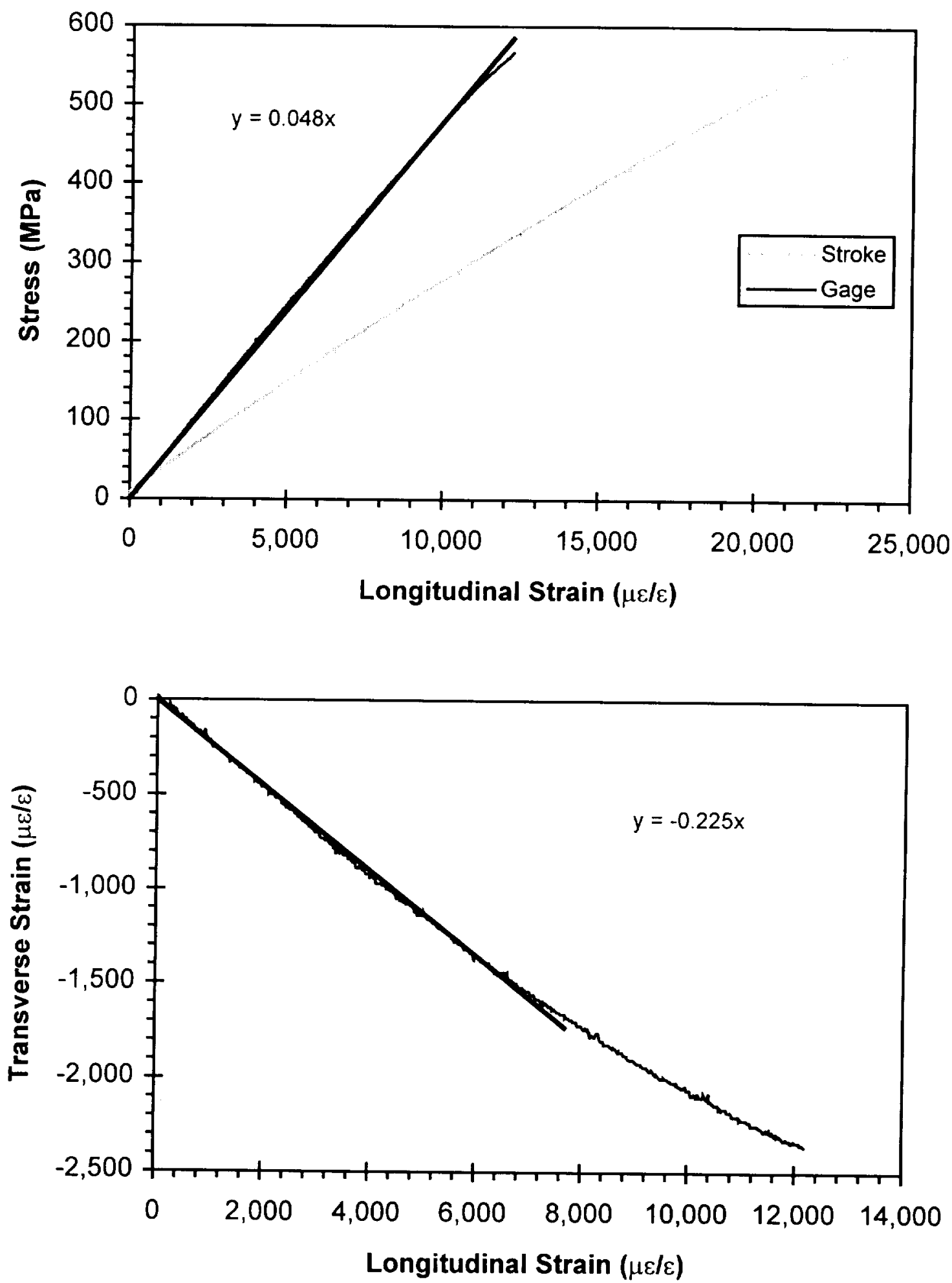

Figure C.2: Tensile test 3-4-1-b 

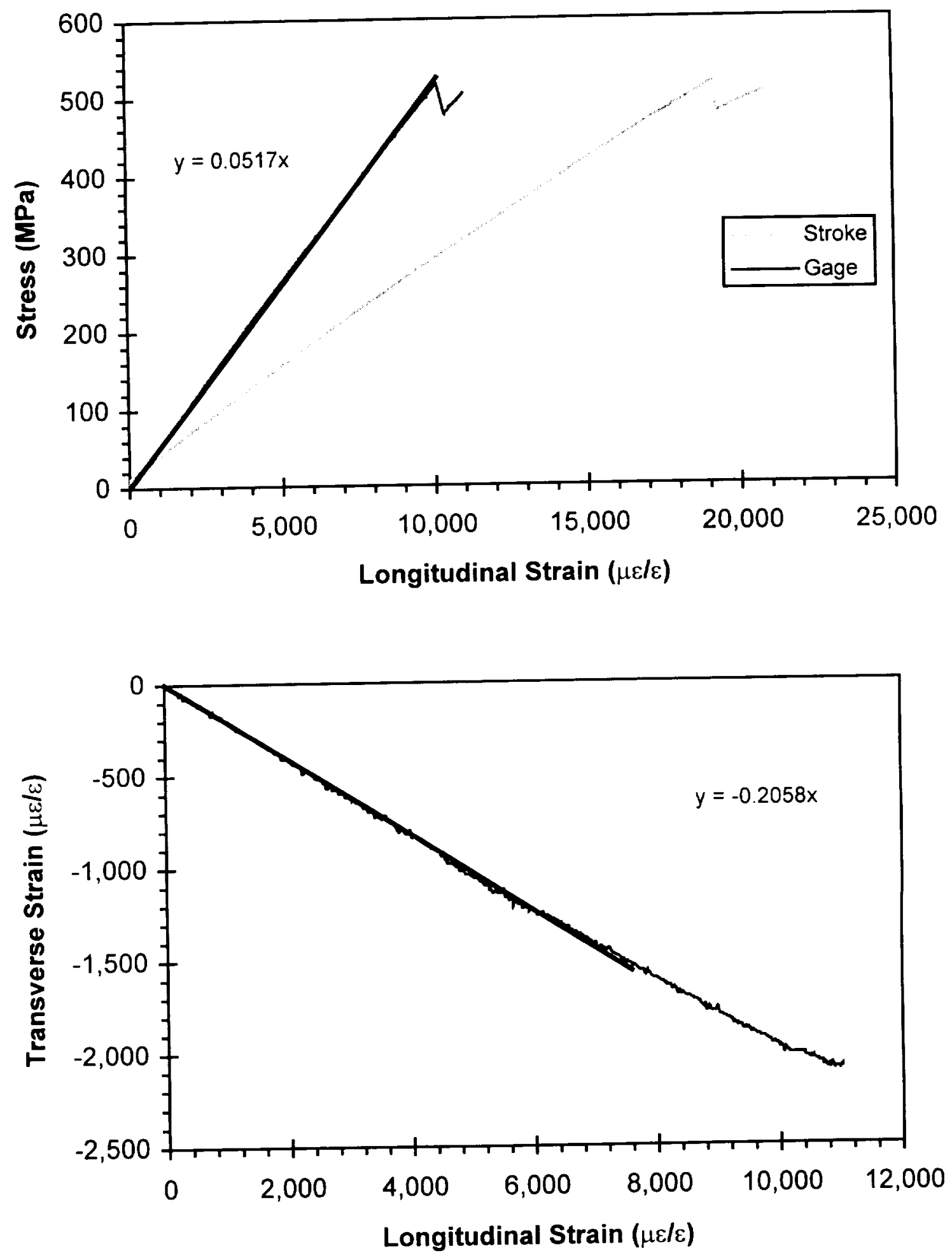

Figure C.3: Tensile test 3-4-2-a 

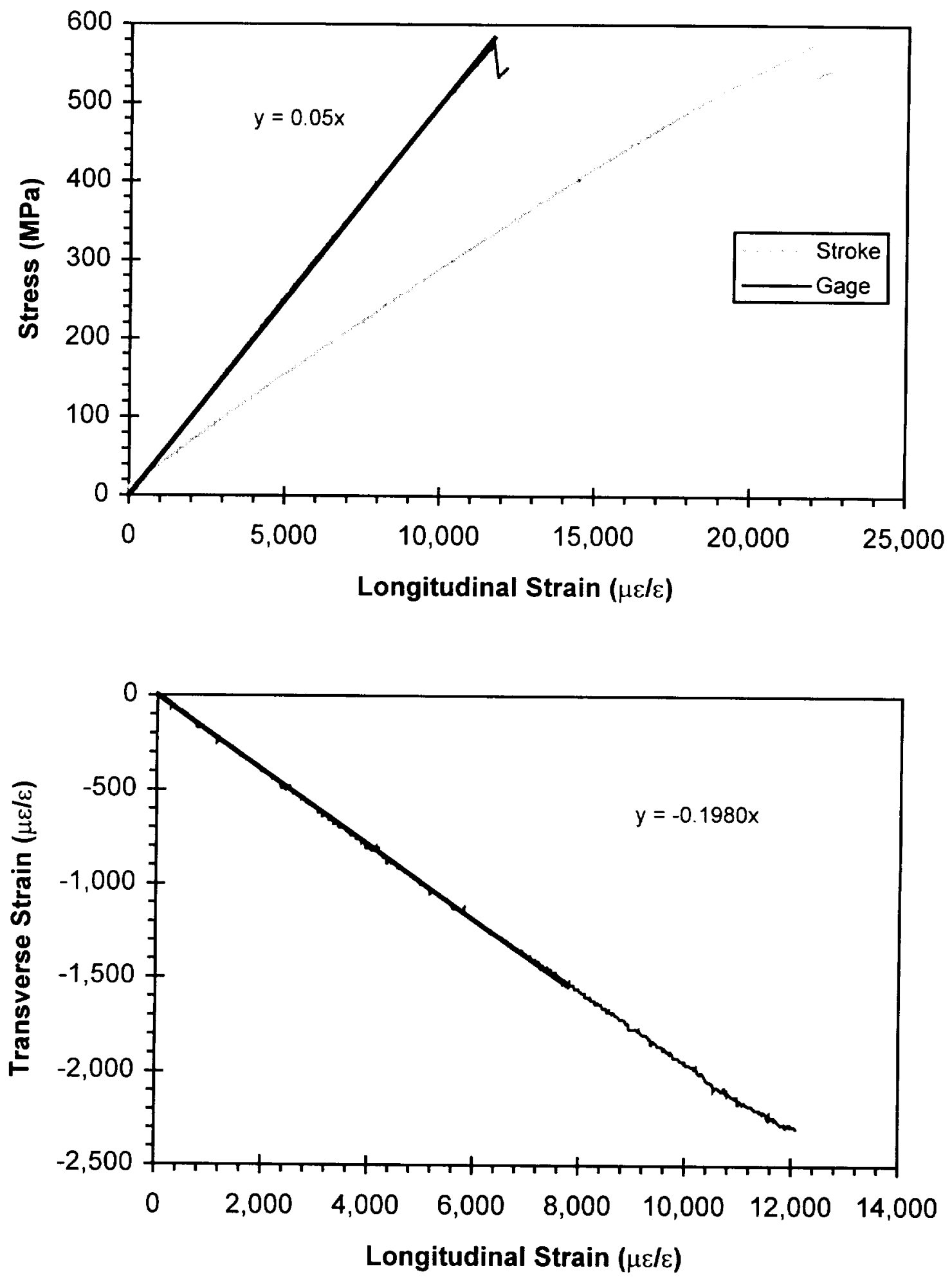

Figure C.4: Tensile test 3-4-2-b 

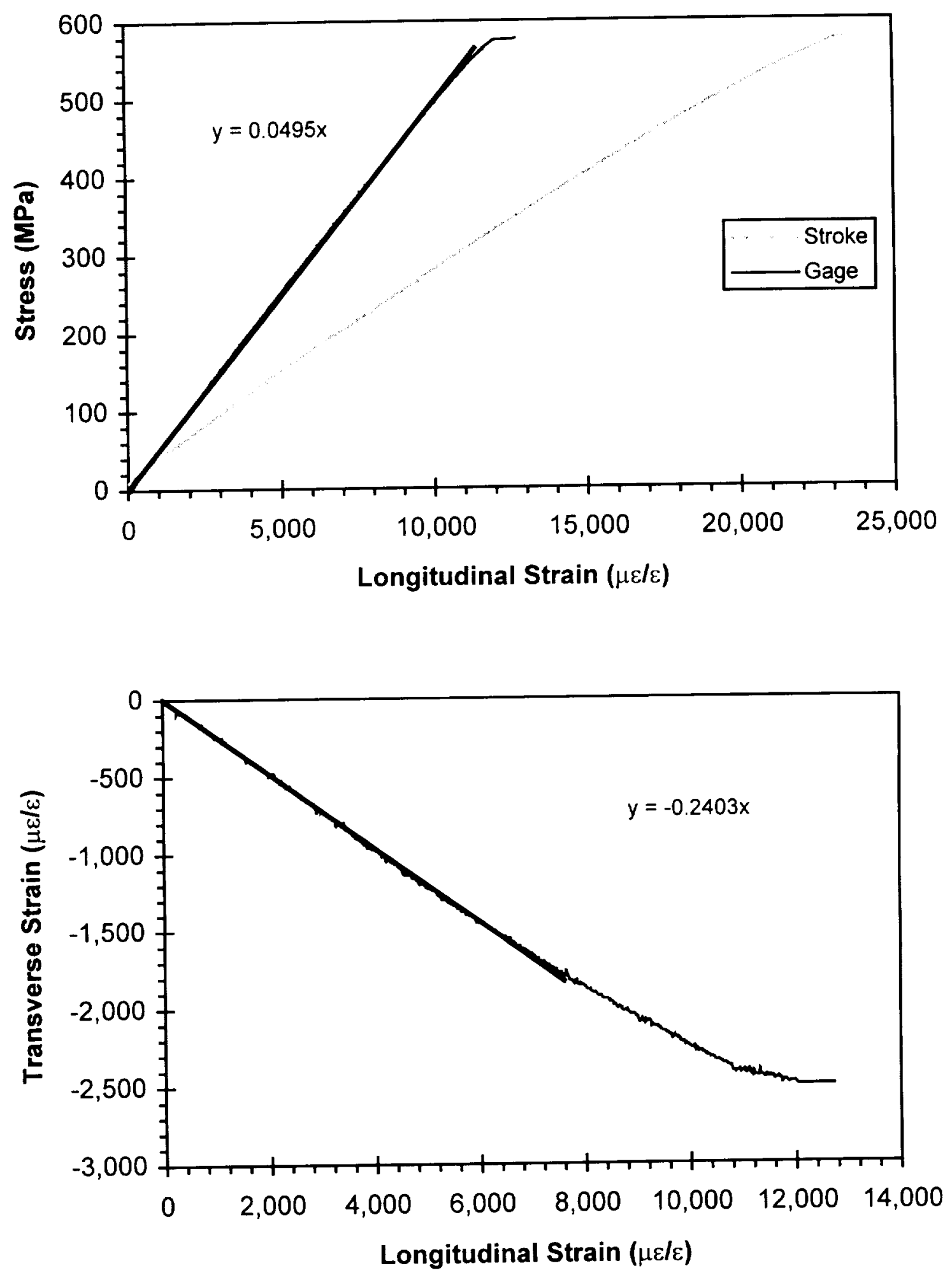

Figure C.5: Tensile test 3-4-3-a 

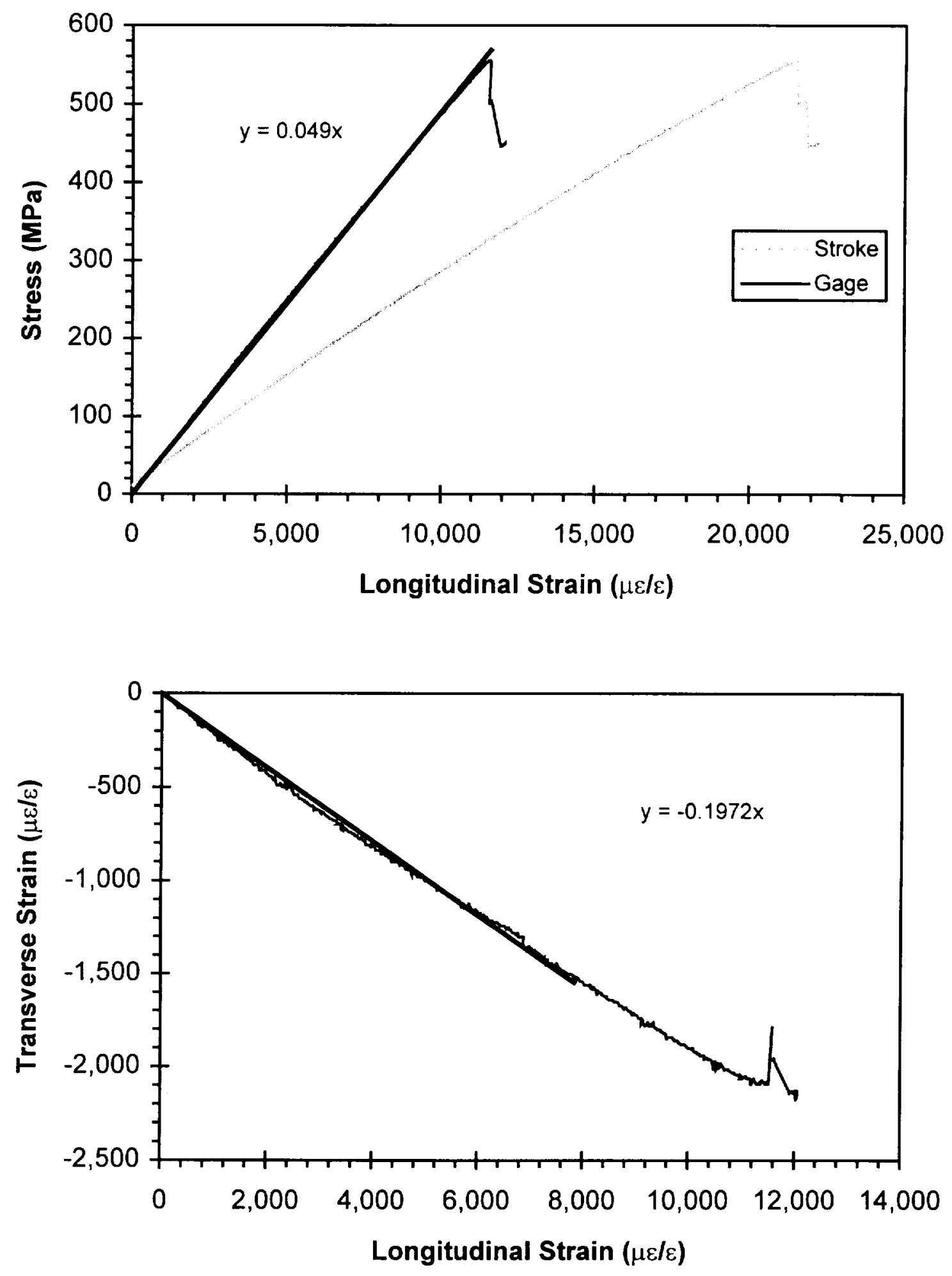

Figure C.6: Tensile test 3-4-3-b 

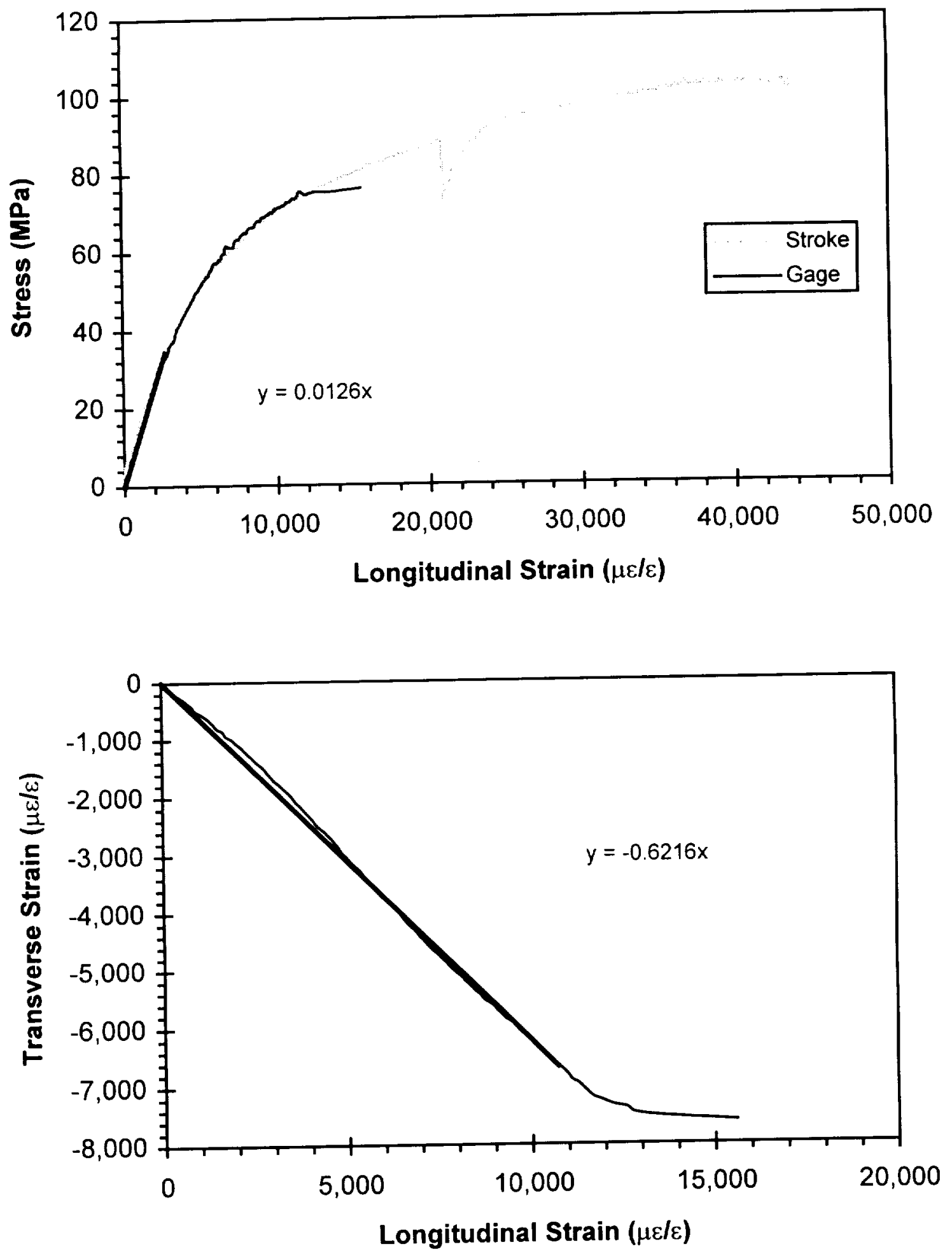

Figure C.7: Tensile test 4-4-1-b 

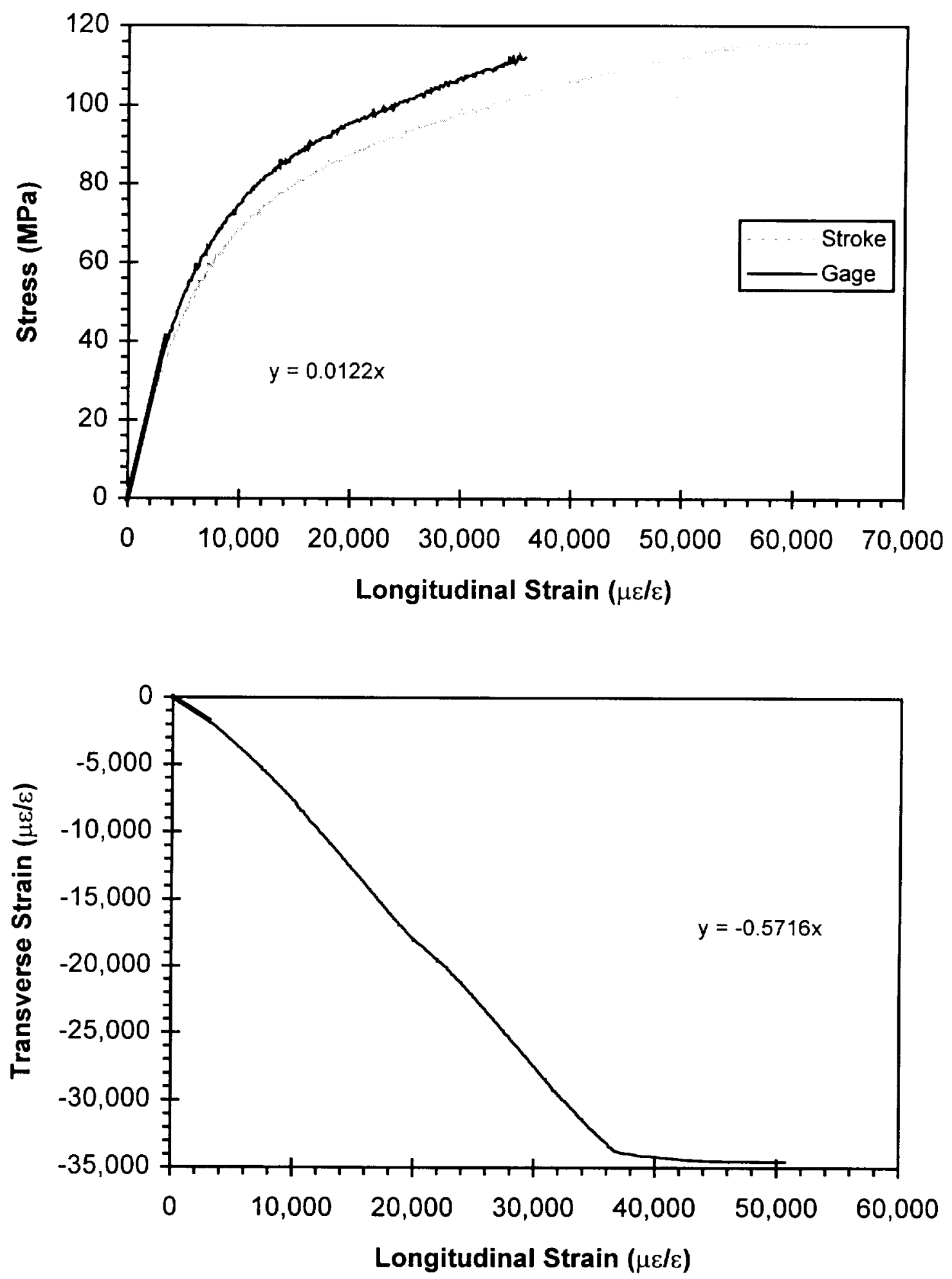

Figure C.8: Tensile test 4-4-1-c 

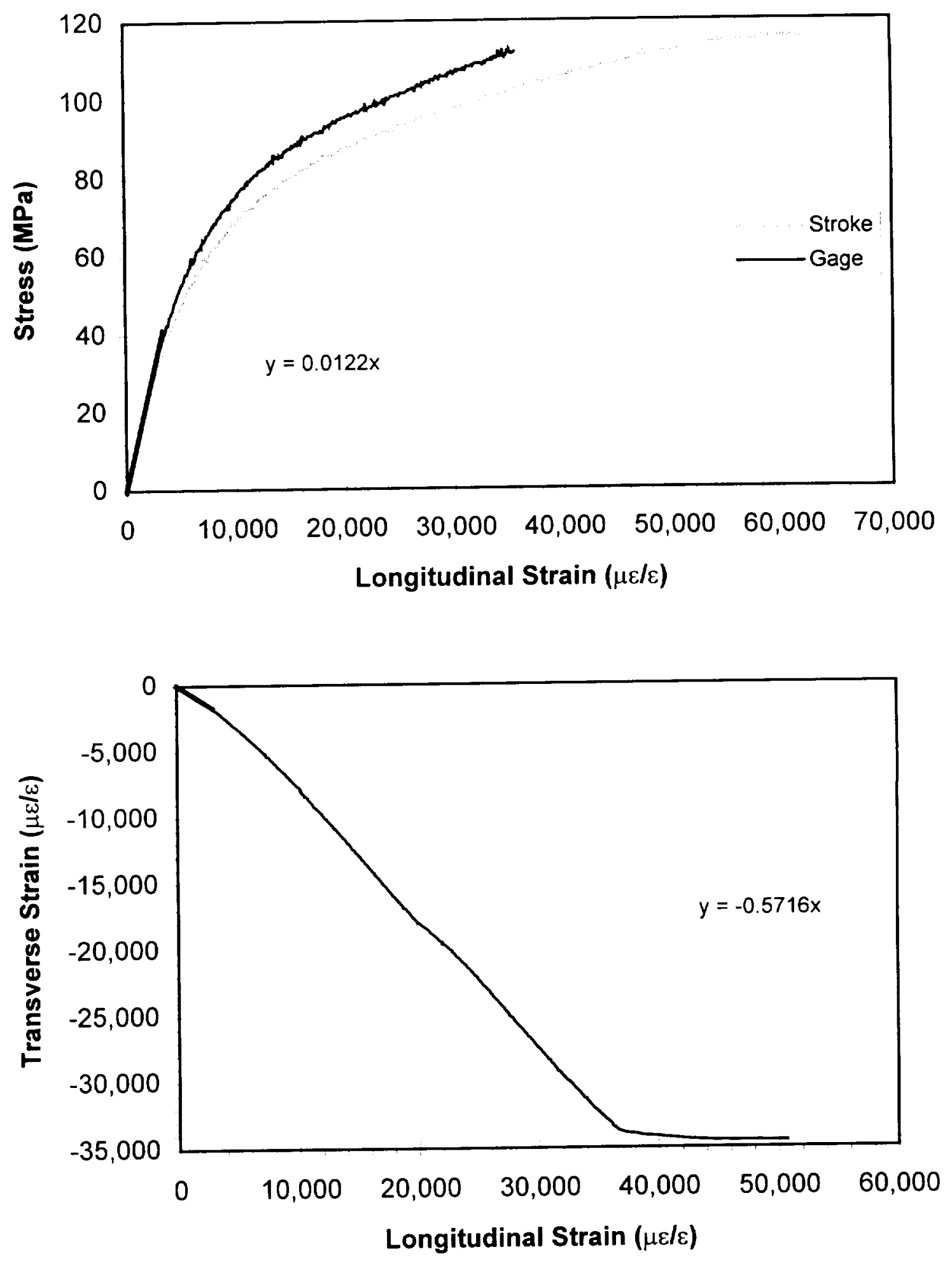

Figure C.9: Tensile test 4-4-2-a 

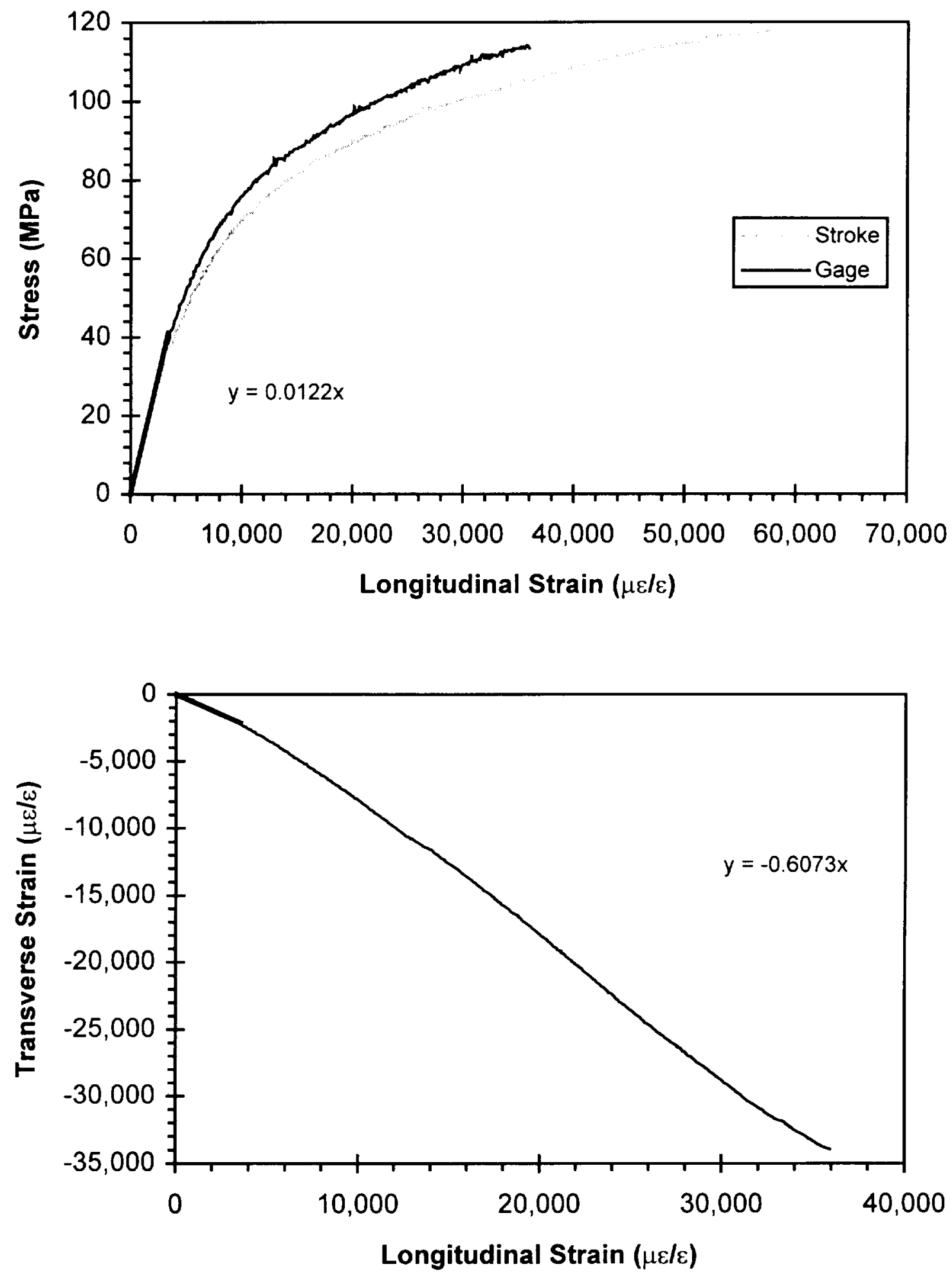

Figure C.10: Tensile test 4-4-2-b 

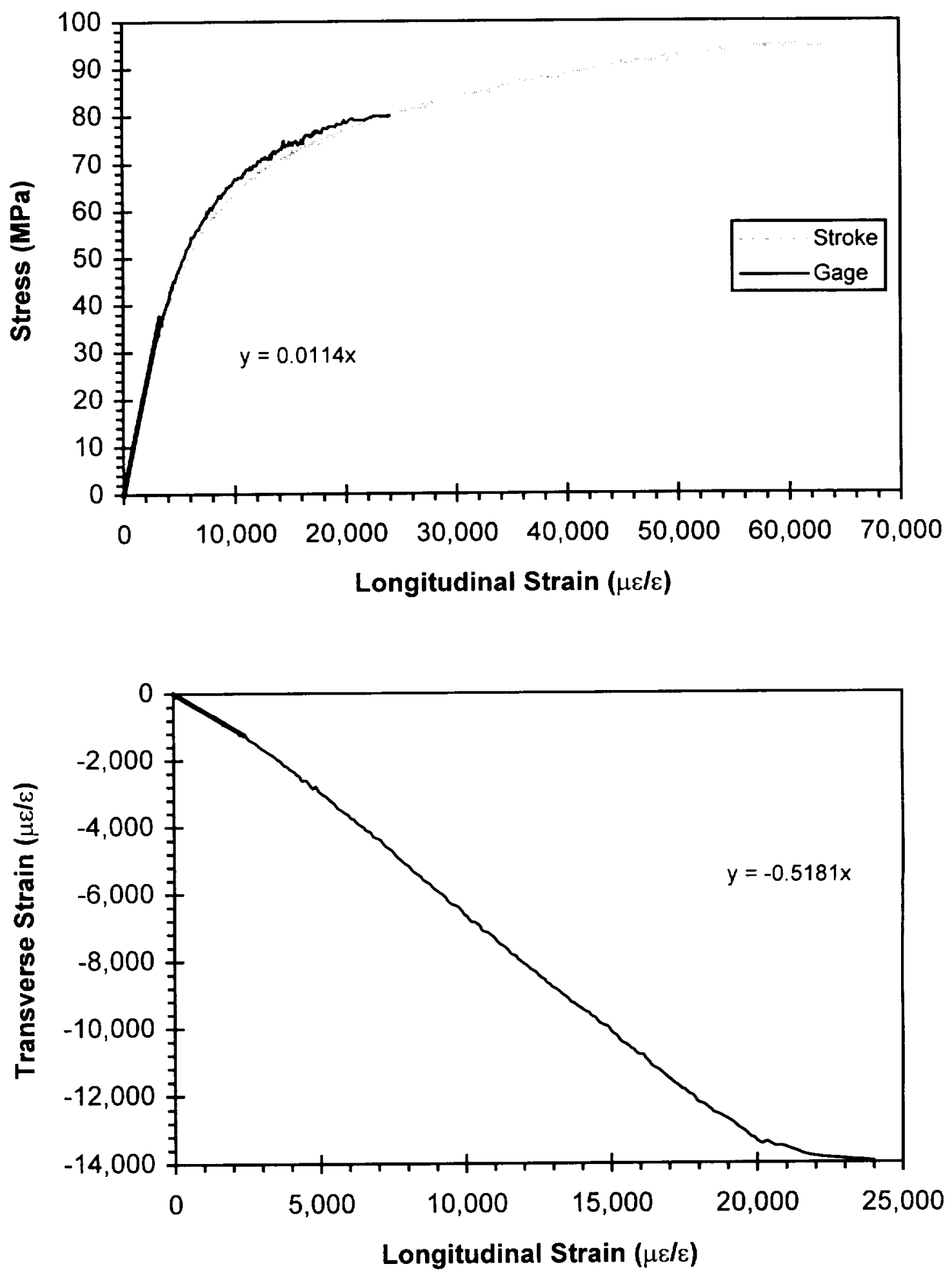

Figure C.11: Tensile test 4-4-3-a 

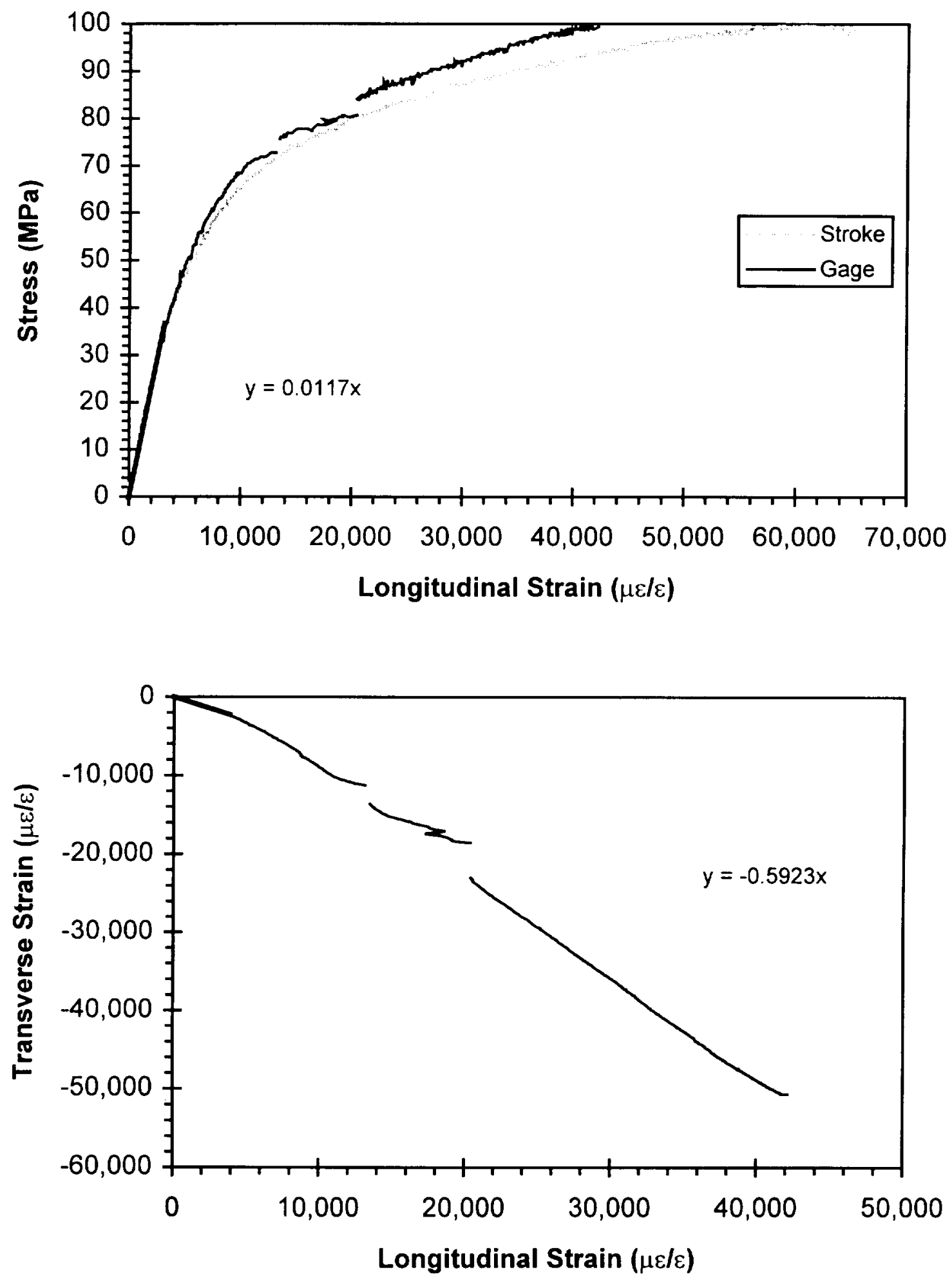

Figure C.12: Tensile test 4-4-3-b 

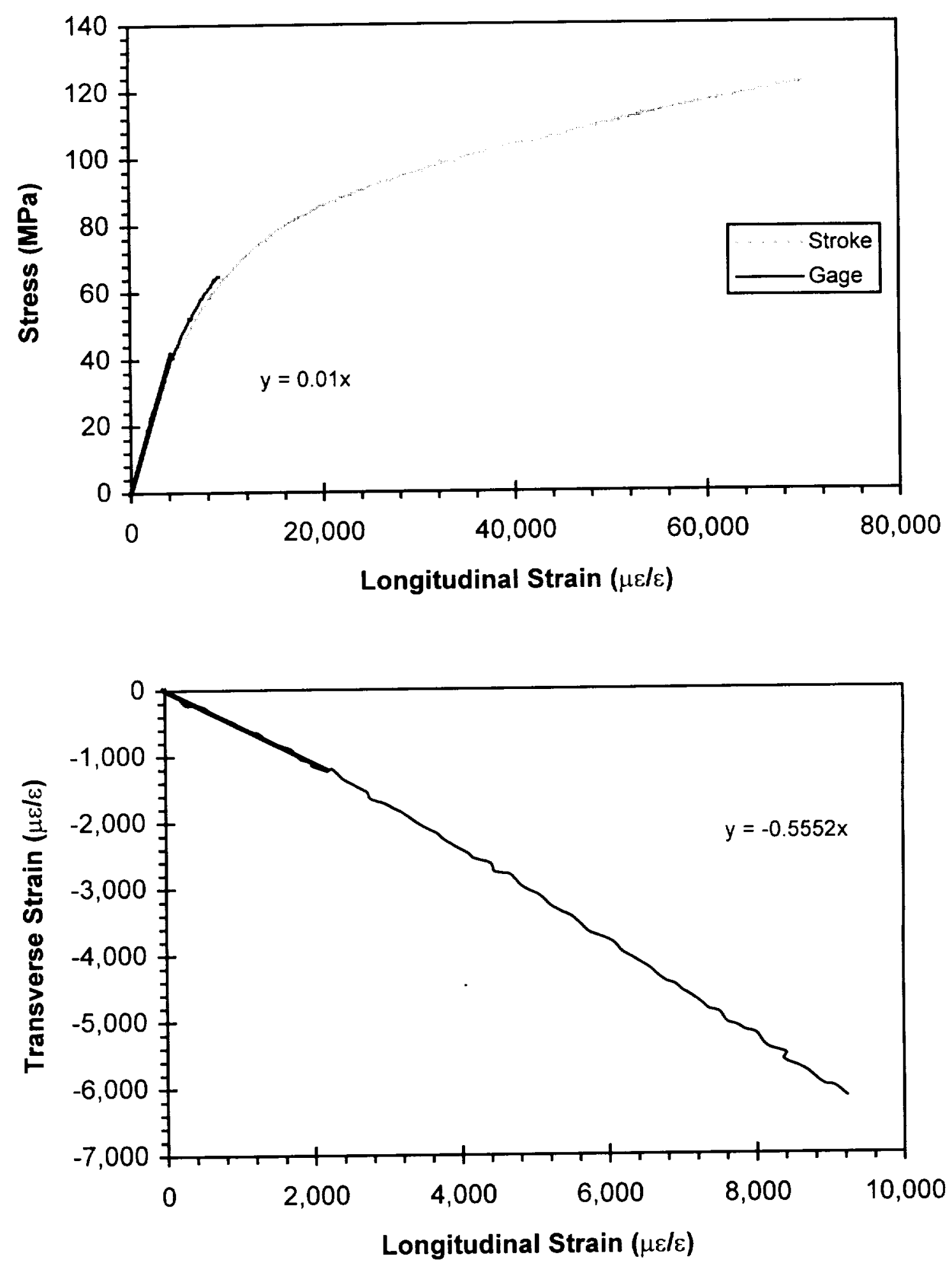

Figure C.13: Tensile Test 5-4-1-a 

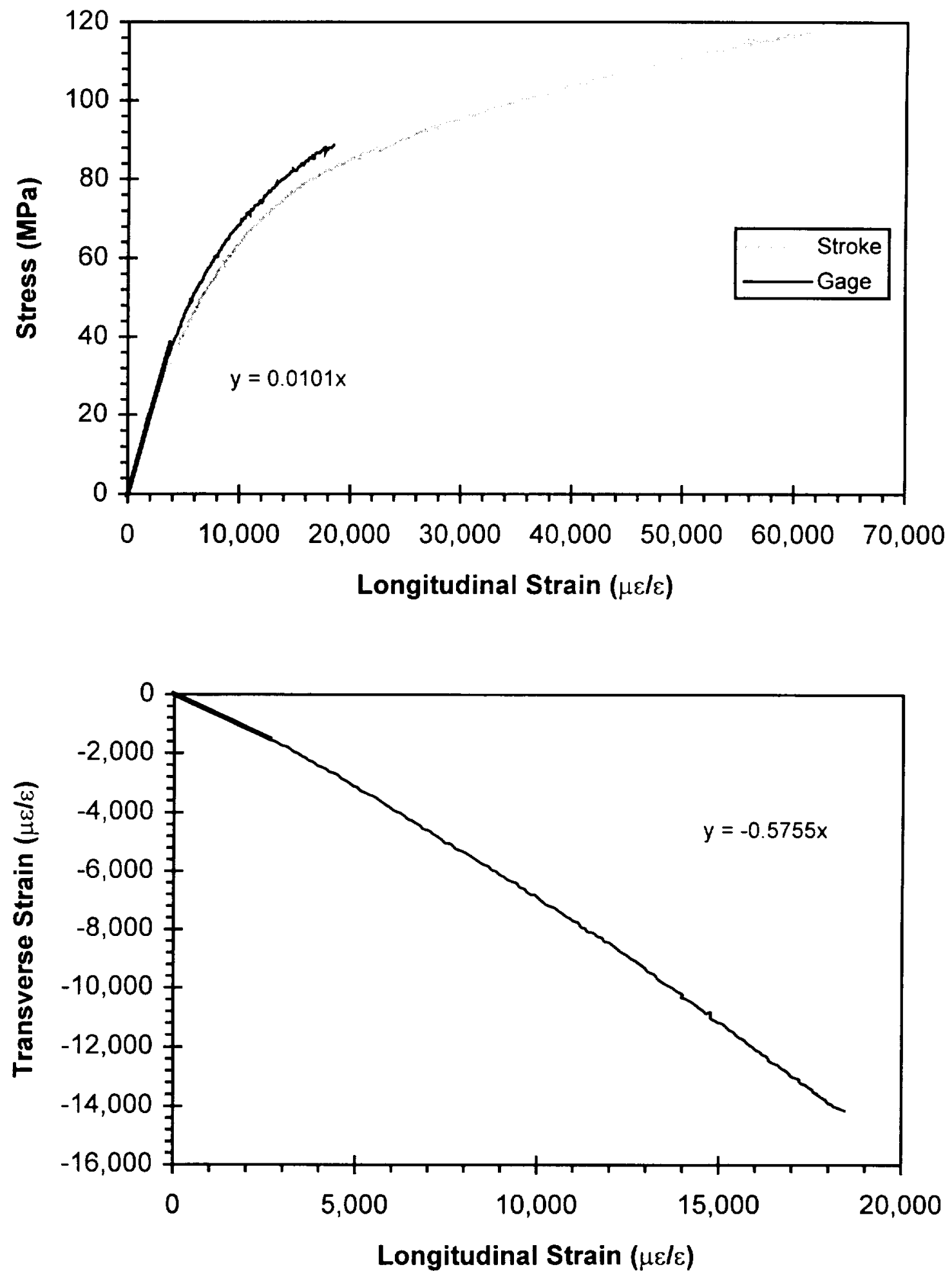

Figure C.14: Tensile test 5-4-1-b 

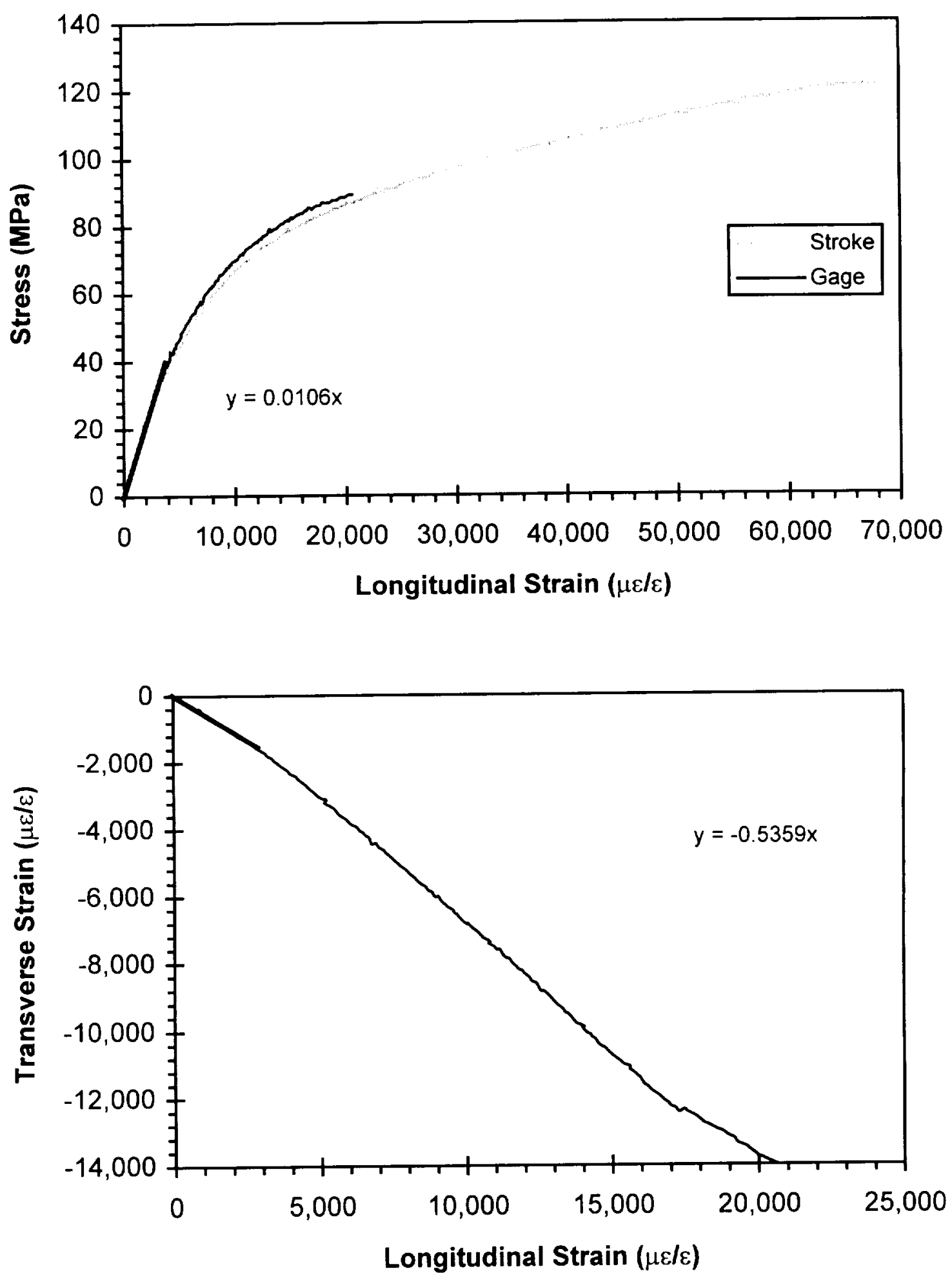

Figure C.15: Tensile test 5-4-2-a 

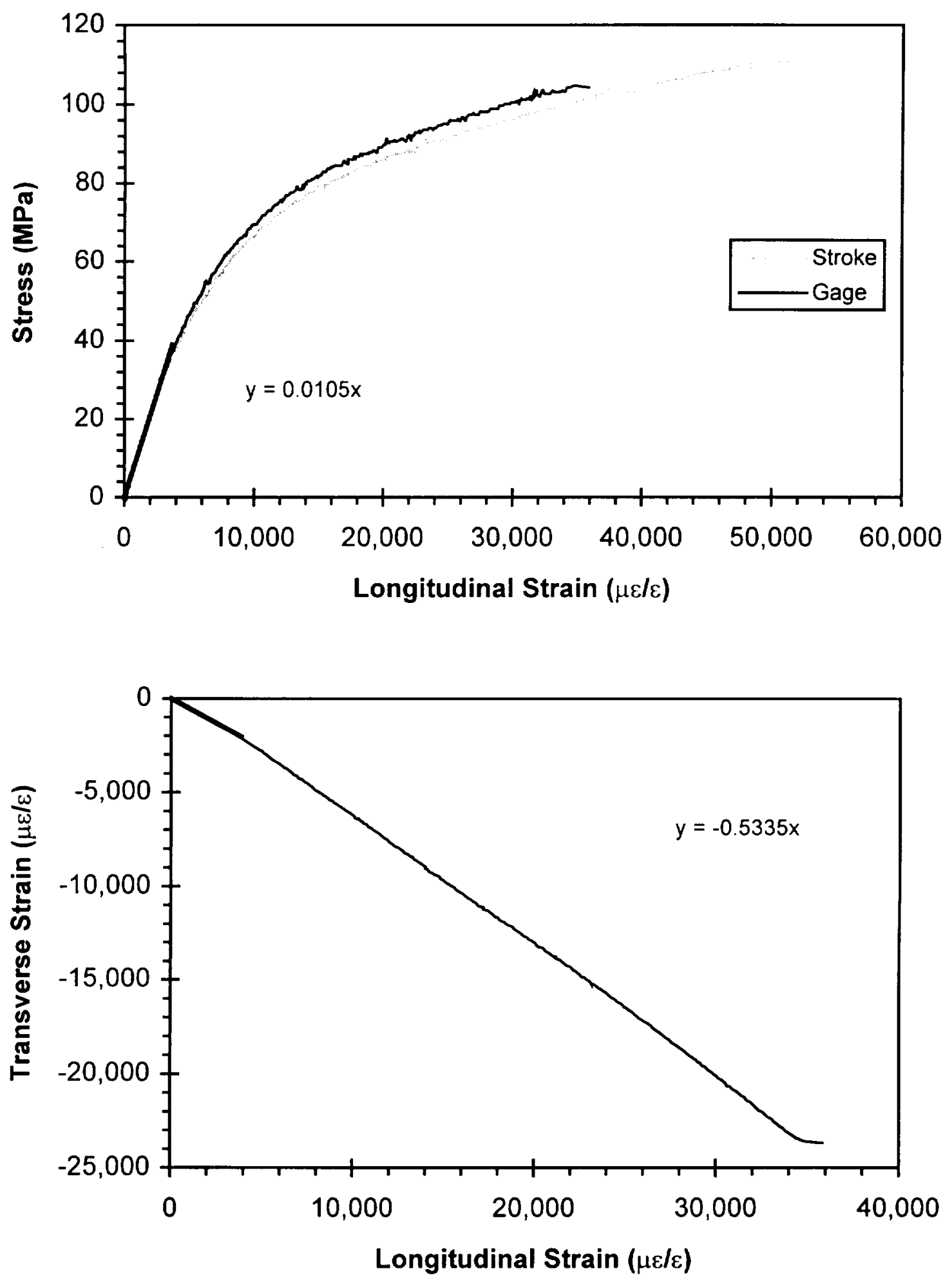

Figure C.16: Tensile test 5-4-2-b 

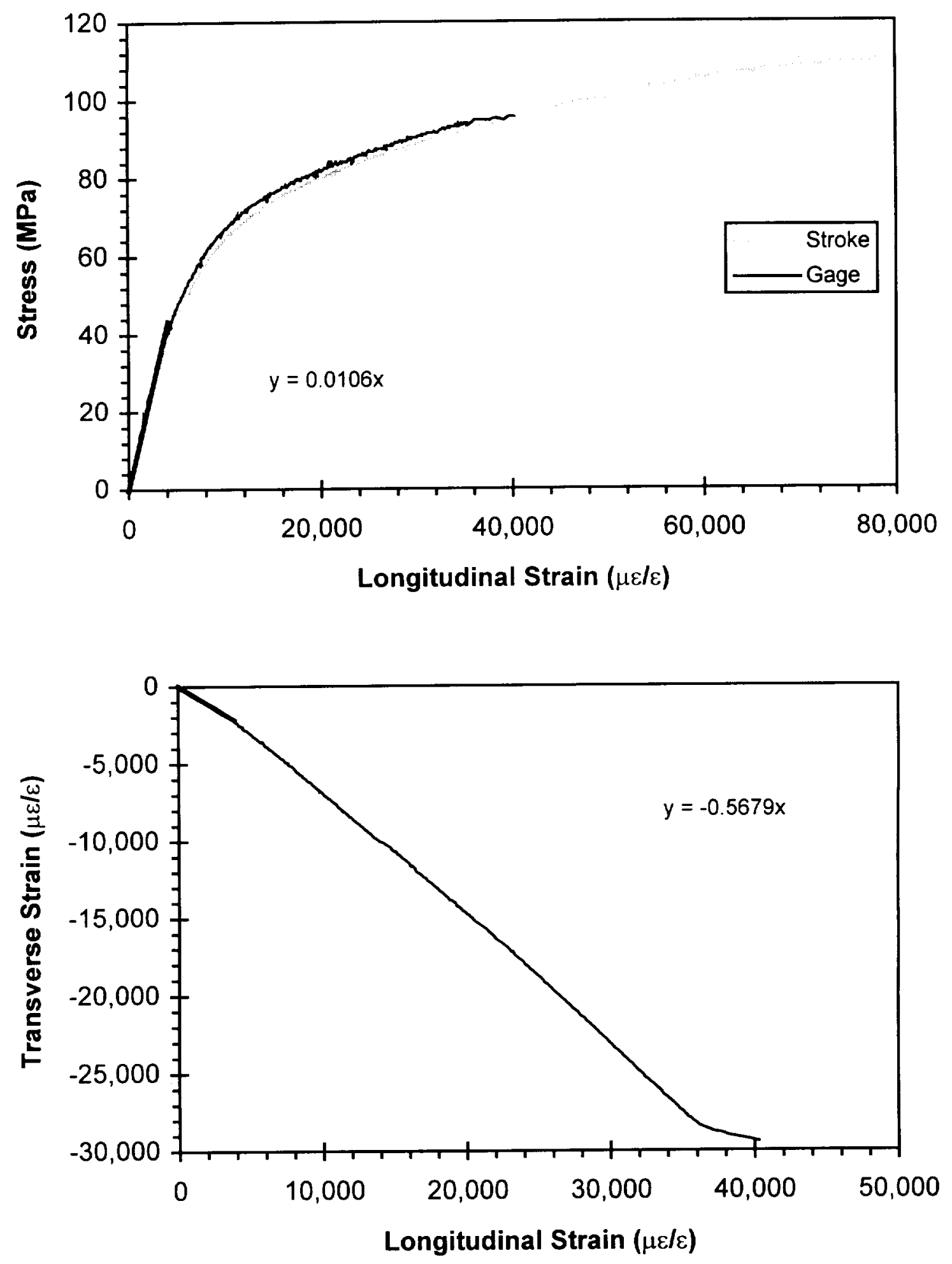

Figure C.17: Tensile test 5-4-3-a 

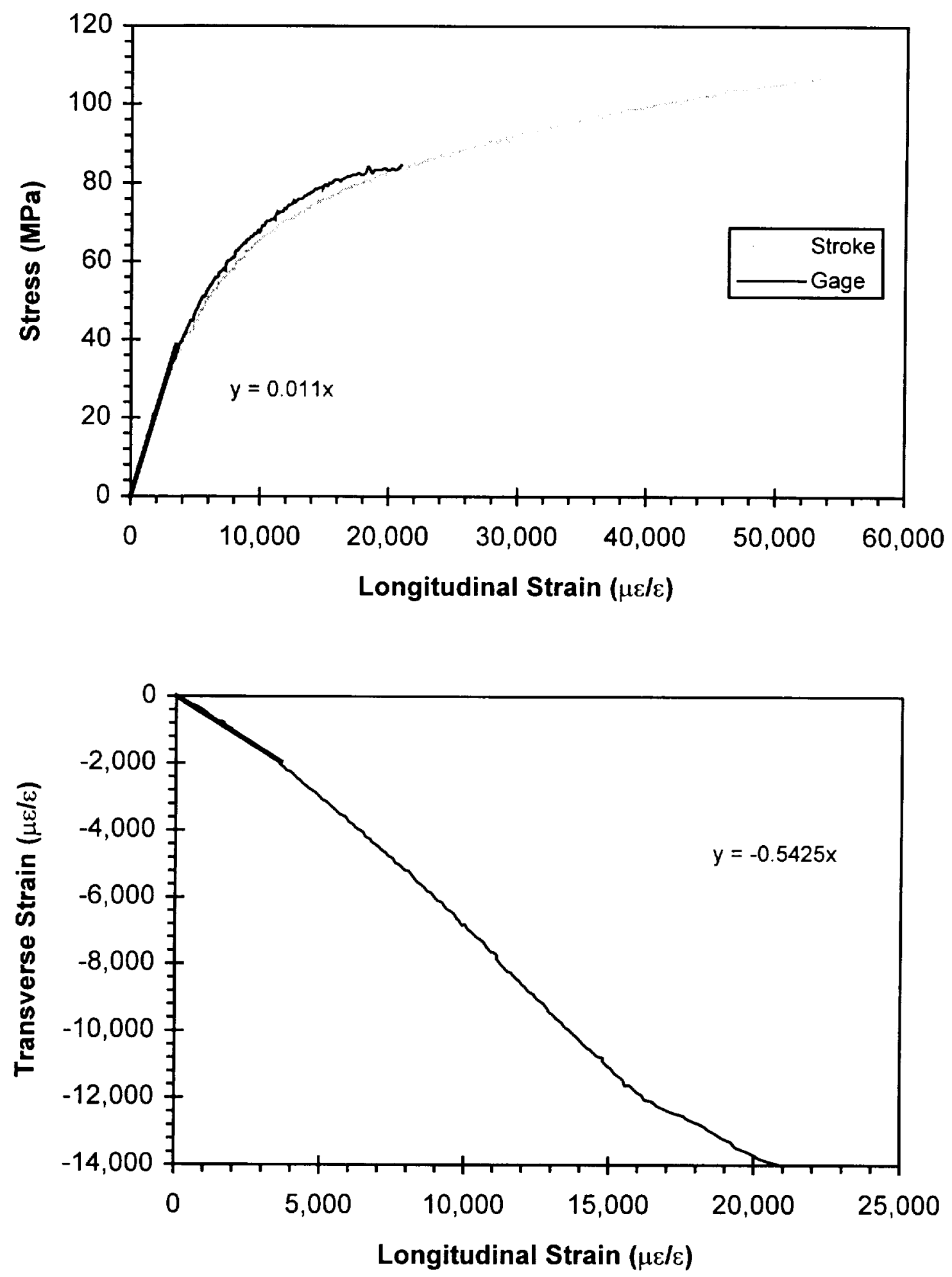

Figure C.18: Tensile test 5-4-3-b 

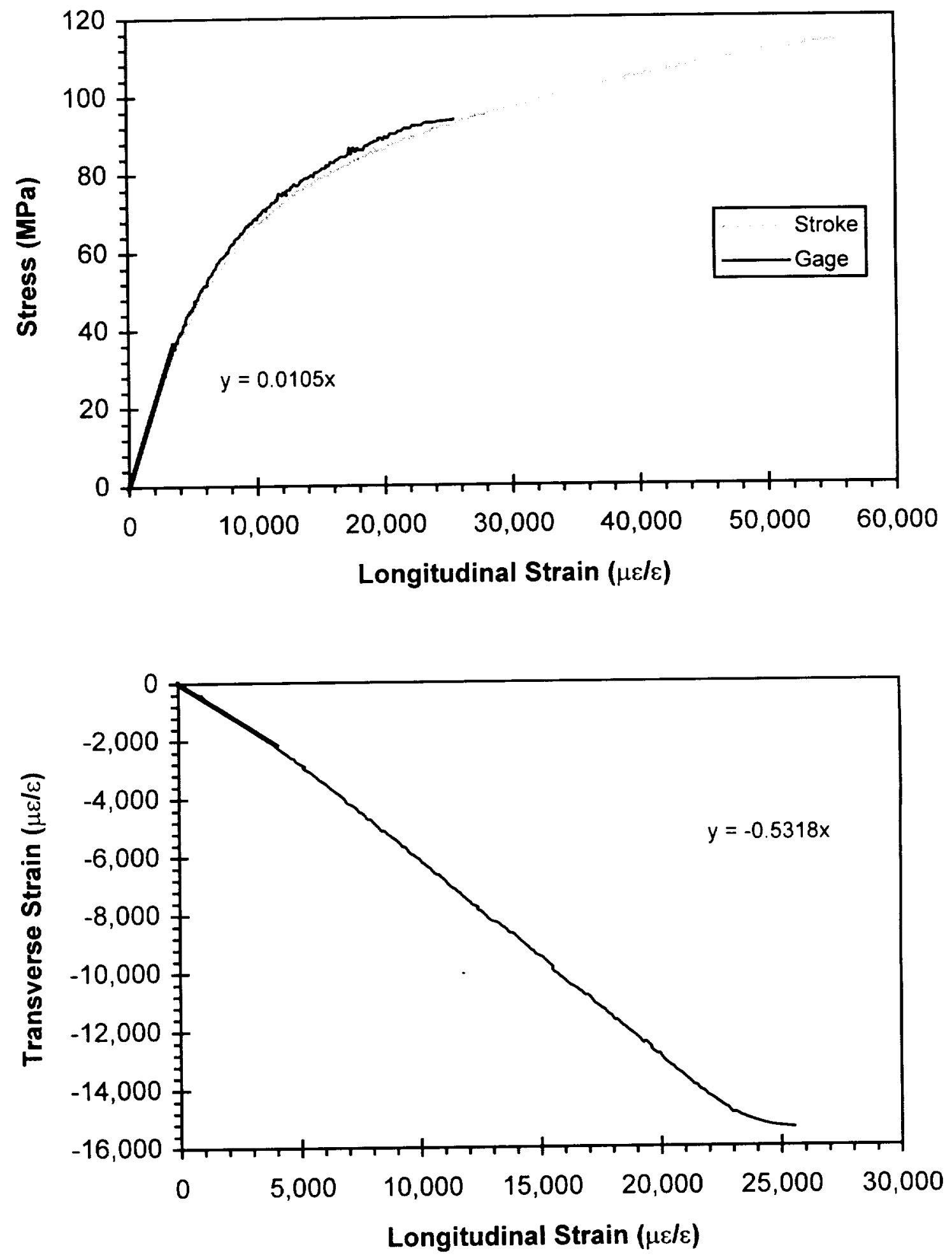

Figure C.19: Tensile test 6-4-1-a 

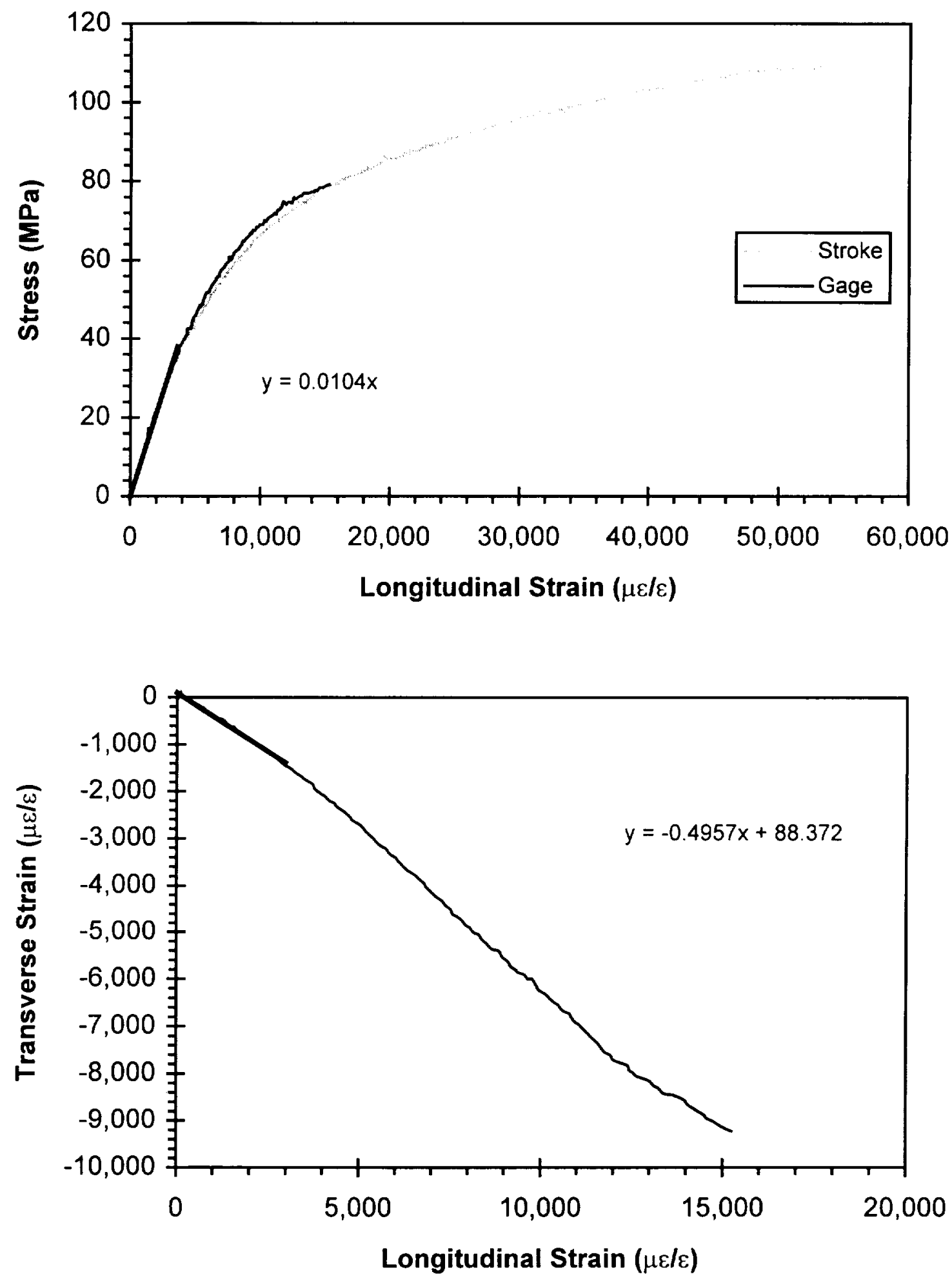

Figure C.20: Tensile test 6-4-1-b 

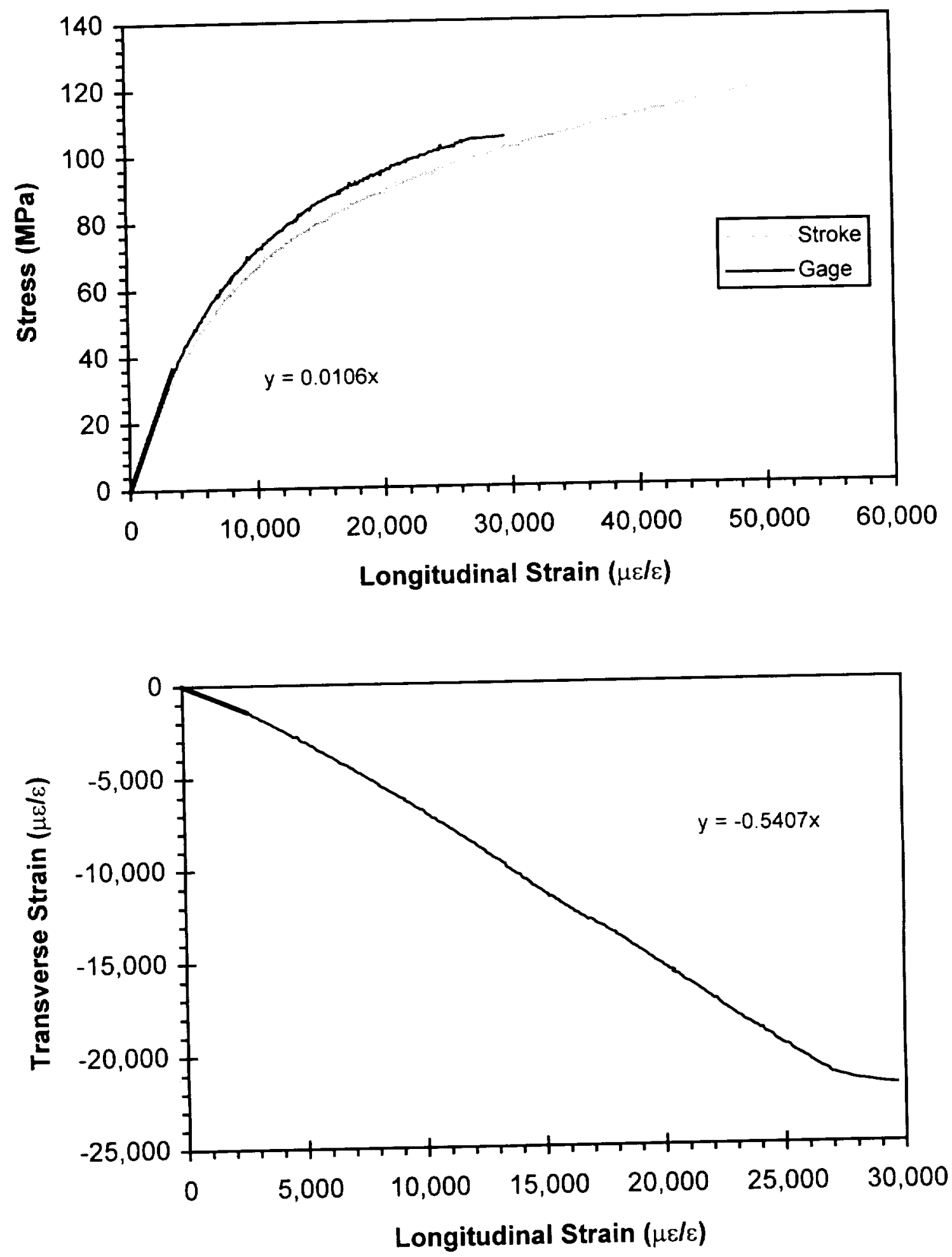

Figure C.21: Tensile test 6-4-2-a 

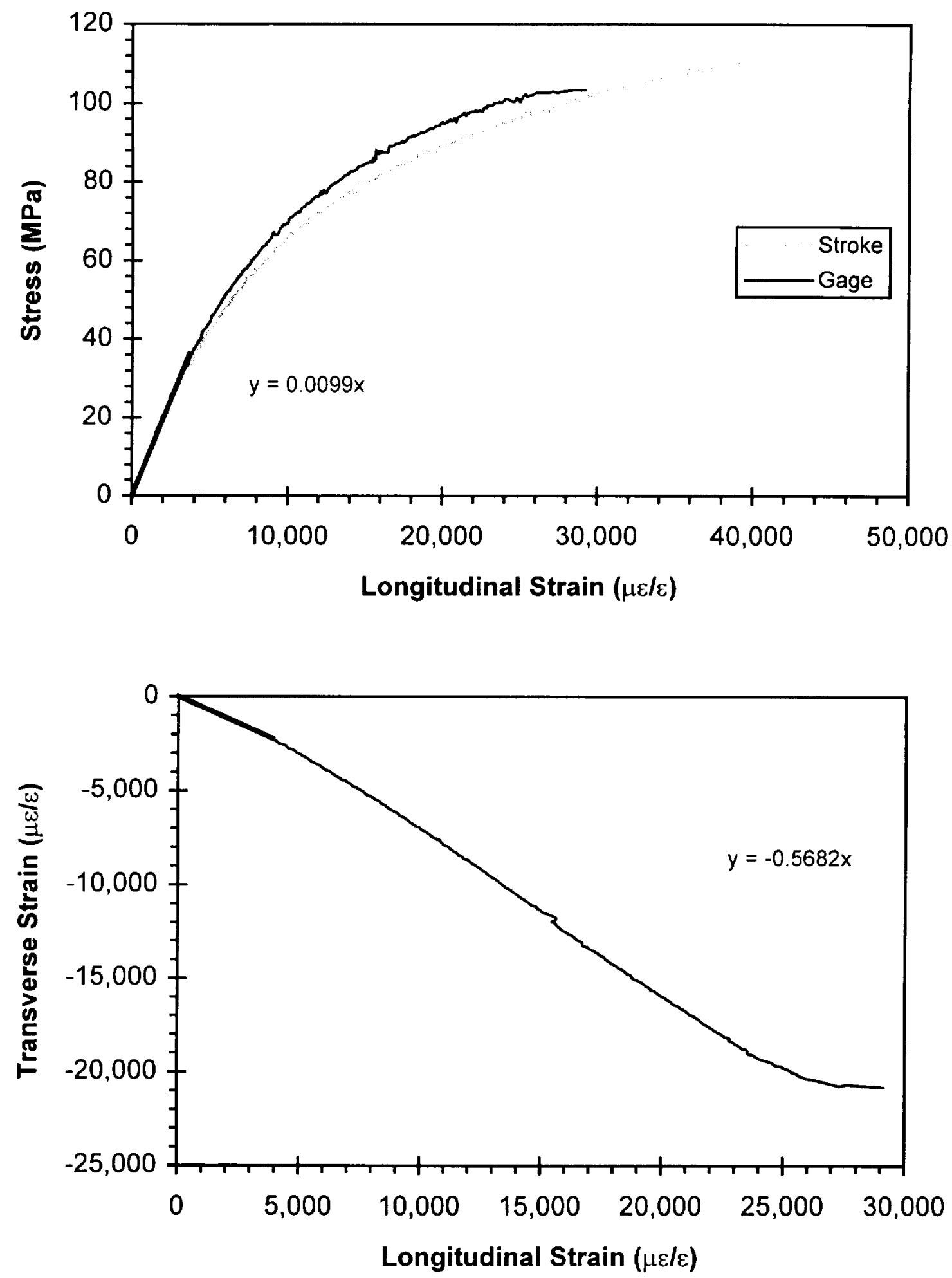

Figure C.22: Tensile test $6-4-2-b$ 

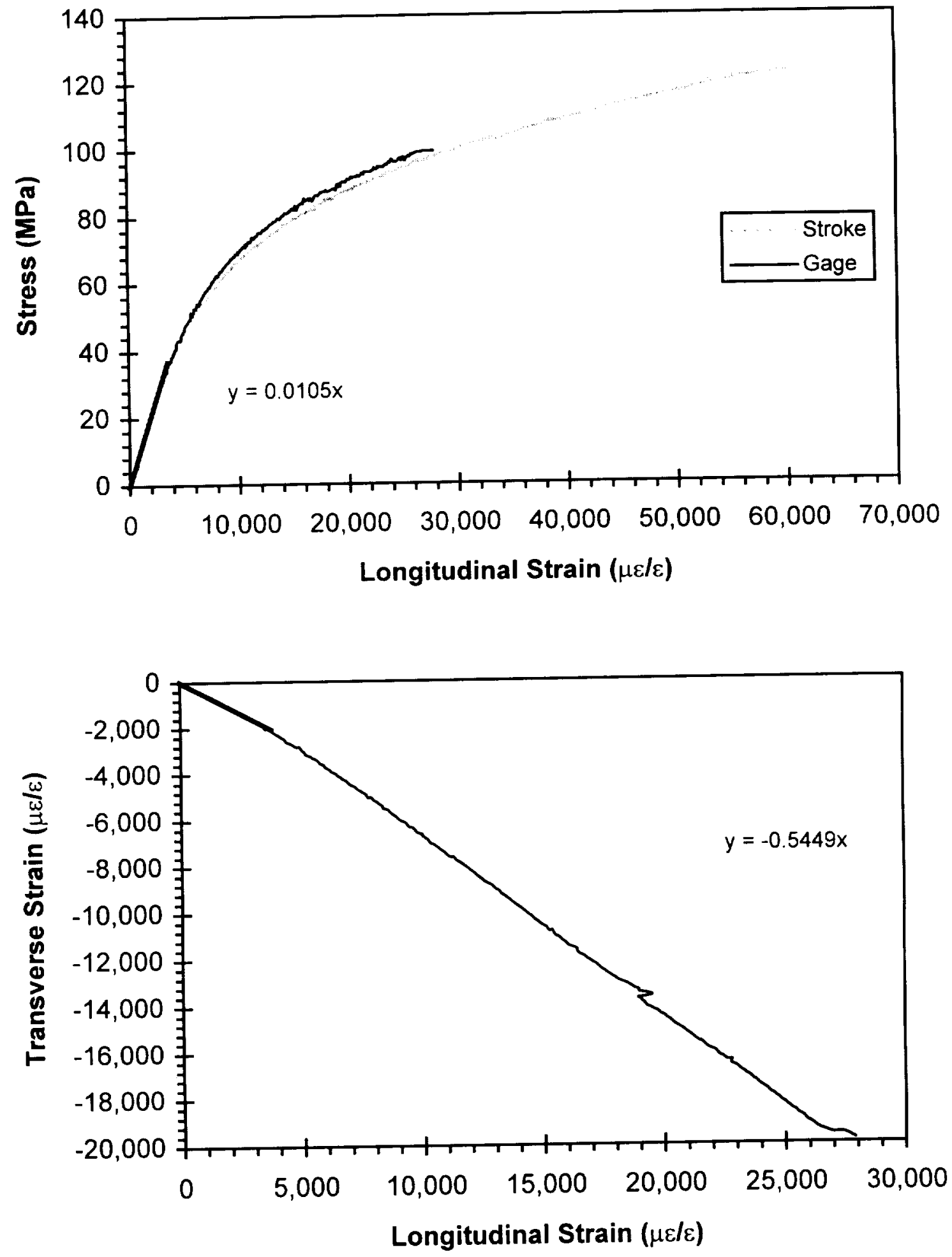

Figure C.23: Tensile test 6-4-3-a 

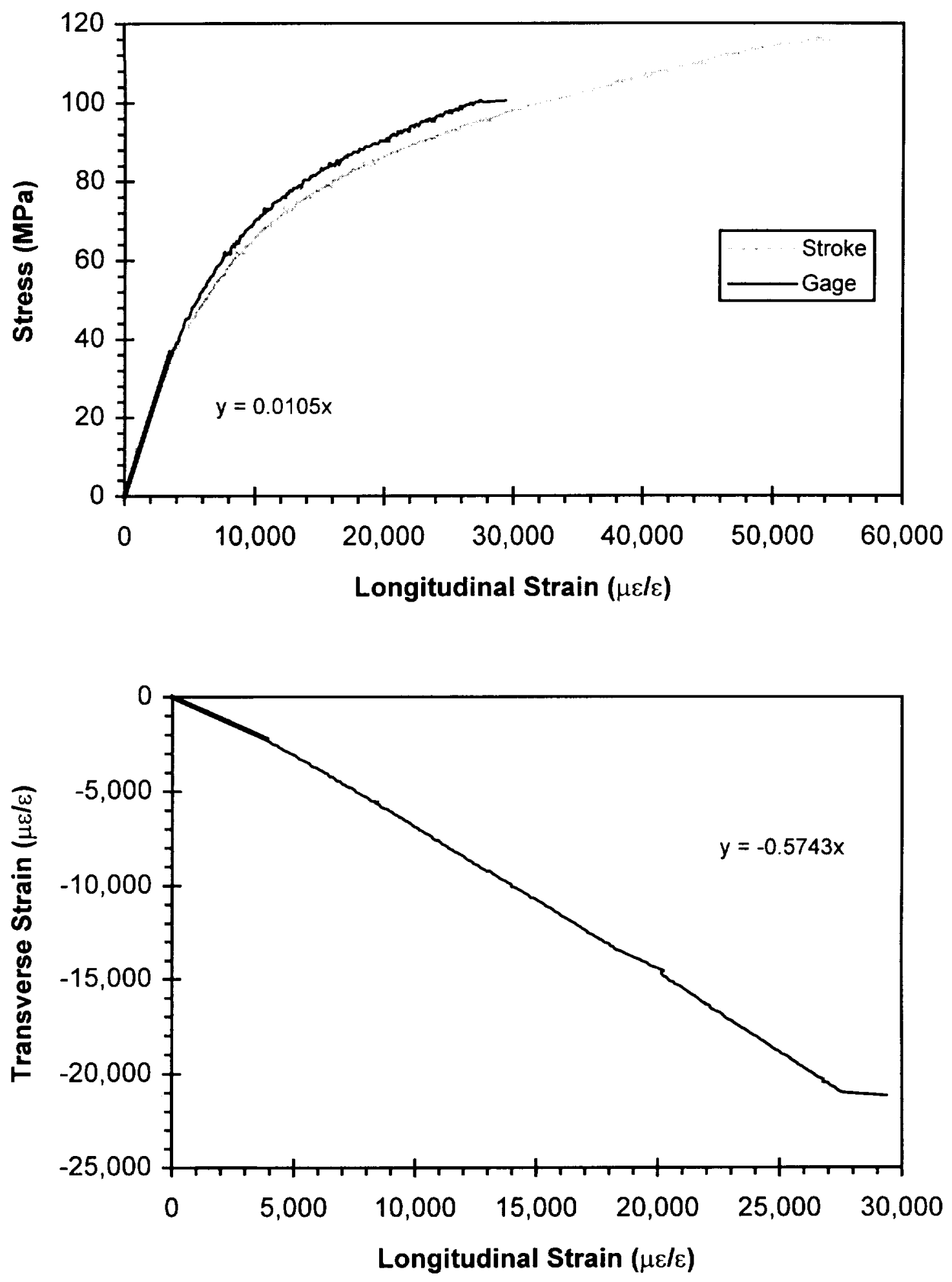

Figure C.24: Tensile test 6-4-3-b 

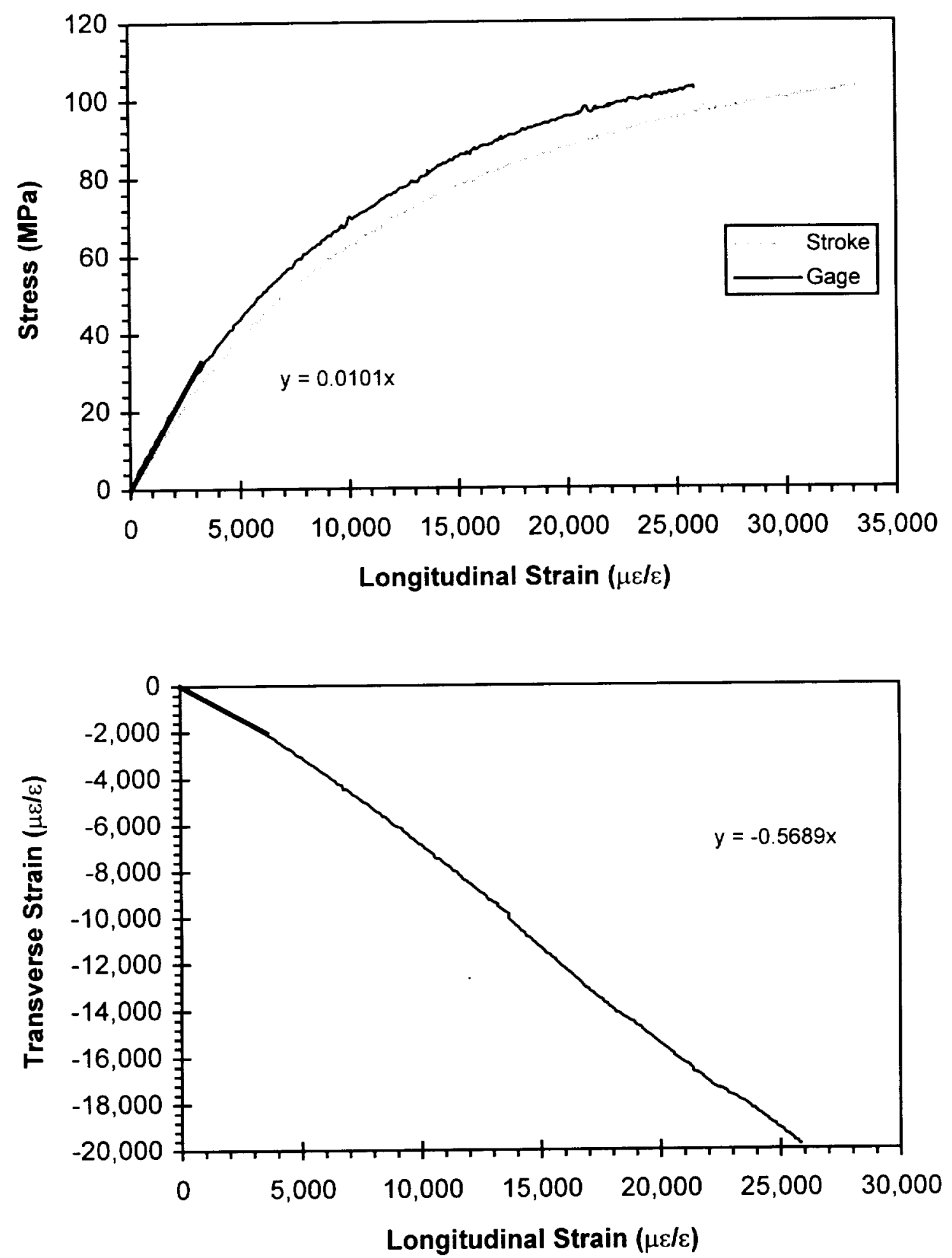

Figure C.25: Tensile test 7-4-1-a 

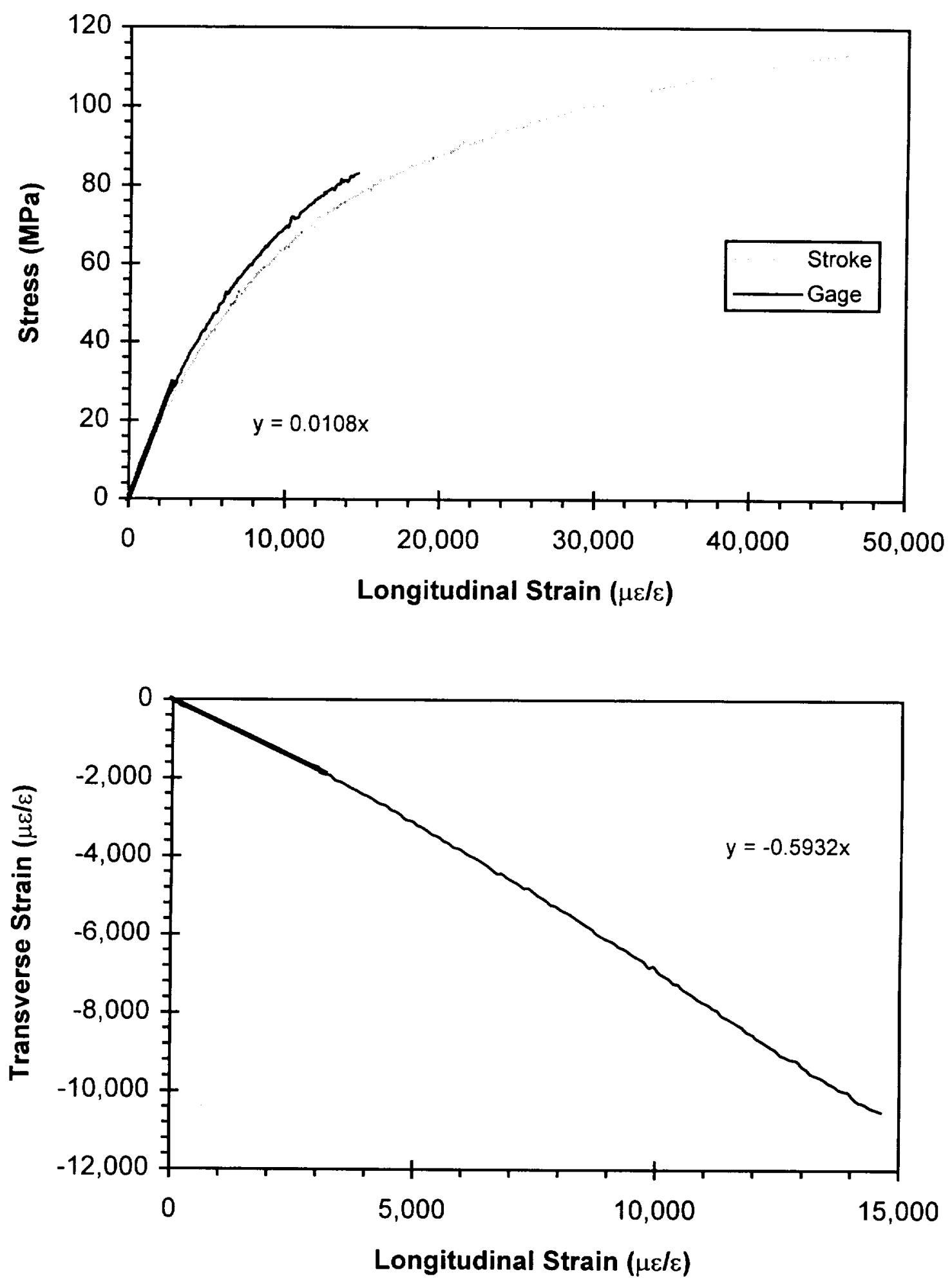

Figure C.26: Tensile test 7-4-1-b 

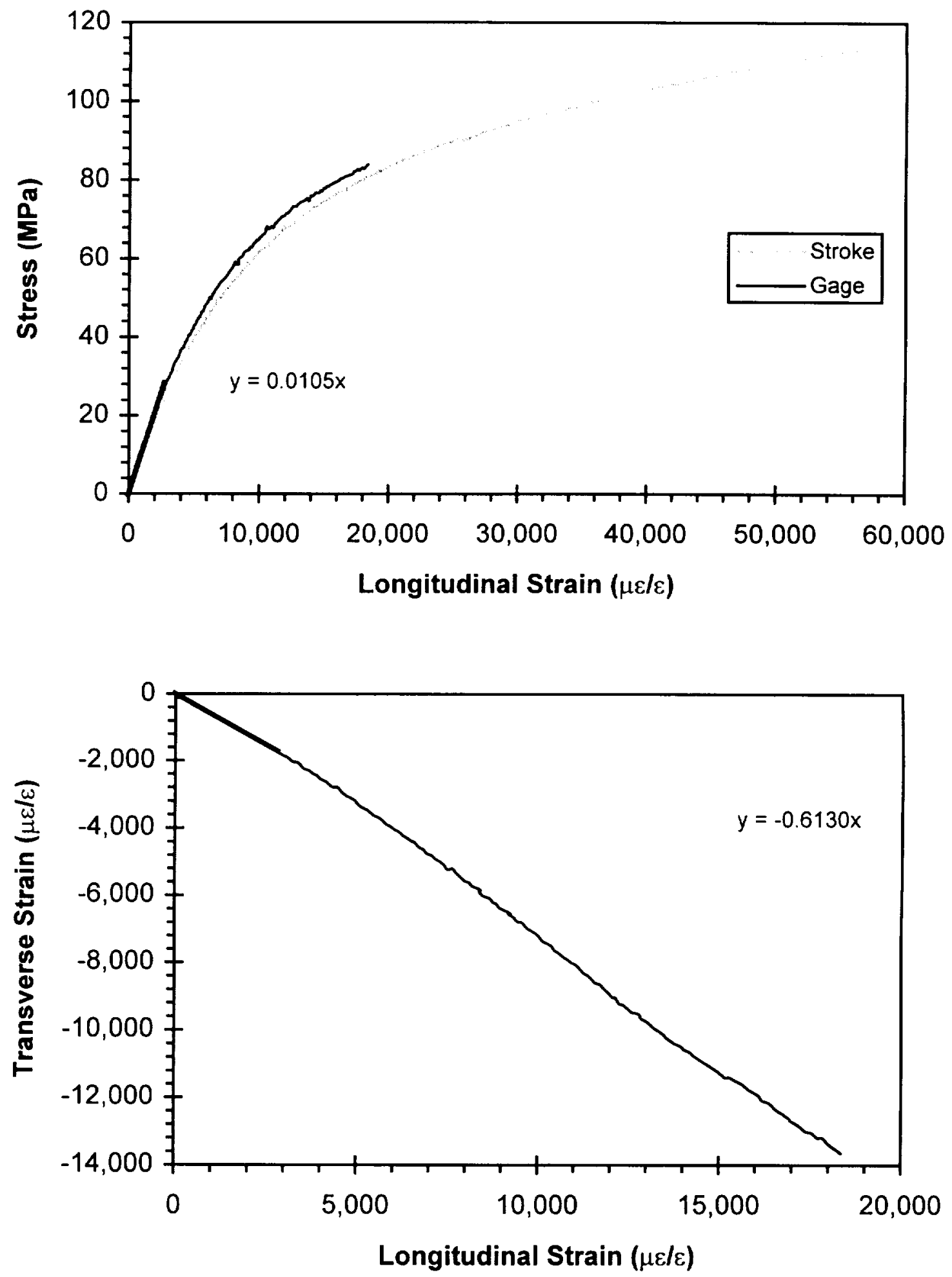

Figure C.27: Tensile test 7-4-2-a 

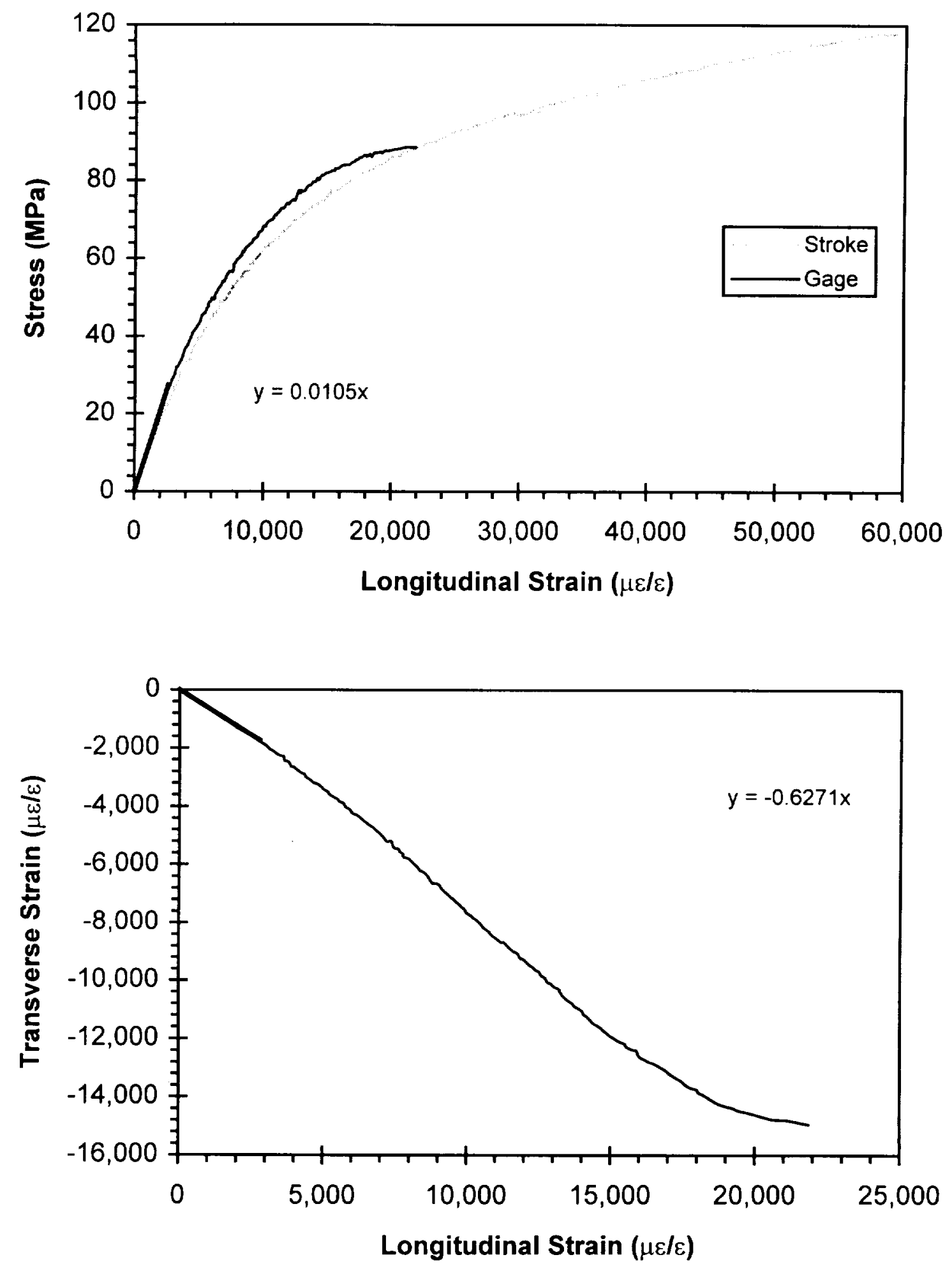

Figure C.28: Tensile test 7-4-2-b 

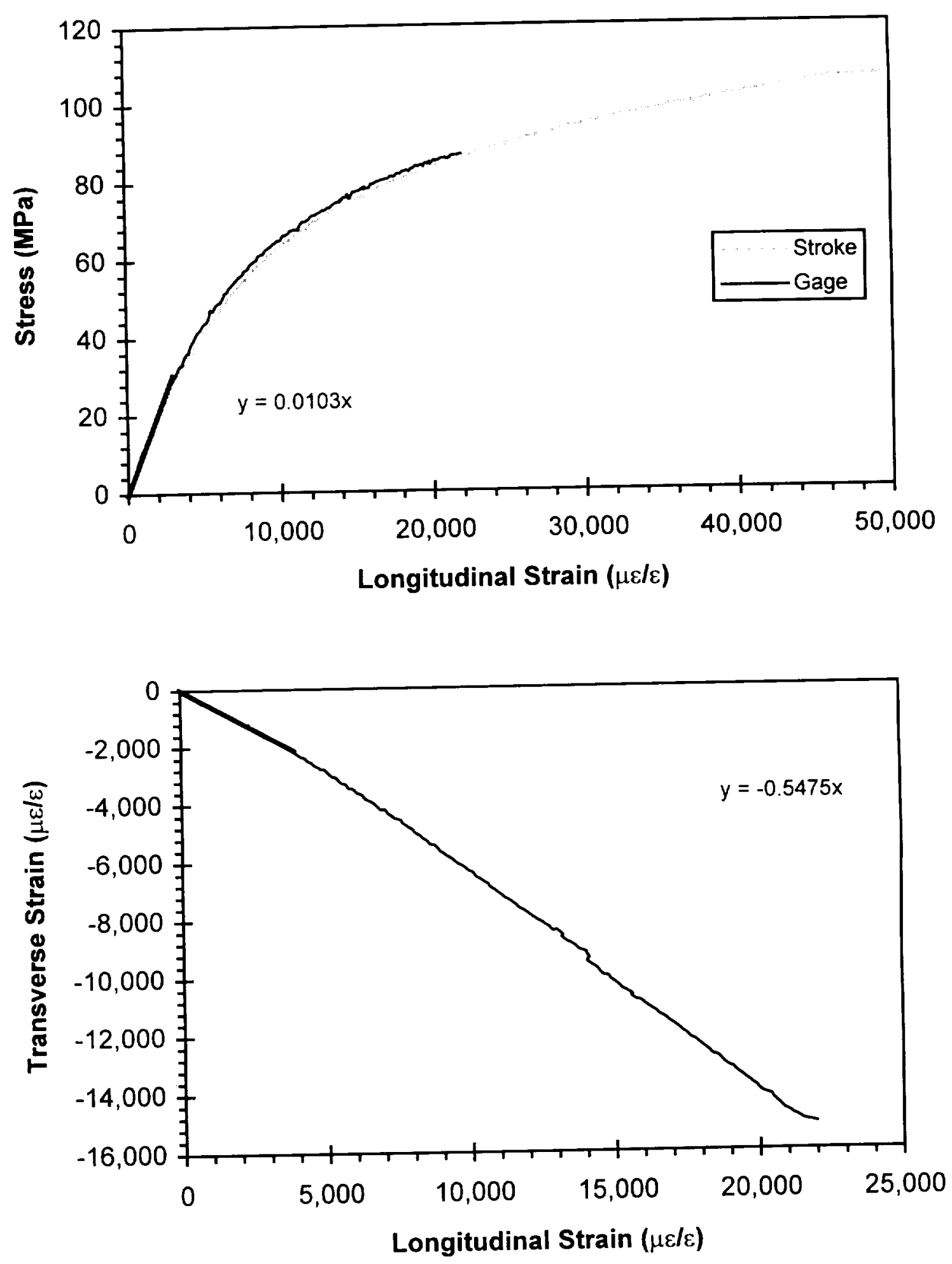

Figure C.29: Tensile test 7-4-3-a 

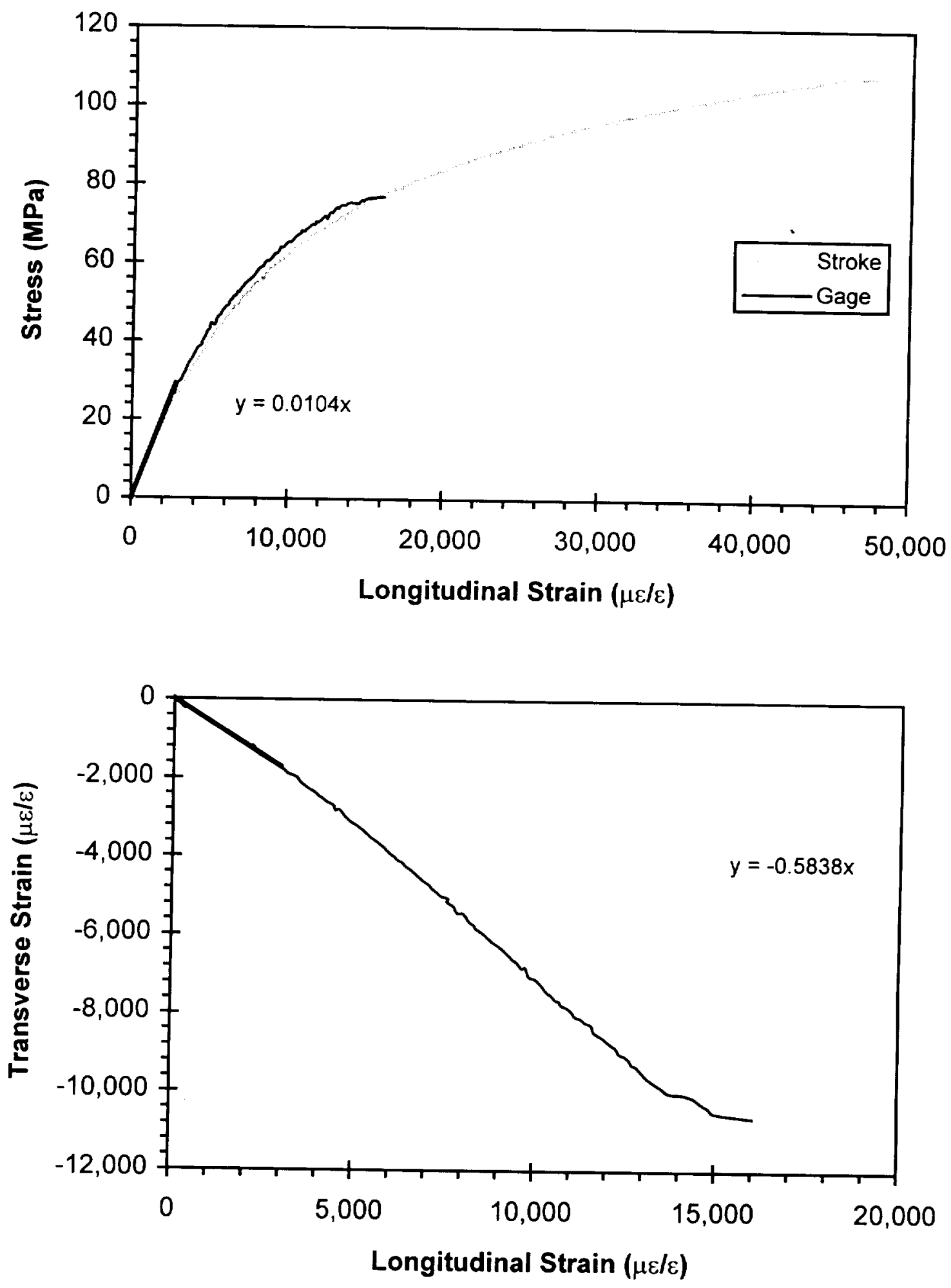

Figure C.30: Tensile test 7-4-3-b 

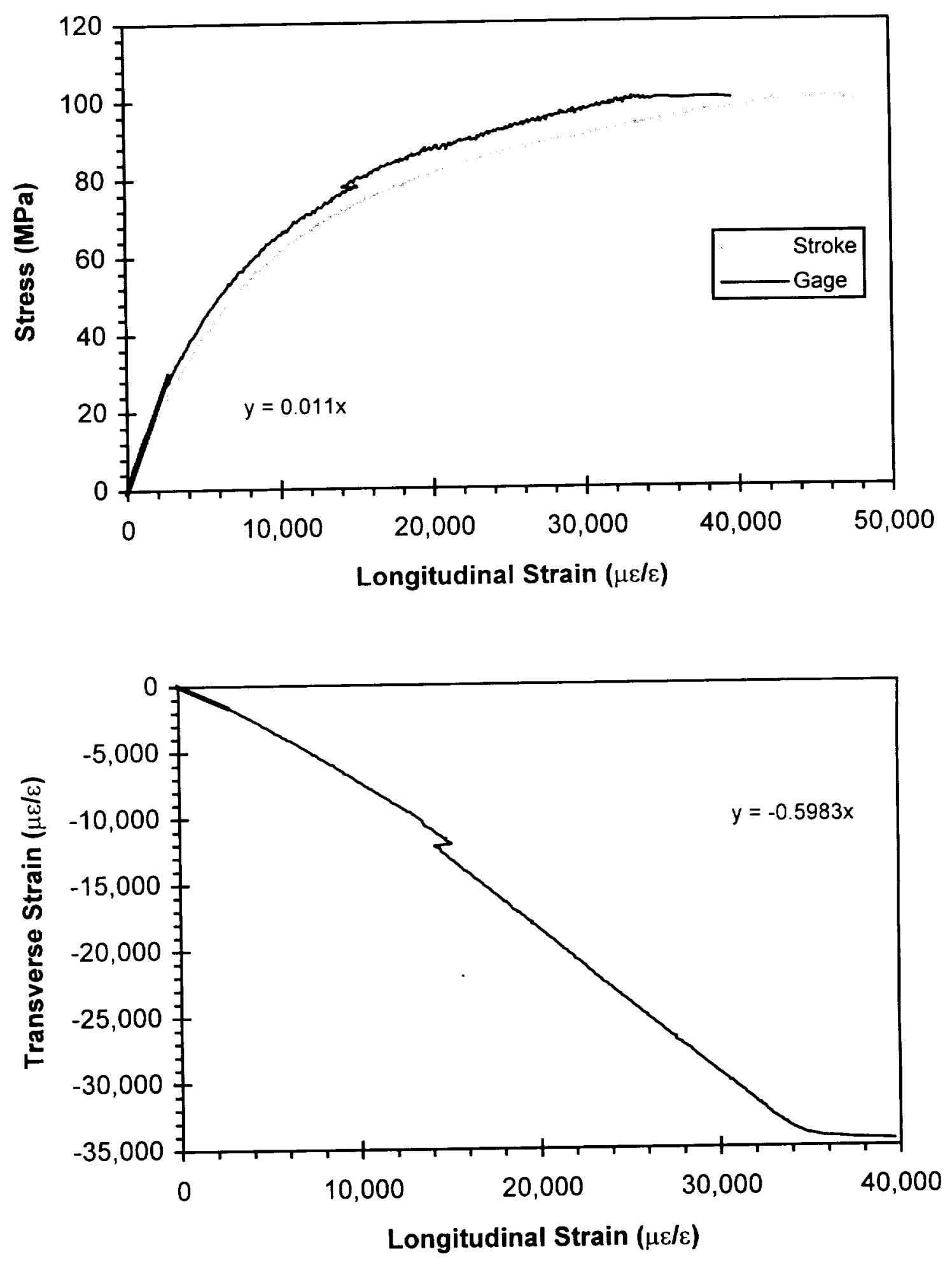

Figure C.31: Tensile test 8-4-1-a 

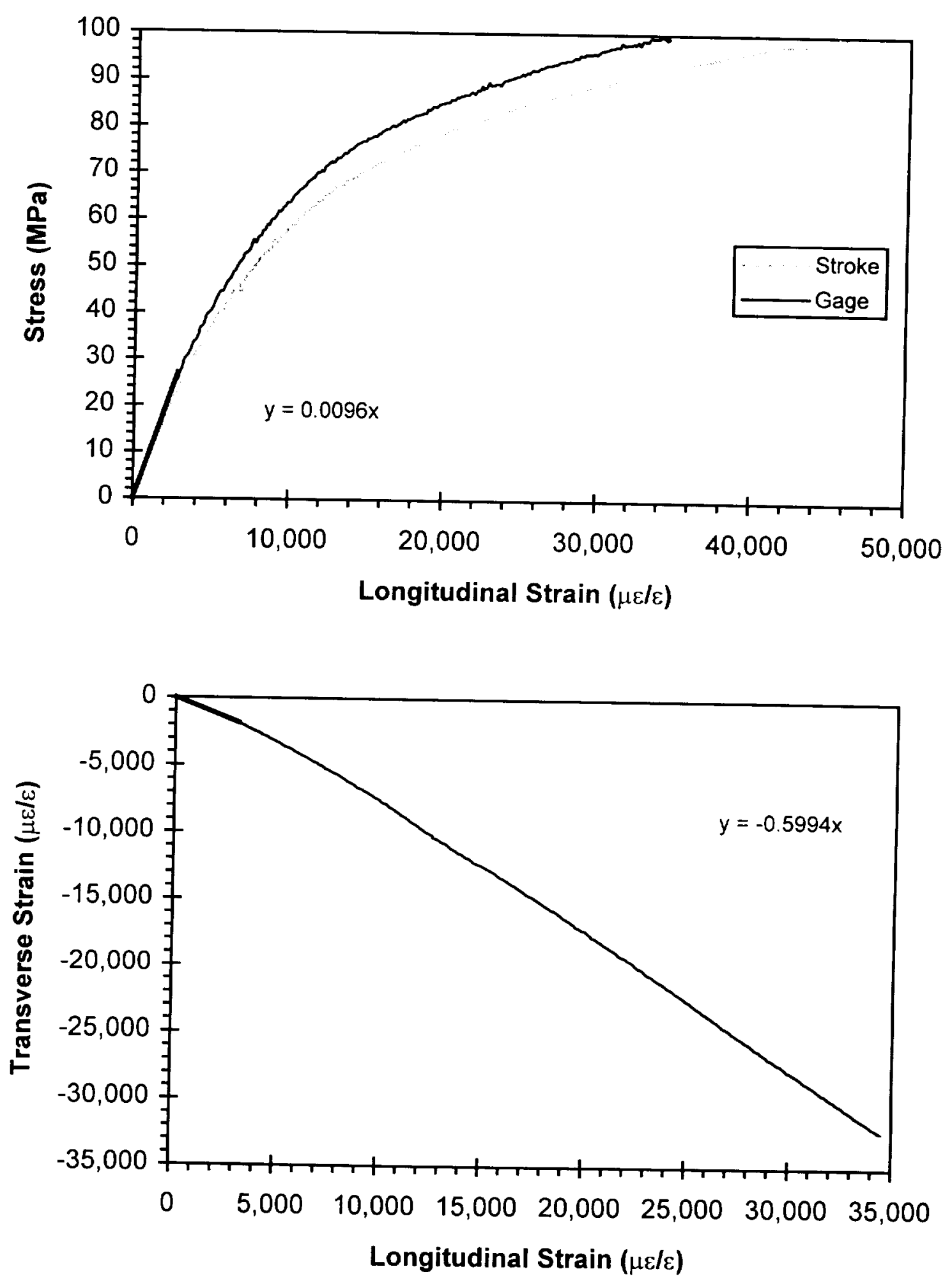

Figure C.32: Tensile test 8-4-1-b 

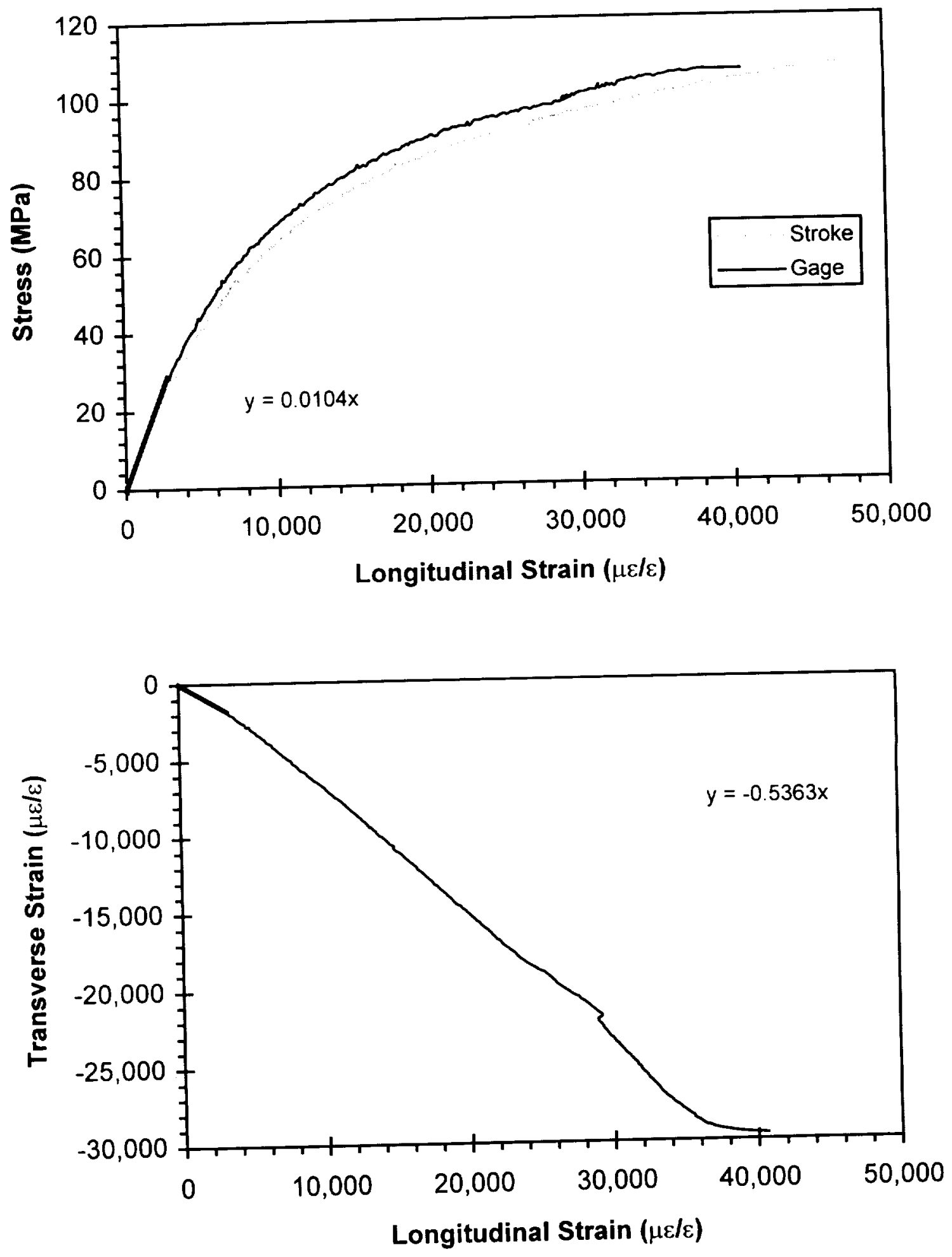

Figure C.33: Tensile test 8-4-2-a 

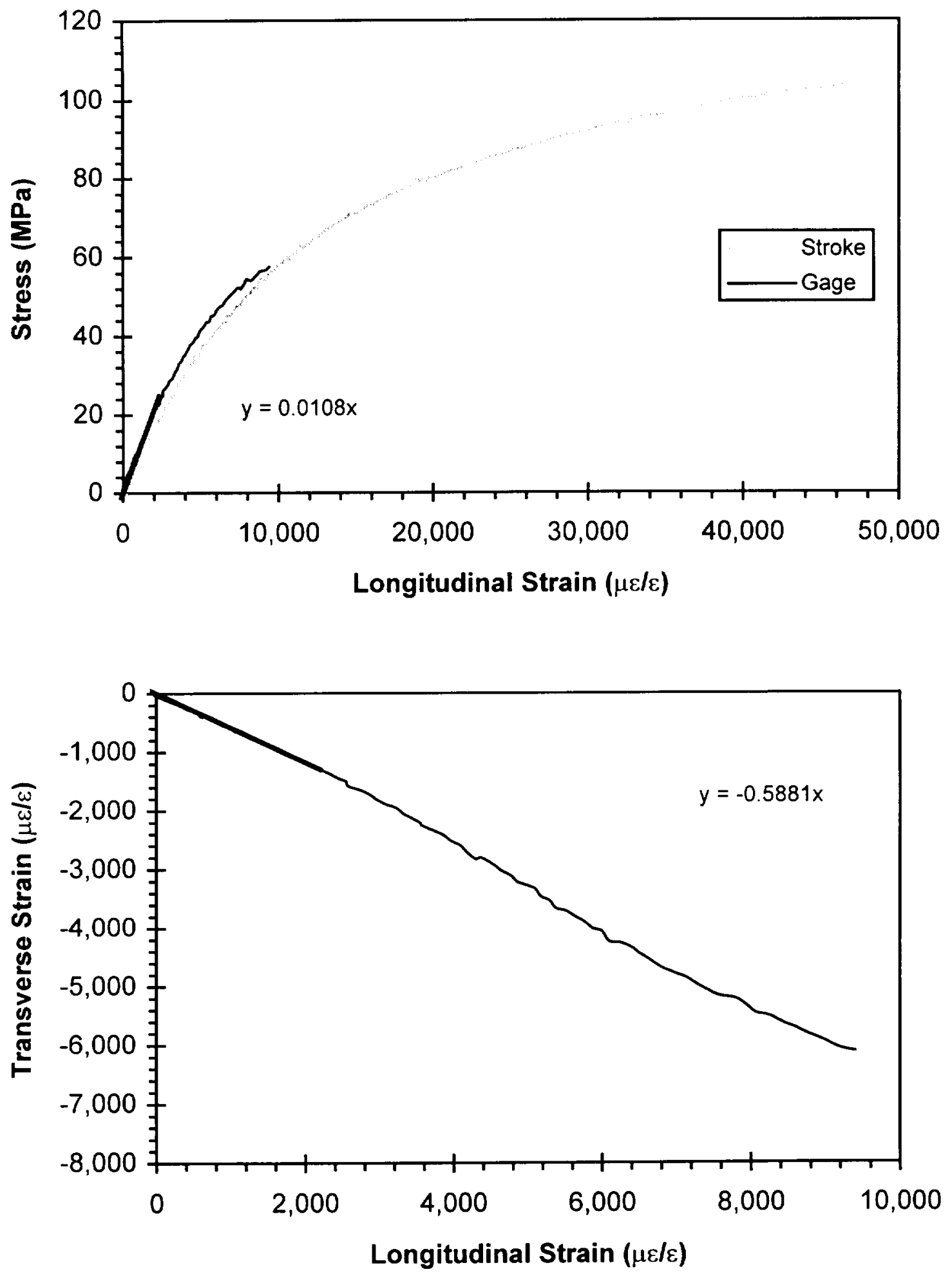

Figure C.34: Tensile test 8-4-2-c 

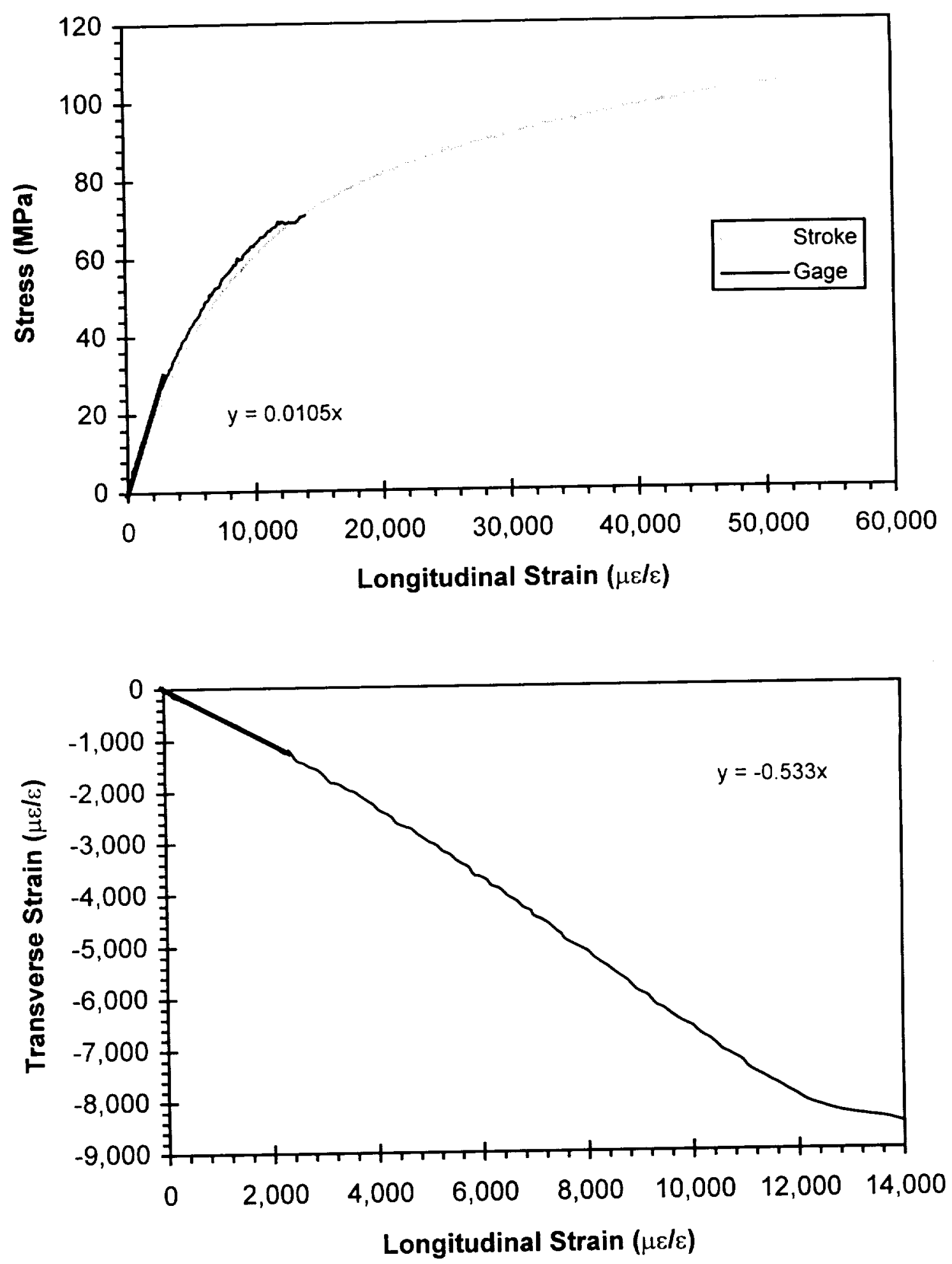

Figure C.35: Tensile test 8-4-3-a 

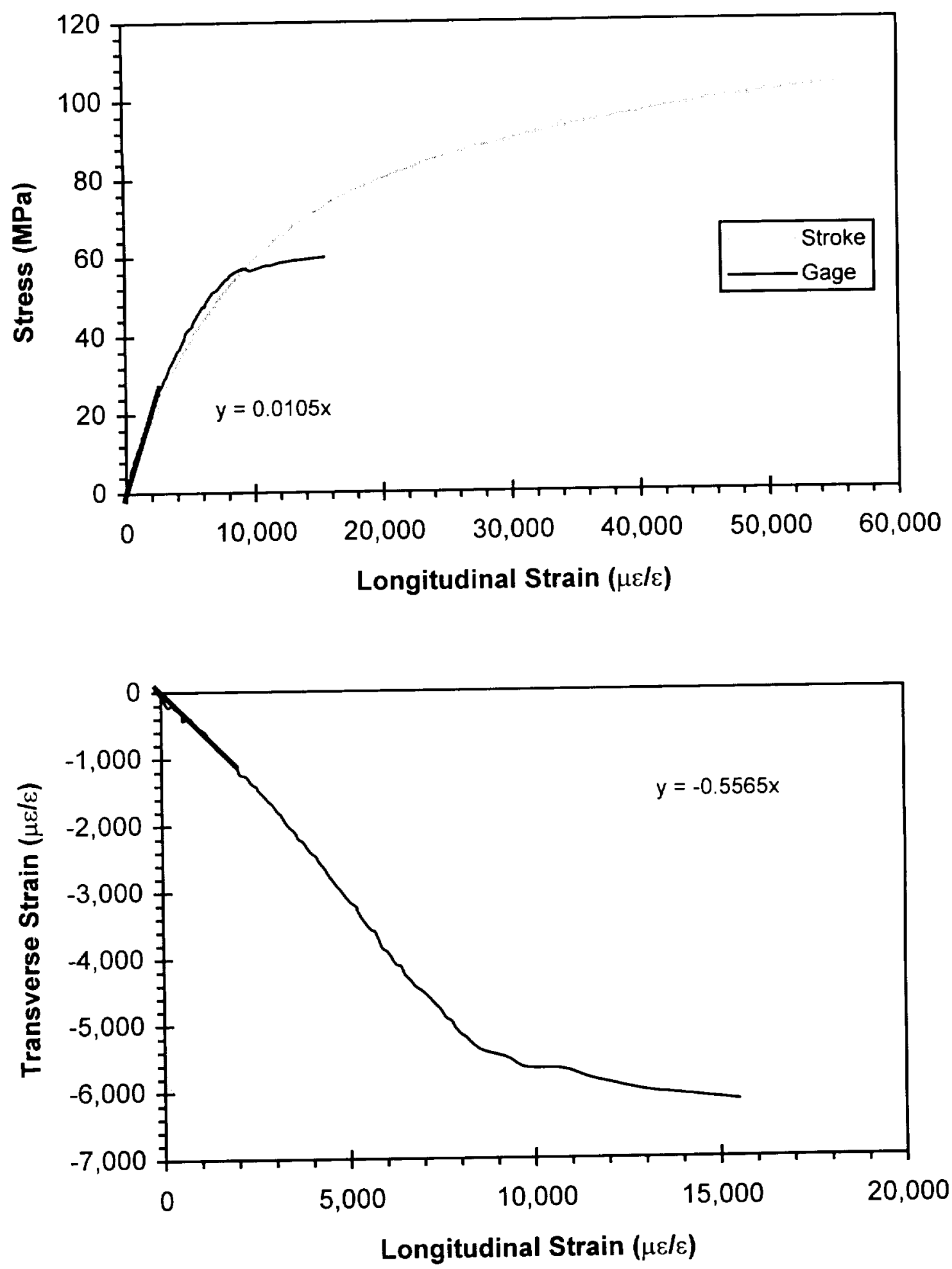

Figure C.36: Tensile test 8-4-3-b 
